\title{
Development of Fiber Optic Aerodynamic Sensors for High Reynolds Number Supersonic Flows
}

\author{
by \\ Wade J. Pulliam \\ Dissertation submitted to the Faculty of the \\ Virginia Polytechnic Institute and State University \\ Doctor of Philosophy \\ In \\ Aerospace Engineering \\ APPROVED: \\ Dr. Joseph A. Schetz, Chairman \\ Dr. Bernard Grossman \\ Dr. Kent Murphy \\ Dr. Thomas Diller \\ Dr. James Marchman
}

In partial fulfillment of the requirements for the degree of

February 2, 2000

Blacksburg, VA

Keywords: fiber optics, skin friction, pressure, temperature, heat flux 


\title{
Development of Fiber Optic Aerodynamic Sensors for High Reynolds Number
}

\section{Supersonic Flows}

\author{
Wade J. Pulliam \\ Dr. Joseph Schetz, Chairman \\ Aerospace Engineering
}

\begin{abstract}
(ABSTRACT)
The purpose of the project was to examine fiber optic sensors for the measurement of pressure, skin friction, temperature, and heat flux in high Reynolds number, supersonic flow. Using a standard fiber optic signal conditioning unit (specifically a broadband interferometric system using spectra), the work centered around determining under what conditions these sensors will work effectively and quantifying the total system limitations.

An interferometric-based, fiber optic skin friction sensor was developed for the measurement of wall shear stress in complex, supersonic flows. This sensor type was tested successfully in laminar, incompressible flow, and supersonic flow up to Mach 1.92, Mach 2.4 and 3.0 flow, in which the sensor operated with varying success. A micromachined, fiber optic pressure sensor was also tested in these supersonic conditions, also with varying success. The accurate operation of these sensors was found to be tied to the flow conditions and the fiber optic, signal processing system.

A correlation was found to exist between the energy of the flow, either through its dynamic pressure or through external disturbances such as shocks or separation, and the noise in the signals, expressed by the variance of the gap estimate, for the pressure and skin friction sensors in these flows. The energy of the flow couples with the mechanical properties of the sensor reducing the fringe contrast of the signal used by the optical signal processing system to determine a gap estimate. As the energy of the flow is increased and the sensor is excited, the fringe contrast is reduced. A practical limit of a normalized fringe contrast of 0.10 was found for producing accurate gap estimates in real flows. A consequence is that there is a limit to the dynamic pressure of the flow for the sensors to operate accurately, which is demonstrated by the varying success of the supersonic wind tunnel tests. This correlation is sensor specific, meaning that sensors can be designed to operate successfully in any flow. Also, the signal processing system, which forms the other end of the total system, could be improved to allow accurate measurements with the current sensors.
\end{abstract}




\section{Acknowledgements}

I'd first like to acknowledge the help and mentoring of my advisor, Dr. Joseph A. Schetz. His guidance and support were essential through some of the dark periods of this research and educational effort, and his dedication to my professional skills went well beyond what was required and is greatly appreciated. My mother and the Jesuits also deserve credit between them for teaching me responsibility, dedication, and a desire for perfection that was vital in the present effort.

I'd also like to recognize the friendship and help of Michael Novean and Raymond Fuller. We ran the gauntlet of the first few years of graduate school together and remained loyal in a time when the competition was cutthroat. The skill of the machinists in the shop, Bruce Stanger, Kent Morris, and Greg Duding and the electronics technician, Gary Stafford, have made this research possible. The help of Kevin Shinpaugh for explaining fiber optics and related demodulation systems should also not go unnoticed. Also, the support received from my colleagues at Luna Innovations, especially Michelle Wilson, Dr. Kent Murphy, Pat Russler, Mark Jones, Steve Poland, Tom Wavering, Jason Borinski, Carrie Kozikowski, and Mike Miller, have allowed me the freedom necessary during the final push to get this done.

This research was supported by the U. S. Air Force out of the Air Force Research Laboratories with Dr. Diana Glawe and Lt. Lars Johnsen serving as contract monitors. 


\section{Table of Contents}

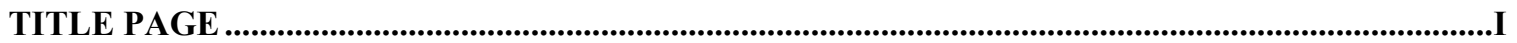

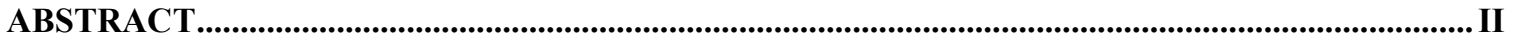

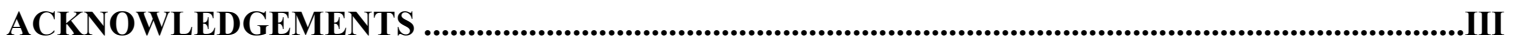

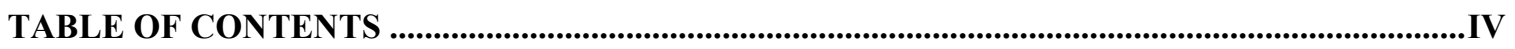

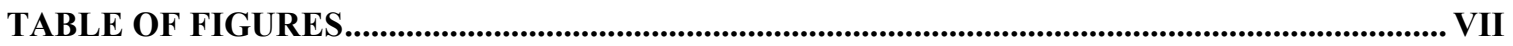

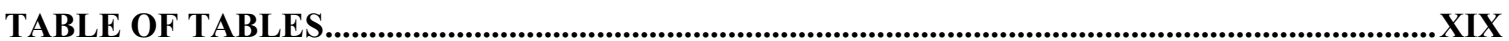

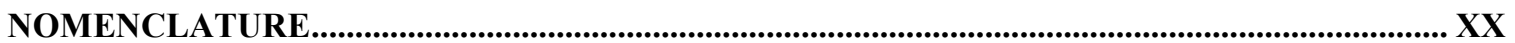

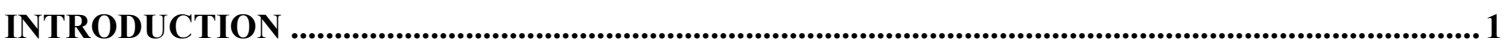

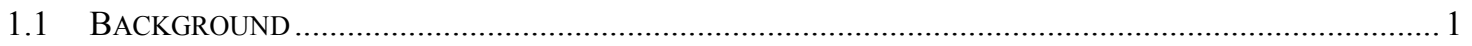

1.2 SKIN FRICTION EXPERIMENTAL TECHNIQUES ........................................................................... 1

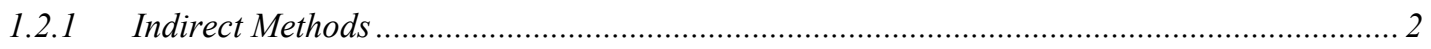

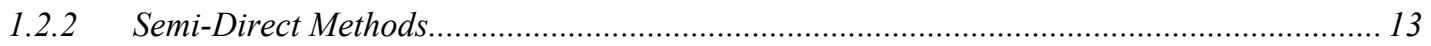

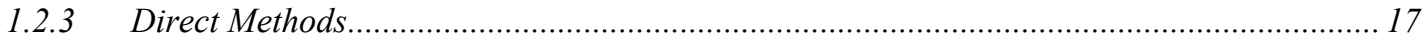

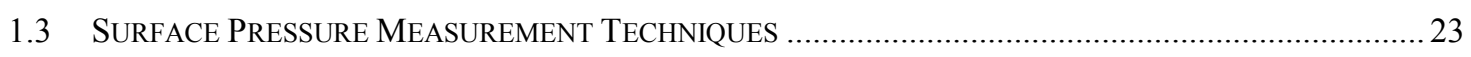

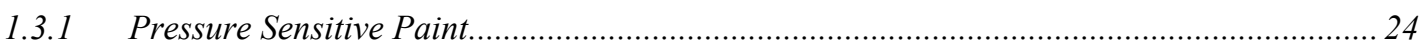

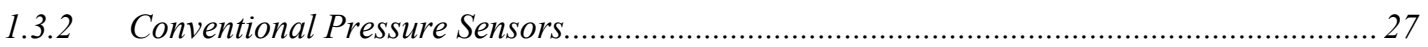

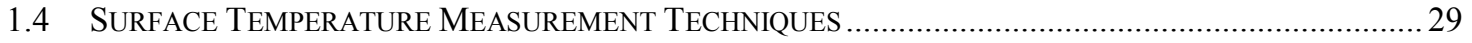

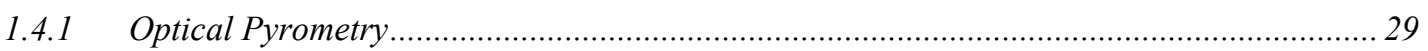

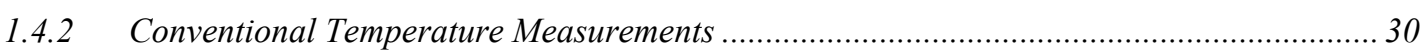

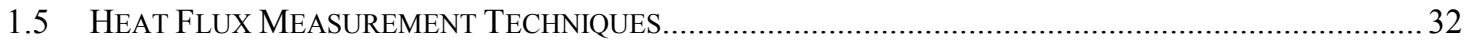

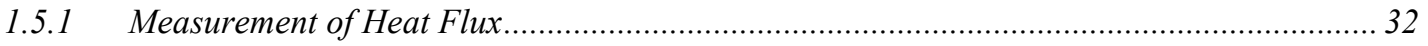

1.5.2 Calculation of Heat Flux from Surface Temperature Time History .................................... 33

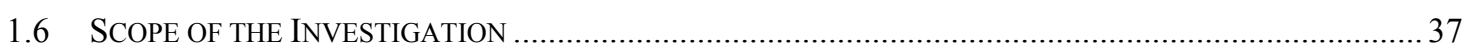

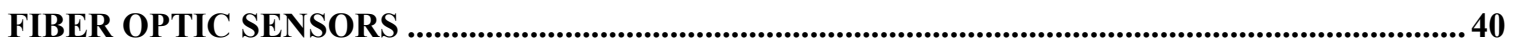

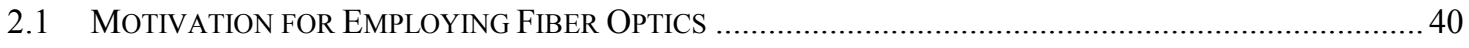

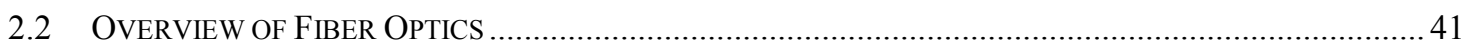

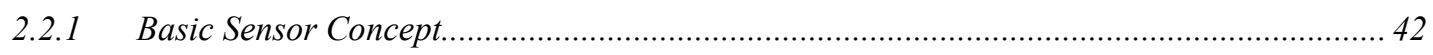

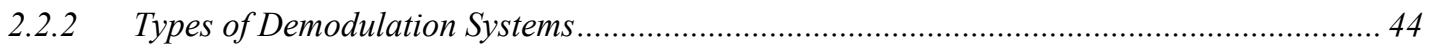

SENSOR DESIGN .................................................................................................................................5 53 


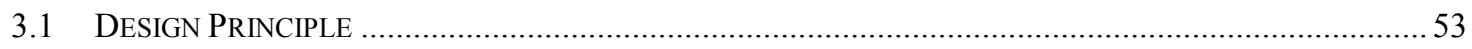

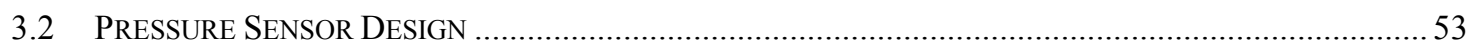

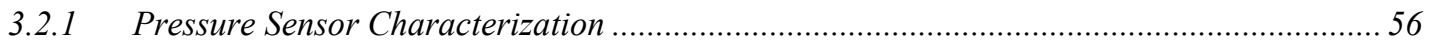

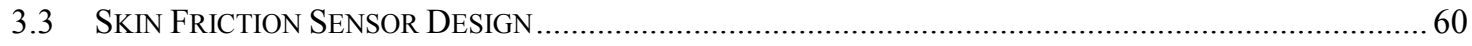

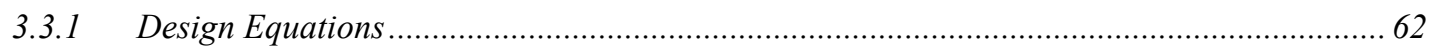

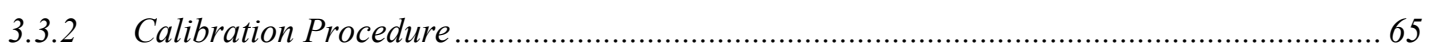

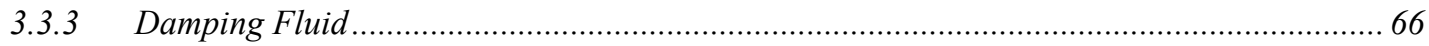

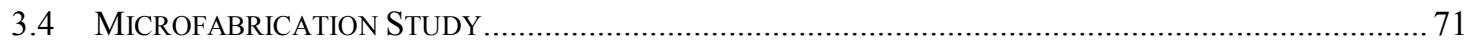

3.4.1 Motivation for Microfabrication of Skin Friction Sensor …………….............................. 72

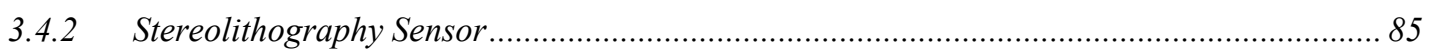

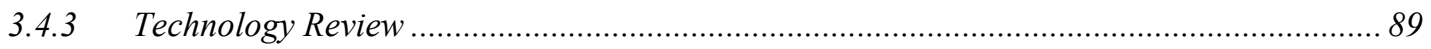

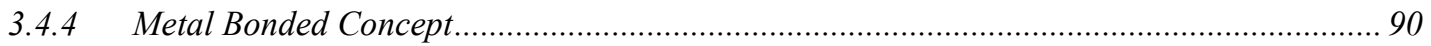

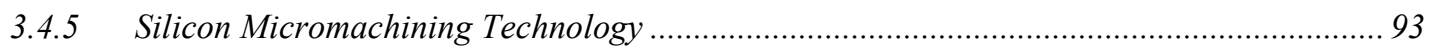

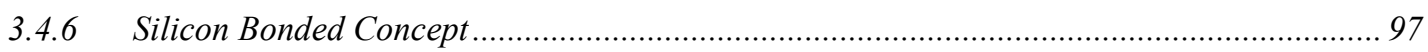

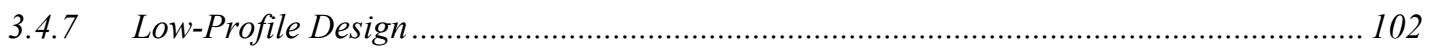

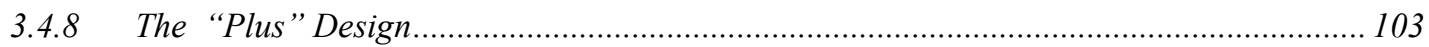

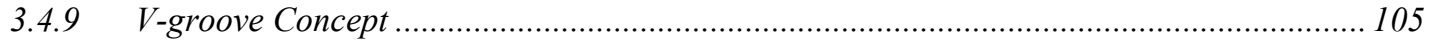

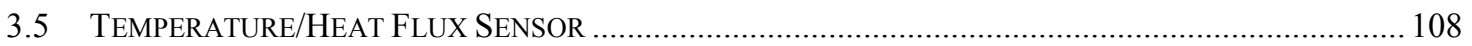

WIND TUNNEL TESTING OF PROTOTYPE SENSORS.............................................................111

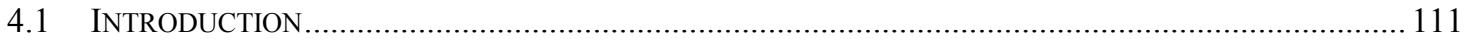

4.2 MicromachINEd Fiber OPTIC PRESSURE SENSOR................................................................. 112

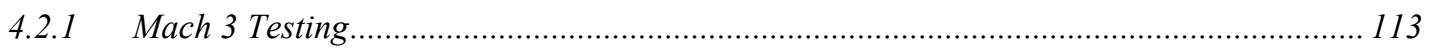

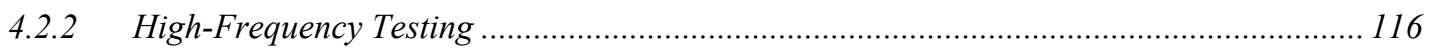

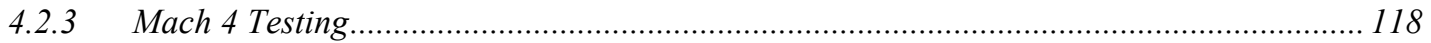

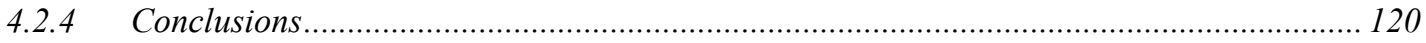

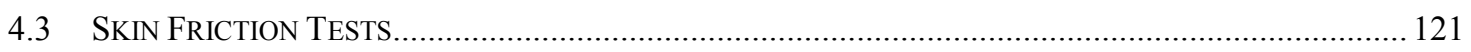

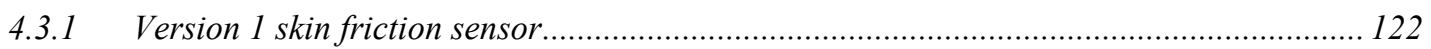

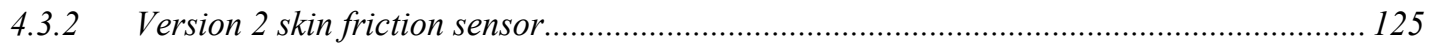

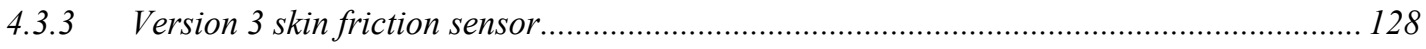

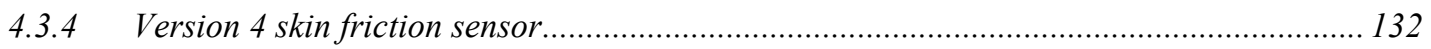

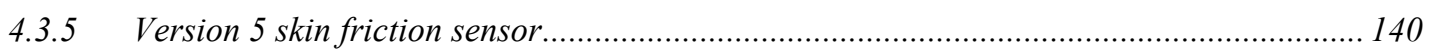

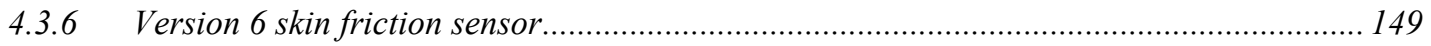

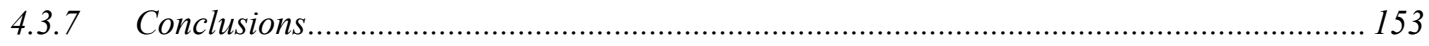

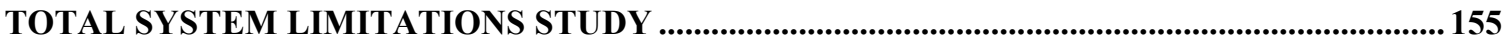

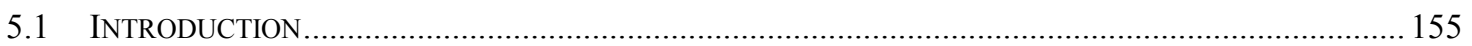

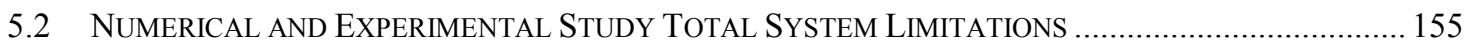




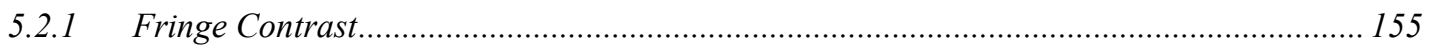

5.2.2 Error Sources in Spectral Interrogation Signal Processing System ................................. 156

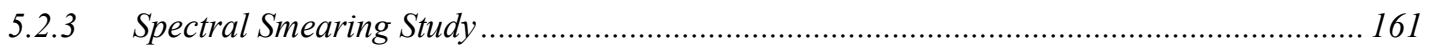

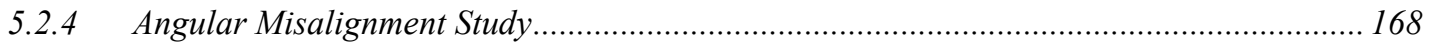

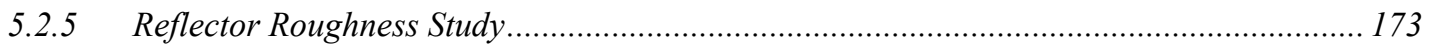

5.2.6 Combined Effects .................................................................................................. 178

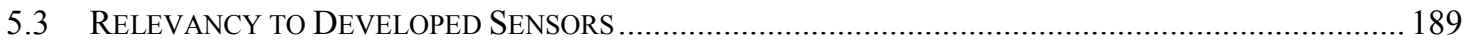

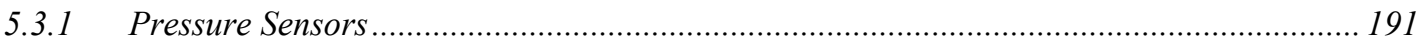

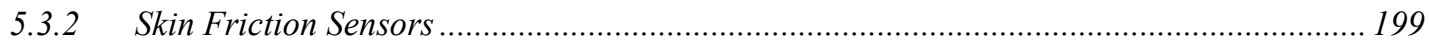

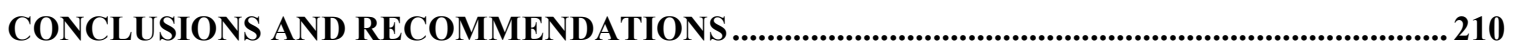

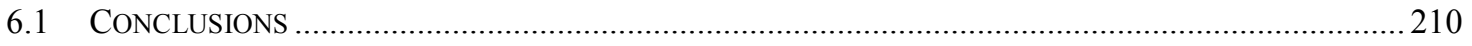

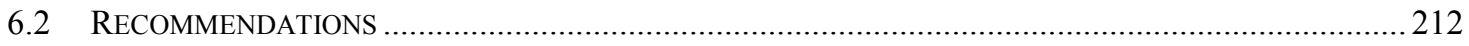

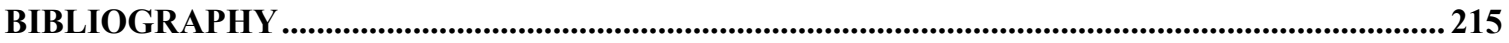




\section{Table of Figures}

Figure 1. Indirect shear stress measuring techniques............................................... 4

Figure 2. Sensitivities of various obstacles as skin friction meters................................. 7

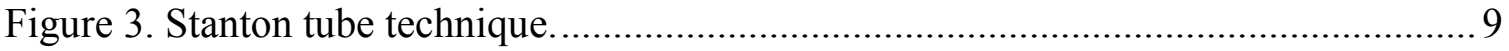

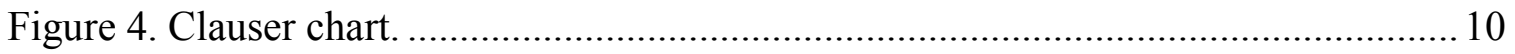

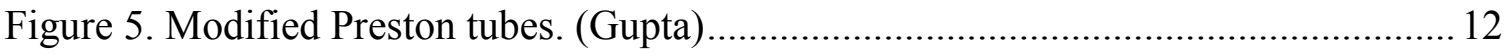

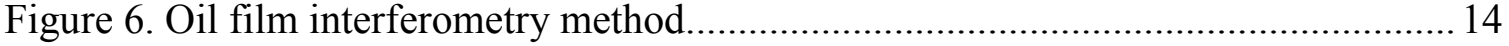

Figure 7. Wavelength of light scattered from liquid crystals under shear stress. (Klein and

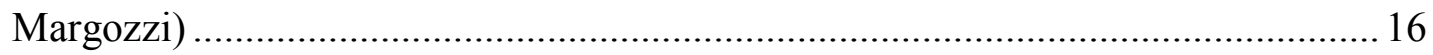

Figure 8. Apparatus used by Froude to measure skin friction on planks........................ 18

Figure 9. Example of nulling design. (Kistler)......................................................... 19

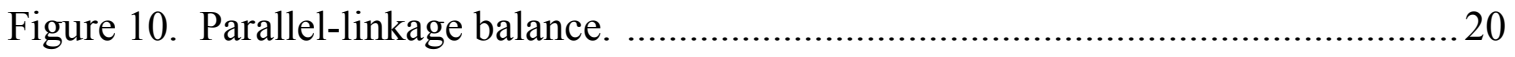

Figure 11. Silicon micromachined version of a table-top design................................... 21

Figure 12. Non-nulling cantilever beam concept. ........................................................ 22

Figure 13. a) Photograph of nulling skin friction sensor design that uses an intensity-

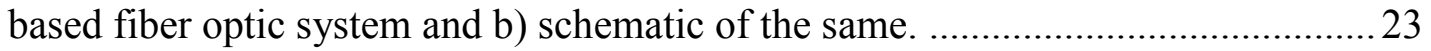

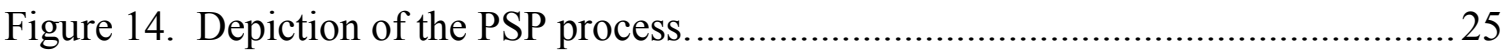

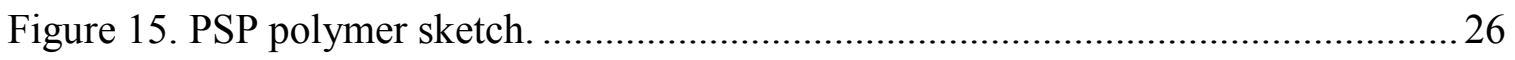

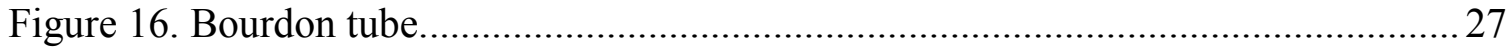

Figure 17. Strain-gage balance rosette on a diaphragm. ......................................... 28

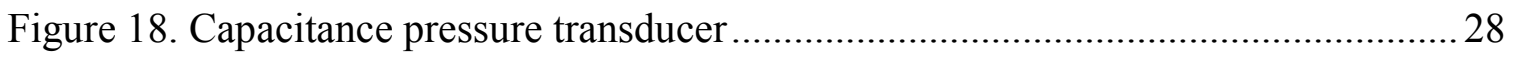

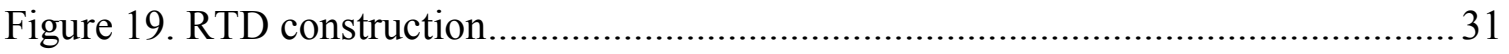

Figure 20. Current fiber optic temperature sensor design. ...................................... 31

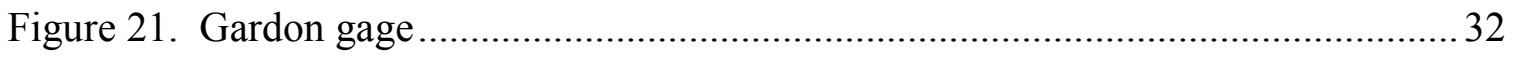

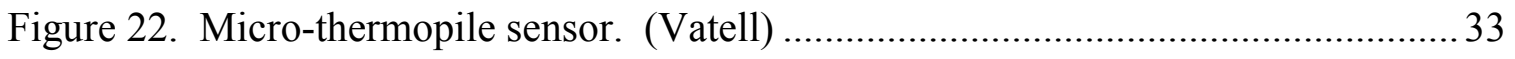

Figure 23. Sketch of aluminum-constantan thermocouple design. ............................... 34

Figure 24. Diagram of the null-point calorimeter concept ........................................ 35

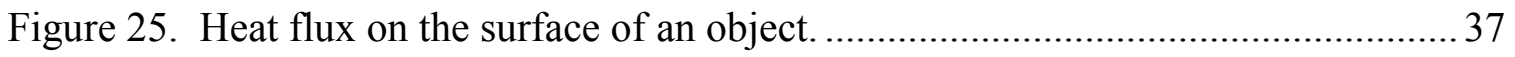

Figure 26. Sketch of the concept of the guiding of light by an optical fiber...................42

Figure 27. Extrinsic Fabry-Perot interferometer concept............................................ 43

Figure 28. Variation of intensity for changes in optical gap ..................................... 46 
Figure 29. Output of an intensity-based interferometric signal over a period. 46

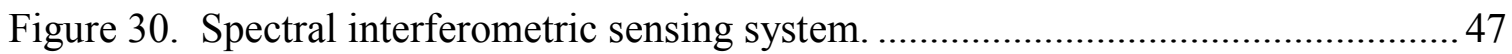

Figure 31. Depiction of spectral interrogation system method.

Figure 32. Schematic of Fizeau interferometer-based absolute demodulation approach. 50

Figure 33. Theoretical outputs of quadrature signals obtained from a dual wavelength system.

Figure 34. Two- $\lambda$ system theoretical output showing the phase lead/lag relationship at a direction change. .51

Figure 35. Idealized Lissajous figure of dual-wavelength output.................................51

Figure 36. Micromachined sensing element for the fiber optic pressure sensor..............53

Figure 37. Assembled fiber optic pressure sensor........................................................55

Figure 38. Representative pressure characteristics of fiber-optic pressure sensors. ........56

Figure 39. Temperature characterization data for a representative large area sensor......57

Figure 40. WSU shock tube (a) and sensor fixture mounted in the shock tube (b)........58

Figure 41. Time history (a) and power spectrum (b) of fiber optic sensor data from WSU

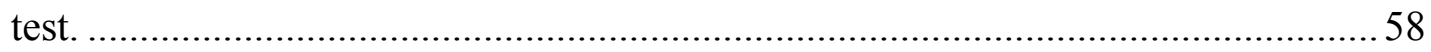

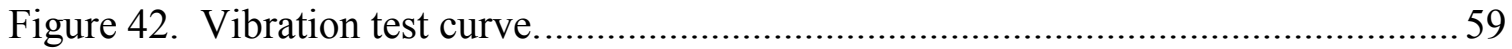

Figure 43. Reference frame (a) and test results (b) for the pressure sensor vibration test.

Figure 44. Conceptual model of skin friction sensing head and cantilever beam........... 61

Figure 45. Sketch of fiber locations in skin friction sensor.

Figure 46. Conventional calibration procedure, a) sketch of procedure, photograph of setup, and c) sample calibration result.

Figure 47. Comparison of the effect of a change in index of refraction with pressure on a theoretical optical path length for air, oil, and glycerin.

Figure 48. Comparison of the effect of a change in index of refraction with temperature on a theoretical optical path length for air, oil, and glycerin. 71

Figure 49. Sketch of the geometric variation which Allen studies for pressure gradient effects for floating head skin friction sensors. .73

Figure 50. Depiction of the effect of pressure gradient on a cantilever beam skin friction sensor for producing possible errors to the measurement. 75 
Figure 51. Computational skin friction results for a Mach 3 flow and $60{ }^{\circ} \mathrm{C}$ temperature difference. Note that the flow direction is from left-to-right 80

Figure 52. 2-D plot of the $\mathrm{K}-\Omega$ results for a Mach 3 flow and a $60{ }^{\circ} \mathrm{C}$ temperature difference 82

Figure 53. Comparison of skin friction coefficients for different grids for full sensor of a laminar flow calculation for a Mach 3 flow and a $60{ }^{\circ} \mathrm{C}$ temperature difference..... 83

Figure 54. Comparison of skin friction coefficient for different sensor sizes for a Mach 3 flow and a $60{ }^{\circ} \mathrm{C}$ temperature difference 84

Figure 55. Stereolithography process .87

Figure 56. Photographs of the (a) top and (b) bottom pieces of the SLA gage...... .88

Figure 57. Photographs of the side view (a) and top view (b) of the bottom piece of the SLA gage with strain gages attached. 88

Figure 58. Calibration of a stereolithography skin friction sensor for both axes including linear fit equations. 89

Figure 59. Depiction of the metal bonded gage design. 91

Figure 60. Design trade-off of a bonded metal design. 92

Figure 61. (100) silicon wafer with reference to its relevant planes. 93

Figure 62. Bulk micromachining of silicon wafer, a) unaltered Si wafer, b) applied photoresist, c) photoresist removed in a pattern, d) wafer after etching, and e) final etched wafer after photoresist is removed. 94

Figure 63. Examples of isotropic and anisotropic etch in silicon. 95

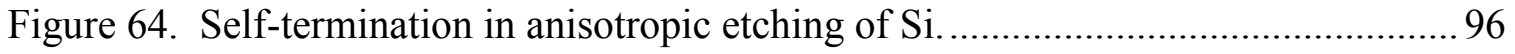

Figure 65. Etch planes in silicon, a) into the 100 plane and b) into the 110 plane.......... 96

Figure 66. Devices using anisotropy, exampled by a typical pressure sensor fabrication process. 96

Figure 67. Example of a fabrication processing using surface micromachining. 97

Figure 68. Sketch of the design of the Si bonded sensor. 98

Figure 69. Design space for Si bonded concept. 99

Figure 70. 400X detail of the prototype silver halide mask used in the proof-of-concept tests where the beam meets the sensing head. 100 
Figure 71. Successful etch test on a $<100>$ silicon wafer using the mask in Figure 70 in an attempt to produce a sheet similar to the right sketch of Figure 68.

Figure 72. Test etching into a 1-1-0 wafer $100 \mu \mathrm{m}$ deep showing the beginning of underetching.

Figure 73. Test etching into a 1-1-0 wafer $300 \mu \mathrm{m}$ deep showing the degradation of the wafer at this etch depth. 101

Figure 74. (a) Cut-away schematic diagram of the fiber optic skin friction sensor high aspect ratio cantilever beam with lateral platform and sapphire optical fiber, and (b) The cantilever, platform and fiber are fabricated within an Si substrate and flush with the wafer surface.

Figure 75. Manufacturing steps of the "Plus" micromachined design........................... 104

Figure 76. Design space of the "plus" design. 104

Figure 77. New, simple micromachined concept for a silicon micromachined skin friction sensor.

Figure 78. Design space of v-groove sensor concept.............................................. 106

Figure 79. Photograph of a v-groove sensor test article.............................................. 106

Figure 80. A calibration of a v-groove test article. .................................................. 107

Figure 81. Photograph of fiber optic temperature sensor using metal wire.................. 108

Figure 82. Sketch of the fiber optic heat flux/surface temperature sensor..................... 109

Figure 83. Photo of the fiber optic heat flux/surface temperature sensor prototype...... 109

Figure 84. Optical path length vs. temperature of a prototype fiber optic heat flux/surface

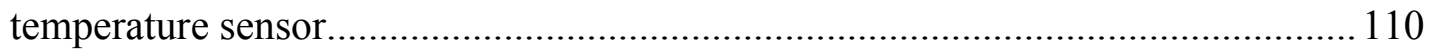

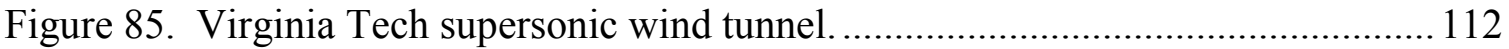

Figure 86. Micromachined, fiber optic pressure sensor used for Mach 2.4 tests, a) bare sensor and $b$ ) housed sensor.

Figure 87. Flush-mounted test of the micromachined, fiber optic pressure sensor in a Mach 2.4 flow.

Figure 88. Photograph of the linear array of micromachined, fiber optic pressure sensors. 114

Figure 89. Sketch of shock generation in supersonic tunnel used for the pressure sensor tests. 115 
Figure 90. Flush-mounted test of the micromachined, fiber optic pressure sensor in a Mach 3.0 flow (with the shock). 115

Figure 91. Flush-mounted test of the micromachined, fiber optic pressure sensor in a Mach 3.0 flow (without the shock). 116

Figure 92. Representative raw intensity data (amplified photodiode output) of the fiber optic pressure sensor using the intensity interferometric signal processing system.

Figure 93. Frequency spectrum of representative raw intensity data showing sensor natural frequency $(140 \mathrm{kHz})$. 118

Figure 94. Frequency spectrum of representative raw intensity data showing aerodynamic signal content (low frequency detail). 118

Figure 95. Cone model in Virginia Tech supersonic wind tunnel with the Mach 4 nozzle.

Figure 96. Pressure results of cone experiment at Mach 4 showing the comparison of fiber optic and conventional pressure sensors. 120

Figure 97. Standard Luna EFPI strain sensors depicting size and construction technique...

Figure 98. Sketch of the Version 1 skin friction sensor using EFPI strain sensors integrated with an optical pressure transducer in the sensing head.

Figure 99. Photograph of the Version 1 integrated pressure-skin friction sensor.

Figure 100. Skin friction coefficient for Test 8 measured using the Version 1 skin friction sensor demonstrating the large magnitude of the typical result.

Figure 101. Results of the fiber optic pressure sensor in the sensing head of the Version 1 sensor. 125

Figure 102. Schematic of the Version 2 skin friction sensor. ................................... 126

Figure 103. Photograph of the Version 2 skin friction sensor. ................................... 127

Figure 104. Design space of the Version 2 skin friction sensor................................... 127

Figure 105. Test 1 of Version 2 skin friction sensor in a Mach 3.0 flow at a total pressure of $690 \mathrm{kPa}(100 \mathrm{psi})$. 128

Figure 106. Test 2 of Version 2 skin friction sensor in a Mach 3.0 flow at a total pressure of $690 \mathrm{kPa}(100 \mathrm{psi})$. 128 
Figure 107. Photographs of the Version 3 skin friction sensor...................................... 129

Figure 108. Design space of Version 3 skin friction sensor.......................................... 130

Figure 109. Test 1 of Version 3 skin friction sensor in a Mach 3.0 flow at a total pressure

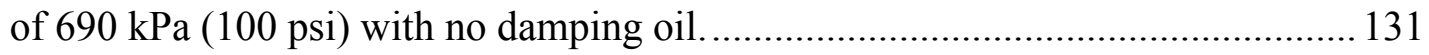

Figure 110. Test 16 of Version 3 skin friction sensor in a Mach 3.0 flow at a total pressure of $690 \mathrm{kPa}$ (100 psi) with damping oil

Figure 111. Photograph of the Version 4 skin friction sensor with rendering of the centerbody.

Figure 112. Design space of Version 4 skin friction sensor. 133

Figure 113. Test 11 of Version 4 skin friction sensor in Mach 3.0 flow without damping fluid.

Figure 114. Test 2 shear results of Version 4 skin friction sensor in Mach 2.4 flow at a total pressure of $345 \mathrm{kPa}(50 \mathrm{psi})$.

Figure 115. Test 2 skin friction coefficient results of Version 4 skin friction sensor in Mach 2.4 flow at a total pressure of $345 \mathrm{kPa}$ (50 psi) 135

Figure 116. Test 4 skin friction coefficient results of Version 4 skin friction sensor in Mach 2.4 flow at a total pressure of $345 \mathrm{kPa}$ (50 psi). 135

Figure 117. Schematic of the channel flow shear calibrator. 136

Figure 118. Pressure distribution along channel of calibration rig.

Figure 119. Photograph of calibration rig. 138

Figure 120. Test 2 of the Version 4 skin friction sensor in a fully-developed, incompressible glycerin flow.

Figure 121. Test 3, variable loading test of the Version 4 skin friction sensor in a fullydeveloped, incompressible glycerin flow.

Figure 122. Test 5 of the Version 4 skin friction sensor in a fully-developed, incompressible glycerin flow in which the sensor has been rotated $180^{\circ}$ 140

Figure 123. Design space of Version 5 skin friction sensor. 141

Figure 124. Photograph of the Version 5 skin friction sensor.

Figure 125. Test 3 shear results of the Version 5a skin friction sensor in Mach 2.4 flow at a total pressure of $345 \mathrm{kPa}$ (50 psi). 
Figure 126. Test 3 skin friction coefficient results of the Version 5a skin friction sensor in Mach 2.4 flow at a total pressure of $345 \mathrm{kPa}$ (50 psi).

Figure 127. Test 4 skin friction coefficient results of the Version 5a skin friction sensor in Mach 2.4 flow at a total pressure of $345 \mathrm{kPa}(50 \mathrm{psi})$.

Figure 128. Test 10 shear results of the Version 5a skin friction sensor in a Mach 3.0 flow at a total pressure of $520 \mathrm{kPa}(75 \mathrm{psi})$.

Figure 129. Test 10 skin friction coefficient results of the Version 5a skin friction sensor in a Mach 3.0 flow at a total pressure of $520 \mathrm{kPa}(75 \mathrm{psi})$ 144

Figure 130. Test 11 skin friction coefficient results of the Version 5a skin friction sensor in low pressure, Mach 3.0 flow at a total pressure of $520 \mathrm{kPa}$ (75 psi). 145

Figure 131. Test 24 cross-flow shear results of the Version 5a skin friction sensor in Mach 3.0 flow at a total pressure of $550 \mathrm{kPa}(80 \mathrm{psi})$ 145

Figure 132. Test 6 of the Version 5a skin friction sensor in a Mach 3.0 flow at a total pressure of $750 \mathrm{kPa}(110 \mathrm{psi})$. 146

Figure 133. Test 3 shear results of the Version 5a skin friction sensor at Wright Pat in Mach 1.92 flow at a total pressure of $345 \mathrm{kPa}$ (50 psi).

Figure 134. Test 3 skin friction coefficient results of the Version 5a skin friction sensor at Wright Pat in Mach 1.92 flow at a total pressure of $345 \mathrm{kPa}$ (50 psi).

Figure 135. Test 6 shear results of the Version 5a skin friction sensor at Wright Pat in Mach 1.92 flow at a total pressure of $345 \mathrm{kPa}(50 \mathrm{psi})$. 148

Figure 136. Test 6 skin friction coefficient results of the Version 5a skin friction sensor at Wright Pat in Mach 1.92 flow at a total pressure of $345 \mathrm{kPa}$ (50 psi). 148

Figure 137. Test 17 shear results of the Version 5b skin friction sensor in Mach 3.0 flow at a total pressure of $550 \mathrm{kPa}(80 \mathrm{psi})$.

Figure 138. Design Space of Version 6 skin friction sensor.......................................... 150

Figure 139. Photograph of the Version 6 skin friction sensor. 150

Figure 140. Test 25 shear results of the Version 6 skin friction sensor in Mach 3.0 flow at a total pressure of $690 \mathrm{kPa}(100 \mathrm{psi})$.

Figure 141. Test 25 skin friction coefficient results of the Version 6 skin friction sensor in high pressure, Mach 3.0 flow at a total pressure of $690 \mathrm{kPa}$ (100 psi). 151 
Figure 142. Test 46 shear results of the Version 6 skin friction results in Mach 2.4 flow at a total pressure of $240 \mathrm{kPa}(35 \mathrm{psi})$. 152

Figure 143. Test 46 skin friction coefficient results of the Version 6 skin friction results in Mach 2.4 flow at a total pressure of $240 \mathrm{kPa}$ (35 psi). 152

Figure 144. Test 44 shear results of the Version 6 skin friction sensor in Mach 2.4 flow at a total pressure of $240 \mathrm{kPa}(35 \mathrm{psi})$.

Figure 145. Test 48 shear results of the Version 6 skin friction sensor in Mach 2.4 flow at a total pressure of $240 \mathrm{kPa}(35 \mathrm{psi})$ 153

Figure 146. Explanation of fringe contrast. 156

Figure 147. Calculation of absolute error due to pixel shift for random and constant pixel shifts at 50 and $100 \mu \mathrm{m}$ for the spectral interrogation signal processing system.... 158

Figure 148. A/D noise absolute error calculation for the spectral interrogation signal processing system. 158

Figure 149. Absolute error of source ripple error calculation for the spectral interrogation signal processing system.

Figure 150. Loss of fringe contrast for source ripple calculation for the spectral interrogation signal processing system.

Figure 151. Calculated spectra of system error conditions, a) typical system errors and b) maximum values of study.

Figure 152. Sketch of the fiber optic accelerometer.

Figure 153. Fiber optic accelerometer data demonstrating the reduction of fringe contrast due to smearing.

Figure 154. Depiction of error calculations due to spectral smearing showing the progression of the reflection surface during the integration time of the CCD array.

Figure 155. Results of numerical study of spectral smearing for the spectral interrogation signal processing system, a) absolute error in terms of gap speed and b) absolute error in terms of percent smearing. 165

Figure 156. Detail of the calculation of absolute error due to spectral smearing for the spectral interrogation signal processing system. 166 
Figure 157. Calculation of absolute error of the spectral interrogation signal processing system due to spectral smearing beyond the first harmonic. 166

Figure 158. Grid convergence study of calculations of spectral smearing. 167

Figure 159. Loss of normalized fringe contrast due to spectral smearing for the spectral interrogation signal processing system. 168

Figure 160. Calculation of fringe contrast of the spectral interrogation signal processing system due to spectral smearing beyond first smearing harmonic. 168

Figure 161. Depiction source of power and fringe contrast loss from angular misalignment of the reflector.

Figure 162. Power loss due to angular misalignment of the reflector.

Figure 163. Measurement of fringe contrast loss due to angular misalignment, a) in air and b) glycerin. 171

Figure 164. Depiction of error calculations due to angular misalignment showing the progression of the reflection surface and the basis of the weighting function.

Figure 165. Results of angular misalignment calculations for the spectral interrogation signal processing system, a) absolute error and b) loss of normalized fringe contrast.

Figure 166. Photograph of the AFM setup. 174

Figure 167. AFM of pressure sensor diaphragm surface (darkening of shade indicates a deeper surface). 175

Figure 168. AFM of portions of typical skin friction sensor reflector (darkening of shade indicates a deeper surface).

Figure 169. Worst-case of measured fringe contrast of the Version 6 skin friction sensor during calibration. Spike indicates that bad refection sections exist on these surfaces.

Figure 170. Depiction of error calculations due to reflector roughness for the spectral interrogation signal processing system.

Figure 171. Roughness calculation for the spectral interrogation signal processing system, a) Absolute error and b) loss of normalized fringe contrast. 178

Figure 172. Sketch of the PZT experimental setup. 179 
Figure 173. PZT experimental results for a range of gap speeds with the stored spectra for each speed on the right and the resulting FFT calculation on the left.

Figure 174. Vibration data of skin friction sensor demonstrating that reduction of fringe contrast due to more that spectral smearing. For the nominal spectrometer,

Figure 175. Numerical simulation of total system error for the fiber optic pressure sensor using the spectral interrogation signal processing system (using nominal system errors and a standard deviation of $9.353 \mathrm{~nm}$ for surface roughness), a) detail of absolute error and b) overall system error. 185

Figure 176. Numerical simulation of loss of fringe contrast for the fiber optic pressure sensor using the spectral interrogation signal processing system (using nominal system errors and a standard deviation of $9.353 \mathrm{~nm}$ for surface roughness). 186

Figure 177. Depiction of skin friction sensor total system numerical simulation showing the progression of the reflection surface during the integration time of the CCD array

Figure 178. Numerical simulation of total system error for the fiber optic skin friction sensor (using nominal system errors, a standard deviation of $25.0 \mathrm{~nm}$ for surface roughness, and angular movement tied to gap movement), a) detail of absolute error and b) overall system error.

Figure 179. Numerical simulation of loss of normalized fringe contrast for the skin friction sensor (using nominal system errors, a standard deviation of $25.0 \mathrm{~nm}$ for surface roughness, and angular movement tied to gap movement),

Figure 180. Example of fringe contrast calculation software for a pressure sensor, a) spectrum of stationary gap, b) fringe contrast of stationary gap, c) spectrum of totally smeared spectrum and d) fringe contrast of totally smeared spectrum.

Figure 181. Run 20 of the micromachined pressure sensor array in a Mach 3.0 flow without the shock.

Figure 182. Comparison of fringe contrast to change in gap estimate for Run 20 of the micromachined pressure array in a Mach 3.0 flow without the shock, a) upstream pressure sensor and b) downstream pressure sensor.

Figure 183. Run 15 of the micromachined pressure sensor array in a Mach 3.0 flow with the shock. 
Figure 184. Comparison of fringe contrast to change in gap estimate for Run 15 of the micromachined pressure array in a Mach 3.0 flow with the shock, a) pressure sensor upstream of shock and b) pressure sensor downstream of shock.

Figure 185. Comparison of fringe contrast and algorithm correction factor in Run 20 of the pressure sensor array in a Mach 3.0 flow. 196

Figure 186. Semi-log plot of fringe contrast vs. variance of gap change for pressure sensor in various flow conditions.

Figure 187. Linear plot of fringe contrast vs. variance of gap change for pressure sensor in various flow conditions showing fringe contrast cut-off.

Figure 188. Plot of fringe contrast vs. variance of algorithm correction factor for pressure sensor in various flow conditions 198

Figure 189. Run 2 of the Version 5a skin friction sensor in a Mach 2.4 flow at a total pressure of $345 \mathrm{kPa}(50 \mathrm{psi})$.

Figure 190. Fringe contrast change compared to change in gap estimate for Run 2 of the Version 5a skin friction sensor in a Mach 2.4 flow at a total pressure of $345 \mathrm{kPa}(50$ psi), a) channel 2 and b) channel 4 .

Figure 191. Run 9 of the Version 5a skin friction sensor in a Mach 3.0 flow at a total pressure of $520 \mathrm{kPa}(75 \mathrm{psi})$. 201

Figure 192. Fringe contrast change compared to change in gap estimate for Run 9 of the Version 5a skin friction sensor in a Mach 3.0 flow at a total pressure of $520 \mathrm{kPa}(75$ psi), a) channel 2 and b) channel 4 . 202

Figure 193. Run 5 of the Version 5a skin friction sensor in a Mach 3.0 flow at a total pressure of $750 \mathrm{kPa}(110 \mathrm{psi})$. 202

Figure 194. Fringe contrast change compared to change in gap estimate for Run 5 of the Version 5a skin friction sensor in a Mach 3.0 flow at a total pressure of $750 \mathrm{kPa}$ (110 psi), a) channel 2 and b) channel 4. 203

Figure 195. Comparison of fringe contrast and algorithm correction factor estimate for Run 5 of the Version 5a skin friction sensor in a Mach 3.0 flow at a total pressure of $750 \mathrm{kPa}(110 \mathrm{psi})$ demonstrating the correlation of fringe contrast and phase correction RMS variation. .204 
Figure 196. Comparison of fringe contrast and algorithm correction factor for channel 3 of the Version 6 skin friction sensor in a Mach 3.0 flow at a total pressure of 240 $\mathrm{kPa}$ (35 psi) showing the correlation of the two, a) Run 41 and b) Run 48........... 205

Figure 197. Clear depiction of correlation of gap artifacts and changes in fringe contrast factor for channel 3 of the Version 6 skin friction sensor in a Mach 3.0 flow at a

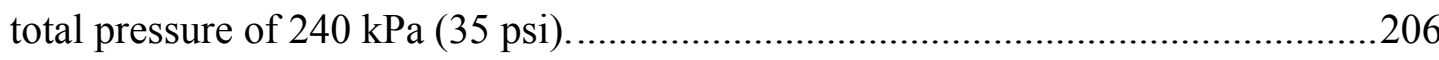

Figure 198. Semi-log plot of fringe contrast vs. variance of gap change for the Version 5a skin friction sensor for various flow conditions. 207

Figure 199. Linear plot of fringe contrast vs. variance of gap change for the Version 5a skin friction sensor for various flow conditions showing fringe contrast cut-off. .. 207

Figure 200. Plot of fringe contrast vs. variance of algorithm correction factor for the Version 5a skin friction sensor for various flow conditions. 208

Figure 201. Reduction in the Version 5a skin friction sensor fringe contrast due to energy of the flow as measured by dynamic pressure 208 


\section{Table of Tables}

Table 1. Advantages and Disadvantages of Skin Friction Measurement Techniques ........ 3

Table 2. Comparison of different sensing technologies............................................... 40

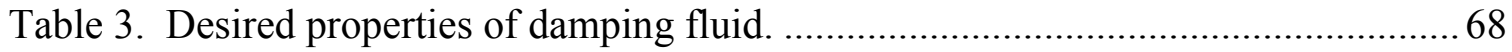

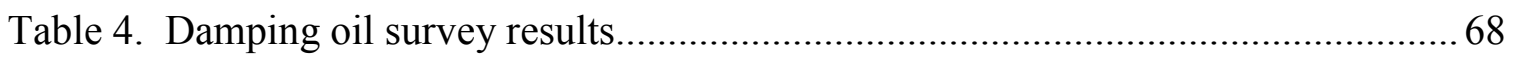

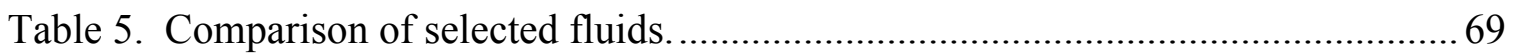

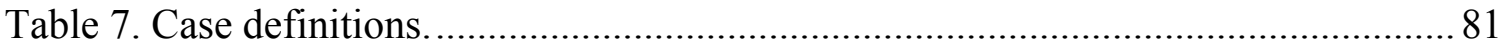

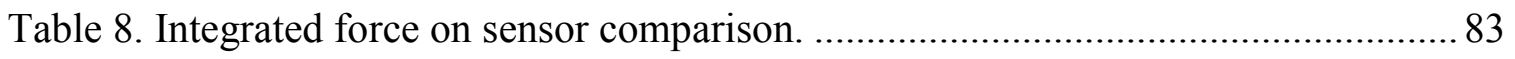

Table 9. Technology overview for bonded micro-skin friction design.......................... 93

Table 10. Summary of Skin Friction Sensor Versions.................................................. 154

Table 12. Correlation of gap change, fringe contrast change, and correction factor change

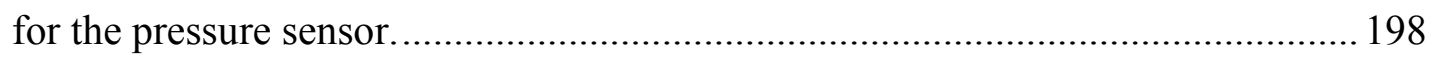

Table 13. Correlation of gap change, fringe contrast, and correction factor change for the

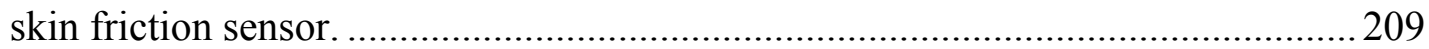




\section{Nomenclature}

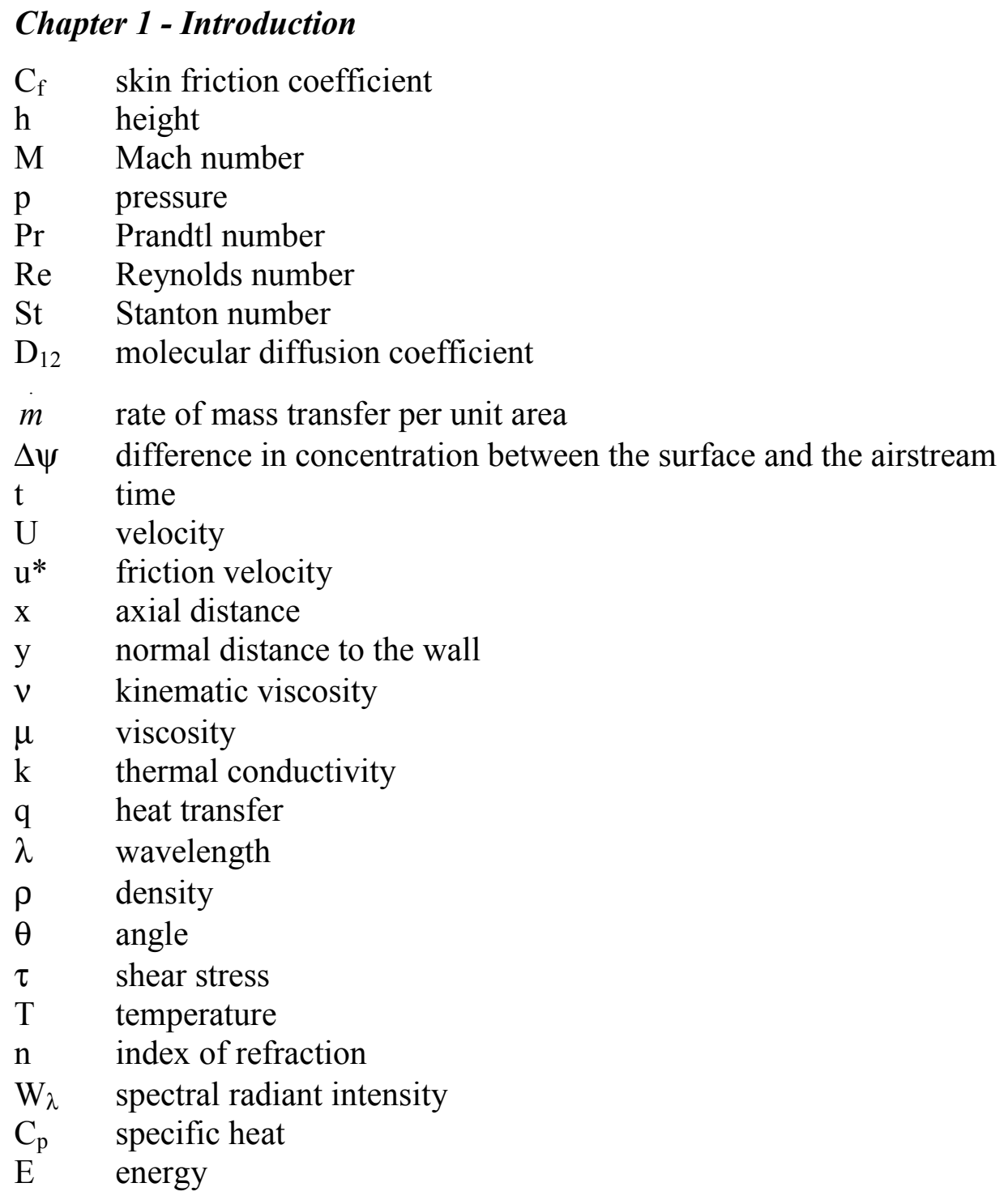

\section{Chapter 2 - Fiber Optics}

OPL optical path length

$\mathrm{n}$ index of refraction

I intensity

A amplitude

$\phi \quad$ phase angle

\section{Chapter 3 - Sensor Design}

y deflection

d diameter 


$\begin{array}{ll}\text { P } & \text { applied load } \\ \text { E } & \text { modulus of elasticity } \\ \text { I } & \text { area moment of inertia } \\ \text { L } & \text { length } \\ \mu & \text { Poisson's ratio } \\ \rho & \text { density } \\ \text { X } & \text { distance along the beam } \\ \theta & \text { bending angle } \\ \Delta & \text { deflection } \\ \delta & \text { optical gap } \\ \delta & \text { boundary layer thickness } \\ \mathrm{r} & \text { radius } \\ \omega_{\mathrm{n}} & \text { natural frequency } \\ \mathrm{f}_{\mathrm{n}} & \text { natural frequency } \\ \mathrm{m} & \text { mass } \\ \mathrm{F} & \text { force } \\ \mathrm{A} & \text { area } \\ \mathrm{M} & \text { moment } \\ \mathrm{t} & \text { length } \\ \mathrm{h} & \text { thickness } \\ \mathrm{OPL} & \text { optical path length } \\ \mathrm{n} & \text { index of refraction } \\ \mathrm{T} & \text { temperature } \\ & \end{array}$

\section{Chapter 4 - Wind Tunnel Testing}

$\mathrm{C}_{\mathrm{f}} \quad$ skin friction coefficient

M Mach number

$\mathrm{p}$ pressure

$\tau \quad$ shear stress

$\gamma \quad$ ratio of specific heats

\section{Chapter 5 - Total System Limitation}

FC fringe contrast

I intensity

$\mathrm{S}$ percent smearing,

$\Delta \mathrm{s} \quad$ change in optical gap

$\lambda_{\mathrm{c}} \quad$ center wavelength of source

d diameter

$\quad$ weighting function

\section{Subscripts}

eff effective

$\mathrm{x}$ distance in freesteam direction

w condition at wall 
e conditions at edge of boundary layer

o stagnation condition

$\infty \quad$ freestream condition

f fence

h hot-wire

t total

$r \quad$ reflector location

\section{Superscripts}

$+\quad$ laminar sublayer nondimensionalization 


\section{Development of Fiber Optic Aerodynamic Sensors for High Reynolds Number Supersonic Flows}

\section{Chapter 1}

\section{Introduction}

\subsection{Background}

Acquiring accurate, transient measurements in harsh environments, especially of pressure, skin friction, temperature, and heat flux, has always pushed the limits of available sensor technology. Recent developments in advanced system and process controls have increased the need for sensors capable of acquiring measurements in extreme environments, such as supersonic flows. The large changes in multiple parameters can introduce uncertainties and errors into measurements of these quantities even for a simple supersonic flow. High temperatures and heat fluxes often accompany such flows causing temperature-induced errors in electrical-based instrumentation. Until recently, the technology to measure certain properties (such as pressure, skin friction, temperature, or heat flux) in high temperature and high EMI environments has simply not existed. Smaller sizes, especially in normally small supersonic test facilities, are desired to make more detailed and accurate measurements. In an effort to improve such measurements, instrumentation is moving to modern construction techniques, such as micromachining, and advanced querying techniques, such as fiber optics, which when combined will greatly improve accuracy, repeatability, and resolution while reducing sensitivity to electromagnetic radiation and temperature.

\subsection{Skin Friction Experimental Techniques}

The accurate measurement of skin friction is vital to the development of turbulence models for future generations of computational fluid dynamic simulations. This quantity has been shown to be much more difficult to accurately predict numerically than, say, wall pressure. In particular, wall shear stress has a more direct input into turbulence models as the key component of friction velocity, 


$$
u^{*}=\sqrt{\frac{\tau_{w}}{\rho}}
$$

which is used as a scaling parameter in the correlation of turbulent boundary layer profiles. Hence, accurate skin friction measurements are required to generate improved turbulence models. Experimentally, the knowledge of the wall shear stress provides more insight for situations such as reverse flow into the condition of the flowfield than, for example, a pressure measurement. Finally, the determination of skin friction greatly assists the test engineer in assessing the performance and design of fluid machinery devices.

A number of techniques for the measurement of wall shear stress have been devised over the years, ranging from inferring the skin friction by measuring the boundary layer profile to using some correlation/analogy to the direct measurement of the force on a surface. Although all of these techniques can be shown to work for some flow regimes, indirect methods have not been shown to be reliable for complex flows such as 3D and/or unsteady cases with rough wall, curved walls, flows with injection or suction, or high-speed flows, especially those with high enthalpies, combustion and/or impinging shocks. On the other hand, direct measurements do not require any foreknowledge of the flow or its properties and can provide accurate results for all the regimes mentioned above. ${ }^{1}$ Table 1 presents the various methods for measuring wall shear stress and their respective advantages and disadvantages. A further description of these methods, their regimes of accuracy, and their limitations is given below.

\subsubsection{Indirect Methods}

Numerous indirect techniques have been developed over the years to infer skin friction values from other measurements. Although they are not a direct measurement of the shear on the wall, these techniques have been used in many simple flows. These methods fall within three categories: velocity profile measurement, where the slope of the profile is approximated and then shear calculated from Stokes Law; analogies, such as Reynolds Analogy, where another quantity is measured at the wall and related to shear via one of these analogies; and flow around an obstacle, where pressure is measured in various ways within the boundary layer and then related through calibration to the shear. Obviously, all of these methods, at one level or another, rely on assumptions about the 
flow and each individual method has its own advantages and disadvantages, as outlined in Table 1. A detailed discussion of many of these indirect methods is included in Nitche, et al. ${ }^{2}$, from which Figure 1 is taken.

Table 1. Advantages and Disadvantages of Skin Friction Measurement Techniques ${ }^{3,2,10,13,15}$

\begin{tabular}{|c|c|c|c|}
\hline \multicolumn{2}{|c|}{ Measurement Technique } & Advantages & Disadvantages \\
\hline \multicolumn{2}{|c|}{$\begin{array}{l}\text { Direct Measurements } \\
\text { (Force) }\end{array}$} & $\begin{array}{l}\text { - Flow and fluid independent } \\
\text { - Able to determine direction } \\
\text { - Non-intrusive }\end{array}$ & $\begin{array}{l}\text { - Small force } \\
\text { - High cost }\end{array}$ \\
\hline \multicolumn{4}{|c|}{ Semi-Direct Measurements } \\
\hline \multicolumn{2}{|c|}{ - Oil Film Interferometry } & $\begin{array}{l}\text { - Flow condition independent } \\
\text { - Provides a global measurement } \\
\text { - Non-intrusive }\end{array}$ & $\begin{array}{l}\text { - Temperature sensitive } \\
\text { - Shear stress, shear gradient, time limited } \\
\text { - } \text { Requires optical access }\end{array}$ \\
\hline \multicolumn{2}{|l|}{ - Liquid Crystals } & $\begin{array}{l}\text { - Flow condition independent } \\
\text { - Provides a global measurement } \\
\text { - Non-intrusive }\end{array}$ & $\begin{array}{l}\text { - } \text { Temperature and pressure sensitive } \\
\text { - Requires optical access } \\
\text { - Low sensitivity } \\
\text { - Limited time window } \\
\text { - } \text { Shear stress limited } \\
\end{array}$ \\
\hline \multicolumn{4}{|c|}{ Indirect Measurements } \\
\hline \multirow[t]{2}{*}{ - Analogy } & $\begin{array}{l}\text { Heat Transfer } \\
\text { (Reynolds } \\
\text { Analogy) }\end{array}$ & $\begin{array}{l}\text { - Dual purpose sensor } \\
\text { - Low cost } \\
\text { - High frequency response }\end{array}$ & $\begin{array}{l}\text { Low precision measurement } \\
\text { - } \text { Limited to determine direction } \\
\text { temperature sensitivity } \\
\text { - Requires knowledge of freestream }\end{array}$ \\
\hline & $>$ Mass Transfer & - Low cost & $\begin{array}{l}\text { - Calibration not available } \\
\text { - Low precision measurement } \\
\text { - Not able to determine direction } \\
\text { - Limited temperature range with high } \\
\text { temperature sensitivity } \\
\text { - Requires knowledge of freestream }\end{array}$ \\
\hline \multirow[t]{2}{*}{$\begin{array}{l}\text { - Flow About } \\
\text { Obstacles }\end{array}$} & $\begin{array}{l}>\text { Sub-Layer } \\
\text { Fence }\end{array}$ & $\begin{array}{l}\text { - Simple } \\
\text { - } \text { Low cost }\end{array}$ & $\begin{array}{l}\text { - Difficult calibration } \\
\text { - Susceptible to misalignment } \\
\text { - Requmes Law of the wall } \\
\text { conditions }\end{array}$ \\
\hline & $>$ Stanton Tube & $\begin{array}{ll}\text { - Simple } \\
\text { - Low cost }\end{array}$ & $\begin{array}{l}\text { Susceptible to misalignment } \\
\text { - Rssumes Law of the wall } \\
\text { conditions }\end{array}$ \\
\hline \multirow[t]{3}{*}{$\begin{array}{l}\text { - } \text { Profile } \\
\text { Measurement }\end{array}$} & $>$ Preston Tube & $\begin{array}{l}\text { - Simple } \\
\text { - Low cost }\end{array}$ & $\begin{array}{l}\text { - Flow calibration required } \\
\text { - Susceptible to misalignment } \\
\text { - } \text { Assumes Law of the wall } \\
\text { - Requires layer thickness limited } \\
\text { conditions }\end{array}$ \\
\hline & $>$ Hot-Wire & $\begin{array}{l}\text { Simple } \\
\text { - Provides high frequency data }\end{array}$ & $\begin{array}{l}\text { Assumes Law of the wall } \\
\text { - } \text { Fragile and temperature limited } \\
\text { Requires knowledge of fluid viscosity }\end{array}$ \\
\hline & $\begin{array}{l}\text { Laser Doppler } \\
\text { Anemometry }\end{array}$ & $\begin{array}{ll}\text { - Non-intrusive } \\
\text { Provides high frequency data }\end{array}$ & $\begin{array}{l}\text { Requires optical access } \\
\text { - Requires seed particles } \\
\text { - Requires knowledge of fluid viscosity }\end{array}$ \\
\hline
\end{tabular}


TECHNIQUE

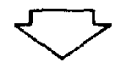

SURFACE HOI FILM:

a

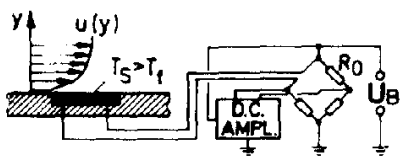

CALIBRATION UNDERLYING PRINCIPLE
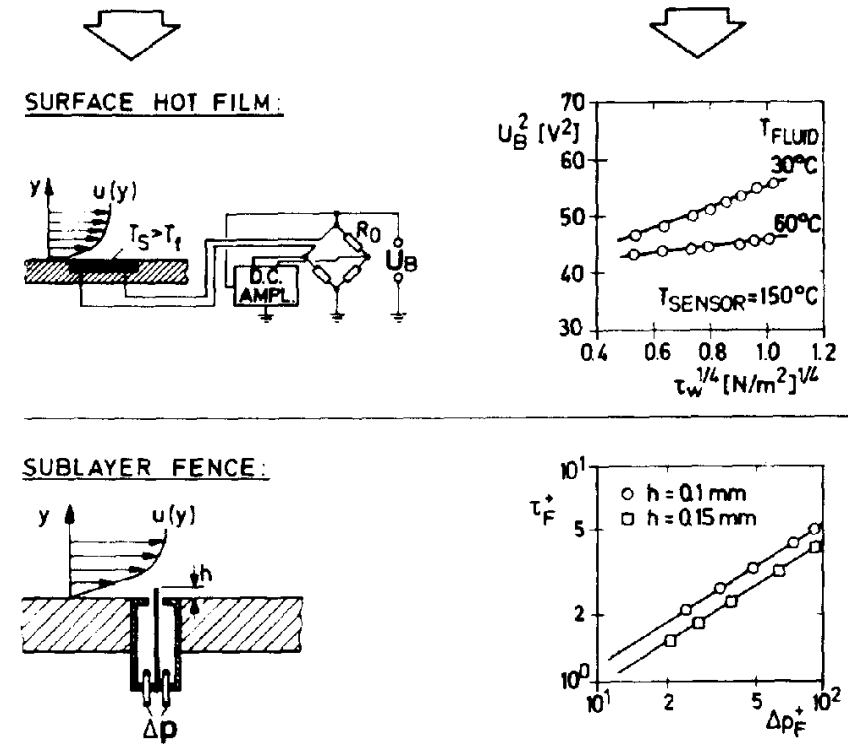

(c)

d
PRESTON TUBE:

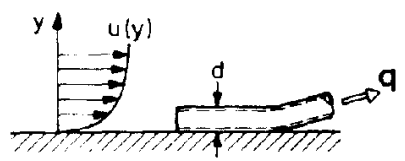

WALL-FIXED HOT-WIRE :
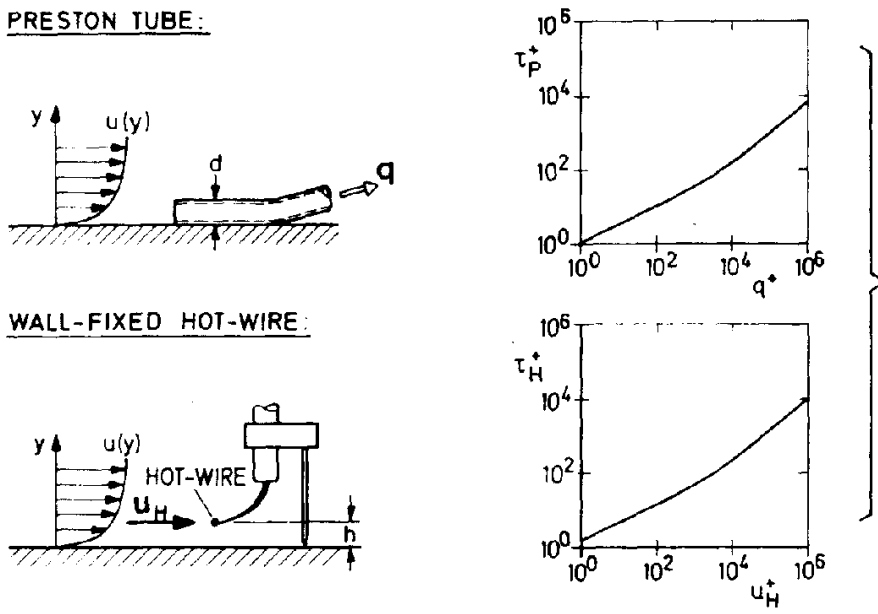

COMPUTAIIONAL PRESTON IUBE:

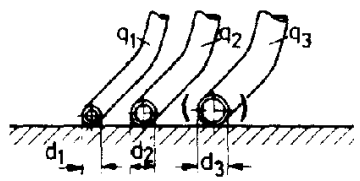

WALL-FIXED DOUBLE HOT-WIRE:

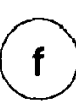

e

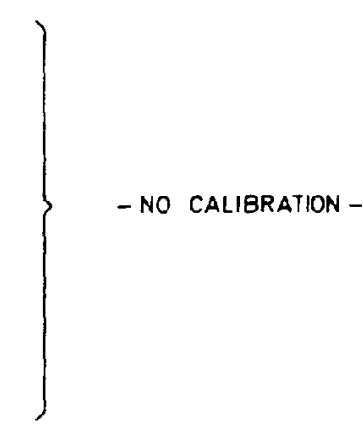

- No calibration -

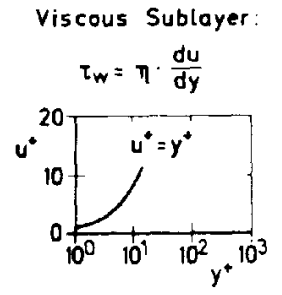

Law of the Wall:

$$
u^{*}=F\left(y^{*}\right)
$$

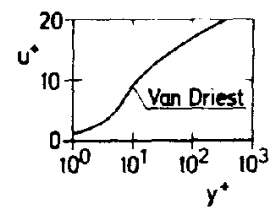

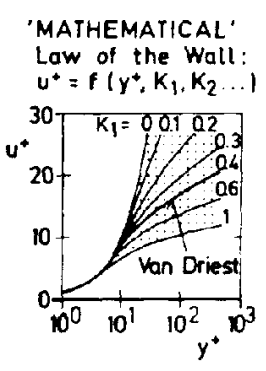

Figure 1. Indirect shear stress measuring techniques. ${ }^{2}$ 


\section{$\underline{\text { Analogies }}$}

The term, analogy techniques, refers to two methods that rely on the interdependency of heat and mass transfer to skin friction. This interdependency stems from the changing conditions within the velocity laminar sublayer, i.e. the altering of the thermophysical properties of the fluid near the wall surface. The first technique is based on Reynolds Analogy, which relates skin friction to heat transfer,

$$
S t=\operatorname{Pr}^{1 / 3} \cdot \frac{C_{f}}{2} .
$$

The second method is akin to the first, but uses the mass transfer of a material from the wall surface with the relation,

$$
S t_{\text {Diff }}=S c^{-2 / 3} \cdot \frac{C_{f}}{2} .
$$

However, as will be seen, these techniques require the knowledge of freestream conditions, which are difficult to define in many complex flow situations. Also, both analogies are very sensitive to flow conditions, especially temperature, and though they both can provide qualitative results (such as transition), their quantitative results are questionable in complex flows.

Surface hot-film or hot-wire techniques, all based on Reynolds Analogy, have been used for a great deal of skin friction studies in the past. Figure 1a depicts the basic concept of these techniques. A small film or wire is heated above the temperature of the flow, and the convective heat losses from the sensor, measured by a constant-temperature anemometer, can be correlated to the wall shear stress by means of a calibration. Brown ${ }^{4}$ showed that the heat transfer from a heated element on a surface to the boundary layer is related to skin friction by Equation 4

$$
\tau_{w}=\frac{19}{10} \frac{\mu^{2}}{\operatorname{Pr} \cdot L^{2}}\left(\frac{q}{k \Delta T}\right)^{3}-\frac{5}{18} L \frac{d p}{d x} \frac{k \Delta T}{q}
$$

where $\mathrm{L}$ is the effective length of the film. However, since a constant-temperature anemometer is used, the calibration usually reduces to a form of King's Law, Equation 5,

$$
U_{B}^{2}=A+B \cdot \tau_{w}{ }^{n} .
$$

where $\mathrm{n}$ is commonly between 0.25 and 0.3 . Obviously, these sensors must be calibrated over the temperature range over which they are to be used, as the rate of convection to the 
flow varies non-linearly with temperature, with an example depicted in the second column of Figure 1. These sensors are usually constructed on low thermal conductivity substrates, such as Pyrex, to limit conduction within the surface, which would effectively increase the size and decrease the sensitivity and time response of the sensor. ${ }^{5}$ As well as being simple, the thin-film/wire gage can be used in facilities with short run times. ${ }^{6}$ This technique performs rather well in cases where Reynolds Analogy may be applied. However, in those flows where combustion, mixing, unsteadiness, or 3D effects make the assumption that there is a simple relation between momentum and heat transfer tenuous, the validity of the method is questionable. There is an obvious temperature limit to this method at a point where the sensor can not carry the necessary current to heat the element above the flow temperature to allow convection from the surface. Conduction through the substrate limits the time over which the calibration can rigorously hold. ${ }^{7}$ Also, in separated regions, even those that are $2 \mathrm{D}$, it is not possible to determine flow direction. In summary, there is a class of flows where the hot-film/wire technique is valid and useful, yet not to a level where quantitative values can be determined, and there are many classes of flows where the sensor can not be utilized.

The use of a sublimating surface coating has, in the past, been commonly used in a qualitative manner to identify transition based on the increased rate of sublimation due to increased skin friction in the turbulent flow. Owen and Ormerod ${ }^{8}$ made a quantitative investigation of this technique for the mass transfer from a small finite region and obtained the following expression,

$$
\frac{\dot{m} l}{\rho \cdot D_{12} \Delta \psi}=0.54\left({\frac{l^{2} \tau_{w}}{D_{12} \mu}}^{1 / 3},\right.
$$

where $m$ is the rate of mass transfer per unit area, $D_{12}$ is the molecular diffusion coefficient, and $\Delta \psi$ is the difference in concentration between the surface and the airstream. Equation 6 only holds for situations where the diffusion layer is smaller than the velocity laminar sublayer. This restriction limits the use of this correlation to small regions, confining the diffusion layer's growth. A more detailed study of the technique was made by Sherwood and Trass ${ }^{9}$, using naphthalene in both subsonic and supersonic 
flows. At this time though, this technique has not been developed to the point of being a useful technique, mostly due to its inadequacies in obtaining accurate quantitative results.

\section{Flow Around Obstacles}

These techniques all use a small obstacle to create a pressure difference and the assumption of wall similarity of flow in a turbulent boundary layer to derive skin friction. Although, as Figure 2 indicates, there are numerous obstacle flow skin friction meters, each of which have various efficacy, only the sublayer fence and Stanton tube are widely used (except for the Preston tube which will be detailed in the next section), therefore only those will be covered in this review. Further detail is provided in Winter ${ }^{10}$ for the interested reader.

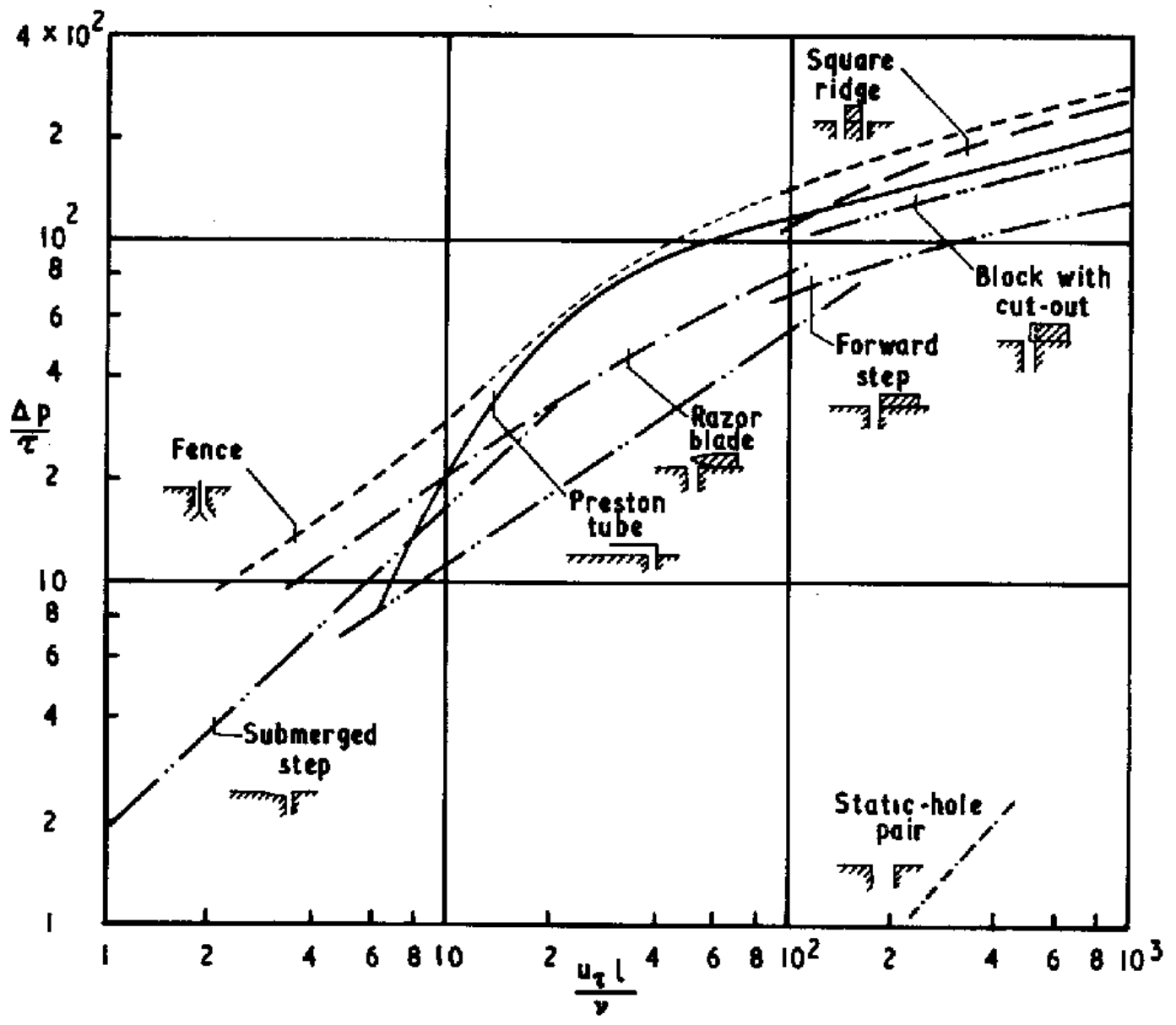

Figure 2. Sensitivities of various obstacles as skin friction meters. ${ }^{10}$ 
The sublayer fence was first suggested by Konstantinov. ${ }^{10}$ The technique, as shown in Figure 1a, consists of a small vertical strip that protrudes into the laminar sublayer of the boundary layer and chambers for pressure reading on either side of the fence. As described in Nitsche, et al. ${ }^{2}$, the sublayer fence technique is based on the laminar sublayer similarity law,

$$
u^{+}=y^{+}, u^{+}=\frac{u}{u^{*}}, y^{+}=\frac{u^{*} \cdot y}{v} .
$$

The differential pressure measured across the fence is correlated to the local wall shear stress through a relationship between the pressure and the velocity distribution in the laminar sublayer. The calibration is determined empirically (a prototypical example is shown in the second column of Figure 1), but the parameters are based on laminar sublayer variables

$$
\tau_{F}^{+}=\left(\frac{u^{*} \cdot h / 2}{v}\right)^{2}, \Delta P_{F}^{+}=\frac{1}{2}\left(\frac{u_{F}}{u^{*}} \frac{u^{*} h / 2}{v}\right)^{2}, u_{F}=\sqrt{\frac{2 \Delta p}{\rho}},
$$

where half the height of the fence is taken as the characteristic wall distance and assuming a fictitious flow velocity proportional to the differential pressure. As well as developing a relatively large pressure difference, the sublayer fence has the advantage that it is small enough to remain within the laminar sublayer, therefore it can be used in a flow with strong pressure gradients. The small size also make compressible flow measurements possible, allowing for only minimal density variation normal to the wall. In addition, the fence's fore and aft symmetry make it useful even in cases with flow reversal, such as separation. However, the small size of the fence makes its geometry difficult to define and, therefore difficult to calibrate. Also, as can be seen in the calibration variables in Equation 8, the properties of the boundary layer must be known $a$ priori and must be able to be defined well, which means flows where the free-stream conditions are difficult to define can not be accurately considered. Finally, the calibration is only valid for steady, 2D flows, removing most complex flows from consideration.

The Stanton tube is actually a cross between an obstacle method and the Preston tube (discussed in the next Section). As shown in Figure 3, the technique consists of a razor blade over a static pressure port. The razor blade makes a total pressure port that 
can be positioned much closer to the surface than a Preston tube, within the laminar sublayer, reducing the effects of pressure gradients and compressibility on the results. Yet, the calibration parameters are very similar to those of the sublayer fence, based on laminar sublayer variables. A big attraction of this technique is the ability to transform any pressure port into a skin friction sensor. However, error sources even in a simple flow abound with the technique, from the size and shape of the pressure hole, to the position and height of the razor, to the difficulty of calibrating a sensor that is already in place in the test fixture. Even if these difficulties were overcome, all the problems stated with the sublayer fence would remain.

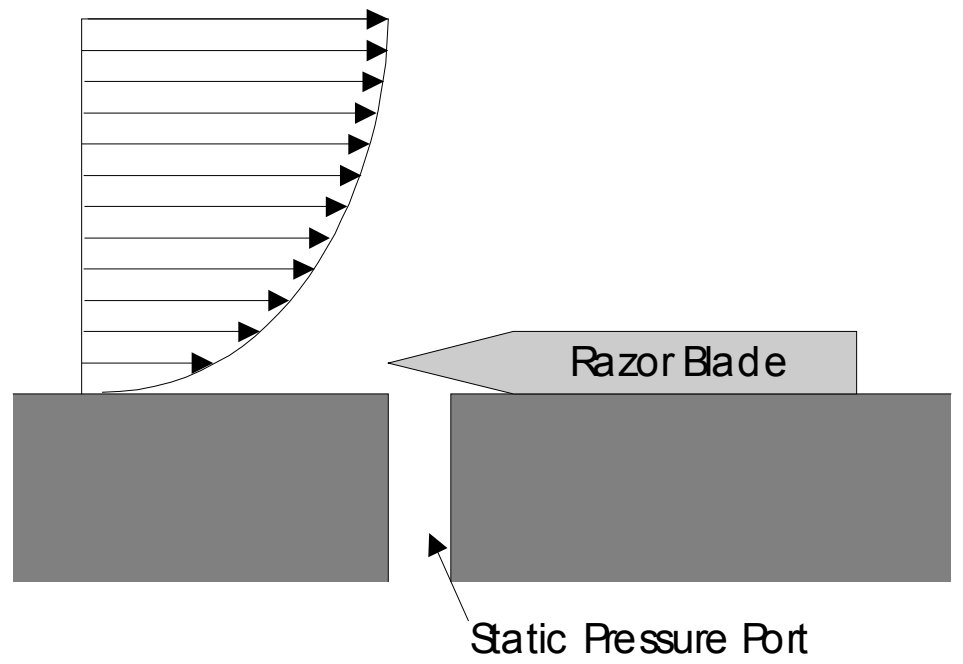

Figure 3. Stanton tube technique.

\section{Profile Measurements}

As defined in this discussion, indirect wall shear stress evaluation using profile measurements are techniques which measure the velocity within the logarithmic region of the law of the wall, and relate that to skin friction through a Clauser plot. The velocity profile in the log region is

$$
\frac{u}{u^{*}}=A \cdot \log \left(\frac{y u^{*}}{v}+B\right.
$$

or

$$
\frac{u}{U} \frac{U}{u^{*}}=A \cdot \log \left(\frac{y U}{v}\right)+A \cdot \log \left(\frac{u^{*}}{U}\right)+B
$$


so that a series of lines relating $\mathrm{u} / \mathrm{U}$ as a function of $\mathrm{yU} / \mathrm{v}$ may be drawn with $u^{*} U=\left(\frac{C_{f}}{2}\right)^{1 / 2}$ as a parameter. The chart in Figure 4 is used by superimposing a measured velocity profile and obtaining the skin friction coefficient by matching. The result is, of course, based on the choice of A and B in Equation 9 and 10, which is of some debate. Although one could attempt to measure many velocities in the log region and use the Clauser plot to determine skin friction, the methods of this section, with the possible exception of laser doppler anemometry, attempt to measure velocity at a known distance from the wall and relate that to wall shear stress. Obviously, these techniques work better in incompressible, turbulent flows where the law of the wall is well defined. In other regimes where it is difficult to correlate boundary layers, such as compressible flows, flows with very large pressure gradients, flows near separation zones or relaminarization, unsteady or 3D flows, or those flows with changing thermophysical properties, the use of these techniques becomes suspect. Also, each of these techniques requires knowledge of the flow properties, especially the viscosity.

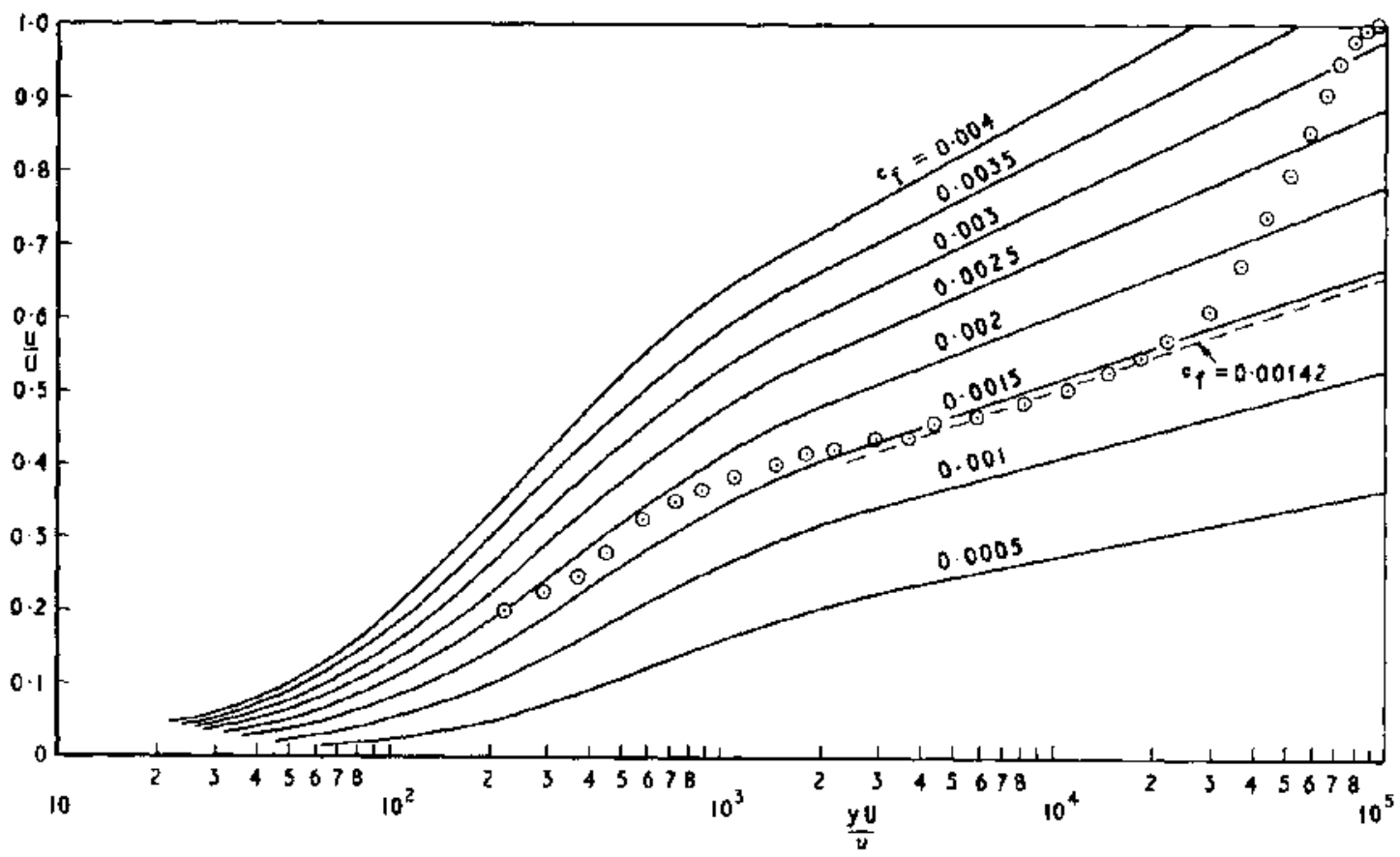

Figure 4. Clauser chart. (from Winter ${ }^{10}$ ) 
The Preston tube, as seen in Figure 1c, is a widely used technique due to its ease of construction and use. Similar to the Stanton tube, in that it attempts to use a single pressure measurement along with the assumed similarity of turbulent boundary layers to determine skin friction, the Preston tube consists of a circular Pitot tube lying on the surface. However, unlike the Stanton tube, the Pitot tube not only senses the laminar sublayer but the overlap and logarithmic regions of the boundary layer, i.e. the entire law of the wall, as well. As with the sublayer fence, the Preston tube calibration parameters arise from the law of the wall variables,

$$
\tau_{P}^{+}=\left(\frac{u^{*} \cdot d / 2}{v}\right)^{2}, q^{+}=\frac{1}{2}\left(\frac{u}{u^{*}} \frac{u^{*} \cdot d / 2}{v}\right)^{2}
$$

with half of the tube's diameter taken where the dynamic pressure is effectively read. The observed relationship between the law of the wall and the calibration curve, an example seen in the second column of Figure 1, indicated that use of the calibration for cases that deviate from the normal equilibrium turbulent boundary layer case is difficult, including all the cases mentioned above.

However, the large user base of Preston tubes has lead to many attempts to determine correction factors for many situations, including misalignment, wall roughness, heat flux, and pressure gradients, and "rules of thumb" for the acceptable uncertainty in those cases. ${ }^{10}$ In compressible flows there is the difficulty of not only defining the correct temperature to use for thermophysical property evaluation (a negative to the technique in itself) but also relying on the assumption that the pressure reading is taken from the center of the tube.

A variation of this technique, as described in detail in Nitsche, et al. ${ }^{2}$, is to include several tubes of different sizes and eliminate the need to rely on the law of the wall variables in the calibration. As depicted in Figure 1 and Figure 5, the different tube diameters effectively read the dynamic pressure at different heights within the boundary layer, and through a computational process arrive at the velocity distribution, and hence the wall shear stress. Also, the last design of Figure 5 is used for 3D flows, where the outer tubes are balanced to find the flow direction, and then used along with the middle tube to determine the velocity distribution. 


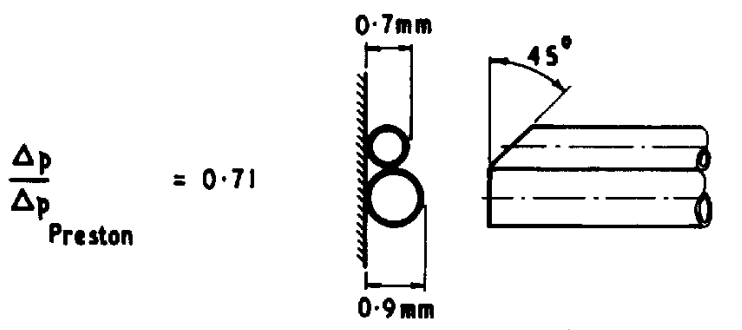

(a)

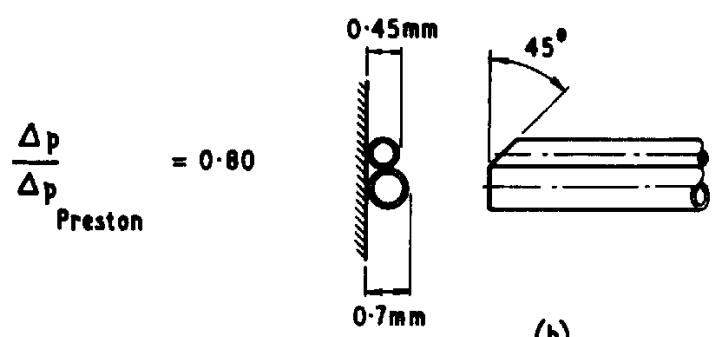

(b)

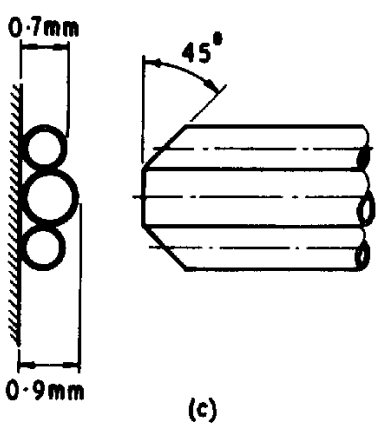

Figure 5. Modified Preston tubes. (Gupta ${ }^{11}$ )

The wall-fixed hot-wire method, as shown in Figure 1d, is very similar to the Preston tube technique as both are based on the law of the wall. However, as the hotwire measures the velocity at a known distance from the wall, instead of the displaced distance measured by a wall mounted Preston tube, the measurements can more readily be correlated to wall shear stress. The calibration parameters are very similar to those of the Preston tube, with the $\mathrm{d} / 2$ of Equation 11 replaced by $\mathrm{h}$, the height of the hot wire and the dynamic pressure parameter replace by a velocity parameter

$$
\tau_{H}^{+}=\left(\frac{u^{*} \cdot h}{v}\right)^{2}, u_{H}^{+}=\frac{1}{2}\left(\frac{u}{u^{*}} \frac{u^{*} \cdot h}{v}\right)^{2} .
$$


Although this technique has the same limitations as those of the Preston tube in flow regimes with pressure gradients or heat flux, the limitations can be regarded as less severe, as the hot wire can be placed much closer to the wall than the Pitot tubes. Also, the hot wires interfere with the flow much less than the Preston tubes, generating a more believable result at the expense of a serious loss of ruggedness. A separate issue with the hot wire method, though, is that flows with high temperatures or varying temperatures in time or space make the measurements much more difficult if not impossible. As with the computational Preston tube technique, the hot wire method can be expanded to multiple wires, as shown in Figure 1f, eliminating the need for calibrations in flows that deviate from the law of the wall.

A final profile measurement technique is Laser Doppler Anemometry (LDA). As with any LDA method, the flow is "seeded" with small particles that follow the flow, A laser beam is split and crossed within the flow, creating a interference pattern. As the particles pass through the interference pattern, light pulses are generated, which are counted and through a statistical method the velocity within the volume of the crossed beams is determined. This method can be used in the same manner as the hot-wire technique, without the concern of changing calibrations due to temperature, as long as the seeds in the flow survive. There is an additional concern that the seed particles will not be able to completely follow the flow when the measurement is near shocks or very near the wall, at least when the $\mathrm{y}^{+}$is of the order of the seed size. This technique also requires optical access, which may be difficult in many test situations.

\subsubsection{Semi-Direct Methods}

The term semi-direct method refers to a class of wall shear stress measurement techniques which do directly measure the shear at the wall, but in ways which, unlike completely directs methods, severely limit their use in all flowfields. These techniques have in common the ability to globally measure skin friction with rather good spatial resolution, while remaining non-intrusive to the flow. The weakness of these methods is large temperature sensitivity, inability to determine flow direction and the requirement for optical access to the test article during the test. These restrictions obviously limit 
their use in complex, 3D, and high enthalpy flows, but the benefits in simple cases do make these techniques useful and advantageous. These techniques include oil film interferometry, sometimes referred to as a Laser Interferometer Skin Friction (LISF) Meter, and shear-sensitive liquid crystals.

Oil film interferometry, based on work by Tanner and Blows ${ }^{12}$, is based on the time rate of thinning of an oil film on a polished surface subjected to aerodynamic shear. Under proper conditions, the skin friction can be deduced without reference to the properties of the boundary layer. The concept is relatively simple. A thin oil film is applied to the test surface with the leading edge perpendicular to the streamline. The oil thickness decreases with time due to the wall shear stress of the air flow, as depicted in Figure 6. A laser beam is directed to the film, with a portion of the laser light reflected at the air-oil interface, R1, and the rest reflected at the surface of the test article, R2. The path length of the light from the laser reflected from these two interfaces differs according to the oil film thickness. As the oil film thins, a time sequence of interference fringes occurs due to this path length difference.

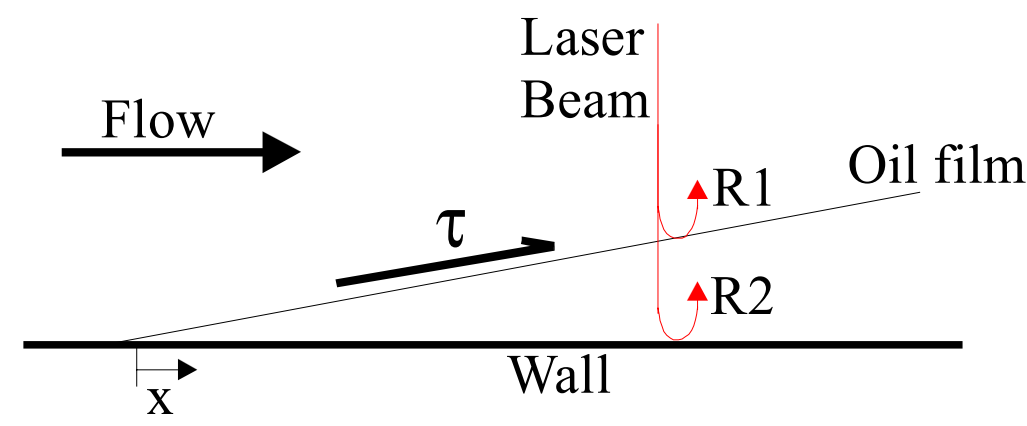

Figure 6. Oil film interferometry method.

Tanner and Blows showed that the time variation of the thickness of an oil film, $\mathrm{h}$, in a $2 \mathrm{D}$ flow is given by,

$$
\frac{\partial h}{\partial t}=\frac{1}{3 \mu} \cdot \frac{\partial}{\partial x}\left(h^{3} \cdot \frac{\partial p}{\partial x}\right)-\frac{1}{2 \mu} \frac{\partial}{\partial x}\left(h^{2} \tau_{w}\right) .
$$

Now, an oil film subjected to a constant shear stress will assume a wedge shape. Taken from Wideman, et al. ${ }^{13}$, Equation 13 reduces to the basic hydrodynamic LISF equation which for this case is 


$$
\tau=\frac{\rho_{o} \cdot v_{o} \cdot x}{h \cdot t} .
$$

From refraction theory, the oil thickness can be determined from the equation

$$
h=\frac{N \cdot \lambda}{2 \cdot n_{o} \cdot \cos \left(\theta_{t}\right)}
$$

Combining Eqns. (14) and (15), the expression for the wall shear stress becomes

$$
\tau=\frac{2 \cdot n_{o} \cdot \rho_{o} \cdot v_{o} \cdot x \cdot \cos \left(\theta_{t}\right)}{N \cdot \lambda \cdot t},
$$

where $\mathrm{N}=$ effective fringe number, $\mathrm{t}=$ effective oil flow time, $\mathrm{n}_{\mathrm{o}}=$ oil refractive index, $\rho_{\mathrm{o}}=$ oil density, $\nu_{\mathrm{o}}=$ oil viscosity, $\lambda=$ laser wavelength, $\theta_{\mathrm{t}}=$ oil refraction angle, and $\mathrm{x}=$ distance in the streamline direction. It can be concluded that given a time history of the fringe pattern, one could, in principle, determine the shear.

However, there are obvious problems which limit the use of this technique. ${ }^{14}$ First, the viscosity of the oil is very temperature sensitive, therefore one would require accurate temperature readings to remove this effect and be able to take measurements in even moderately high-speed flows. In addition, the underlying oil flow equations assume zero shear and pressure gradients and a constant wall shear stress. Although corrections are available for these gradients and small run time would limit changes in shear stress, this obviously limits this technique's use in complex flows, such as separation regions. Also, in high shear flows, above $1000 \mathrm{~Pa}$, surface waves can appear on the oil, disrupting the laser coherence, and preventing usable interference fringes from being obtained until the oil becomes very thin. However, the thin oil leads to a loss of fringe pattern. If the film becomes too thin and approaches the wavelength of the laser, one can lose the ability to determine many fringes, which limits both spatial and measurement resolution. This situation would be more likely to occur for longer test runs, and this effectively limits the viable testing time for this technique. Finally, the reduction of the fringe pattern requires a priori knowledge of the streamline pattern, complicating a test even further.

Shear-sensitive liquid crystals provide another semi-direct method, pursued early on by Klein and Margozzi ${ }^{15}$, to measure skin friction on a test article. Liquid crystals are mostly derivatives of cholesterol which appear as viscous liquids and yet show many of the features of solid crystals. One of these features is selective light scattering which 
means that when illuminated by unpolarized white light, liquid crystals strongly reflect only one wavelength at each viewing angle.

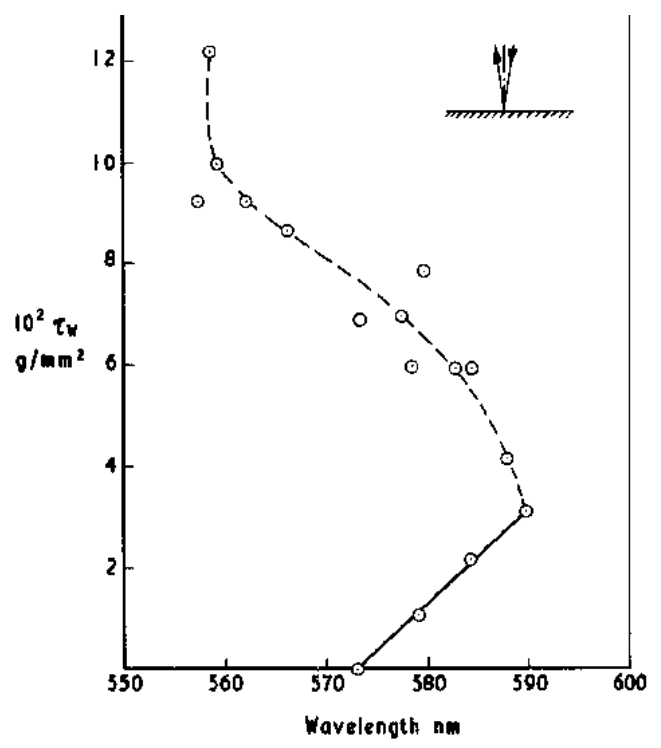

Figure 7. Wavelength of light scattered from liquid crystals under shear stress. (Klein and Margozzi)

The liquid crystals can be formulated so that small changes in surface shear stress can cause a change in the molecular structure of the liquid crystal and shift the wavelength of the reflected light in a continuous manner, as seen in Figure 7. By a careful calibration, these color patterns can be converted back into a shear pattern for the whole test article. Obviously, this technique is non-intrusive to the flow and does not require any knowledge of the boundary layer. The difficulty is formulating a liquid crystal which is highly sensitive to shear while being insensitive to temperature and pressure, and this has proven to be a large hurdle to the wider adoption of this technique. Plus, the necessary calibration has proven difficult to conduct accurately, and, without the calibration, this technique is reduced to yielding qualitative results of such things as areas of high and low shear and the location of transition. ${ }^{16}$ Also, the liquid crystal does within a matter of a day crystallize and lose its light scattering ability, and would have to be stripped off and newly applied. Since this method relies on a liquid on the test surface, high shear levels cause the same surface waves seen in the oil interferometry technique. 
Finally, as with the LISF, this method requires optical access, which may be impossible with the given geometry of the test piece.

\subsubsection{Direct Methods}

The first recorded direct measurement of skin friction was made over 125 years ago by Froude (Figure 8) who was performing research on the resistance of ship hulls, followed by Kempf (1929) and Schoenherr (1932), whose data formed the basis of the incompressible flow skin friction estimations. Other than the work of Schutz-Grunow (1940), the interest in direct skin friction measurements did not peak until aircraft speeds required precise measurements in compressible flow. A much more detailed history of direct measurements of skin friction is presented in Winter. ${ }^{10}$ Direct measurements, as defined in this discussion, refer to techniques that separate a small element, referred to as a floating head, from the wall and measure the tangential force that the flow imparts on it. Direct measurements are the most believable of all the techniques. The sensor is measuring the actual shear on the surface, no matter what the fluid is, what the state of the boundary layer is, or what chemical reaction is occurring. Also, since the floating head is level with the wall, the measurement is non-intrusive to the flow. Of course the forces are very small, sometimes requiring large floating heads and expensive instrumentation to obtain accurate results. The direct measurement technique generally falls into two categories, nulling and non-nulling.

In the nulling design, called this because the floating head is returned to its null position, the floating head is acted upon by shear, but the sensor provides a restoring force to the head to keep it in place. The magnitude of that restoring force is monitored, which is equal to the shear force acting on the floating head. An example of a nulling design is shown in Figure 9. In this balance, the floating head was supported by a beam which pivoted around a spring near the base. The movement of the head was sensed by a pair of capacitance plates, with an electromagnet used to supply the restoring force. Other nulling designs have used Linear Variable Differential Transformers (LVDT) to determine position and mechanical linkages and motors to return the head to the null position. Although the nulling design does remove some of the possible error introduced 
by the movement of the head, the sensors are very complex, mechanically unreliable, and have a slow time response.

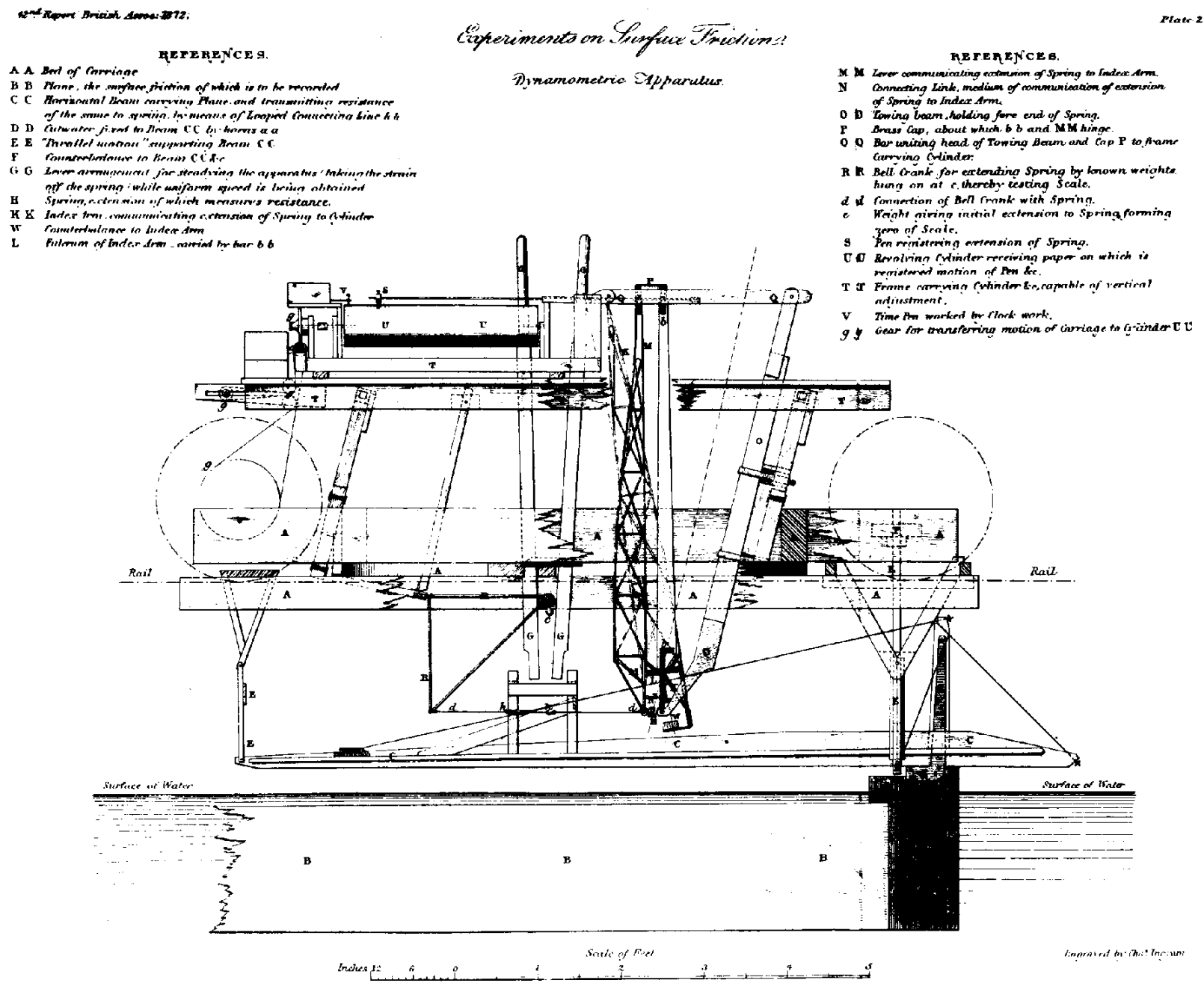

Figure 8. Apparatus used by Froude to measure skin friction on planks. 


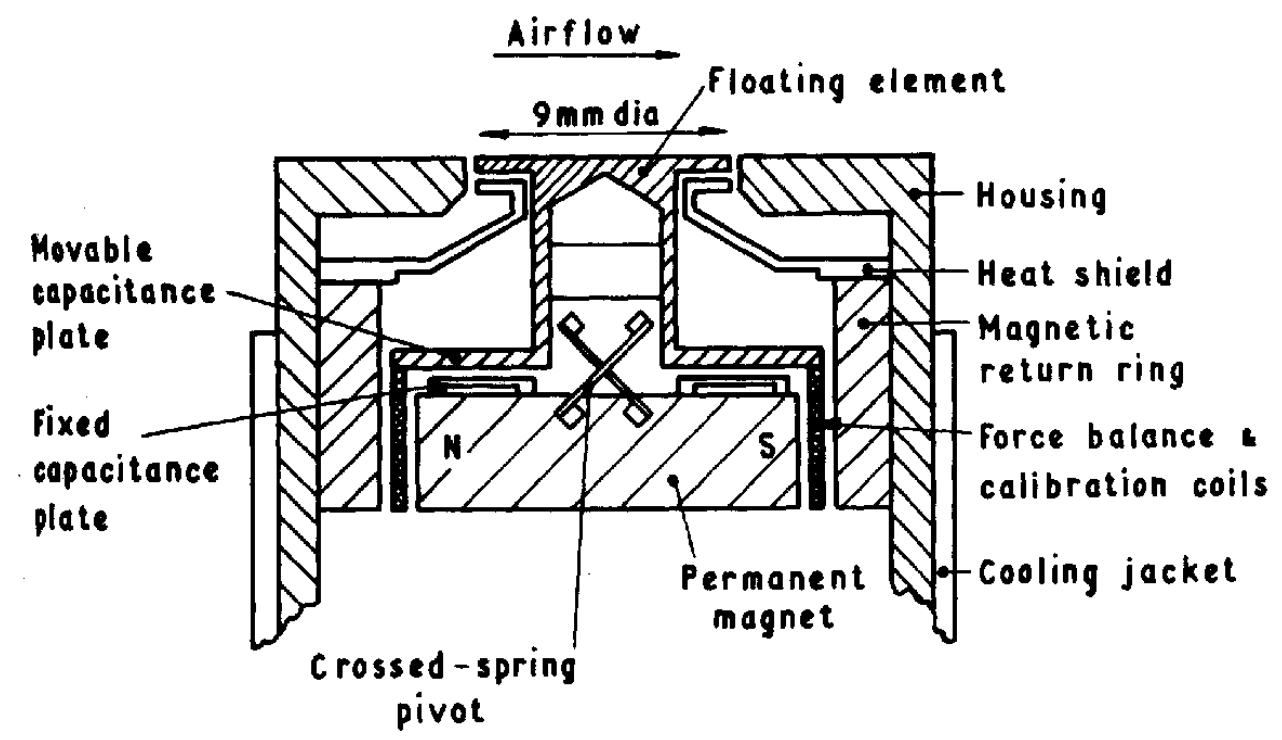

Figure 9. Example of nulling design. $\left(\operatorname{Kistler}^{17}\right)$

In a non-nulling design, the floating head is allowed to deflect slightly under the shearing load. This type of design is much simpler than a nulling design, allowing the removal of the restoring force mechanism, thus making much smaller time responses possible. However, this arrangement does allow the structure of the sensor to flex and this variation may introduce spurious results, as studied by numerous researchers and explained in a later section. One early arrangement, as shown in Figure 10, was a parallel linkage design, where the floating head was supported by two beams that flex with the applied shear. In this design, the movement was monitored by an LDVT near the base. Unfortunately, the two flexures require that the sensor be large and not as sensitive as other designs. 


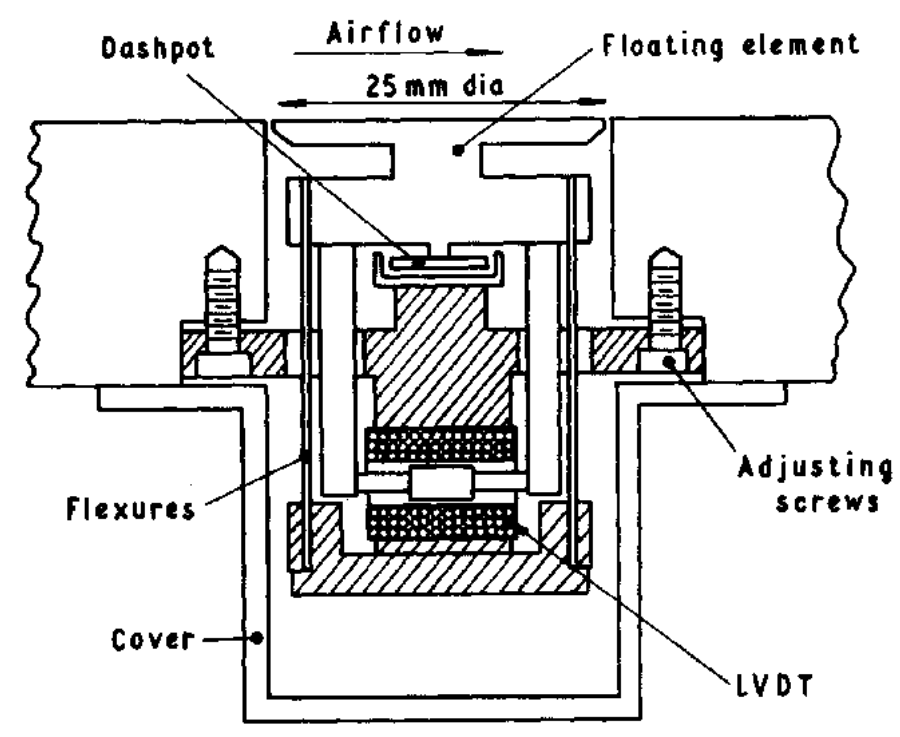

Figure 10. Parallel-linkage balance. ${ }^{10}$

Another type of non-nulling gage is a design where the floating head is supported by tethers around its periphery. Figure 11 is a photograph of a recent micromachined version of this type. ${ }^{18}$ The difficulty with this design is that it is sensitive to normal pressure changes, as the tethers are more sensitive to motion normal to the wall than parallel to it. In macro-designs, where the displacement was measured by strain gages mounted on the tethers, the sensor was not only sensitive to normal pressures, but also to temperature changes as the strain gages were very near the flow. Also, in supersonic flows, wall temperature can change significantly during a test. However, the heat flow boundary conditions are significantly different at the floating head than the rest of the wall, leading to significant temperature differences and, as will be demonstrated later, significant errors in the shear measurement. In present micromachined designs, the strain gages have been replaced by capacitance, as in the sensor in Figure 11, or by an external laser/photodiode system. ${ }^{19}$ The drawbacks of the table-top design remain, limiting the use of the sensor to simple flows without large temperature or pressure variation. In fact, the recent pursuit of the table-top design has more to do with the state of current surface micromachining technology than the soundness of the approach. 


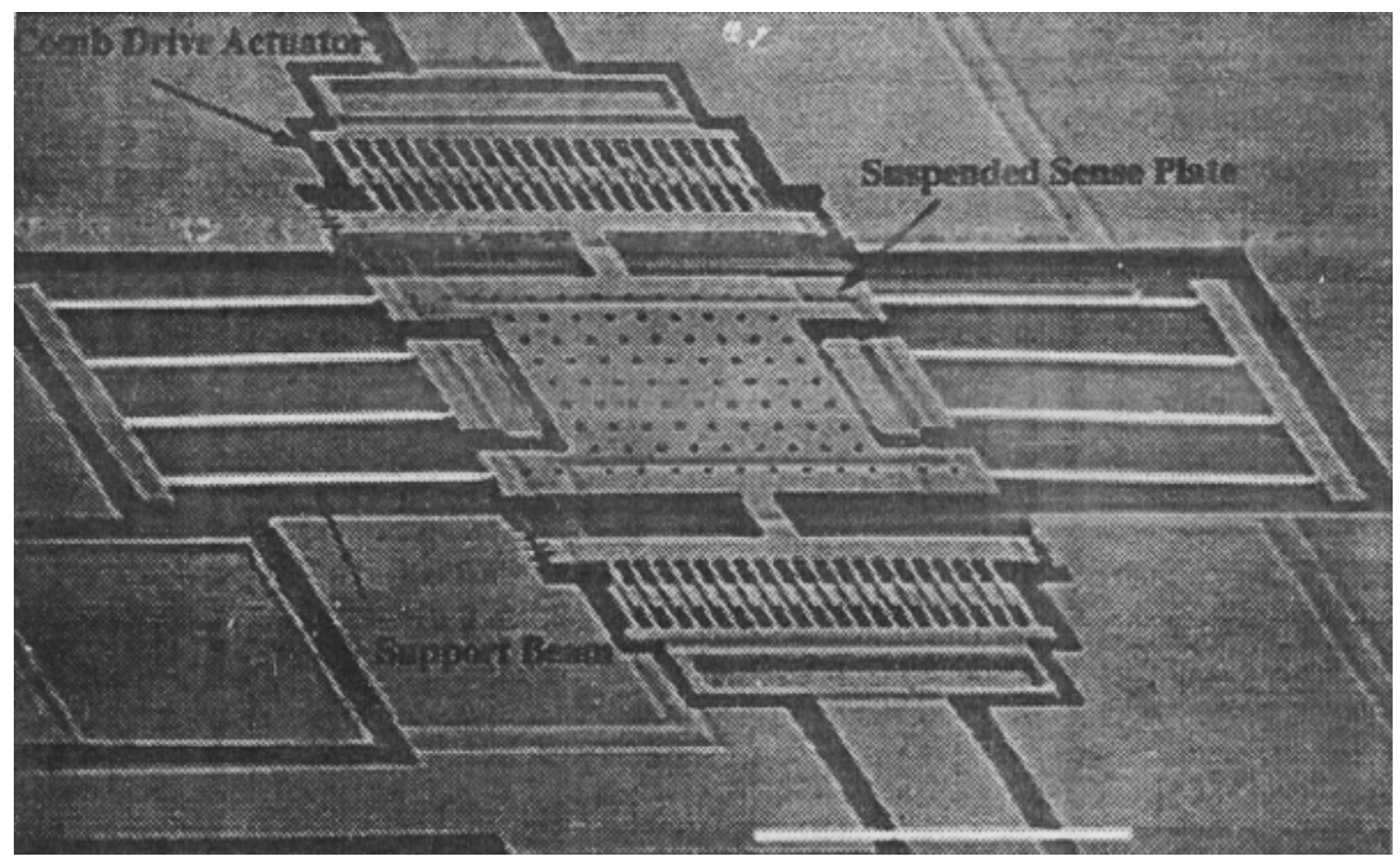

Figure 11. Silicon micromachined version of a table-top design.

Another non-nulling design, and the one used in this study, is a cantilever beam concept. As depicted in Figure 12, the floating head is attached to a single cantilever beam that flexes with the application of shear to the head. In the earlier work at Virginia Tech, the displacement was measured by the strain that it caused at the base of the beam. If the displacements are kept to a minimum, the issue of head protrusion into the flow is negligible. This design offers high stiffness for normal forces, while being relatively weak for tangential forces, providing a sensor that is insensitive to normal pressure variations. The concept is very simple, rugged and has a short time response. If the sensing head is constructed of similar materials as the surrounding wall, errors from temperature mismatches will be minimized. Also, the concept can be easily extended to measure in two directions, removing the directional ambiguity of other designs. 


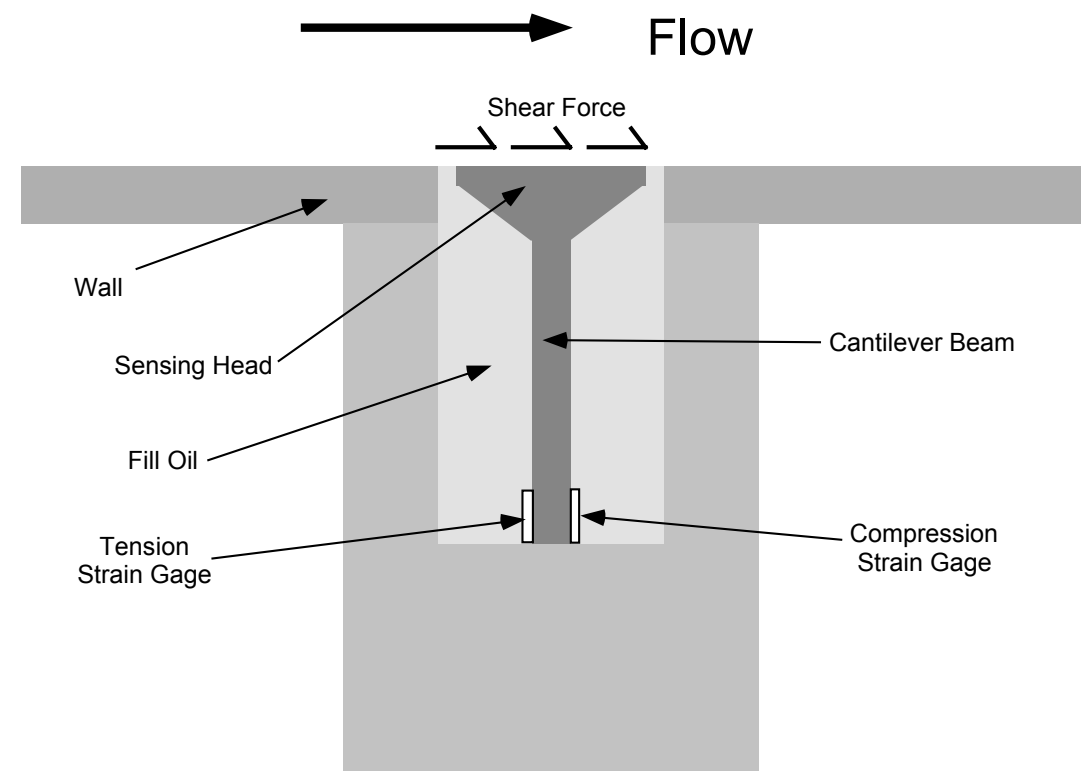

Figure 12. Non-nulling cantilever beam concept.

MacArthur $^{20}$ supported his floating element on a piezoelectric crystal, and although this is a very sensitive method, it only provides transient or dynamic data. Winter and Gaudet ${ }^{10}$ were able to use resistance strain gages in a large balance, which allowed sensitive measurements in a static environment. Schetz and Nerney made the next step to semi-conductor strain gages, improving the output 100 fold. ${ }^{1}$ In fact, Schetz's research group has produced a series of skin friction sensors based on strain gages mounted to the cantilever beam in a non-nulling arrangement. ${ }^{21,22,23}$

As with any sensor, new design concepts take advantage of more sensitive transducing technology as it evolves. In the current study, the sensing technology has moved to fiber optics, allowing a design principle based on deflection instead of strain, which is new in non-nulling cantilever beam designs. Fiber optics offer not only increased sensitivity to displacement but also new design concepts that increase the sensitivity to shear, permitting the area of floating head and length of the cantilever beam to be decreased, therefore reducing the size of the whole sensor package. The use of fiber optics also brings with it all the normal advantages that they have over conventional semi-conductor or foil strain gages, including temperature and electromagnetic radiation insensitivity (useful in weakly ionized gases), increased temperature ceilings and elimination of the spark hazard (important in explosive environments). 
Fiber optics have been used in the past in the operation of a skin friction gage, but this gage was a nulling type in which the fiber optics were used for feedback to the motor. $^{24}$ The gage, as pictured in Figure 13a, uses an optical fiber to shine light on a target attached to the back of the sensing head. This target is reflective on half the surface and absorbing on the other half, as depicted in Figure 13b. The fiber is aligned at the border between the two sides. As the head is deflected, the intensity of the reflected light is changed as the target moves to either side. The motor then counteracts that movement, returning the head to the null position. This concept is markedly different than the current design in both concept and results. The size of the sensor is very large (approximately $135 \mathrm{~mm}$ in diameter with a sensor head $20 \mathrm{~mm}$ in diameter) and as is the case with all nulling designs, the response of the instrument is slow (natural frequency of $0.5 \mathrm{~Hz}$ ). The use of interferometric sensing in the current design allows for very accurate measurements that are not prone to spurious inputs, unlike intensity-based systems, and it does not require the careful alignment to a target, unlike the concept developed in this project.

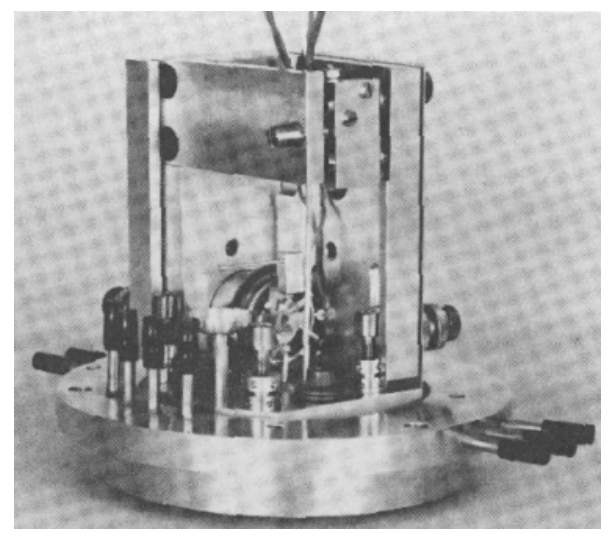

(a)

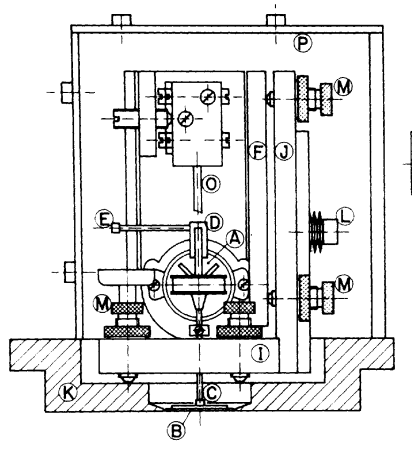

(b)

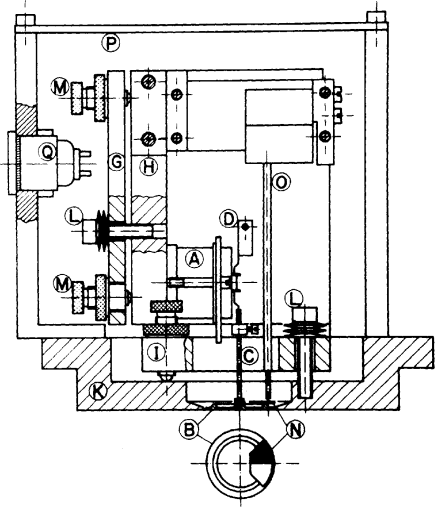

Figure 13. a) Photograph of nulling skin friction sensor design that uses an intensity-based fiber optic system and b) schematic of the same. ${ }^{24}$

\subsection{Surface Pressure Measurement Techniques}

Pressure sensors have been the most used and versatile instruments in flowfield measurements. Pressure measurements can be used to calculate or estimate many other 
flow properties. Typically, pressure measurements are the most cost-effective method of determining much of the flowfield. Therefore, pressure sensors have undergone the most development and changes during the last 50 years. Current pressure sensing technology includes electrical-based transducers, such as piezo-resistive and piezo-electric sensors, and pressure sensitive paint. Simon and Goldstien ${ }^{25}$ and Doeblin ${ }^{26}$ provide interesting and informative reviews of pressure sensing technology.

\subsubsection{Pressure Sensitive Paint}

Traditional measurement techniques for acquiring surface pressure distributions on wind tunnel models have utilized embedded arrays of pressure taps. This method requires construction of a very complicated and expensive model. Yet the data obtained with pressure taps is limited. The pressure is obviously only obtained where there is a pressure tap. Taps can be placed only so close together, limiting the spatial resolution possible. A model has very limited room for the lines that run from the pressure tap to the pressure transducer, limiting the number of taps that can be placed in the model. Also, there are geometric restrictions where a pressure tap can be placed, edges and large curvatures proving to be the most difficult. It is in these regions where the pressure data is desired the most, though. Therefore, the engineer has to be very careful where taps are placed. The judicial placement of the pressure taps is more of an art than science, requiring a great deal of experience.

Frequency response can also be a problem with pressure taps. Fluctuations in the pressure must travel through the lines back to the transducer to be measured. The longer and thinner the line, the greater the time it takes for the new pressure to reach the transducer, limiting the rate at which pressures can be measured. Also, in most pressure tap measurements, the taps are not connected to individual pressure transducers, but are chained together to a limited number of transducers which sample from each line in turn, reducing again the data rate possible. These limitations put a restraint on the data which can be measured with pressure taps, eliminating the high frequency content that may exist in the pressure field.

The development and use of pressure sensitive coatings, that change visual properties depending on the pressure level, promise to transform the art of pressure 
measurement in wind tunnel testing. Conventional wind tunnel testing provides integrated forces and moments through balance measurements and pressure at specific points through pressure taps. Pressure sensitive coatings on wind tunnel models have the promise of providing pressure data at higher spatial resolution and data rates than conventional pressure taps. By determining pressure distributions, pressure sensitive coatings have the potential to predict loads early in the design process with aircraft models. The nearly continuous pressure distribution provided by this method can be integrated over individual model components (e.g. wings, fuselage, control surfaces) to provide detailed load information. These pressure data will allow a greater understanding of the whole flowfield and of the details of the flow leading to the measured forces and moments than is now possible. Successful development of these techniques could also provide great cost savings. Since an aerodynamic force and moment model could be coated, providing the pressure distribution, the construction of a separate, complicated pressure model would be unnecessary, saving time and money. ${ }^{27}$

A pressure sensitive coating, that changes visual properties with pressure level, could solve all the above difficulties. The normal model used for measurements of forces and moments via a 6-component balance could now also be used for the pressure measurements. Images of the model, coated with a pressure sensitive material, would provide a complete view of the pressure field, as depicted in Figure 14. These images would provide the pressure to a spatial resolution equal to the resolution of the camera and a frequency response equal to the refresh rate of the camera. ${ }^{28,29}$ This method is also remote, meaning that wiring does not have to be brought out from the model. This fact means that the method can be used in rotating machinery or other applications where telemetry had to be used in the past. ${ }^{30}$

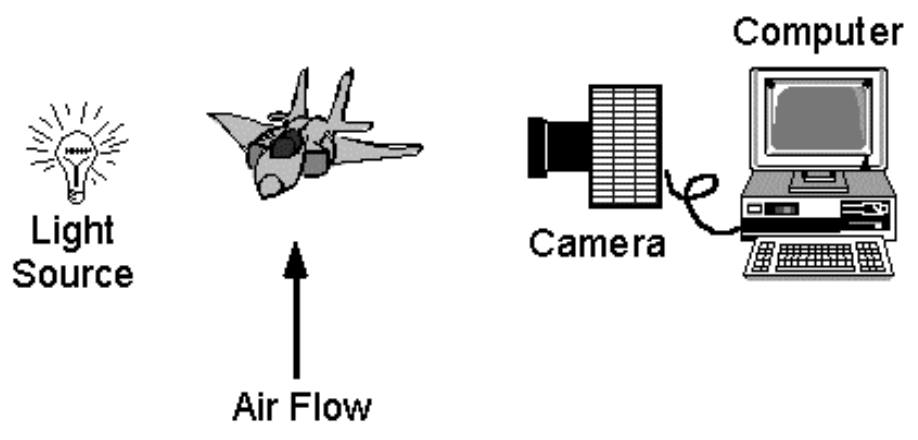

Figure 14. Depiction of the PSP process. 
In the past, pressure sensitive paint (PSP) has been used with varying success. ${ }^{31,32}$ PSP consists of an oxygen permeable polymer binder in which a luminescent dye is dispersed, as depicted in Figure 15. The dye is excited by absorbing light (generally UV or blue) and relaxes by emitting red shifted light. An alternate decay process is through the interaction of the dye with an oxygen molecule (called oxygen quenching). As the pressure above the layer increases, the oxygen concentration within the layer will increase, and the luminescence intensity will decrease. Thus, with more oxygen present, less luminescence is observed, all other variables remaining constant. This change in intensity can be converted to a change in pressure via an appropriate calibration.

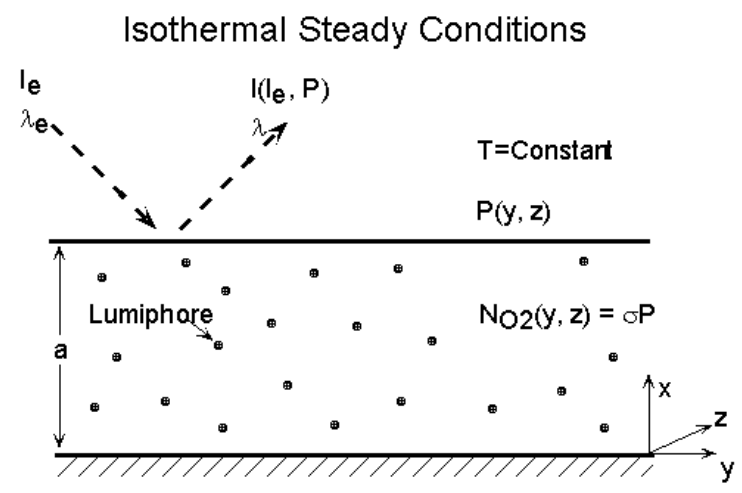

Figure 15. PSP polymer sketch.

Although PSP does provide a complete pressure field at a high data rate, there are some difficulties that limit its usefulness. The paints are temperature sensitive and decoupling the effect is difficult. Therefore, temperature variations across the surface will introduce errors into the measurements. Additionally, unsteady pressures introduce problems, since the oxygen concentration across the layer is no longer uniform due to finite diffusion times. Even when relative pressure measurements are obtained on a model, accurate absolute measurements have been difficult. Also, some or the paint and/or solvents used are toxic, requiring special cleanup and safety procedures, which increase the cost and time required. 


\subsubsection{Conventional Pressure Sensors}

Although pressure sensitive paint has made great strides over the last decade, most pressure measurements are still made with what will be termed conventional pressure sensors, defined as transducers having a portion that deflects with pressure change. One of the oldest technologies is elastic pressure transducers, such as the Bourdon tube (Figure 16), and this is the basis of many mechanical pressure gages. The basic element of the Bourdon tube, is a tube of non-circular cross-section. A pressure difference between the inside and outside of the tube distorts it, which moves an indicator. Similar gages can be made of numerous shapes, yet they all operate similar to the Boudon tube. These sensors, by necessity, are physically large, leading to a slow response.

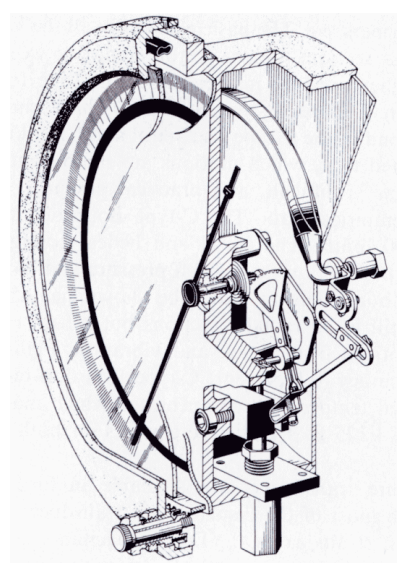

Figure 16. Bourdon tube. ${ }^{26}$

More current transducers use the deflection of a diaphragm to determine the pressure. The deflection is measured in a variety of manners, each with its advantages and disadvantages. A widely used technology is the application of strain gages onto the diaphragm, as depicted in Figure 17. The strain gages, which can either be attached with adhesive or sputtered onto the diaphragm, are then connected through a Wheatstone bridge that provides a voltage output, which is proportional to the strain on the diaphragm. Another option is piezoelectric pressure transducers in which the diaphragm deflection is transmitted to a quartz crystal, and the piezoelectric effect of the compressed crystal creates a charge. Or, the diaphragm can be part of a capacitor, as shown in Figure 
18, changing the distance across the dielectric, which through a simple circuit, can be converted to a voltage. Both of these sensors require little movement to create a signal, therefore they are useful for high frequency measurements. All three of these sensor types are mature technology, so they are repeatable and relatively inexpensive. However, for all, the electronics are internal to the sensor. Large changes in temperature can affect these electronics, and cause significant drift in the data, invalidating results in certain flow conditions, especially harsh ones like supersonic flows. Also, the piezoelectric and capacitance sensors only produce AC signals, which make them useless for slowly changing pressures. It can be seen that a pressure sensor that can be temperature insensitive and have a high frequency response would be useful in harsh environments.

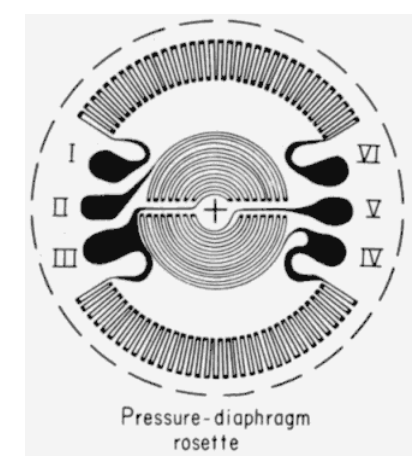

Figure 17. Strain-gage balance rosette on a diaphragm.

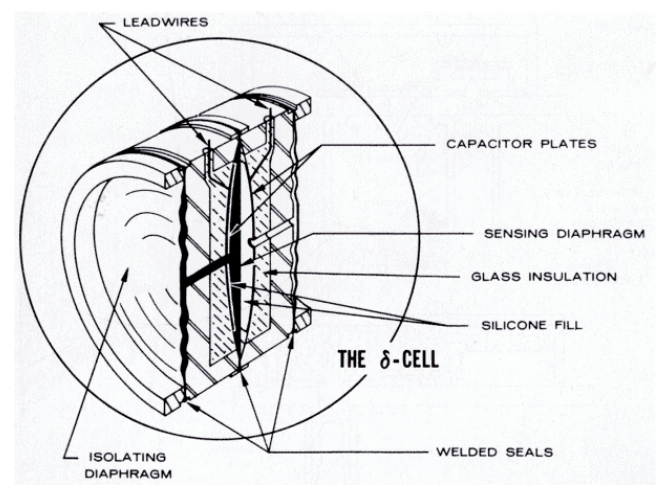

Figure 18. Capacitance pressure transducer. ${ }^{26}$

Due to the large possible market, there has been significant prior work done in developing a fiber optic pressure sensor. Fiber optics promise smaller, more temperature 
and EMI insensitive transducers. These earlier fiber optic pressure sensors either use a simple optical signal processing system that cause as many problems as they solve, or they are not constructed using micromachining techniques. ${ }^{33,34}$

\subsection{Surface Temperature Measurement Techniques}

Temperature measurements are also a long-standing technology that has been developed into many successful transducer types. Two main types exist: those that remotely measure temperature in excess of the limits of normal methods via the radiation the body emits, such as optical pyrometry, and conventional exposure types that use a physical effect to create a voltage change. Diller and $\operatorname{Tien}^{35}$ and Doeblin ${ }^{26}$ provide interesting and informative reviews of temperature sensing technology.

\subsubsection{Optical Pyrometry}

Although there are numerous types of radiation temperature measurements systems, optical pyrometry is a standard industry device and will be reviewed briefly here. All of these sensors measure the radiation emitted by a surface at high temperatures, an ideal being a blackbody that has an emissivity and absorptivity of unity. $^{26}$ The law governing this ideal type of radiation is Planck's law,

$$
W_{\lambda}=\frac{C_{1}}{\lambda^{5}\left(e^{C_{2} /(\lambda T)}-1\right)}
$$

where $\mathrm{W}_{\lambda}=$ spectral radiant intensity

$\lambda=$ wavelength of radiation

$\mathrm{T}=$ absolute temperature of blackbody.

As can be seen, this law indicates that energy is emitted at all wavelengths, but not the same magnitude of energy at all wavelengths. The peak of the curve shifts with temperature, and the total power emitted increases with the fourth power of the temperature. Optical pyrometers, which require high temperatures (above $700^{\circ} \mathrm{C}$ ), utilize the principle that the radiant intensity varies with temperature at a given wavelength. A tungsten filament is then heated by the operator until the brightness matches that of the body as seen through a narrow-band filter. The power needed to heat 
the filament is then compared to a calibration that uses a perfect blackbody. The temperature of the object is then calculated with an estimate of its emittance. This inexact knowledge of the emittance is the major source of error for this method. Also, beside the temperature limit, the need for the operator feedback makes this system useless for applications in which the temperature is quickly changing. Although other radiation measurement systems exist, each suffers from an inability to determine the true emittance of the surface. Therefore, as long as the transducer will survive the temperature, exposure type sensors are typically more accurate.

\subsubsection{Conventional Temperature Measurements}

Although there are a great deal of direct temperature measurement methods, only the most widely used, thermocouples and resistance thermometer detectors (RTD), will be reviewed here.

The thermocouple is the most widely used temperature sensor due to is simplicity and ruggedness. The sensor consists of two dissimilar metals joined at two junctions. When those junctions are at different temperatures, a voltage is created between the junctions. This phenomenon is known as the Seeback effect. Typically, well-known combinations of metals are used, with one of the junctions kept at a reference temperature (normally an ice-bath), providing a known output voltage for the temperature of the other junction. The largest drawback of the thermocouple is the small voltage output, which makes signal noise and grounding major issues. Also, ensuring good physical and thermal contact with the surface to be measured is a major difficulty. A solution is sputtering the thermocouple material on the surface, guaranteeing good contact with the surface and a good junction between the materials. However, as the sputtered material typically has different properties than the bulk material, additional junctions are formed where the lead wires are connected to the sputtered material. ${ }^{25}$

The resistance-based temperature sensors take advantage of the fact that the resistance of most materials changes with temperature. RTD are typically metal wires wrapped around an insulator, as seen in Figure 19. Thin films of metal deposited on an insulator can also be used. The resistance of metals ( $\mathrm{Pt}, \mathrm{Ni}, \mathrm{Cu}, \mathrm{W}$ are commonly used) typically change less than $1 \% /{ }^{\circ} \mathrm{C}$. Platinum is most commonly used due to its precision, 
linearity, and stability. The output of the sensor can be improved by at least two orders of magnitude by using semiconductor materials instead of metals, but this introduces a large non-linearity to the results. ${ }^{25}$ All of these sensors, as was with thermocouples, are sensitive to signal noise and grounding. Additionally, the thin films can be affected by particles or liquid in the flow and at higher temperatures by oxidation of the film, all of which change the resistance independent of the temperature.

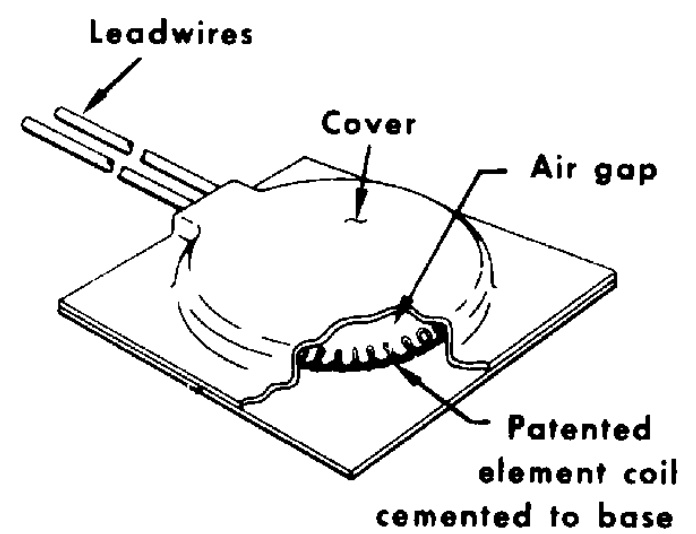

Figure 19. RTD construction. ${ }^{26}$

Previous research has also been performed with fiber optic temperature sensors. These sensors typically work by measuring the growth of a material, such as metal, as its temperature increases, as depicted in Figure 20. These sensors, although capable of operating in some environments in which conventional temperature transducers can not (especially electrically noisy or radiating environments), are too large to make fast measurements with exact knowledge of where the measurement was made. ${ }^{36,37}$

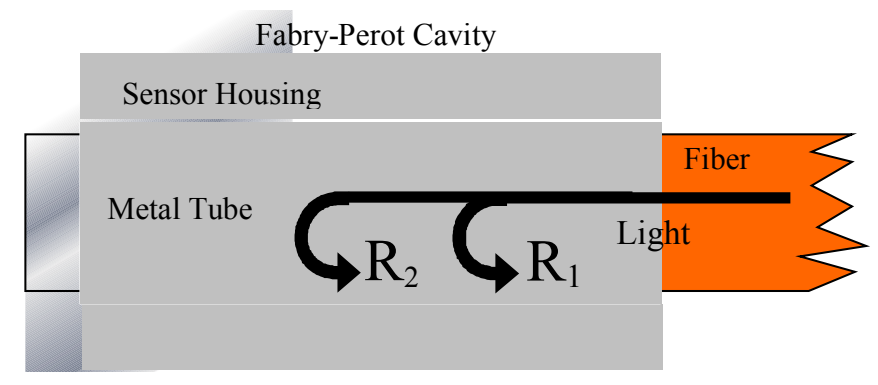

Figure 20. Current fiber optic temperature sensor design. 


\subsection{Heat Flux Measurement Techniques}

The need to measure local convective or total heat transfer has led to the development of several types of heat flux sensors. As with skin friction, heat flux is a quantity that has proven very difficult to accurately determine. The measurement is often affected by the sensor itself, altering heat flux at the sensor from the surrounding surface. Several methods have been developed that provide a direct indication of heat flux, such as Gardon gages and the layered heat flux sensor. However, these sensors operate effectively only in certain types of flows and suffer from the problem stated above. Another method is to use a measurement of the time-history of temperature in the body, which can be obtained through a variety of methods, and then calculate the heat flux through Fourier's Law. Each of these methods will be described below.

\subsubsection{Measurement of Heat Flux}

Although used in a variety of situations, the Gardon gage, as depicted in Figure 21 , is accurate only in constant heat flux cases. The gage works by forming a differential thermocouple between the thin constantan disk and the copper wire. An equilibrium temperature difference is quickly established in a constant heat flux case between the center and edges of the disk that is proportional to the heat flux. If the heat flux is varying, no equilibrium can be established, which is why the gage is limited to a constant heat flux. However, a major drawback of the Gardon gage for any situation more than a moderate heat flux is that the temperature of the center of the disk quickly becomes different than that of the surrounding wall, with the obvious effect on the heat flux measurement.

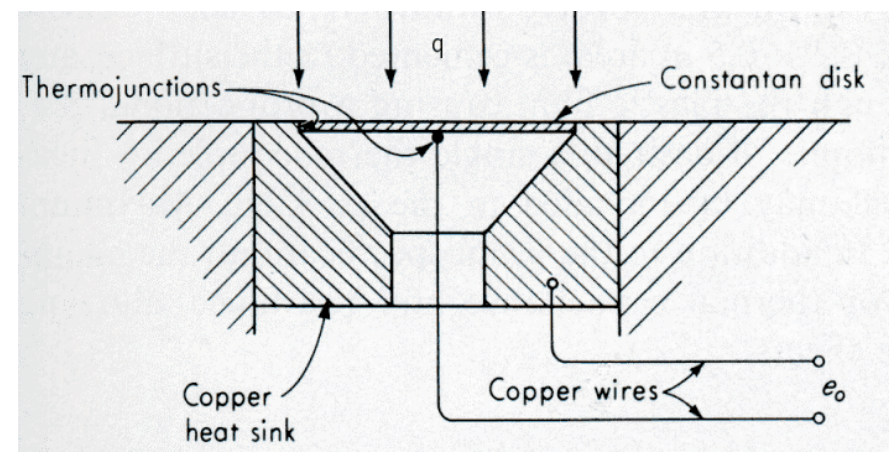

Figure 21. Gardon gage. ${ }^{26}$ 
Heat flux can also be measured using a layered design, such as the one manufactured by Vatell, which operates by having two layers of sputtered thermocouples separated by a known thermal resistance. These sensors can measure both heat flux and surface temperature accurately with a very high frequency response due to the thin layers involved. In order to get this high frequency response, though, the sensor must cover a relatively large area. This large surface area counteracts the frequency response, in that any flow phenomenon that would cause the heat flux to change as quickly as the sensors can measure would be significantly smaller than the area of the sensor. The small sizes of these phenomena cause the fluctuations to average out, mitigating some of the advantages the high frequency response brings.

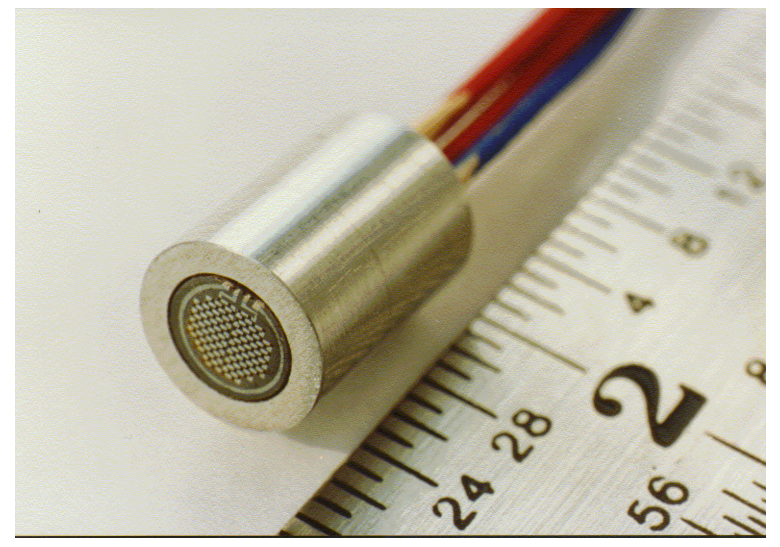

Figure 22. Micro-thermopile sensor. $\left(\right.$ Vatell) ${ }^{38}$

\subsubsection{Calculation of Heat Flux from Surface Temperature Time History}

As mentioned above, the temperature time-history is often used to calculate heat flux. Several methods have been developed for placement of the temperature measurement within the body, which will be discussed. The algorithms used to calculate the heat flux for the temperature time-history will then be reviewed.

\subsubsection{Sensing Types}

A common method of making surface measurements is surface resistance thermometers, which, as described above, consist of a thin film of metal deposited on top of a thin film of insulator on top of the surface to be measured. The resistance of the 
metal coating, such as platinum, is proportional to the temperature. During the test, the resistance of the coating is recorded and related back to temperature through a calibration. One problem for researchers is obtaining a consistent calibration of the coating, as the calibration of sputtered materials tends to change over time.

Surface thermocouples, as depicted in Figure 23, work by forming a thermocouple junction right at the surface. A major difficulty with this method is forming the junction at the surface, and only the surface. If the junction is not at the surface, both the wall temperature and the heat flux measurements are compromised.

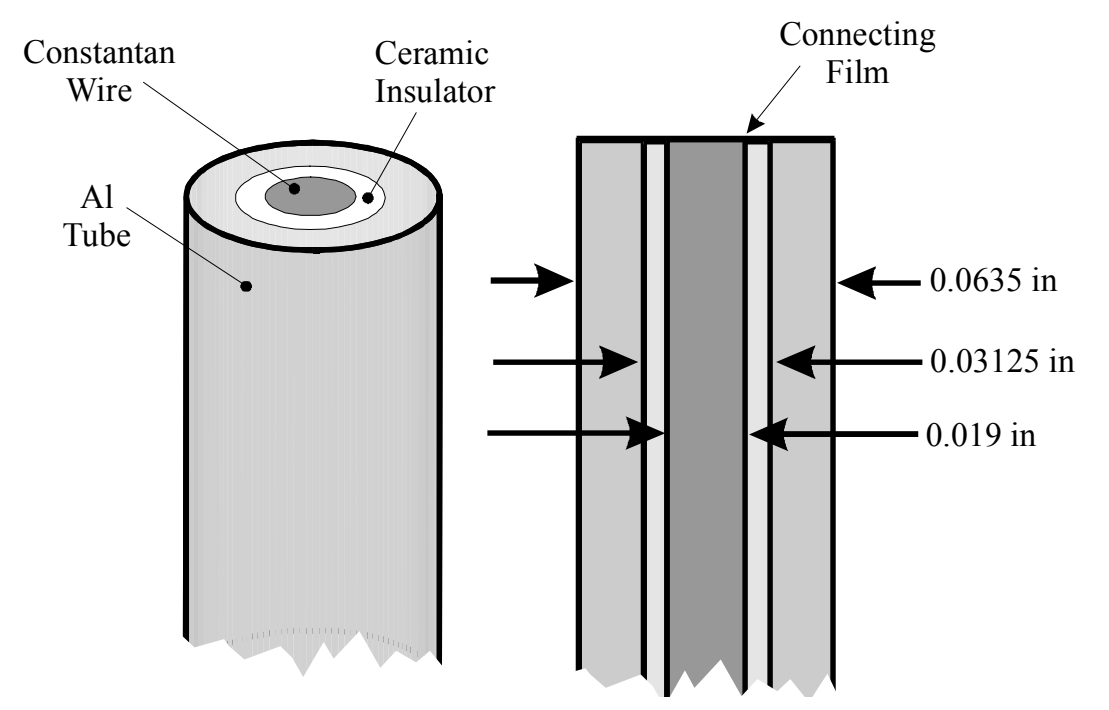

Figure 23. Sketch of aluminum-constantan thermocouple design.

Another option examined is the null point calorimeter, as shown in Figure $24 .^{39}$ In this case, the thermocouple is placed at the end of a hole drilled through the back of the plug. If the geometry is correct, that point will experience the same time history as the surface. Many researchers in the past have used this method, and there is a large information base on making these measurements correctly. Also, with the thermocouple junction below the surface, there is much less chance of the sensor being damaged. 


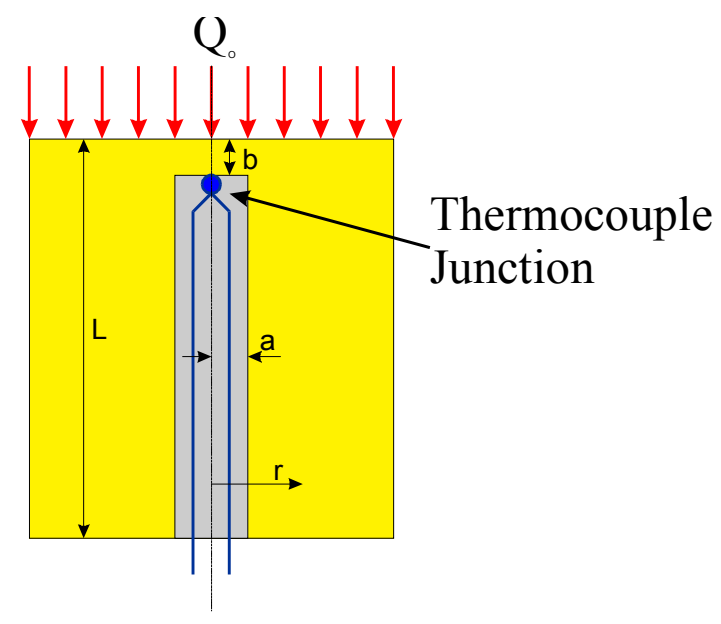

Figure 24. Diagram of the null-point calorimeter concept

\subsubsection{Algorithms}

\section{Cook-Felderman Algorithm}

Heat flux can be deduced from the surface temperature data, by differentiating.

Given,

$$
Q=\left(\frac{d E}{d t}\right),
$$

The solution of this equation in this case has to be done numerically since the temperature is a function of the time. To do this the Cook-Felderman technique ${ }^{40}$ was used as the starting point of the work. According to their formulation:

$$
q\left(t_{n}\right)=\frac{2 \sqrt{k \rho C_{p}}}{\sqrt{\pi}}{ }_{j=1}^{n} \frac{T_{j}-T_{j-1}}{\sqrt{t_{n}-t_{j}}+\sqrt{t_{n}-t_{j-1}}},
$$

where:

$$
\begin{aligned}
& \mathrm{q}=\text { Heat flux }\left(\mathrm{W} / \mathrm{m}^{2}\right), \\
& \mathrm{k}=\text { Thermal conductivity }(\mathrm{W} / \mathrm{m} \cdot \mathrm{K}), \\
& \rho=\text { Density }\left(\mathrm{kg} / \mathrm{m}^{2}\right), \\
& \mathrm{C}_{\mathrm{p}}=\text { Specific heat capacity }(\mathrm{J} / \mathrm{kg} \cdot \mathrm{K}), \\
& \mathrm{T}_{\mathrm{j}}=\text { Temperature at time } \mathrm{j}, \text { and } \\
& \mathrm{t}_{\mathrm{j}}=\text { Time at the end of the } \mathrm{j}^{\mathrm{th}} \text { of } \mathrm{n} \text { time intervals. }
\end{aligned}
$$


This formulation assumes that the interior temperature far from the surface does not change in time; what is termed a semi-infinite solid assumption. This assumption is valid for varying lengths of test time, depending on the thickness of the material, the thermophysical properties of the material and the heat flux expected. The temperature interior to the material can be monitored to determine when the calculations are valid. Unfortunately, as with any differentiation method, noise in the input signal is amplified during the processing. Filtering of the input signal is usually necessary to obtain reasonable results.

By using a "modified" Cook-Felderman ${ }^{41}$ technique the noise problem can be improved through the following formulation:

$$
q(t)={\frac{\left(k \rho C_{p}\right)^{\frac{1}{2}}}{(\pi \Delta t)^{\frac{1}{2}}}}_{I=1}^{m}\left[\frac{T_{I}-T_{I-1}}{\sqrt{m+1-I}}+\left(T_{m+1}-T_{m}\right)+\frac{1}{3}\left(T_{m}-T_{m-1}\right)\right]
$$

This technique offers slightly increased stability and a reduction in noise amplification.

\section{Walker-Scott Algorithm}

Although the Cook-Felderman technique is useful for most situations, the method is not capable of incorporating temperature dependent properties, which can be a major drawback when working with large temperature variations. One possibility is the inverse heat conduction approach developed by Walker and Scott of Virginia Tech. ${ }^{42}$ Walker $^{43}$ performed a similar analysis using this technique, therefore much of his work has been used to formulate an approach to this particular problem. Unfortunately, this problem is not a true inverse problem. ${ }^{44}$ The inverse heat conduction approach generally uses a temperature measured a known distance from the surface (Figure 25). In this case, the temperature is measured on the surface. 


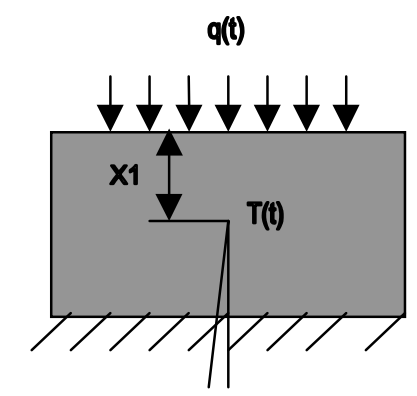

Figure 25. Heat flux on the surface of an object.

One solution is to break the problem into two parts and in this manner, the problem is uncoupled into an inverse and a direct problem. The two parts can be solved separately to arrive at an overall estimate of heat flux. This method is an improvement over the Cook-Feldermann technique, because it avoids calculation of the derivative of the temperature distribution. Thus, the effects of noise in the temperature measurements are reduced.

The problem is broken down into a series of time steps. The inverse part of the problem uses an initial guess for the heat flux and calculates an estimate of the surface temperature. The estimated temperature is then compared to the actual measured temperature. Through iteration, the heat flux is changed until the estimated temperature matches the measured temperature within some predetermined tolerance. The temperature profile through the object is calculated for several steps, and the heat flux is recalculated using the calculated temperature to estimate temperature dependent material properties. Essentially, the first part of the solution estimates a heat flux based on the surface temperature. The second part then calculates a temperature profile based on the estimated heat flux, and then recalculates the heat flux using the calculated temperature profile and temperature dependent material properties, iterating until a solution is found.

\subsection{Scope of the Investigation}

The following chapters will present the process for design, construction, and testing of miniature, fiber optic sensors and their limitations. In Chapter 2, a description of the ideas and principles behind fiber optic instrumentation are discussed. In Chapter 3, 
design principles of the pressure, temperature/heat, and skin friction sensors are explained, as well as the effort to micromachine the designs. In Chapter 4, the results from various testing of the sensors in environments ranging from incompressible to supersonic flows will be presented and discussed. A study of the limitation of these fiber optic sensors due to the signal processing system and aerodynamic inputs is given in Chapter 5. Finally, the conclusions and recommendations of the author are given in Chapter 6. The Appendices include the mechanical drawing of the sensors, their calibrations, the entire tunnel testing results, and an uncertainty analysis.

As with any large project, a certain amount of collaboration occurred during the course of the work, including work performed by others and work performed by others at the direction of the author, all of which will be delineated here. Skin friction sensor work performed by the author, included the design equations, mechanical drawings, troubleshooting, wind tunnel testing, and date analyzing. Construction and calibration of these skin friction sensors was performed at the direction of the author. The damping fluid survey and study was performed at the direction of the author. Design, fabrication, and characterization of the pressure sensor was a team effort, which included the author. All Virginia Tech test of the pressure sensor was accomplished by the author. As mentioned in the text, the shock tube and vibration tests of the pressure sensor were performed by other team members. The fiber optic temperature/heat flux sensor was conceived by the author, constructed at his direction and tested by another engineer.

The microfabrication skin friction sensor work, including creating new concepts, performing design trade-off studies, identifying fabricators, and testing of completed sensors, was completed by the author. The silicon etching was done by Litton PolyScientific in consultation with the author. The pressure gradient study for microfabricated sensors was also original. The computation fluid dynamic and analytical work into temperature errors with microfabricated skin friction sensors was done by others with input and suggestions from the author. The developed fully-developed flow calibration rig was developed by other with original design input and help from the author.

All spectral wind tunnel data was reduced and analyzed by the author for both pressure and skin friction sensors, including the examination of gap estimates, correction factor, and fringe contrast. All numerical simulations were written and performed by the 
author. The accelerometer experiment and skin friction sensor vibration data in Chapter 5 was taken by another engineer. The angular misalignment experiment was performed at the direction of the author, while the PZT experiment was completed by the author. Finally, all statistical work used to examine wind tunnel data was accomplished by the author. 


\subsection{Chapter 2}

\section{Fiber Optic Sensors}

\subsection{Motivation for Employing Fiber Optics}

As stated earlier, the direct measurement of skin friction by a cantilever beam has made progress as new sensing technology has become available. First with Winter and Gaudet (1977) using foil strain gages, and then with Schetz and Nerney (1977) using semi-conductor strain gages, these classes of skin friction sensors were designed to generate strain at the base of the cantilever, which is measured by these electrical-based sensing devices, and then through a calibration is converted to a wall shear stress measurement. The Schetz research group has produced a series of successful skin friction sensors based on strain gages mounted on the cantilever beam for environments ranging from low-speed to turbomachinery to hypersonic flows. Both foil and semi-conductor strain gages have their relative advantages and disadvantages for this application. ${ }^{45,46,47}$

Also, current pressure sensor designs are not able to make accurate high-speed measurements in harsh environments, including high temperature, high Mach number flows which can cause ionization of the gas. Currently, measurements in such an environment are made through pressure taps that protect the sensor, but the process greatly reduces the frequency response. Most of the pressure transducers used today employ foil or semiconductor strain gages. A good summary of these two types of gages and the related electronics can be found in Chadwick (1993) ${ }^{48}$ Table 2 presents a comparison of foil and semi-conductor strain gages in relation to fiber optic sensing.

Table 2. Comparison of different sensing technologies.

\begin{tabular}{|c|ll|l|}
\hline Sensing Technology & \multicolumn{1}{|c|}{ Advantages } & \multicolumn{1}{c|}{ Disadvantages } \\
\hline Foil Strain Gages & - $\begin{array}{l}\text { low temperature sensitivity } \\
\text { insensitive to pressure changes } \\
\text { relatively low cost }\end{array}$ & $\begin{array}{l}\text { - low output } \\
\text { EMI sensitive }\end{array}$ \\
\hline $\begin{array}{c}\text { Semi-Conductor } \\
\text { Strain Gages }\end{array}$ & - & $\begin{array}{l}\text { high output } \\
\text { insensitive to pressure changes } \\
\text { relatively low cost }\end{array}$ & - high temperature sensitivity \\
\hline Fiber Optics & - $\begin{array}{l}\text { low temperature and EMI sensitivity } \\
\text { high sensitivity } \\
\text { operability at extreme temperatures }\end{array}$ & - & $\begin{array}{l}\text { higher cost } \\
\text { possible pressure sensitivity }\end{array}$ \\
\hline
\end{tabular}


In the current study, the sensing technology has moved to fiber optics, allowing a design principle based on deflection instead of strain, which for the specific case of the skin friction sensor is new in non-nulling cantilever beam designs. Fiber optics not only offer increased sensitivity to displacement but also allow new design concepts that increase the sensitivity to shear, permitting the area of the floating head and the length of the cantilever beam to be decreased, reducing the size of the whole sensor package. These advantages lead to increased sensitivity and reduction of the size of the pressure sensor design as well. The use of fiber optics brings with it all the advantages that they have over designs which employ conventional semi-conductor or foil strain gages, including temperature and electromagnetic radiation insensitivity.

\subsection{Overview of Fiber Optics}

Optical fiber systems have been developed during the past twenty-five years for primary applications in long-distance, high-speed digital information communication. Sensors using optical fiber have been developed over the past fifteen years for applications in the characterization of aerospace and hydrospace materials and structures, civil structures, and industrial process control and biomedical systems. ${ }^{49,50}$ Optical fibers are used as the field-sensitive elements in sensors for the measurement of environmental parameters such as strain, temperature, vibration, chemical concentrations, and electromagnetic fields. Their advantages for such measurements include 1) an inherent immunity to electromagnetic interference, 2) avoidance of ground loops, 3 ) the capability of responding to a wide variety of measurands, 4) excellent resolution compared to conventional foil strain gages, 5) the avoidance of sparks, which is especially important for applications within explosive environments, and 6) operation at temperatures of approximately $1073 \mathrm{~K}$ for silica waveguides and above $2173 \mathrm{~K}$ for sapphire waveguides.

The spatial modes that exist in an optical fiber can be described as standing wave patterns oriented orthogonal to the axis of propagation. The energy distribution in these modes is based upon solutions to Maxwell's equations and the application of boundary conditions. In an optical fiber, injected light is guided by a dielectric cylindrical core surrounded by a dielectric cladding, see Figure 26. Although not completely accurate, 
one can look at the light transmitted down the fiber as being reflected at the core/cladding interface depending on what is termed the critical angle, a function of the index ratio according to Snell's law. As long as the critical angle is not exceeded, the light will reflect and stay confined in the core. If the critical angle is exceeded, the light will not be reflected and will continue into the cladding where it is absorbed. This angle defines the acceptance angle and, conversely, the angle of the cone of the light that exits the fiber. In reality, the light is transmitted as a field down the fiber, which acts solely as a waveguide, with energy mostly confined in the core, but with an evanescent field that extends into the cladding. If the angle becomes too great, the waveguide can not contain the energy, and the light energy starts to be attenuated in the cladding. Electric field continuity across the core/cladding interface, particularly in step-index fibers, dictates the allowable modes in a given fiber. Although a great deal of work has been done in fibers that carry a range of boundary solutions, called multimode fibers, this study was performed only with singlemode fibers, in which only a narrow range of wavelengths are carried, with the rest being attenuated in the cladding. ${ }^{51}$

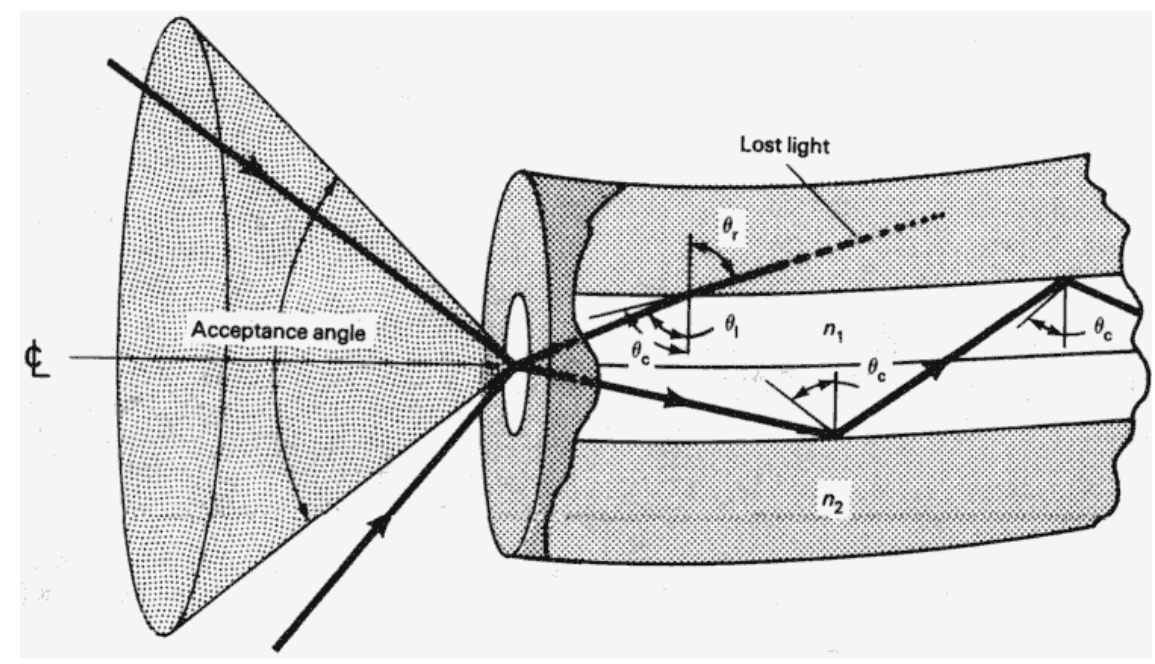

Figure 26. Sketch of the concept of the guiding of light by an optical fiber. ${ }^{52}$

\subsubsection{Basic Sensor Concept}

Fiber optic sensors are considered by many researchers as the best available technology for acquiring measurements in harsh environments. As a result, these sensors 
have begun to replace conventional electrical sensors in specific applications. For example, the transducing technique used by fiber optic sensors does not involve electrical signals, so they are essentially immune to electromagnetic interference (EMI). This fact is especially advantageous in environments where EMI is unavoidable, such as near radar emitters or on the skin of spacecraft and re-entry vehicles. These sensors have also been demonstrated at higher temperatures than their electrical counterparts. In all, there are many operational environments in which the employment of fiber optic sensors is advantageous. $^{49}$

A variety of fiber optic sensing techniques have been put to practical use in the last two decades, including intensity-based interrogation and interferometry. One the most versatile techniques for a variety of fiber optic sensor applications is extrinsic Fabry-Perot interferometry, or EFPI, invented by Murphy et al. ${ }^{49}$. EFPI-based sensors use a distance measurement technique based on the formation of a low-finesse FabryPerot cavity between the polished end face of a fiber and a reflective surface, shown schematically in Figure 27. Light is passed through the fiber, where a portion of the light (determined by the difference between the index of refraction of air and the fiber) is reflected at the fiber/air interface $\left(\mathrm{R}_{1}\right)$. The remaining light propagates through the optical path between the fiber and the reflective surface and is reflected back into the fiber $\left(\mathrm{R}_{2}\right)$.

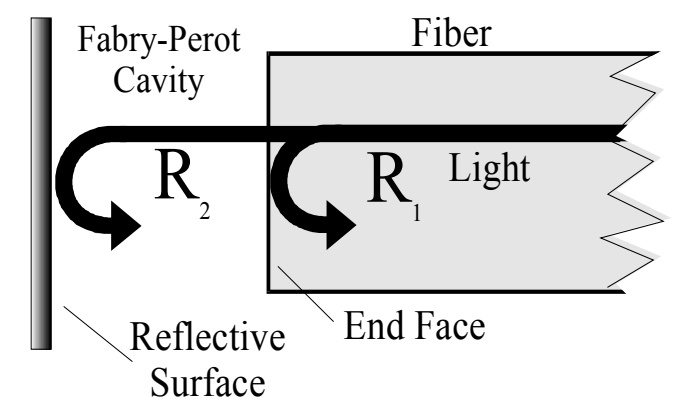

Figure 27. Extrinsic Fabry-Perot interferometer concept.

These two light waves interfere constructively or destructively based on the optical path length difference traversed by each and their wavelength. Optical path length (OPL) is defined, as in Equation 21, as the physical gap between the end of the 
fiber and the reflective surface multiplied by the index of refraction of the material in the gap. For air $\mathrm{n}=1$, however for other materials the value can be significantly different.

$$
O P L=G a p * n
$$

In other words, the interaction between the two light waves in the Fabry-Perot cavity is modulated by a change in the gap distance or change in refractive index of the material in the gap. This change in optical path length is more extensively defined in Equation 22,

$$
\Delta O P L=\Delta G a p * n+(G a p * \Delta G a p){ }^{*}{ }_{i=1}^{y} \frac{\partial n}{\partial x_{i}} * \Delta x_{i}
$$

where, Gap - the physical distance between the fiber and reflector, termed gap or optical gap

$\Delta \mathrm{Gap}$ - the change in the physical distance between the fiber and reflector

$\mathrm{n}$ - index of refraction, and

$\mathrm{x}$ - any parameter with which refractive index changes (an example would be temperature).

It should be noted, by examining these equations, that the returning signal is a function of the optical path length, not just the physical optical gap, therefore it is sensitive to changes in refractive index as well. The resulting light signal then travels back through the fiber to a detector where the signal is converted into an electrical signal and then demodulated to produce a distance measurement by various signal processing systems developed at Luna Innovations, a commercial fiber optic sensing company that sponsored and assisted in this work.

\subsubsection{Types of Demodulation Systems}

The demodulating of the signals from an EFPI cavity can be performed with a variety of methods. The methods either considered or used in the study are detailed below. A more comprehensive overview can be found in Liu and Measures. ${ }^{53}$

\section{$\underline{\text { Intensity-Based Interferometric Systems }}$}

A basic demodulation system using single wavelength interrogation is shown in Figure 28. A laser diode supplies coherent light to the sensor head, and the reflected light 
is detected at the second leg of the optical fiber coupler. The output can then be approximated as a low-finesse Fabry-Perot cavity in which the intensity at the detector is,

$$
I_{r}=\left|A_{1}+A_{2}\right|^{2}=A_{1}^{2}+A_{2}^{2}+2 A_{1} A_{2} \cos \Delta \phi
$$

if $A_{1}$ and $A_{2}$ are the amplitudes of $R_{1}$ and $R_{2}$, and $\Delta \phi$ is the phase difference between them. The output is a sinusoid with a peak to peak amplitude and offset that depends on the relative intensities of $A_{1}$ and $A_{2}$, as depicted in Figure 29. A phase change of 360 degrees in the sensing reflection corresponds to one fringe period. If a source wavelength of $1.3 \mu \mathrm{m}$ is used, the change in gap for one fringe period is $0.65 \mu \mathrm{m}$. The drop in detector intensity is due to the decrease in coupled power from the sensing reflection as it travels farther away from the single-mode input/output fiber. By tracking the output signal, minute displacements are determined. The disadvantage of this type of demodulation system is the non-linear transfer function and directional ambiguity of the sinusoidal output. If gap changes occur at a peak or valley in the sinusoid as shown in Figure 29 at $\pi, 2 \pi, 3 \pi, \ldots$, they will not be detected because the slope of the transfer function is zero at those points. The sensitivity of the system correspondingly decreases at points near multiples of $\pi$. If the direction of gap movement changes at a peak or valley, that information is lost, which causes directional ambiguity in the signal.

One approach to solving these problems is to design the sensor so that at the maximum gap the signal does not exceed the linear region of the transfer function. The linear region of the sinusoidal transfer function in shown in Figure 29. Confining operation to the linear region places difficult manufacturing constraints on the sensor by requiring the initial gap to be positioned at the Q-point of the transfer function curve. In addition to the difficult manufacturing constraints, the resolution and accuracy are limited when the signal output is confined to the linear region. To solve the non-linear transfer function and directional ambiguity problems, alternative signal demodulation approaches can be used such as white light interferometry and dual wavelength interrogation, described next. ${ }^{54}$ 

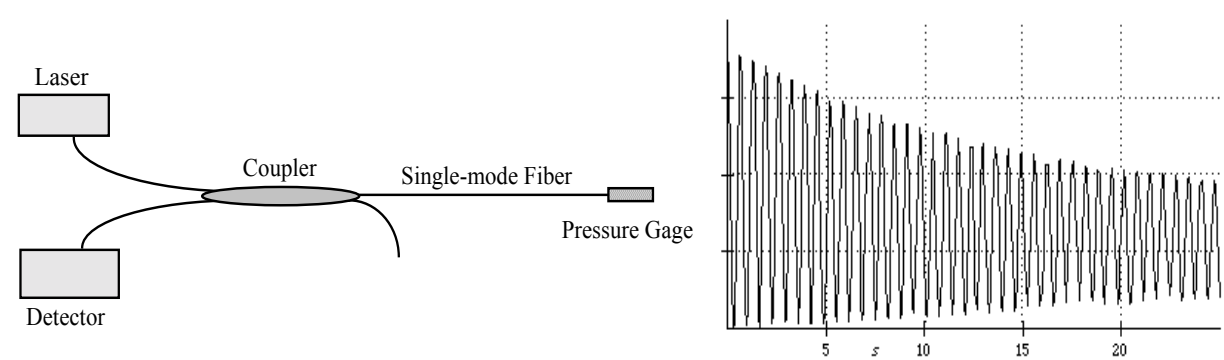

Diaphragm Discplacment (microns)

Figure 28. Variation of intensity for changes in optical gap. ${ }^{\mathbf{5 0}}$

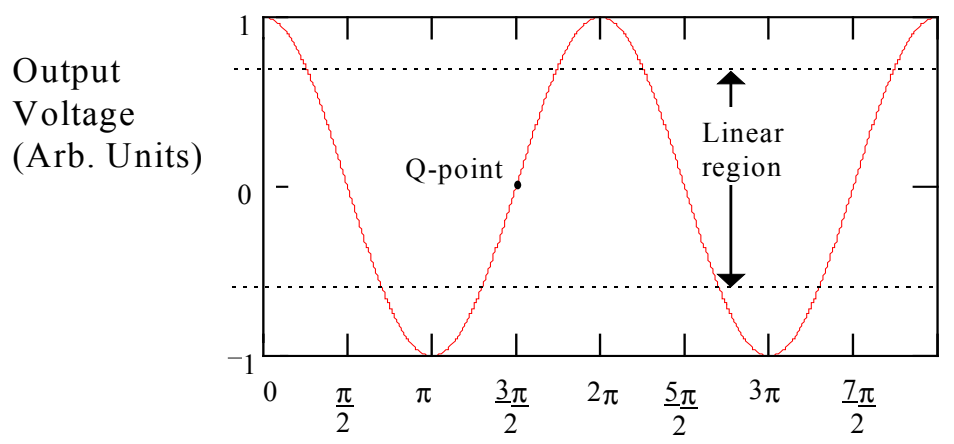

Phase of signal

Figure 29. Output of an intensity-based interferometric signal over a period.

\section{$\underline{\text { Spectral Interrogation Systems }}$}

White-light interferometry is an optical cross-correlation technique capable of determining very accurately the path imbalance between two arms of an interferometer. $^{55,56}$ For the case of the EFPI sensor, white-light interferometric techniques provide the exact optical path length between the fiber endfaces that form the Fabry-Perot cavity. The basic configuration of the absolute EFPI system is shown in Figure 30 . 


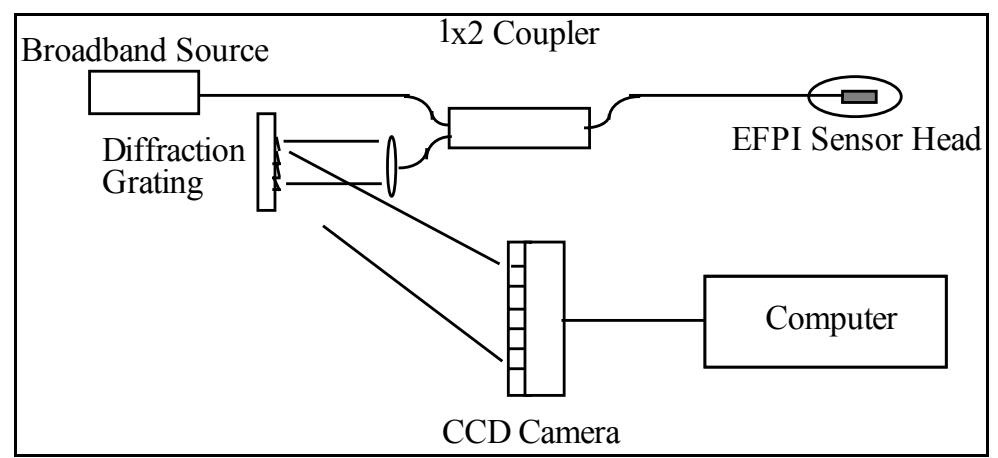

Figure 30. Spectral interferometric sensing system.

In more detail, a broadband, white light source, as depicted in the upper left of Figure 31, is transmitted to the sensor, where it becomes modulated by the Fabry-Perot cavity. The modulated spectra, as shown in the upper right of Figure 31, are then physically split into its component wavelengths by a diffraction grating, which are measured by a charged-coupled device (CCD) array, as shown in Figure 30. The spectra are then off-loaded from the CCD array, and an optical path length is calculated from the spectra using the Luna algorithm. The main part of the algorithm is an FFT, which transforms the signal from a wavelength domain to a gap domain. The output of this is a plot that has numerous peaks, as shown in the bottom of Figure 31. The location of the maximum of the main peak, found with a peak search, is the absolute optical gap of the EFPI cavity.

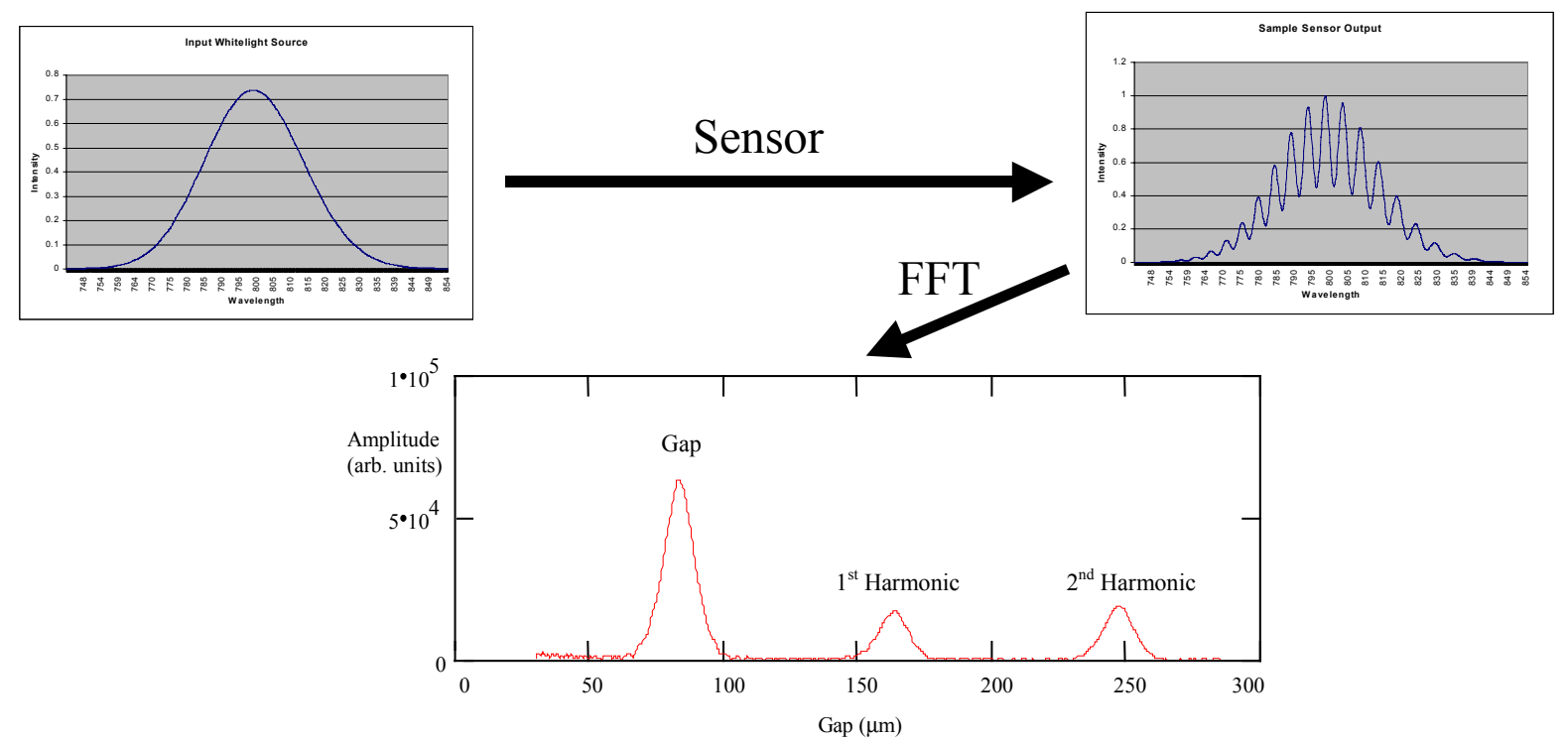

Figure 31. Depiction of spectral interrogation system method. 
Spectral interrogation has become the preferred method and the system type that is primarily used in this study, as well as the system for which the limitation study is done. The determination of an absolute gap is extremely useful. Although most transducers, and for that matter most information that is measured, are of a relative nature to a nominal value, the absolute gap removes the ambiguity seen with the previous system. Also, the system can be turned off, and back on, and the data can be gathered again from that point, without having to determine the nominal point. Because the system is gathering data from a range of wavelengths, and not just one, the resolution and accuracy of the system are robust where other systems are prone to error, such as microbends in the fiber. For the current system used in this study, the measurement resolution is estimated to be 200 picometers, or $0.2 \times 10^{-9}$ meters.

Finally, the per channel cost of the system can be reduced through multiplexing. Multiplexing an electrical signal is relatively simple, as the querying of the multiplexer does not affect the circuit holding each individual signal. However, with a relative optical system, as the intensity system above, the loss of the signal from query to query renders the measurement useless. With the spectral interrogation system, and its ability to determine an absolute gap, the system can be moved from fiber to fiber with a mechanical optical switch, and one can record accurate data on each channel. Another multiplexing method is to inject the signal of multiple fibers into one system simultaneously. If the gaps of the sensors are significantly different from each other, the combined spectrum can be demodulated into separate peaks through the FFT process, referred to as gap division multiplexing, with each peak in the plot corresponding to a gap of one of the sensors. It has been demonstrated that up to four gaps can be tracked simultaneously by one system with this method. ${ }^{57}$ These advantages over other optical signal processing systems make spectral interrogation systems attractive, and that is the reason for its primary use in this study.

Although there are numerous benefits to spectral interrogation systems, there are some drawbacks that affect measurements in aerospace environments. The first and foremost drawback is the speed of the system, which is at least three orders of magnitude slower than the other systems $(\sim 100 \mathrm{~Hz}$ compared to $>1 \mathrm{MHz})$. This is a problem in that many aerospace-type events occur faster than $100 \mathrm{~Hz}$, up to hundreds of kiloHertz. The source of the problem is the speed of the spectrometer internal to the system, which uses 
a CCD array to measure the intensities of the wavelengths. Since this is a chargedcoupled device, there is a certain integration time necessary for the pixels to receive enough light to create a charge that can be read by the analog/digital converters. During this time the sensor will still be moving. The movement can become so great, that the CCD array inside the spectrometer of the signal processing system can not receive a clean signal during its integration time. This is termed spectral "smearing". As the spectral smearing becomes more pronounced, the system can no longer determine an accurate optical path length. Another problem of the spectral interrogation system as compared to other systems is that it is more susceptible to mechanical imperfections in the sensor such as misalignment or roughness of the reflector. Each of these error sources will create its own constructive and destructive pattern which, when combined, may not give strong peaks and valleys for the spectra, so it does not change much from the Gaussian input. This lack of contrast, in turn, reduces the size of the FFT peak, making the determination of where the peak occurs difficult. Therefore, the error limitations of the entire system are a combination of sources from the system and the sensor. It is this total system limitation, referring specifically to those applicable to aerospace environments and its individual inputs from the optical signal processing system and the various sensors in different flowfield, that is the purpose of this study.

The software and hardware details of the fiber optic signal processing system are proprietary to Luna Innovations. In summary, the optical gap is determined by an estimate using the FFT of the returned spectrum and a calculated correction factor.

An alternative white-light interrogation approach is shown in Figure 32. Here, an optical cross correlation technique is employed using a Fizeau wedge interferometer. ${ }^{58}$ The optical signal reflected from the sensor is transmitted through the Fizeau interferometer where a fringe pattern forms on the CCD array at a point where the Fizeau wedge gap matches the Fabry-Perot cavity within the pressure sensor head. A simple algorithm is then used to determine the peak intensity on the CCD array corresponding to the displacement of the sensor head. In either case, the use of an absolute displacement will make the design and success of any sensors developed simpler. Not only will it allow comparison to the Si micromachined and micromachined counterparts of these sensors, but will also allow the leverage of these previous systems to lower costs and improve reliability. Unfortunately this is a patented process and was not available here. 


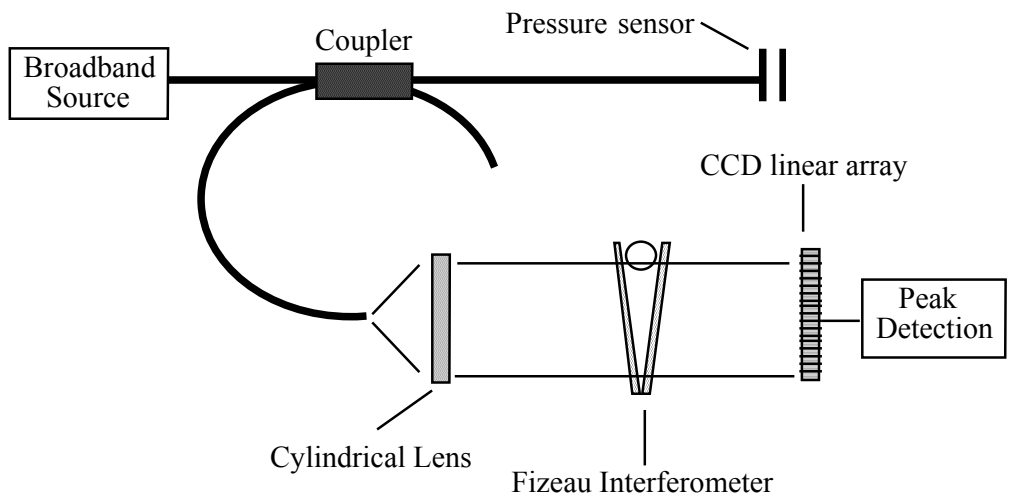

\section{Figure 32. Schematic of Fizeau interferometer-based absolute demodulation approach.}

\section{Dual-Wavelength-Based Systems}

The white-light interferometric system described above is ideal for low speed $(<100 \mathrm{~Hz})$ sensing applications, but it does not have the speed to work in a highly transient flow. One approach to a high-frequency demodulation system is based on dualwavelength interrogation and that is suitable for measurements at frequencies up to 10 $\mathrm{kHz}$ and above. By properly choosing the wavelengths (90 phase difference), quadrature outputs can be obtained, therefore the ambiguity in the interferometric intensity based demodulations systems can be removed. ${ }^{59,60}$ Figure 33 shows a theoretical plot of the output signal that can be obtained with this system. As seen from the graph, when one signal is at a peak or valley, the other signal is in the linear region. In this manner, one signal always has a linear response. By monitoring the phase lead/lag relationship between the signals, the direction of gap movement is unambiguously determined. An output showing the phase lead/lag relationship at a direction change is shown in Figure 34. 


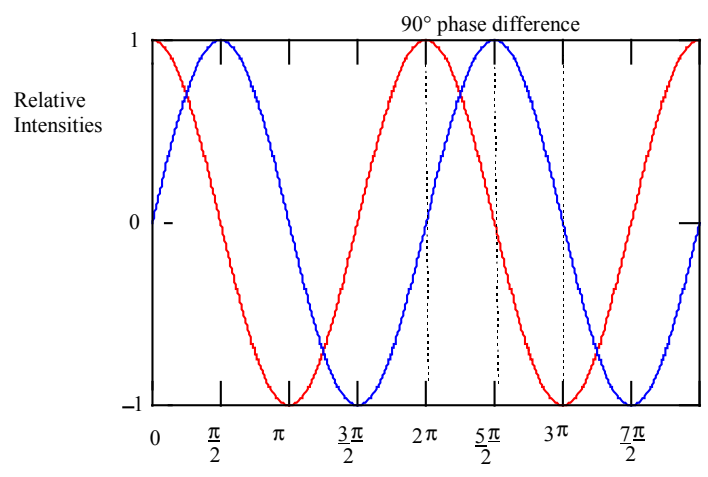

Figure 33. Theoretical outputs of quadrature signals obtained from a dual wavelength system.

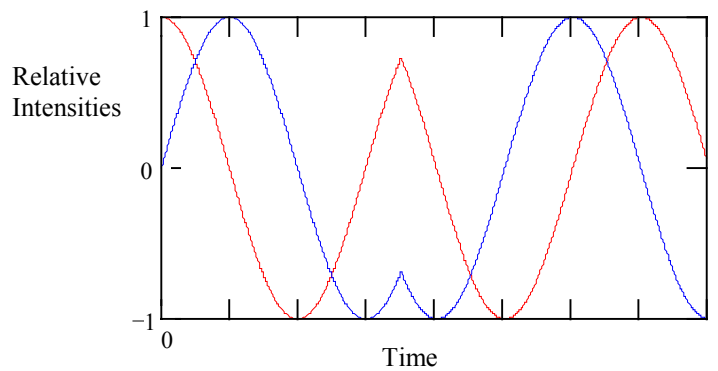

Figure 34. Two- $\lambda$ system theoretical output showing the phase lead/lag relationship at a direction change.

In the region of $90^{\circ}$ phase difference, gap values and directional changes can be monitored by plotting the two signals on opposite axes to create a Lissajous figure, as shown in Figure 35. By choosing source wavelengths a few nanometers apart, the range of unambiguous phase detection increases. By tracking the rotations around the circle and converting $\alpha$ to a displacement value, one finds the perturbation in terms of gap change. This relative displacement is then converted into a proportional voltage.

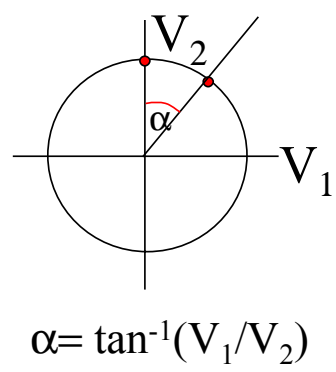

Figure 35. Idealized Lissajous figure of dual-wavelength output. 
This system is very useful in aerospace environments in which flow parameters, and therefore the gaps, can change very quickly. However, as was the case with the spectral system, if the gap changes between points more than $180^{\circ}$ on the Lissajous figure, the direction and magnitude information is lost. Since this system is relative between data points, in that the movement of one step is added to the movement from the previous steps to determine the overall movement, once a point is missed, all subsequent data is in error. Also, in a practical system, the signal-to-noise ratio has been a problem, with the noise filling a significant portion of the circle. If the circle becomes too small or the noise too large, the signal can cross over to another quadrant without the gap moving. And as before, once the system loses track, it can not recover to an accurate gap. 


\subsection{Chapter 3}

\section{Sensor Design}

\subsection{Design Principle}

A description of the design and development of the fiber optic sensors for measurement of pressure, skin friction, temperature, and heat flux is presented below. Design equations and physical trade-offs are examined, as are the efforts to micromachine the designs.

\subsection{Pressure Sensor Design}

The sensing element of the fiber optic pressure sensor designed and developed by Luna Innovations is a micromachined structure fabricated by outside contractors. Shown in Figure 36, the sensing elements are mass produced with an ultrasonically machined Pyrex base wafer and a silicon wafer, which were anodically bonded together and diced into individual sensing elements. For each sensing element, the machined portion of the Pyrex base forms a cavity with the silicon wafer bonded to the surface. The portion of the silicon wafer that remains after dicing acts as a diaphragm and is designed to deform under pressure. The deformation is measured with an optical fiber. ${ }^{61}$

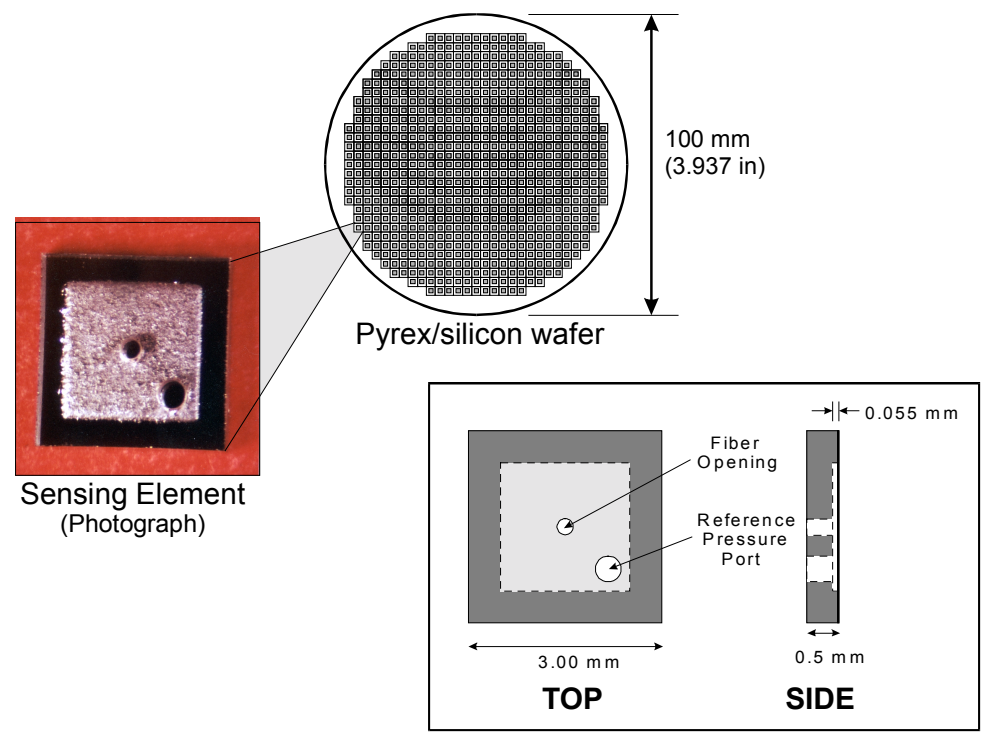

Figure 36. Micromachined sensing element for the fiber optic pressure sensor. 
The size of the machined cavity, the diaphragm material properties and the diaphragm thickness are critical design criteria in determining the measurement range of the sensor. These three characteristics were considered carefully during the design process. Their relationship to one another is governed by the deflection equation below along with the equation for the natural frequency, ${ }^{62}$

$$
\begin{gathered}
y=\frac{0.0151 P L^{4}\left(1-\mu^{2}\right)}{E h^{3}}, \\
w_{n}=10.4 \sqrt{\frac{E h^{2}}{\rho L^{4}\left(1-\mu^{2}\right)}}
\end{gathered}
$$

where;

$$
\begin{aligned}
& \mathrm{y}=\text { deflection of the center, } \\
& \left.w_{n}=\text { natural frequency ( } \mathrm{rad} / \mathrm{sec}\right), \\
& \mathrm{h}=\text { thickness of the diaphragm, } \\
& \mathrm{E}=\text { Young's Modulus of the diaphragm material (silicon), } \\
& \mathrm{P}=\text { applied pressure, } \\
& \mathrm{L}=\text { length of the cavity (assumed square), and } \\
& \mu=\text { Poisson's Ratio for the diaphragm material (silicon). } \\
& \text { and }, \rho=\text { density. }
\end{aligned}
$$

Generally, linearity is maintained so long as the deflection does not exceed $1 / 3$ the diaphragm thickness. However, to maintain good measurement resolution, a minimum full-range deflection of approximately 4 to $5 \mu \mathrm{m}$ was maintained. Using Equation 24, the resolution and linearity constraints, and the available wafer thickness of 20 microns, designs for two sensing elements were developed for two sensors with linear ranges of $20.684 \mathrm{kPa}$ (3 psi) and $137.895 \mathrm{kPa}(20 \mathrm{psi})$. The cavity dimensions are the only difference between the two designs, thus creating sensing elements with two different active sensing areas. Active areas are $4.0 \mathrm{~mm}^{2}$ for the $20.684 \mathrm{kPa}$ (3 psi) design and $1.96 \mathrm{~mm}^{2}$ for the $137.895 \mathrm{kPa}(20 \mathrm{psi})$ design.

The fully assembled sensor is shown in Figure 37. An optical fiber and glass tube fiber spacer are bonded in the center opening of the sensing element using epoxy. The 
optical gap between the bottom of the diaphragm and the end face of the fiber is a FabryPerot cavity. As described in Chapter 2, light interference resulting from the internal reflection of light at the fiber end face $\left(\mathrm{R}_{1}\right)$ and reflected light off of the bottom surface of the diaphragm $\left(\mathrm{R}_{2}\right)$ is used to monitor the optical path length. The optical gap varies with diaphragm deflection, which in turn varies with applied pressure. The reference port on the bottom of the sensing element acts as a vent through which air can pass to maintain a constant pressure on the reference side of the diaphragm. Representative room temperature performance characteristics for both sensor designs are shown in Figure 38. The large area sensor $\left(4.00 \mathrm{~mm}^{2}\right.$ active area) is linear up to approximately $20.684 \mathrm{kPa}(3$ psi) and the small area sensor $\left(1.96 \mathrm{~mm}^{2}\right.$ active area) is linear up to $137.895 \mathrm{kPa}$ (20 psi). Generally, sensitivities in the linear range of the large and small area sensors range between 3.4474 to $4.8264 \mathrm{kPa} /$ micron and 20.6844 to $34.474 \mathrm{kPa} /$ micron, respectively.

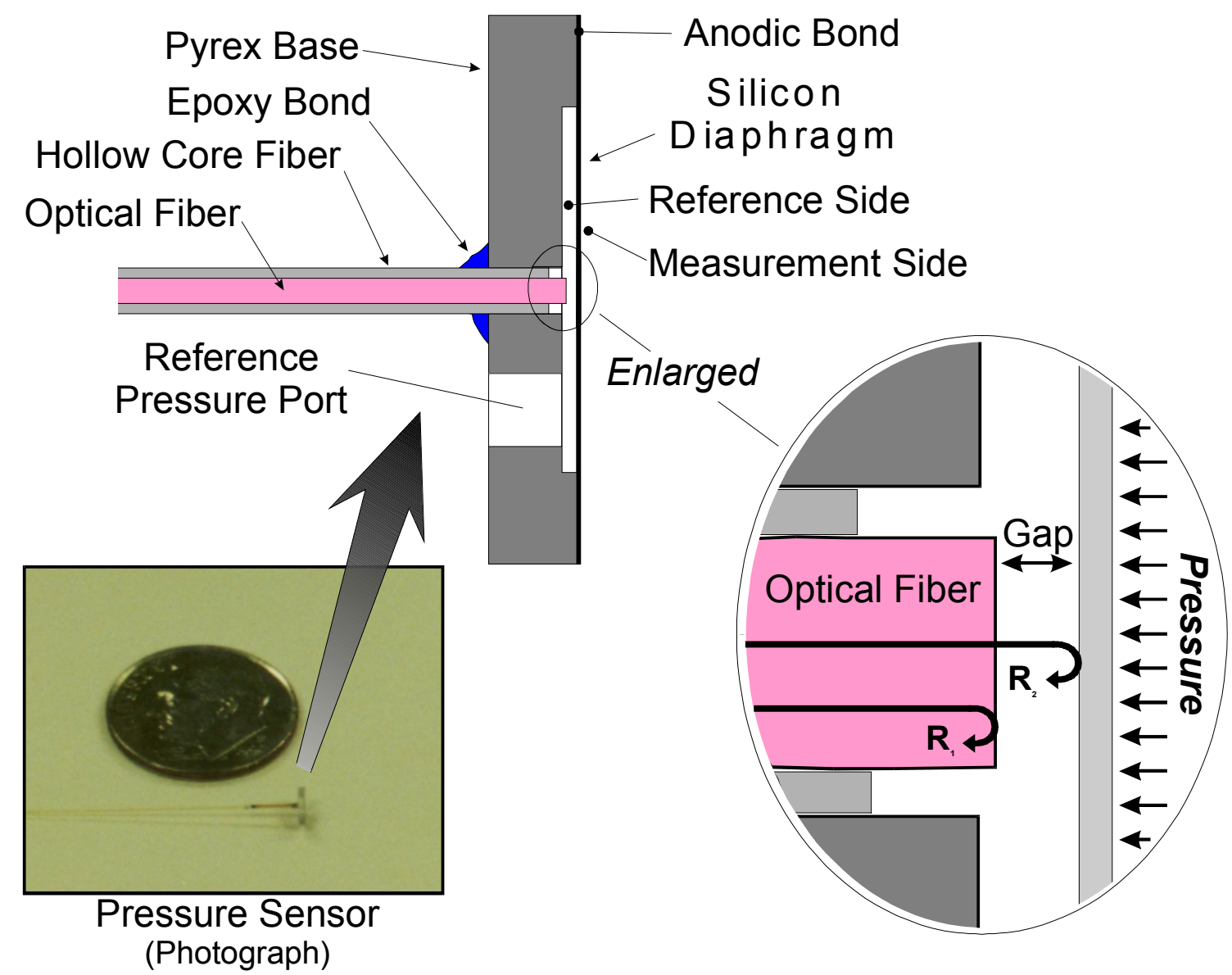

Figure 37. Assembled fiber optic pressure sensor. 


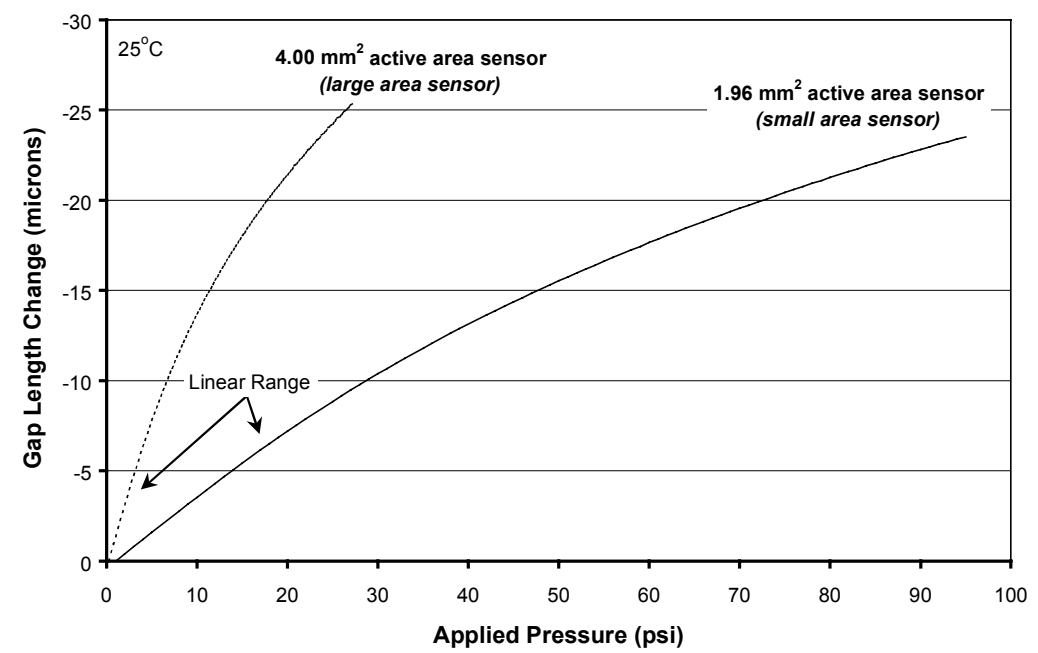

Figure 38. Representative pressure characteristics of fiber-optic pressure sensors.

\subsubsection{Pressure Sensor Characterization}

Some laboratory characterization of the pressure sensor was performed prior to the first aerodynamic tests. As temperature insensitivity is an advantage of this technology, the stability of the sensor to temperature change was examined. Determining the temperature behavior eliminates a possible source of uncertainty when examining the wind tunnel data. The sensor was also vibration tested to eliminate that as a source of spurious effects. Finally, the frequency response of the sensor was experimentally determined by a standard shock tube test.

\section{Temperature Behavior}

The temperature characteristics of the pressure sensor design were determined using a dual temperature/pressure testing apparatus constructed for this purpose at Luna. It was anticipated that the sensor design would show at least some temperature sensitivity due to thermal expansion and temperature-related changes in the index of refraction of the air in the optical path length. To determine this sensitivity, several large and small area sensors were tested by running the sensors through several pressure cycles in much the same manner as the repeatability tests but at several different temperatures. The data shown in Figure 39 were acquired during one such series of tests. Starting at room temperature $\left(25^{\circ} \mathrm{C}\right)$, this sensor was pressure cycled once at each of the temperatures shown up to $500^{\circ} \mathrm{C}$. Visual analysis confirms that significant changes in sensitivity and 
zero offset occurred as the temperature was increased. However, for moderate temperature variations that occur in the Virginia Tech supersonic wind tunnel, the sensor is very stable, eliminating this as a concern for the aerodynamic tests.

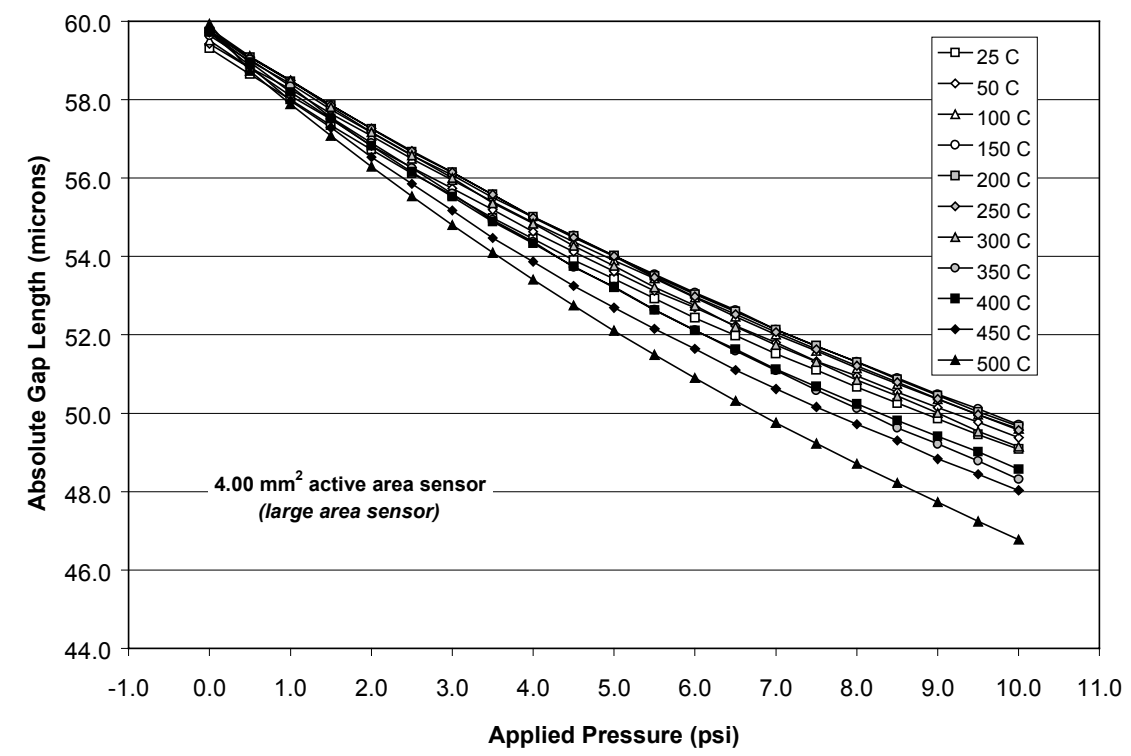

Figure 39. Temperature characterization data for a representative large area sensor.

\section{Frequency Response}

The frequency response characteristics of the fiber optic, micromachined, pressure sensor design were determined during a shock tube test performed by Russler and Wavering of Luna at Wright State University (WSU) during tests in November 1998. The WSU shock tube, shown in the photograph in Figure 40a, is a simple expendable diaphragm design that can be automatically triggered to coincide with high-speed data acquisition. A $137.895 \mathrm{kPa}$ (20 psi) range (1.96 $\mathrm{mm}^{2}$ active area), fiber optic pressure sensor was installed in a fixture with a co-located high-frequency, piezoresistive pressure sensor. The fixture was mounted on the side wall of the WSU shock tube for testing, as shown in Figure 40b. Data from the fiber optic sensor were acquired using the dual wavelength system described in Chapter 2. This system possessed adequate frequency response characteristics to resolve the high-frequency behavior of the fiber optic pressure sensor. Conventional high-speed signal processing equipment was used to acquire data from the piezoresistive pressure sensor. Data from the best test run in the shock tube are 
shown in Figure 41. Data from both sensors are smoothed (filtered) using a 10-point moving average to eliminate spurious signals. The data from both sensors show virtually the same response to a moving shock wave. The piezoresistive pressure sensor is known to possess a natural frequency greater than $200 \mathrm{kHz}$, so data from this sensor should show the general behavior of the shock wave. Since both sets of data agree reasonably well, the high frequency capability of the fiber optic pressure sensor seems to be at least as good as the piezoresistive sensor. A power spectrum of the unfiltered fiber optic sensor data is shown in Figure 4lb. The data show the resonant response of the fiber optic sensor, which occurred at approximately $137 \mathrm{kHz}$.

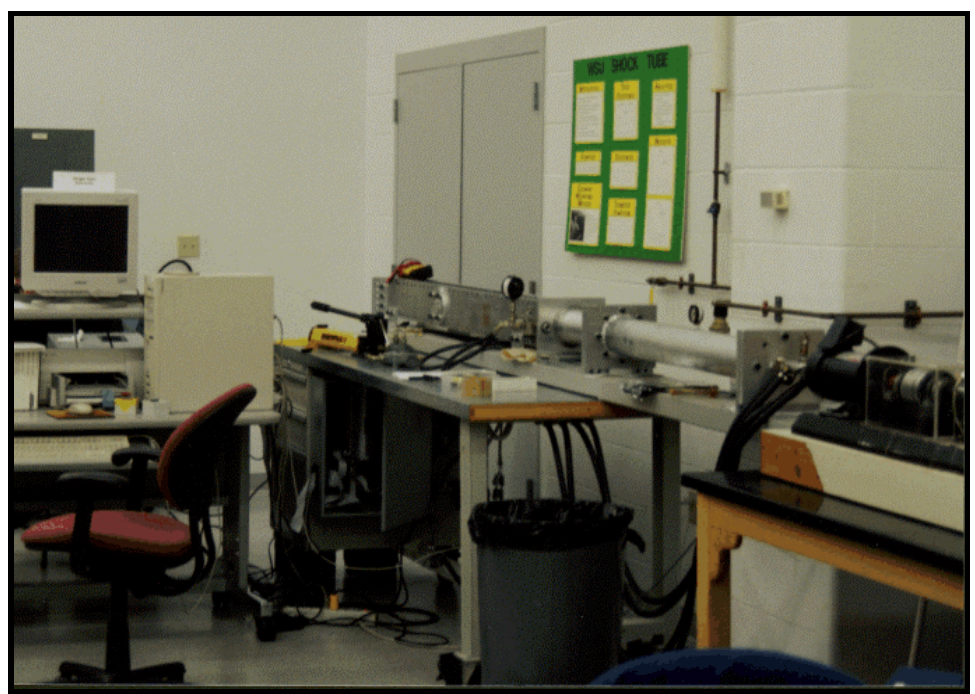

(a)

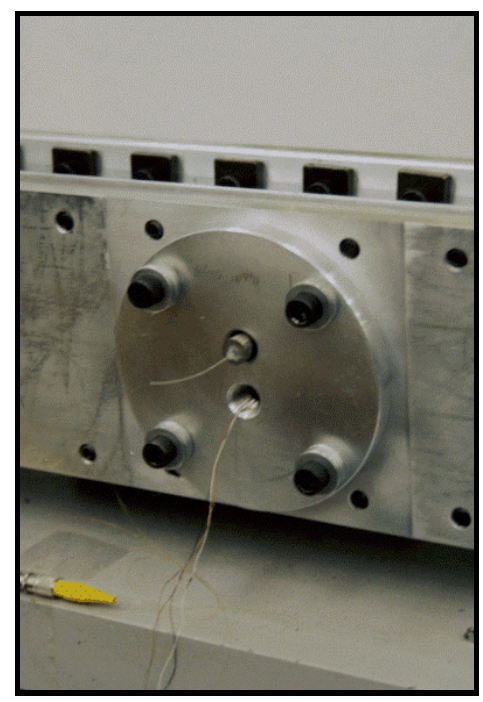

(b)

Figure 40. WSU shock tube (a) and sensor fixture mounted in the shock tube (b).

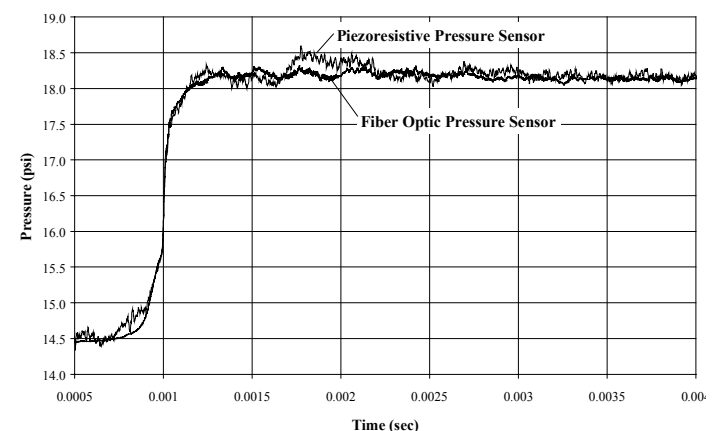

(a)

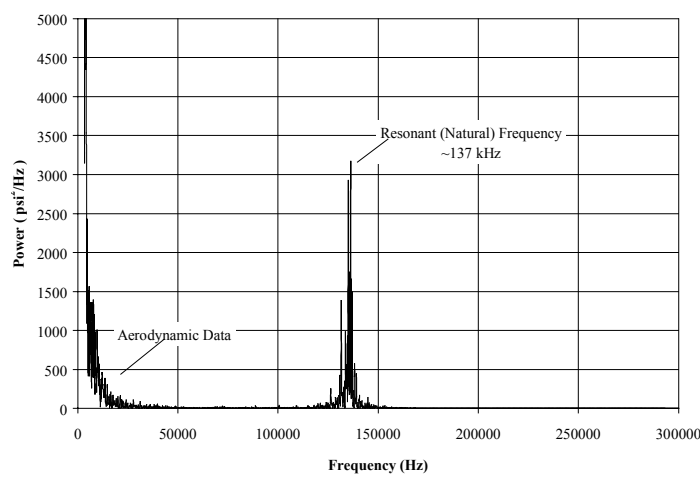

(b)

Figure 41. Time history (a) and power spectrum (b) of fiber optic sensor data from WSU test. 


\section{Vibration}

The pressure sensor was vibration tested to determine the effect moderate vibration levels would have on the sensor measurements. Testing was conducted per NASA PS 21-2, "Environmental Testing Electronic and Electromechanical Equipment". A representation of the vibration curve called out in this specification is shown in Figure 42. The pressure sensor was tested using the "curve D" vibration characteristic shown in the figure from the NASA report.

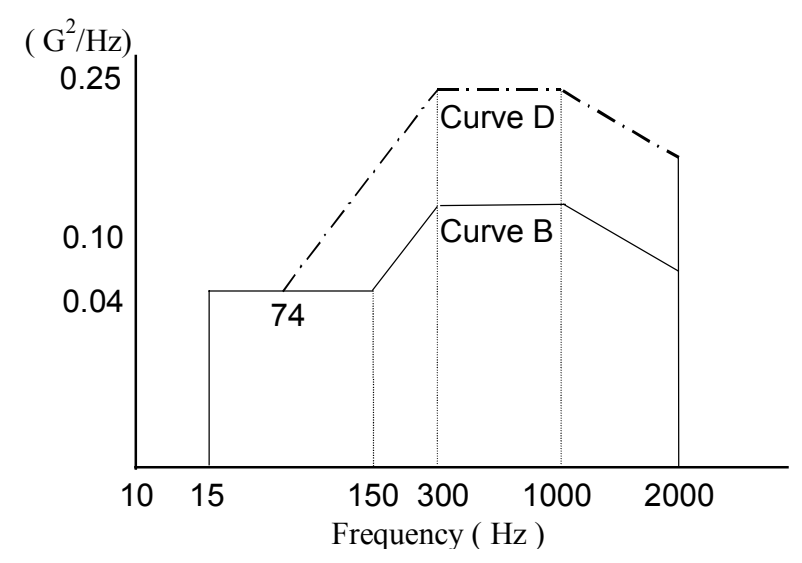

Figure 42. Vibration test curve.

The vibration test stand used to run these tests is located at Litton PolyScientific in Blacksburg, Virginia. This test stand is capable of generating random, unidirectional vibration in the required frequency and amplitude ranges. To test vibration tolerance in all 3 axes, the sensor had to be re-oriented on the test stand. The directional reference frame used during testing of the sensor is shown in Figure 43a. Two of the three reference frames were used since two are identical due to symmetry of the sensor. Due to the fact that the spectral interrogation signal processing system operates at only $66 \mathrm{~Hz}$ and the NASA specification extends to $2000 \mathrm{~Hz}$, it became necessary to use statistical means to the effect of the vibration. The method used was to take long data segments at each test condition and then find the standard deviation of the result. Although the low data rate of the optical signal processing system precludes capturing the waveform, a long data segment statistically will record the correct proportion of the spurious signals if any exist. These spurious signals directly relate to increasing the standard deviation of the record. Therefore, if the standard deviation does not change significantly from the static 
case, there is not a large effect. Test results, shown in Figure 43b, show variations of less than a nanometer in the optical gap measurement throughout the vibration range, which is less than the uncertainty currently associated with the spectral interrogation signal processing systems used with the sensor.

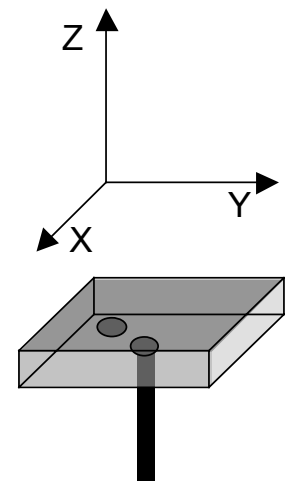

(a)

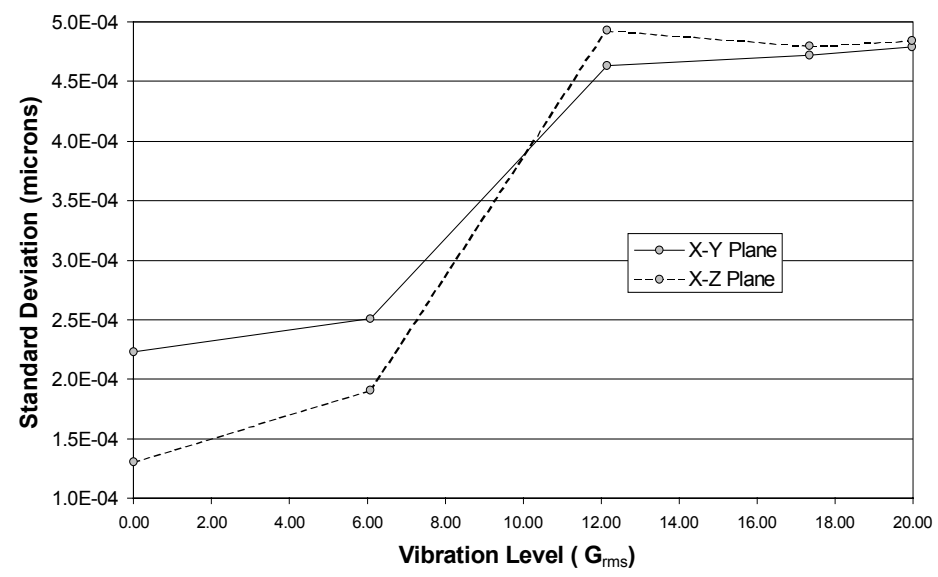

(b)

Figure 43. Reference frame (a) and test results (b) for the pressure sensor vibration test.

\subsection{Skin Friction Sensor Design}

The fiber optic skin friction sensor is based on the EFPI measurement technique to monitor the optical gap between the bottom of the sensing head and the end face of the optical fiber. Figure 44 shows that as the cantilever beam, to which head is attached, bends, the optical path length changes. The bending force is produced by the shear stress on the surface of the head. Thus, the change in path length is directly related to the magnitude of the shear stress on the head. Calibration of the sensor allows shear stress to be determined by monitoring the optical path length change. Two optical path measurements are used in each direction the shear is to be determined. By having a pair of fibers in each orthogonal direction of the head, the wall shear stress can be resolved in both direction and magnitude, as shown in Figure 45. The pair of fibers has two benefits. The first is the obvious redundancy, both in the measurement and in reliability. The second is to reduce or eliminate the effects of temperature and pressure in the shear measurement. If one imagines a pair of fibers opposite each other while the sensor has 
one of its sensing head axis aligned in the streamwise direction, with each fiber measuring an optical gap change, the shear measurement is just the increase of the path in the upstream fiber or the decrease in path of the downstream fiber. However, if a significant temperature change were to occur, each of these paths will be affected the same, both either increasing or decreasing with the temperature. The same is true for any effect due to change in the imposed pressure. If one then subtracts the two optical path length changes, the temperature and pressure effects would be eliminated, or at least minimized, while the shear effect remains. Therefore, it has been the design philosophy here to have two opposing fibers in the measurement direction in all skin friction sensors.

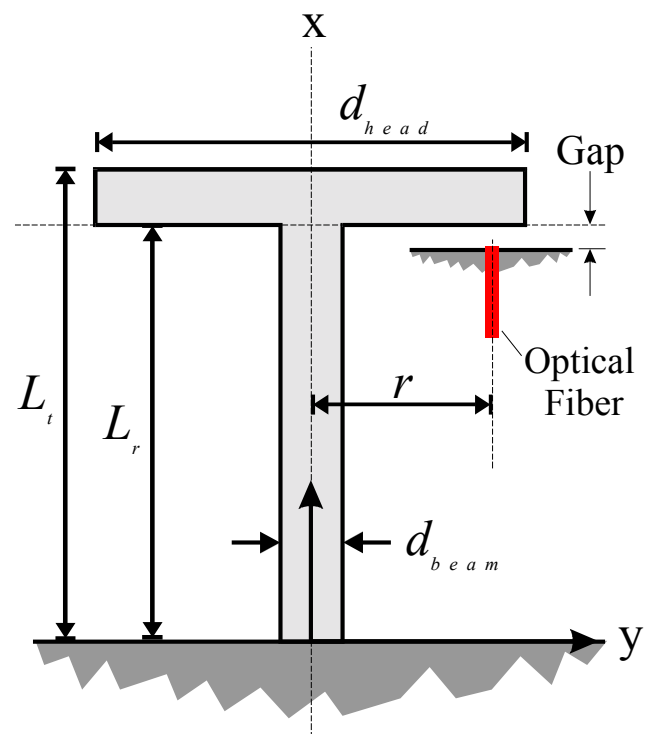

No Load

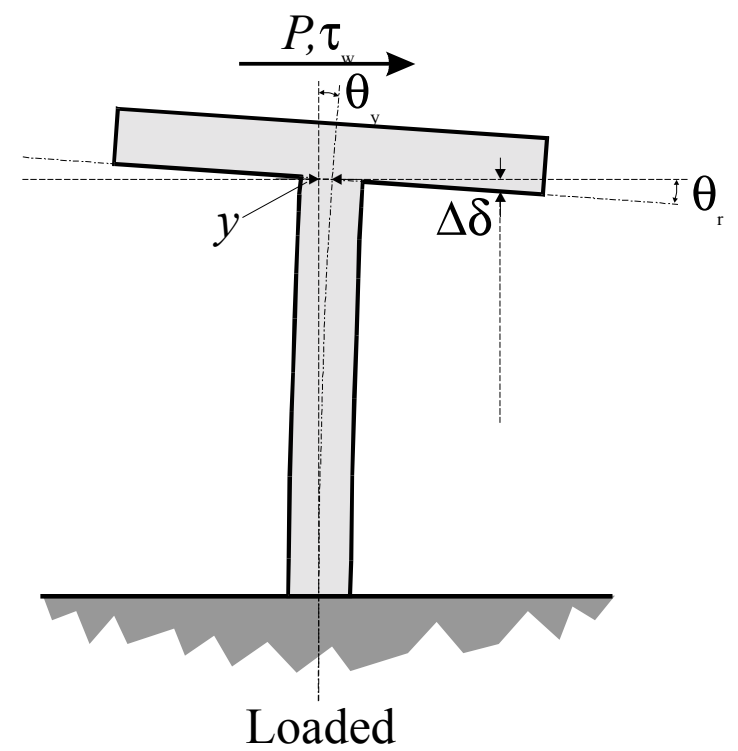

Figure 44. Conceptual model of skin friction sensing head and cantilever beam.

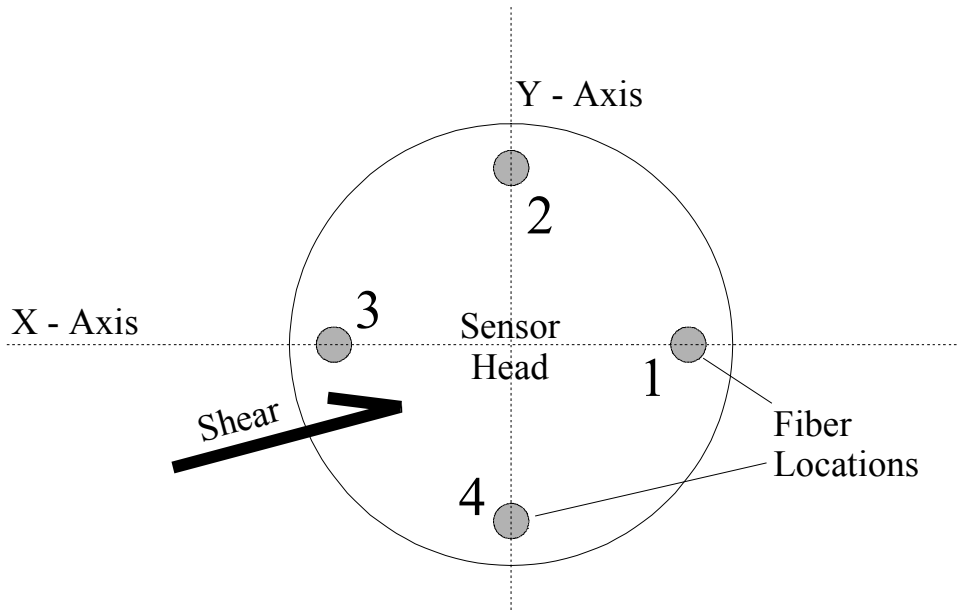

Figure 45. Sketch of fiber locations in skin friction sensor. 


\subsubsection{Design Equations}

The sensing head and beam can be modeled as a simple cantilever beam with a rigid circular disk on the end. The equation for the deflection of a beam loaded at the end point is, ${ }^{63}$

$$
y(x)=\frac{P}{6 E I}\left(x^{3}-3 L_{t} x^{2}\right)
$$

where,

$$
\begin{aligned}
& y(x)=\text { deflection, } \\
& P=\text { point load or force, } \\
& E=\text { Young's Modulus (Modulus of Elasticity), } \\
& I=\text { moment of inertia, } \\
& x=\text { distance to point of deflection, and } \\
& L_{t}=\text { length of the beam, }
\end{aligned}
$$

The moment of inertia, I, for a circular cross section is given as,

$$
I=\frac{\pi}{64} d_{\text {beam }}^{4}
$$

where,

$$
d_{\text {beam }}=\text { diameter of the beam. }
$$

The angle of deflection, $\theta_{y}$, is,

$$
\theta_{y}=\theta_{r}=\left(\frac{d y}{d x}\right)_{r},
$$

where,

$\theta_{r}=$ deflection angle of the y-plane.

If the angle of deflection is assumed small, then the optical gap change between the bottom of the head and the end face of the optical fiber, $\Delta \delta$, can be calculated,

$$
\Delta \delta \approx \theta_{r} r,
$$

where, $r=$ distance from the beam centerline to the optical fiber centerline.

By taking the derivative of Equation 26 and substituting Equation 29, we get 


$$
\theta_{r}=\frac{P}{6 E I}\left(3 x^{2}-6 L_{t} x\right)
$$

The force, $P$, on the end of the beam is directly related to the shear stress, $\tau_{w}$, on the surface of the circular head and the diameter of the head, $d_{\text {head }}$. Force equals stress multiplied by the area, or,

$$
P=\tau_{w}\left(\pi \frac{d_{\text {head }}^{2}}{4}\right)
$$

Substituting Equations 27 and 31 into Equation 30,

$$
\Delta \delta=\frac{8 \tau_{w} r d_{\text {head }}^{2}}{E d_{\text {beam }}^{4}}\left[L_{t}{ }^{2}\left(2 \alpha-\alpha^{2}\right)\right],
$$

where,

$\alpha=\frac{L_{r}}{L_{t}}$, and $L_{r}=$ the distance from the base of the beam to the optical reflector which is attached to the beam in some manner. Usually, the reflector is just the bottom of the head).

Also important is the deflection of the head within the physical gap between the head and the housing so that interference with the housing can be determined. Equation 33 describes the bending of a cantilever beam at its free end, ${ }^{63}$

$$
y_{\max }=\frac{P L_{t}^{3}}{3 E I} \text {. }
$$

If the variables for the optical gap deflection are inserted, the equation becomes,

$$
y_{\max }=\frac{16 \tau_{w} d_{\text {head }}^{2} L_{t}^{3}}{3 E d_{\text {beam }}^{4}} .
$$

For conventionally machined sensors, the movement is inconsequential compared to typical physical gaps (generally a few thousands of an inch). However, the micromachined designs have smaller physical gaps between the head and housing, but still need the same movement in the optical gap. Equation 34 becomes important for these designs. 
Equation 32 can be used as a mathematical model of the sensing head and beam. The design shear stress, $\tau_{\mathrm{w}}$, is one of the specified design criteria. Typically this was chosen here as $300 \mathrm{~Pa}$, as this is a shear level of a common test condition. Young's modulus, $E$, is a physical property of the material used, which for temperature stability has been chosen here as Invar36, E =140 GPa. The other parameters will depend upon the chosen sensing head size, $d_{\text {head }}$, and the interrelationship between the remaining variables. This design concept also allows the placement of the fibers away from the cantilever beam, providing greater sensitivity. It is this concept, especially when referring to head size, which led to these fiber optic sensors being smaller, than previous skin friction sensors produced by the Virginia Tech research group. This analytical model was used during the program to design every skin friction sensor, and good agreement between the model and the calibrations of the completed sensors was obtained.

The cantilever beam is the most critical component in the skin friction sensor design. The optical path length measurement technique offers optimum resolution over a minimum path length. Generally, the greater the change in optical path length, the higher the measurement resolution will be for the shear stress measurement. However, if the deflection is too large, the head will interact with the sensor body and/or the lip of the sensor head will protrude into the flow, invalidating the shear stress measurement. Therefore, the sensing head and cantilever beam must be designed to produce an optimum deflection for a given shear stress based on increased measurement resolution and the dimensional limitations put on the movement of the sensing head.

It should be noted that the design considerations go beyond the mechanical aspects described above. The sensing head size is an important characteristic. Too small a head size, and the force generated by the flow is too small to measure. Too large a head, and the sensor become susceptible to pressure gradient errors. It has generally been desirable to have as small a head as feasible. An examination of the design equation will show that the minimum head size is defined, outside of material selection, by the ability to machine parts accurately. There is a limit to how long a thin beam can be machined, or said another way how thin a beam of certain length can be lathed, without introducing a distortion in the beam. A straight beam is necessary so that the gap between the sensing head and the housing can be kept to a minimum, which has been shown to minimize errors due to various aerodynamic effects. 
Another important characteristic of the skin friction sensor is its natural frequency. The skin friction sensor head can be modeled as a cantilever beam fixed at the base with a mass on the free end. The governing equation for this model ${ }^{64}$ is,

$$
f_{n}=\left(\frac{1}{2 \pi L_{t}^{3 / 2}} \sqrt{\frac{12 E I}{\left(m_{\text {beam }}+4 m_{\text {head }}\right)}},\right.
$$

where,

$$
\begin{aligned}
& f_{n}=\text { the natural frequency }(\mathrm{Hz}), \\
& m_{\text {beam }}=\text { the mass of the beam, and, } \\
& m_{\text {head }}=\text { the mass of the head. }
\end{aligned}
$$

Equation 35 was used during the design process to estimate the natural frequency of the skin friction sensor. The estimation was often used in determining the susceptibility of the sensor to input vibration, either from a mechanical or aerodynamic source.

\subsubsection{Calibration Procedure}

The normal method of calibrating the skin friction gages has been by hanging weights from the sensing head, as depicted in Figure 46. By taking care to align the sensing axis and the head to the gravity vector, this calibration procedure can yield accurate and repeatable results. Because the application of weight has a linear effect on the results, the weight of the string and mass holder can be ignored, and the relative optical gap change can be measured for each mass. When aligned, one optical gap will decrease (compression) due to additional mass, and the other increase (tension). Typically, a calibration will consist of measuring the change in optical gap for a series of masses that encompass the design load condition. At each of these mass points, the gap before, during and after the application of weight is recorded so that the change in gap and stability of the measurement can be ascertained. The gap change is determined for both the tension and compression fiber, the sensor is rotated around, and the procedure is then repeated until a calibration in tension and compression is obtained for each fiber. One would expect that the slopes of opposing fibers would be the same (i.e. the slope of Fiber 1 in tension is the same as the slope of Fiber 3 in compression). In addition, the 
channels should all have nearly the same slope if the cantilever beam is fabricated well. The better both of these items are, the more confidence one has that the sensor was constructed well and the results will be repeatable. Both of these calibration checks continually improved during the course of the study as machining, design, and fabrication were all refined. The calibrations of all the skin friction sensors in this study are included in Appendix B.

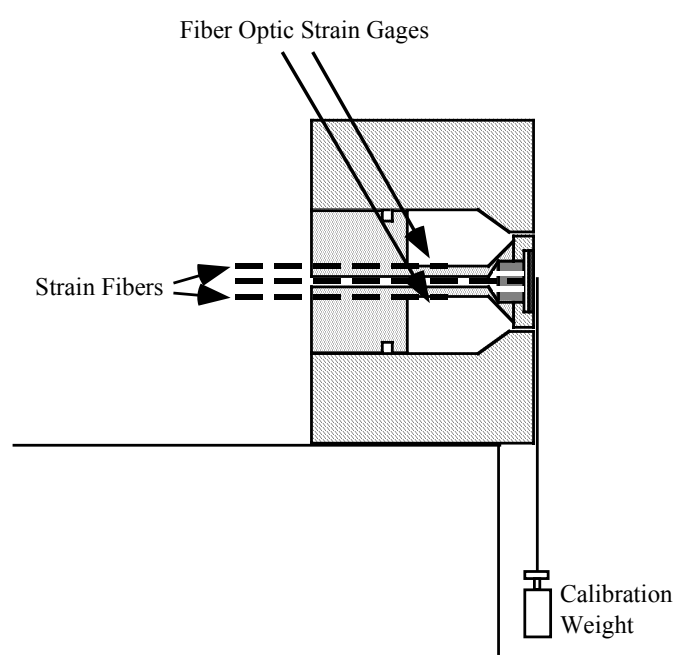

(a)

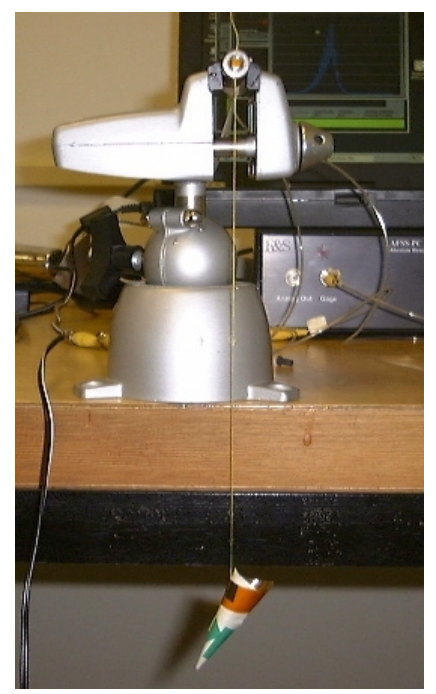

(b)

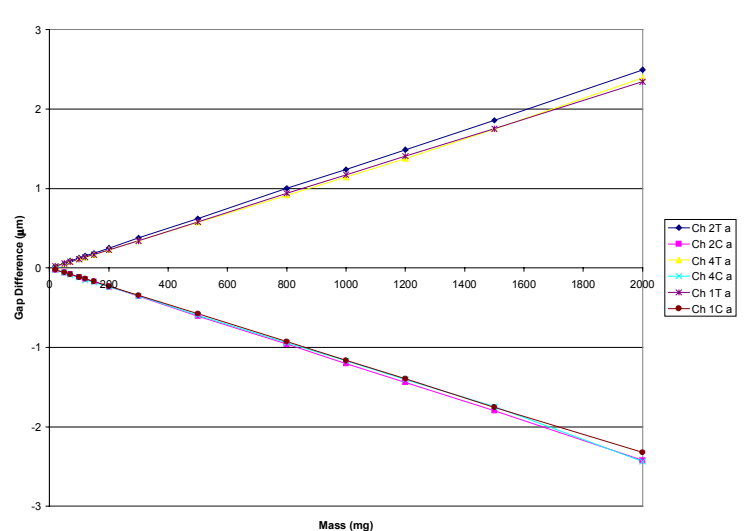

(c)

Figure 46. Conventional calibration procedure, a) sketch of procedure, photograph of setup, and c) sample calibration result.

\subsubsection{Damping Fluid}

In previous studies, a fluid has been used to fill the interior volume between the sensing beam and the housing. The fluid serves a few purposes; minimizing pressure 
gradient effects, providing a continual surface for the flow between the head and the housing, providing protection of the interior of the sensor from large temperature change, and supplying damping to the cantilever beam.

It was quickly realized that using the same fluid [Dow Corning 203 silicon oil] that had been used at Virginia Tech in the past might not be optimal for the fiber optic design. Previously, viscosity and maximum temperature had been important. However, because the current design requires that the fluid be in the optical path, changes in the index of refraction of the fluid affect the measured optical path length, and therefore the indicated shear, as indicated by Equation 22. Using two fibers in each measurement direction minimizes the errors due to perturbations, but reducing the perturbation effect before compensation seemed prudent. Therefore, a search was conducted to determine the best damping fluid available.

Table 3 presents the desired properties of the fluid. The high viscosity was desired in an effort to prevent the fluid from leaking out of the sensor during testing. Index of refraction of the fluid is also an important quantity, as changes in index will appear as changes in the optical path length, even when one has not occurred. It is desirable to have a fluid that does not change index with temperature or pressure, so as to minimize the compensation necessary. A limit on the absolute value of the index is also important so that the first reflection in the EFPI cavity will take place. If the index of refraction is close to the index of refraction of the optical fiber, no reflection will take place, and the interference pattern will not occur. The fluid also needs to be as transparent as possible in the wavelengths where the signal processing systems operate (700-950 nm and $1300 \mathrm{~nm})$. Compatibility between the fluid and a silicon rubber sheet sometimes used to top the sensor to keep the fluid in and provide damping is important. The results of the survey are presented in Table 4. The properties of the selected fluids, Dow Corning 203 silicon oil and glycerin, are in

Table 5. The research group has used the silicon oil for many years. However, this choice was due to its high temperature capabilities. If the temperature requirement is relaxed, glycerin becomes a more desirable choice, and it was used as the damping fluid in all the later sensors here. 
Table 3. Desired properties of damping fluid.

\begin{tabular}{|l|c|}
\hline \multicolumn{1}{|c|}{ Property } & Desired property value \\
\hline Viscosity & $>100 \mathrm{cSt}$ \\
\hline Change of path with temperature $(\mathrm{dn} / \mathrm{dT})$ & $<0.04$ \\
\hline Change of path with pressure $(\mathrm{dn} / \mathrm{dP})$ & 0.0 \\
\hline Index of refraction $(\mathrm{n})$ & Below 1.4 \\
\hline Flash point & As high as possible \\
\hline Boiling point & As high as possible \\
\hline Pour point & $<-65^{\circ} \mathrm{F}$ \\
\hline Percent transmission & As high as possible \\
\hline Compatible with silicon rubbers? & Yes \\
\hline
\end{tabular}

Table 4. Damping oil survey results.

\begin{tabular}{|c|c|c|c|c|c|}
\hline Manufacturer & Product & $\underline{\mathbf{n}}$ & $\frac{\text { Viscosity }}{\text { (cSt) }}$ & Pour Pt (F) & $\frac{\text { Flash Pt }}{(\mathbf{F})}$ \\
\hline Gelest & FMS-123 & 1.381 & $300-350$ & -52.6 & 500 \\
\hline Gelest & FMS-221 & 1.387 & $80-120$ & -67 & $>392$ \\
\hline Specialty Silicones & ssp-1400 & 1.403 & 100 & -60 & $400 \mathrm{~F}$ \\
\hline Specialty Silicones & $"$ & 1.4032 & 350 & -60 & $?$ \\
\hline Specialty Silicones & $"$ & 1.4033 & 500 & -60 & $?$ \\
\hline Specialty Silicones & $"$ & 1.403 & 1000 & -60 & $658 \mathrm{~F}$ \\
\hline Wacker & AK & 1.403 & 100 & -67 & $>527$ \\
\hline Wacker & $"$ & 1.403 & 150 & -58 & $>572$ \\
\hline Wacker & $"$ & 1.403 & 200 & -58 & $>572$ \\
\hline Wacker & $"$ & 1.403 & 250 & -58 & $>572$ \\
\hline Wacker & $"$ & 1.4035 & 350 & -58 & $>572$ \\
\hline Wacker & " & 1.4035 & 500 & -58 & $>572$ \\
\hline \multirow[t]{3}{*}{ Wacker } & $"$ & 1.4035 & 1000 & -58 & $>608$ \\
\hline & $"$ & 1.4037 & 2000 & -58 & $>608$ \\
\hline & $"$ & 1.4037 & 5000 & -58 & $>608$ \\
\hline DOW & $\begin{array}{c}\text { OPTIM Glycerine } \\
(99.7 \%)\end{array}$ & 1.474 & $1410 \mathrm{cp}$ & $-62.6($ fr. $\mathrm{Pt})$ & 384 \\
\hline DOW & $\begin{array}{c}\text { Dow Glycerine } \\
(96 \%)\end{array}$ & 1.4675 & $624 \mathrm{cp}$ & -46.4 (fr. Pt) & $>390$ \\
\hline DOW & 200 Fluid & 1.403 & 100 & -85 & $>620$ \\
\hline DOW & 201 Fluid & 1.4032 & 200 & -85 & $>620$ \\
\hline DOW & 202 Fluid & 1.4034 & 350 & -85 & $>620$ \\
\hline DOW & 203 Fluid & 1.4034 & 500 & -58 & $>620$ \\
\hline
\end{tabular}


Table 5. Comparison of selected fluids.

\begin{tabular}{|c|c|c|}
\hline Manufacturer & Dow & Various \\
\hline Product & $203 \mathrm{fluid}$ & glycerin \\
\hline Viscosity & $500 \mathrm{cSt}$ & $950 \mathrm{cp}$ \\
\hline Surface tension $\left(\right.$ dynes/cm at $\left.\mathbf{7 7 ^ { \circ }}{ }^{\mathbf{F}}\right)$ & 20.8 & 63.0 \\
\hline D(OPL)/dT (measured or calculated) & -0.04 & -.024894 \\
\hline D(OPL)/dP (measured or calculated) & $5.2099 \mathrm{e}-4$ & $2.5258 \mathrm{e}-4$ \\
\hline Index of refraction $(\mathbf{n})$ & 1.4034 & 1.474 \\
\hline${\text { Pour point }\left({ }^{\circ} \mathbf{F}\right)}^{\text {Flash point }\left({ }^{\circ} \mathbf{F}\right)}$ & -58 & 17 \\
\hline Compatible with silicon rubbers? & $>213.98$ & unk \\
\hline
\end{tabular}

Some of the needed property values were not known by the manufacturers, therefore it was necessary to obtain these through experiment or analysis. A Luna fiber optic refractometer was used to measure the change of optical path length as temperature and pressure were varied in separate experiments. With this knowledge, the effect of optical path change due to shear forces could be distinguished from that of the temperature and pressure, as well as an understanding of the susceptibility to these physical properties. An additional theoretical analysis for air was completed to determine the effect on the optical path length measurement when temperature and pressure were varied. This analysis is summarized here..

First, remember that for a stationary gap,

$$
\Delta O P L=\operatorname{Gap}^{* *}{ }_{i=1}^{y} \frac{\partial n}{\partial x_{i}} * \Delta x_{i} .
$$

Index of refraction is function of density,

$$
n \approx 1+\alpha^{*} \rho,
$$

or substituting the ideal gas law,

$$
n \approx 1+\alpha * \frac{P}{T} .
$$


Now, the index of refraction is 1 for a vacuum and 1.00029 for air at standard pressure and temperature. Using these two data points, the index of refraction for air can be expressed as,

$$
n_{\text {air }}=1+\left(2.9 * 10^{-4}\right) * \frac{P / 1 \text { atm }}{T / 298 K} .
$$

Using Equation 36 and taking the appropriate partial derivatives, the realtionship between change of index of refraction and either temperature or pressure for air is given by,

$$
\begin{aligned}
(\Delta O P L)_{a i r} & =G a p *\left(\frac{\left.\left(-2.9 \times 10^{-4}\right) * 298\right)}{298^{2}} *(\Delta T(K))\right. \\
(\Delta O P L)_{a i r} & =\frac{G a p *(0.00029) * \Delta P(\mathrm{kPa})}{101.3} .
\end{aligned}
$$

To compare experimental and analytical results, calculations were performed on a theoretical $100 \mu \mathrm{m}$ physical gap to determine the changes in the optical path length with the various filling fluids due to changes in temperature and pressure, and then to compare the calculations to design movements due to shear. The results are in Figure 47 and Figure 48. The effect on optical path length due to pressure is greatest for air, as it is the most compressible. However, the change in optical path due to a large pressure change is still small compared to design gap movements due to shear, and they should easily be compensated. The temperature effect is much greater for both the silicon oil and the glycerin, with a $100{ }^{\circ} \mathrm{C}$ change producing the same change in optical path length as a typical design gap movement for shear. Through compensation, this change will also be mitigated for the modest temperature changes seen in the test at the Virginia Tech supersonic wind tunnel. The conclusion is that if one is concerned about changes in temperature, one would wish to have air in the optical path, and if pressure if pressure is a concern, glycerin. Overall, since in typical flow environments the temperature effect is so much greater than the effect of change of pressure, air is preferable, although implementing this remains difficult until another source of damping can be developed. 


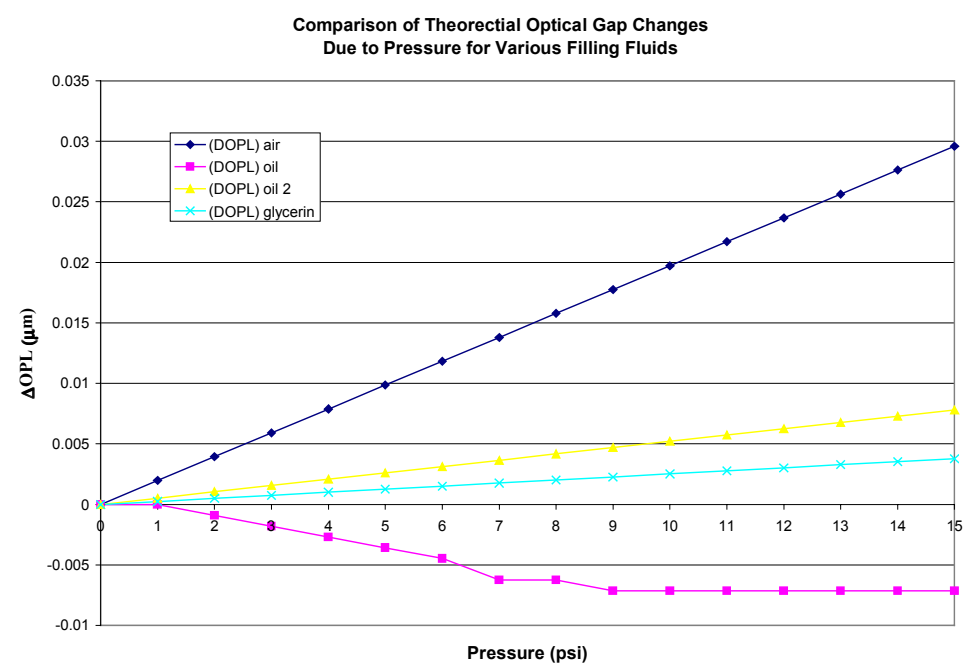

Figure 47. Comparison of the effect of a change in index of refraction with pressure on a theoretical optical path length for air, oil, and glycerin.

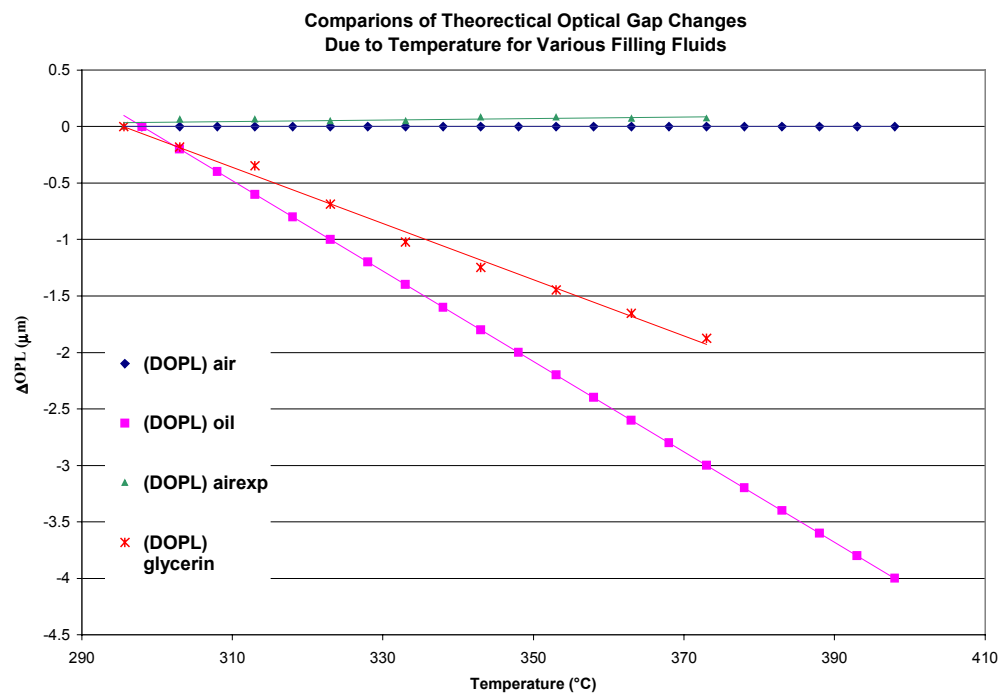

Figure 48. Comparison of the effect of a change in index of refraction with temperature on a theoretical optical path length for air, oil, and glycerin.

\subsection{Microfabrication Study}

A research effort was undertaken to find an inexpensive method to micromachine the skin friction sensor. This proved to be a difficult task, considering the ever-changing capabilities that encompass microfabrication technology. There has been great interest in developing a microfabricated skin friction sensor recently. Small physical size is 
important for skin friction measurements, because the boundary layer thickness in many practical applications is itself small. To put this idea in perspective, note that the boundary layer thickness in a scramjet combustor would be several millimeters while that on a typical turbine blade is a fraction of a millimeter. This size requirement means that the optimal "footprint" of a gage should be on the order of a millimeter and leads directly to a consideration of micromachining techniques. As mentioned in Chapter 1, most of the prior work has centered on either the table-top design or a hot wire sensor. ${ }^{65,66,67}$ Below is a synopsis of the research, design work, and tests that were performed here in an effort to produce a micromachined version of a cantilever beam skin friction sensor, the first known study into the possibility of microfabricating such as sensor.

\subsubsection{Motivation for Microfabrication of Skin Friction Sensor}

For all fluid dynamic measurements, it is preferred that sensor be a small as possible to approximate measurements at a point. This is especially true of skin friction sensors, as the small size tends to reduce many of the typical errors in wall shear stress measurements. Microfabrication technology allows the construction of very small sensors, with inexpensive batch techniques, increasing frequency response and repeatability. Another possible benefit from the microfabrication of the skin friction sensor, would be the reduction of two commonly studied error sources for floating head skin friction sensors, pressure gradients and differences between the sensing head temperature and that of the wall. It would seem clear that as the head size decreases, the error due to pressure gradient would decrease as well. In addition, as the size of the sensing head decreases compared to the boundary layer thickness, it tends to reason that step-temperature differences, which affect the measurement by altering the boundary layer, would decrease as well. Both of these error sources were studied during this study and the work is described below.

\subsubsection{Pressure Gradient Effects}

The design and error sources involved with making measurements using a floating head skin friction sensor have been studied by a great number of researchers. These design concerns include misalignment and geometry of the floating head ${ }^{68,69,70}$, 
surface tension of the fluid in the gap introducing spurious forces ${ }^{71,72}$, and pressure gradient effects ${ }^{72,73}$. Allen ${ }^{69}$ performed extensive studies in supersonic flows of the errors for various physical gap sizes around the head, head diameters, size and shape of the floating element lip, and protrusion or recession of the head, as depicted in Figure 49. Allen's first conclusion was that the possible error sources are all zero if the head is perfectly aligned with the surrounding wall. Also, the error from protrusion or recession of head is the same. In studying the effect of possible misalignment, Allen concluded that head diameter should be as small as possible, and the lip around the edge of the floating element should be as small as possible. Both of these conclusions are derived from his study of misalignment of the sensing head, but they are also valid for pressure gradient effects, the additional force that a spatially varying pressure will impose on a floating element. Filling the sensor with an incompressible fluid eliminates the pressure gradient effect, as it provides a constant pressure around and under the floating head.

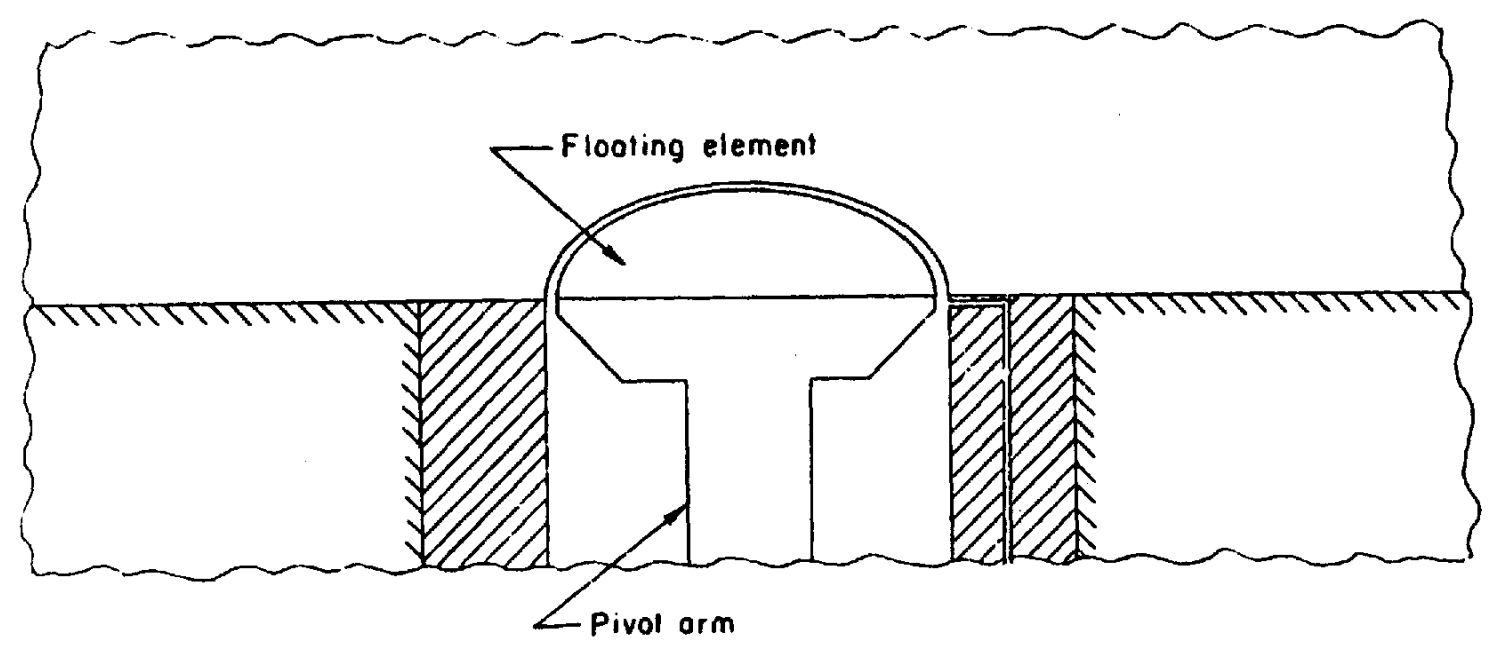

Geometric variables
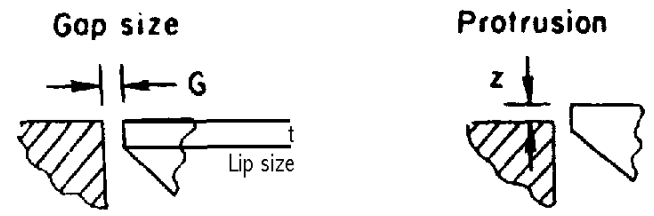

Figure 49. Sketch of the geometric variation which Allen ${ }^{68}$ studies for pressure gradient effects for floating head skin friction sensors. 
However, Frei ${ }^{71}$ and Hirt $^{72}$ both studied the force that the surface tension of the fluid imparts on the head. As stated above, a high surface tension fluid is usually desirable for filling the sensor, because the tension is what retains the fluid to the interior of the gage. The conclusion of these studies is that properly aligned sensors are not susceptible to these surface tension errors. It should be noted that there is some disagreement between workers in the field about if it is better to have the gap round the floating element to be large or small. Allen indicated that a larger gap reduces misalignment errors, while most others tend to try to disturb the flow as little as possible by having as small a gap as possible. From a practical point, a larger gap eases the machining constraints on a sensor, but a small gap is necessary to keep as much of the filling fluid as possible in the gage during testing.

Some of the conclusions obtained by these researchers have interesting consequences for micromachined versions. First, most micromachining technologies have tolerances measured in micrometers, meaning that the sensor will always have what is considered perfect alignment. Second, the head and lip size will decrease dramatically. Finally, the small gaps desired by some in the field are simple for the technology. In fact, it has been hypothesized that having a small gap around the whole cantilever beam will eliminate the necessity for the filling oil to remove pressure gradient effects. ${ }^{74}$

As work continued on microfabricating these sensors, an examination of the effect of the reduction of the head size in comparison to the effects of a pressure gradient seemed in order. The forces caused by the pressure gradient can be expressed as a lip force and a moment force, as depicted in Figure 50. Given an adverse pressure gradient, a moment will be generated that tends to increase the skin friction results, while a lip force (the integration of the force of the pressure on head lip of the head) tend to decrease the results. 


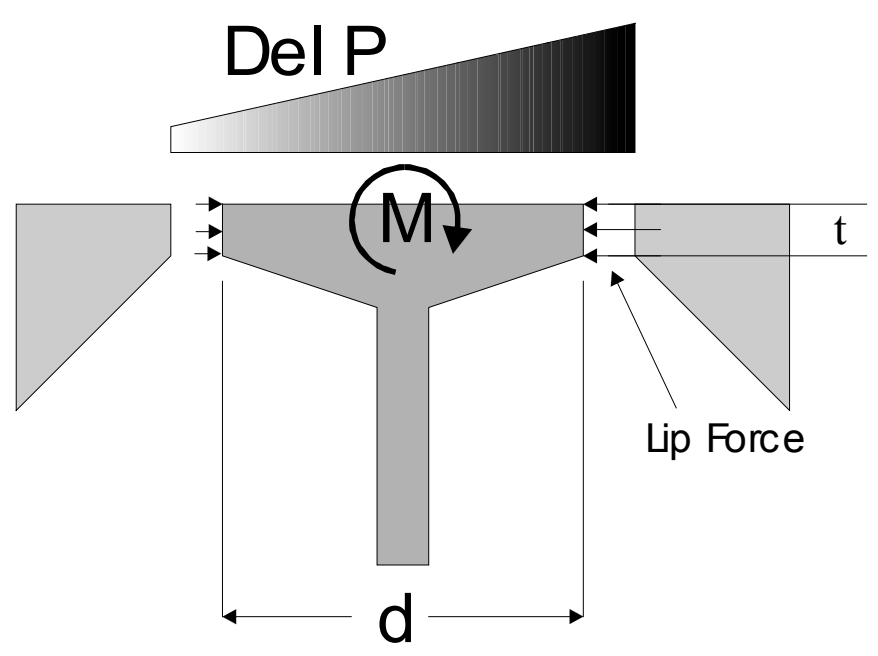

Figure 50. Depiction of the effect of pressure gradient on a cantilever beam skin friction sensor for producing possible errors to the measurement.

As the study is for microfabrication, one can assume the pressure gradient is locally linear, and for the purposes of this study, a circular head. The change in pressure for a given distance due to the gradient is given by,

$$
\Delta P=\nabla P^{*} x .
$$

The variables for this work are defined as,

$\mathrm{t}$ - thickness of the lip of the sensing head

$\mathrm{f}$-factor describing the linear pressure drop from the surface to the interior of the sensor

$\mathrm{F}$ - force

$\mathrm{P}$ - pressure

$\mathrm{d}$ - diameter of sensing head

$\mathrm{x}$ - gradient direction

$\mathrm{y}$ - orthogonal to gradient direction

$\mathrm{M}$ - moment

A - area of sensing head

Previous researchers ${ }^{72,24}$ developed an equation for the lip force assuming a linear drop of the pressure difference along the lip at the surface to the average pressure in the 
interior of the sensor. This developed relation did not resolve the component of the pressure in the direction of the pressure gradient, but instead used the total magnitude, leading to the incorrect results of

$$
F_{l}=f^{*} t^{*} \nabla P^{*} \pi * d^{2} / 4 .
$$

In this study, the component of the pressure was taken in the direction of the gradient with the lip force determined by the integral of the pressure around the diameter of the head,

$$
F_{l}=2 t * \int_{0}^{d / 2} 2 x * \nabla P \frac{x}{d / 2} d y
$$

which reduces to,

$$
F_{l}=\frac{8 t^{*} f^{*} \nabla P^{d / 2}}{d} \int_{0}\left(\frac{d^{2}}{4}-y^{2}\right) d y,
$$

and solved as,

$$
F_{l}=-\frac{2}{3} t^{*} f^{*} \nabla P^{*} d^{2} .
$$

As expected, the force is negative for the adverse gradient.

Now, the moment force can be determined by the integral of the pressure over the area of the head,

$$
M=x^{*} p(x) d A,
$$

which in the current variables is

$$
M={ }_{-d / 2}^{d / 2} x * \nabla P *(x+d / 2) * 2 * \sqrt{\left(d^{2} / 4-x^{2}\right)} d x,
$$

which solves to

$$
M=\frac{\nabla P^{*} \pi * d^{4}}{64} .
$$

If this moment is then converted into the apparent force to counteract it, the results would be,

$$
F_{M}=\frac{\nabla P^{*} \pi * d^{4}}{64^{*} L_{t}} .
$$


As stated above, this force is positive for the adverse gradient. As these forces oppose each other, it could actually be possible to design a skin friction sensor that would be insensitive to pressure gradient, although depending on the flow regime, the relative forces involved may be so different as to make the geometry of such a sensor impractical. Clearly though, the head geometry is the dominant effect. If the lip size is decreased at the same rate as the head diameter, the lip force decreases with the third power of the diameter, while the moment decreases with a fourth power. The measured force will obviously decrease with the second power, meaning that as the sensor is decreased in size, the pressure gradient effects have decreasing importance. This conclusion is one of the reasons that researchers measuring skin friction strive for as small a head as possible (the main reason is to get as close to a point measurement as possible). Both of these reasons is a major impetus to microfabricate these sensors.

\subsubsection{Step-Temperature Effects}

Another concern for making accurate skin friction measurements is the potential for a temperature mismatch between the head and the surrounding wall. This temperature mismatch is unavoidable if different materials are used for the wall and sensor for even a moderate heat flux. Matching the thermal properties of the sensing head of the gage with the surrounding wall is very important in correctly measuring wall temperature, heat flux, and skin friction. It is easy to see the effect a mismatch would have on the wall temperature and heat flux, but it may not be so obvious for skin friction. In fact, it can be expected that an error in any of the quantities would lead to errors in all of them. Heat flux and skin friction are related through Reynolds Analogy, $\mathrm{St} \approx \mathrm{C}_{\mathrm{f}} / 2$.

A different temperature of the sensing head has two possible effects on the flow, which in turn affect the shear acting on the sensing head. The different temperature of the sensing head changes the viscosity, $\mu$, of the fluid flowing over the head compared to that of the rest of the flow. This directly changes the shear through Stokes' Law, $\tau_{\mathrm{w}}=\mu$ $\partial \mathrm{u} /\left.\partial \mathrm{y}\right|_{\mathrm{w}}$. But, for compressible flows, the momentum and energy boundary layer equations are coupled, therefore a change in the energy balance at the wall caused by a temperature mismatch will lead to a change in the boundary layer profile. This boundary layer profile change affects the measured shear through the $\partial \mathrm{u} /\left.\partial \mathrm{y}\right|_{\mathrm{w}}$ term. 
Numerous researchers have examined the effect of the step-temperature change

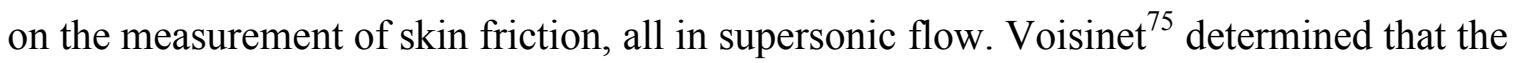
error due the temperature mismatch is a function of both Reynolds number and Mach and can be as great as $0.45 \%$ per $\mathrm{K}$ difference between the wall and the sensing head. As expected from the discussion above, the error is positive for higher sensor temperatures. The absolute error is not that sensitive to wall shear stress level, therefore the skin friction measurement error becomes large when the wall shear stress becomes low. Westkaemper found little error for the temperature mismatch for small differences, as opposed to the large differences of the Voisinet work. ${ }^{76}$ However, when one reinterprets the Westkaemper data in the terms Voisinet used, the two correlate. An extensive computational and experimental study of this problem was undertaken by Debieve, et al. ${ }^{77}$ The major conclusion is that the error is caused largely through changes in the local thermal properties and not on any in the boundary layer profile.

Because of the simplicity of constructing heated thin-film sensors for the purpose of inferring skin friction, many researchers have examined the effect of the heat transfer from the heated films to the air on the measurement. ${ }^{78,79,80}$ This research area is beneficial to the examination of floating head sensor errors, with conclusions similar to those discussed here.

As the sensor is constructed to the scale of microfabrication technology, the effect of this temperature mismatch error compared to the shear measurement as the head size decreases was previously unknown. It was hypothesized that as the head size becomes smaller, there is less of a possibility of the temperature mismatch to cause changes in the boundary layer profile. If the head size were decreased enough, the thermal boundary layer formed would not be able to grow outside of the laminar sublayer for turbulent boundary layers, increasing the likelihood that the effect is only with the thermal properties. Computational and analytical studies, performed by other researchers in conjunction with this project, have been performed to gain insight to this problem

\section{CFD Study of Step-Temperature Change}

Using current micromachining technologies, the skin friction sensor must be constructed from different materials than will likely used as the wall of the test article, so a step temperature change will develop even for moderate heat fluxes, leading to the 
errors discussed above. A computational effort was undertaken in coordination with the current work by Dr. Eric Fuller of AeroSoft Inc. ${ }^{81}$ to examine the effect of the step temperature change as the size of the sensor is decreased.

A sample of that study is included here. The simulation was run using the General Aerodynamic Simulation Program (GASP) version 3.2.3, a widely utilized CFD code written by AeroSoft. The calculation, using the $\mathrm{K}-\Omega$, two-equation turbulence model, was made over a flat plate over with a $2500^{\circ} \mathrm{C}\left(4540^{\circ} \mathrm{F}\right)$ total temperature, Mach 3 flow. The wall temperature was set to $280^{\circ} \mathrm{C}\left(540^{\circ} \mathrm{F}\right)$, with a $1 \mathrm{~mm}$ by $1 \mathrm{~mm}$ section raised to $340^{\circ} \mathrm{C}\left(640^{\circ} \mathrm{F}\right)$. This discontinuity simulates a typical difference in temperature between the sensor, with a typical size for a micromachined device and the surrounding wall.

Figure 51 depicts the predicted skin friction along the flat plate with the flow moving from left to right. The skin friction coefficient was found to be lower on the sensor, as compared to the surrounding wall. Surface integration of $\mathrm{C}_{\mathrm{f}}$ along the sensor gave a value of $2.585 \times 10^{3}$ while the surrounding value was $2.561 \times 10^{3}$, a difference of $1 \%$ due to the temperature change. This difference is opposite in sign to that seen in previous studies, and of much smaller magnitudes. A more detailed study was needed to determine the reason for the difference. 


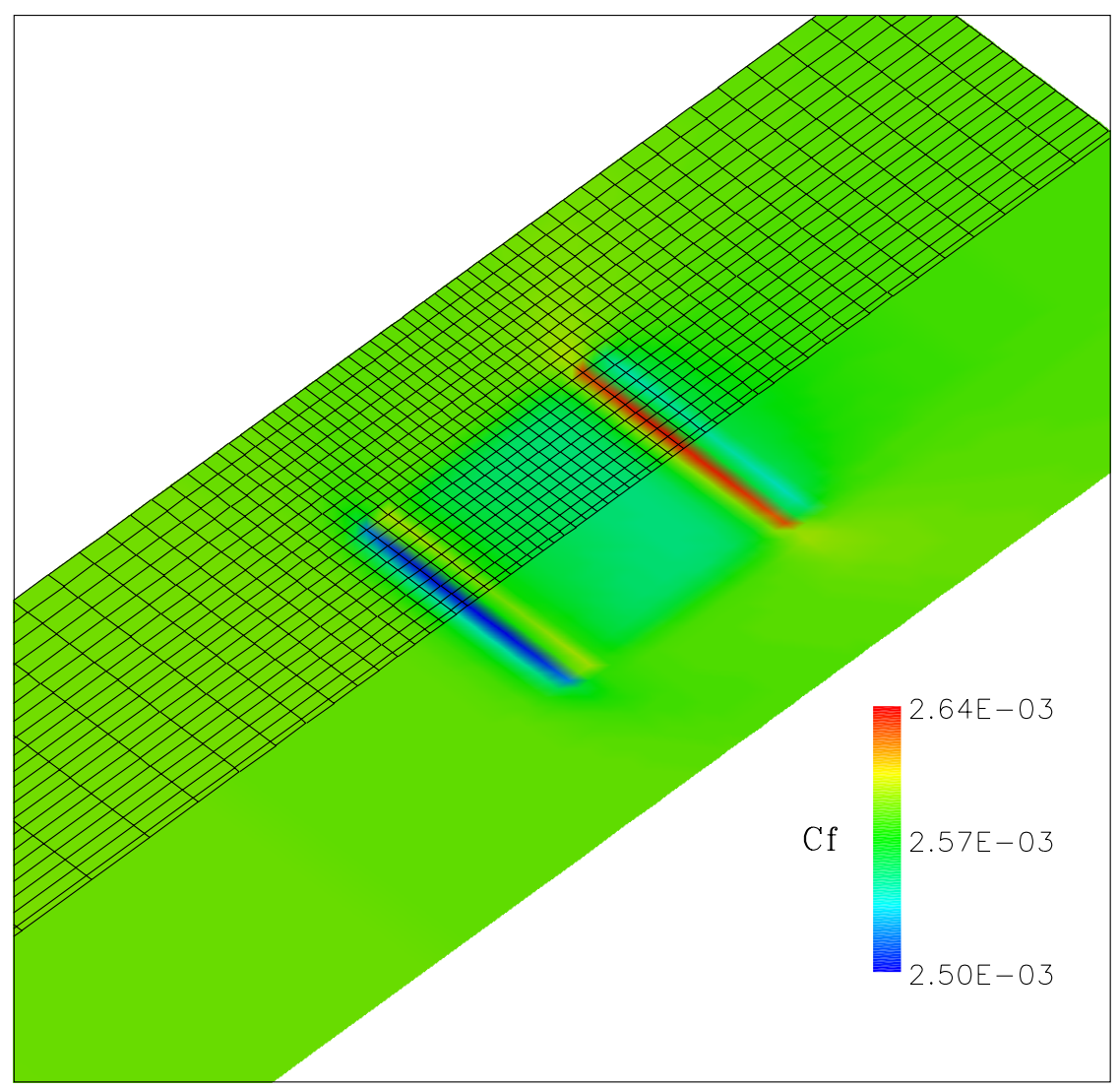

Figure 51. Computational skin friction results for a Mach 3 flow and $60{ }^{\circ} \mathrm{C}$ temperature difference. Note that the flow direction is from left-to-right. ${ }^{81}$

The lack of any significant change in the skin friction as compared to the surrounding wall does not correlate well with the experimental data available from numerous authors, as indicated above. As all evidence points to the fact that a majority of the effect is due to the change of the fluid thermophysical properties, it was hypothesized that the grid may have not been fine enough to allow this change to propagate enough cells off the wall to see an effect. A grid study was then performed in a 2-D case to determine what the effect of this grid coarseness might be.

The extended study was performed in $2 \mathrm{D}$, to allow the greatest study without the expenditure of a large amount of computational time. As Figure 51 shows, there is not much variation in the cross-flow direction, and therefore it was determined that the $2 \mathrm{D}$ simulations would model the correct physics at a fraction of the cost. The test conditions for the simulations were as followed: Mach $3, \mathrm{~T}_{\infty}=992 \mathrm{~K}, \mathrm{P}_{\infty}=101325 \mathrm{~Pa}, \mathrm{~T}_{0}=2777.77$ 
$\mathrm{K}$, and $\mathrm{P}_{0}=3.72169 \mathrm{e} 6 \mathrm{~Pa}$. The wall temperature was set to $280^{\circ} \mathrm{C}\left(540^{\circ} \mathrm{F}\right)$, with a $1 \mathrm{~mm}$ long section raised to $340^{\circ} \mathrm{C}\left(640^{\circ} \mathrm{F}\right)$.

Three different grids were run, a described in Table 6, for each turbulence model tested: a standard case, one with more cells on the head, and one with more cells in the boundary layer. The grid convergence study was necessary due to concerns about the results of the standard case. The first concern was that the new thermal boundary layer on the head would not grow into sufficient cells in the velocity layer to affect it, and therefore the number of cells in the layer were doubled. The second concern was that the results showed a discontinuity at the beginning and end of the head, therefore the number of grid points over the head should be decreased so that the integrated values of shear were correct. As shown in Figure 52, which used a K- $\Omega$ turbulence model, the different grids only showed slight differences, and all had the same trend - lower shear for the higher temperature. This lower shear is counter-intuitive, the higher head temperature should start a thermal boundary in which the viscosity of the fluid should be greater and therefore the shear should be higher. However, as seen in Table 7, the differences were negligible. As is usually the case with CFD results, the turbulence model was questioned, and so the cases were re-done using a Baldwin-Lomax algebraic model,. These results were also showed negligible effects, and so the cases were performed for a laminar case, removing the question of a turbulence model and the ability to capture the effect of the thermal boundary layer for this case. As seen in Table 7, the laminar case does show a significant, and positive error in the shear. The error is affected by the changes in grids, with an increase in shear as the y-grid is refined, and then again when the $\mathrm{x}$-grid is refined and the y-grid is relaxed back to its original size. Results of the laminar case for the different grid spacings are shown in Figure 53.

Table 6. Case definitions.

\begin{tabular}{|c|c|c|c|}
\hline $\begin{array}{c}\text { Grid } \\
\text { Number }\end{array}$ & $\begin{array}{c}\text { Number of Cells on } \\
\text { Sensor Head }\end{array}$ & $\begin{array}{c}\text { Number of Cells in } \\
\text { Boundary Layer }\end{array}$ & Definition \\
\hline 1 & 20 & 40 & Standard Grid \\
\hline 2 & 20 & 80 & Y-refined \\
\hline 3 & 80 & 40 & X-Refined \\
\hline
\end{tabular}




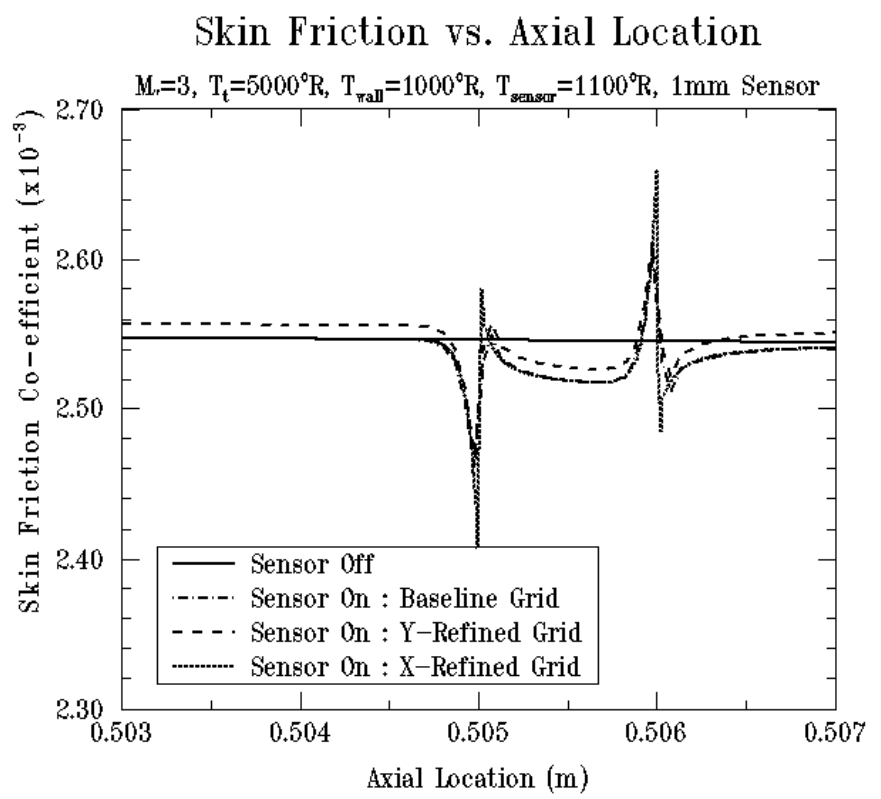

\section{Figure 52. 2-D plot of the $\mathrm{K}-\Omega$ results for a Mach 3 flow and a $60{ }^{\circ} \mathrm{C}$ temperature difference. ${ }^{81}$}

The original intent of the CFD work was to demonstrate that as the size of the sensing head decreased, the error from the temperature mismatch would also decrease. The physical reasoning is that as the head size decreases in terms of the boundary layer thickness, any effects that the head causes must decrease. In order to quickly test this hypothesis, the sensing head was halved and then halved again without re-gridding to simulate a head at half and quarter size. Only laminar cases were run, as only the laminar case above seemed to show any effect of the temperature change. Table 7 presents the results of all the 2-D calculations. The error actually increases as the head size decreases, as shown in Figure 54. This caused a great deal of concern, as it seemed counterintuitive. However, after some study it was concluded that because the cases were not regridded, the solution actually became coarser as the head size decreased. Therefore, the increase in error is the same artifact of the grid size for the different head sizes as it was with grid spacing within the original laminar case. After discovering this, a literature search into laminar flow/step temperature difference cases was performed, and it was found that much finer grids had been used in previous work. ${ }^{82}$ In the end, the results seem to indicate that the x-grid spacing is very important. The researchers have determined that the grid is most likely fine enough in the $\mathrm{y}$-direction, but that the $\mathrm{x}$ - 
spacing need to be greatly increased to get accurate results for this boundary layer within a boundary layer case.

Table 7. Integrated force on sensor comparison.

\begin{tabular}{|c|c|c|c|c|c|c|c|c|c|c|c|}
\hline \multirow[b]{2}{*}{$\begin{array}{c}\text { Grid } \\
\#\end{array}$} & \multirow[b]{2}{*}{ Sensor } & \multicolumn{2}{|c|}{ K-Omega } & \multicolumn{2}{|c|}{$\begin{array}{l}\text { Baldwin- } \\
\text { Lomax }\end{array}$} & \multicolumn{2}{|c|}{ Laminar } & \multicolumn{2}{|c|}{ Laminar (Half) } & \multicolumn{2}{|c|}{$\begin{array}{l}\text { Laminar } \\
\text { (Quarter) }\end{array}$} \\
\hline & & $\begin{array}{c}\text { Force } \\
(N)\end{array}$ & $\begin{array}{c}\% \\
\text { Diff. }\end{array}$ & $\begin{array}{c}\text { Force } \\
(N)\end{array}$ & $\begin{array}{c}\% \\
\text { Diff. }\end{array}$ & $\begin{array}{l}\text { Force } \\
(N)\end{array}$ & $\begin{array}{c}\% \\
\text { Diff. }\end{array}$ & Force (N) & $\begin{array}{c}\% \\
\text { Diff. }\end{array}$ & Force (N) & $\begin{array}{c}\% \\
\text { Diff. }\end{array}$ \\
\hline 1 & Off & 1.622 & \multirow{2}{*}{-0.55} & 1.783 & \multirow{2}{*}{-2.86} & 0.146 & \multirow{2}{*}{8.22} & 0.07310 & \multirow{2}{*}{9.7} & 0.0365 & \multirow{2}{*}{10.7} \\
\hline 1 & On & 1.613 & & 1.732 & & 0.158 & & 0.00802 & & 0.0405 & \\
\hline 2 & Off & 1.628 & \multirow{2}{*}{-0.61} & 1.681 & \multirow{2}{*}{-2.92} & 0.152 & \multirow{2}{*}{5.26} & 0.00762 & \multirow{2}{*}{6.5} & 0.0381 & \multirow{2}{*}{7.5} \\
\hline 2 & On & 1.618 & & 1.632 & & 0.160 & & 0.00812 & & 0.0410 & \\
\hline 3 & Off & 1.622 & \multirow{2}{*}{-0.49} & 1.489 & \multirow{2}{*}{-2.22} & 0.161 & \multirow{2}{*}{4.97} & 0.00817 & \multirow{2}{*}{6.3} & 0.0411 & \multirow{2}{*}{3.7} \\
\hline 3 & On & 1.614 & & 1.456 & & 0.169 & & 0.00869 & & 0.0426 & \\
\hline
\end{tabular}

Laminar Skin Friction $\mathrm{Co}^{-}$-efficient

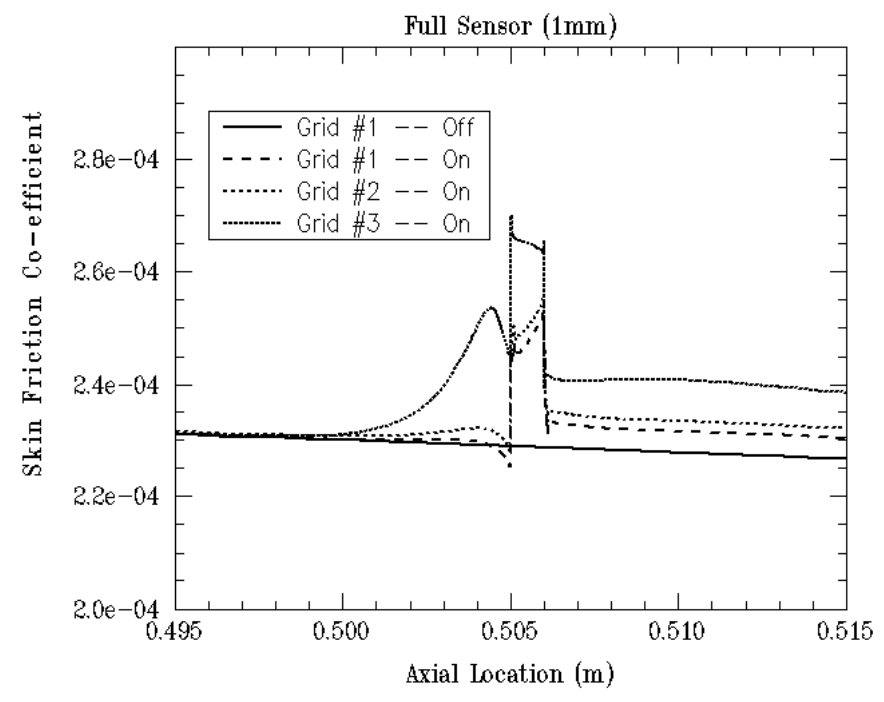

Figure 53. Comparison of skin friction coefficients for different grids for full sensor of a laminar flow calculation for a Mach 3 flow and a $60{ }^{\circ} \mathrm{C}$ temperature difference. $^{81}$ 


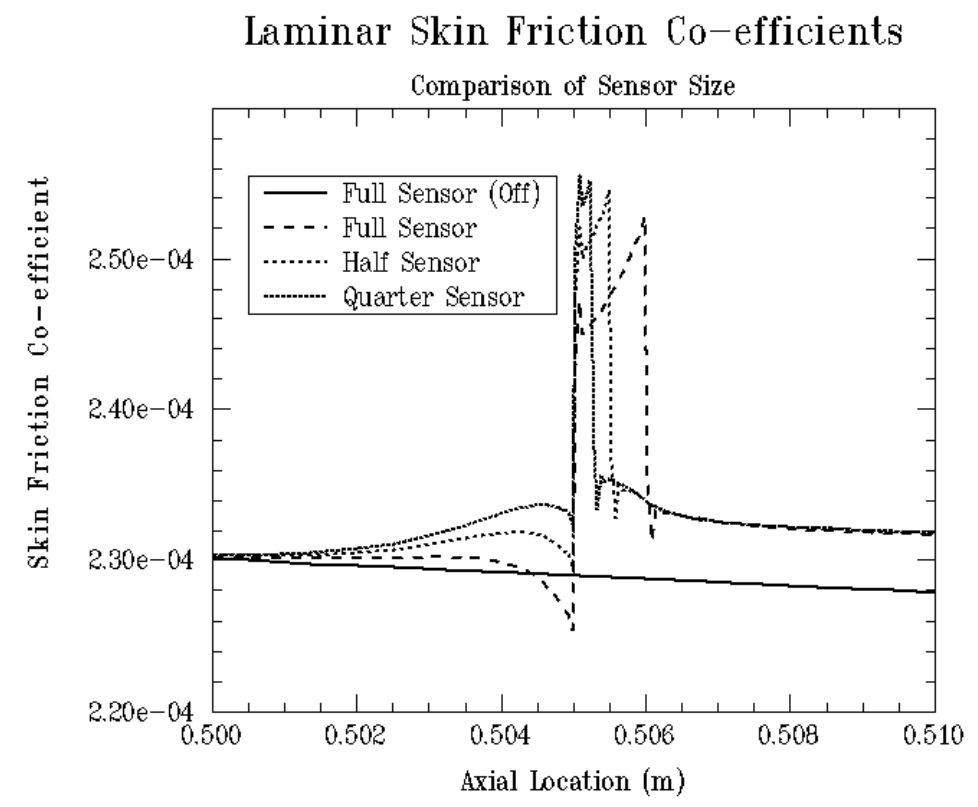

\section{Figure 54. Comparison of skin friction coefficient for different sensor sizes for a Mach 3 flow and a $60{ }^{\circ} \mathrm{C}$ temperature difference. ${ }^{81}$}

It has also been hypothesized that as the volume of the sensor decreases, the less likely it is that it can support a large temperature difference. The thermal mass would become so small that it would follow the temperature of the wall closely, just as a small thermocouple bead reacts more quickly to a temperature change.

\section{Analytical Study of Step-Temperature Change}

The study of the measurement errors caused by a step-temperature change on the wall continued with an analytical study performed in collaboration to this study by Inger. ${ }^{82}$ As before, the purpose of the work was to get an indication of the relationship between the head size and the magnitude of the error. The real question is if the error scales linearly with the head size, as the shear does, and if the percentage error will increase or decrease as the skin friction sensor concept is miniaturized through microfabrication technology. Again, the analytical study was performed on a laminar supersonic boundary layer so as to avoid the turbulence closure problem. In this study, the effects of the step change on pressure and heat transfer were also examined. 
Although skin friction was the main concern of the work, the other results are of interest to the concurrent microfabrication efforts for the pressure and heat flux sensors.

The results of the work are too extensive to detail here, see Appendix D, but the conclusion will be outlined. ${ }^{83}$ The positive step-temperature change causes a compression field upstream of the change, and then there is a pressure decay due to the negative step-temperature change at the rearward edge. The combination produces a rise and fall that balances at a point on the higher temperature patch. For the case of wall shear stress on the higher temperature patch, the front and rear edge effect reinforce each other, producing a higher shear stress on the patch than the surrounding wall. This result is the same as the CFD results and those of previous researchers. An important point is that the analytical solution shows the discontinuities at the front face that had been a concern during the CFD study. This shear stress artifact is a result of the discontinuity of the induced pressure gradient that occurs at the front edge. Finally, as expected, the heat transfer is reduced along the higher temperature patch. This result bolsters confidence in the CFD work, and this will allow the work to be expanded to turbulent cases in the future.

\subsubsection{Stereolithography Sensor}

For very short-duration testing as in shock tubes or tunnels $\left(0.5-10 \times 10^{3} \mathrm{sec}\right.$.), the priorities for the material selection of skin friction sensors change. For this type test, the facility acceleration loads are so large and the test time so short, that it is necessary for the natural frequency of the sensor to be very high so that the sensor can react and measure the shear while not exhibit ringing. Previous researchers attempted to solve this problem by using light materials such as plastics, which proved to be rather effective in solving the above problems. An effort was made, as a first foray into microfabrication technology, to make such a sensor through the stereo-lithography process. ${ }^{84}$ Although most users either employ the results of stereo-lithography for demonstrations or for making molds to produce the part in other materials, the hardened resin from the stereolithography process, which has many similar properties as the plastics, was used here as the final sensor material. In most environments, this solution is not practical due to the low temperature ceilings of these materials. But in short run time facilities, even the high 
heat fluxes and enthalpies seen in high Mach number, combusting flows do not have time to transmit much energy to the surface. A sputtered coating should be enough to protect the surface of the sensor from both high-speed particulates in the flow and the high temperatures seen briefly during the run.

The process of stereolithography (SLA) uses exposure to UV light to solidify a special liquid resin (DuPont Somos 7100, in our case) into a desired 3D shape. Some concepts illustrating the technology are shown in Figure 8. The liquid resin is kept either in the free surface mode (Figure 8a) or in the fixed surface mode (Figure 8b). The latter has a resin container with a transparent window plate for exposure. The solidification always happens at the stable window/resin interface. An elevator is pulled up over the thickness of one additional layer above the window for each new exposure (Figure 8c). This scanning method has the advantage of point by point controllability, avoiding unevenness of solidification leading to nonuniform shrinking of the works. When applying the scanning technique, a laser beam is used to solidify one microscopic polymer area at a time to arrive at complicated 3D shapes by stacking thin films of hardened polymer layer upon layer. Process control is simply directed from a CAD system containing the 'slice' data. The laser beam is focused down to 200-250 $\mu \mathrm{m}$ spot size and typical fabrication times range from 30 minutes to an hour, allowing for very quick turn-around times. The position accuracy for the laser beam spot is $1 \mu \mathrm{m}$ in the zaxis and $0.25 \mu \mathrm{m}$ in the $\mathrm{x}$ and $\mathrm{y}$ directions. 


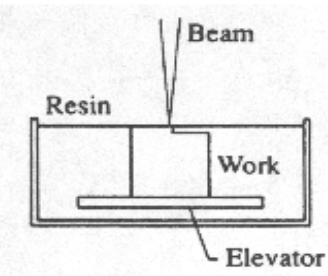

Free surface method

a
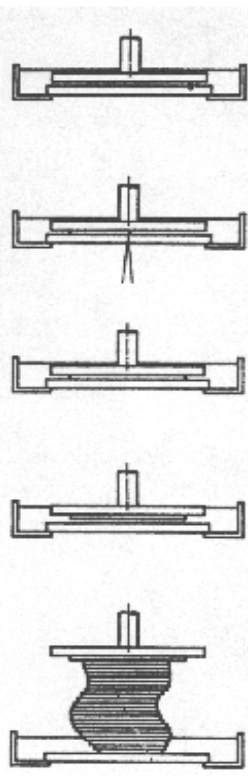

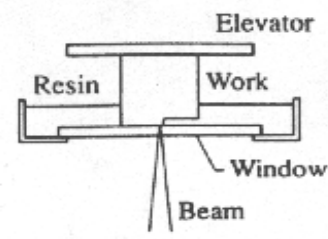

Fixed surface method

b

c

Position the elevator

near the window

Scan the beam

along the first layer

Finish the first layer

Pull up the elevator

for thickness of one layer

Repeat these operations

to make the object shape

\section{Figure 55. Stereolithography process. ${ }^{85}$}

The sensor was constructed in two parts with the stereo-lithography process, which snap together for final construction, as seen in Figure 56, for a shock tube environment. The bottom piece was then sent off for attachment of conventional strain gages, as described in the next section. Conventional strain gages were used in this effort because of the data rate needed to make measurements in a shock tube. At the time, the needed fiber optic signal processing systems were not yet available.

In an effort to minimize the sensor size, semi-conductor strain gages were selected over metal foil gages due to their smaller physical size and much higher gage factor. The larger signal generated by these gages allowed the design of smaller beams and sensing heads, producing not only a physically smaller sensor but also one with a higher natural frequency, which, as discussed above, is of paramount importance for this application. While the Virginia Tech research group has attached the strain gages for 
past designs themselves, that has met with mixed results and it was decided to contract out the attachment of the strain gages to an outside company.

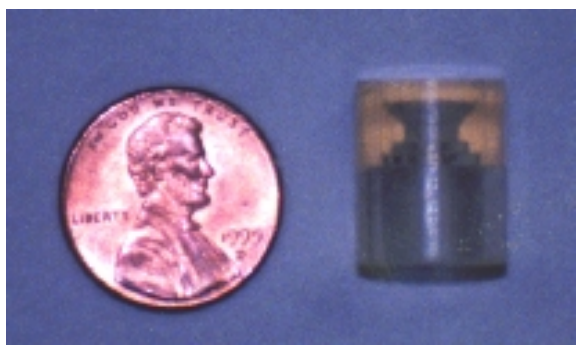

(a)

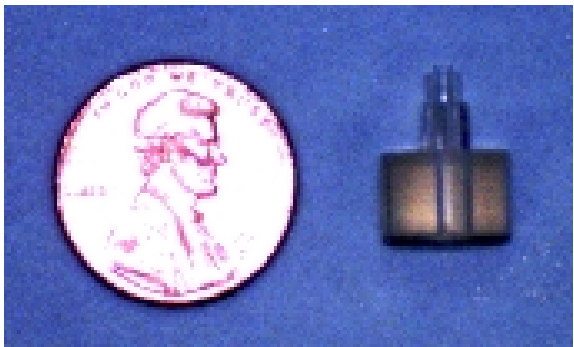

(b)

Figure 56. Photographs of the (a) top and (b) bottom pieces of the SLA gage

The research group selected Micron Instruments' SS-090-060-1150P bar semiconductor strain gages. The dimensions of the gage are $2.9 \times 0.2 \mathrm{~mm}(0.090 \times 0.008$ inches). This size could easily fit on the beam of the sensor, and the resistance of $1150 \Omega$ allows greater bridge voltage and therefore output without self-induced heating of the strain gages, which would cause drift. Figure 57 pictures the base with attached strain gages.

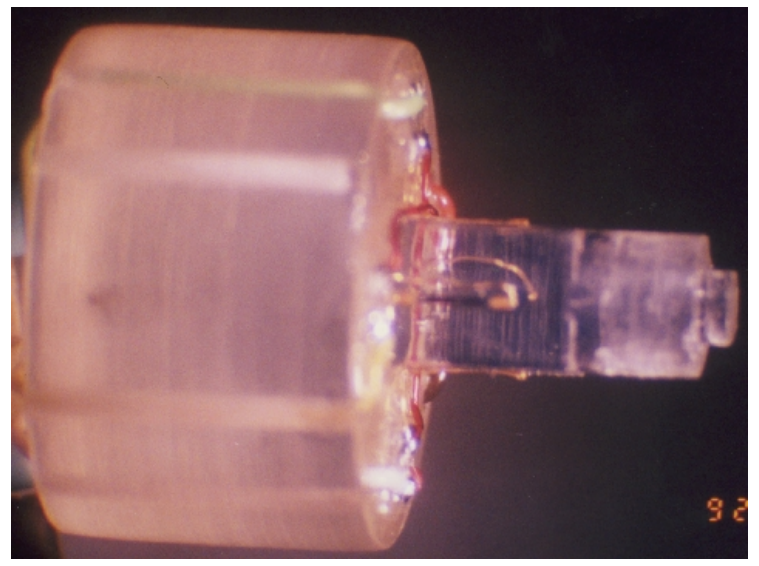

(a)

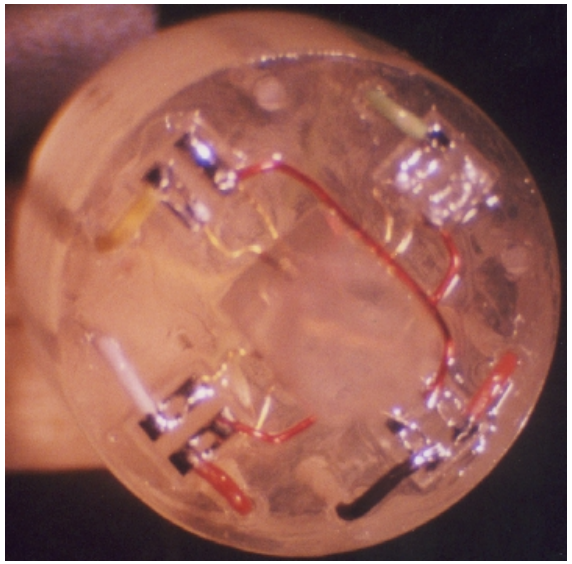

(b)

Figure 57. Photographs of the side view (a) and top view (b) of the bottom piece of the SLA gage with strain gages attached. 
A sample calibration curve of a constructed SLA sensor can be seen in Figure 58.

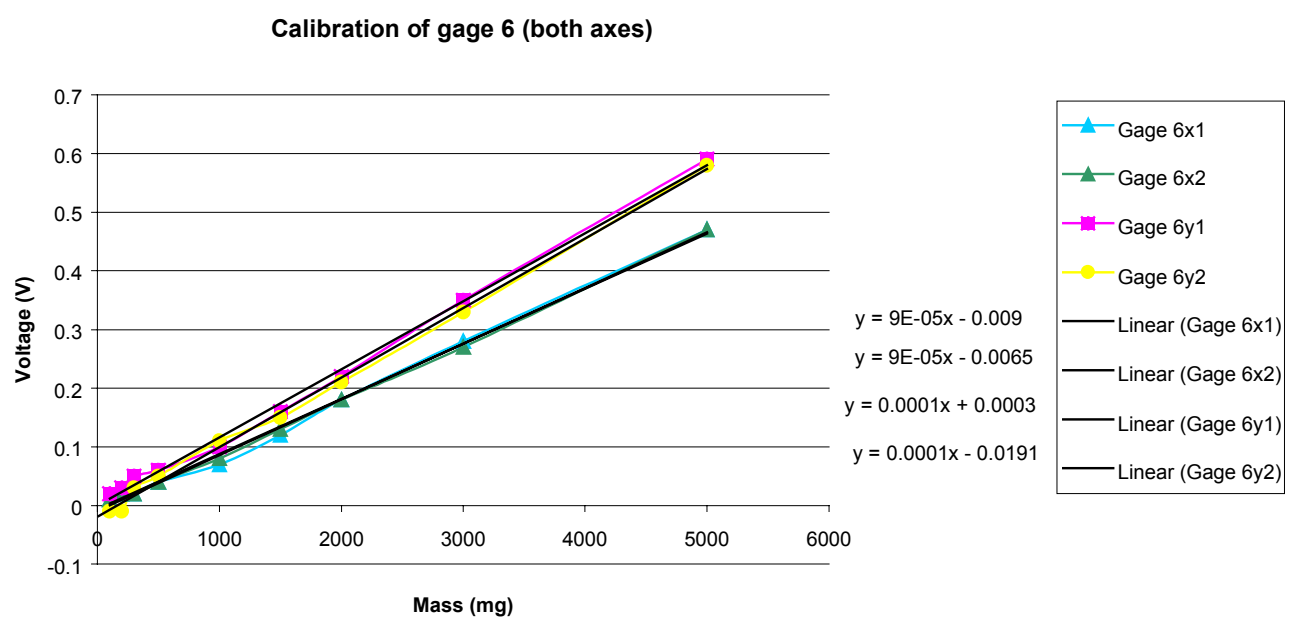

\section{Figure 58. Calibration of a stereolithography skin friction sensor for both axes including linear fit equations.}

The SLA process demonstrated great possibilities for the construction of skin friction sensors for measurement in highly transient flows. The construction of the sensors was much more repeatable and simple than with conventional machining. Additionally, once a design has been worked out, large numbers of the sensors can be fabricated at relatively low cost. However, the tunnel testing of the sensor was not successful; this was attributed to poor adhesion of the strain gages to the SLA resin. Future sensors will have to solve this problem. Also, the material is not very useful outside the stated environment. It has been suggested that the SLA process be used to fabricate molds in which the sensor is made from a higher temperature material. Finally, newer processes, such as selective laser sintering, could be used to make the part directly from metal. Due to the temperature limitations, though, other microfabrication techniques were explored.

\subsubsection{Technology Review}

A detailed investigation was conducted into the possibility of finding a different method of fabricating miniaturized gages. The first idea had been to micromachine the 
skin friction sensors from silicon. Although this option should be successful, is does involve a great deal of time and money. At first, it was thought that a simpler and more inexpensive alternative method could be found.

The first alternative was to laser cut thin metal sheets, and then diffusion bond the sheets together to form a part. The pieces could be made inexpensively, repeatably, very small, and out of metal, which is desirable so as to increase the operating temperature and to match thermal properties with the wall. The drawbacks are that the sensor would only be to measure in one direction and bonding the sheets together may not be a simple or accurate process. However, the metal sheet bonding appeared to be the best alternative, and was it explored in detail.

\subsubsection{Metal Bonded Concept}

Previously, the pieces of a skin friction sensor were made on conventional machining tools and then assembled, which meant that the pieces had to have clearance to slide together during assembly. Unfortunately, this leaves a great deal of internal volume for air to move around and for pressure changes and gradients to cause spurious forces. Filling the gage with a liquid alleviates these problems, but as mentioned earlier causes others. It was hypothesized that by having the housing of the sensor match the geometry of the beam with only a small gap, that the volume inside the sensor will be very small, making it difficult for air to flow into the sensor and, in turn, making the oil unnecessary.

A depiction of the metal bonded concept is presented in Figure 59. Cuts are made in each sheet for the desired geometry and clearances. Then, the sheets are put together and bonded. In the sketch on the left, the gage would be only sensitive vertically. The optical fibers are placed in channels indicated by the red squares. This design also takes advantage of placing the fibers away from the beam, increasing sensitivity, and that is what makes the concept feasible in the first place. 


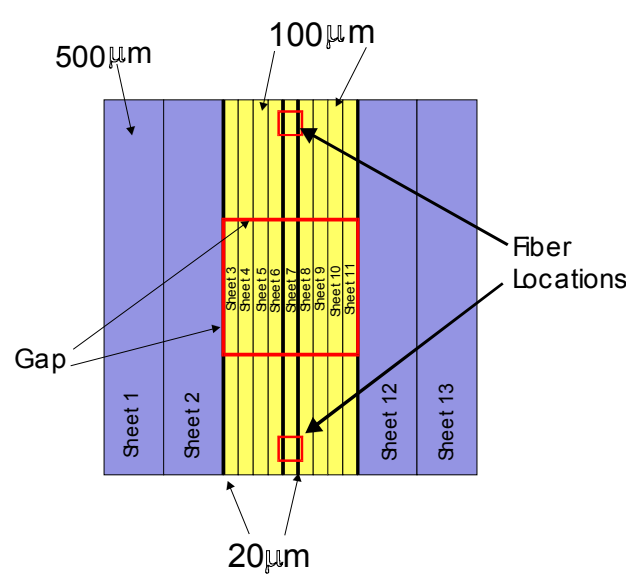

Top View of the Gage

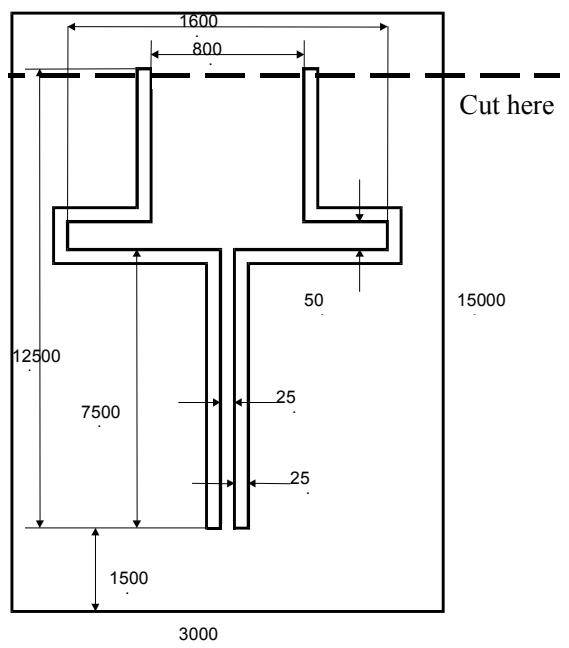

Side View of a Sheet

Figure 59. Depiction of the metal bonded gage design.

The design space of the selected geometry in Figure 59 is presented in Figure 60. The design equation for the bonded sensors is slightly different than those for a circular crosssection. This is mostly due to the different moment of inertia, but also to the larger freedom this design affords the head and reflector geometry. The pertinent design equation for bonded sensors is

$$
\Delta \delta=\frac{6 \tau_{w} r t}{E h^{3}}\left[L_{t}{ }^{2}\left(2 \alpha-\alpha^{2}\right)\right],
$$

where, $\mathrm{t}$ - length of the sensing head

$\mathrm{h}$ - the thickness of the beam in the bending direction.

It should be noted that because of the concept, the width of the sensor does not matter, since the width of the head and the beam must be the same. Also, as mentioned above, the maximum deflection becomes more important as the size of the sensor decreases. Using the same variables, the new equation for tip deflection in the gap between the head and the housing is

$$
y_{\text {max }}=\frac{4 \tau_{w} t L_{t}^{3}}{E h^{3}} .
$$

If $\mathrm{r}$ is place at a reasonable location compared to the overall sensor, the design equation reduces to a trade-off between the beam width and the head length as seen in the figure. 


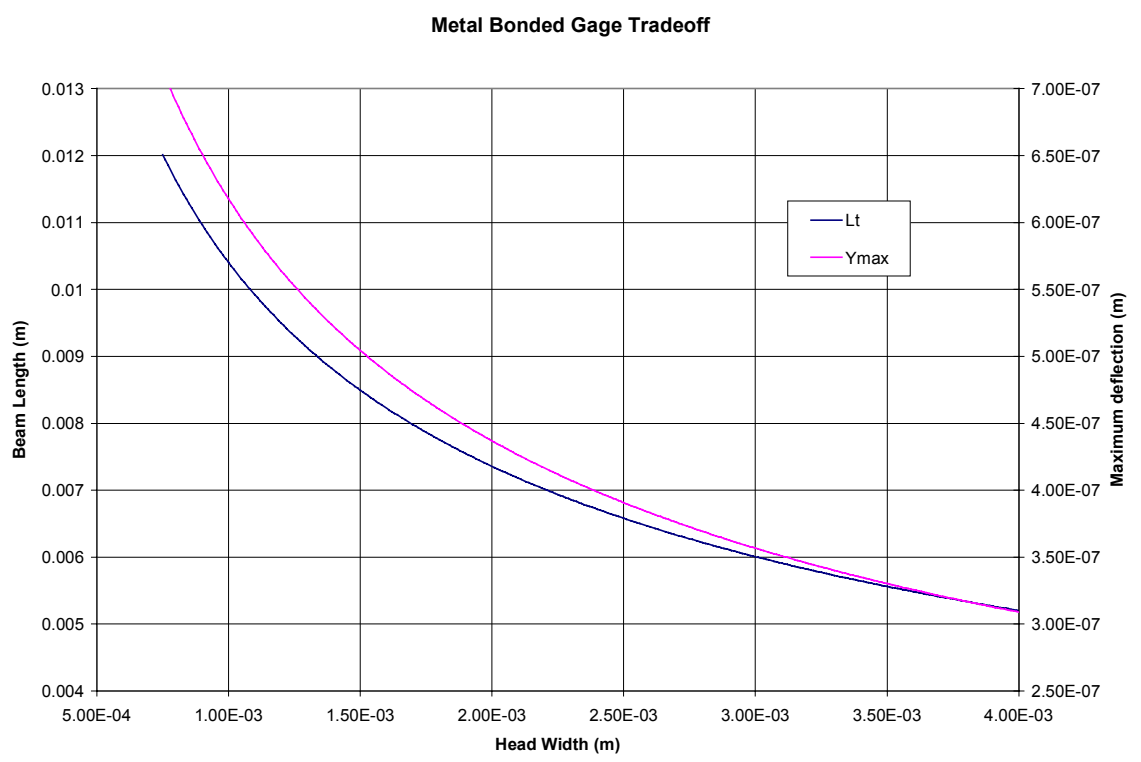

Figure 60. Design trade-off of a bonded metal design.

A survey of all the possible technologies to fabricate the sheets was undertaken, and the results are summarized in Table 8. Etching of metal is very inexpensive, but the minimum cut is $127 \mu \mathrm{m}$ (0.005 in.) which is much too large. The excimer laser design is more likely to allow the elimination of fill liquid with its smaller cutting widths, but the expense of this technology makes it unfeasible. The LIGA process is still under development, and therefore it is very expensive. The YAG laser option appears to be the only alternative for metal construction. This option was pursued, but the bonding became a larger concern. Litton Polyscientific in Blacksburg worked extensively on the problem, but they were unable to develop a process that would remove all voids between the sheets, rendering the successful use of metal sheets improbable. However, Litton was confident that they could bond Si wafers together without voids, therefore the Si wafer option was explored and it is described further in Section 3.4.6. This exploration led to the examination of numerous Si micromachining technologies for the construction of the skin friction sensor. 
Table 8. Technology overview for bonded micro-skin friction design.

$\begin{array}{llllll} & \text { Option A } & \text { Option B } & \text { Option C } & \text { Option D } & \text { Option E } \\ & \text { YaG Laser-Metal } & \text { Eximer Laser-Metal } & \text { Etch - Silicon } & \text { Etch - Metal } & \text { LIGA } \\ \text { Cost } & \text { Expensive } & \text { Very expensive } & \text { Inexpensive } & \text { Inexpensive } & \text { Very expensive } \\ \text { Materials } & \text { Invar, SS } & \text { Any } & \text { Si, possibly SiC } & \text { Any } & \text { Ni, Cu } \\ \text { Minimum cut width } & 50 & 13 & 10 & 127 & 5 \\ \text { Cut ratio } & 1 \text { to } 1 & 8 \text { to } 1 \text { (approx) } & 30 \text { to } 1 & .8 \text { to } 1 & 50 \text { to } 1 \\ \text { Partial cuts? } & \text { No } & \text { Yes } & \text { Yes } & \sim 5 & \text { Yes } \\ \text { Accuracy } & \sim 127 & \sim 127 & \sim 5 & \text { excellent } & \text { excellent } \\ \text { Repeatability } & \text { low } & \text { low } & \text { excellent } & \text { fewest cuts } \\ \text { Bonding } & \text { difficult, lots of cuts } & \text { fewer cuts } & \text { simple and proven fewer cuts } & \text { can't get small cuts } & \text { unknown } \\ \text { Problems } & \text { must be very thin } & \text { slow and expensive } & \text { not metal } & & \end{array}$

\subsubsection{Silicon Micromachining Technology}

As all of the following concepts utilize Si micromachining technology in their conceptualization, an overview for the reader is warranted. The interested reader can find a good review in either Madou or Peterson. ${ }^{85,86}$ Crystalline silicon forms a covalent bond structure similar to diamond. The lattice structure is configured into planes that adhere to the Miller indice rules. Figure 61 depicts a silicon wafer sliced to the (100) plane and the relevant crystal structure oriented to that plane is included. Depending on the micromachining technique, $\mathrm{Si}$ etches at different rates into each plane, which really means that the acid used in the process reacts at different speeds into the crystal structure defined by its orientation to the various crystal planes. It is that fact and the ability to mask portions of the Si from the etching, that allows various forms to be constructed into the crystal.

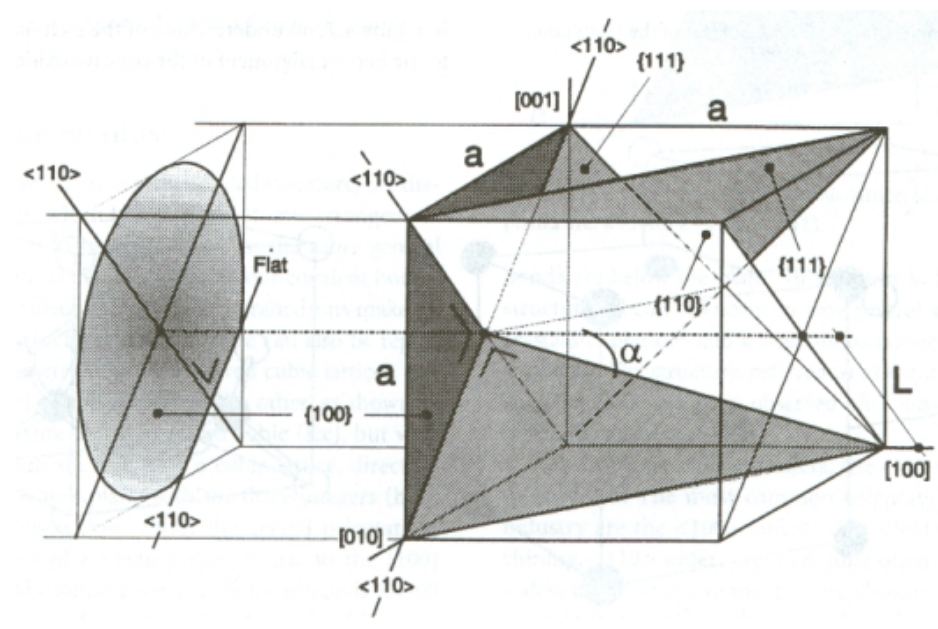

Figure 61. (100) silicon wafer with reference to its relevant planes. ${ }^{87}$ 
Single crystal wafers can be used to fabricate microstructures in two basic ways: either through bulk micromachining or through surface micromachining.

In bulk micromachining, the wafer itself will be used as the final device. Figure 62 shows the basic steps in the process of bulk micromachining. The initial state of the silicon wafer is shown in Figure 62a. A thin oxide layer is thermally grown over the (silicon) surface. Photolithography begins with the application of a thin layer of photosensitive polymer (photoresist), which is applied using a spin coater and then baked to produce a relatively hard thin coating over the oxide layer. The photoresist is exposed to appropriate radiation (UV light in most cases) through a mask. Figure $62 \mathrm{~b}$ shows the photoresist layer applied over the oxide, and Figure 62c shows the result of exposure and development of the photoresist, followed by etching of the oxide layer to produce the final etch mask in the oxide itself.

(a)

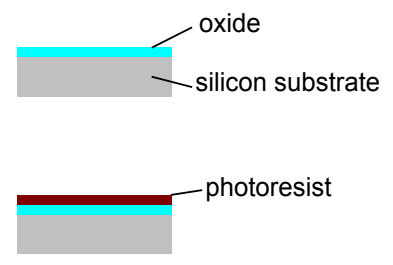

(e)

(b)

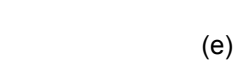

(c)

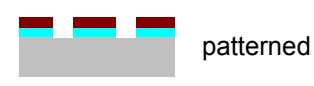

(d)

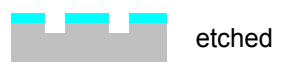

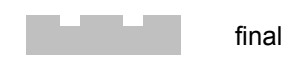

Figure 62. Bulk micromachining of silicon wafer, a) unaltered Si wafer, b) applied photoresist, c) photoresist removed in a pattern, d) wafer after etching, and e) final etched wafer after photoresist is removed.

A different chemical is used to etch through the exposed oxide layer in these areas, leaving the base silicon exposed. The remaining photoresist is removed at this point, it being no longer useful in the process. Figure $62 \mathrm{~d}$ shows how the oxide layer masks portions of the silicon substrate while allowing the other areas to be attacked by the silicon etchant. Etch rates in silicon can be as high as several hundreds of angstroms per minute. Once the desired dimensions are obtained, the oxide layer is chemically removed and the wafer is either diced on a dicing saw or held for additional processing.

Depending on the application, two types of etching may be performed on the wafer: isotropic or anisotropic. The basic difference between the two types of etching is the degree to which the wafer material preferentially etches along certain crystal planes. 
When the etching can proceed along all planes at the same rate, it is said to be isotropic. If etching occurs exclusively along only one crystal plane, it is said to be completely anisotropic.

In most cases, the degree of anisotropy is determined by the selectivity of the etchant (for wet etching). Figure 63a shows a typical isotropic etch result, while Figure $63 \mathrm{~b}$ shows completely anisotropic etching. The effective difference is that the anisotropic system produces extremely smooth and straight sidewalls due to the preferential etching along this crystal plane, while the isotropic system produces rounded sidewalls. Anisotropic etching is generally more difficult to control, but it is extremely useful for producing micromachined optical surfaces and controllable angles. Figure 64 shows how different shapes can be obtained using anisotropic etching with specifically oriented (silicon) crystal planes. In Figure 65a, the slower etch rate along the silicon (111) crystal plane is used to produce angled features at 54.7 degrees to the surface. This feature was used here to produce v-grooves for fluidic channels or positioning features for holding optical fibers. By obtaining wafers with the angle between the (111) plane and the surface controlled properly, it is possible to produce 45 degree smooth surfaces in the silicon, which can be used as corner reflectors for optical sensors. Because the etch rate for the (111) direction is typically 30 - 60 times slower than that for the (100) direction, this system is effectively self-limiting in nature, allowing relaxation of the time controls for production. Figure 64 illustrates this feature. Figure $65 \mathrm{~b}$ shows how the relatively slow etch rate along the (111) plane compared to the (110) plane can be used to produce smooth vertical sidewalls, again for fluidic and optical applications. Figure 66 shows how different shapes can be obtained using anisotropic etching with specifically oriented (silicon) crystal planes.

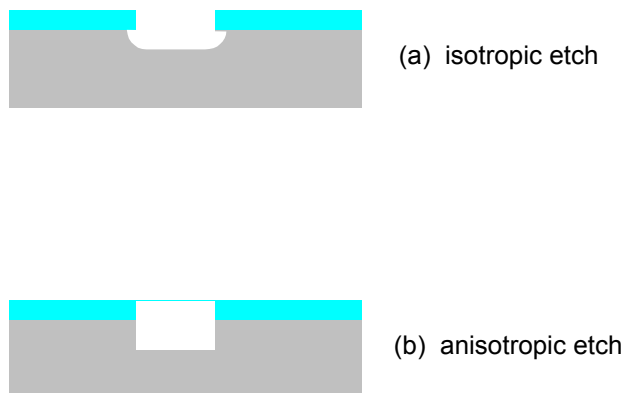

Figure 63. Examples of isotropic and anisotropic etch in silicon. 


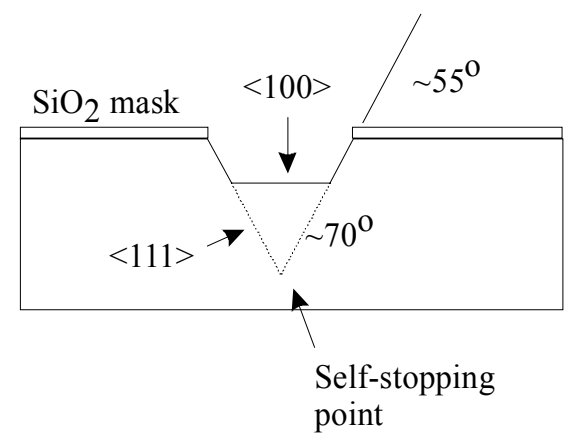

Figure 64. Self-termination in anisotropic etching of Si.

(a)

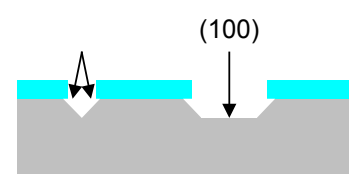

(b)

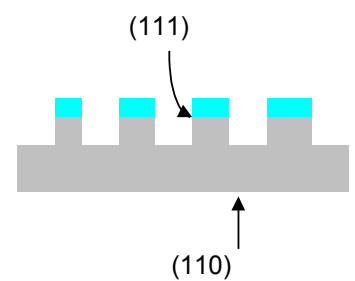

(a)

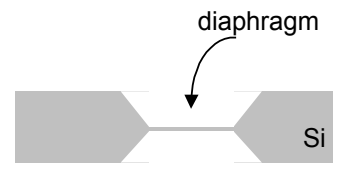

(b)

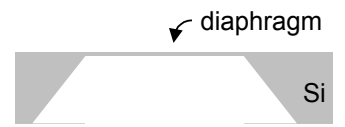

(c)

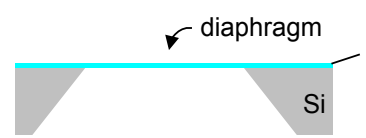

Figure 65. Etch planes in silicon, a) into the 100 plane and $b$ ) into the 110 plane.
Figure 66. Devices using anisotropy, exampled by a typical pressure sensor fabrication process.

In surface micromachining, the substrate is only used as a base for construction of surface structures which are applied through film deposition and etching steps. The process used is similar to "lost wax" casting, in that a temporary layer (called the sacrificial layer) is used to separate a structural layer from the substrate material. The sacrificial layer is removed in subsequent processing steps, leaving free-standing structures. The most common material system used is polycrystalline silicon as the structural layer with phosphosilicate glass (PSG - silicon dioxide with 1-10 percent phosphorous by weight) as the sacrificial layer. Hydrofluoric acid (HF) will preferentially etch the sacrificial layer, leaving the polysilicon nearly untouched. Figure 67 shows the basic steps in the process of fabrication of a cantilevered beam. Figure 67a shows the silicon substrate with a polysilicon layer and a photoresist pattern already 
applied. Figure 67b shows polysilicon layer etched away except for the base structure for the beam. In Figure 67c and Figure 67d the application and planarization of the PSG sacrificial layer is depicted. Planarization of the surface is necessary prior to application of the second polysilicon layer which will form the beam, as shown in (e). Finally, as shown in Figure 67f, the PSG sacrificial layer is etched away, leaving the polysilicon free-standing beam.

(a)

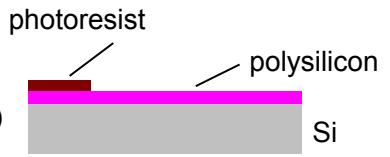

(b)

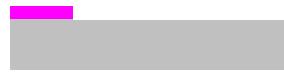

(c)

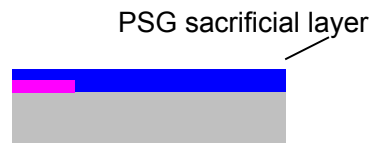

(d)

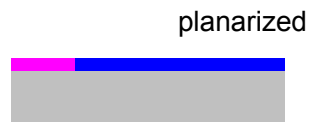

2nd polysilicon

(e)

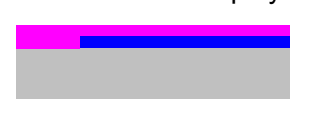

(f)

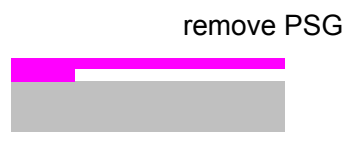

\section{Figure 67. Example of a fabrication processing using surface micromachining.}

In most cases, micromachining can be accomplished using a "dry" etch process, in which the etching is performed by exposing the surfaces to various types of plasmas. Plasma etching equipment is required in this case, to replace the wet chemical etching process. One such process is Deep Reactive Ion Etching (DRIE), which uses an ion beam and a reactive plasma to remove $\mathrm{Si}$ from the wafer in any manner desired. The high cost of the process limits its use.

For the current project, an extensive study was performed to use bulk micromachining to fabricate the slices of the bonded gage concept. Design concepts using other processes were also explored, with an overview of the work provided below.

\subsubsection{Silicon Bonded Concept}

The Si wafer design is very similar as that of the laser cutting metal wafer design except that the use of bulk micromachining etching will allow a great deal fewer slices, is much less inexpensive, and has repeatability unmatched by laser cutting. After some test wafers were etched, the design was slightly changed. The new design is shown in Figure 
68. The sensor was to be constructed of four wafers bonded together, which have been etched using three masks. The center-pieces, as shown on the right of Figure 68, will anisotropically etch all the way through a 0-1-1 Si wafer. Although this orientation will give straight side walls, they will be at a slight angle and the corners of certain features will etch away at an angle as shown in the figure. By selecting the correct wafer orientation, where these angles occur can be known, and the sensor could look as shown. The fiber channel can be isotropically etched. This procedure would be performed on two wafers. The outer pieces can have reliefs etched in them to allow movement of the sensing head. The two center-pieces can then be anodically bonded back to back, and then the outer pieces bonded on to them. The four bonded wafers can then be diced up into numerous identical sensors. The fibers can then be attached and the sensors calibrated. This approach should allow the manufacture of hundreds of identical sensors at a time, very inexpensively. It has been estimated that over 200 sensors can fit on a four inch silicon wafer during mass production.

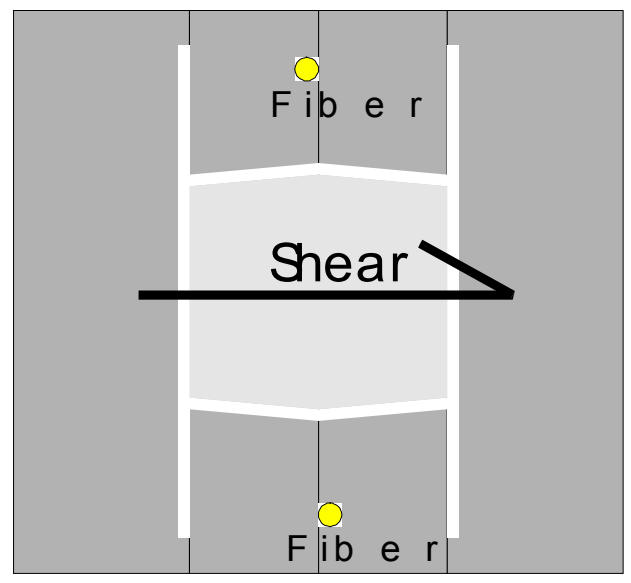

Top view of gage

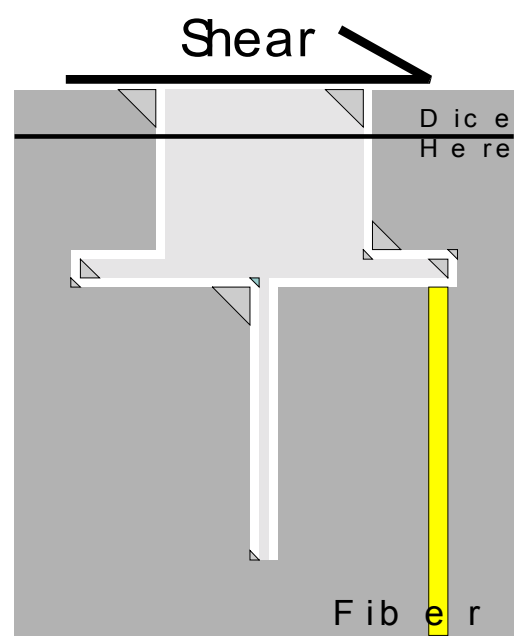

Side view of sheet

Figure 68. Sketch of the design of the Si bonded sensor.

As with the metal bonded concept, the design space for the Si wafer sensor collapses to only two variables, beam width and beam length. The limits of the technology impose an envelope around possible geometry selections. In this particular case, care had to be taken not to shrink the cantilever beam and head portion too small so that there was sufficient area for bonding between the slices to be possible. Again, the 
maximum deflection of the beam was important as the gap between the head and housing was to be minimized.

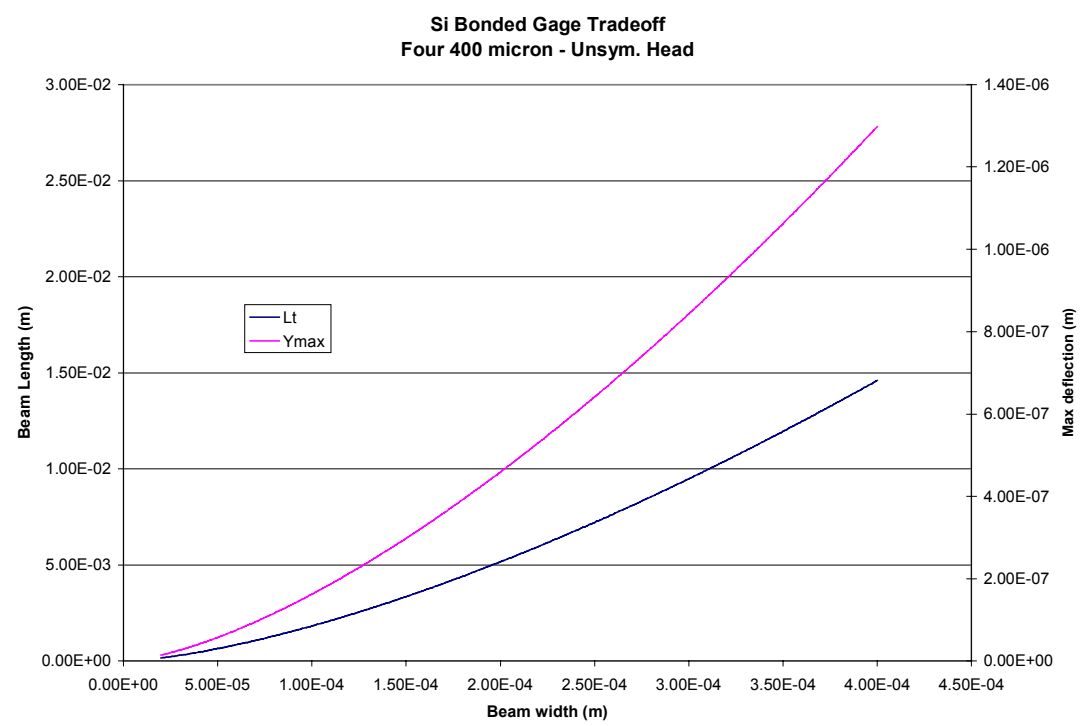

Figure 69. Design space for Si bonded concept.

Litton Polyscientific was able to demonstrate repeatable anodic bonding of $\mathrm{Si}$ wafers, leading to more intensive wafer etching experiments. Temperature testing of the anodic bonding demonstrated sustained temperatures of over $1000^{\circ} \mathrm{C}$. Litton also performed the mask and Si etching experiments.

\section{1-0-0 Crystal Orientation Tests}

Figure 70 shows a close-up of a mask used in the etching process. An inexpensive $(\sim 20)$ silver halide mask was used for the first tests. More accurate, and more expensive $(\sim 500)$, chromium/quartz masks could be used in future production. As can be seen in the figure, the mask does not produce lines that are perfectly uniform. This non-uniformity was a concern, but the results shown in Figure 71 were positive. This first etch test was performed on a wafer with a 1-0-0 crystal orientation as this orientation is more forgiving of mask variations. The etching away of the corners was expected in the 1-0-0 wafer, and should be reduced in a 1-1-0 wafer. Also, inspection of the wafer indicated that the sidewalls were not perpendicular, but again the walls should be with a 1-1-0 wafer. This crystal orientation will produce perpendicular sidewalls with aspect ratios of approximately 30:1. 


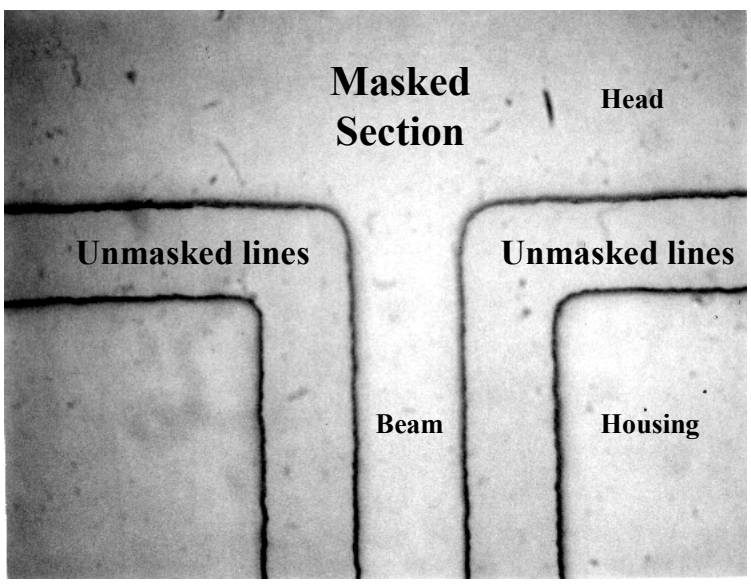

Figure 70. 400X detail of the prototype silver halide mask used in the proof-ofconcept tests where the beam meets the sensing head.

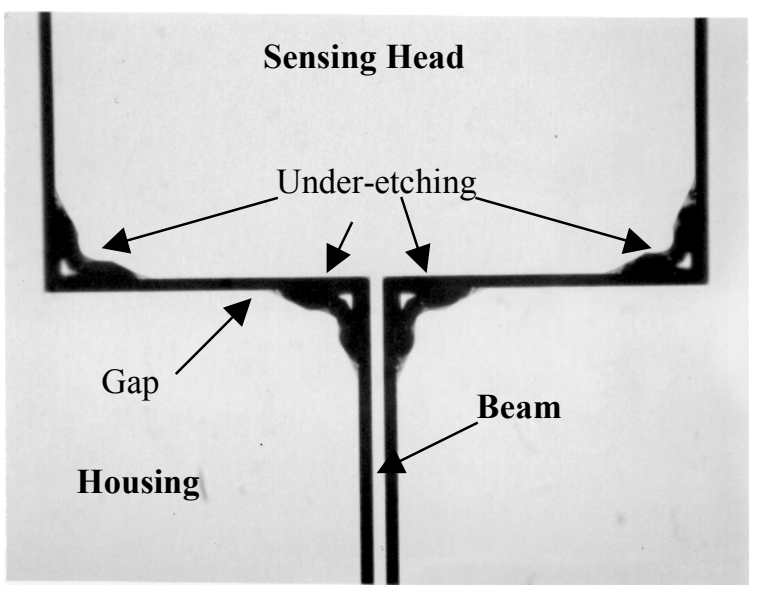

Figure 71. Successful etch test on a $<100>$ silicon wafer using the mask in Figure 70 in an attempt to produce a sheet similar to the right sketch of Figure 68.

\section{1-1-0 Crystal Orientation Tests}

The majority of the effort was concentrated on perfecting the processes necessary to etch the wafer from both sides. As stated above, the test began on a silicon wafer orientated to the 1-0-0 crystal plane. That etching was only carried out for a short time and, therefore, not very deep into the wafer. But as those tests were positive, Litton moved on to etching the structure in a wafer orientated to the 1-1-0 crystal plane. This plane should provide straight walls on features that line up with the 1-1-1 plane in the wafer, and then etch similar to the previous tests with any other feature. Below in Figure 72 is a photograph of a test where the proposed structure was etched into a 1-1-0 wafer to 
a depth of $100 \mu \mathrm{m}$. As can be seen, the structure remains intact to that depth, with very little etching around corners.

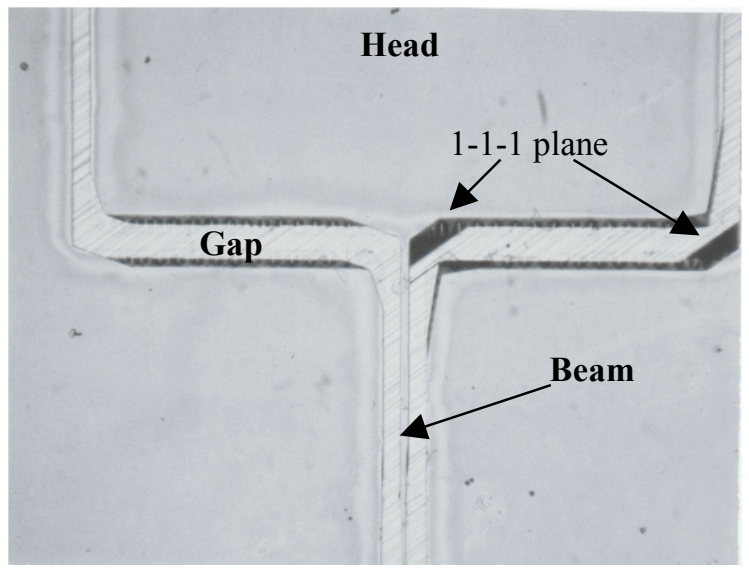

\section{Figure 72. Test etching into a 1-1-0 wafer $100 \mu \mathrm{m}$ deep showing the beginning of} under-etching.

Figure 73 shows the continuation of the above etch to $300 \mu \mathrm{m}$. Etching to this depth has allowed the structure to break out of the shape defined by the mask, and is quickly moving to the 1-1-1 planes. Obviously this shape is unusable. It was determined that the resolution needed to make a successful etch to this depth was out of reach with the equipment on hand. To continue down this path, the etching would have to be limited to around $100 \mu \mathrm{m}$. Therefore, the Litton began concentrating the effort with $200 \mu \mathrm{m}$ thick wafers and perfecting the procedures necessary to etch from both sides and have the structures meet, and bonding these wafers together. This work is not yet complete.

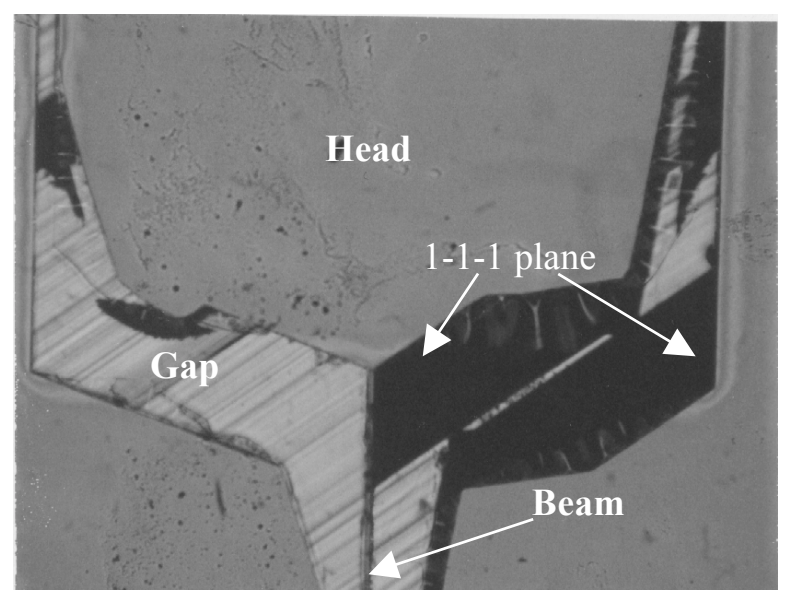

Figure 73. Test etching into a 1-1-0 wafer $300 \mu \mathrm{m}$ deep showing the degradation of the wafer at this etch depth. 


\subsubsection{Low-Profile Design}

An alternate concept is a low profile design that uses surface micromachining technology. The sensor consists of a high aspect ratio, cantilever beam with the sensing head on its side and an optical fiber that comes in from the side and reflects off the side of the beam, as shown in Figure 74. The design is meant to be as flat as possible and to be used in surface mount applications. The high aspect ratio beam is still strong to a normal force and yet pliable to a shear force. Aspect ratios of 30:1 are possible, with the width of the beam being only $10 \mu \mathrm{m}$.

As was discussed above, construction of this sensor would be through typical surface micromachining technology. First, using a (1-1-0) oriented wafer, the cavity that forms the interior of the sensor is etched away. Second, a sacrificial layer is grown that provides a $10 \mu \mathrm{m}$ buffer around the etched cavity. Then, polycrystalline Si is laid down to form the cantilever beam and floating head. Finally, the sacrificial layer is removed, leaving the beam and head free standing. The beam anchors at the base with a locking finger arrangement that is common in these surface micromachined devices. ${ }^{88}$ The fiber is laid into a trough that is etched from the other side of wafer. Another option is to have two beams, so as to remove the possibility of twisting. This fabrication technique requires none of the bonding or alignment difficulty of the previous concept. However, the number of masks and steps in the procedure is much greater than the previous design, raising the cost of a completed sensor. 


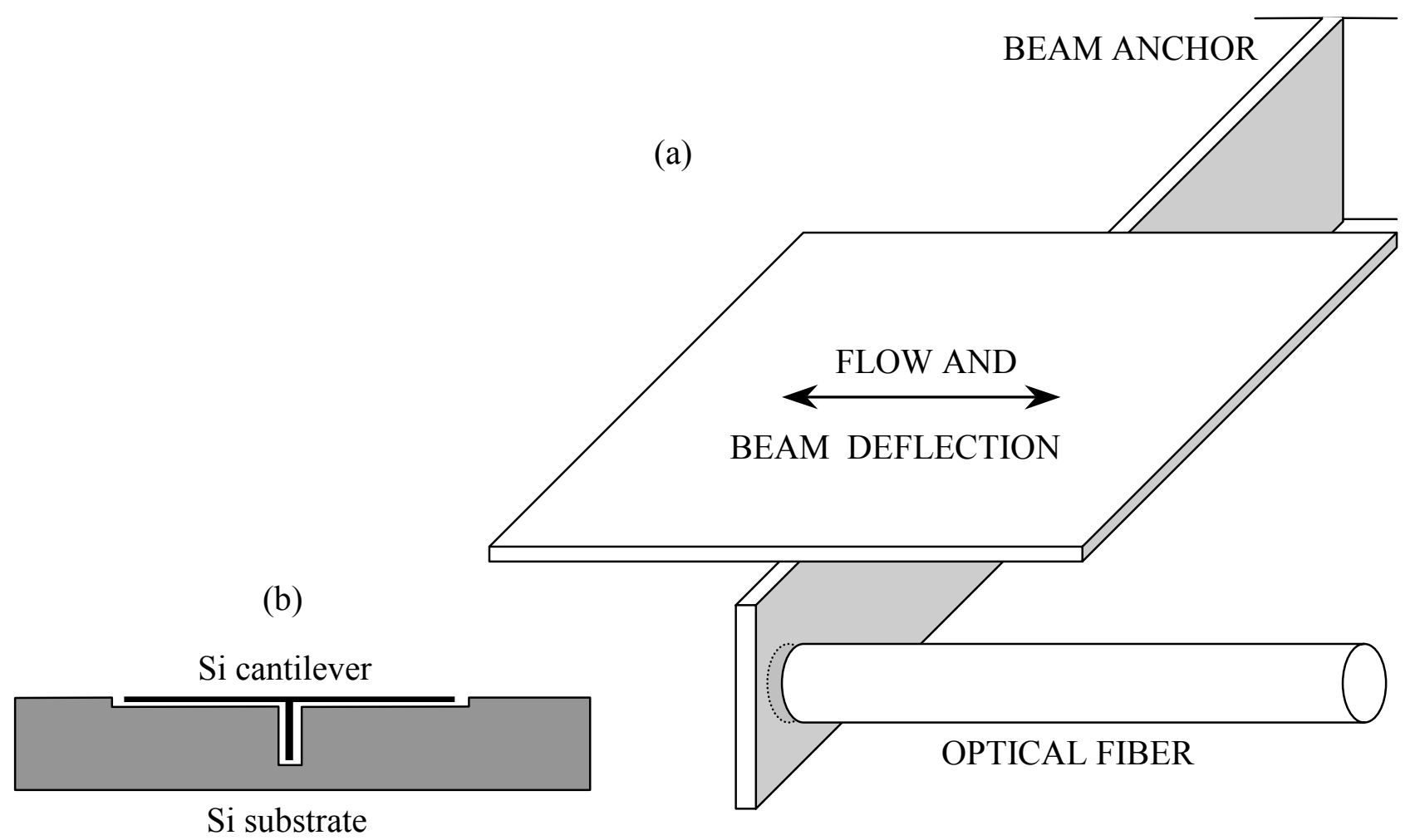

Figure 74. (a) Cut-away schematic diagram of the fiber optic skin friction sensor high aspect ratio cantilever beam with lateral platform and sapphire optical fiber, and (b) The cantilever, platform and fiber are fabricated within an Si substrate and flush with the wafer surface.

\subsubsection{The "Plus" Design}

An early design concept was to use bulk micromachining techniques to fabricate individual pieces of the sensor and then adhere them together to construct the sensor. The envisioned steps of the fabrication and construction are presented in Figure 75. Beams are etched into a plus shape, with mating shapes in the heads and bases. The heads and bases would have to be ultrasonically machined, as this is the only way to cut circles into silicon as envisioned. The optical fibers are placed at the corners of the plus as seen in the figure. It was quickly realized that there were too many steps and attachments that had to go correctly for this concept to work properly. In addition, the design space of the "plus" concept, presented in Figure 76, clearly demonstrated that the concept could not have the desired sensitivity. The fibers are too close to the neutral axis 
and the arms of the "plus" increase the moment of inertia making the beam too stiff. Yet, both of these lessons were useful when the following concept, the v-groove, was conceived.

1

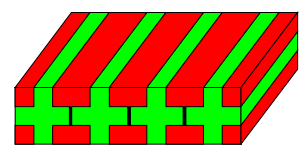

2

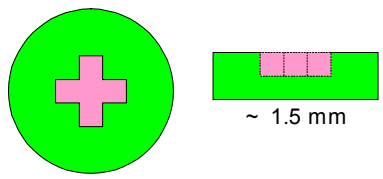

3

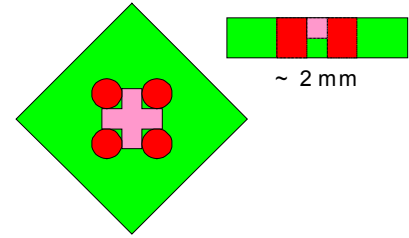

4

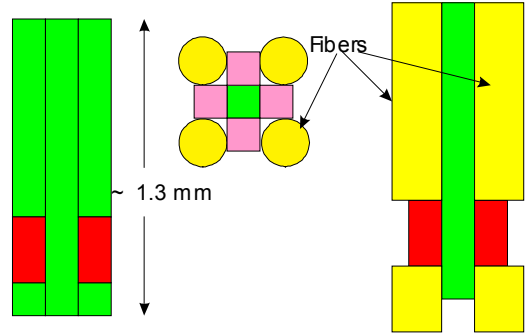

Ech away silicon

and then cut plus shape

Cut Head and then Ech plus out to fit on top of beam

Cut Base and then Ech plus out to fit the bottom of beam Then etch out holes for the fibers

Ech away the arms of the plus in the gap region between the fibers

Assemble the gage, bonding pieces together and adding fibers

Figure 75. Manufacturing steps of the "Plus" micromachined design.

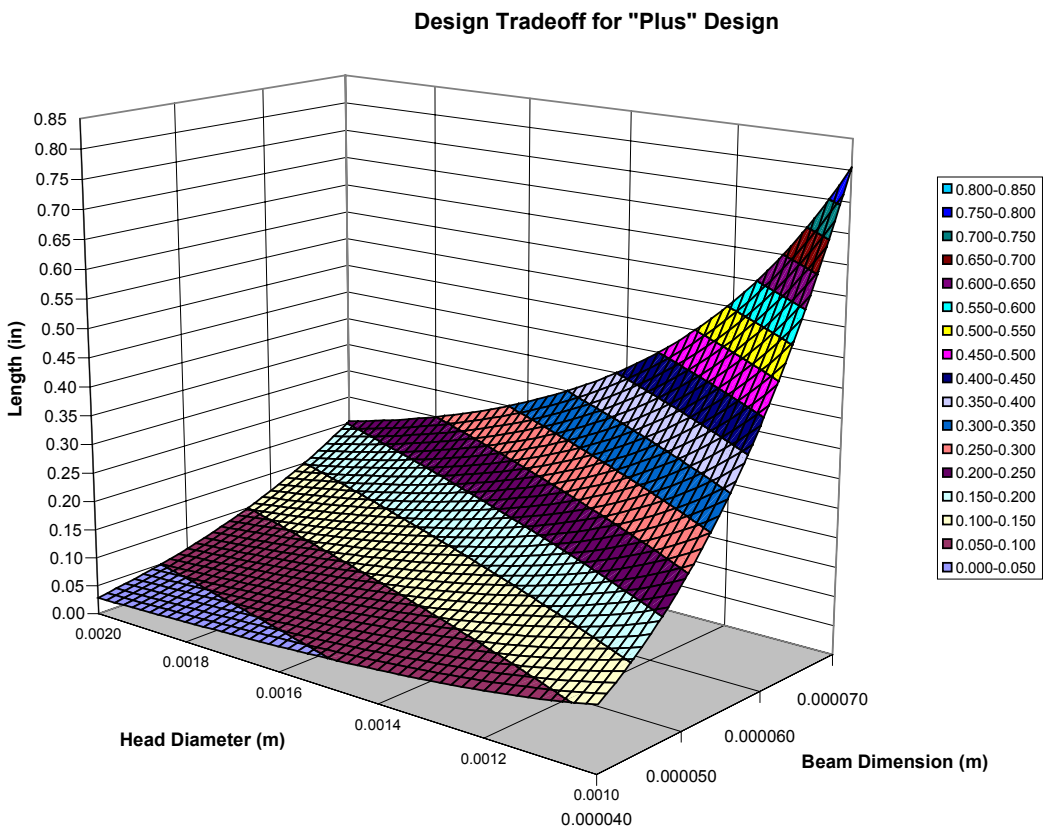

Figure 76. Design space of the "plus" design. 


\subsubsection{V-groove Concept}

During examination of a possible conventionally machined design, a new concept was conceived, as depicted in Figure 77. The design took advantage of micromachining work performed to construct a fiber optic refractometer for another program at Luna. A surface micromachined v-groove is the basis of this concept, providing a solid placement for the fibers. The beam can be made extremely repeatably and inexpensively through the already designed processes. A head, also using inexpensive processes, can be made out of silicon and attached to end of the beam as was conceived in the "plus" design. The fiber runs the length of the beam, only being attached to the beam at the top, and riding within a v-groove cap at the base. Having a fiber run the length of the sensor allows the use of the total integration of the strain for the beam, which is solidly placed into a base piece where the v-groove cap is attached. One of the best qualities of this concept is that because it can be fabricated with precision diamond saws, it can be extended to many different materials, including alumina, $\mathrm{SiC}$, and metals.

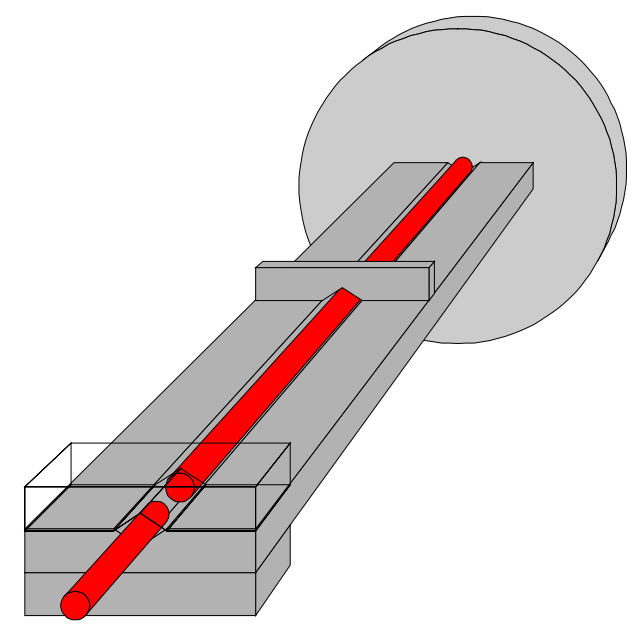

Figure 77. New, simple micromachined concept for a silicon micromachined skin friction sensor.

The design space of a particular version of the v-groove concept is presented in Figure 78. As with the plus design, finding a sensitive design is difficult because the fiber is not far from the neutral axis of the beam. However, the technique allows the fabrication of long thin beams, which can be used to counteract the small radius. As can 
be seen, there is a "kink" in the design space around a head width of $0.0025 \mathrm{~m}$, where a small sensing head can be had for a relatively short beam. Any constructed sensors using this concept would be designed around this kink.

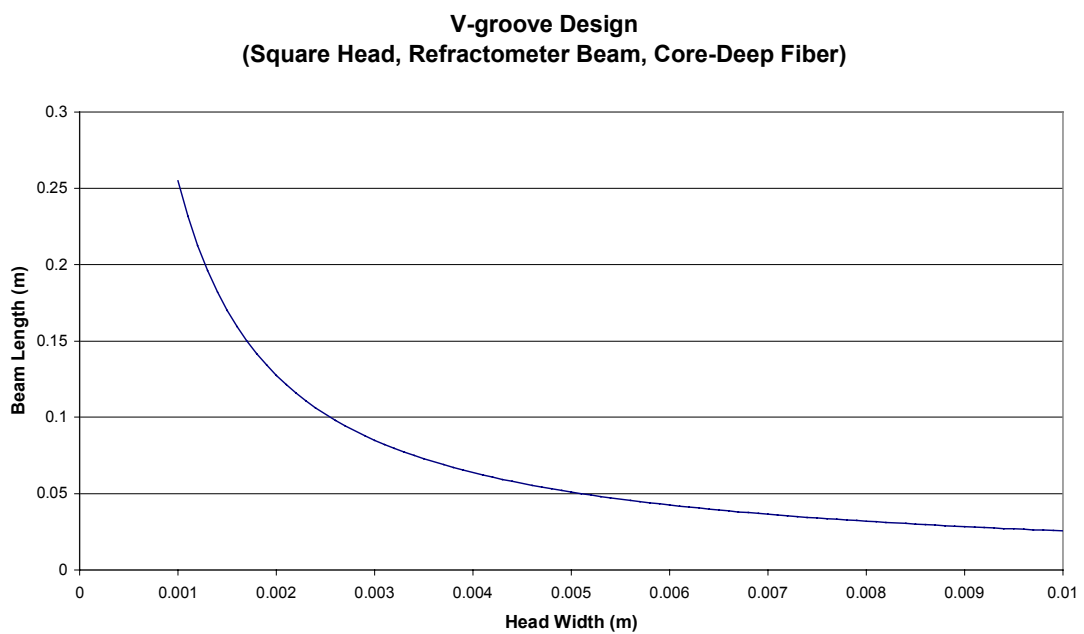

Figure 78. Design space of v-groove sensor concept.

Figure 79 presents a photograph of a v-groove concept gage. This test piece has a v-groove etched into a $400 \mu \mathrm{m}$ Si wafer to a depth so that the fiber rested half in and half out of the groove. The piece was $50.8 \mathrm{~mm}$ ( 2 in.) long with a cap piece $38.1 \mathrm{~mm}$ (1.5 in.) from the free end which guides the fiber in the groove, as well as a small cap piece about half way up the piece to keep the fiber in place. The fiber is affixed by epoxy on the wafer at the free end, with the optical path occurring under the cap piece.

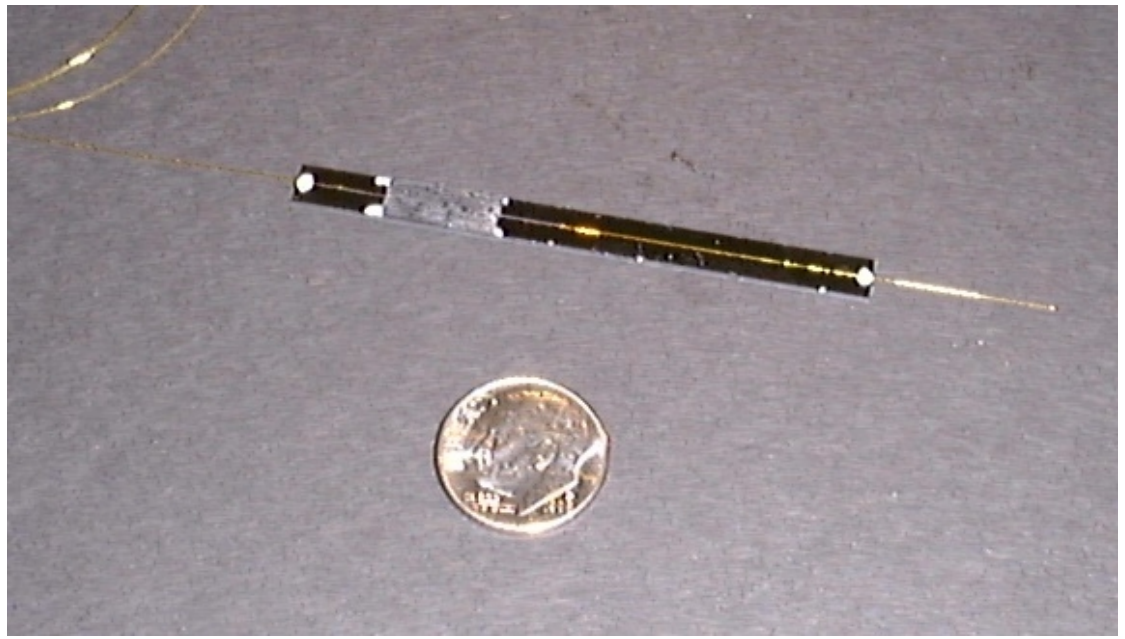

Figure 79. Photograph of a v-groove sensor test article. 
Figure 80 shows the calibration of the v-groove test article with mass, both in tension and compression. Some creep was observed in the data during the testing. It is hypothesized that this is due to friction in the v-groove. The creep was seen as movement of the starting point during each application of a force in the direction of the movement, i.e. during tension, the starting optical path would get larger, conversely during compression the optical path would get smaller. If the calibration is calculated to take into account this moving starting point, the calibration becomes remarkably linear. This friction effect will have to be solved if this concept is to be developed further, although typical vibration environments shake the sensor through any friction points. It is this last concept, the v-groove design, which seems the most likely to be inexpensive and successful.

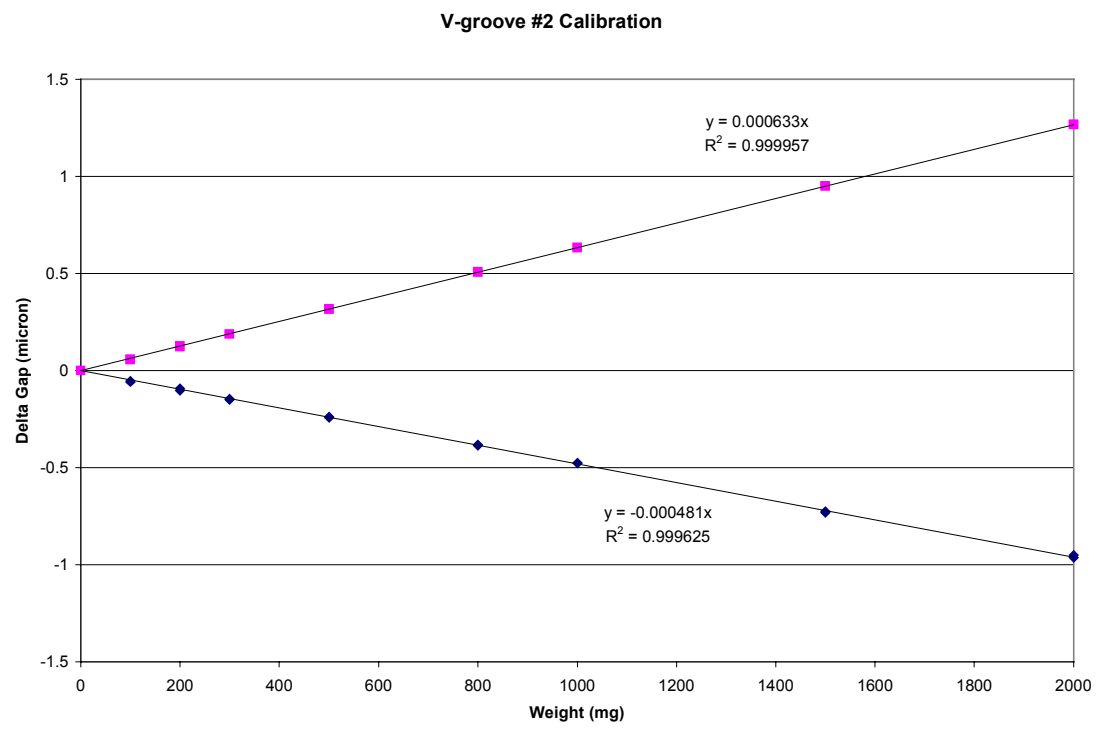

Figure 80. A calibration of a v-groove test article.

In the end, it was determined that although a few of the explored methods would be feasible and worth exploring further, there was not sufficient effort available in the current program to actually produce a micromachined skin friction sensor. 


\subsection{Temperature/Heat Flux Sensor}

A fiber optic heat flux/surface temperature sensor was constructed and tested for evaluation purposes during the study. The current commercially available Luna fiber optic sensor, pictured in Figure 81, which uses the change in length of a metal wire with a change in temperature, does not have a small enough active length to make a measurement that could be used for an accurate evaluation of heat flux or surface temperature.

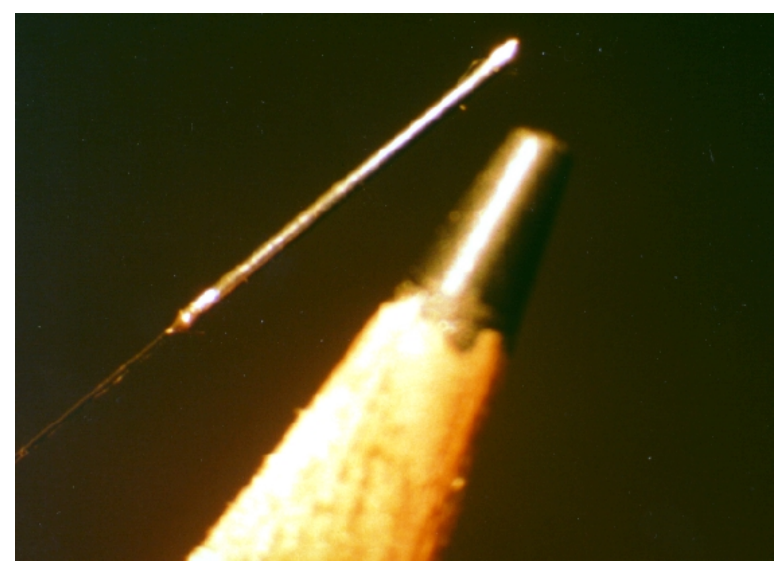

Figure 81. Photograph of fiber optic temperature sensor using metal wire.

An alternative to that sensor was made by attaching 2 silicon wafers with different thickness on the end of a fiber with index-matching epoxy, see Figure 82 (adhesive that has the same index of refraction as the silicon wafer, and therefore does not cause a reflection). By tracking the reflections off each surface, Luna can measure the optical path length change of each wafer with temperature, as defined by Equation 53, and through a calibration, correlate that to an average temperature across that wafer.

$$
\Delta O P L=\left(\frac{\partial n}{\partial T} * \Delta T+n\right) * C . T . E . * \Delta T * \text { Thickness }+ \text { Thickness } * \frac{\partial n}{\partial T} * \Delta T
$$

This design has been made possible by recent improvements in multiplexing of the signal processing system described above. By designing the sensor system so that the reflections all have optical path lengths that are significantly different, each optical path can be tracked separately, a concept known as gap division multiplexing. ${ }^{89}$ Figure 82 depicts the concept of the heat flux/surface temperature sensor, and Figure 83 presents a photograph of the sensor. By correctly isolating the temperature change along the axis of 
the fiber, one can determine the differences in the temperatures between the wafers and calculate heat flux. The calculated heat flux could then be easily used to extrapolate back to obtain the surface temperature.

A number of prototype sensors were constructed to determine the best method of construction and which variation gave the best results. The thickness of the wafer is a tradeoff; too thick and the time response of the sensor is poor, too thin and the temperature resolution is poor. Thicknesses of 20 and 30 microns were chosen, producing a sensor with a time response of $0.247 \mathrm{sec}$. (calculated as time to $95 \%$ full scale for a step change in temperature) and a temperature resolution of $0.1{ }^{\circ} \mathrm{C}$. Only after numerous attempts was a successful sensor constructed. Figure 84 presents the results. Both optical path lengths are relatively linear with temperature, but OPL2 changes much more than expected. It is felt that this was due to imperfect bonding of the thin wafer, which in this sensor was the farther from the fiber and that the wafer is bending from the temperature change and introducing index changes due to strain.
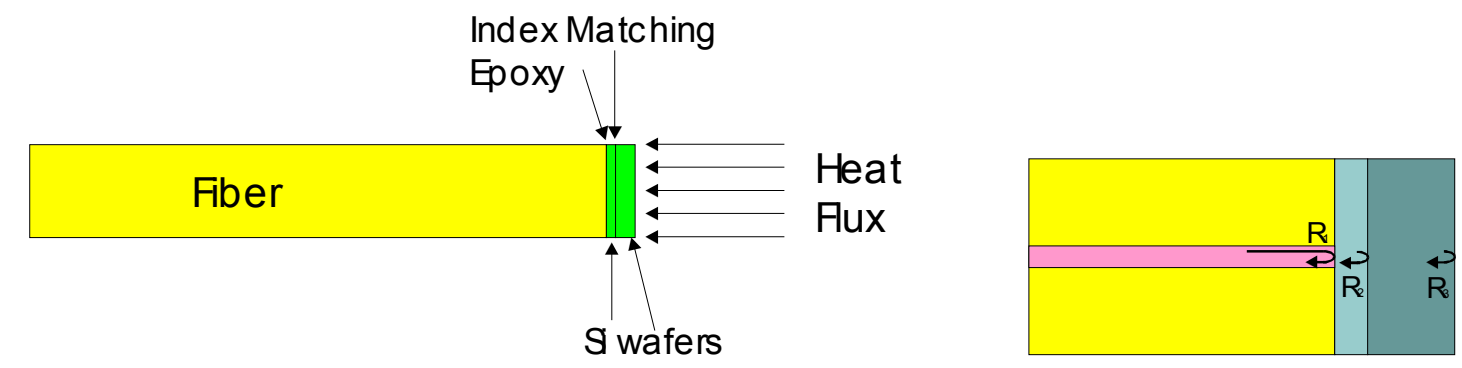

Figure 82. Sketch of the fiber optic heat flux/surface temperature sensor.

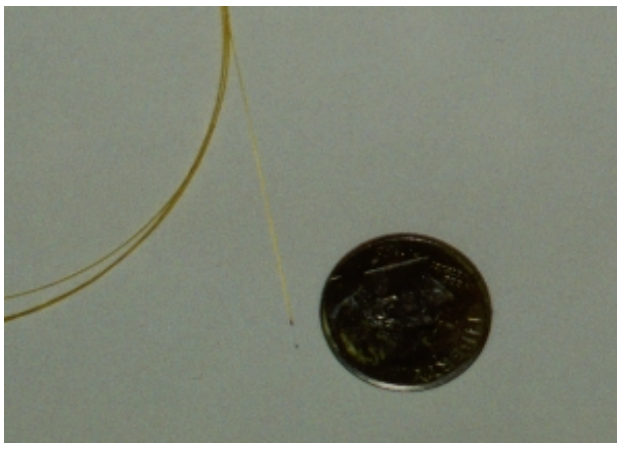

Figure 83. Photo of the fiber optic heat flux/surface temperature sensor prototype. 


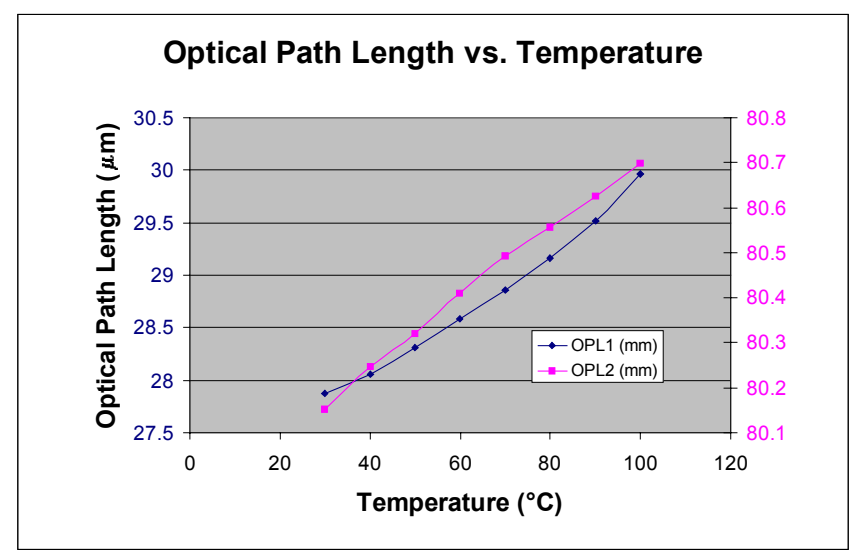

Figure 84. Optical path length vs. temperature of a prototype fiber optic heat flux/surface temperature sensor.

Some positive results were obtained with the fiber optic heat flux sensor, however, the difficulty of constructing and correctly packaging the sensor for use indicated that another solution was necessary for the current work. Another review of the available technologies led to the selection of the surface thermocouple, as described in Chapter 1, as the most likely to be successful. It is this sensor type which was used in the Version 3 skin friction sensor described in Chapter 4. 


\subsection{Chapter 4}

\section{Wind Tunnel Testing of Prototype Sensors}

\subsection{Introduction}

Several versions of the skin friction and pressure sensors were tested extensively in the wind tunnel to ascertain their performance. Early testing of the skin friction concept with a simple prototype was very positive, leading to a false sense of security. The design was put aside as completed so that other portions of the project could be moved forward. When testing of the design resumed with a presumably refined design, the results were not as positive as had been seen during the previous testing. This difficulty led to a large effort to determine what the problems were with the design and correct them. On the other hand, testing of the pressure sensor proceeded without many problems. Below is a synopsis of the testing, providing the results that were important in the development of the skin friction and pressure sensors. A complete collection of the testing data obtained during the project is presented in Appendix A.

The tests were performed in the Virginia Tech supersonic wind tunnel, seen in Figure 85. This facility is a blowdown tunnel that has interchangeable nozzles and a test section size of $23 \times 23 \mathrm{~cm}$, allowing Mach 2.4, 3.0, and 4.0 conditions with total pressures ranging from 240 to $760 \mathrm{kPa}$ (35 to $110 \mathrm{psia}$ ) and total temperatures of approximately $285 \mathrm{~K}$. Taken all together, these characteristics led to very high Reynolds numbers (approximatley $4.5 \times 10^{7} / \mathrm{m}$ ). The run time for this tunnel is between $5-10$ secs., depending on the conditions. 


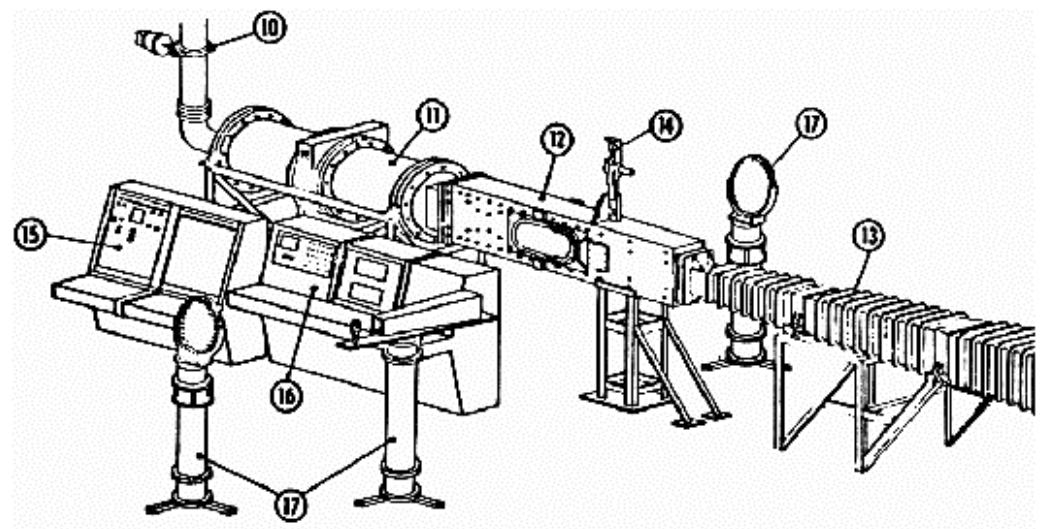

Figure 85. Virginia Tech supersonic wind tunnel.

\subsection{Micromachined Fiber Optic Pressure Sensor}

The micromachined, fiber optic pressure sensor, described in Chapter 3, was tested in various supersonic flows in an effort to characterize and evaluate performance in this type of environment. The first tests took place with the housed pressure sensor seen in Figure 86 (calibration data of this pressure sensor, as well as those of the rest of the pressure data presented here can be found in Appendix B). The sensor was flush mounted in the floor of the tunnel and tested in a Mach 2.4 flow at a total pressure of 550 $\mathrm{kPa}(80 \mathrm{psia})$.

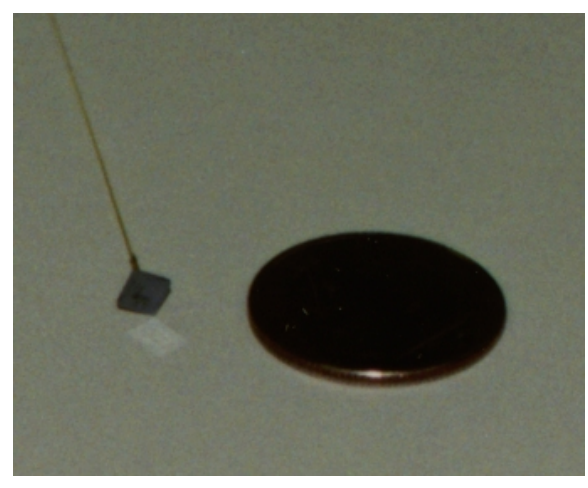

(a)

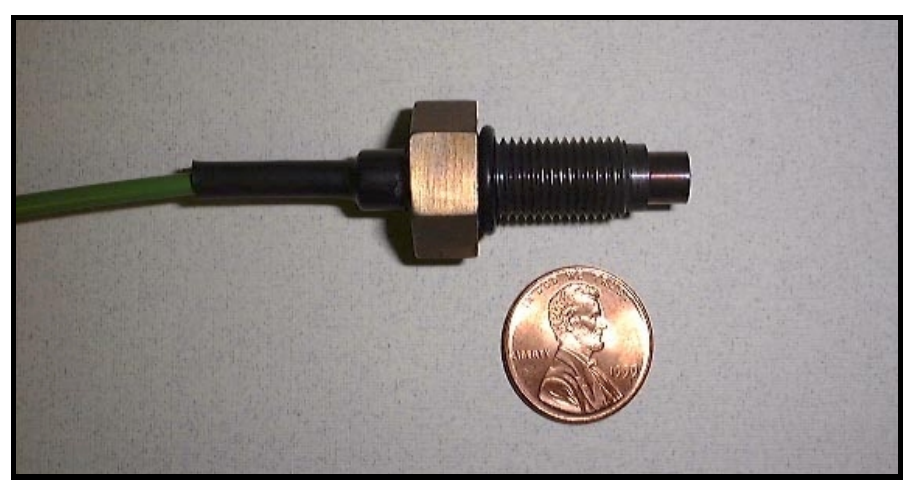

(b)

Figure 86. Micromachined, fiber optic pressure sensor used for Mach 2.4 tests, a) bare sensor and b) housed sensor.

Typical results of the pressure tests are presented in Figure 87. The blue line is the tunnel total pressure, the magenta line is the static pressure, and the green line is the 
optical pressure sensor. The tunnel static pressure is measured by a standard pressure transducer that was connected to the tunnel floor by approximately one meter of pressure line tubing. As can be seen, the optical pressure sensor and the pressure tap give similar results during the steady part of the tunnel pressure profile. There is greater noise in the optical data, which is attributed to the optical sensor being flush-mounted in the tunnel and the tap being connected by tubing, which provided natural aerodynamic damping. It should be noted that it is not usually possible to flush-mount pressure sensors and acquire reliable results. Typically, the temperature drift of a conventional pressure sensor is so large as to ruin the data. However, due to the nature of the fiber optic pressure sensor, this temperature drift difficulty is reduced. One will also notice the large noise during the start up and stopping of the tunnel. During this time, the diaphragm of the pressure sensor is excited more than during the stable part of the run, leading to the large noise content. This effect will be discussed further below.

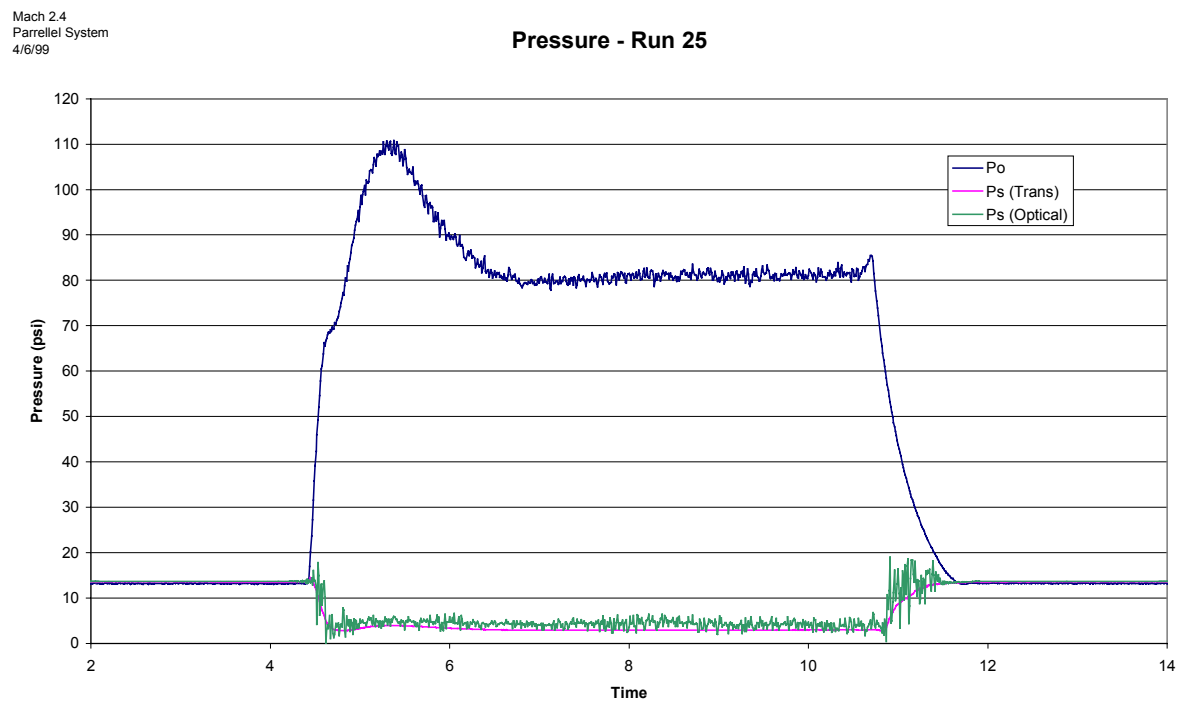

\section{Figure 87. Flush-mounted test of the micromachined, fiber optic pressure sensor in} a Mach 2.4 flow.

\subsubsection{Mach 3 Testing}

Following the successful test performed in the Mach 2.4 flow, an array of pressure sensors was prepared to examine pressure gradients and pressure fluctuations in a shock-wave/boundary layer interaction region. The array is pictured in Figure 88. As 
can be seen, the array has 5 sensors, and the total array is mounted in the tunnel floor so that the sensors are flush with the surface.

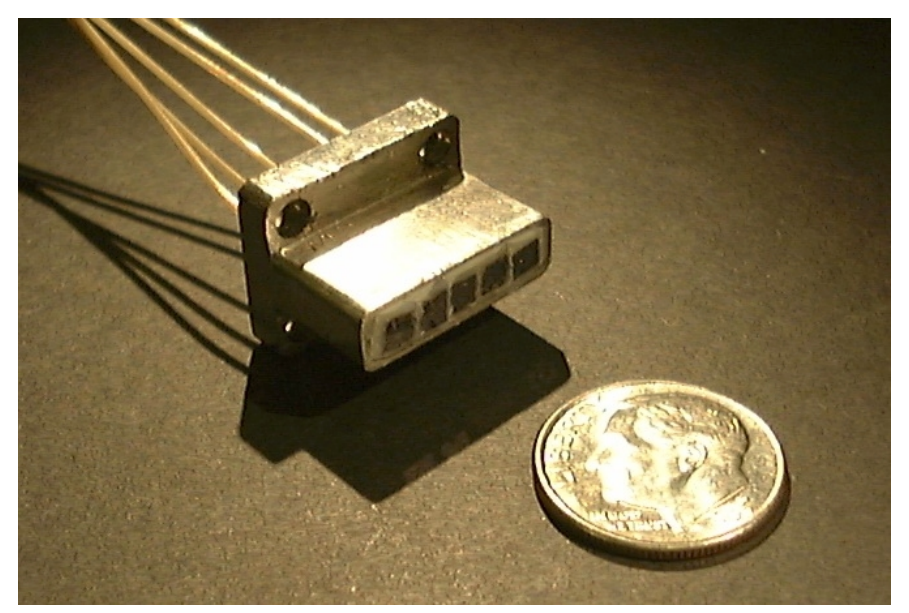

Figure 88. Photograph of the linear array of micromachined, fiber optic pressure sensors.

A shock/boundary layer interaction was created by inserting a $12.7 \mathrm{~mm}(1 / 2 \mathrm{in}$.) cylinder $25.4 \mathrm{~mm}(1 \mathrm{in}$.) through the tunnel floor into the flow $(\delta=8 \mathrm{~mm})$, as depicted in Figure 89. A typical result of a case in a Mach 3.0 flow with a shock impinging on the array is presented in Figure 90. The shock interaction with the boundary layer has caused the natural frequency of the pressure sensors to be excited, a problem that occurs in conventional pressure sensors as well. If one looks closely, the higher noise on the second sensor, termed Press 2, than the first, would seem to indicate that the shock most likely was centered on the second sensor. In explanation, when the natural frequency of the sensor is excited, the diaphragm, and therefore the optical path length in the sensor, moves very quickly. The movement can get so large that the CCD array inside the spectrometer of the signal processing system can not get a clean signal during its integration time. This is termed spectral "smearing", as described in Chapter 2. As the spectral smearing becomes more pronounced, the system can no longer determine an accurate optical path length, therefore the apparent noise in the results appears. This was seen to a smaller degree in the results from the Mach 2.4 flow. But now with the combination of a higher passing frequency of large eddies in the boundary layer due to the higher Mach number, and the shock wave and unsteady local separation imposed on 
the system, the spectral smearing has become such a problem that no useful information can be obtained from the first sensor. If one examines the plot carefully, though, the signal of the first sensor, termed Press 1, can be seen to match the surface pressure tap well, even though with high noise content.

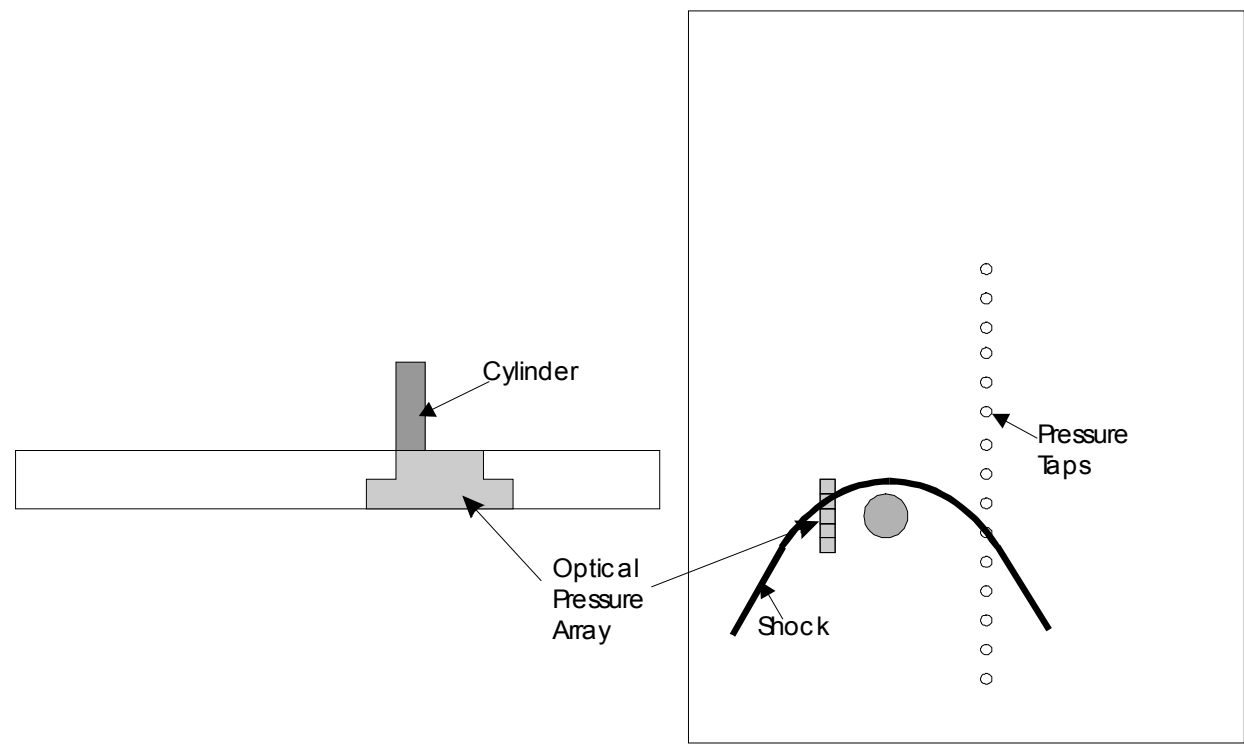

Figure 89. Sketch of shock generation in supersonic tunnel used for the pressure sensor tests.

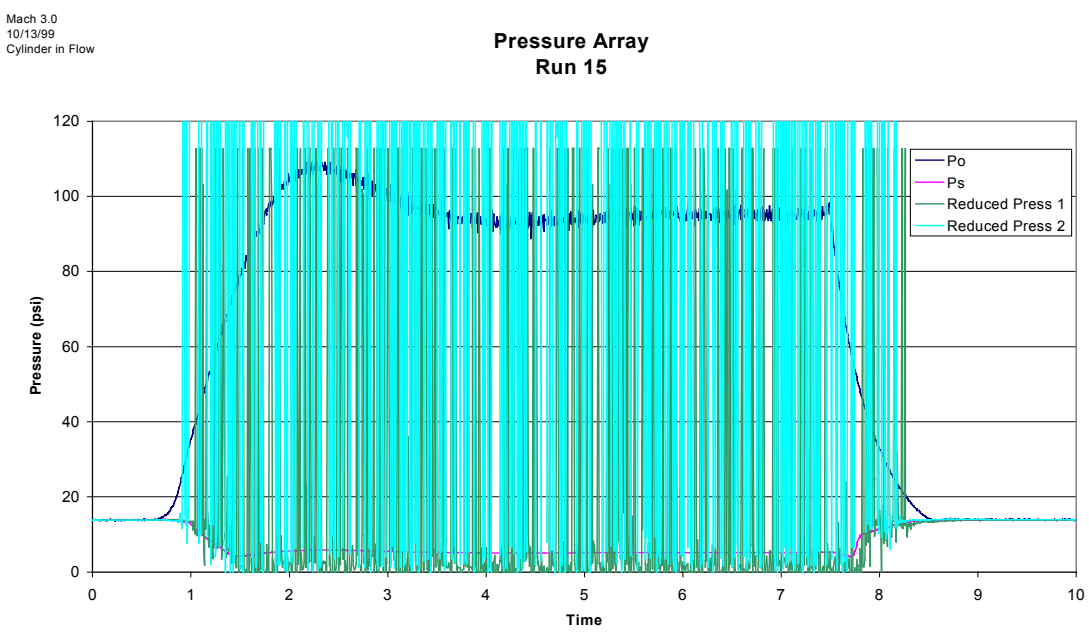

Figure 90. Flush-mounted test of the micromachined, fiber optic pressure sensor in a Mach 3.0 flow (with the shock). 
Tests were then performed in the Mach 3.0 flow with the shock generator removed. This decreased the energy in the flow near the natural frequency, and as one can see in Figure 91, much improved results were obtained. Only during tunnel start up and stopping is the spectral smearing pronounced. In all, these results indicated that the micromachined, fiber optic pressure sensor can operate in supersonic flow while flushmounted, as long as the natural frequency is not excited. There are numerous solutions to this problem (increasing the CCD array speed or damping the pressure sensor by slightly recessing it), which will be explored in the future and described in Chapter 6 .

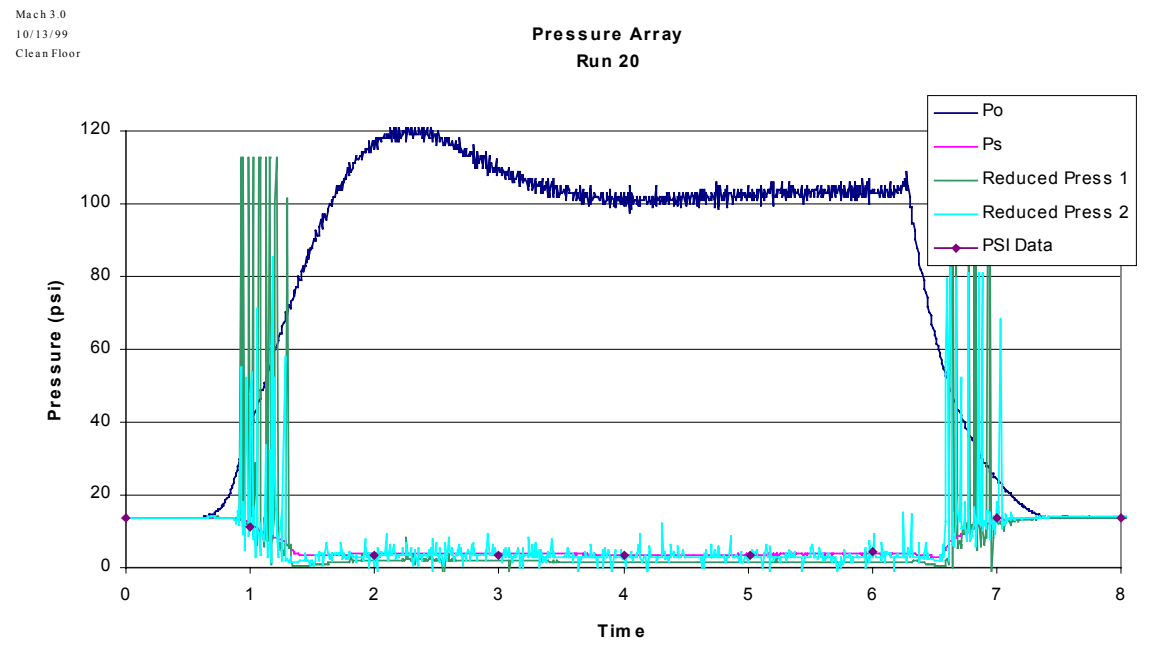

Figure 91. Flush-mounted test of the micromachined, fiber optic pressure sensor in a Mach 3.0 flow (without the shock).

\subsubsection{High-Frequency Testing}

During the tests of the fiber optic pressure sensor array, high-frequency pressure measurements were acquired in the same Mach 3 supersonic flow (no shock) in which the above measurements were made. The third (central) sensor in the array was constructed in a configuration to allow the use of the dual wavelength $(2-\lambda)$ or the intensity-based interferometric systems. These systems are theoretically capability of monitoring gap changes with frequency responses on the order of $1 \mathrm{MHz}$ or greater. Yet, as with the spectral interrogation system, successful measurements with these systems in this flow proved difficult with the intensity-based interferometric system providing the most useful results. 
Since the sensors were flush mounted, the diaphragm natural frequency $(140 \mathrm{kHz})$ was excited by high-frequency pressure disturbances in the flow. This means that the sample rate must be at least twice the natural frequency, approximately $300 \mathrm{kHz}$, in order to avoid signal aliasing. Several data sets were acquired using the intensity-based interferometric system over time periods as low 200 microseconds at sample rates as high as $50 \mathrm{MHz}$. An example of the data acquired during these tests is shown in Figure 92. The fluctuations in static pressure that occurred were at such high frequencies that it was difficult to determine whether individual peaks and valleys in the signal represent multiple interferometric fringes or turn-around points, a potential difficulty mentioned in Chapter 2. Therefore, it was not possible to process the data to produce a time history of the static pressure during these tests. The data was instead processed through a direct Fourier transfer (DFT) algorithm to produce frequency spectrum plots characterizing the frequency content in the raw signals, as the representative plots in Figure 93 and Figure 94 show. The prominent peak in Figure 93 is the natural frequency of the pressure sensor. Assuming that this is the only purely mechanical (or structural) contribution to the signal, the remaining signal content must be the result of static pressure fluctuations in the supersonic flow. Figure 94 shows that a majority of the remaining signal content occurs at frequencies below $20 \mathrm{kHz}$ with the typical roll-off of power that one would expect at the frequency increased.

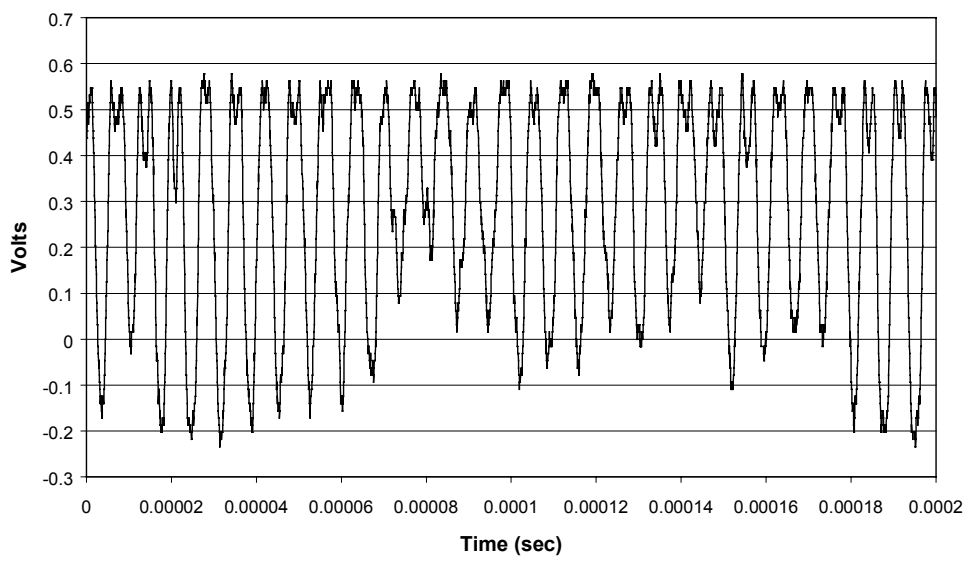

Figure 92. Representative raw intensity data (amplified photodiode output) of the fiber optic pressure sensor using the intensity interferometric signal processing system. 


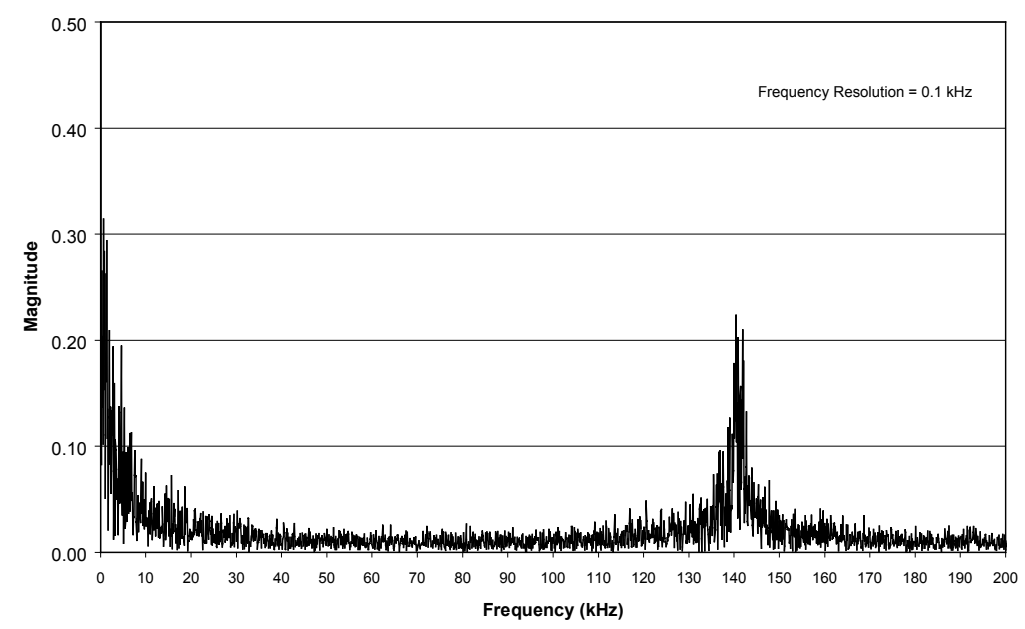

Figure 93. Frequency spectrum of representative raw intensity data showing sensor natural frequency $(140 \mathrm{kHz})$.

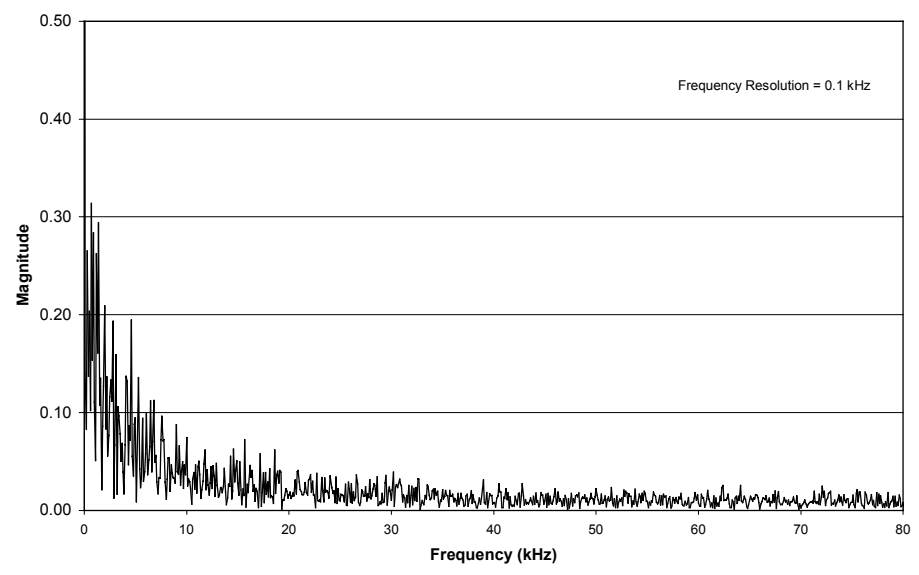

Figure 94. Frequency spectrum of representative raw intensity data showing aerodynamic signal content (low frequency detail).

\subsubsection{Mach 4 Testing}

The micromachined, fiber optic pressure sensor was also installed in a model to demonstrate its capability of making in situ measurements on a complex shape. The pressure sensors were installed in a blunt-nosed cone model $\left(10^{\circ}\right.$ half-angle, $18.8 \mathrm{~cm}$ long, $7.6 \mathrm{~cm}$ base diameter and a nose with a $0.635 \mathrm{~cm}$ radius of curvature), which had been chosen as the test geometry for a separate project. The pressure sensors used were a combination of conventional pressure transducers (for comparative purposes) connected to the model via pressure taps and internally-installed optical pressure sensors. These pressure sensors were installed in a ring around the one side of the cone at an axial 
location of $12.7 \mathrm{~mm}(0.5 \mathrm{in}$.) from the nose. In addition, a pressure tap was located at the nose. The instrumented model was tested in the Virginia Tech supersonic wind tunnel using the Mach 4 nozzle. A photo of the model installed in this facility is shown below in Figure 95.

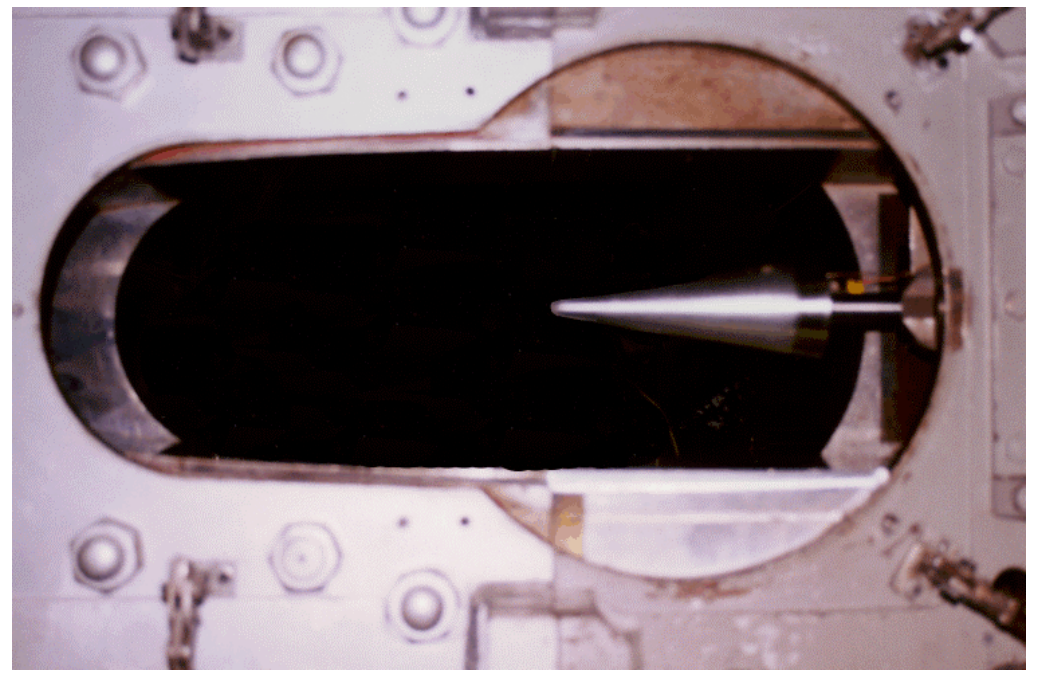

Figure 95. Cone model in Virginia Tech supersonic wind tunnel with the Mach 4 nozzle.

The measurements included pressure data from the static taps and the optical pressure sensor (using the spectral interrogation signal processing system), as well as the nose pressure and the wind tunnel conditions, including both the static and stagnation pressures. A plot of one of the runs is shown in Figure 96. As can be seen in Figure 96, there is good agreement during the steady part of the run between the conventional pressure sensors taken with a PSI, Inc pressure measurement system (represented by data points) and the optical pressure sensor (represented by a purple line), with an average pressure of $10.3 \mathrm{kPa}(1.5 \mathrm{psi}$ ) (compared to a CFD value of $11.7 \mathrm{kPa}(1.7 \mathrm{psi})$ ). One can notice a response lag in the conventional pressure sensors, owing to their external mounting as compared to the internal mounting of the optical sensor. Again, the noise in the signal from the optical sensor during start up and shut down of the tunnel is attributed to spectral smearing. 


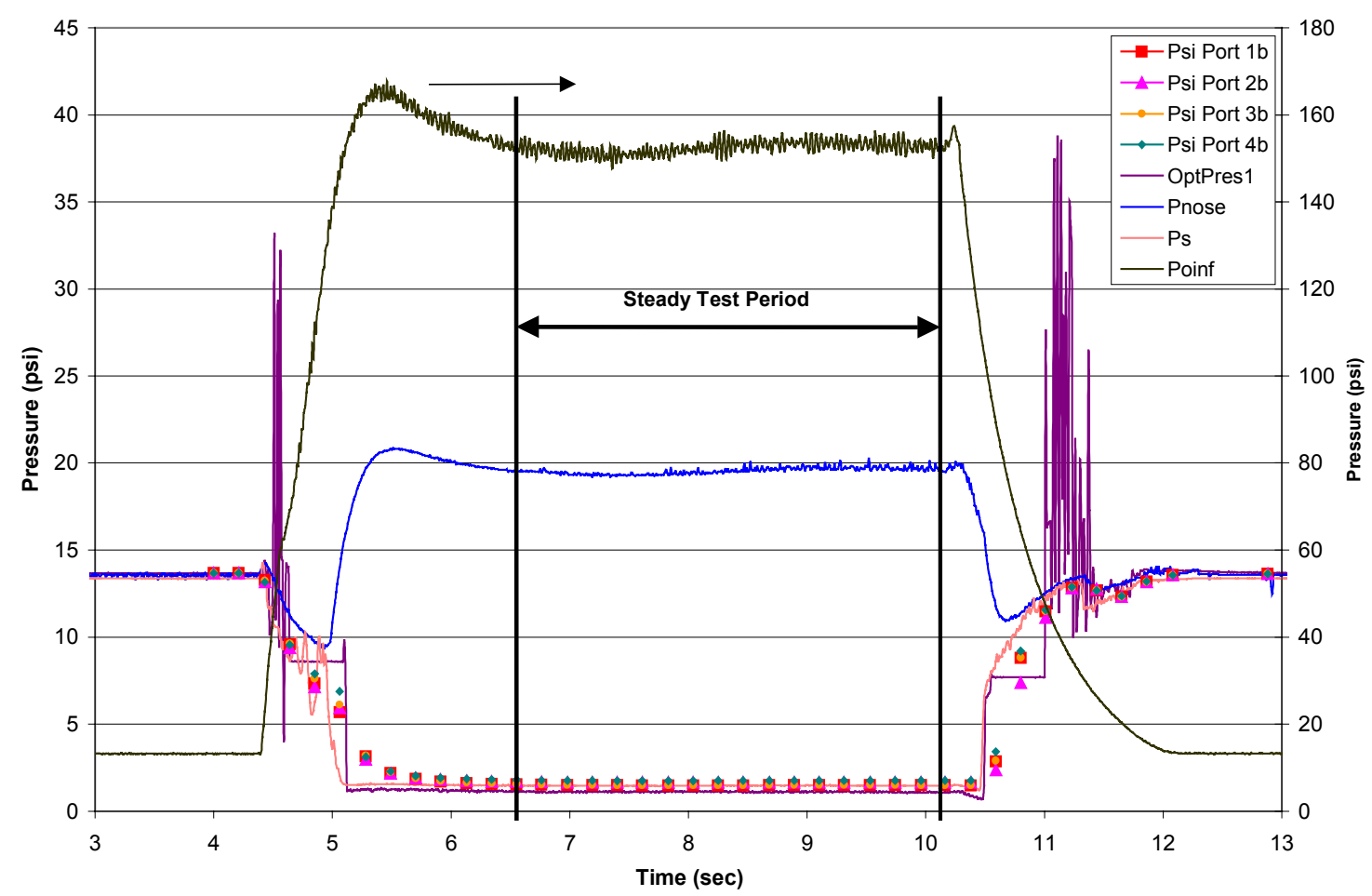

Figure 96. Pressure results of cone experiment at Mach 4 showing the comparison of fiber optic and conventional pressure sensors.

\subsubsection{Conclusions}

The fiber optic pressure sensor reliably and accurately performs in all but the most harsh flow environments. In addition, the sensor has demonstrated the potential to be an improvement in terms of temperature stability and high-frequency measurements than its counterparts. However, in certain high-energy flow conditions, the pressure sensor diaphragm, combined with the spectral interrogation signal processing system, can produce noise to the point of gather no discernable data. This increased noise is due to spectral smearing when the natural frequency of the sensor is excited by the flow. 


\subsection{Skin Friction Tests}

A series of skin friction sensors were constructed in an evolution of design philosophy to demonstrate the validity of the fiber optic concept. The mechanical drawings and calibrations to shear (both with and without fluid in the optical path) and temperature for each version are included in Appendix B and C, respectively.

The various versions of the fiber optic, skin friction sensor were also tested in the Virginia Tech supersonic wind tunnel. Most of the testing took place at two conditions, Mach 2.4 at a total pressure of $345 \mathrm{kPa}$ (50 psia), and Mach 3.0 at a total pressure of 520 $\mathrm{kPa}(75$ psia). Experience in this facility and calculations using the Schultz-Grunow correlation $^{90}$ for turbulent boundary layers has led to expected shear conditions, $\tau_{\mathrm{w}}=175$ $\mathrm{Pa}\left(\mathrm{C}_{\mathrm{f}}=0.0018\right)$ in the Mach 2.4 flow and $\tau_{\mathrm{w}}=300 \mathrm{~Pa}\left(\mathrm{C}_{\mathrm{f}}=0.0015\right)$ in the Mach 3.0 flow. As explained in the design section, the skin friction sensors are designed with two fibers in each deflection direction. The two fibers will measure opposite signals for changes in shear, but will measure the same signal for changes in temperature or pressure. By taking the two signals and subtracting them, any effect due to temperature or pressure is minimized, while the effect of shear is reinforced. Using this method means that one signal will be positive and one will be negative, ideally by the same amount, and this is evident in some of the results. The shear signals should begin with the pressure rise in the tunnel and return to the starting value when the tunnel stops. Shear results are typically non-dimensionalized into a coefficient of friction, $\mathrm{C}_{\mathrm{f}}$, through the following equation:

$$
C_{f}=\frac{\tau_{w}}{\frac{\gamma}{2} P_{e} M_{e}^{2}},
$$

where, the shear stress $\tau_{\mathrm{w}}=\mathrm{F} / \mathrm{A}$ and ' $\mathrm{F}$ ' is the force, ' $\mathrm{A}$ ' is the area of the skin friction gage head, $\gamma$ is the specific heat ratio, $P_{\infty}$ is ambient pressure, and $M_{\infty}$ is the Mach number. This non-dimensionalization allows for correlation of various turbulent boundary layer flows, and provides another check with the above expected values. 


\subsubsection{Version 1 skin friction sensor}

The original skin friction balance design, termed the Version 1 skin friction sensor, was a non-nulling cantilevered beam made out of high-temperature plastic with a sensing head attached to the end and is similar to other designs previously constructed by Schetz and his graduate students. ${ }^{91,92,93,94}$ Attached to the beam were two Luna EFPI fiber optic strain sensors, as pictured in Figure 97. The concept was to construct a skin friction sensor as close as possible to previously fabricated conventional strain gage designs that have been successful. For the initial experiments on this program, a fiber optic pressure gage was substituted as the sensing head in the typical skin friction gage design as shown in Figure 98. The fiber optic pressure sensor was a prototype constructed using the same physical principle as the aforementioned micromachined version. A photograph of the combined pressure-skin friction gage is given in Figure 99. For both the skin friction and pressure sensor in this design, the intensity-based interferometric fiber optic signal processing system manufactured by Luna was used. At the time of the experiments, this system offered greater speed and resolution than any other Luna optical signal processing system.
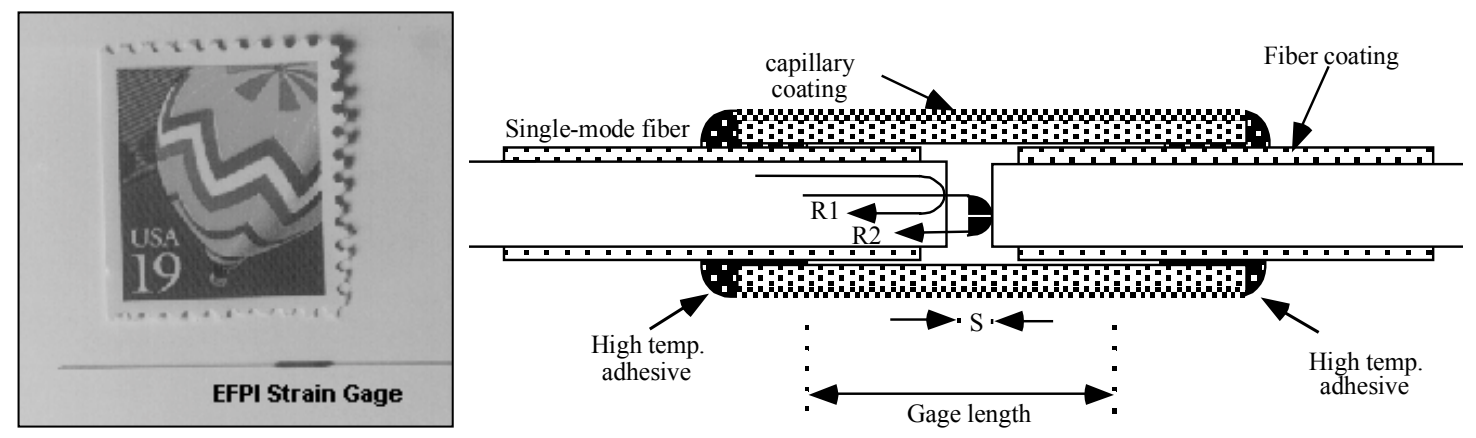

Figure 97. Standard Luna EFPI strain sensors depicting size and construction technique. 

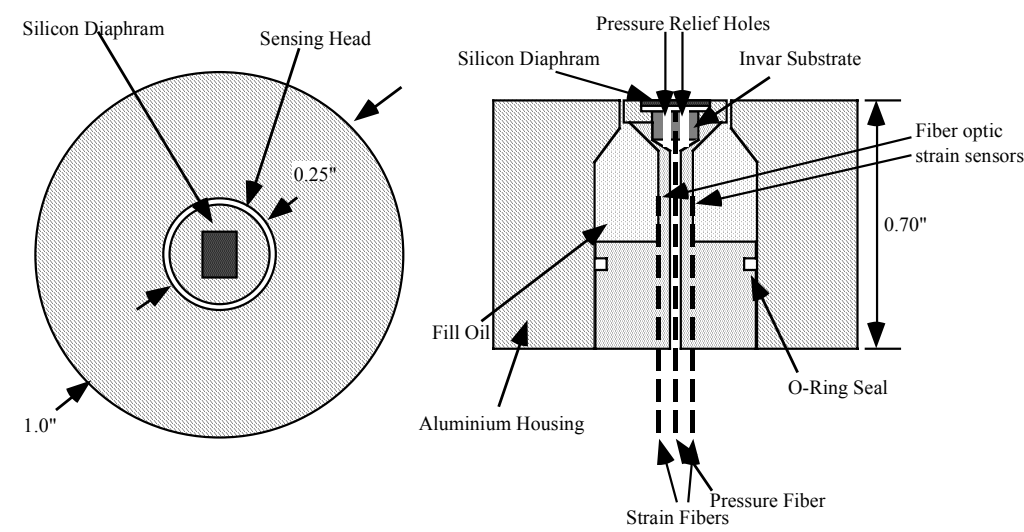

Figure 98. Sketch of the Version 1 skin friction sensor using EFPI strain sensors integrated with an optical pressure transducer in the sensing head.

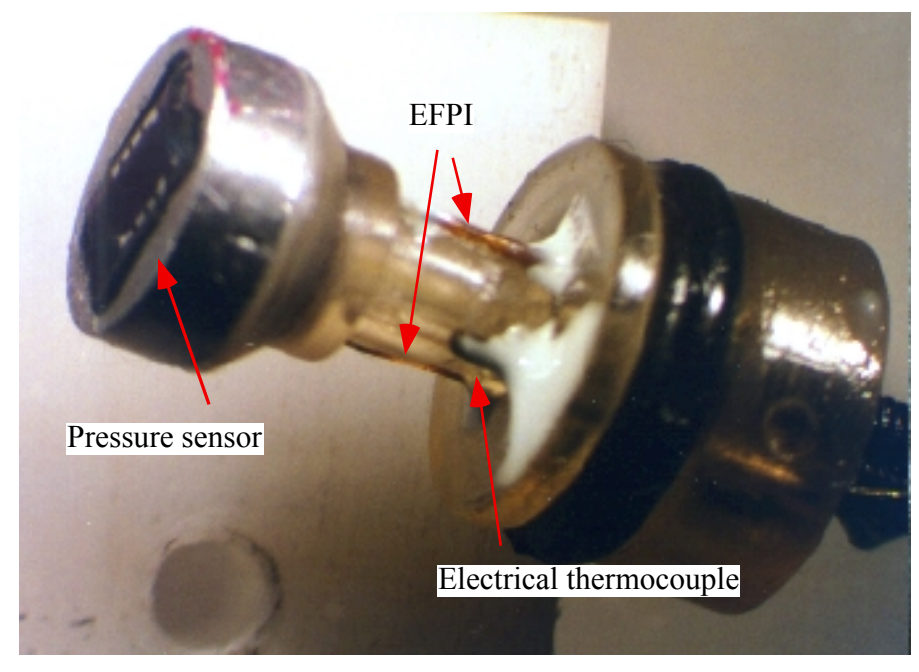

Figure 99. Photograph of the Version 1 integrated pressure-skin friction sensor.

Initial tests of the Version 1 sensor were performed at the Virginia Tech supersonic wind tunnel in July 1996. A typical output from these tests is presented in Figure 100. Through experience and calculations, the coefficient of friction was expected to be 0.0017 at these test conditions. As can be seen, the value obtained in these tests was much higher than anticipated, around six times greater. Further testing revealed that the gage was nearly an order of magnitude more sensitive to normal pressure than shear stress. It is thought that this is due to the fact that the standard EFPI strain sensor is sealed, see Figure 97. The two fibers that form the optical gap are epoxied in a glass tube, which seals in atmospheric pressure. However, these sensors were exposed to the low static pressures of the test facility, which proved to cause great difficulties. It is 
believed that the high pressure inside the EFPI sensor caused the glass tube to deflect on the beam of the skin friction gage in response to pressure changes and not just shear stresses.

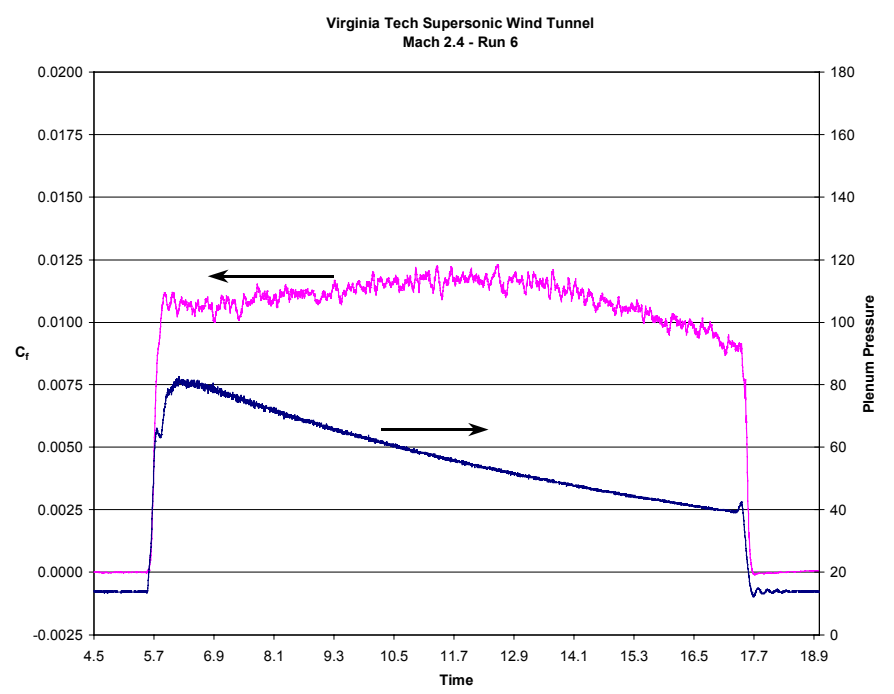

\section{Figure 100. Skin friction coefficient for Test 8 measured using the Version 1 skin friction sensor demonstrating the large magnitude of the typical result.}

Figure 101 presents typical results from the fiber optic pressure sensor that encompassed the sensing head of the skin friction sensor. The results are qualitatively good, but quantitatively a problem. The difference is attributed to the difficulty in reducing data from intensity-based interferometric fiber optic signal processing. As mentioned in Chapter 2, if a signal changes direction while near a peak or valley in the sinusoidal output, it is difficult to differentiate from simple noise, and that can easily lead to the results being a fringe off the correct value. Another problem is if the sensor moves through multiple fringes between samples from the data acquisition, where total fringe movements can be missed for a fast changing sensor. It is for reasons such as these and also due to large improvements in the competing optical signal processing system types, that all later sensors used the spectral interrogation signal processing described in Chapter 2, where the absolute optical gap is determined, eliminating the need for complex and error-prone data reduction. 


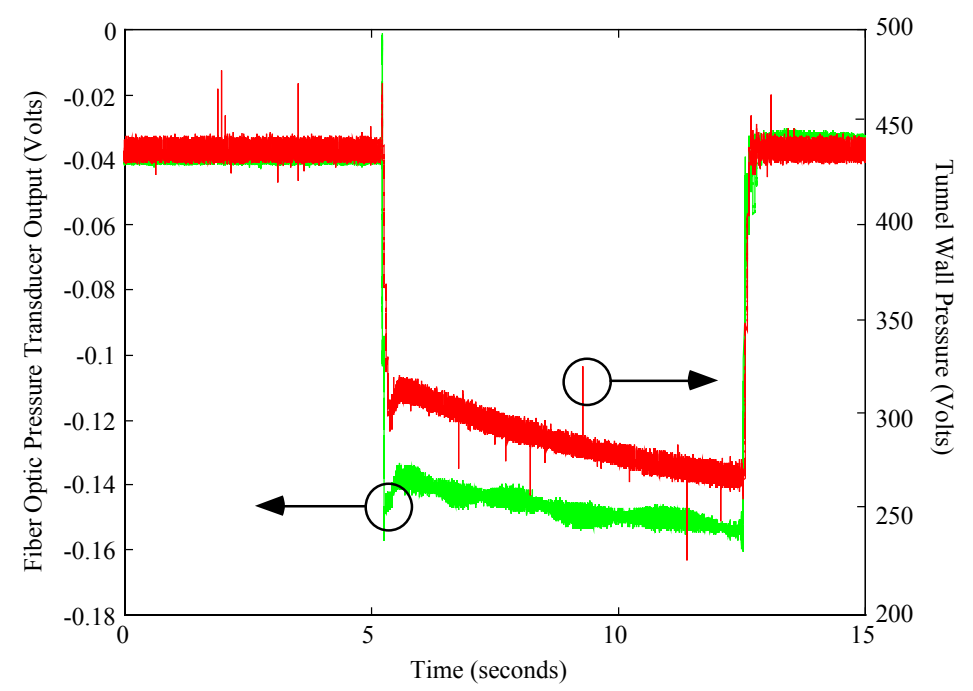

Figure 101. Results of the fiber optic pressure sensor in the sensing head of the Version 1 sensor.

\subsubsection{Version 2 skin friction sensor}

The general concept for the second fiber optic skin friction gage is depicted in Figure 102, and it results from the design equations developed in Chapter 3. The reflector for the EFPI, which produces the second reflection in this case, is a disk placed about half way down the cantilever sensing beam. By not enclosing sensing fibers in a glass tube, as was done in the Version 1 sensor, the gage was made insensitive to pressure changes. Because a concurrent goal of the project is to construct a fiber optic skin friction gage for high temperature applications, it was decided that this sensor would be machined out of a metal. Invar was selected for its low coefficient of thermal expansion (C.T.E.) (C.T.E. $\approx 1.88 \mathrm{E}-6 \mathrm{~m} / \mathrm{m}{ }^{\circ} \mathrm{C}$ ) and its low thermal conductivity. In fact, Invar was used as the material in all later designs due to the temperature stability it affords.

Using the design equations developed in Chapter 3, a design study was performed to examine the trade-offs that this design concept exhibits. By looking at Equation 32, one can see that that $\mathrm{E}$ and $\tau_{\mathrm{w}}$ are set by the selection of the sensor material and design shear. The design movement, $\Delta \delta$, is determined by the system resolution and the acceptable resolution uncertainty. Therefore, the variables left are $\mathrm{L}_{t}, \mathrm{~d}_{\text {beam }}, \mathrm{d}_{\text {head }}, \mathrm{r}_{\mathrm{fiber}}$ and $\alpha$. Design goals of skin friction sensors are usually to decrease the head diameter as much as possible, which has the consequence making the beam diameter as small as 
possible and the length as long as possible, both determined by machining technology. All of the variables were examined in a series of plots, as in Figure 104, to determine the various trade-offs. For this sensor design, the generated plot uses a beam length of 1.1 inches, chosen as a limit of conventional machining for the beam diameters studied, and includes the design surface for the remaining three variables. It was first determined that the radial location of the fiber was not that important as it was a linear effect. From this point on, this radius was set as a percentage of the reflector diameter to reduce the number of variables. The radius was large enough to get the largest response possible, but not so large as to endanger being at a rounded edge of the reflector once it is machined. The selected design has a sensing head diameter of $6.35 \mathrm{~mm}$ (0.25 inches), using a design movement at $300 \mathrm{~Pa}$ of $100 \mathrm{~nm}$. The overall dimensions of $9.525 \mathrm{~mm}$ (0.375 in.) by $31.75 \mathrm{~mm}\left(1 \frac{1}{4} \mathrm{in}\right.$.) make this one of the smaller skin friction sensors produced by this research group. The completed gage is pictured in Figure 103.

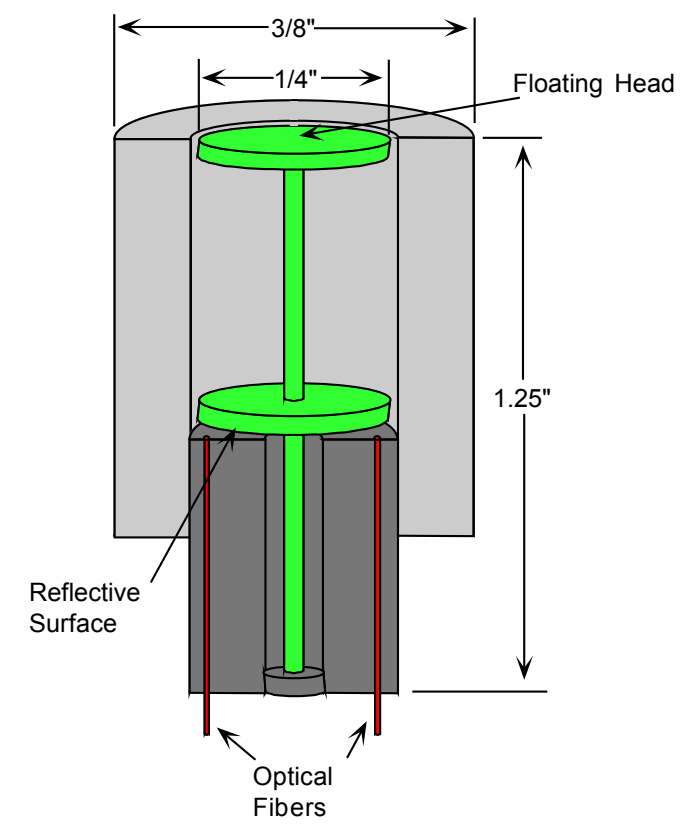

Figure 102. Schematic of the Version 2 skin friction sensor. 


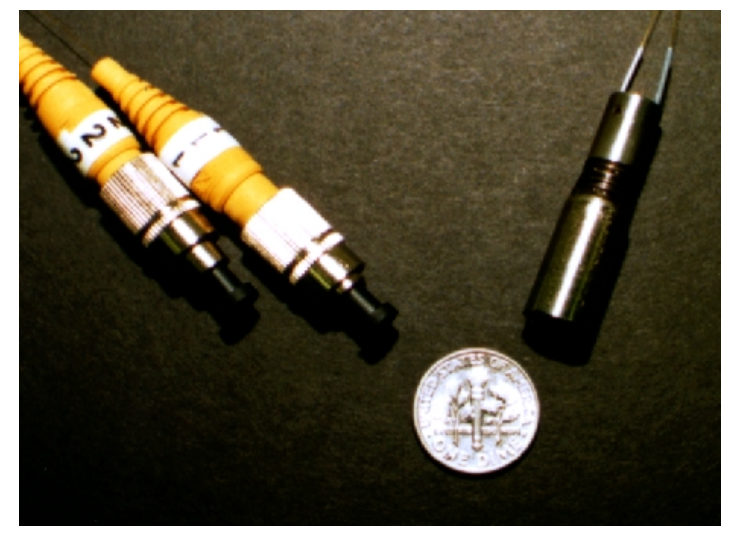

Figure 103. Photograph of the Version 2 skin friction sensor.

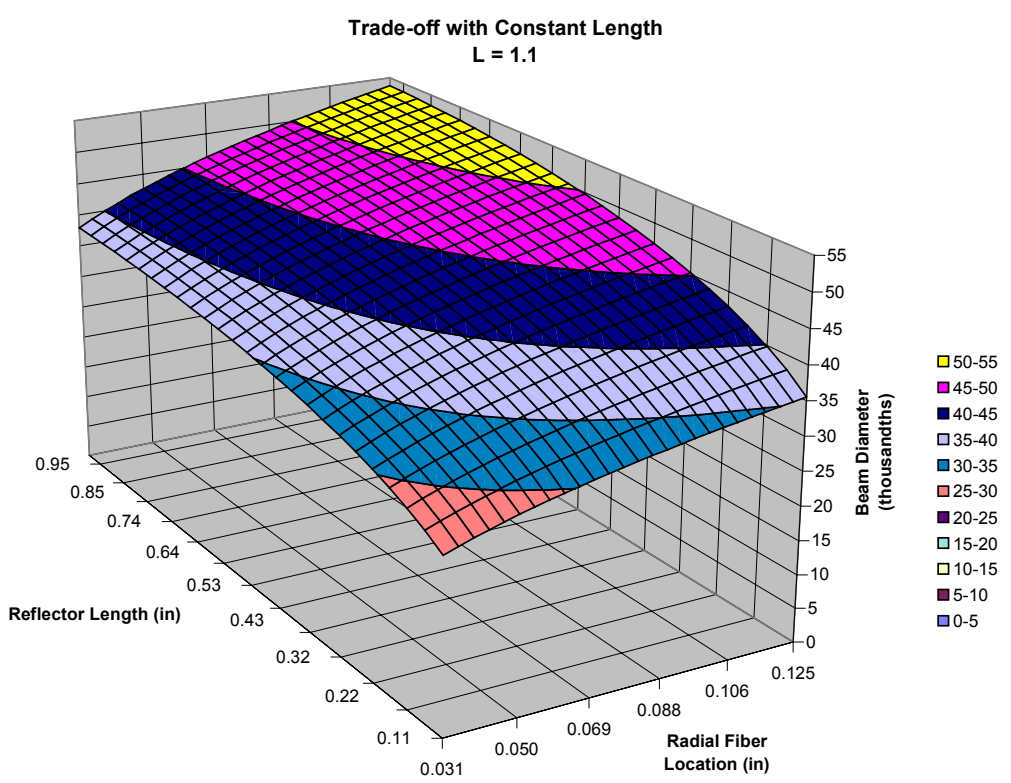

Figure 104. Design space of the Version 2 skin friction sensor.

Figure 105 and Figure 106 present results of tests performed with the Version 2 skin friction sensor, which was filled with Dow Corning 203 silicon oil, at the Virginia Tech supersonic wind tunnel. During this entry, the Mach 3 nozzle was installed and run at a total pressure of $690 \mathrm{kPa}(100 \mathrm{psi})$. As shown in the figures, the testing with the fiber optic gage indicated the coefficient of friction to be 0.00125 and 0.0121 respectively for Figure 105 and Figure 106. These results compare reasonably well with the expected value of 0.0015 . The output of the sensor for the complete test shows important characteristics - a near constant value during the steady portion of the run and a return to zero at the end of the run. 


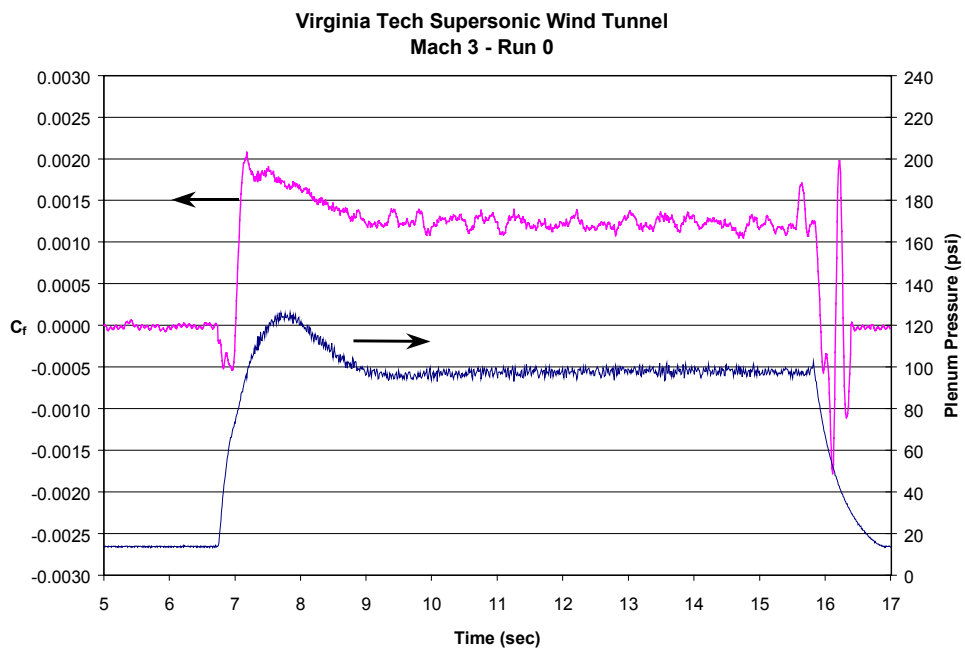

Figure 105. Test 1 of Version 2 skin friction sensor in a Mach 3.0 flow at a total pressure of $690 \mathrm{kPa}(100 \mathrm{psi})$.

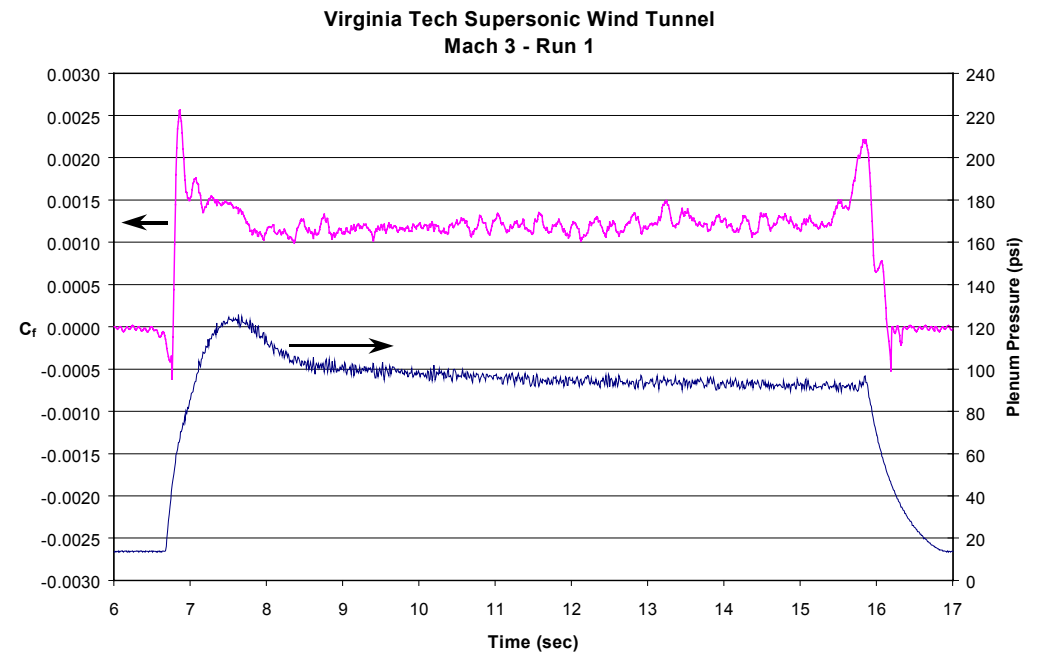

Figure 106. Test 2 of Version 2 skin friction sensor in a Mach 3.0 flow at a total pressure of $690 \mathrm{kPa}(100 \mathrm{psi})$.

\subsubsection{Version 3 skin friction sensor}

The Version 2 sensor results led to a false sense of confidence with this design concept. The development of the skin friction sensor was put aside to concentrate on other aspects of the study. When the research returned to this problem, it was decided to make the sensor more complex with the addition of a pressure sensor and a thermocouple 
so that measurements of skin friction, pressure, surface temperature and heat flux would be possible. The thermocouple was used to measure a time-history of surface temperature, which would then be used to calculate heat flux through the CookFelderman algorithm, as described in Chapter 1. A sensor to measure all the desired measurands, skin friction, pressure, surface temperature, and heat flux, was constructed, as shown in Figure 107. The surface thermocouple for measuring temperature and calculating heat flux can be seen, as well as the flush-mounted micromachined, fiber optic pressure sensor. In this sensor, the reflector disk was removed, and the second reflection was obtained from the underside of the sensing head. The design space for this sensor is presented in Figure 108. The selected geometry has sensing head of $6.35 \mathrm{~mm}$ (0.25 inches) in diameter, with a design movement of $150 \mathrm{~nm}$ at $300 \mathrm{~Pa}$. The overall dimensions were larger to accommodate the thermocouple and pressure sensor. A detaailed mechinical drawing of this sensor is presented in pages 275-6 in Appendix C.
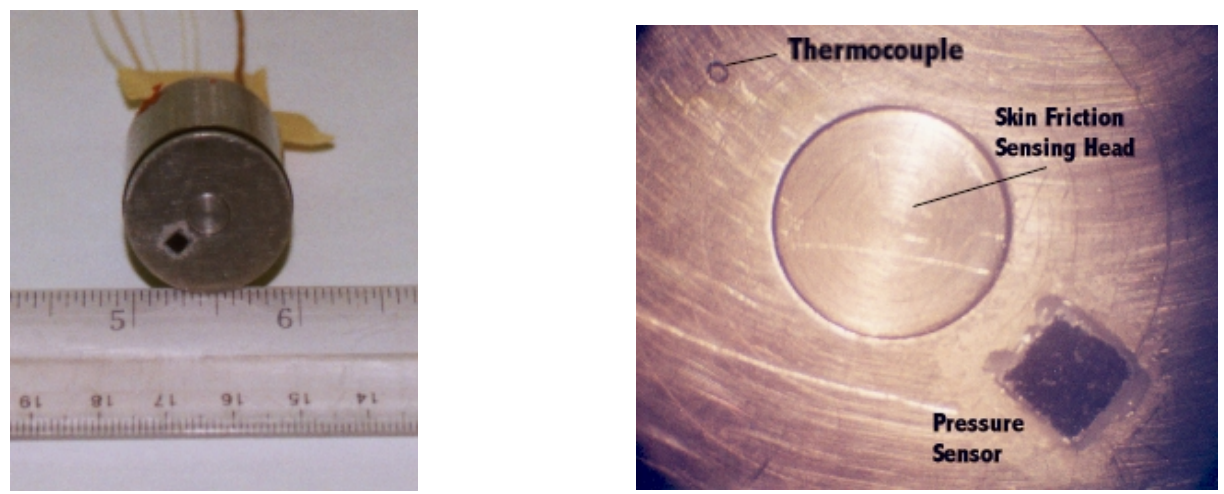

Figure 107. Photographs of the Version 3 skin friction sensor. 


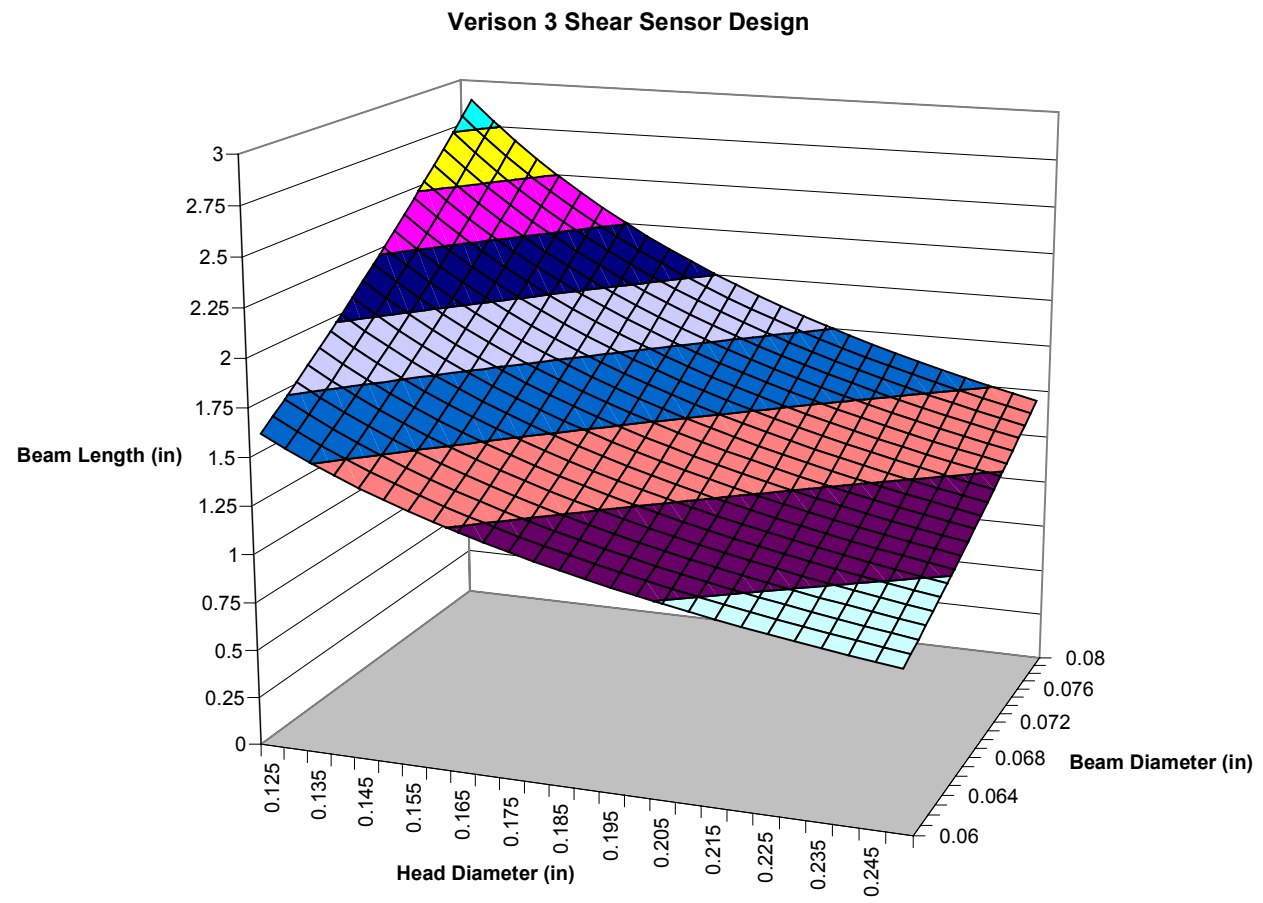

Figure 108. Design space of Version 3 skin friction sensor.

The Version 3 skin friction sensor began testing in the Mach 3.0 flow in March 1999. The first tests, shown in Figure 109, were performed without the customary silicon oil in the sensor. This fill oil had been used to mitigate pressure gradients, protect the sensor from extreme temperatures, provide damping, and provide a smooth surface for the flow to travel over the sensor. However, this oil was an extreme operational problem, as it tended to flow out and require periodic filling. As stated in Chapter 3, there is the concern that changes in the environment will change the index of refraction of the oil and, therefore, introduce error into the shear measurement. In the testing, though, it was hoped that the optical sensors would not need the oil, thereby removing the operational problem and potential error source. However, the results in Figure 109, which are typical, show that the sensor vibrates too much without the oil to provide damping. This is due to the same problem that affected the pressure sensor in certain conditions. The movement of this sensor type is much larger, and the cantilever beam design makes it prone to such movement. 


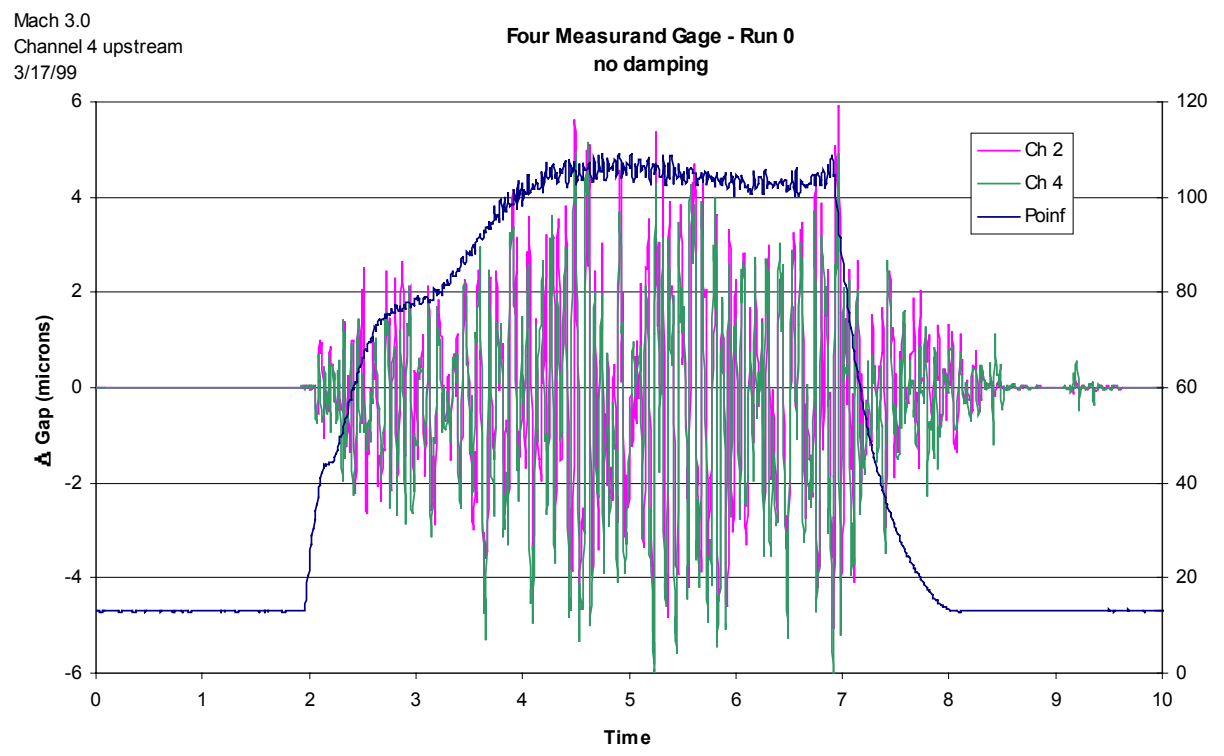

Figure 109. Test 1 of Version 3 skin friction sensor in a Mach 3.0 flow at a total pressure of $690 \mathrm{kPa}(100 \mathrm{psi})$ with no damping oil.

The previous results indicated that oil was necessary, at least until another method of damping could be developed. Typical results of the Version 3 skin friction sensor with damping oil are presented in Figure 110. The results show a mirror-image from the two opposing fibers with little noise, which is positive. However, the change in gaps is too small, and often the optical gap changes indicated that the sensor was moving in the wrong direction. After much deductive effort, it was determined that the sensor was assembled poorly, allowing the center body to rock in its connection to the rest of the sensor. The design was altered for Version 4, where a slip fit was specified rather than a thread for the connection between the center body and the base. 


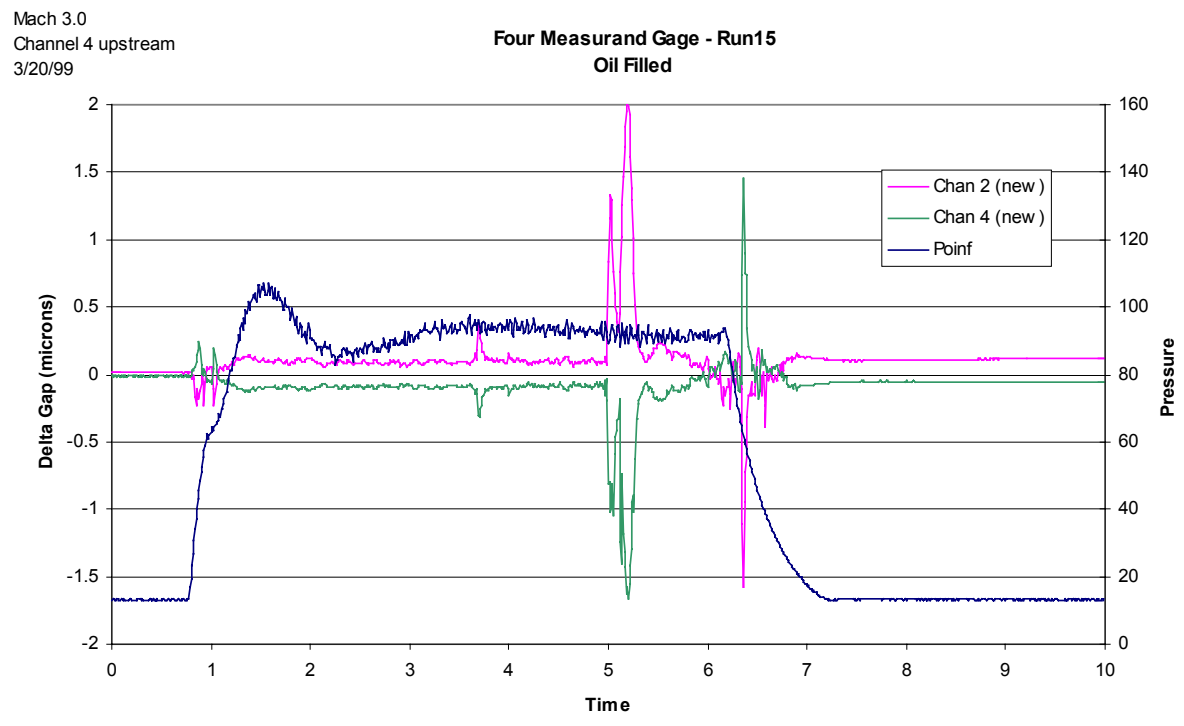

Figure 110. Test 16 of Version 3 skin friction sensor in a Mach 3.0 flow at a total pressure of $690 \mathrm{kPa}(100 \mathrm{psi})$ with damping oil.

\subsubsection{Version 4 skin friction sensor}

During the construction and calibration of the Version 3 sensor, it was observed that it was difficult to align all the fibers to the underside of the head while they were all fixed in the base piece. To alleviate this problem, the next design had each fiber in a separate holder that could be installed and aligned separately. This concept proved very effective, as the optical signal was much improved as compared to the Version 3 sensor. The Version 4 skin friction sensor, Figure 111, was designed for possible experiments in a high pressure gradient flow. Therefore, the sensing head size was reduced as much as was feasible. By having a larger shelf under the head, as pictured in Figure 111, the sensitivity could be maintained as the force from the head was reduced. In addition, a notch was put in the beam at the base to provide a stress concentration and more bending for the same force. The sensing head was only $4.2 \mathrm{~mm}$ (0.165 inches) in diameter, with a design gap movement of $150 \mathrm{~nm}$ for a $300 \mathrm{~Pa}$ shear with the design space presented in Figure 112. The overall dimensions are $12.7 \mathrm{~mm}\left(1 / 2 \mathrm{in}\right.$.) by $38.1 \mathrm{~mm}\left(1 \frac{1}{2} \mathrm{in}\right.$. $)$, still making this a very small sensor in comparison to conventional strain gage-based designs, which are typically $12.7 \mathrm{~mm}(1 / 2$ in.) by $152.4 \mathrm{~mm}$ (6 in.). A detaailed mechinical drawing of this sensor is presented in pages 277-9 in Appendix C. 

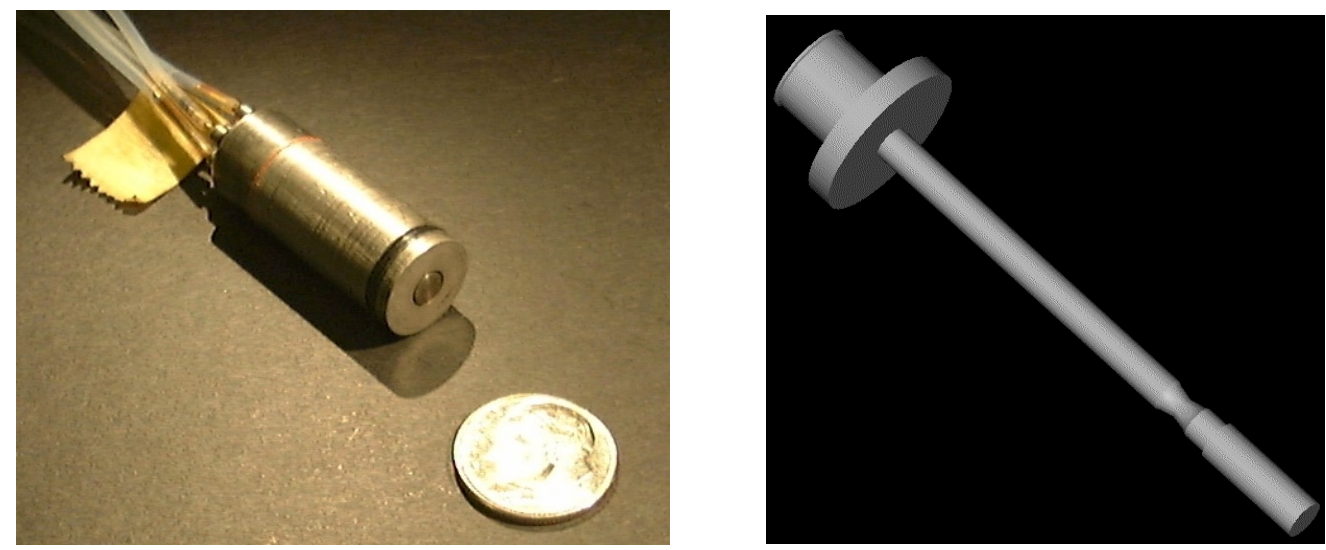

Figure 111. Photograph of the Version 4 skin friction sensor with rendering of the centerbody.

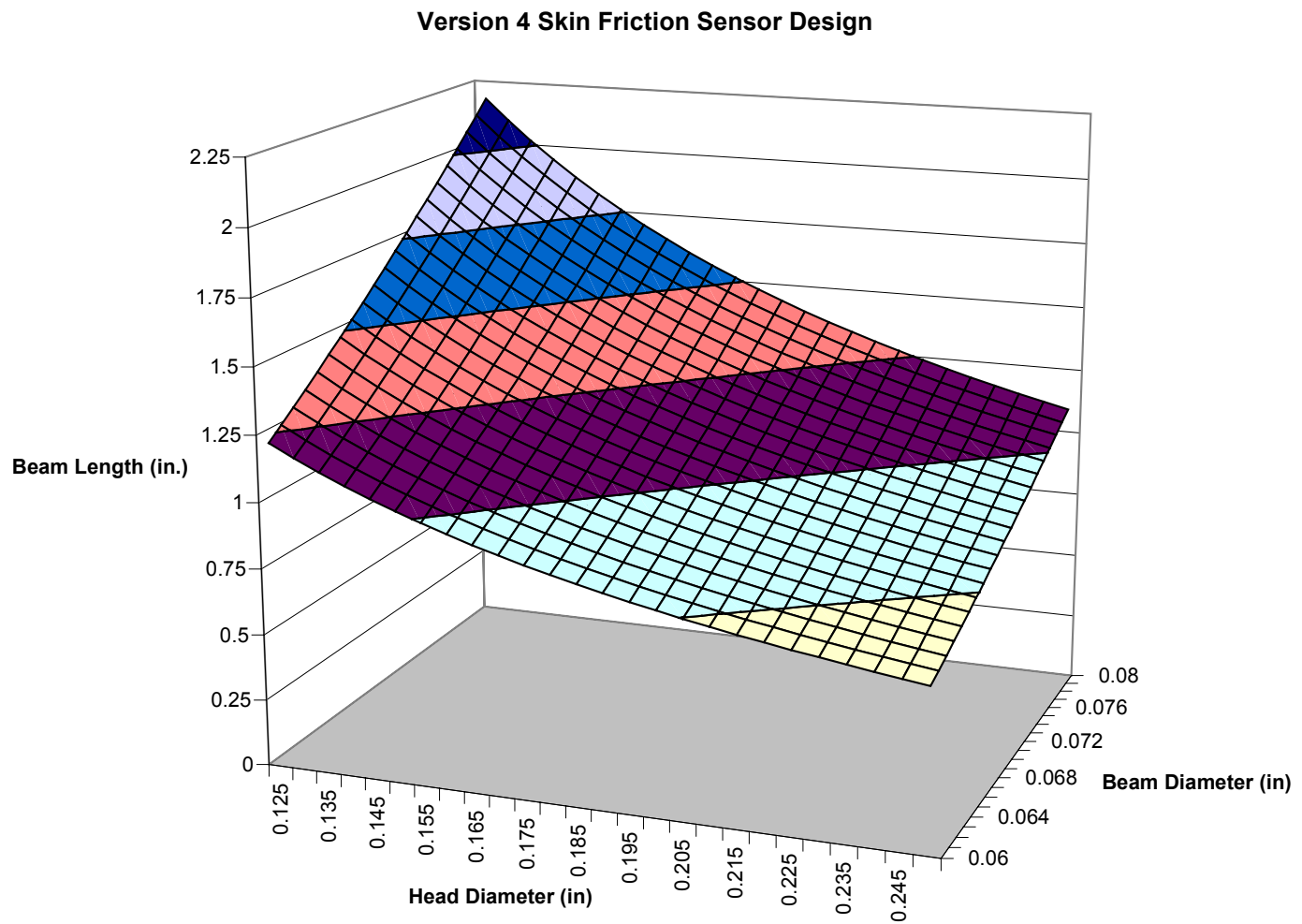

Figure 112. Design space of Version 4 skin friction sensor.

The Version 4 skin friction sensor was also tested at Mach 3 with a total pressure of 550 $\mathrm{kPa}(80 \mathrm{psi})$. Results for a test without oil fill are presented in Figure 113. As before, the lack of damping led to spectral smearing and a high noise content. 


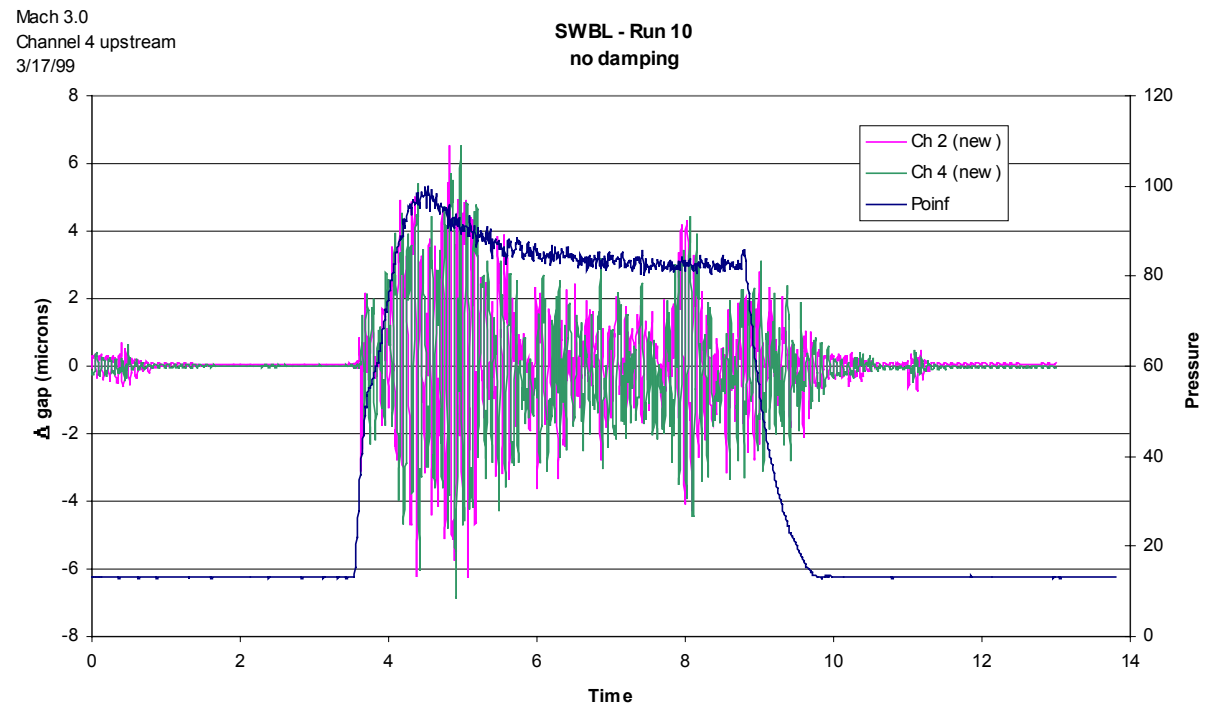

Figure 113. Test 11 of Version 4 skin friction sensor in Mach 3.0 flow without damping fluid.

The sensor was then filled with glycerin (used so that is matched a calibration rig fluid, which is described below) Typical shear and friction coefficient results for a Mach 2.4 flow are presented in Figure 114 and Figure 115, respectively. The results during the steady part of the run are positive, with skin friction values approximately correct. The spike at the beginning of the run is probably due to the fast change in flow conditions during tunnel start up. However, it was not known why the sensor was slow to return to zero after the run. Figure 116 presents similar results.

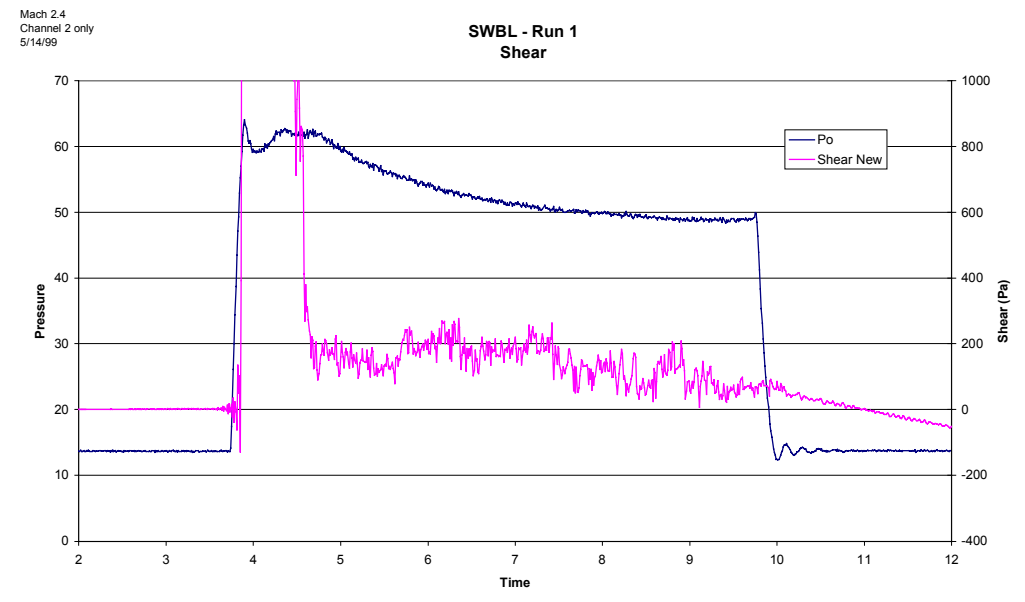

Figure 114. Test 2 shear results of Version 4 skin friction sensor in Mach 2.4 flow at a total pressure of $345 \mathrm{kPa}(50 \mathrm{psi})$. 


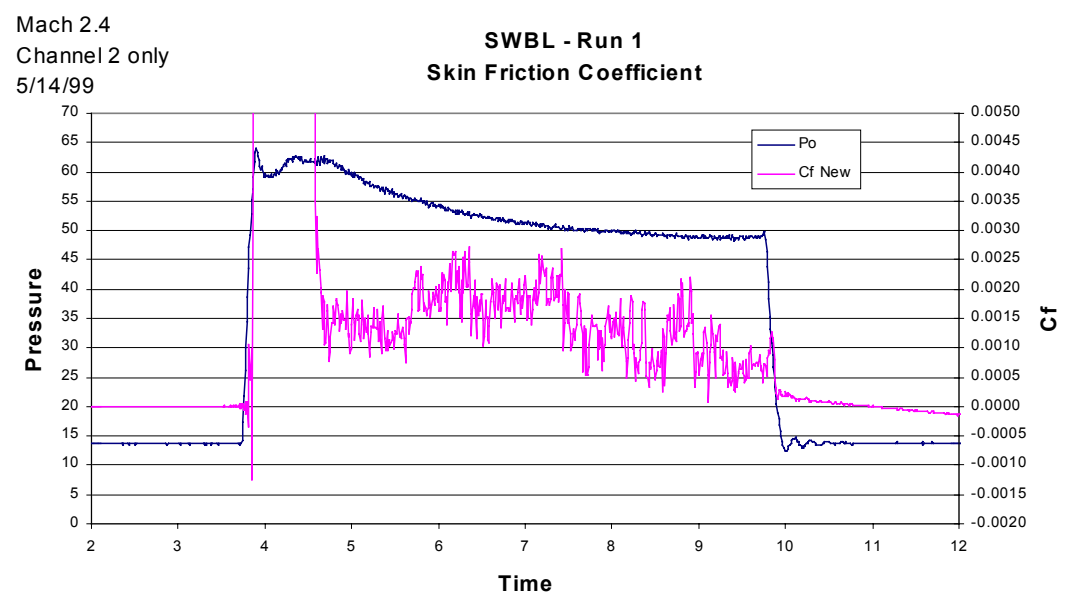

Figure 115. Test 2 skin friction coefficient results of Version 4 skin friction sensor in Mach 2.4 flow at a total pressure of $345 \mathrm{kPa}(50 \mathrm{psi})$.

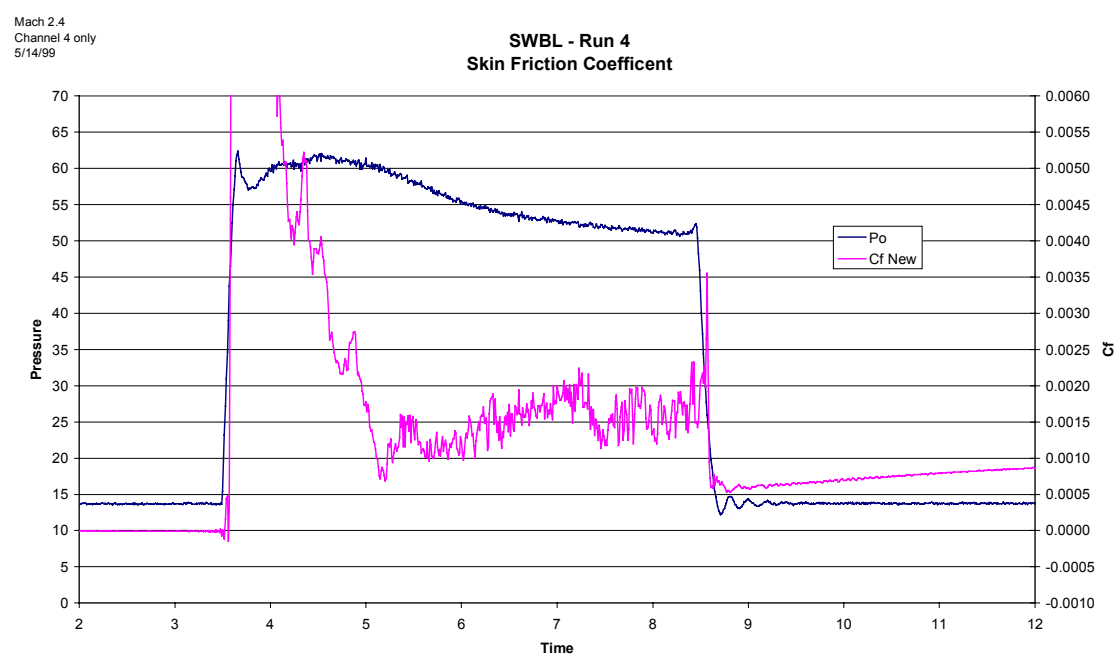

Figure 116. Test 4 skin friction coefficient results of Version 4 skin friction sensor in Mach 2.4 flow at a total pressure of $345 \mathrm{kPa}(50 \mathrm{psi})$.

During tests with this sensor, it was sometimes observed that the sensor would appear to be moving in the wrong direction, as if it were in a strong flow reversal. As this was not likely in the flat plate section of the tunnel floor and shadowgraphs of the flow confirmed that this was not occurring, other possibilities were examined. It was determined that the electronics/software for data acquisition was configured such that it 
was possible to mix up the channels. Although it was never conclusively proven that any channels had been mixed, a re-writing of the acquisition software seemed to solve this problem.

To assist in solving this problem, it was decided to test the sensor in a benign flow, in this case the incompressible glycerin flow of the calibration rig described below. The normal method of calibrating the skin friction gages has been by hanging weights from the sensing head, as depicted in Chapter 3. This method is not practical in many instances: micromachined gages are too small to attach a weight, sensors with integral pressure diaphragms are likely too fragile to attach weights, as well as gages with undefined sensing head sizes such as a rubber filled gage. Therefore, an alternate method for strain calibration was devised and constructed. The new method, seen in Figure 117, actually creates a shear force on the gage head rather than a point force, which more accurately models the forces the sensor experiences in operation.

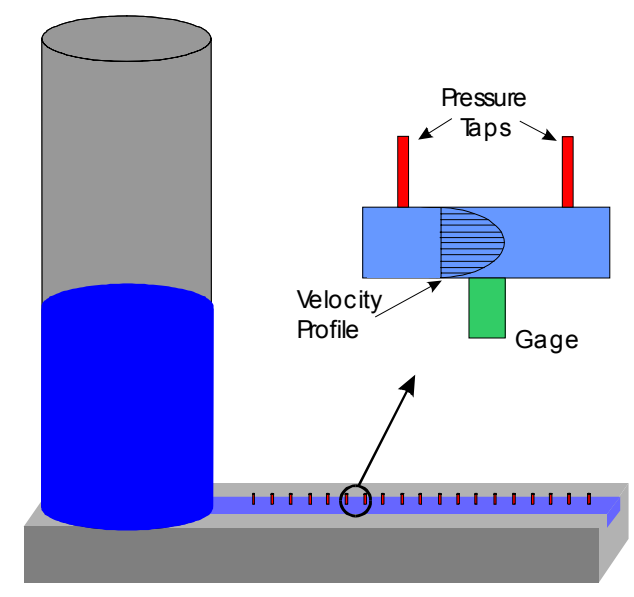

Figure 117. Schematic of the channel flow shear calibrator.

The calibrator has a cylindrical holding tank that feeds glycerin through a thin, rectangular channel. The flow quickly becomes fully-developed, and the aspect ratio $(\mathrm{L}=$ $400 \mathrm{~mm}$ (16 in.) and $\mathrm{h}=6.35 \mathrm{~mm}(0.25 \mathrm{in})$.$) of the channel is large enough to ensure 2-D$ flow in the center region of the channel. A fully developed, 2-D, channel flow is well understood. Once a flow is fully developed, the pressure drop becomes linear with distance along the channel. Therefore, monitoring of the wall pressure along the channel, not only indicates when the flow becomes fully-developed, but also allows easy 
calculation of the average shear between the pressure measurements for the calibration as described in the following procedure. If the forces acting on a two-dimensional fluid element in fully-developed flow are analyzed where $\mathrm{p}$ and $\tau$ denote pressure and shear stress, respectively, then the average shear between the pressure measurements can be obtained by,

$$
f=\left(-\frac{\partial p}{\partial x}+\frac{\partial \tau}{\partial y}\right) \Delta x \Delta y
$$

then,

$$
\tau=-\frac{d p}{d x} h
$$

where, $\mathrm{h}=0.125 \mathrm{in}$. and is the channel height measured from the centerline axis.

Glycerin was chosen because of its dramatic changes in viscosity as a function of temperature. For instance, glycerin at $20^{\circ} \mathrm{C}$ has a viscosity of $1410 \mathrm{cp}$, whereas at $30^{\circ} \mathrm{C}$, it has a viscosity of $612 \mathrm{cp}$. With this in mind, a heat exchanger consisting of copper tubing snaked throughout the tank and channel carrying tap water was constructed, allowing the control of glycerin temperature and therefore viscosity.

Steady, fully-developed flow was obtained by implementing a constant-head tank. To reach a steady state flow, the fluid level in the tank had to remain constant to ensure a constant pressure drop (see Figure 118). To achieve this, a large reservoir was needed to supply the tank with the glycerin. Figure 119 is a photograph of the entire calibration setup.

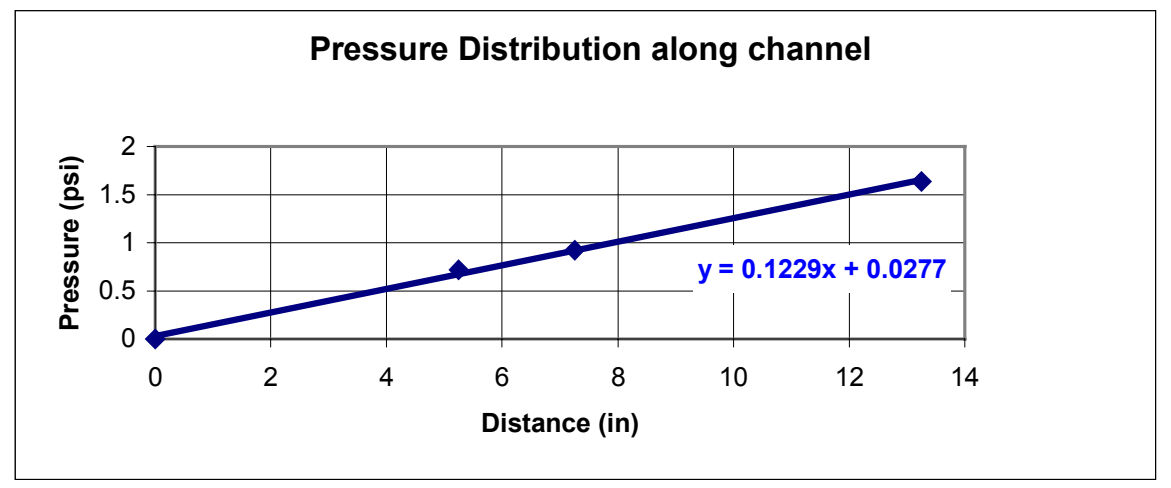

Figure 118. Pressure distribution along channel of calibration rig. ${ }^{95}$ 


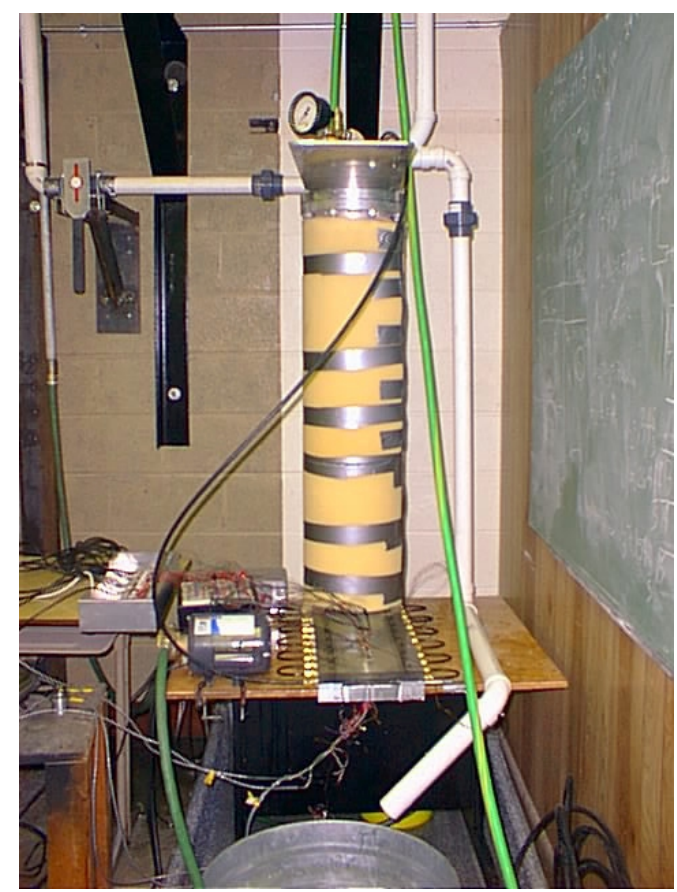

Figure 119. Photograph of calibration rig.

By using a fully-developed flow, a simple pressure reading can determine the shear in a channel. The use of glycerin allows reasonable shears $(100 \mathrm{~Pa})$ for a relatively slow flow. The results of the first test are shown in Figure 120, in which the facility was turned on and off twice. The results show both the correct shape and magnitude with very little noise. Next, the facility was modulated to provide a variable shear, as shown in Figure 121. This result was also very positive. The sensor also moved in the correct direction, showing that the problems of the past had been overcome. In order to test that the sensor was moving in the right direction, the sensor was physical turned $180^{\circ}$ around so that it would react in the opposite manner. Figure 122 shows that this was also a success. One could conclude from these tests that the fiber optic skin friction sensor would work very well in incompressible flow. 


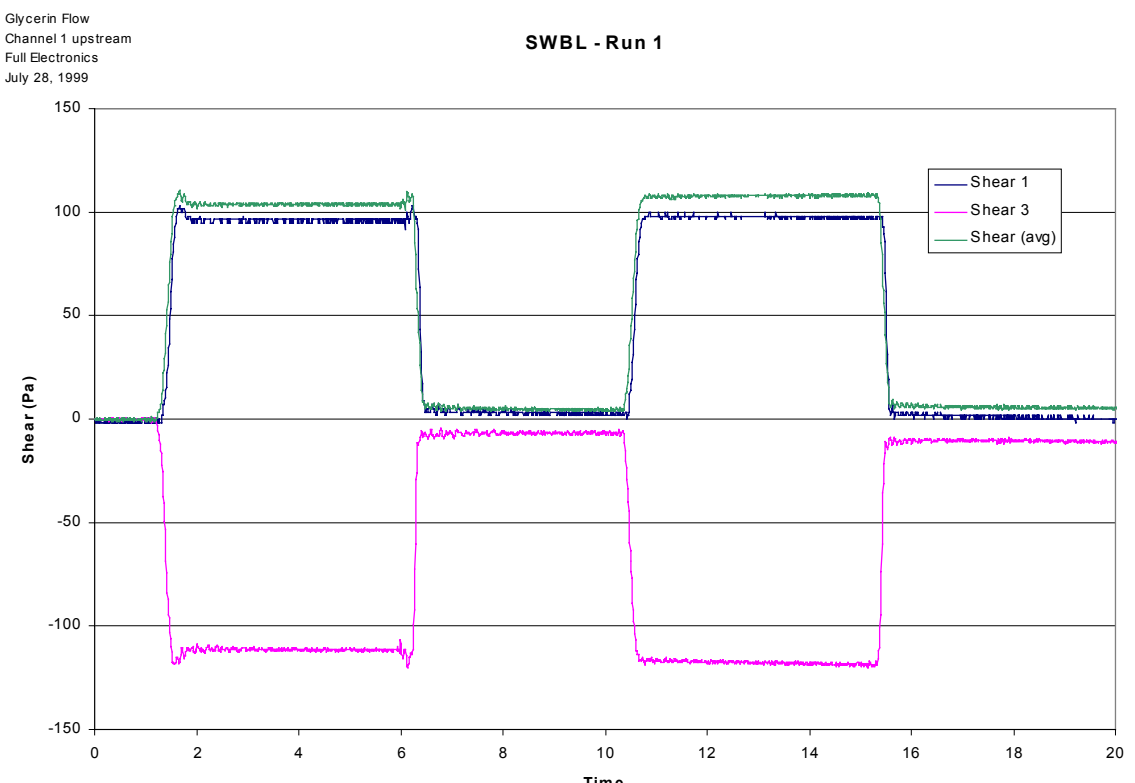

Figure 120. Test 2 of the Version 4 skin friction sensor in a fully-developed, incompressible glycerin flow.

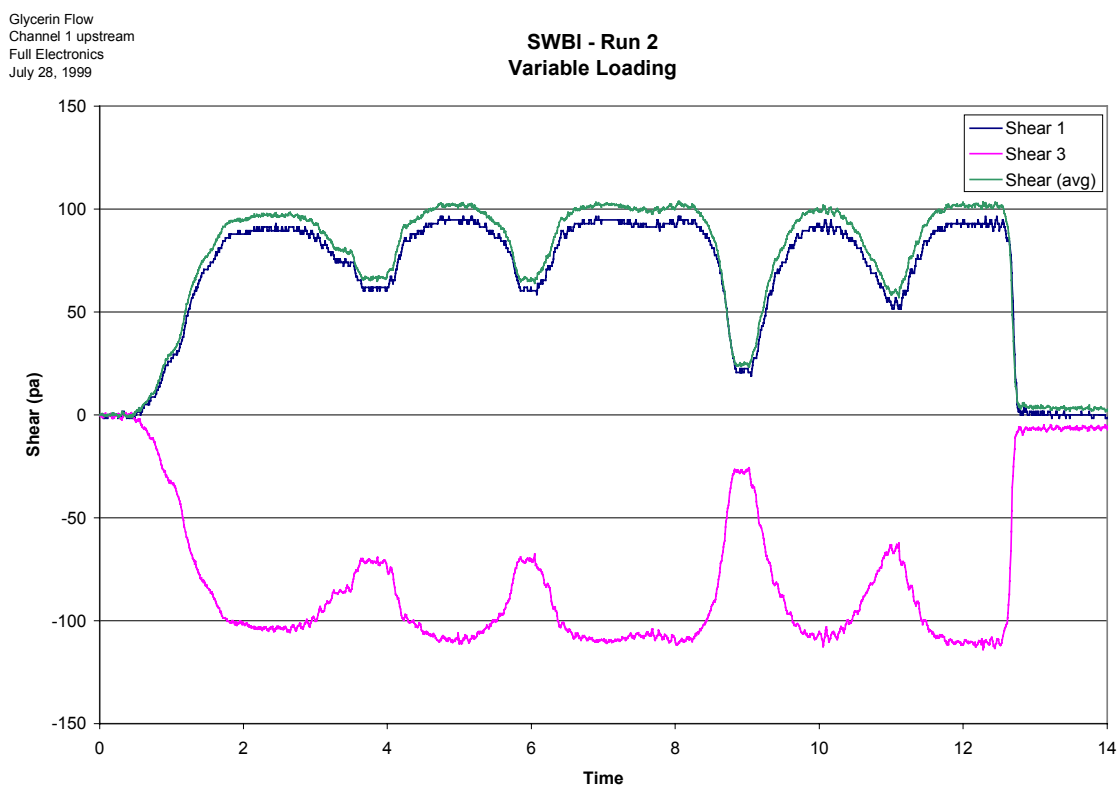

Figure 121. Test 3, variable loading test of the Version 4 skin friction sensor in a fully-developed, incompressible glycerin flow. 


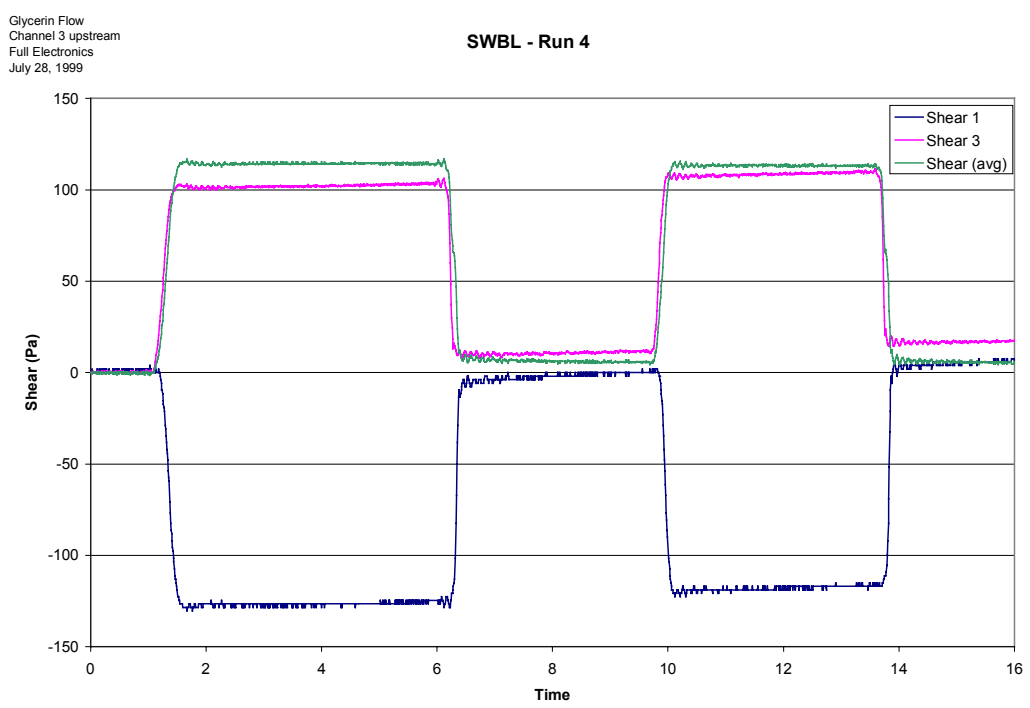

Figure 122. Test 5 of the Version 4 skin friction sensor in a fully-developed, incompressible glycerin flow in which the sensor has been rotated $180^{\circ}$.

\subsubsection{Version 5 skin friction sensor}

The fiber holders of the Version 4 sensor were made of stainless steel, the only tube material readily available. However, the material mismatch between the stainless steel and the Invar of the rest of the sensor led to a large temperature sensitivity. There was a concern that this large temperature sensitivity would present a problem. A redesign replaced the stainless steel tubing with Invar, which increased the temperature stability by a factor of 30 . The design of the center body remained the same, although we now took advantage of numerical machining processes which made thinner, straighter, and more repeatable beams possible. The numerical machining allowed a design gap movement of $350 \mathrm{~nm}$ at $300 \mathrm{~Pa}$, for the same $4.19 \mathrm{~mm}$ (0.165 in.) diameter sensing head, see the design space in Figure 123. It should be noted that with this sensor, the fact that the gages would be filled with glycerin was taken into account in the design equations. For any movement of the sensing head, and therefore the reflector, the physical gap is is multiplied by the index of refraction of the fluid in the optical gap, in this case 1.47, for the determination of the optical path length, as described in Chapter 3. This benefit is slight compensation for the temperature sensitivity that is inherent with the glycerin. The overall size is the same as that of the Version 4 sensor. The shear calibration of the 
Version 5 skin friction sensors are much more linear and consistent between channels than previous designs, due to the improved machining and increased experience with the construction. Two sensors of this version were made, one termed Version 5a and the other Version $5 \mathrm{~b}$. A detaailed mechinical drawing of this sensor is presented in pages 280-2 in Appendix C.

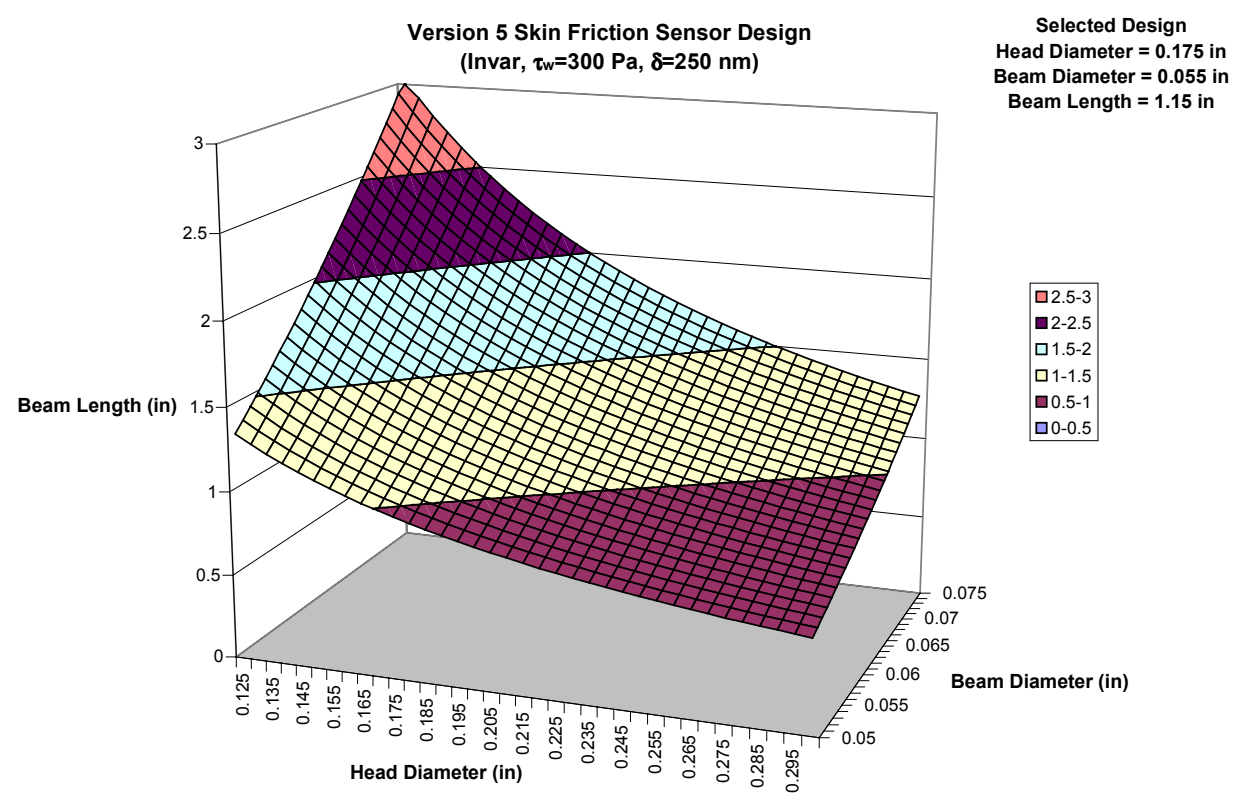

Figure 123. Design space of Version 5 skin friction sensor.

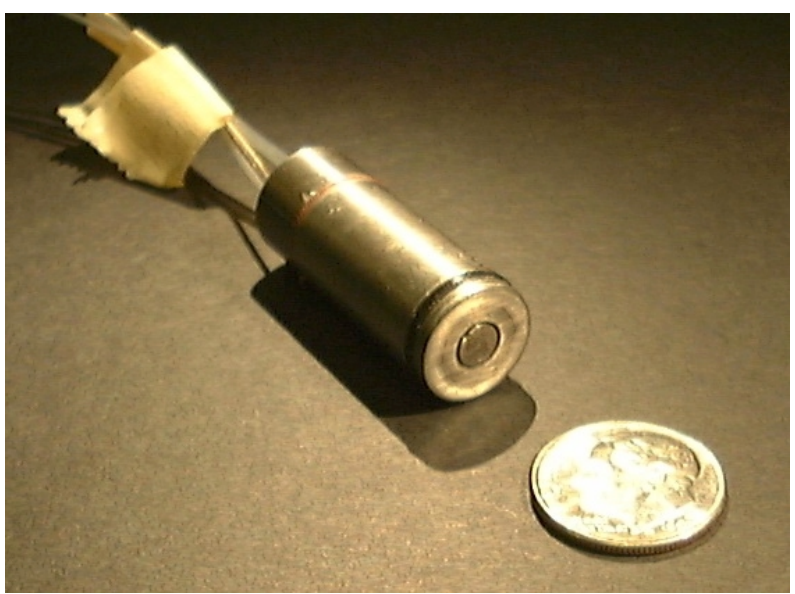

Figure 124. Photograph of the Version 5 skin friction sensor. 
Figure 125 and Figure 126 presents typical results from the Version 5a skin friction sensor in a Mach 2.4 flow at a total pressure of $345 \mathrm{kPa}$ (50 psia). The shape, including the return to zero and temperature stability, is excellent. The noise in the signal is also low, which is expected when one examines the small changes in the spectra. The results are approximately $40 \%$ high. It is not known why this is, but it was considered a promising result considering the past problems. Figure 127 presents data from another test of the same sensor, with similar results.

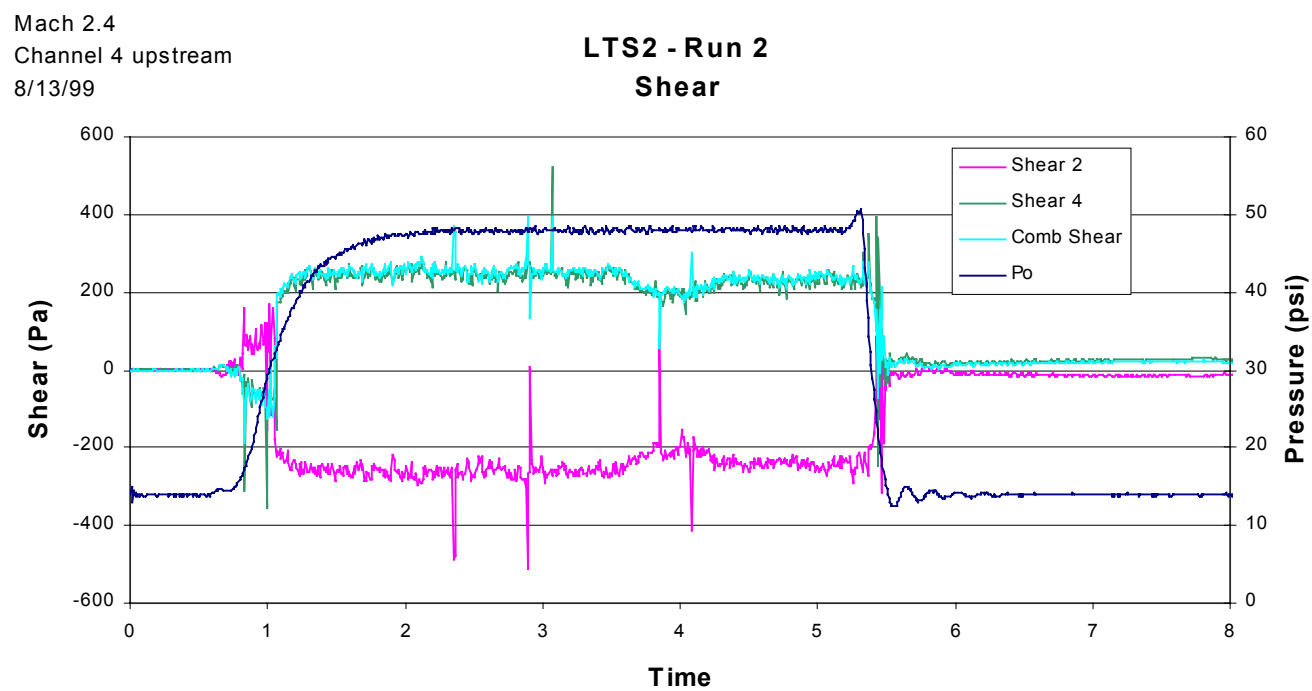

Figure 125. Test 3 shear results of the Version 5a skin friction sensor in Mach 2.4 flow at a total pressure of $345 \mathrm{kPa}$ (50 psi). 


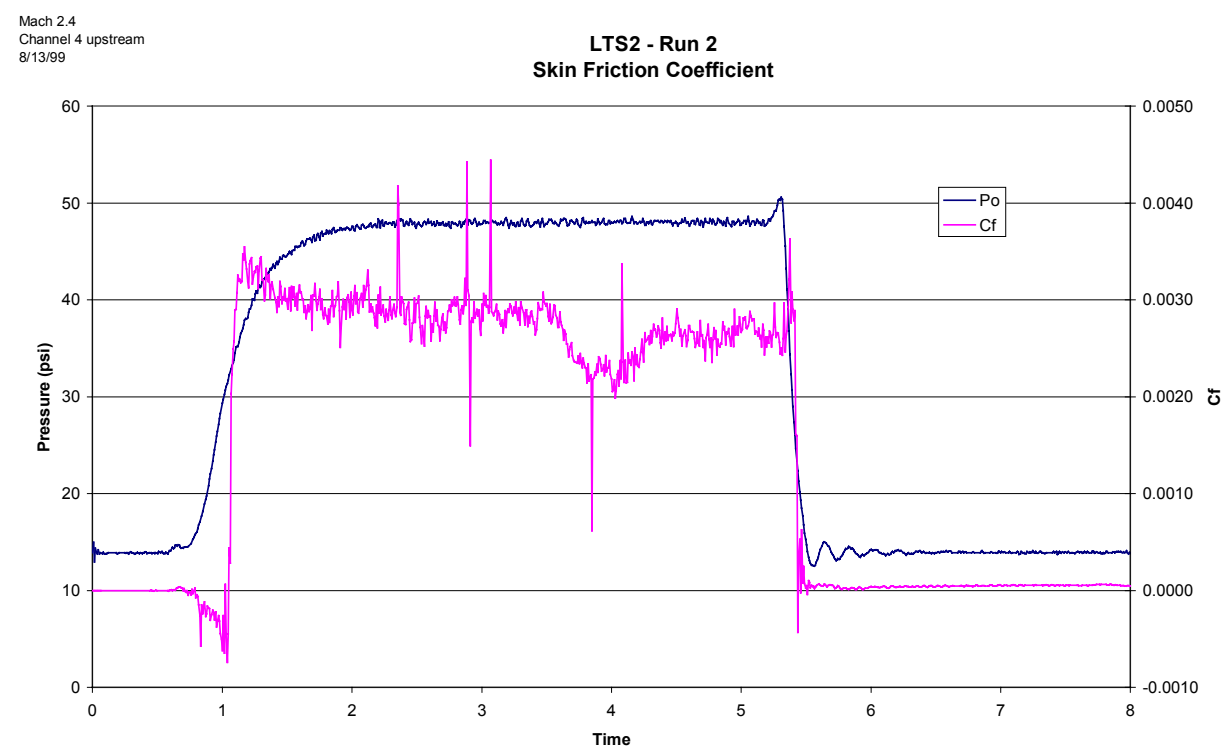

Figure 126. Test 3 skin friction coefficient results of the Version 5a skin friction sensor in Mach 2.4 flow at a total pressure of $345 \mathrm{kPa}$ (50 psi).

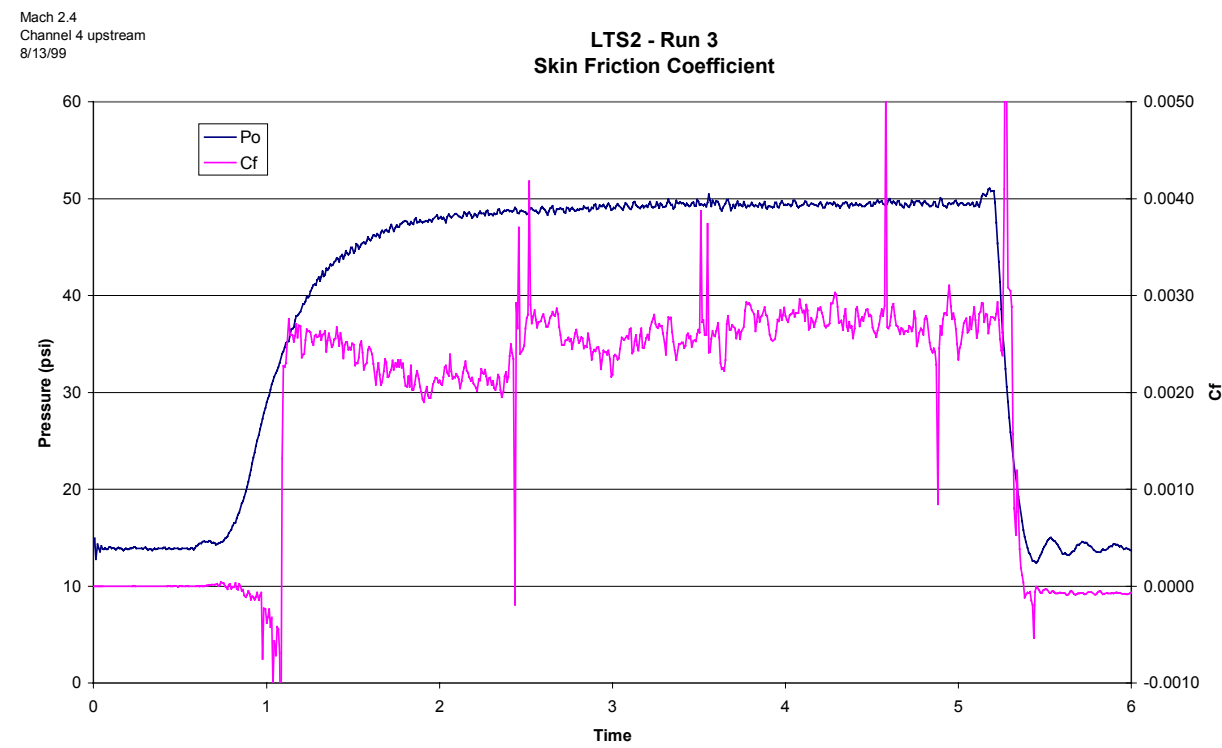

Figure 127. Test 4 skin friction coefficient results of the Version 5a skin friction sensor in Mach 2.4 flow at a total pressure of $345 \mathrm{kPa}$ (50 psi).

The same sensor was then tested in a Mach 3.0 flow at a total pressure of $520 \mathrm{kPa}$ (75 psia). Figure 128 through Figure 130 present results at this condition. The shape and magnitude of the results are both good for this flow. As one can observe, the noise of the 
shear results is much greater in this flow than the previous Mach 2.4 flow. It was hypothesized that the greater aerodynamic energy in the Mach 3.0 flow induced more vibration in the sensor, and that increased the noise.

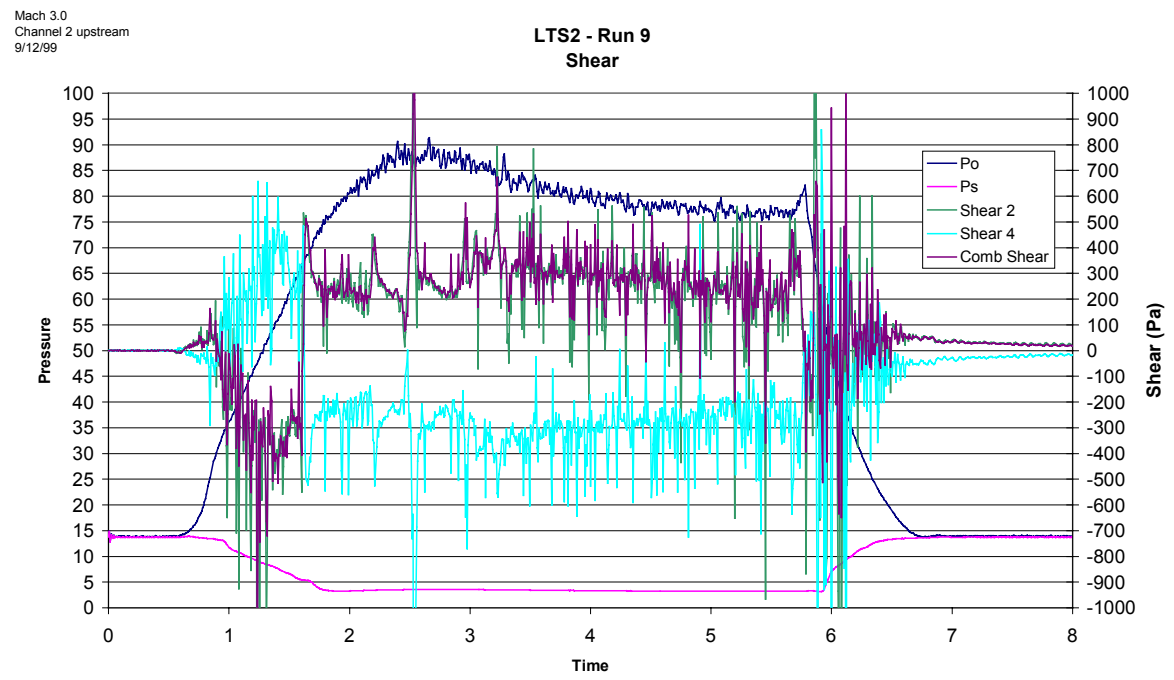

Figure 128. Test 10 shear results of the Version 5a skin friction sensor in a Mach 3.0 flow at a total pressure of $520 \mathrm{kPa}(75 \mathrm{psi})$.

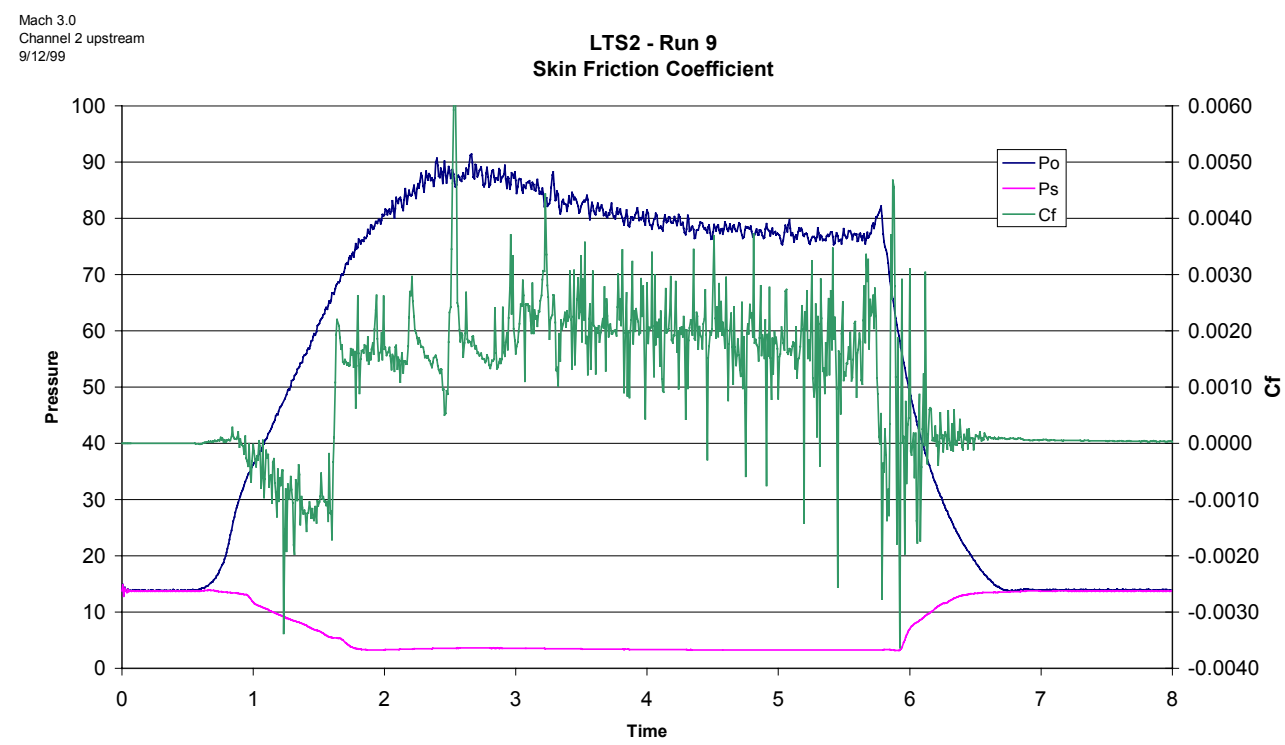

Figure 129. Test 10 skin friction coefficient results of the Version 5a skin friction sensor in a Mach 3.0 flow at a total pressure of $520 \mathrm{kPa}(75 \mathrm{psi})$. 


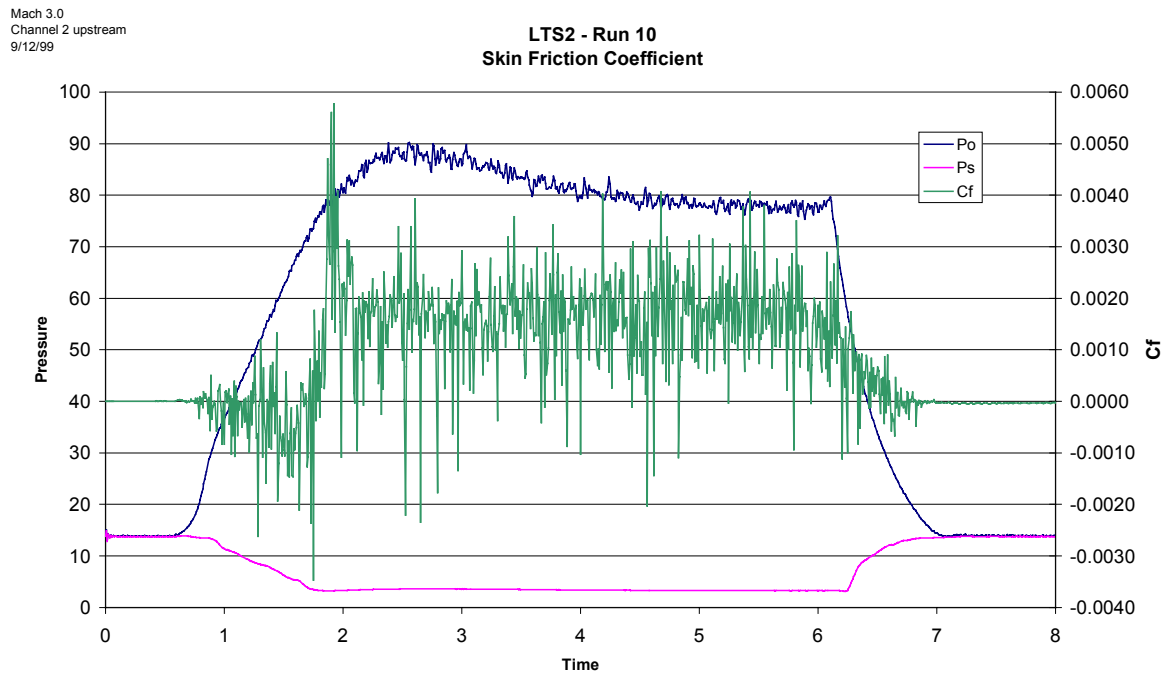

Figure 130. Test 11 skin friction coefficient results of the Version 5a skin friction sensor in low pressure, Mach 3.0 flow at a total pressure of $520 \mathrm{kPa}(75 \mathrm{psi})$.

Figure 131 presents results from a test in which one monitored channel was in the flow direction and one was in the cross-flow direction. This procedure can be used to determine the shear magnitude and direction on the tunnel floor. In this case, the shear is all along the axis of the tunnel due to the 2-D flow configuration, and this particular test was performed to demonstrate that the results would also be in that same direction. As one can see, the cross-flow channel remained at zero during the test, while the flow channel produced good results. These tests indicate that the Version 5a skin friction sensor performs well in this flow condition.

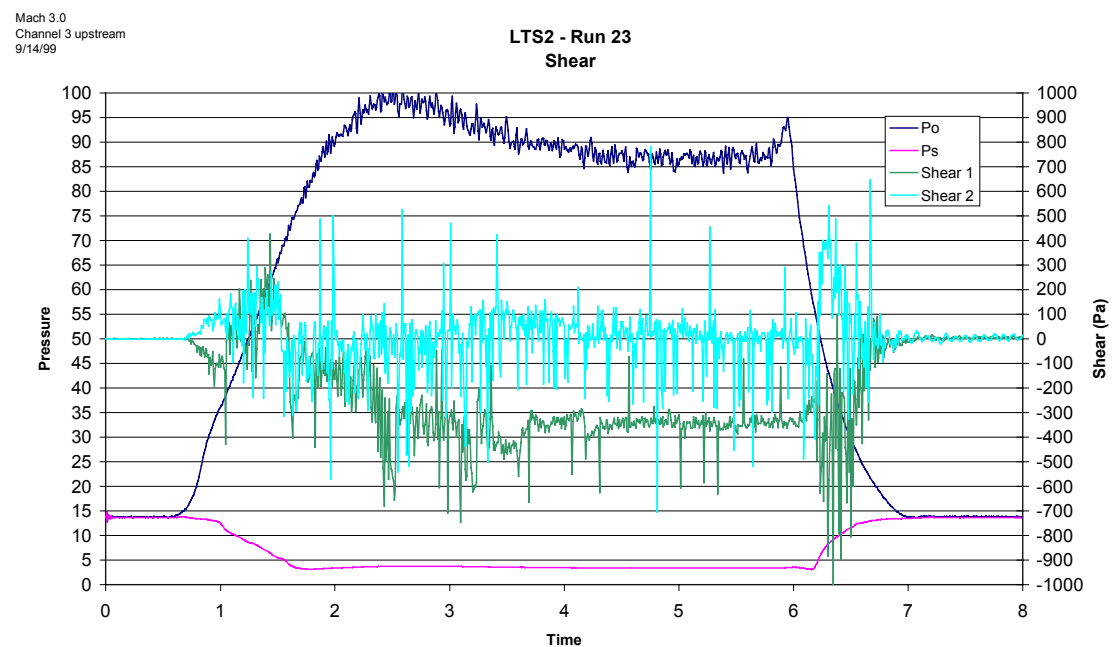

Figure 131. Test 24 cross-flow shear results of the Version 5a skin friction sensor in Mach 3.0 flow at a total pressure of $550 \mathrm{kPa}(80 \mathrm{psi})$. 
The Version 5a sensor was then tested in a Mach 3.0 flow at a total pressure of $690 \mathrm{kPa}(100 \mathrm{psia})$. The results are presented in Figure 132. The energy of this flow has now excited the sensor so much that spectral smearing ruins the results even though it was filled with glycerin as a damping fluid. These results indicated that the sensor has an aerodynamic input envelope in which it can effectively operate. Improved damping or electronics are necessary to expand this envelope.

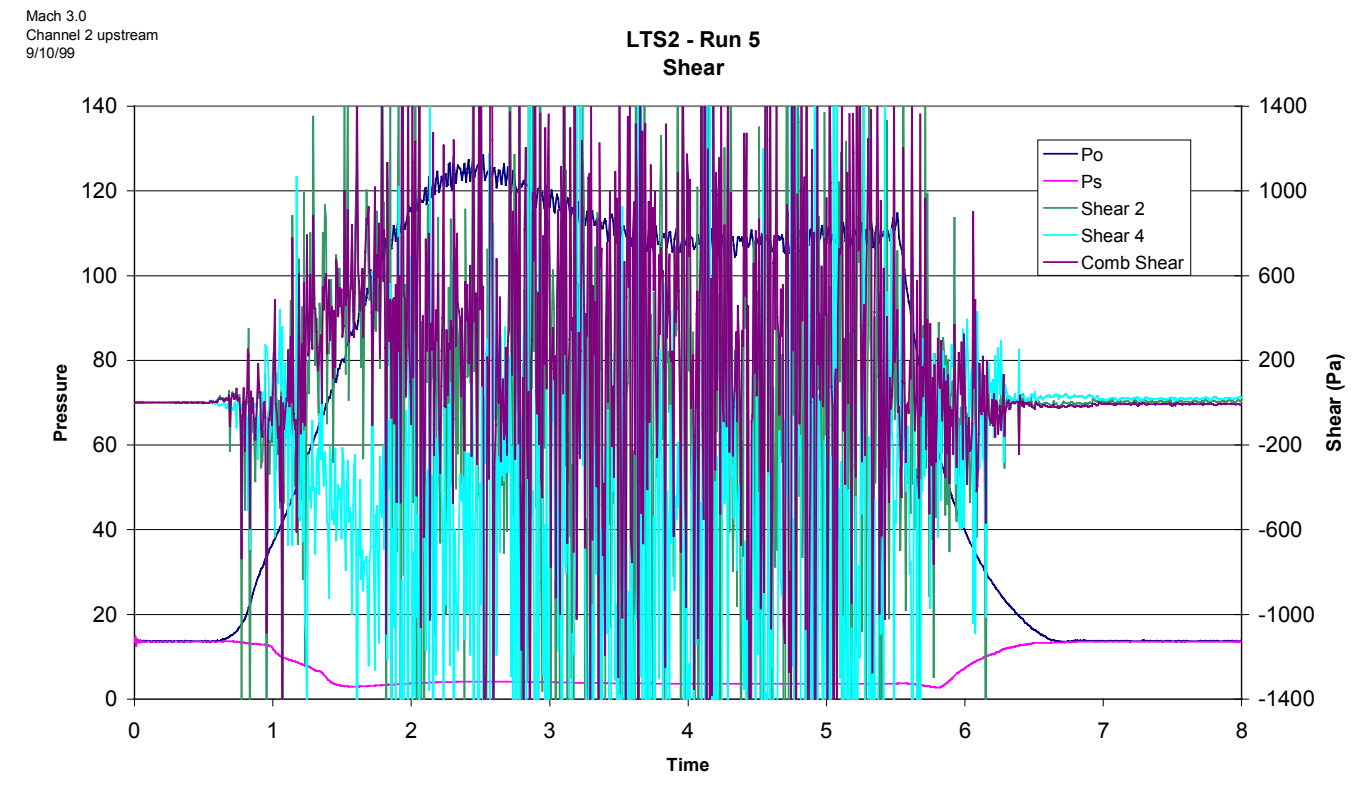

Figure 132. Test 6 of the Version 5a skin friction sensor in a Mach 3.0 flow at a total
pressure of $750 \mathrm{kPa}(110 \mathrm{psi})$.

Finally, this skin friction sensor was tested at the Air Force Research Laboratories (Wright-Patterson Air Force Base) in the Test Cell 19 facility. This tunnel was configured to produce a Mach 1.92 flow at a $345 \mathrm{kPa}(50 \mathrm{psi})$ total pressure. Calculations using the Schultz-Grunow correlation predict a skin friction coefficient of 0.0020 and, given the dynamic pressure, a shear of $300 \mathrm{~Pa}$. Figure 133 through Figure 136 present the results of these tests. The data acquisition of the first test ended prematurely, but the test was of correct shape and magnitude. The second test also produced good results, although there was a temperature drift during the longer than normal test. This drift could not be compensated by the normal method because data was only acquired on one channel during these tests. In all, the Version 5a skin friction sensor produced results for 
this low-energy flow (a lower energy supersonic flow than can be produced in the Virginia Tech supersonic wind tunnel).

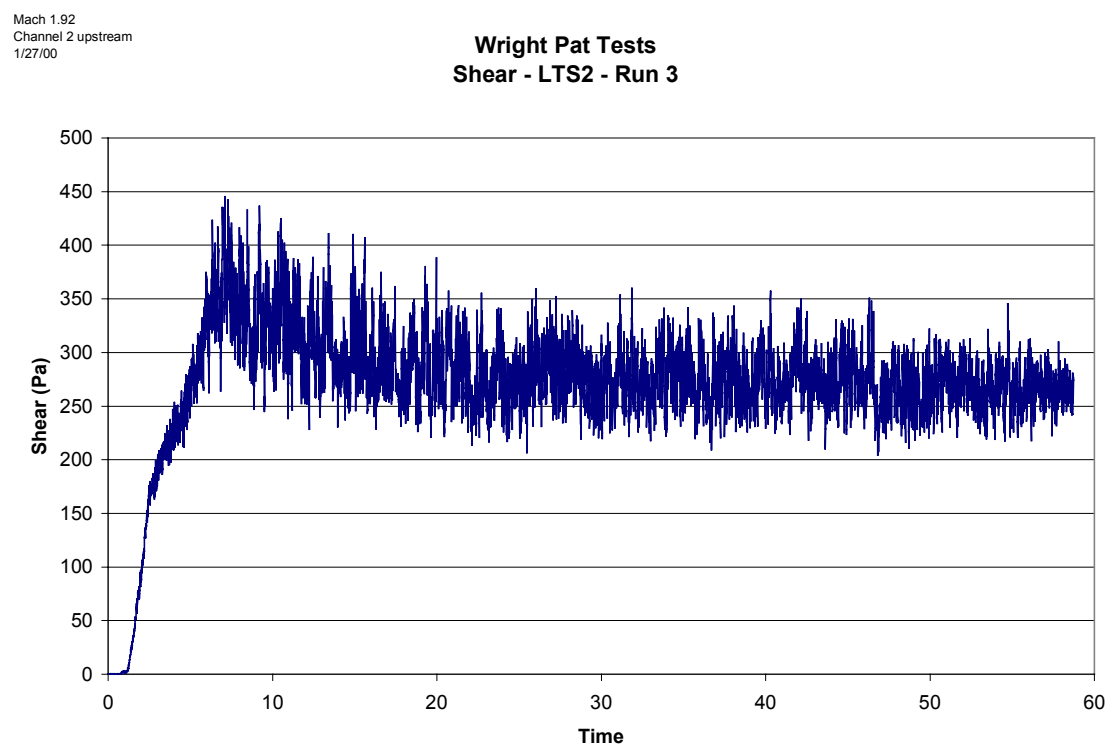

Figure 133. Test 3 shear results of the Version 5a skin friction sensor at Wright Pat in Mach 1.92 flow at a total pressure of $345 \mathrm{kPa}(50 \mathrm{psi})$.
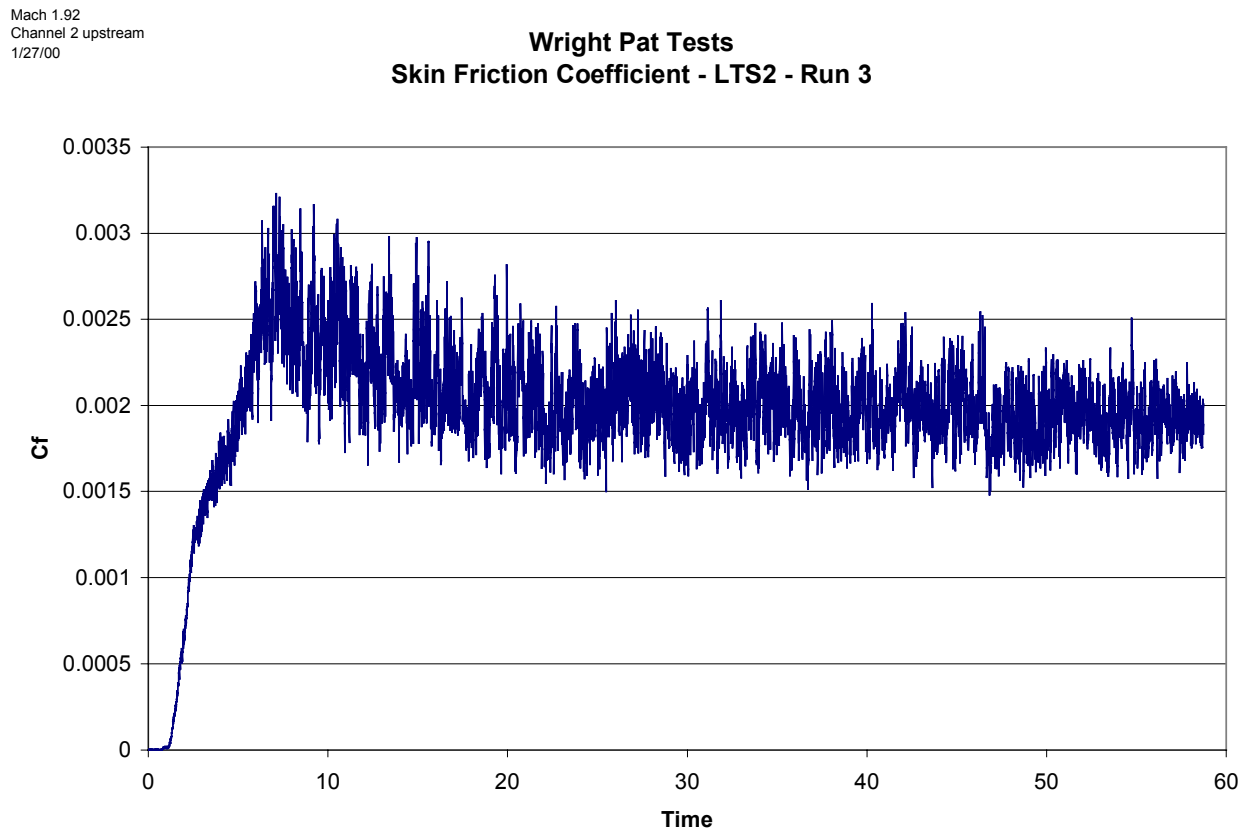

Figure 134. Test 3 skin friction coefficient results of the Version 5a skin friction sensor at Wright Pat in Mach 1.92 flow at a total pressure of $345 \mathrm{kPa}(50 \mathrm{psi})$. 


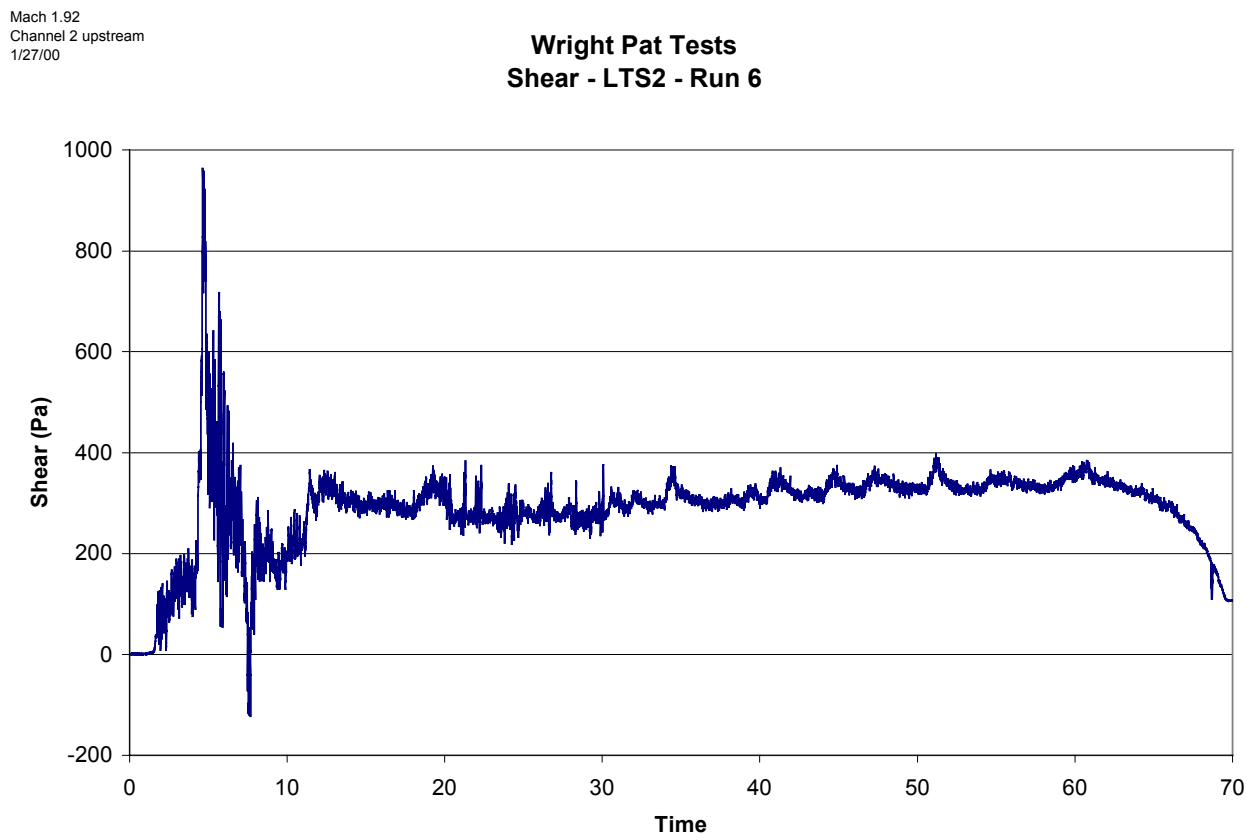

Figure 135. Test 6 shear results of the Version 5a skin friction sensor at Wright Pat in Mach 1.92 flow at a total pressure of $345 \mathrm{kPa}(50 \mathrm{psi})$.

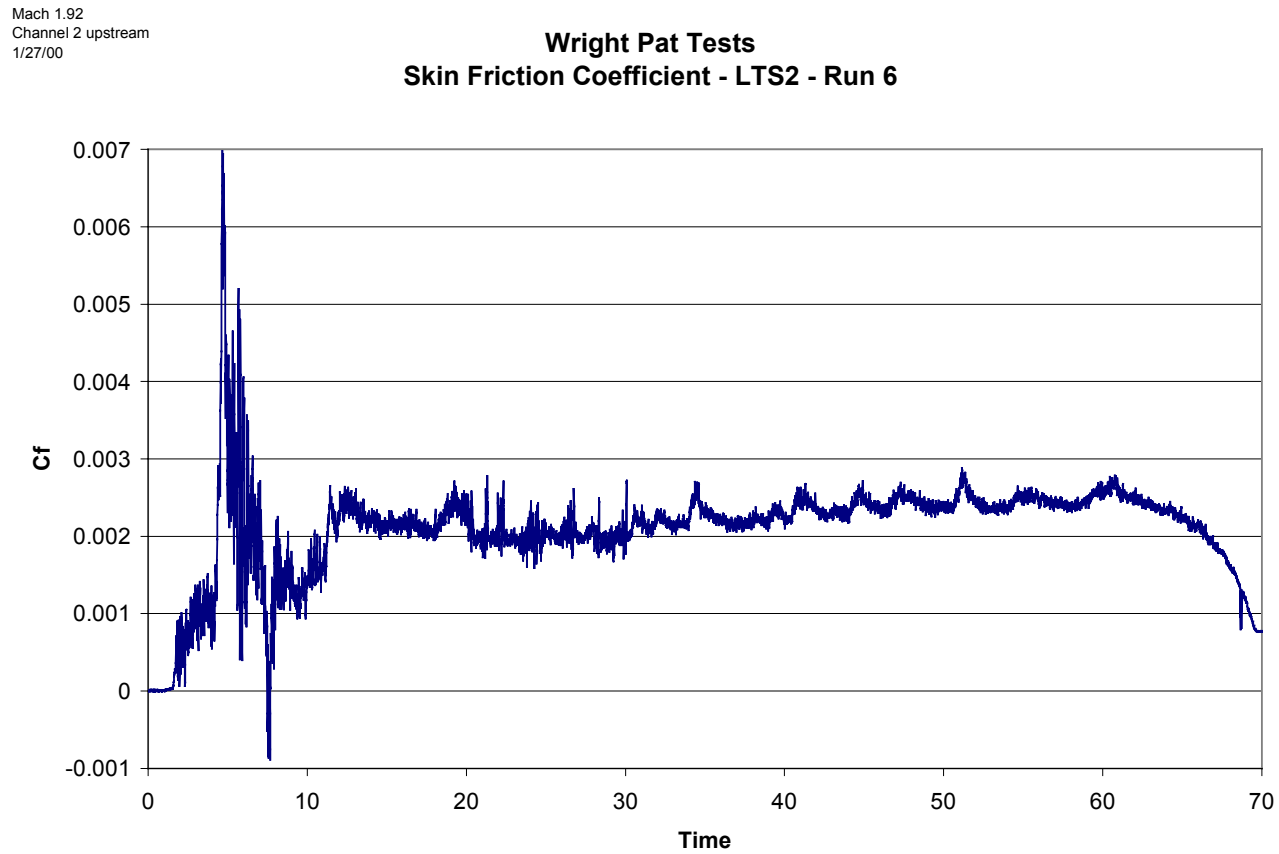

Figure 136. Test 6 skin friction coefficient results of the Version 5a skin friction sensor at Wright Pat in Mach 1.92 flow at a total pressure of $345 \mathrm{kPa}(50 \mathrm{psi})$. 
The Version 5b skin friction sensor was then tested in the Mach 3.0 flow at a total pressure of $550 \mathrm{kPa}$ (80 psia), and these results are shown in Figure 137. This condition produced excellent results with the previous sensor, but although the shape is positive for this sensor, the results are high in shear magnitude. The high results were due to unknown reasons, but pressure and temperature effects were suspected.

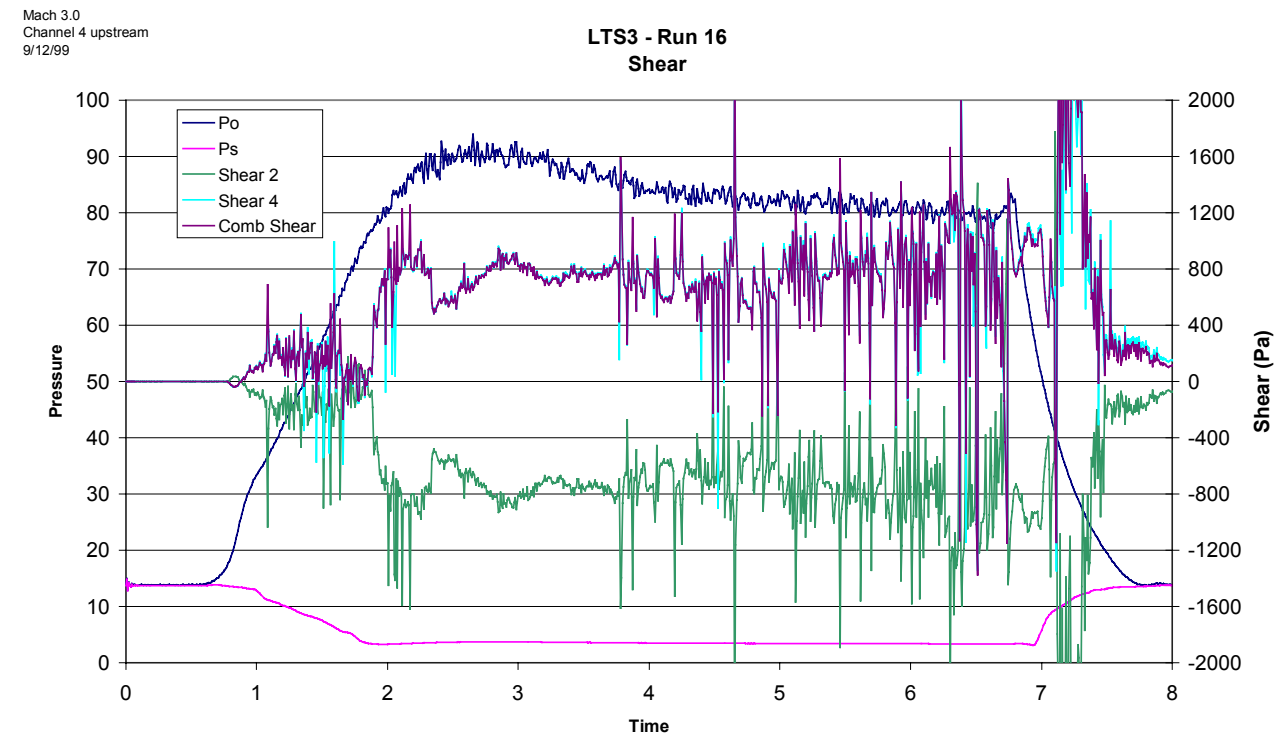

Figure 137. Test 17 shear results of the Version $5 \mathrm{~b}$ skin friction sensor in Mach 3.0 flow at a total pressure of $550 \mathrm{kPa}(80 \mathrm{psi})$.

\subsubsection{Version 6 skin friction sensor}

The results of the previous sensor, the work performed examining the effects of the glycerin in the optical path, and concerns about holding the pieces of the sensor to nanometer tolerances, all indicated that making a sensor with a larger designed optical gap movement would be advantageous. The various spurious effects would be minimized in comparison to the larger movement. The Version 6 sensor was designed (see Figure 138) for a gap movement of $3500 \mathrm{~nm}$ for a $300 \mathrm{~Pa}$ shear. This increase was accomplished by increasing the head diameter to $9 \mathrm{~mm}$ (0.354 in.) as well as increasing the length of the sensor. The completed sensor can be seen in Figure 139. The overall 
size for this sensor was $12.7 \mathrm{~mm}(1 / 2 \mathrm{in}$.) by $47 \mathrm{~mm}$ (1.85 in.). A detaailed mechinical drawing of this sensor is presented in pages 283-4 in Appendix C.

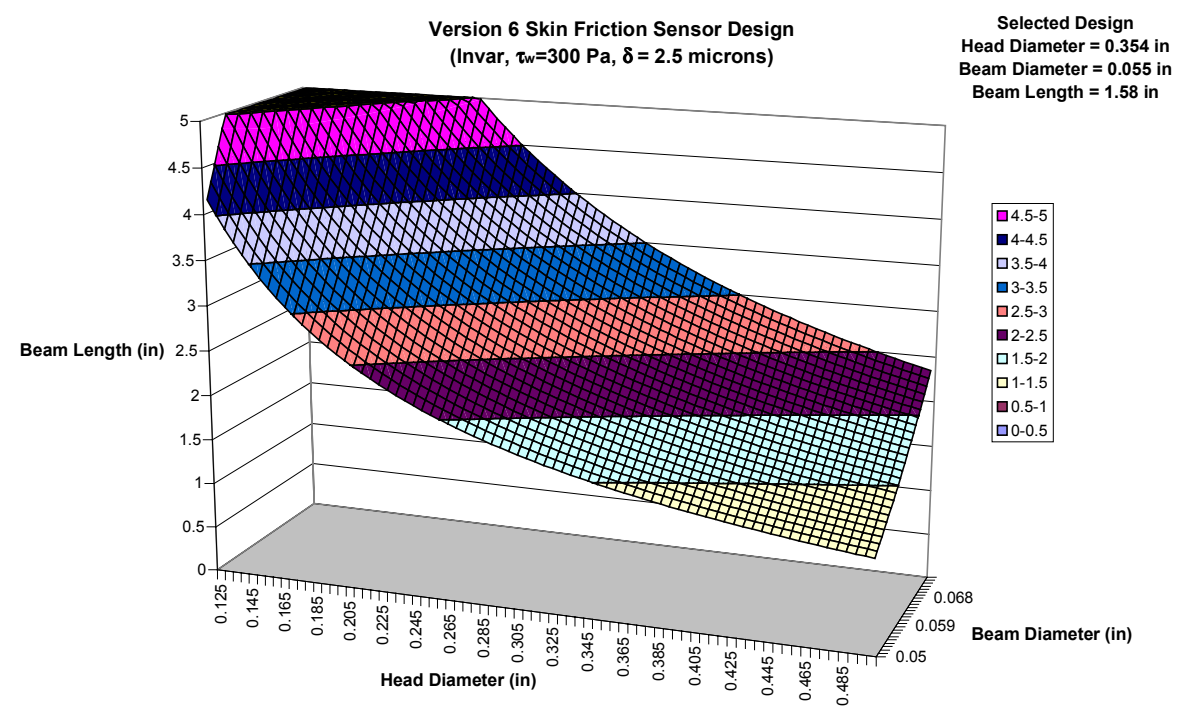

Figure 138. Design space of Version 6 skin friction sensor.

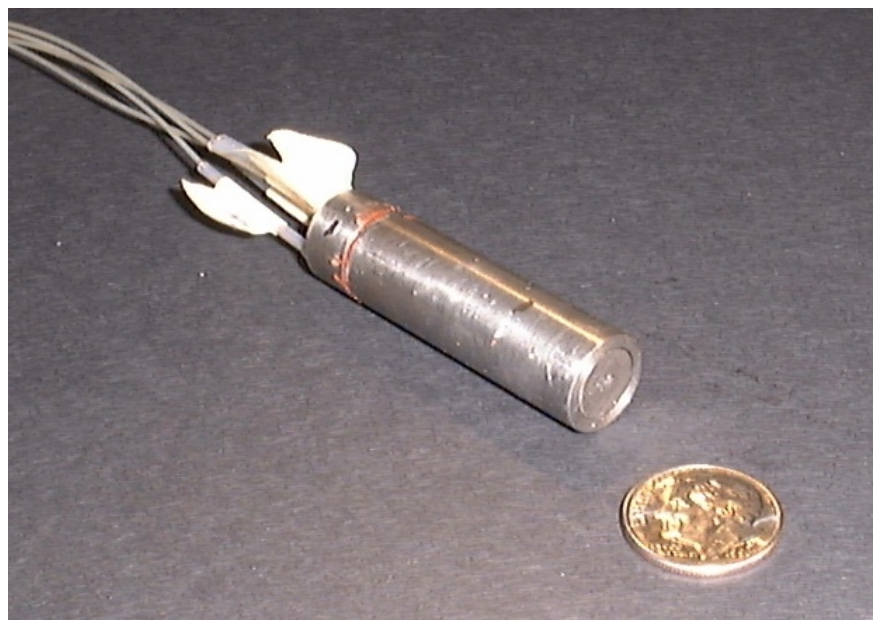

Figure 139. Photograph of the Version 6 skin friction sensor.

Results with this Version 6 skin friction sensor are presented in Figure 140 and Figure 141 for a Mach 3.0 flow at $690 \mathrm{kPa}(100 \mathrm{psia})$. As can be seen, the results have much lower noise. The large gap movement is partly responsible for that, and it has been hypothesized that the lower natural frequency of the sensor might make it easier to damp. The results show a good shape, but they are in the wrong direction and about 50\% low. 
Examination of the sensor showed a small internal leak, which can upset the results of any sensor. The results were presented here to show the low noise that could be obtained at this flow condition with just a change in the sensor design, indicating that a skin friction sensor can be designed to work in any flow condition.

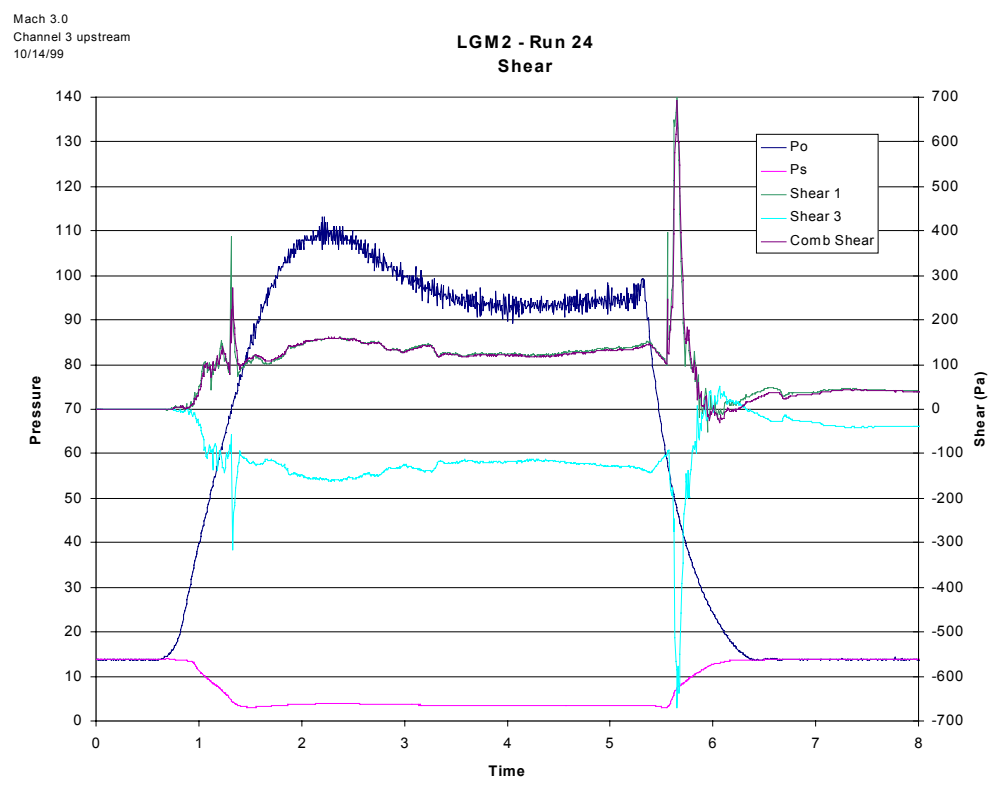

Figure 140. Test 25 shear results of the Version 6 skin friction sensor in Mach 3.0 flow at a total pressure of $690 \mathrm{kPa}(100 \mathrm{psi})$.

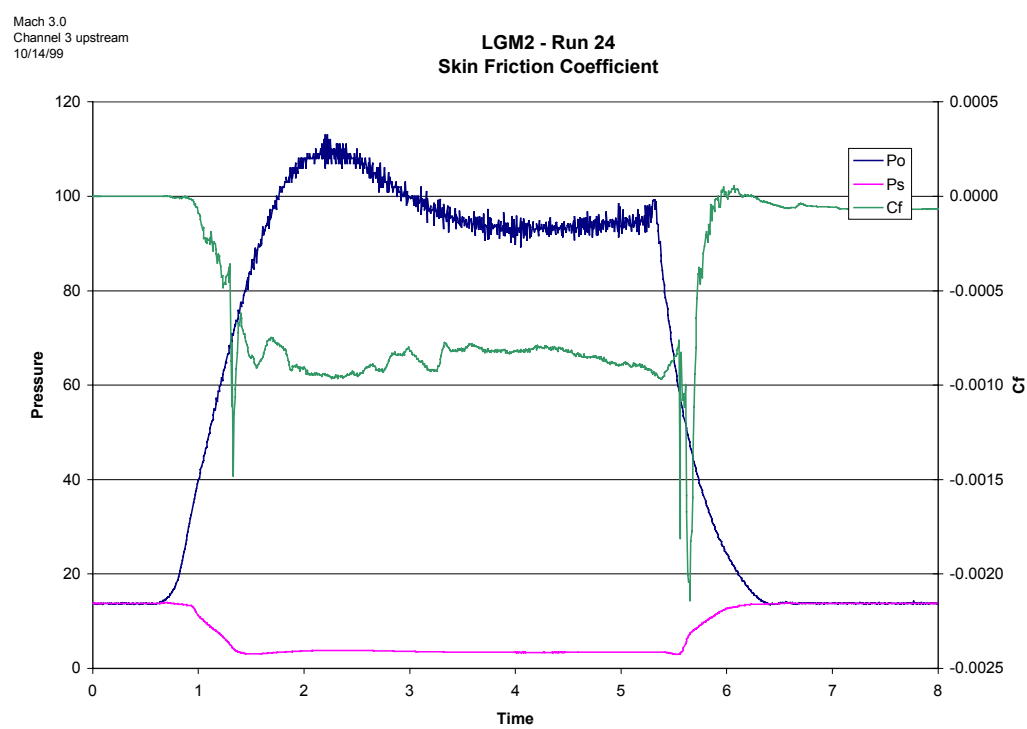

Figure 141. Test 25 skin friction coefficient results of the Version 6 skin friction sensor in high pressure, Mach 3.0 flow at a total pressure of $690 \mathrm{kPa}$ (100 psi). 
The resealed sensor was then testing in a Mach 2.4 flow at a total pressure of 240 $\mathrm{kPa}$ (35 psia). Figure 142 through Figure 145 present results from those tests. The sensor provided good shapes and skin friction values for this flow condition.

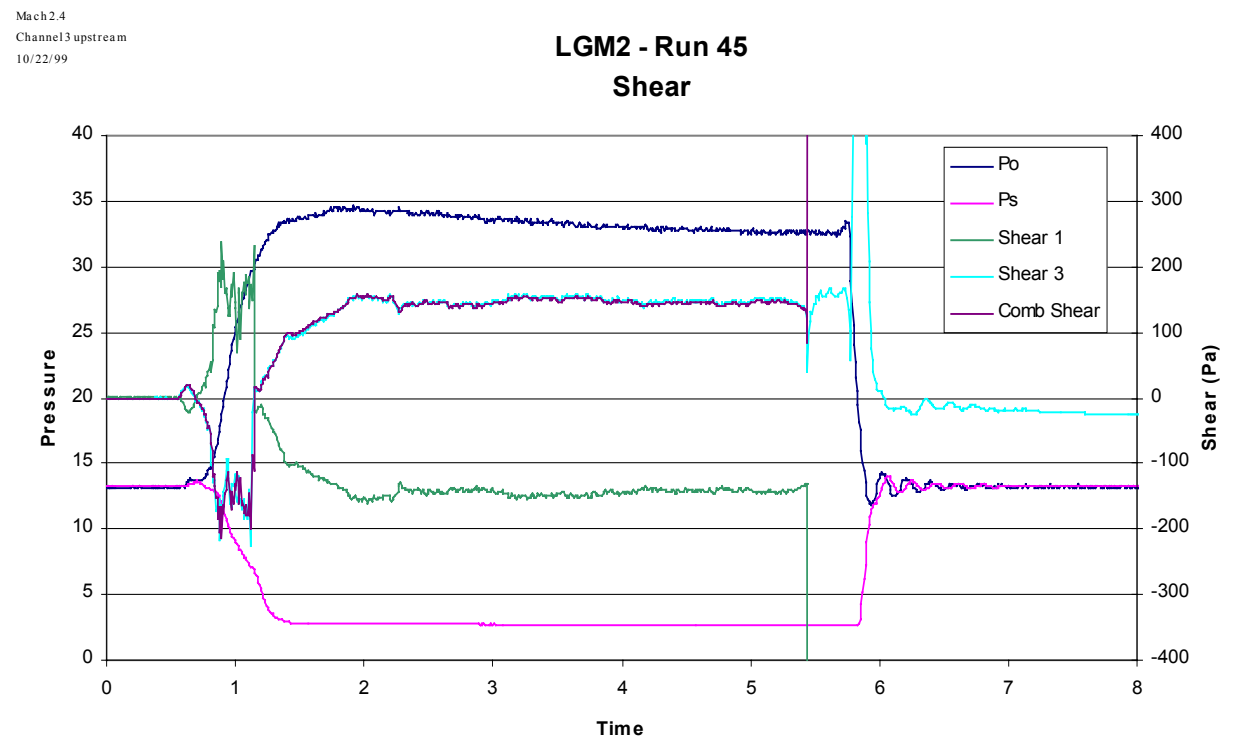

Figure 142. Test 46 shear results of the Version 6 skin friction results in Mach 2.4 flow at a total pressure of $240 \mathrm{kPa}(35 \mathrm{psi})$.

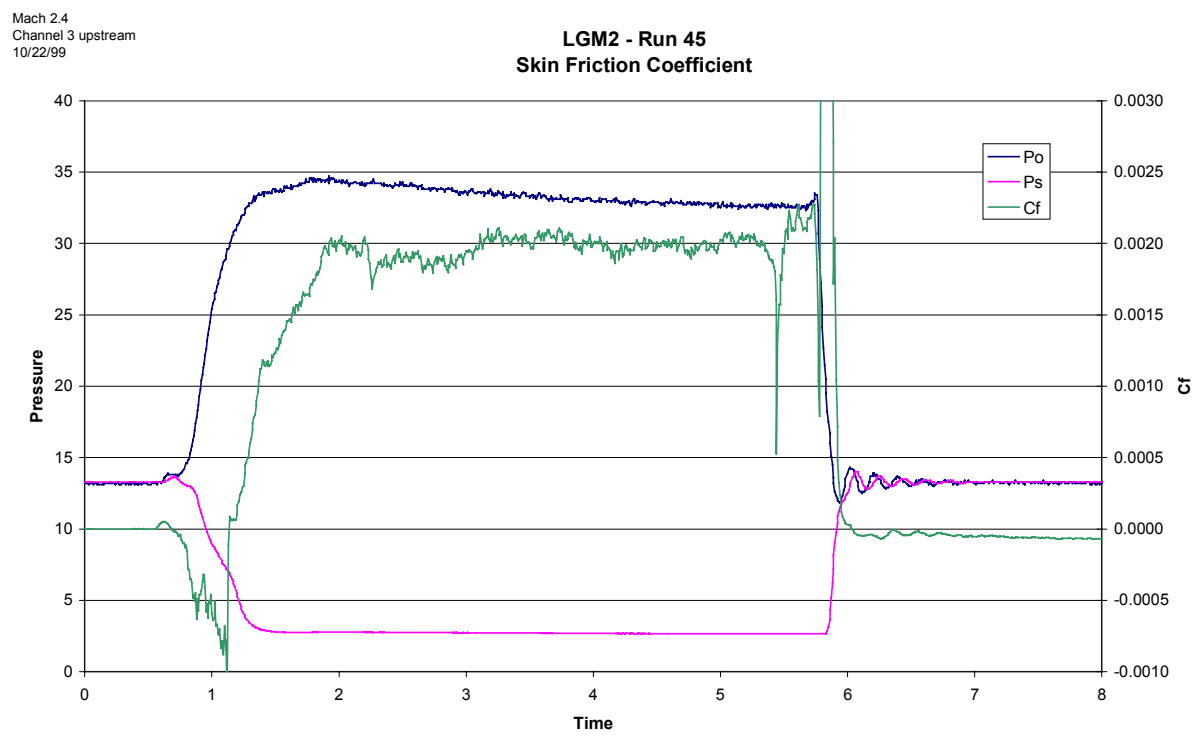

Figure 143. Test 46 skin friction coefficient results of the Version 6 skin friction results in Mach 2.4 flow at a total pressure of $240 \mathrm{kPa}(35 \mathrm{psi})$. 


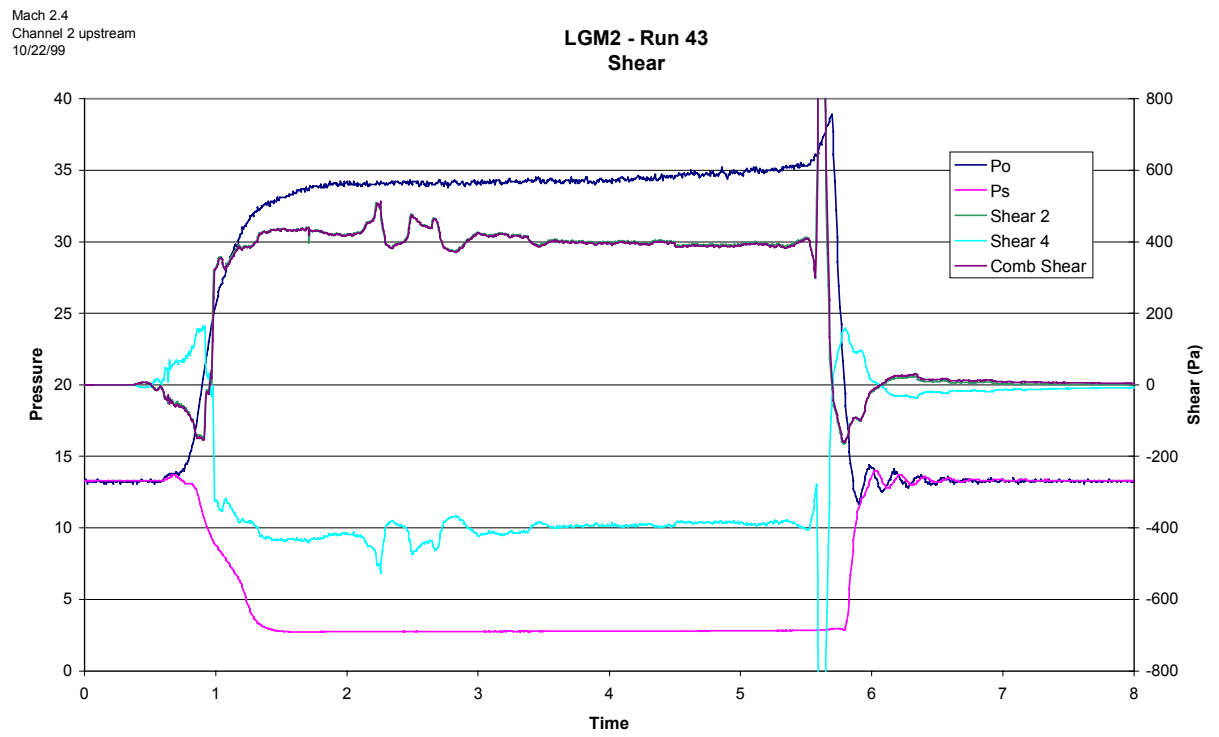

Figure 144. Test 44 shear results of the Version 6 skin friction sensor in Mach 2.4 flow at a total pressure of $240 \mathrm{kPa}(35 \mathrm{psi})$.

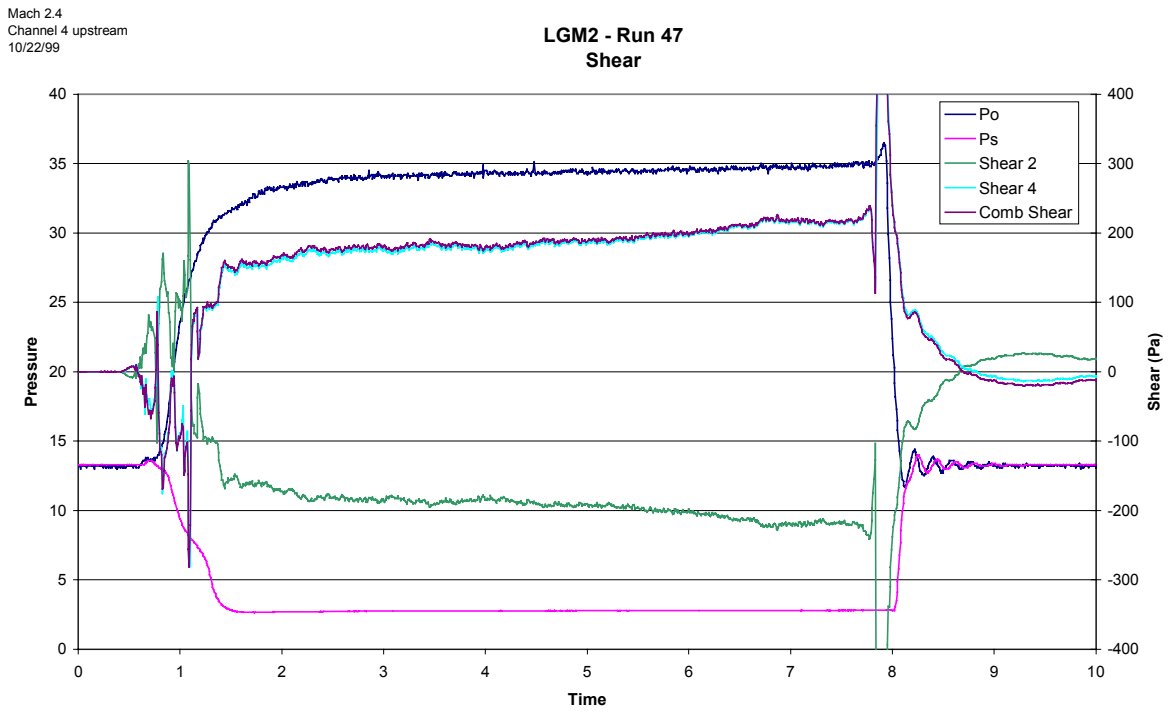

Figure 145. Test 48 shear results of the Version 6 skin friction sensor in Mach 2.4 flow at a total pressure of $240 \mathrm{kPa}(35 \mathrm{psi})$.

\subsubsection{Conclusions}

A series of conventionally machined fiber optic, skin friction sensors were constructed to verify design concepts. It was found that much better fabrication 
techniques were necessary for these fiber optic sensors than had previously been used in other skin friction gages. Also, increasing the design gap movement of the sensor improved results. Finally, the quality of the results depends on the damping of the cantilever beam in the sensor and the input aerodynamic noise. The more input noise or the lower the damping, the worse the results. A summary of the fiber optic, skin friction sensors is provided in Table 9.

Table 9. Summary of Skin Friction Sensor Versions.

\begin{tabular}{|c|c|c|c|c|}
\hline Version & $\begin{array}{c}\text { Design } \\
\text { Movement } \\
{[300 \mathrm{~Pa}](\mathrm{nm})} \\
\end{array}$ & $\begin{array}{c}\text { Overall Size } \\
\text { [Head Diameter] } \\
(\mathrm{mm})\end{array}$ & Performance & What was learned \\
\hline 1 & N/A & {$[6.35]$} & Pressure sensitive & Need open gap \\
\hline 2 & 100 & $\begin{array}{c}9.525 \times 31.75 \\
{[6.35]} \\
\end{array}$ & Worked well & \\
\hline 3 & 150 & $\begin{array}{c}25.4 \times 31.75 \\
{[6.35]}\end{array}$ & Did not work & Needed slip fits and damping \\
\hline 4 & 150 & $\begin{array}{c}12.7 \times 38.1 \\
{[4.19]}\end{array}$ & Worked in simple flows & Can work for some flows \\
\hline 5 & 350 & $\begin{array}{c}12.7 \times 38.1 \\
{[4.19]}\end{array}$ & Worked well & $\begin{array}{c}\text { Needed numerical machining, } \\
\text { will work for some complex } \\
\text { flows }\end{array}$ \\
\hline 6 & 3500 & $\begin{array}{c}12.7 \times 47.0 \\
{[9.0]}\end{array}$ & Low signal noise & $\begin{array}{c}\text { Sensor can be designed for } \\
\text { any flow }\end{array}$ \\
\hline
\end{tabular}

In summary, the measurement of skin friction with an interferometric fiber optic design is possible, but it limited by vibration caused by aerodynamic input. For the very benign flow of the calibration rig, the sensor design works flawlessly. If the sensor was not damped, then no results are possible with the current spectral interrogation signal processing system. For a damped sensor, the results indicate that manufacturing and sealing issues are very important for accurate results. Also, depending on the design, increasing aerodynamic energy levels will cause the sensor to produce more noise, until the results are no longer valid. This conclusion can be seen by examining the results of Version 5a skin friction sensor for the Mach 1.92 flow, Mach 2.4, Mach 3.0 at $520 \mathrm{kPa}$ (75 psia), and Mach 3.0 at $750 \mathrm{kPa}$ (110 psia). Improved fabrication, packaging, and damping of the sensor and improved signal processing systems will allow this sensor design to operate in any flow condition and fulfill the promise of the fiber optic design. 


\subsection{Chapter 5}

\section{Total System Limitations Study}

\subsection{Introduction}

The previous chapter described the signal processing systems and the sensors, as well as the results obtained during testing in various conditions. The sensors demonstrated various difficulties, mostly noise in the output signal, with making measurements in these flows. The sensors and signal processing unit comprise a total system where problems of one impose limits on the other. An experimental and numerical study was initiated to determine these limitations imposed by both the sensor and system.

\subsection{Numerical and Experimental Study Total System Limitations}

Due to some of the testing data presented in Chapter 4, a systematic investigation of error sources and limitations of the sensors and optical signal processing system was undertaken. This study involved bench-top tests of the sensors and electronics, experiments to investigate possible error sources in the electronics, and computer simulations of the signal processing system. The work demonstrates the importance of what is termed "fringe contrast" in the errors of the total system. In addition, the computer simulations of the fiber optic pressure and skin friction sensors demonstrate the characteristics of the same sensors in the wind tunnel.

\subsubsection{Fringe Contrast}

In real terms, fringe contrast is an indication of the deepness of the interference pattern. The fringe contrast is the intensity difference of the peaks of the upper and lower envelopes of the interference pattern. This difference is normalized to allow the comparison of signals with different power, as defined in Figure 146, where I is the intensity. A large fringe contrast occurs when the powers of two reflections that define the interference pattern of the EFPI are nearly the same. If these powers begin to differ 
significantly, the fringe contrast will decrease. As the entire optical gap information is in the interference pattern, any reduction in the ability to see this pattern clearly reduces the ability to accurately determine this gap. In signal processing terms, the pedestal, which is the lower envelope of the fringe pattern, becomes a large portion of the total signal. This lower frequency signal begins to dominate and "leak" into the higher frequency signal, which is the interference pattern. Alternatively, the higher frequency signal lacks the coherence necessary to distinguish itself from the pedestal information. This effect can be seen in the FFT of the fringe pattern as described in Chapter 2. As the fringe contrast decreases, the magnitude of the peaks in the FFT decrease until the peak searching routine fails.

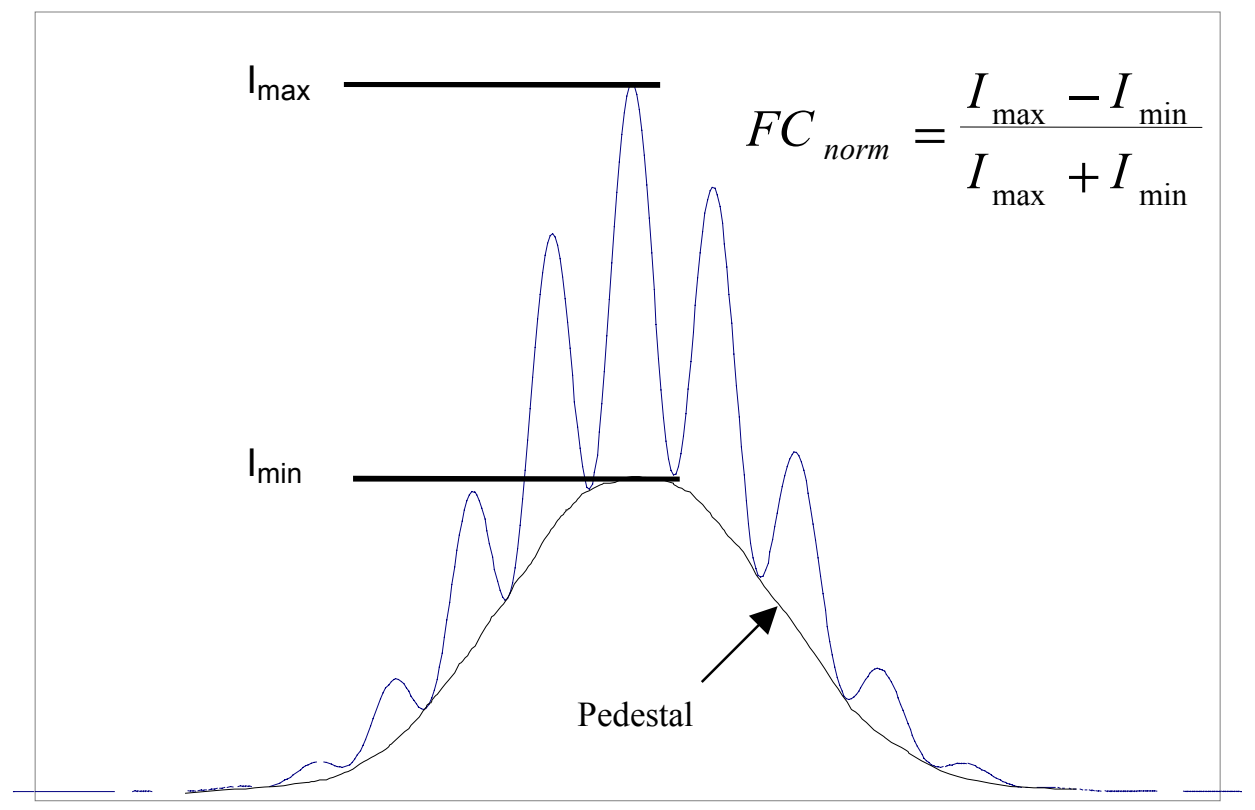

Figure 146. Explanation of fringe contrast.

\subsubsection{Error Sources in Spectral Interrogation Signal Processing System}

As with any electronic system, the Luna spectral interrogation signal processing system has internal noise sources that contribute an error to the output signal. As identified by previous work at Luna, the major system errors are an inexact knowledge of what wavelength is on each pixel in the CCD array of the spectrometer, variations in the LED light source known as source ripple, and noise in the analog/digital converter. ${ }^{96}$ 
Each error source was investigated here through a MatLab system simulation of the spectral interrogation system.

The first error source studied was pixel shift. The spectrometer in the signal processing system uses a diffraction grating to split the component wavelengths of the optical signal along a linear CCD array. This array is carefully calibrated to determine what wavelength strikes which array pixel. However, if something happens to misadjust the spectrometer, this calibration is no longer valid because the correct wavelength does not correspond with the right pixel and any measurement using this calibration would be in error. Two pixel shifting scenarios were examined, a constant pixel shift, where the whole array is moved as a unit and a random shift from pixel to pixel. These shifts correspond to thermal effects on the spectrometer body or imperfection of the diffraction grating. The magnitude of the shift is expressed in terms of number of pixels, with this study ranging from 0 to 1 pixel (the shift magnitude is the standard deviation of the normally distributed shift for the random study). The normally expected value for a wellcalibrated spectrometer is 0.025 pixels.

Figure 147 presents the results of the pixel shift study. As with the entire study, the calculations are performed for both 50 and $100 \mu \mathrm{m}$, the nominal optical gaps used for the pressure and skin friction sensors, respectively. However, none of the error source simulations demonstrated any systematically larger error for one gap than for the other. Neither shifting method produced an appreciable error in the expected range of 0.025 pixels. In fact, the constant pixel shift created little error in comparison to the random shift even at the extreme shift. The random shift produced large errors, but only at the unlikely high shift values. The difference in the magnitude of the shifting types can be explained in two ways. First, the random shift had a magnitude expressed as a standard deviation, meaning that shifts much larger than 1 pixel could occur in that normal distribution. Second, the constant shift only slightly moved the whole spectrum, with the wavelengths still in order. The random shift mixes the wavelengths, producing a much "noisier" spectrum. This noisy spectrum produces large errors at the higher values. 


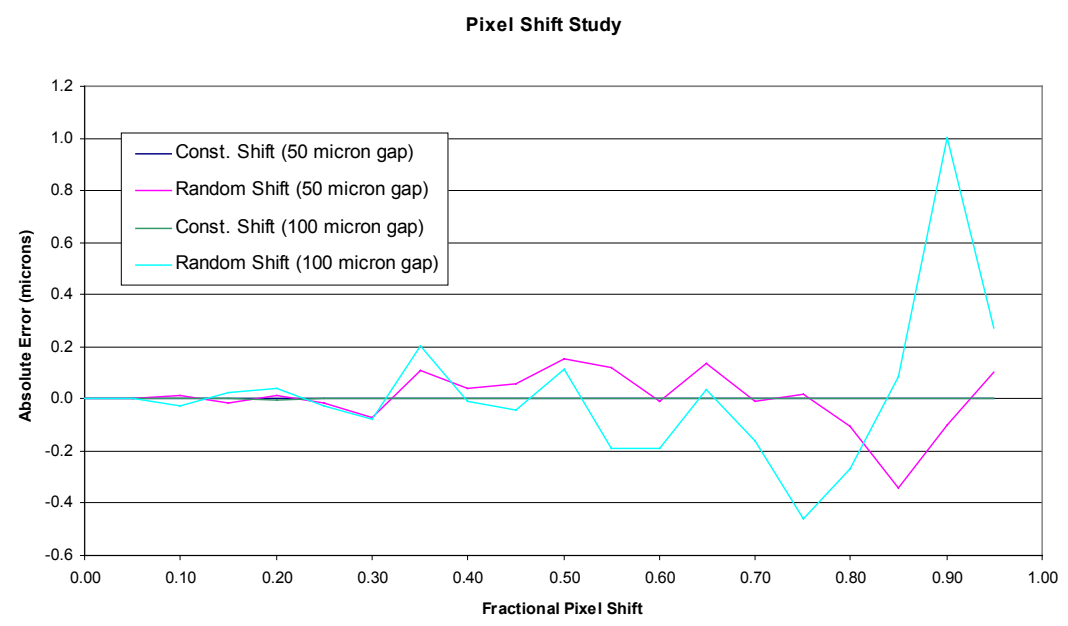

Figure 147. Calculation of absolute error due to pixel shift for random and constant pixel shifts at 50 and $100 \mu \mathrm{m}$ for the spectral interrogation signal processing system.

The second error source investigated is noise in the A/D converter. This error source was simulated by imposing a random noise on the spectrum signal, the standard deviation of which is the number of $A / D$ states to add to the signal. The range of interest was from $1 \mathrm{~A} / \mathrm{D}$ state to 10 (using a 12-bit $\mathrm{A} / \mathrm{D}$ converter). Figure 148 presents the results of the calculation. The absolute error increases along with the noise level in an unbiased manner to over $30 \mathrm{~nm}$. However, for a reasonable noise level of $1 \mathrm{~A} / \mathrm{D}$ state, the error remains at only a few nanometers.

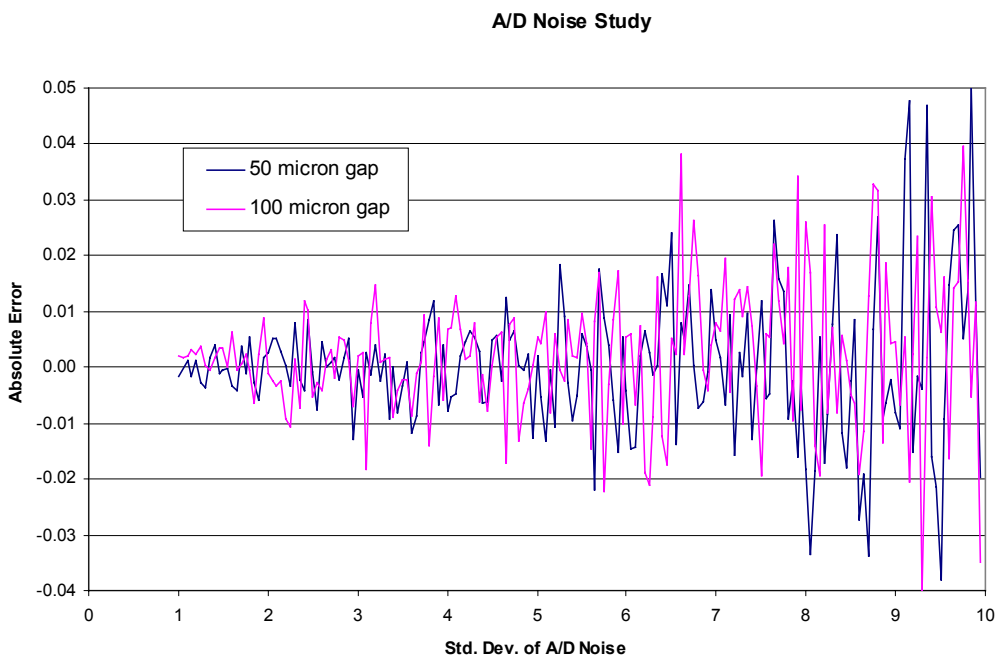

Figure 148. A/D noise absolute error calculation for the spectral interrogation signal processing system. 
A third identified error source in the spectral interrogation signal processing system is source ripple. The LED that provides the broadband white-light source for the system is not a perfect Guassian shape, but has a small ripple imposed on top of it. This ripple creates error by adding what appears to be another fringe pattern onto what is created at the sensor. In this calculation, the ripple was taken from $1 \%$ to $10 \%$, measured as a percentage of the total signal amplitude, with $2 \%$ being nominal with current suppliers at a wavelength of $618 \mathrm{~nm}$. The results of the simulation are presented in Figure 149. As can be seen, the absolute error increases linearly with the magnitude of the ripple, to over $20 \mathrm{~nm}$. The $50 \mu \mathrm{m}$ gap has a positive error, while the $100 \mu \mathrm{m}$ gap is negative, indicating that over a range of gaps the error is unbiased and depends on if the ripple influences the peaks and valley of the spectrum in one direction or the other. The linear loss of fringe contrast can be seen for both gaps in Figure 150, indicating that there may be a connection between the error and the reduction in contrast.

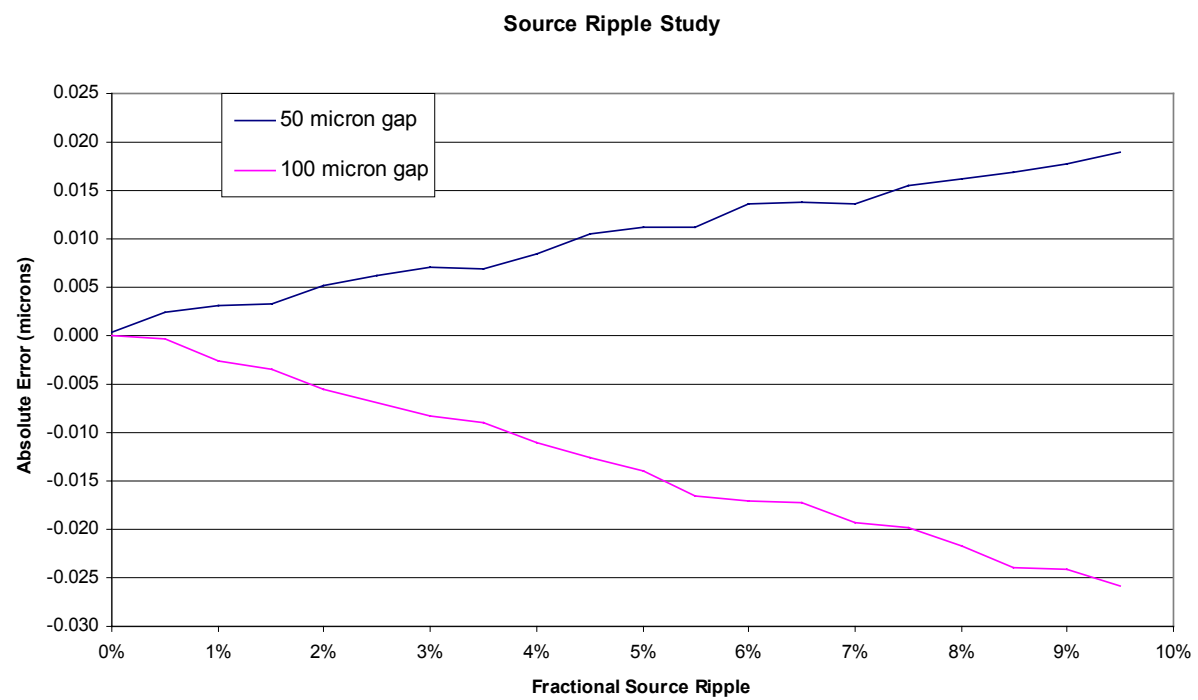

Figure 149. Absolute error of source ripple error calculation for the spectral interrogation signal processing system. 


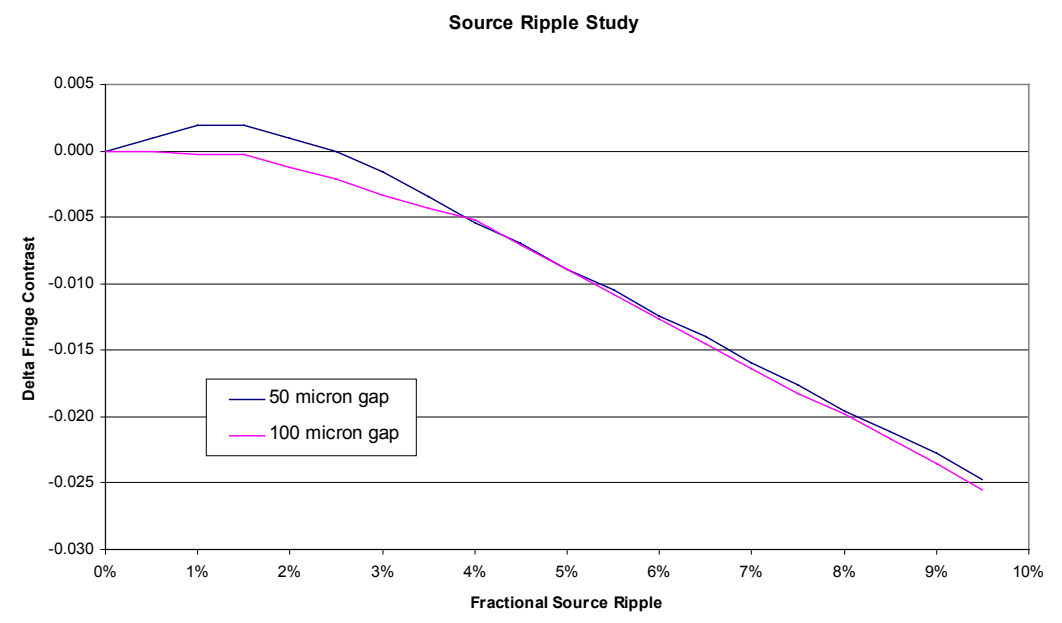

Figure 150. Loss of fringe contrast for source ripple calculation for the spectral interrogation signal processing system.

A pair of spectra was simulated to demonstrate the cumulative effect of the system noise sources, and they are presented in Figure 151. The spectrum on the left is a simulation of the nominal values of the noise sources: a constant pixel variation of 0.025 , a source ripple of $1 \%$, and a standard deviation of $1 \mathrm{~A} / \mathrm{D}$ state of electronic noise. The result is a clean signal with a calculated gap of $50.009963 \mu \mathrm{m}$ from an input $50 \mu \mathrm{m}$ gap spectrum. The figure on the right is a simulated spectrum with the noise sources as large as are conceivable: a random pixel variation of 1 , a source ripple of $10 \%$, and a standard deviation of $10 \mathrm{~A} / \mathrm{D}$ states of noise. The spectrum becomes extremely noisy, with a calculated gap of $50.272996 \mu \mathrm{m}$ for the input gap of $50 \mu \mathrm{m}$, an absolute error of $273 \mathrm{~nm}$.

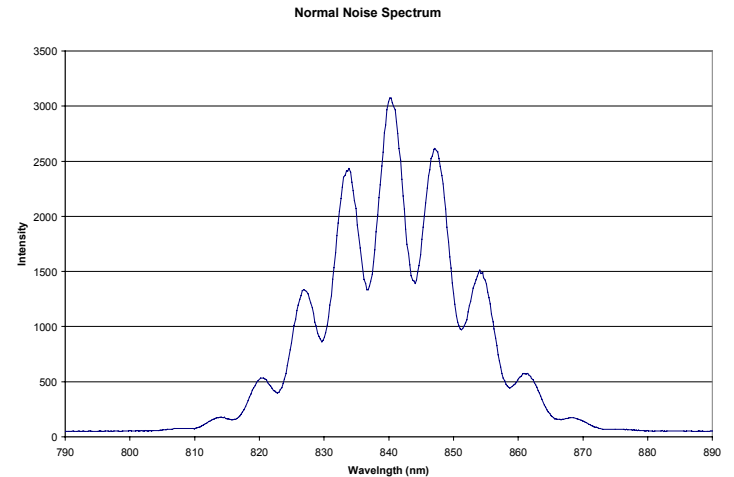

(a)

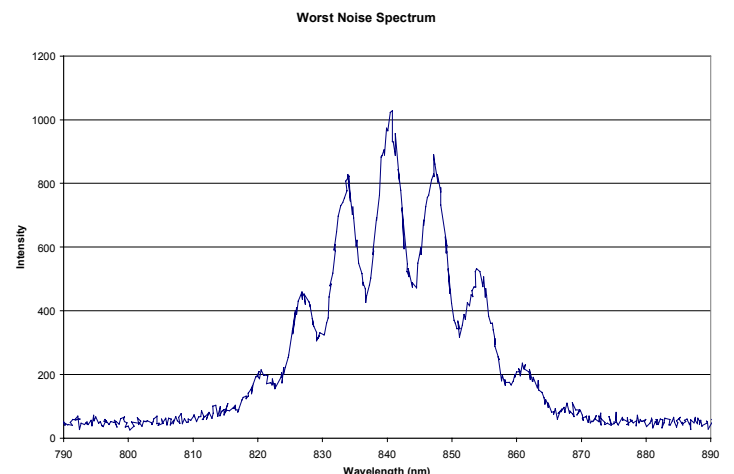

(b)

Figure 151. Calculated spectra of system error conditions, a) typical system errors and b) maximum values of study. 
Although system noise creates error in the optical path measurement, reasonable values of the error sources result in minimal error, on the order of a few nanometers. By using quality components, this system error can be further limited.

\subsubsection{Spectral Smearing Study}

Spectral smearing was the first total sensor/system limitation source studied. Smearing, as described above, is caused by the movement of the gap during the integration time of the CCD array in the spectrometer. If one imagines an increasing gap where the peaks of the fringe pattern are rolling to the right, or to higher wavelengths, over an entire integration period, the intensity of the peaks at the end of the integration time add to where valleys were at the beginning of the period. This superposition causes a reduction in fringe contrast. Now, if the gap moves a distance exactly one-half the center wavelength of the source during the integration time, the beginning peaks will be at the exact pixels that the valleys were, canceling all fringe contrast. This is termed $100 \%$ smearing, defined by the following equation,

$$
S=2 \cdot \frac{\Delta s}{\lambda_{c}},
$$

where $\mathrm{S}=$ percent smearing,

$\Delta \mathrm{s}=$ change in the gap

and $\lambda_{\mathrm{c}}=$ the center wavelength of the source

For a typical center wavelength of $850 \mathrm{~nm}, 100 \%$ smearing will occur for a CCD array with a 0.00125 integration time at a linear gap movment speed of $340 \mu \mathrm{m} / \mathrm{sec}$. Sensors previously designed to use this spectral interrogation system did not operate in environments that induced these gap speeds. Considering that normal full-scale motions for these fiber optic sensors are a few microns, such speeds can not be sustained in linear motion. Only through oscillatory motion can these velocities occur, if only for an integration period or two.

Smearing has become a serious issue as these sensors were tested in supersonic flows and oscillations of the amplitude and frequency necessary to induce spectral smearing were first encountered. Therefore an experimental and numerical study was 
performed to understand the errors involved, what influences those errors, and at what gap movement speed it becomes a problem.

\section{Experimental Efforts Studies}

To study smearing, a method needed to be devised to move the reflector only perpendicular to the light launched from the fiber. The simplest method for producing this gap change without introducing any angular misalignment was to use a fiber optic accelerometer (Figure 152). When placed on a vibration table, the mass deflects the diaphragm and the gap oscillates at a known frequency and amplitude. The purpose of this particular experiment was to verify that under the right conditions smearing could occur with the Luna spectral interrogation system and then, using a system equipped with a faster spectrometer, to see if the smearing is reduced. The results are presented in Figure 153. The low fringe contrast signal is a sample of a smeared signal from the accelerometer. When the faster spectrometer is used, the second, deeper-contrast signal is produced. The fact that a faster spectrometer can eliminate the loss of fringe contrast verifies that smearing was the source of the reduction in the first place, as smearing is the only error source that is dependent on the integration time of the CCD array.

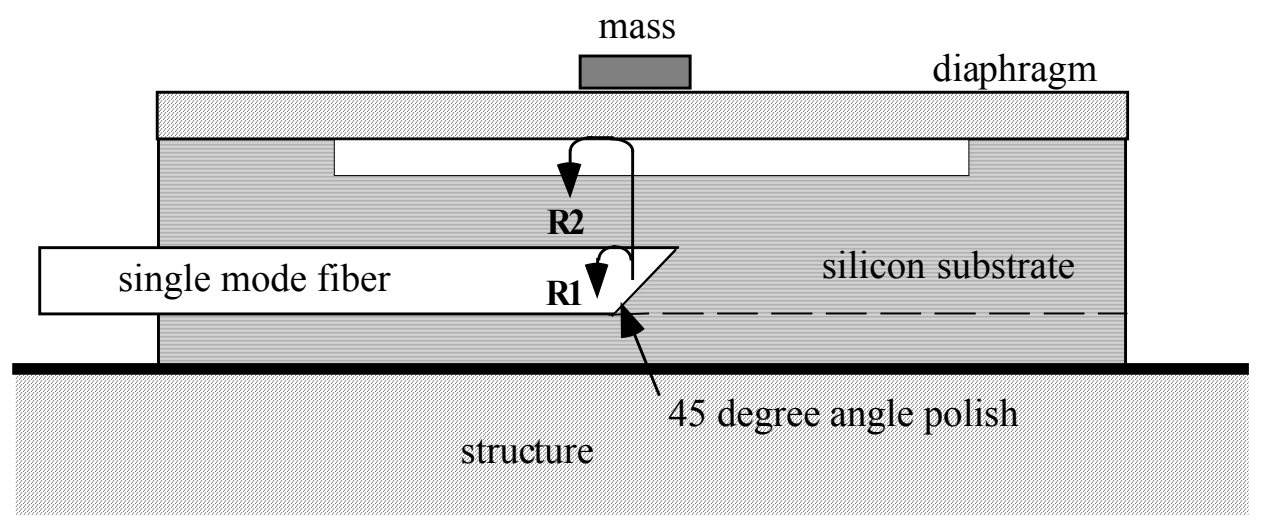

Figure 152. Sketch of the fiber optic accelerometer. ${ }^{97}$ 


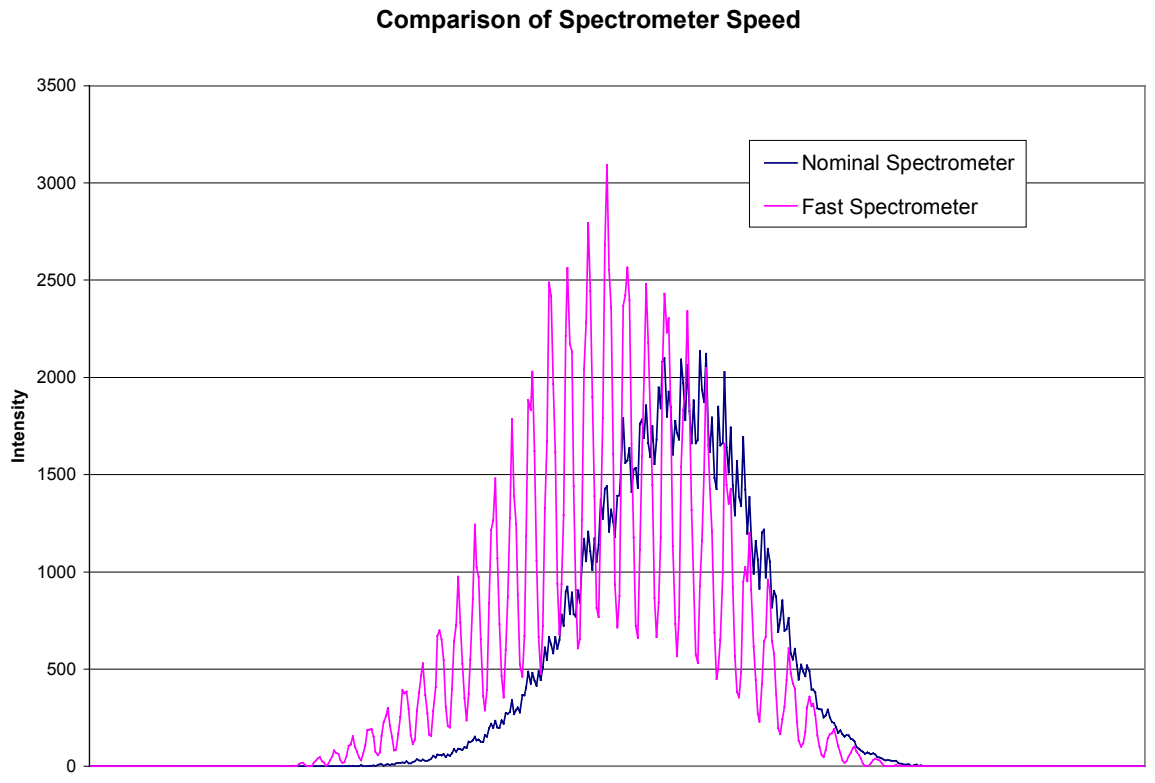

Figure 153. Fiber optic accelerometer data demonstrating the reduction of fringe contrast due to smearing.

\section{Numerical Studies}

The same MatLab routine that was used for examining the system error sources was expanded for the numerical simulation of various sensor sources, including smearing. Figure 154 depicts the method used to simulate smearing. Because the output of the CCD array is the culmination of light to strike each pixel during the integration time, this period was divided into time steps, during which gaps were calculated and a spectrum simulated for that period. At each time step of an integration period, a slightly different gap occurs, and a different spectrum is calculated. All of the spectra from these time steps are added up, and it is this spectrum that is used by the algorithm to determine an effective gap for a particular integration period. Only constant gap speeds were examined in this study, as it is the worst case. A previous study has quantified errors for both a sinusoidal and random movement, but it demonstrated that the constant speed case is the most enlightening. ${ }^{98}$

Results of the smearing simulation are presented in Figure 155 through Figure 159. The first plots show the absolute error due to smearing for a 50 and 100 micron gap with gap speed from 0 to 350 microns/sec. As can be seen, the error increases 
dramatically when $100 \%$ smearing is approached. Figure $155 \mathrm{a}$ presents the results in terms of gap speed, while Figure 155b presents results in terms of percent smearing as defined by Equation 57. As expected the peak of the error occurs at $100 \%$ smearing for both calculated gaps. A detailed look at the error is shown in Figure 156, in which the error remains below $1 \mathrm{~nm}$ for speeds up to $50 \%$ smearing. The calculation was also carried out to gap speeds far in excess of the first instance of smearing. The result, in Figure 157, shows the return to low absolute errors after the first instance of total smearing before increasing again for the harmonic case of 200\% smearing. Although errors are reduced in the gap speeds between the $100 \%$ and $200 \%$ smearing cases, it is clear that sensors can not be allowed to move this quickly, as any oscillation of this magnitude would also go through the first smearing harmonic. Therefore, the rest of the studies are only carried out through this first harmonic.
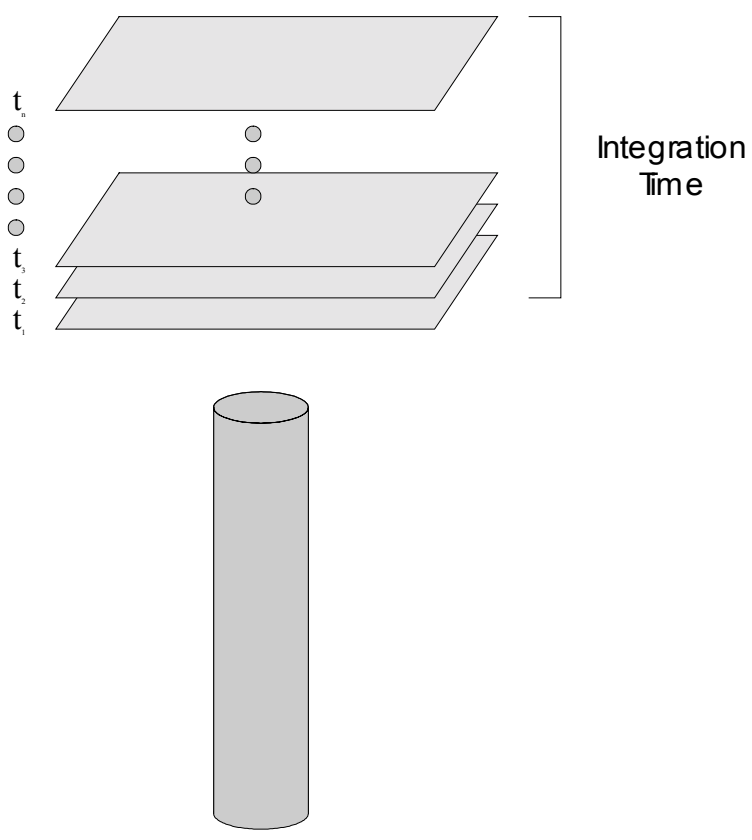

Figure 154. Depiction of error calculations due to spectral smearing showing the progression of the reflection surface during the integration time of the CCD array. 


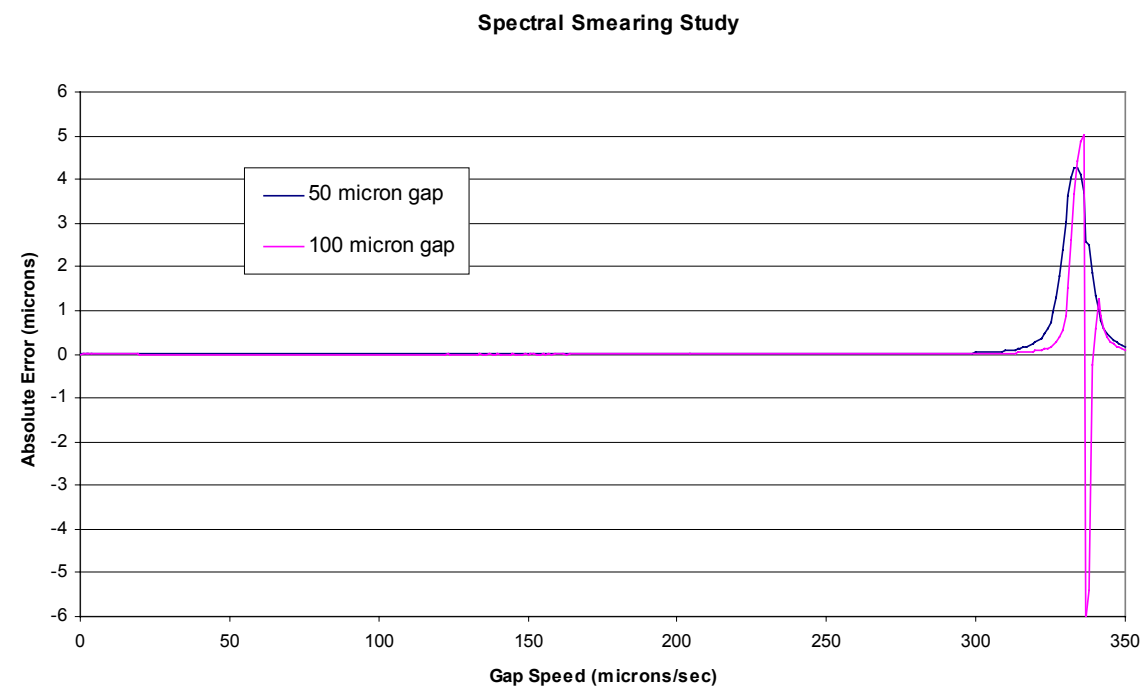

(a)

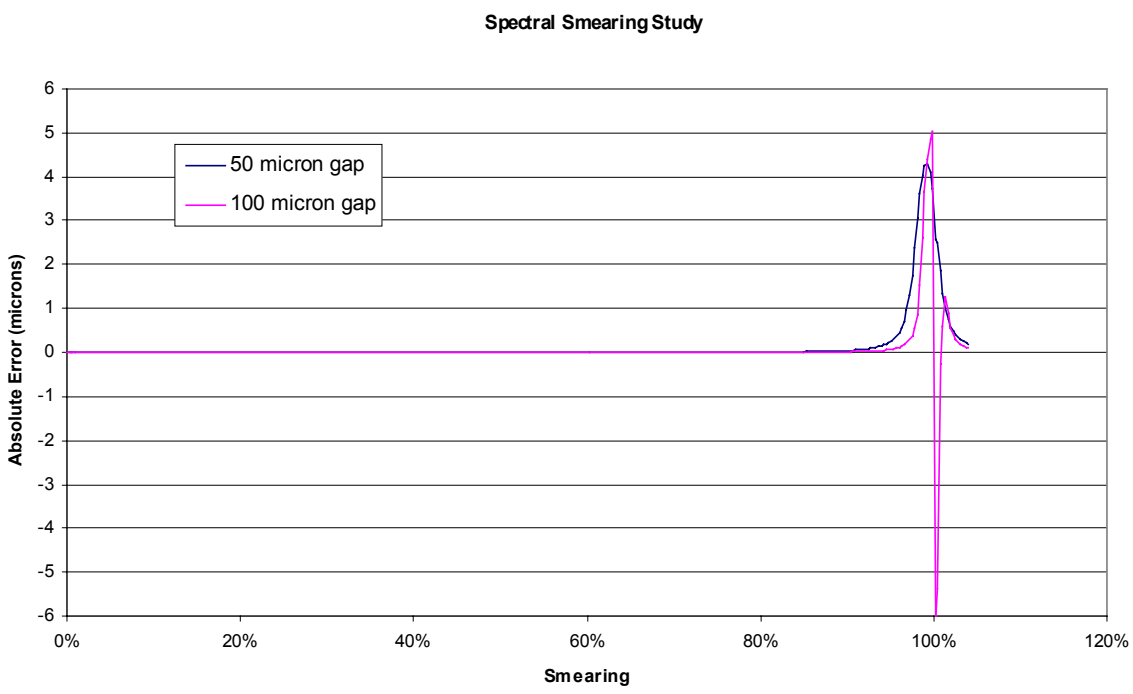

(b)

Figure 155. Results of numerical study of spectral smearing for the spectral interrogation signal processing system, a) absolute error in terms of gap speed and b) absolute error in terms of percent smearing. 


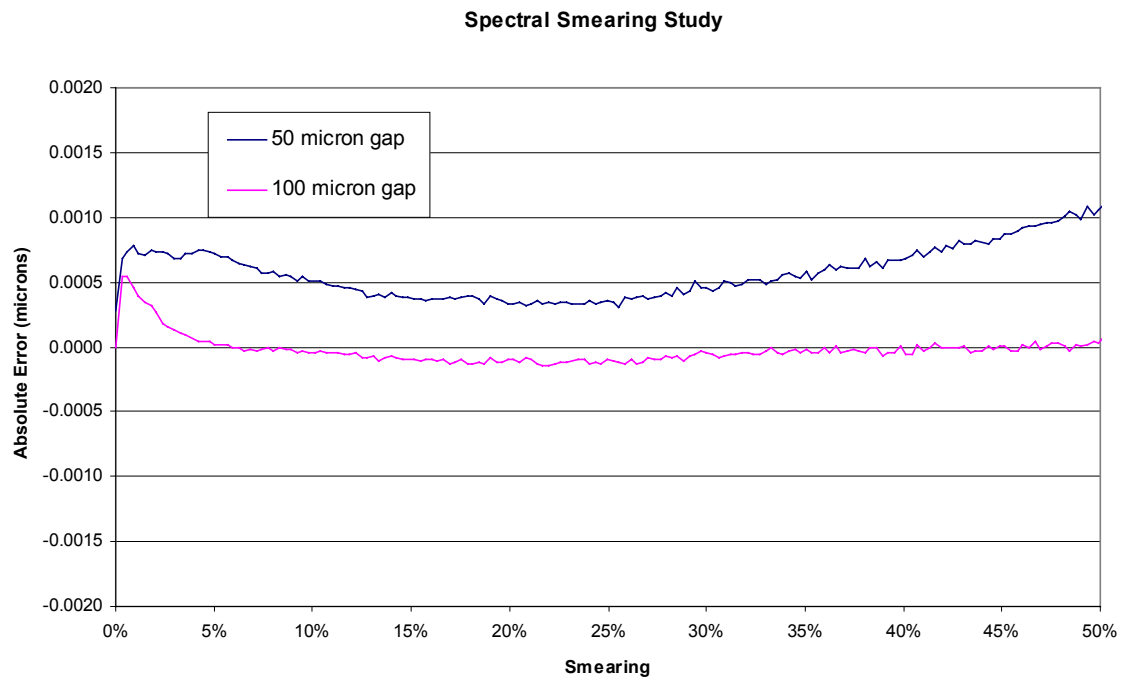

Figure 156. Detail of the calculation of absolute error due to spectral smearing for the spectral interrogation signal processing system.

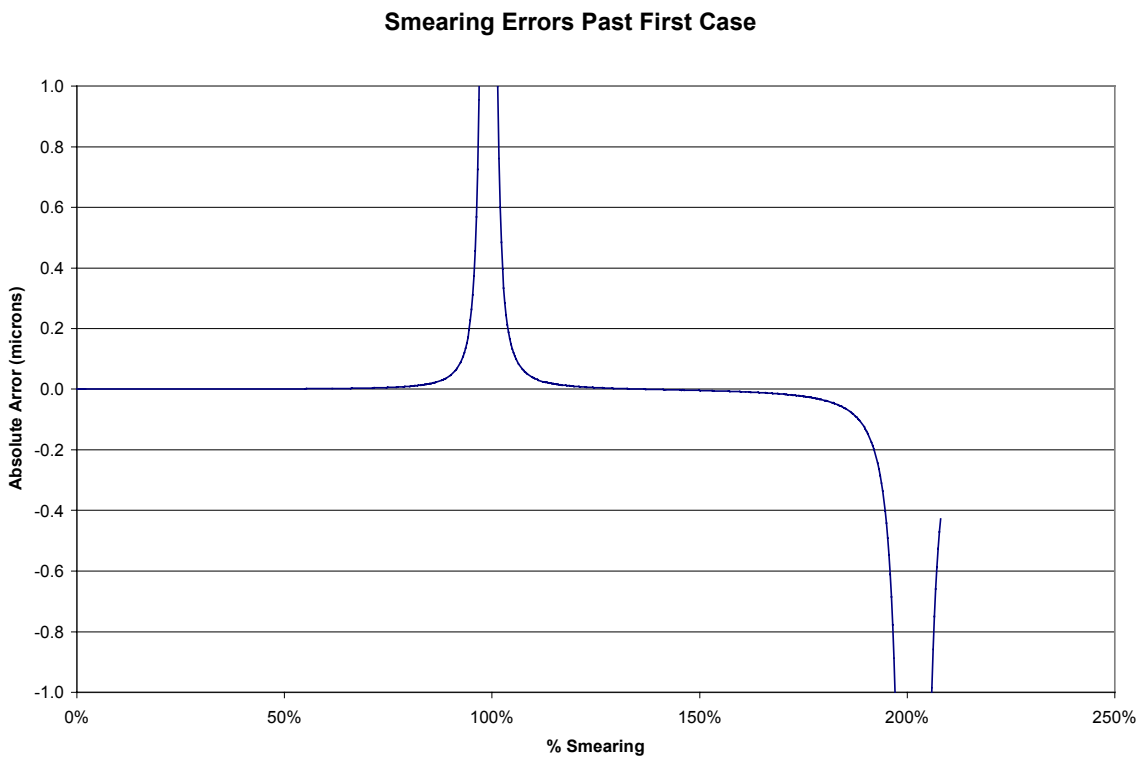

Figure 157. Calculation of absolute error of the spectral interrogation signal processing system due to spectral smearing beyond the first harmonic.

A grid convergence study was performed for the smearing case due to its apparent importance in the overall system limitation. As shown in Figure 158, increasing the number of time steps in the simulation from 50 to 500 steps did not appreciably change 
the location of the peak error. This study added confidence to the total numerical effort, allowing accurate results to be obtained through reasonable computational effort.

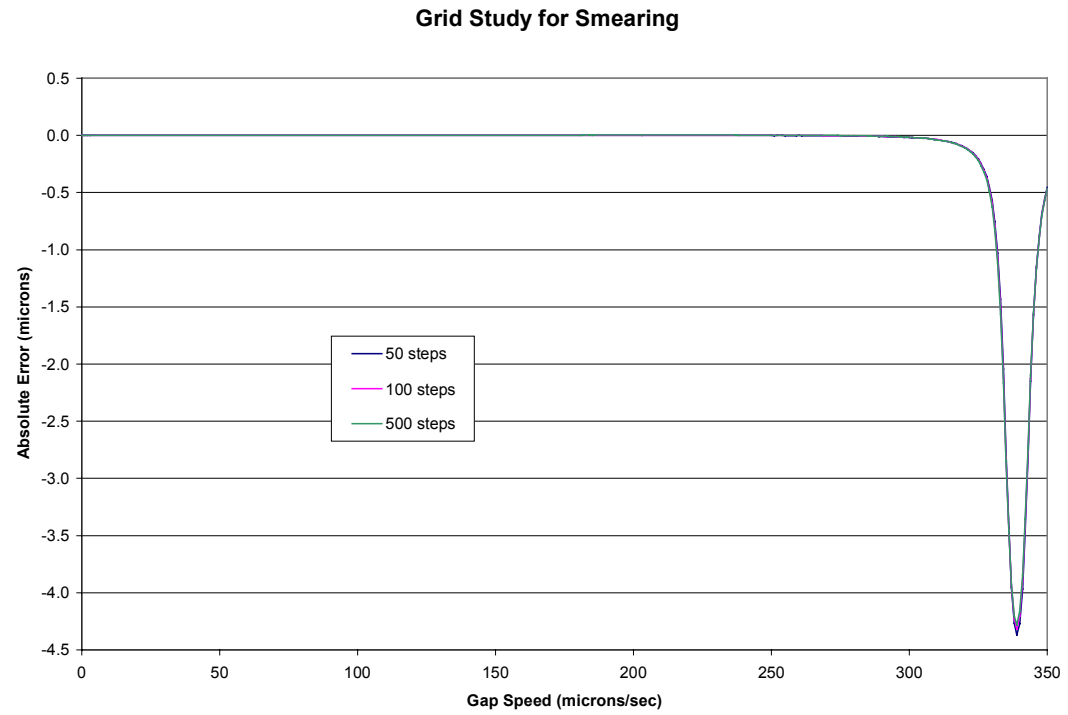

Figure 158. Grid convergence study of calculations of spectral smearing.

Through this computational effort, a correlation between the fringe contrast and error in the gap determination was observed. If the error and the fringe contrast plots (Figure 155a and Figure 159) are compared, the error greatly increases when the fringe contrast drops near or below 0.10. In addition, Figure 160 presents the fringe contrast for the case when the calculation was taken out to the $2^{\text {nd }}$ smearing harmonic. This calculation also shows that peaks in the error plot correspond to the contrast dropping below 0.10. This problematic fringe contrast level holds over a number of different experiments and conditions, as will be detailed below. 


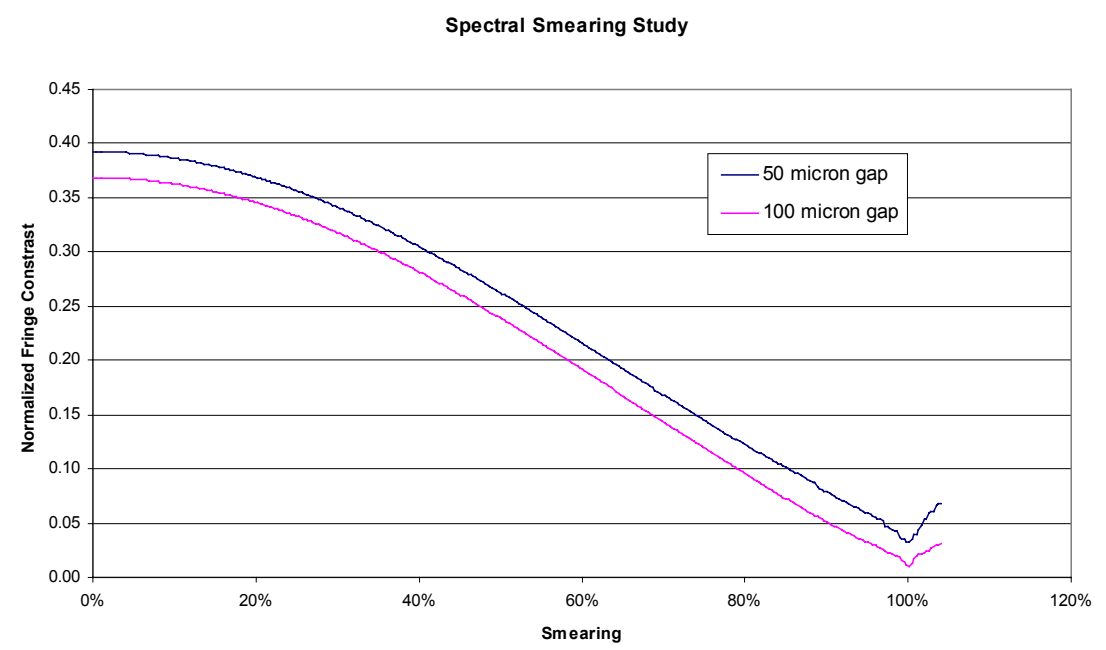

Figure 159. Loss of normalized fringe contrast due to spectral smearing for the spectral interrogation signal processing system.

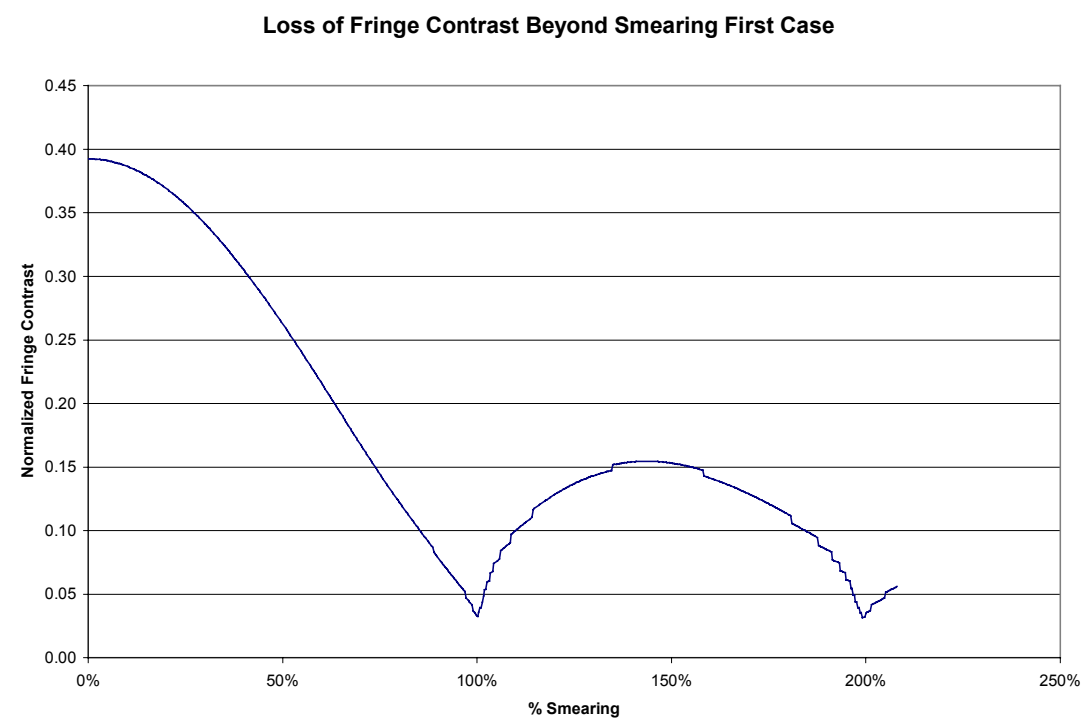

Figure 160. Calculation of fringe contrast of the spectral interrogation signal processing system due to spectral smearing beyond first smearing harmonic.

\subsubsection{Angular Misalignment Study}

Angular misalignment is another possible source of sensor error. As depicted in Figure 161, if the reflector is not aligned properly, the light will not couple correctly back into the fiber. As stated in Chapter 2, the light energy in the fiber forms a Gaussian 
distribution. This distribution persists once the light leaves the fiber. Upon reflection from a misaligned surface, the power of the reflection that re-enters the fiber is seriously degraded, as seen in Figure 162. ${ }^{99}$ Recall, large mismatches of power between the reflection of the fiber/air interface and the one off the reflecting surface seriously degrade fringe contrast and, as seen, possibly induce error. For this reason, the shape of loss of contrast should be similar (Gaussian) as that of the power reduction.

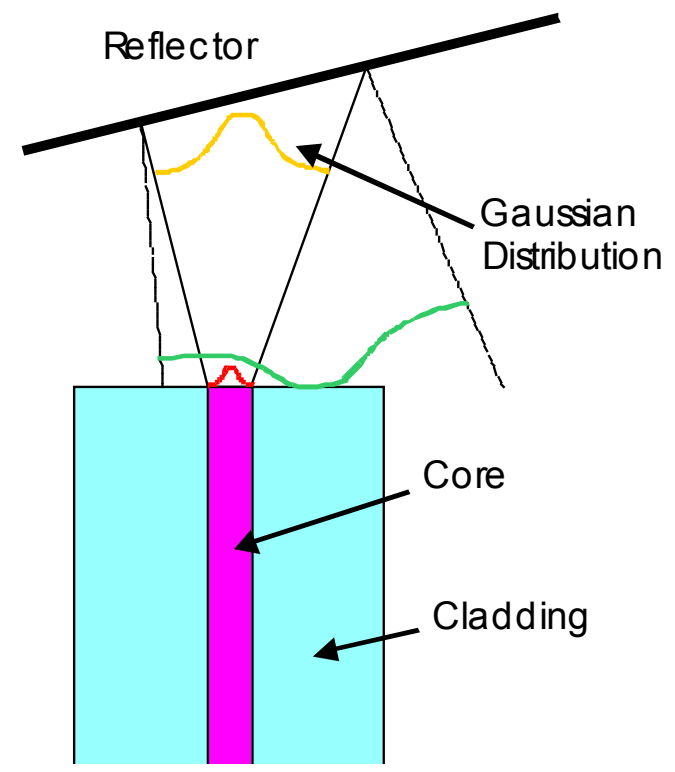

Figure 161. Depiction source of power and fringe contrast loss from angular misalignment of the reflector.

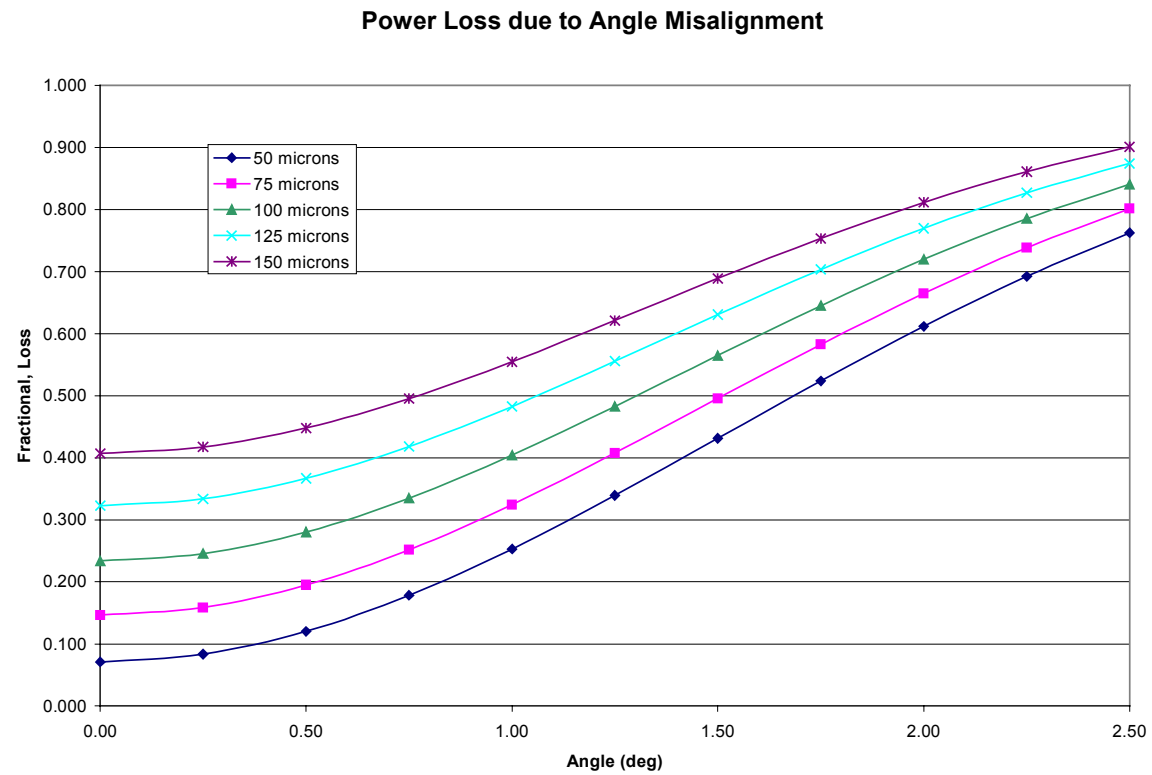

Figure 162. Power loss due to angular misalignment of the reflector. 


\section{Experimental Studies}

In order to verify the loss of fringe contrast due to angular misalignment, an experiment was devised, and the results are presented in Figure 163. A fiber was held at a constant optical gap perpendicular to a mirror mounted on an angle positioner. This positioner was varied over its range and spectra were stored at each angle. The experiment was performed for both air and glycerin in the gap between the fiber and mirror as these were the materials used in this project. The results show a reduction in fringe contrast of a Gaussian shape. It is believed that the dip in the center of the glycerin plot is due to stresses cause by its surface tension slightly altering the light path. It should also be noted that the glycerin fringe contrast decreases more quickly for an angle change than air. It is hypothesized that because the glycerin has a higher index of refraction than air, the cone leaving the fiber does not have as great an angle. The consequence of this smaller angle is that the flat, or high power, region of the Guassian reflection of this tighter cone would move off the core more quickly than a larger cone, causing a power mismatch at a smaller angle than in air.

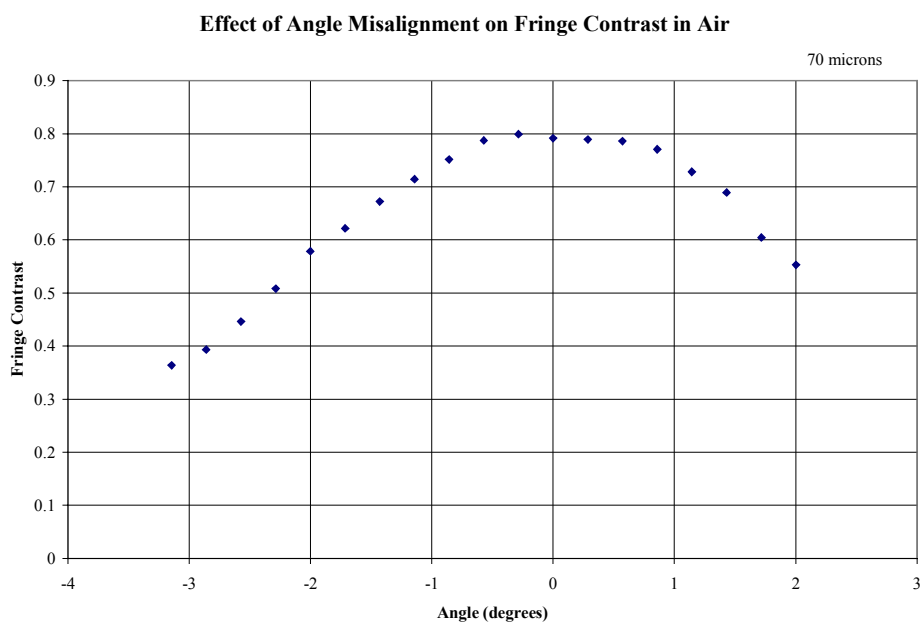

(a) 


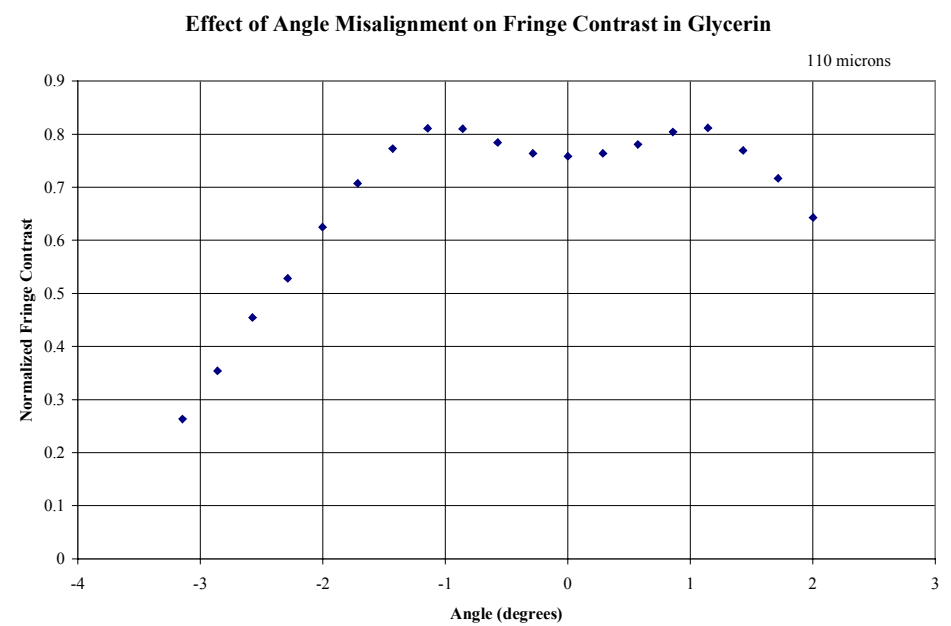

(b)

Figure 163. Measurement of fringe contrast loss due to angular misalignment, a) in air and b) glycerin.

\section{Numerical Studies}

For this simulation, we assume that the misalignment is minor and that only the relatively flat center region of the Gaussian interacts with the air/fiber reflection. In addition, a plane wave assumption, which has been used to simulate all of the spectra, will be used. The consequence of the plane wave approximation is that the cone is ignored, and it is assumed that light which is coupled back into the fiber will only be from light that leaves relatively perpendicular to the fiber endface. This means that the light reflecting off the second surface will only have an influence over the fiber core diameter, in this case $6 \mu \mathrm{m}$. Any angular misalignment will cause part of this circle of influence to be farther than the nominal gap and part of it closer. By using the superposition of light, the gap is split up into numerous gaps that are calculated and superimposed back together for an overall spectrum, similar to the procedure used for the smearing calculations. For this simulation, this means having a number of small steps which simulate the angled surface. Each of these steps must be weighted, as the center steps will have more light reflecting than the outer steps (see Figure 164). This weighting function was determined to be

$$
\varpi=\frac{4}{\# \text { steps }^{*} \pi} * \sqrt{1-\frac{4 x^{2}}{d^{2}}} .
$$




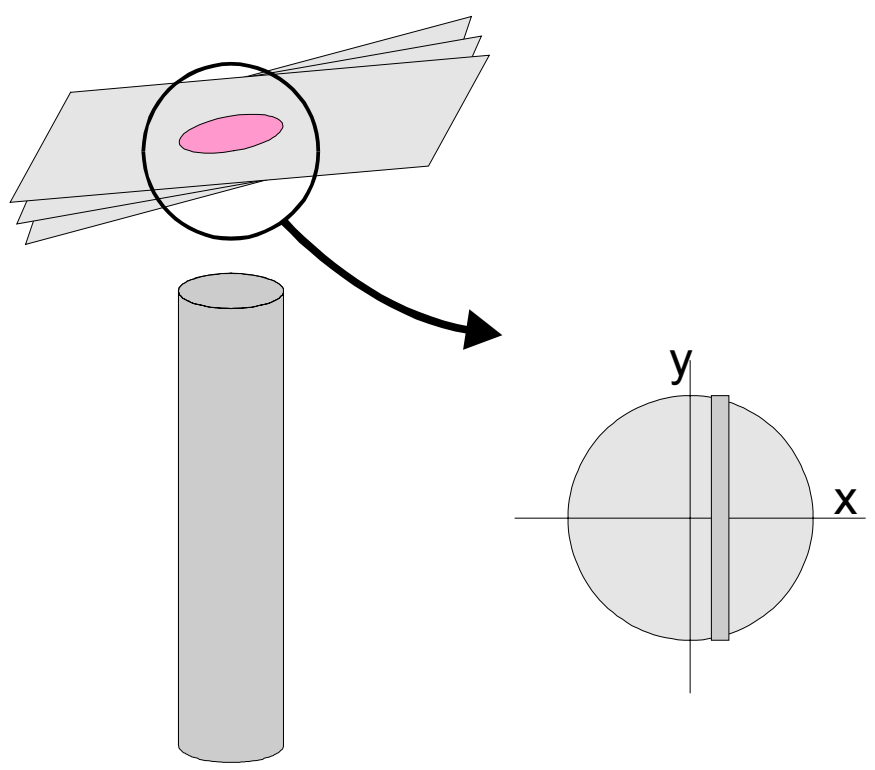

Figure 164. Depiction of error calculations due to angular misalignment showing the progression of the reflection surface and the basis of the weighting function.

Figure 165 presents the results of the angular misalignment simulation. As with smearing, the absolute error remains low until an angle where the spectra is superimposed back to the Gaussian over the $6 \mu \mathrm{m}$ plane wave, at which point all gap information has been lost. This large error, though, occurs far beyond the small angle assumption stated above. For reference, the Version 6 skin friction sensor will have a misalignment of $0.035^{\circ}$ at its design load. Figure $165 \mathrm{~b}$ is a detail of the fringe contrast results. For a misalignment of $0.035^{\circ}$, the reduction in contrast is minimal, and for that matter so is the error. Therefore, although misalignment remains a concern for the skin friction sensor, the experimental and numerical results presented above indicate that it is a small component of the overall error. 


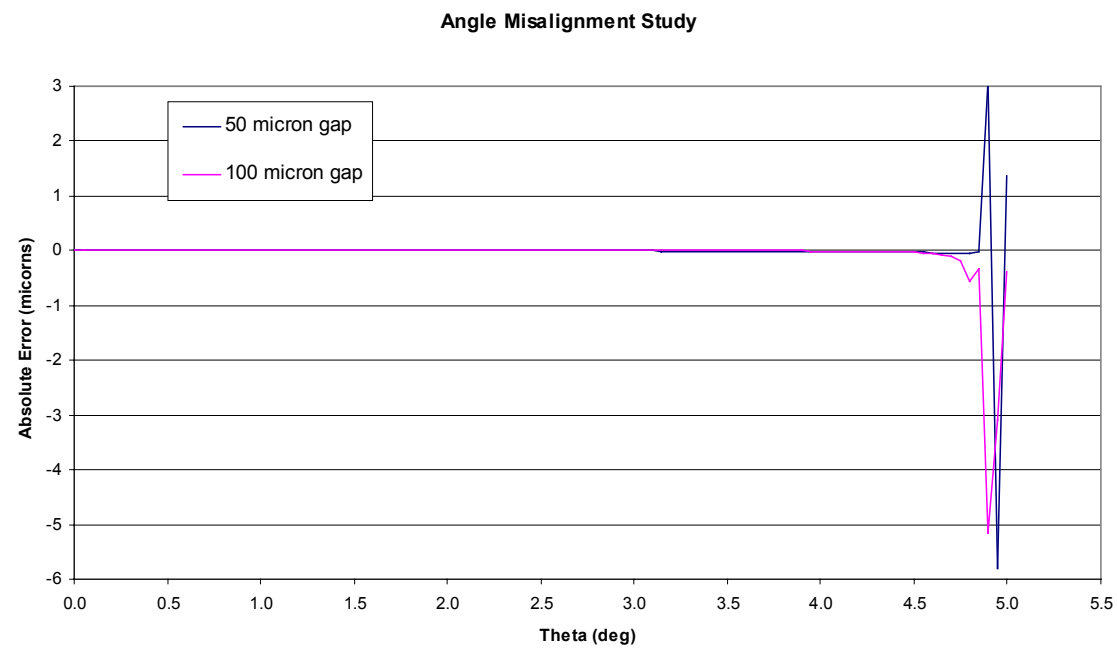

(a)

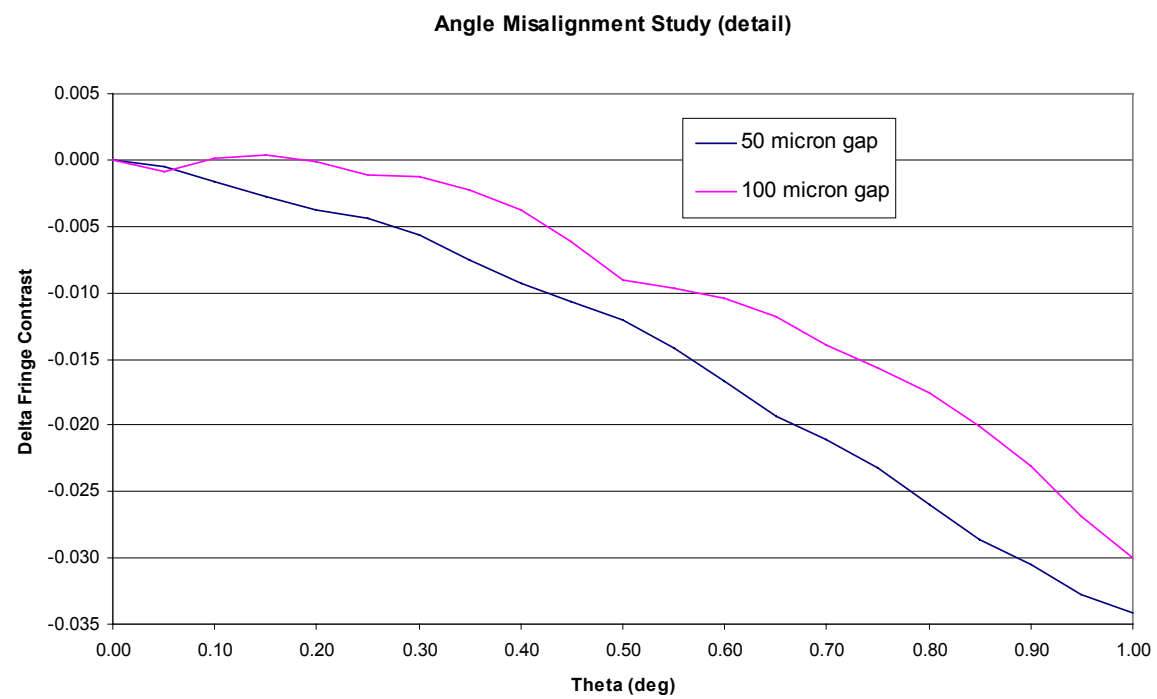

(b)

Figure 165. Results of angular misalignment calculations for the spectral interrogation signal processing system, a) absolute error and b) loss of normalized fringe contrast.

\subsubsection{Reflector Roughness Study}

Another concern raised was the quality of the surface used as the reflector. The majority of the experience at Luna had been with either polished fiber endfaces or micromachined surfaces. The use of polished metal reflectors presented a new variable to be explored. 


\section{Experimental Studies}

In order to quantify the difference between the polished Si diaphragm of the pressure sensor and the polished metal surface used in the skin friction sensor, atomic force microscope (AFM) readings were taken of both surfaces. The AFM setup at the Center for Science and Technology at Virginia Tech is pictured in Figure 166.

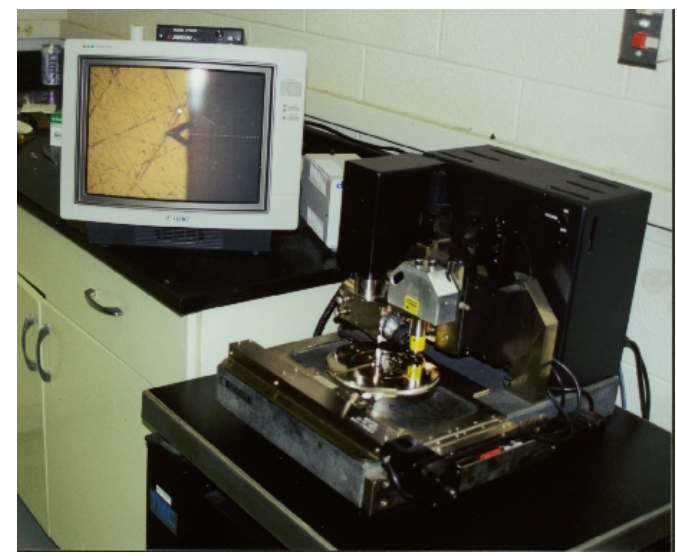

Figure 166. Photograph of the AFM setup.

The results of the AFM readings are pictured in Figure 167 for the pressure sensor diaphragm and Figure 168 for various areas of the skin friction sensing head. The interesting result is the difference in roughness between the two. The pressure sensor had a variance of the roughness over the surface of $9.353 \mathrm{~nm}$, while the polished metal varied from a low of $15.509 \mathrm{~nm}$ for the upper left photograph of Figure 168 to $112.60 \mathrm{~nm}$ for the upper right photograph. This variation is a major concern for the skin friction sensor. As a force is applied to the beam, it not only changes angle, but it deflects, which is seen as a sliding of the impinging light along the surface. For the Version 6 sensor, this "sliding" will be approximately $17 \mu \mathrm{m}$ at the design deflection. As can be seen, this movement would be across half of the pictured area. Clearly a significant change of roughness will have a large effect on the determined gap and in turn the shear measurement. 


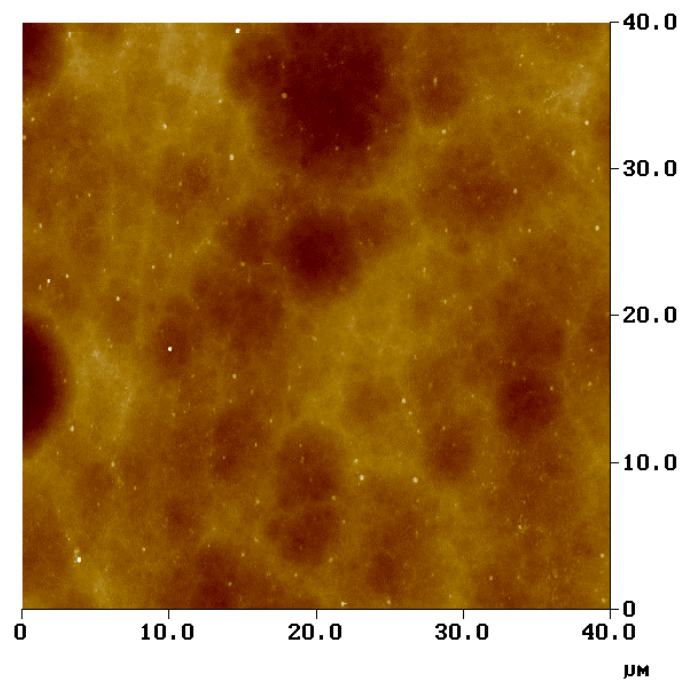

Figure 167. AFM of pressure sensor diaphragm surface (darkening of shade indicates a deeper surface).
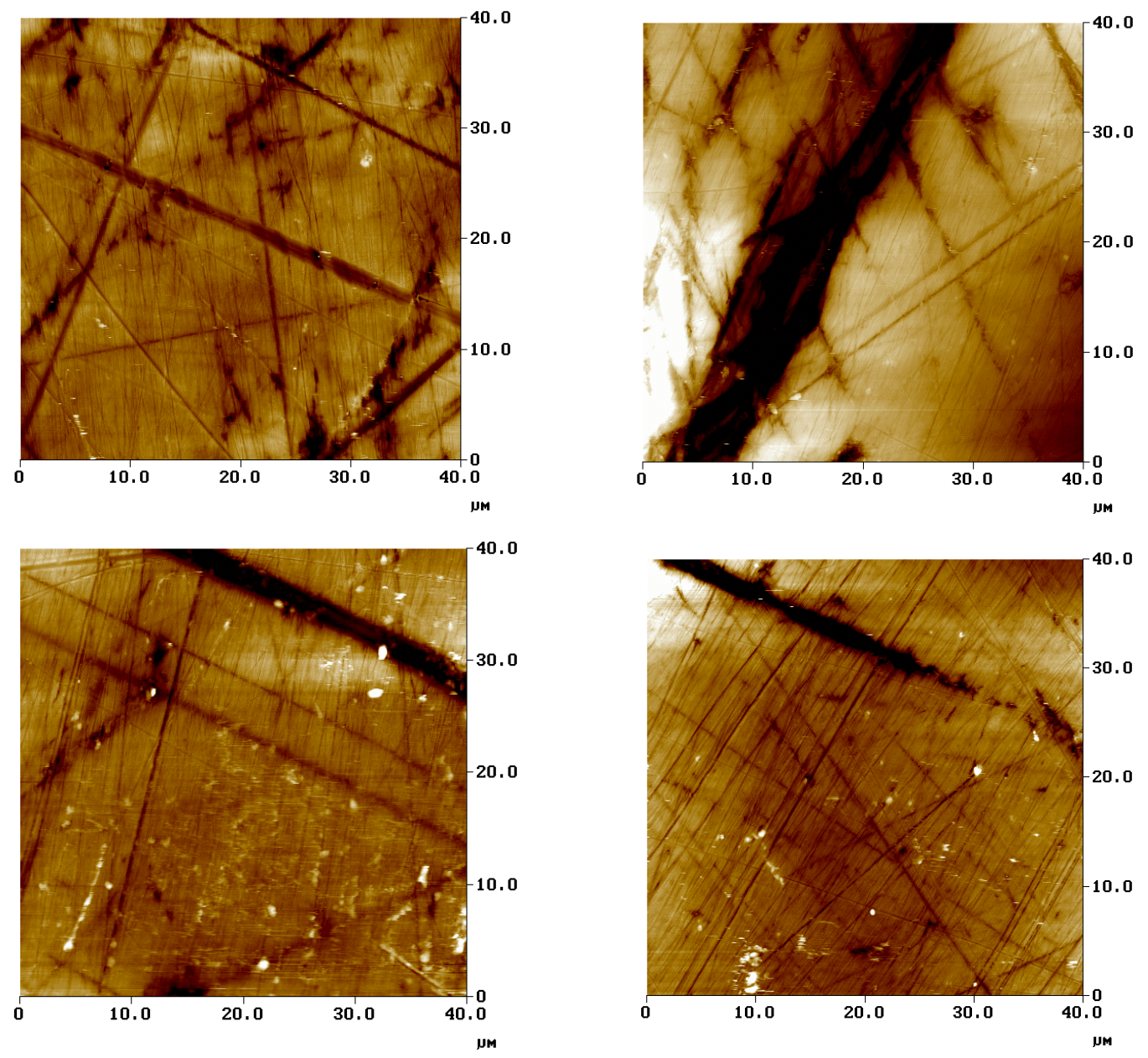

Figure 168. AFM of portions of typical skin friction sensor reflector (darkening of shade indicates a deeper surface). 
To demonstrate the possibility of the roughness on the skin friction sensor being a problem, fringe contrast was measured during a normal calibration of the Version 6 skin friction sensor. Because the data during a calibration is static, and the angle change is extremely small, only surface roughness from sliding is different at each point. Although the calibration of this sensor was extremely linear (see Appendix B), there were variation of the contrast for each channel. The worst of these is presented in Figure 169, where the fringe contrast at $800 \mathrm{mg}$ has decrease to nearly 0.10 , a point at which problems have been demonstrated to occur. Even for channels that do not have a rough point such as this, the variations mean that certain points of reflector are more prone to problems than others.

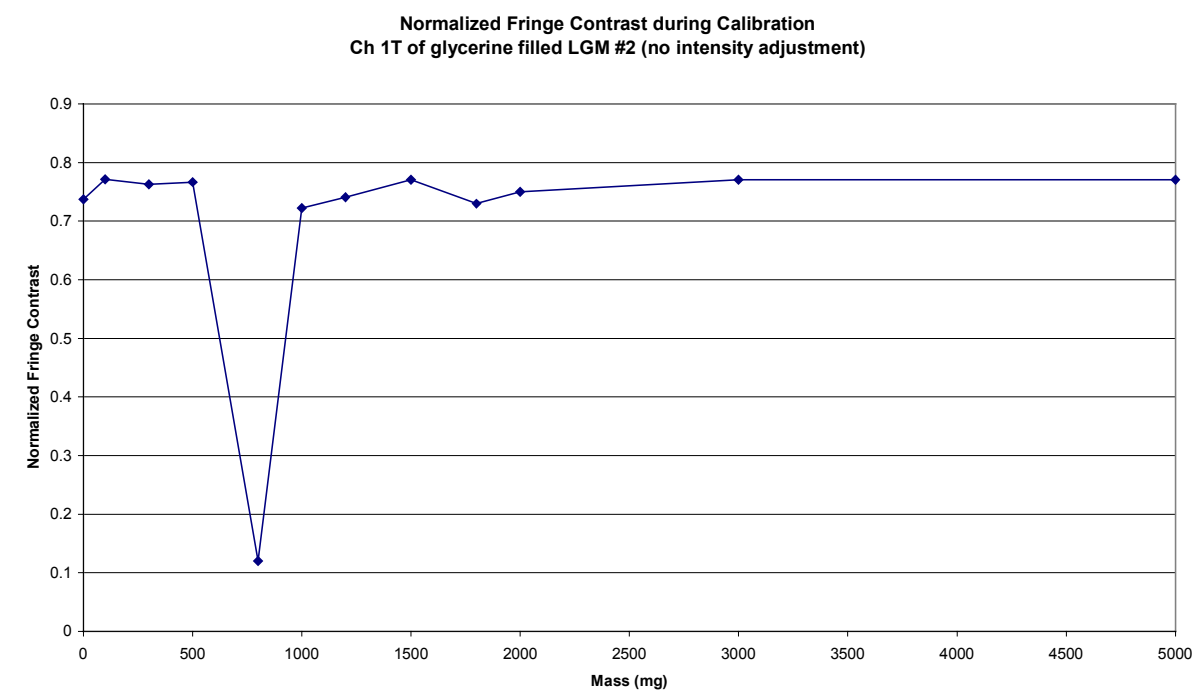

Figure 169. Worst-case of measured fringe contrast of the Version 6 skin friction sensor during calibration. Spike indicates that bad refection sections exist on these surfaces.

\section{Numerical Studies}

As with the previous simulations, the roughness work uses superposition to create the case spectrum. Because the measured roughness was in terms of a standard deviation, the simulation had to use a randomly generated surface. In order to limit the computational time, it was decided to fix a randomly generated surface that would be 
used for all the calculations. Therefore, a 1000 point random surface was generated which was then multiplied by the variance to produce an actual surface geometry for that error estimate. This method also means that the results would be slightly different each time the simulation was performed. However, with the 1000 points used in the normal distribution, the differences should be minor and the results similar.

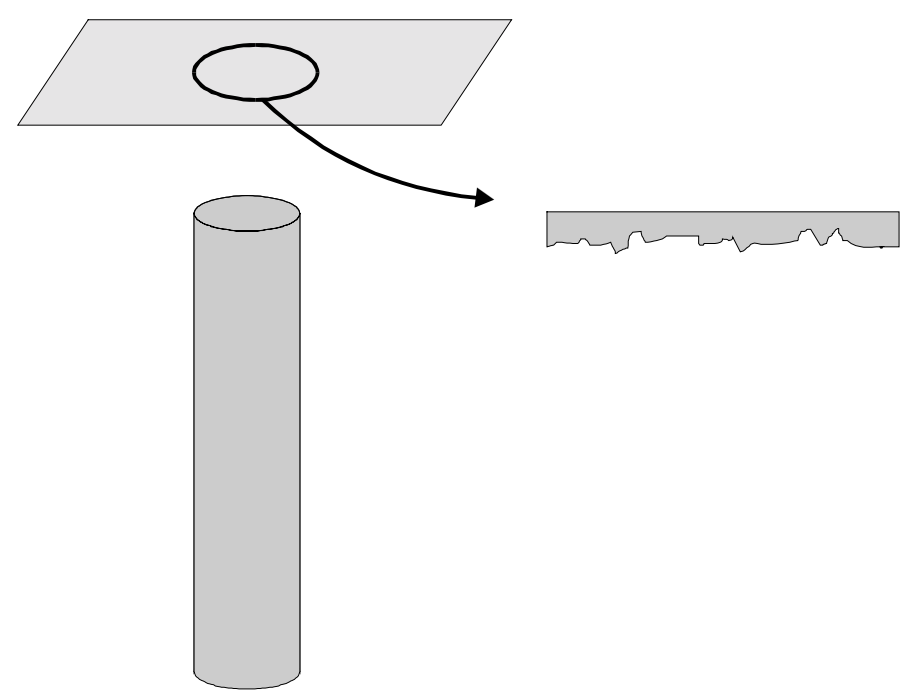

Figure 170. Depiction of error calculations due to reflector roughness for the spectral interrogation signal processing system.

The results of the roughness simulation are presented in Figure 171. Because a separate surface was generated for the 50 and $100 \mu \mathrm{m}$ gaps, comparisons of the two must be carefully done. It can be seen that significant errors do not begin for either gap until a variance of at least 0.01 , which is greater than all but the worst of the AFM data. Also, the fringe contrast approaches 0.10 at a 0.1 variance, which is where the absolute error becomes a problem. This result reinforces the previous concern about normalized fringe contrasts below 0.10 . 


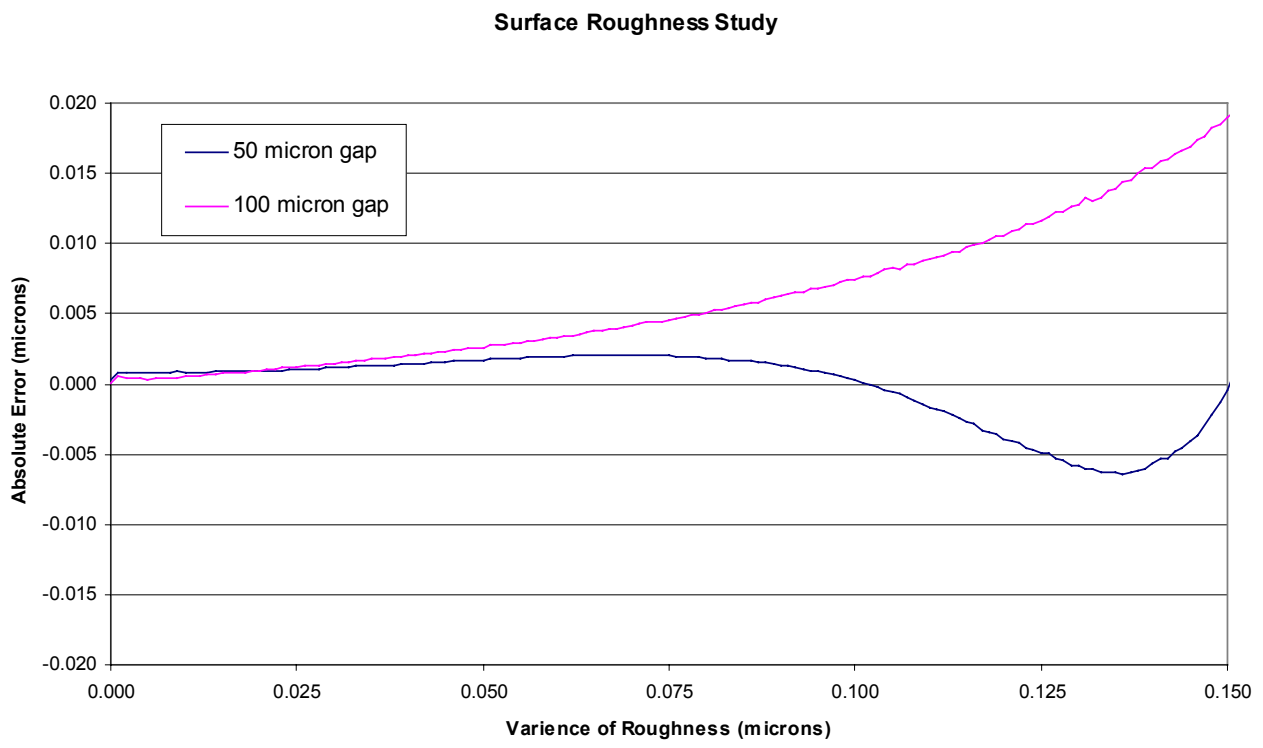

(a)

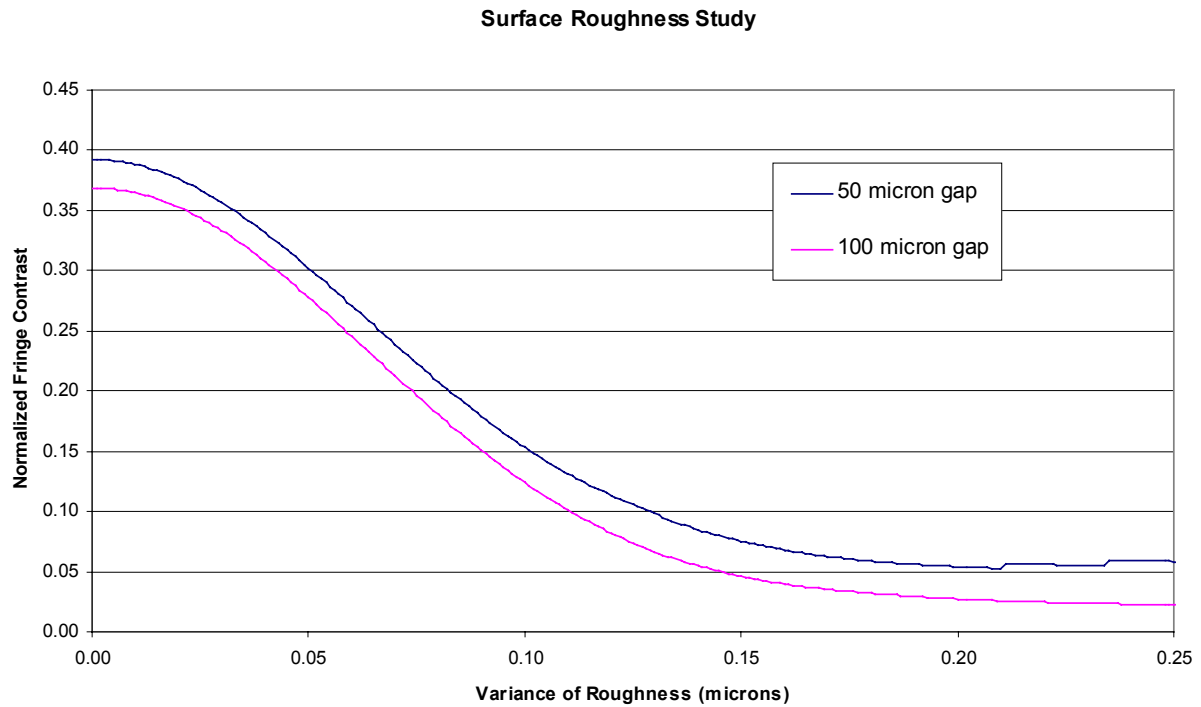

(b)

Figure 171. Roughness calculation for the spectral interrogation signal processing system, a) Absolute error and b) loss of normalized fringe contrast.

\subsubsection{Combined Effects}

The question remains of how these separate error sources combine and if there are any major differences in the results due to combination effects. Two experiments were 
performed to demonstrate the combination of effects. Also, total simulations of the fiber optic pressure and skin friction sensors were developed which added all the effects studied separately above and combined the appropriate ones for the specific sensor.

\section{Experimental Studies}

An experiment, as depicted in Figure 172, was performed to examine the combined effect of smearing and angular misalignment. A mirror was attached to a lead zirconium titanate (PZT) actuator that oscillated due to a triangle wave voltage applied across the PZT sandwich. This signal produced a linear speed in one direction and then the other, determined by the amplitude and frequency of the triangle wave. Because the experiment was designed to examine data sets up to and above spectral smearing speeds, the measurement fiber could not be used to reliable provide feedback to the voltage signal. This problem was overcome by attaching a fiber optic strain gage to the side of the PZT actuator and monitoring its output. A calibration was performed at lower speeds to determine the ratio of strain gage speed to tip speed, resulting in a 36.83:1 ratio. The imposed signal was then adjusted over a range, from low speeds to beyond smearing.

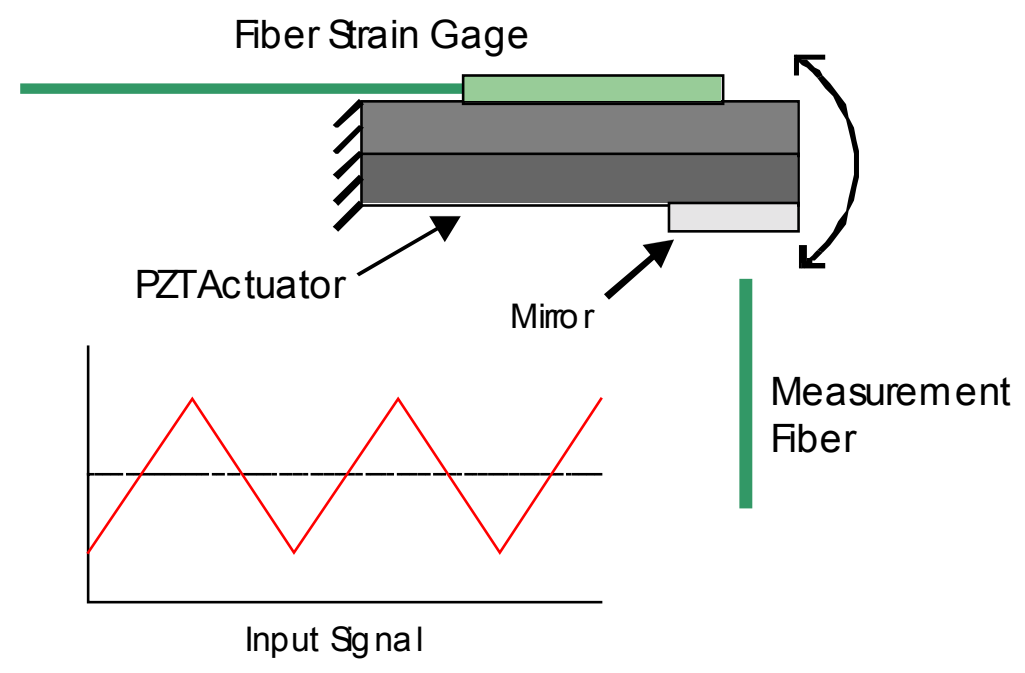

Figure 172. Sketch of the PZT experimental setup.

The spectra from the experiment are presented in Figure 173. A progression of gap speeds is shown, from 10 to $385 \mu \mathrm{m} / \mathrm{sec}$. As can clearly be seen, the fringe contrast degrades as the PZT tip speed increases. To the left of each spectra is its FFT, the peak plot which the system algorithm uses to determine optical gap. The clean spectrum for 
$10 \mu \mathrm{m} / \mathrm{s}$ in Figure 173a produces a peak that has a magnitude of 400000 , the $200 \mu \mathrm{m} / \mathrm{s}$ speed in Figure 173b spectrum of 175000 , and finally the $350 \mu \mathrm{m} / \mathrm{s}$ speed in Figure $173 \mathrm{c}$ spectrum, which is nearly completely smeared, has no discernable peak. Again, this is due to the inability of the little high frequency information left to have the coherence necessary to make a clean peak. This is a general conclusion not limited to the results of this experiment. The main conclusion of this experiment was that lack of significant fringe contrast occurred over a larger range than the smearing analysis demonstrated, indicating that the combined effect of smearing and misalignment spreads out the error problem over a larger range of gap speeds. The smearing simulations show that major error occurs in only a band few microns/sec wide around 100\% smearing. This experiment demonstrates a larger range $(345-385 \mu \mathrm{m} / \mathrm{sec})$.

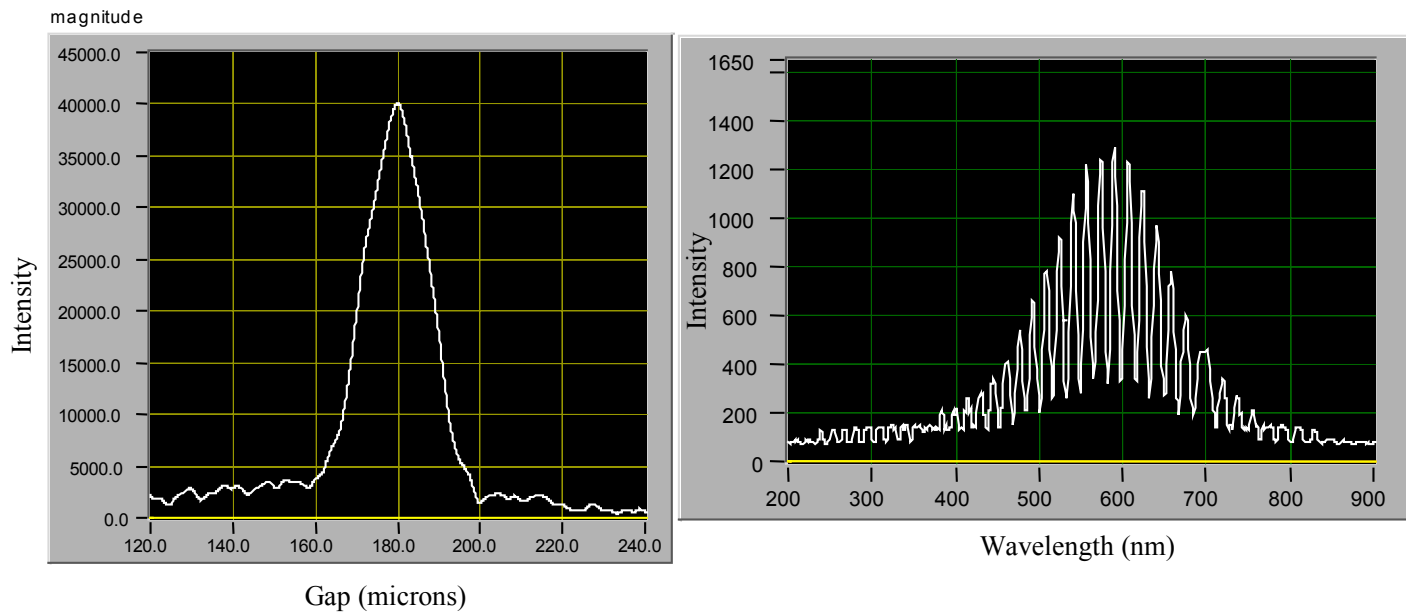

(a) $10 \mu \mathrm{m} / \mathrm{sec}$
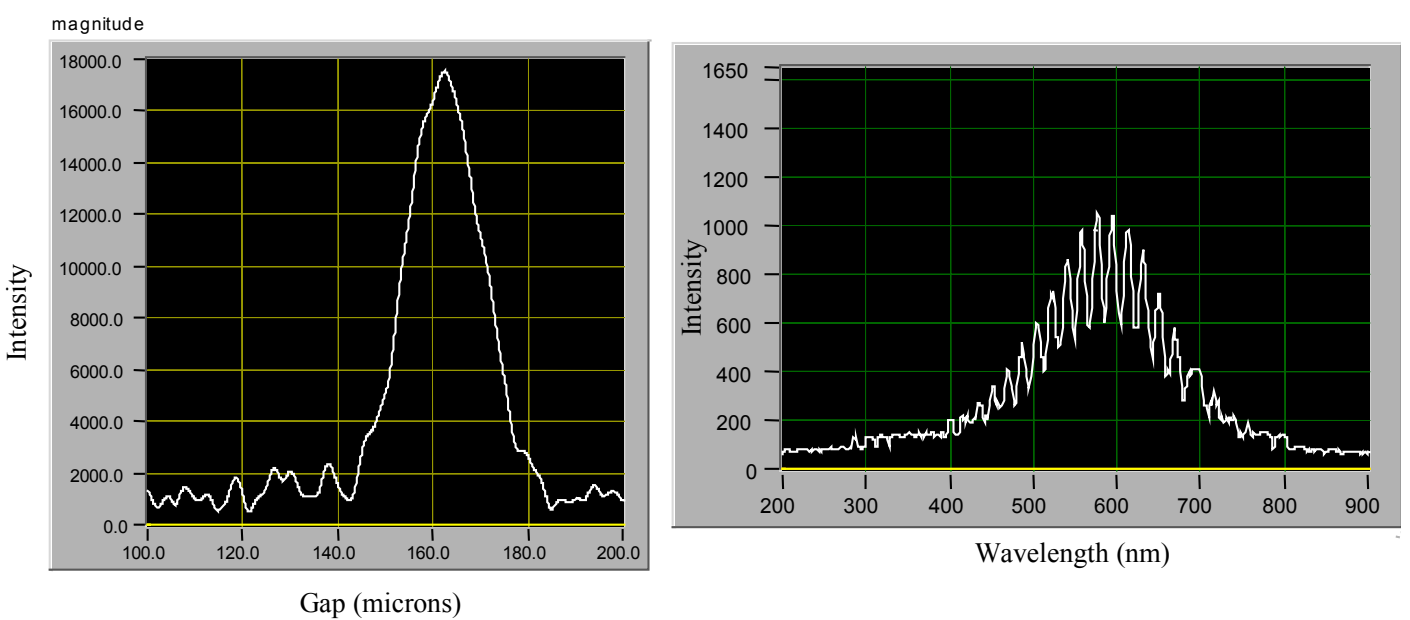

(b) $200 \mu \mathrm{m} / \mathrm{sec}$ 

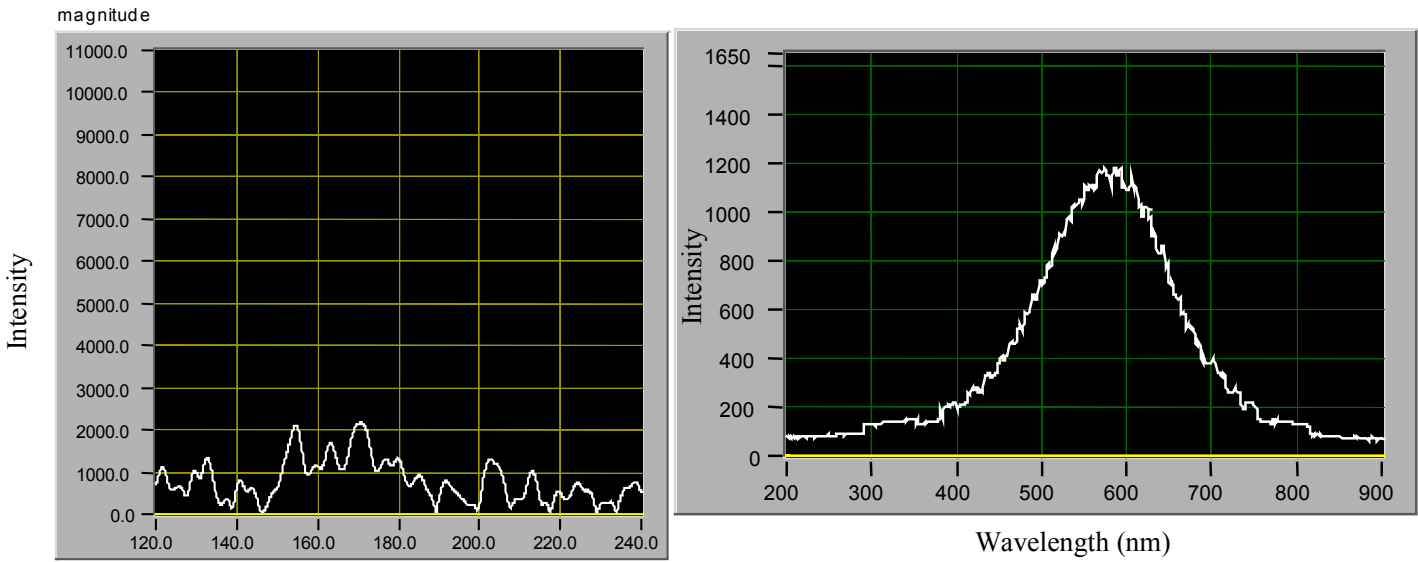

Gap (microns)

(c) $350 \mu \mathrm{m} / \mathrm{sec}$
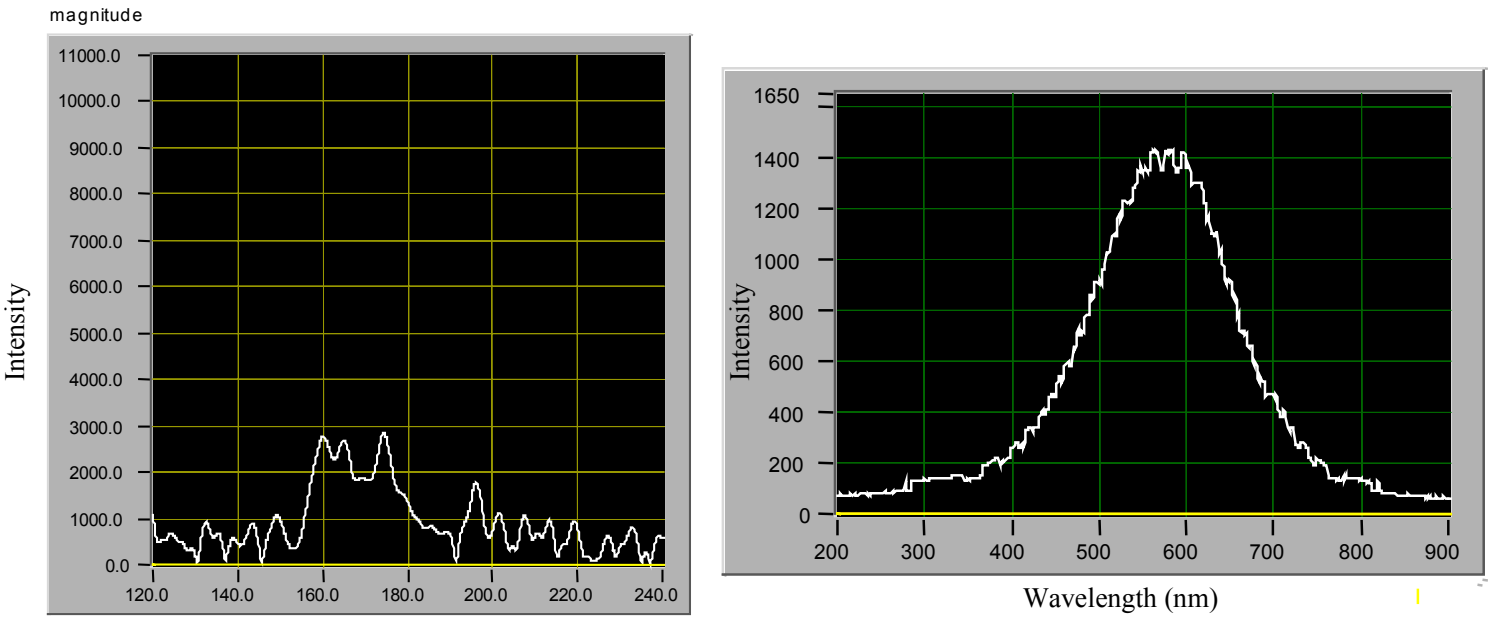

Gap (microns)

(d) $345 \mu \mathrm{m} / \mathrm{sec}$
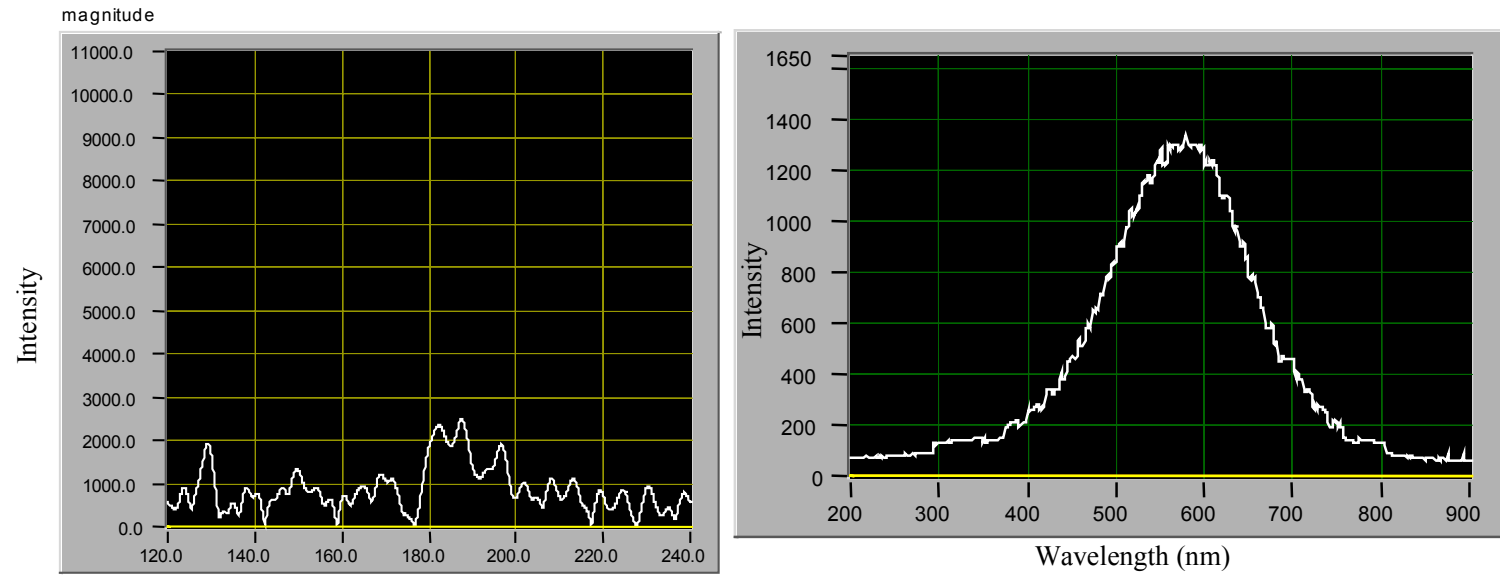

Gap (microns)

(e) $365 \mu \mathrm{m} / \mathrm{sec}$ 


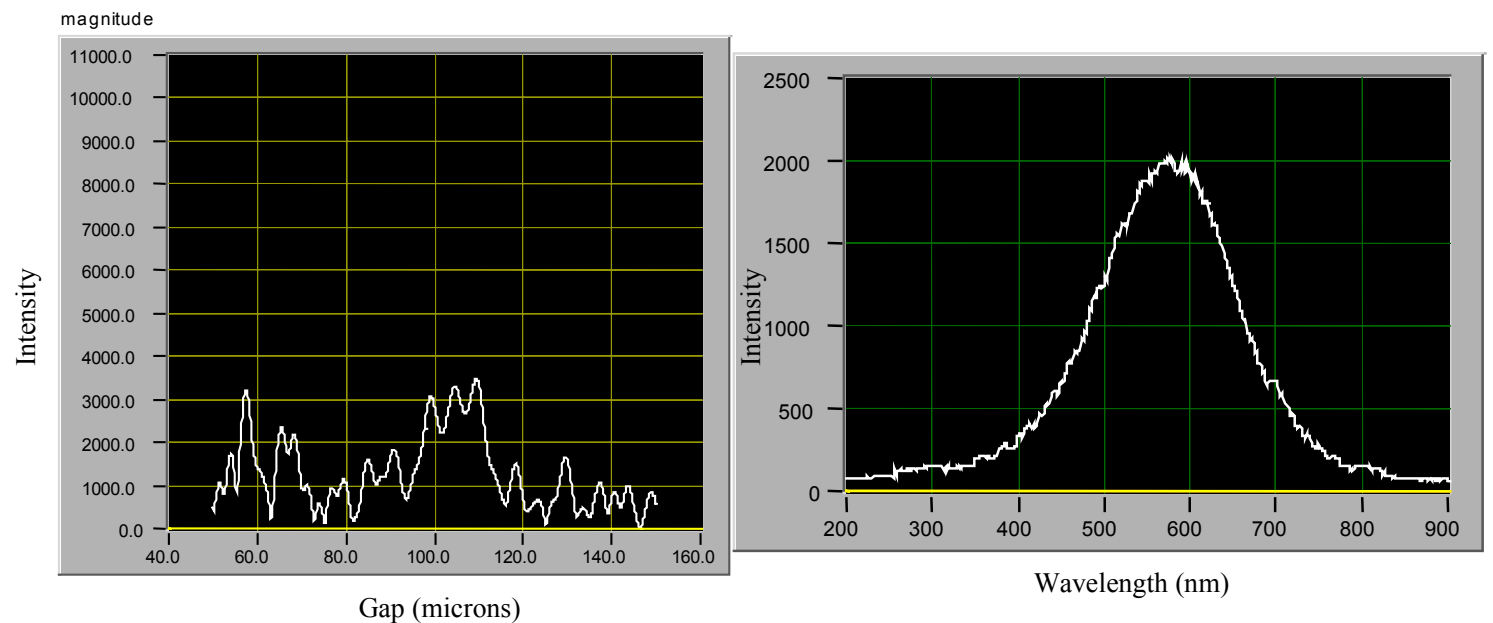

(f) $385 \mu \mathrm{m} / \mathrm{sec}$

Figure 173. PZT experimental results for a range of gap speeds with the stored spectra for each speed on the right and the resulting FFT calculation on the left.

Another experiment also confirms that the loss of fringe contrast for a combined movement and misalignment is not only due to smearing. The Version $5 \mathrm{~b}$ skin friction sensor was mounted on a vibration table and tested at various magnitudes and frequencies. The experiment demonstrated smearing of the skin friction sensor at various conditions, yet the most interesting result was with the fast spectrometer used in the accelerometer experiment above, and this is presented in Figure 174. In the first series of spectra, the sensor loses some fringe contrast and then it returns. This loss occurs when the reflector is traveling through the linear part of its oscillation, where its speed is the greatest. However, when the fast spectrometer is used, the same event and result occurs, as can been seen in the lower section of the figure. The accelerometer experiment demonstrated the ability of the fast spectrometer to reduce or eliminate the reduction of contrast, yet it did not in the current test. As smearing was the only effect present in the accelerometer, and the fast spectrometer eliminated the loss of contrast, the conclusion is that there is some other effect in this swinging motion, other than smearing, that reduces the fringe contrast. 


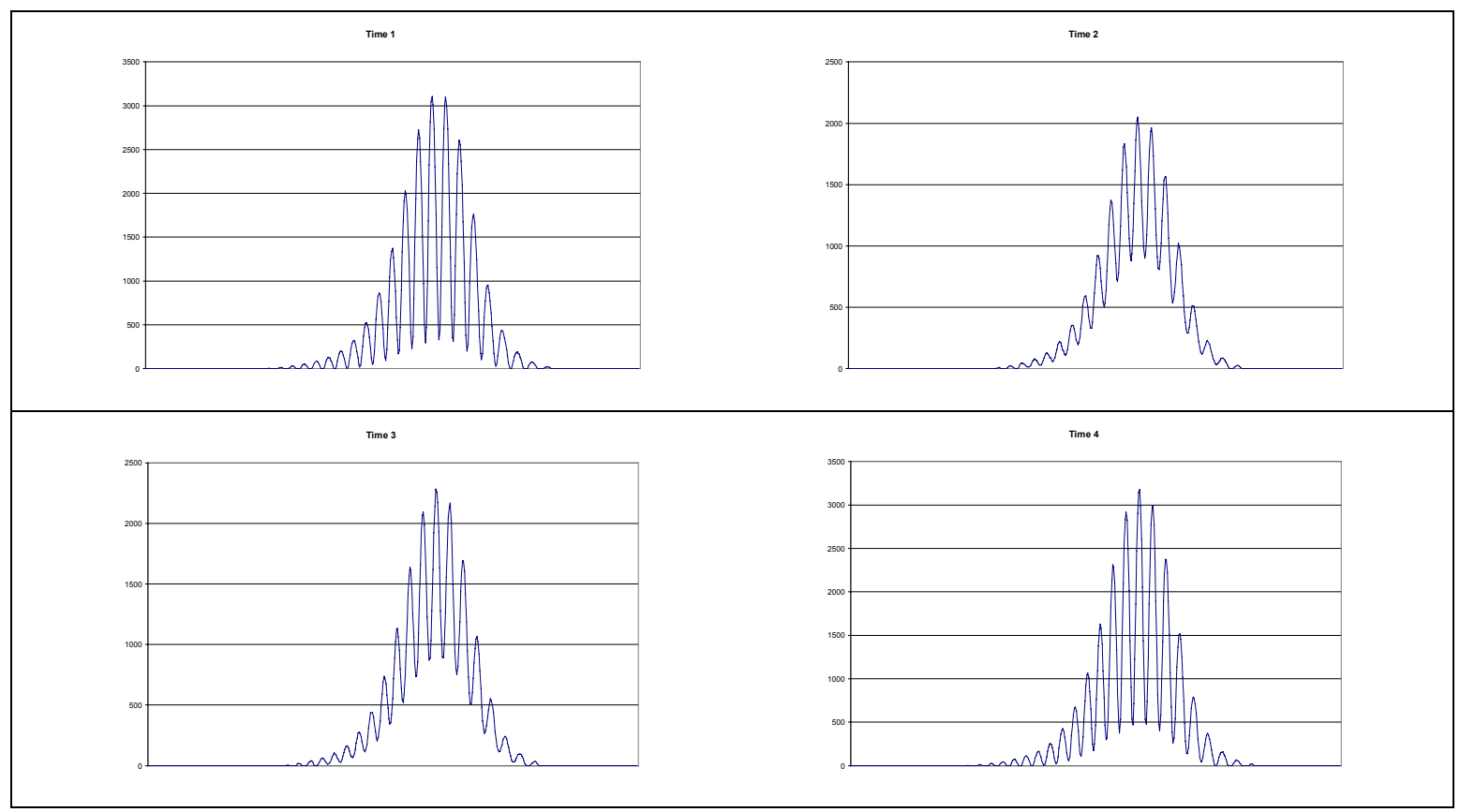

Nominal spectrometer

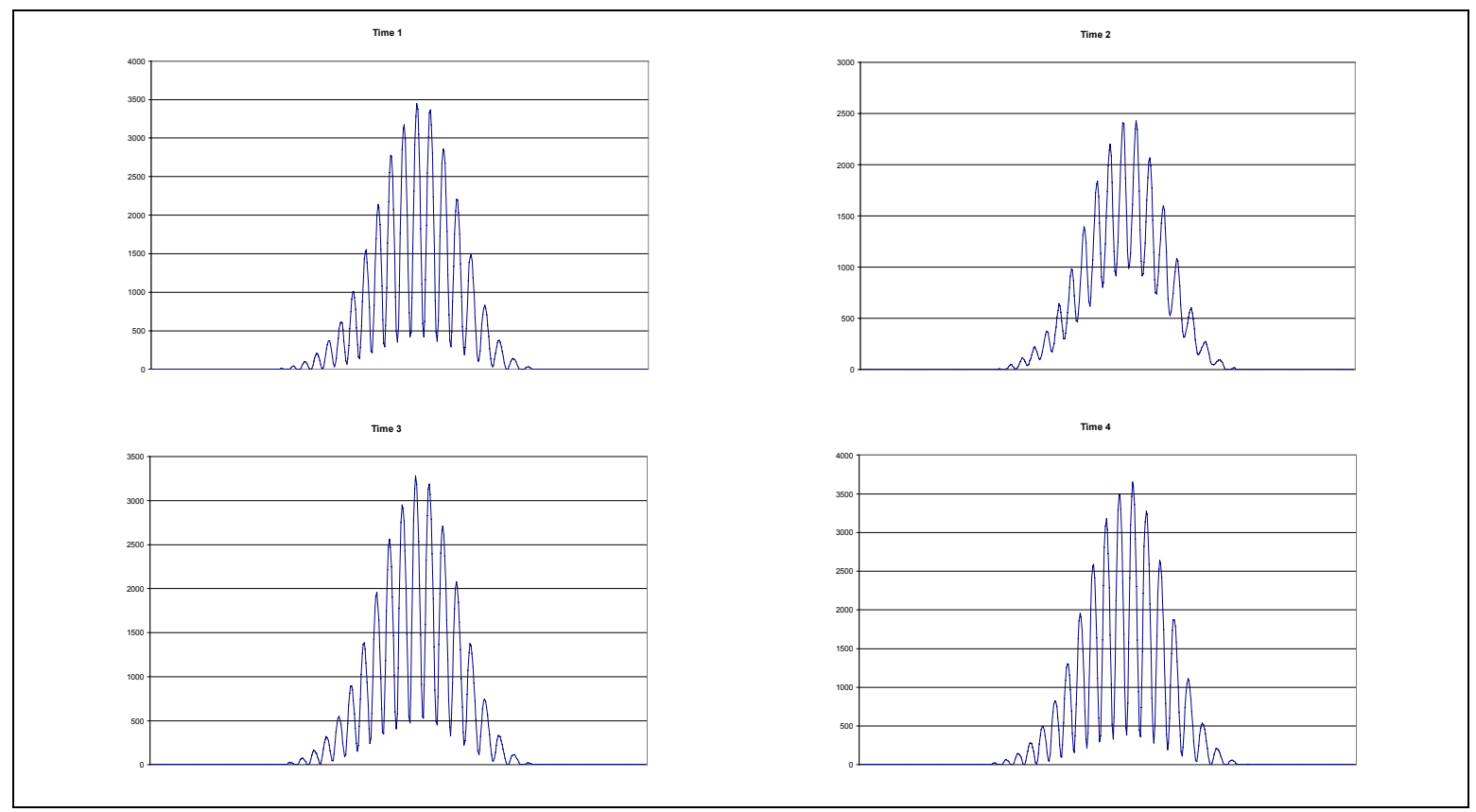

\section{Fast spectrometer}

Figure 174. Vibration data of skin friction sensor demonstrating that reduction of fringe contrast due to more that spectral smearing. For the nominal spectrometer, time 1 is a normal spectrum, time 2 and 3 have a reduction in fringe contrast, and time 4 is back to normal. For the fast spectrometer, time 1 is a normal spectrum, time 2 has a reduction in fringe contrast, while time 3 and 4 are normal spectra. 


\section{Numerical Studies}

Total system simulations were created for the pressure and skin friction sensors. The models differed in the error sources and magnitudes that were included. The pressure sensor included the system noise, smearing, and surface roughness. The skin friction sensor added an angular misalignment that was tied to the smearing to the model. These simulation proved to be successful in matching the experimental results seen in both the lab tests described in this chapter and qualities of the wind tunnel tests in Chapter 5.

\subsubsection{Pressure Sensor}

The pressure sensor model uses the nominal system error sources delineated above, a surface roughness variance of 9.353 (matching the AFM measurements of the diaphragm) and smearing defined by gap speeds from 0 to $350 \mu \mathrm{m} / \mathrm{sec}$. The performance predicted by the model in terms of absolute error is presented in Figure 175. The detailed view in Figure 175a shows a stationary error due to the system noise and surface roughness of approximately $8 \mathrm{~nm}$. This error increases as gap speed increases, but is still below $20 \mathrm{~nm}$ (small compared to full scale) at a speed of $200 \mu \mathrm{m} / \mathrm{sec}$. The interesting result is in Figure 175b. The error does not roll off like in all the previous simulations. An abrupt change occurs at $325 \mu \mathrm{m} / \mathrm{sec}$. This step change has been seen numerous times in tunnel data with this sensor; an example is in Figure 184. The fringe contrast, presented in Figure 176, shows the familiar reduction in contrast as the gap speed increases. It should be noted that the step change in the pressure sensor output occurs at a fringe contrast of 0.06 , but the large error increase begins around $275 \mu \mathrm{m} / \mathrm{sec}$, producing a contrast of a little above 0.10 . This is more confirmation of a fringe contrast limit for the spectral interrogation system to accurately determine optical gaps. 
Total System Simulation of Fiber Optic Pressure Sensor

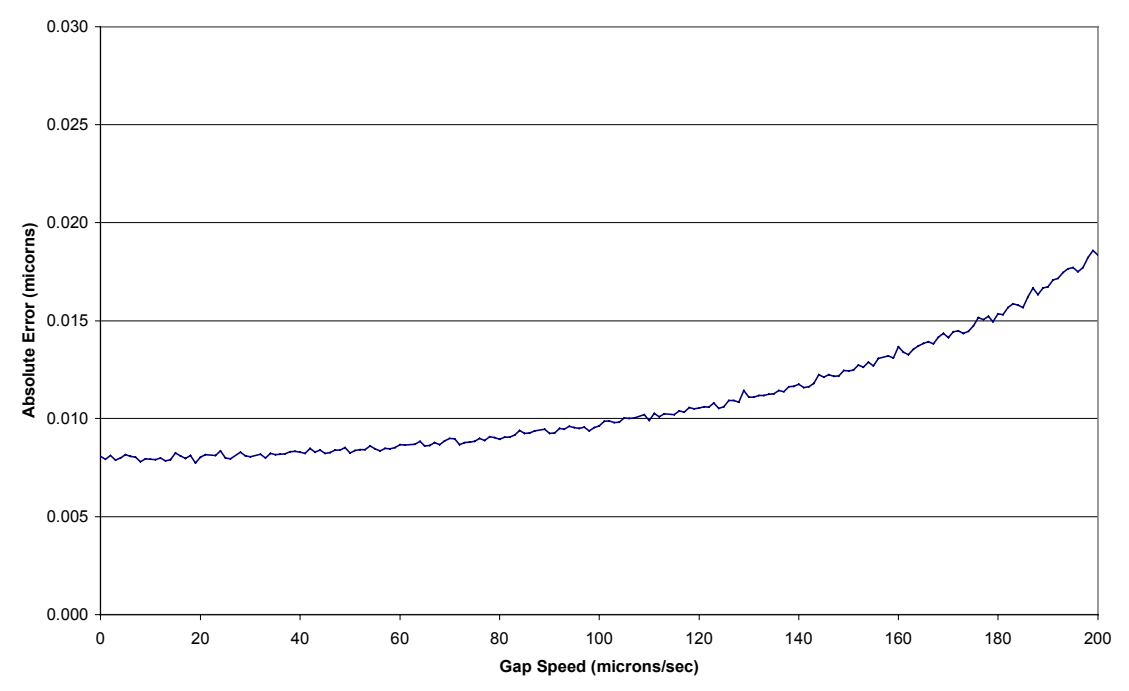

(a)

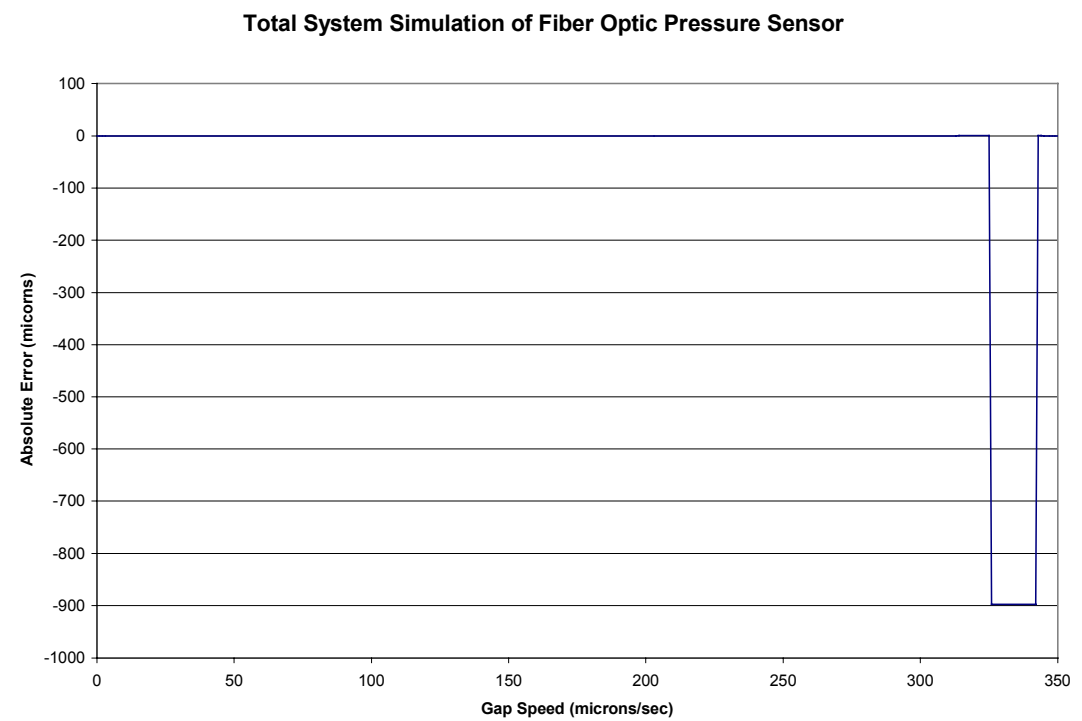

(b)

Figure 175. Numerical simulation of total system error for the fiber optic pressure sensor using the spectral interrogation signal processing system (using nominal system errors and a standard deviation of $9.353 \mathrm{~nm}$ for surface roughness), a) detail of absolute error and b) overall system error. 


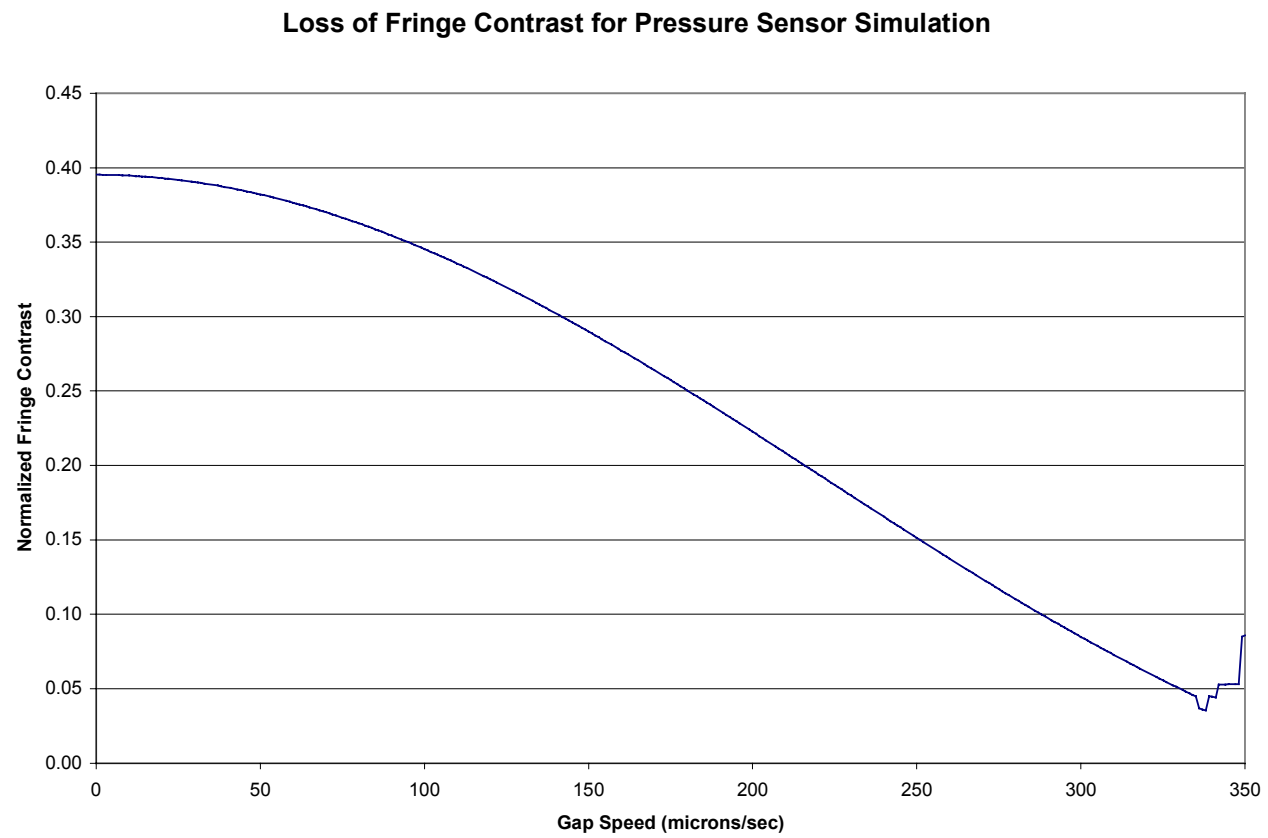

Figure 176. Numerical simulation of loss of fringe contrast for the fiber optic pressure sensor using the spectral interrogation signal processing system (using nominal system errors and a standard deviation of $9.353 \mathrm{~nm}$ for surface roughness).

\subsubsection{Skin Friction Sensor}

A model of the skin friction sensor was also developed. This model included the nominal system noise, surface roughness with a variance of 0.025 (typical of the AFM measurements) gap movement to produce smearing, and angle changes tied to the movement. The simulations proceeded as depicted in Figure 177. The surface starts flat in reference to the fiber, and as it moves away, it rotates by the same amount that the Version 6 skin friction sensor does. 

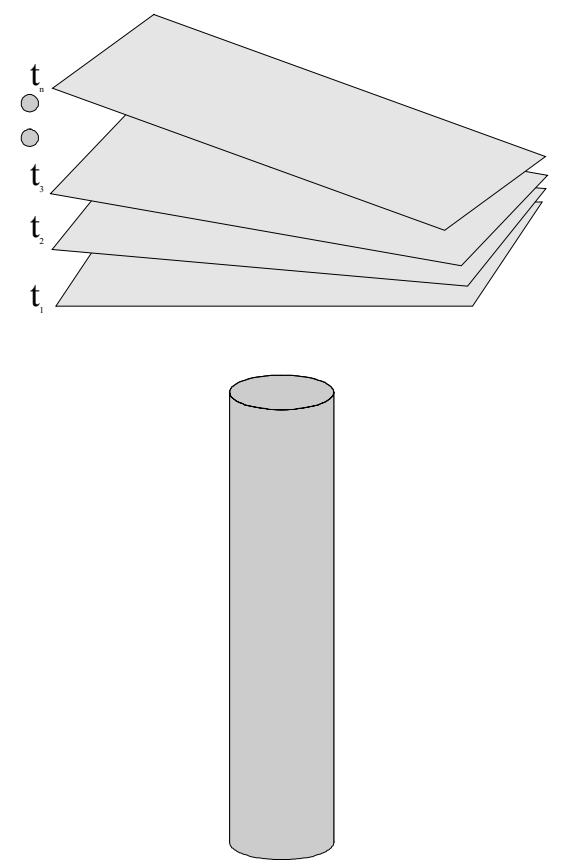

Figure 177. Depiction of skin friction sensor total system numerical simulation showing the progression of the reflection surface during the integration time of the CCD array.

The absolute error results are presented in Figure 178. As with the pressure sensor, there is little error for gap speed up to $200 \mu \mathrm{m} / \mathrm{sec}$. As the gap speed is increased further, the error increases to values similar to those obtained before. However, the change is much less abrupt with this model than with the pressure sensor or the smearing results above. This result agrees qualitatively with the result of the PZT experiment. Also, the speed at which the maximum error occurs has been lowered to $300 \mu \mathrm{m} / \mathrm{sec}$ from the 325 seen in the pressure sensor. 


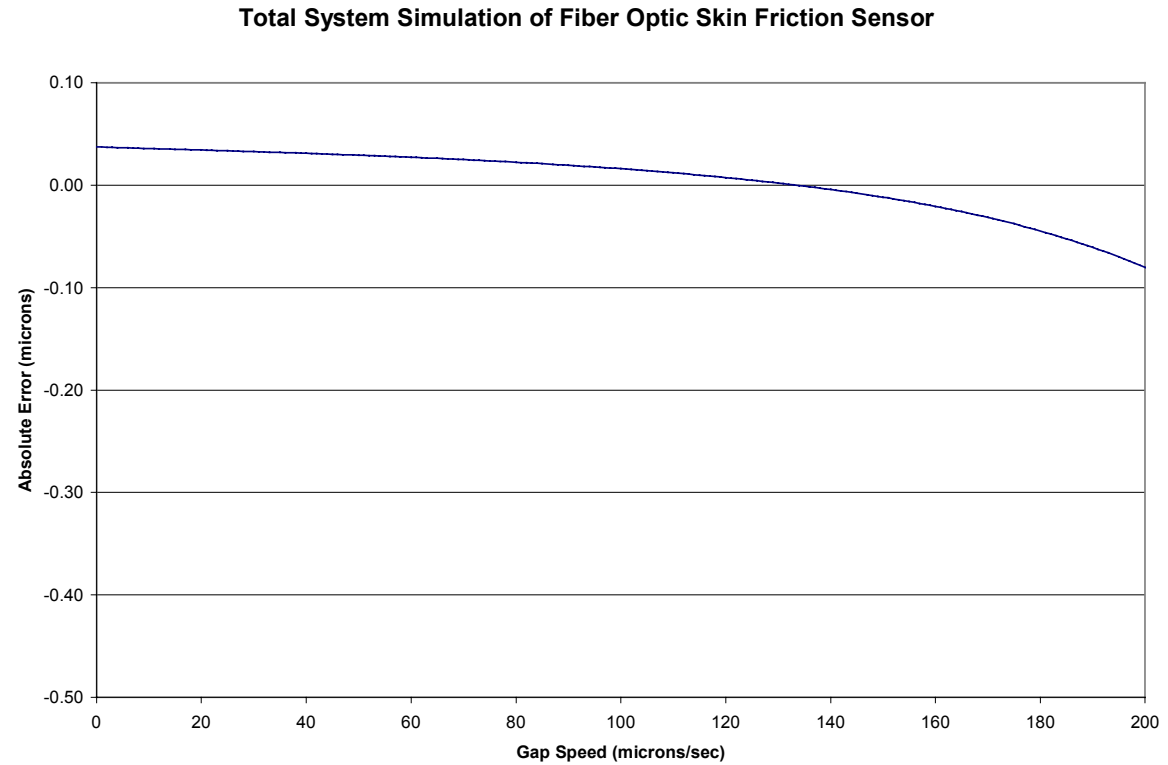

(a)

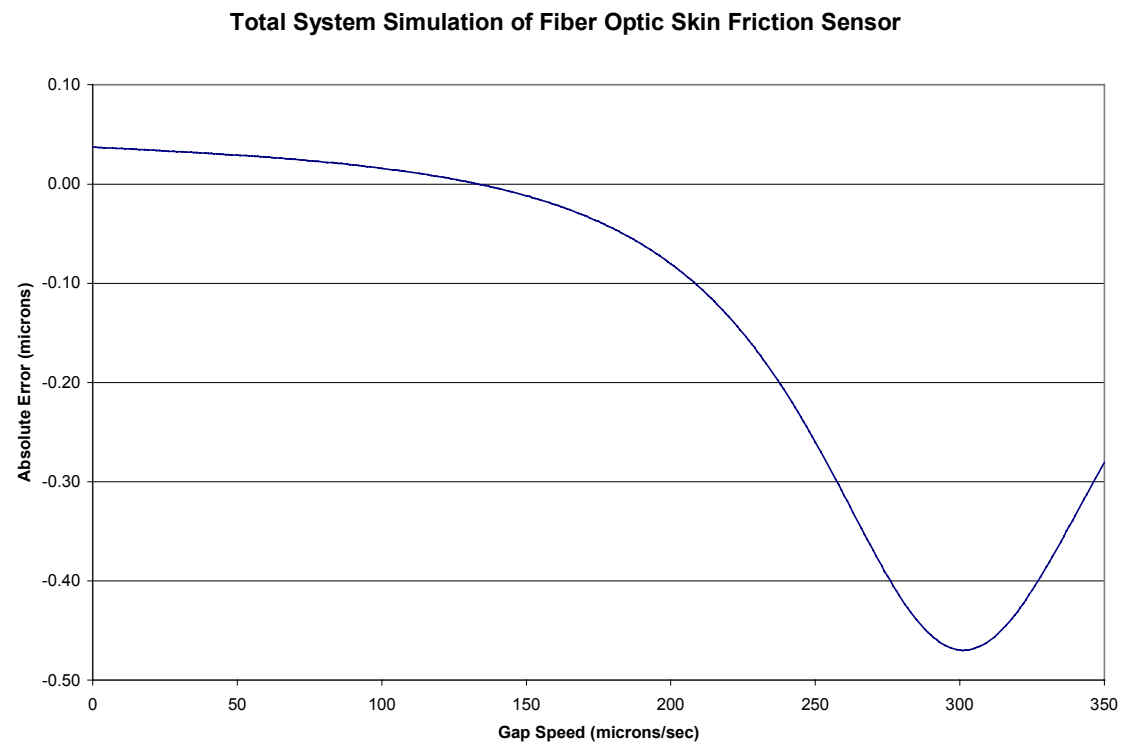

(b)

Figure 178. Numerical simulation of total system error for the fiber optic skin friction sensor (using nominal system errors, a standard deviation of $25.0 \mathrm{~nm}$ for surface roughness, and angular movement tied to gap movement), a) detail of absolute error and b) overall system error. 
The normalized contrast appears to asymptote at 0.12 , never reaching the 0.10 value as shown in Figure 179. This fact may explain why the error result did not increase dramatically, yet produced significant errors over a large range.

Loss of Fringe Contrast for Skin Friction Sensor Simulation

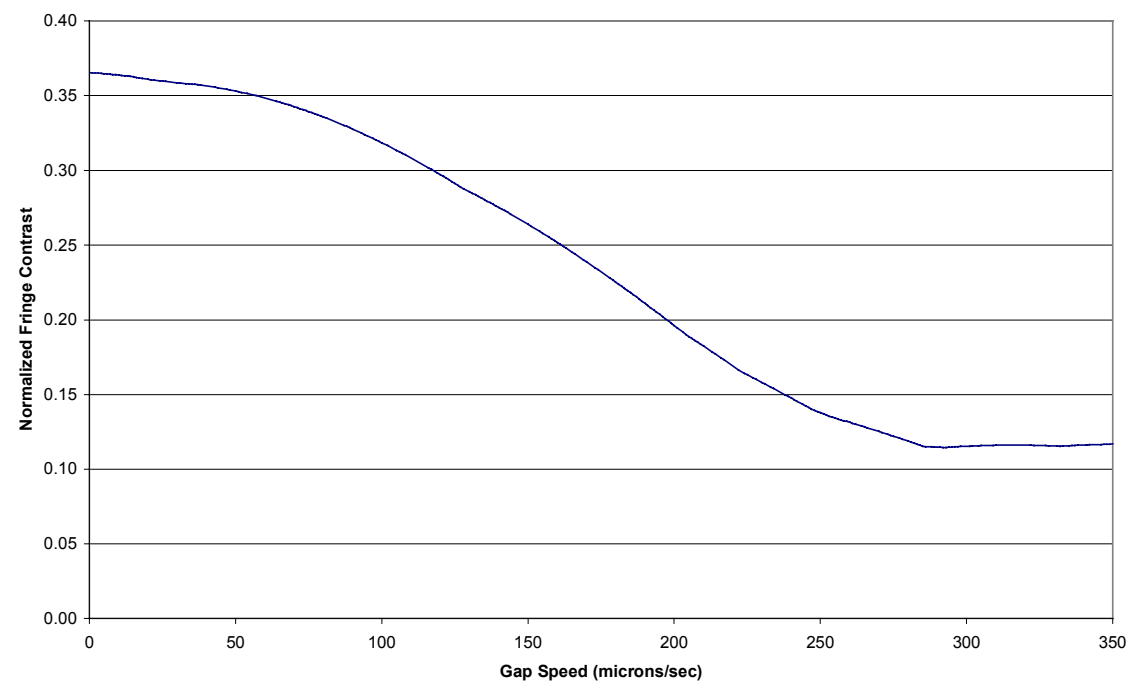

Figure 179. Numerical simulation of loss of normalized fringe contrast for the skin friction sensor (using nominal system errors, a standard deviation of $25.0 \mathrm{~nm}$ for surface roughness, and angular movement tied to gap movement),

In summary, numerical models were constructed for the pressure and skin friction sensors to predict combined error sources and magnitudes during operation. The pressure sensor model predicted a result seen many times in the wind tunnel, while the skin friction model reinforced lab experimental results.

\subsection{Relevancy to Developed Sensors}

The simulations above provided many insights into the operation and errors of the sensor, yet the results only give a perfect numerical indication of what may be occurring in the imperfect real world. Given the results of the simulations, a re-examination of the tunnel data was performed to determine and/or explain the operating boundaries of these sensors in aerospace environments. 
It is clear from the prior results that fringe contrast is an important indicator of uncertainty in the optical gap determined by the spectral interrogation system. As was mentioned in Chapter 5, there was an apparent correlation between the aerodynamic energy of the flow and the noise of the signal. Therefore, a software package was created to re-examine the wind tunnel results by calculating the time-history of fringe contrast for each test. Sample output from a pressure sensor test demonstrates the operation of the software (Figure 180). It would be difficult to determine the pedestal of the fringe pattern in an automatic manner, especially when one considers the possibility of total smearing. The developed routine uses a high-pass filter to take the fringe pattern in Figure 180a and remove the pedestal. It is simple to determine the fringe contrast of the remaining signal as a maximum and minimum of the filtered-spectrum array. Figure $180 \mathrm{~b}$ is a demonstration of the software for a normal fringe pattern, and Figure 180c and $\mathrm{d}$ is for a smeared signal.

Using this software, the recent tunnel data was examined to attempt to find a correlation between: 1) the change in gap during a test and the change of fringe contrast, 2) the change of fringe contrast and the change in a correction factor in the algorithm that is used to obtain higher resolution, 3) the absolute value of the normalized fringe contrast and the variance of the gap change, and 4) absolute value of the normalized fringe contrast and the variance of the correction factor. The analysis demonstrates all of these are true, providing insight to possible improvements for the sensors and/or signal processing system and to the algorithm. Both the pressure and skin friction sensors show good correlation to the above criteria with a flow condition functionality. 


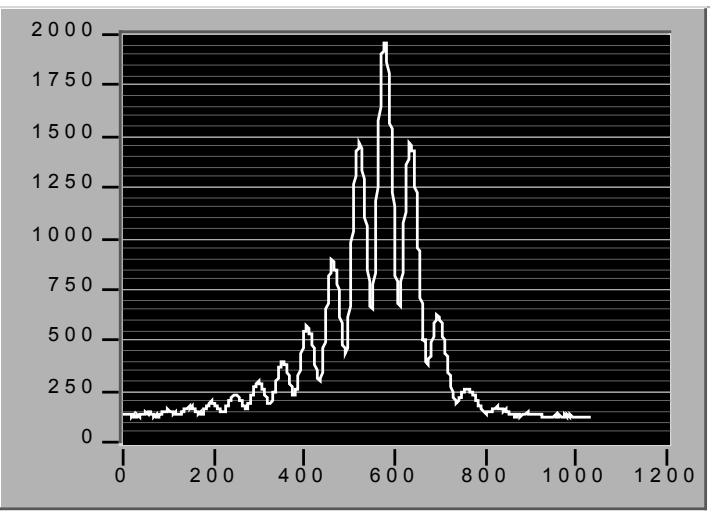

(a)

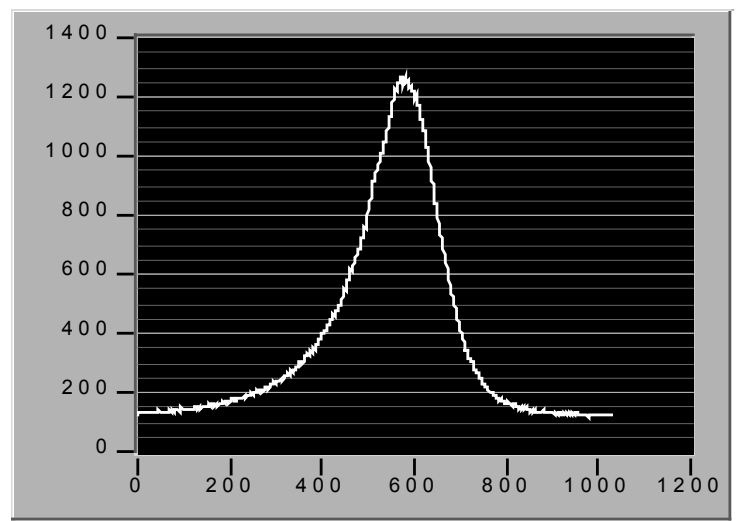

(c)

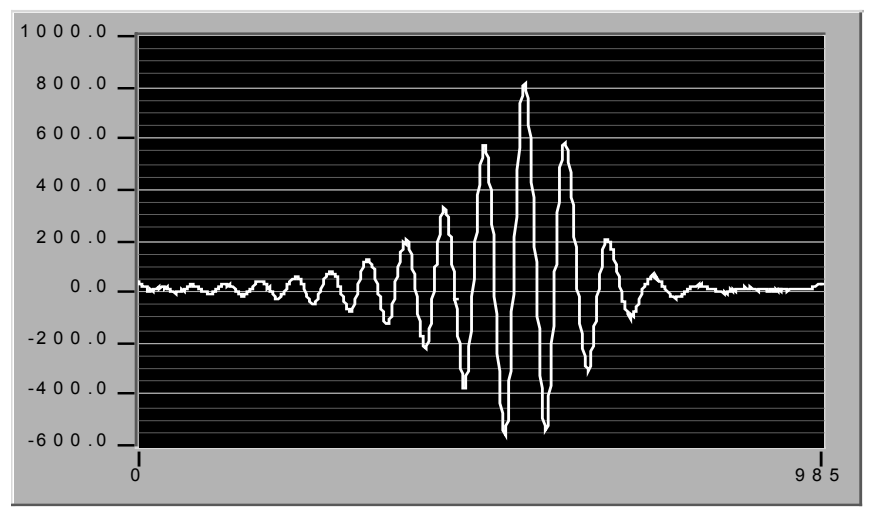

(b)

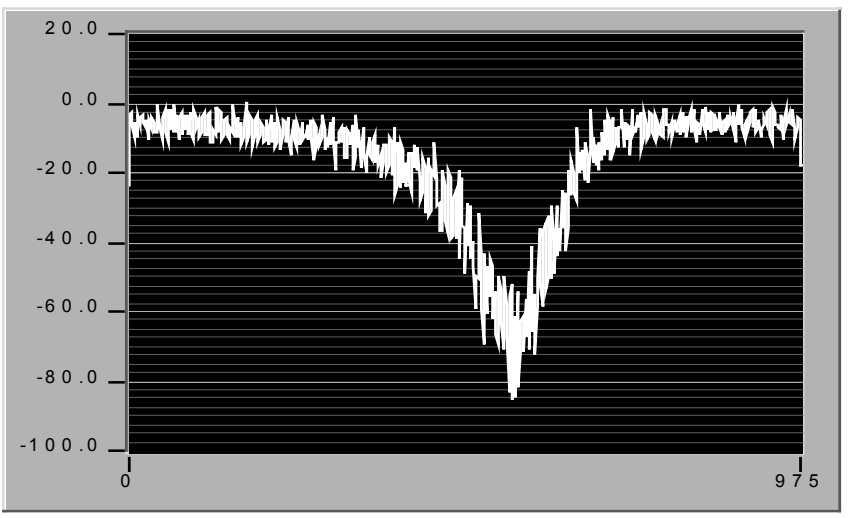

(d)

Figure 180. Example of fringe contrast calculation software for a pressure sensor, a) spectrum of stationary gap, b) fringe contrast of stationary gap, c) spectrum of totally smeared spectrum and d) fringe contrast of totally smeared spectrum.

\subsubsection{Pressure Sensors}

Although the numerical simulations gave useful information about operational criteria for the sensors, a large amount of experimental data needed to be examined to actually determine limits of the sensors to aerodynamic inputs. For the case of the pressure sensor, only two real conditions existed for consideration - the shock/no-shock cases in a Mach 3.0 flow. These conditions provide a low noise/high noise comparison with which the effect of the aerodynamic input could be correlated with fringe contrast and the algorithm correction factor. 
Figure 181 shows the output of the fiber optic pressure sensor in a flat plate, Mach 3.0 flow. On can see the large noise, which is signal processing error, during tunnel startup and shut-down. In addition, there is a noise component during the test that appears as spikes on the pressure signal. A close examination of the plot shows a higher noise for the downstream sensor, Press 2, which may be due to disturbance of the boundary layer by the first pressure sensor.

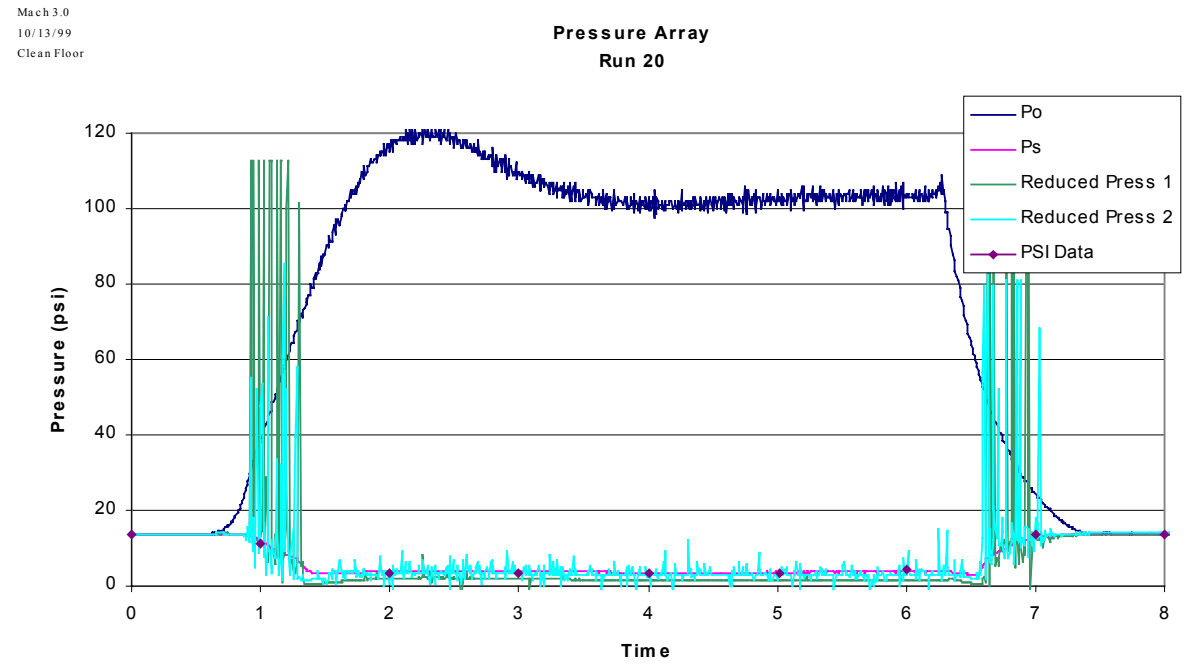

\section{Figure 181. Run 20 of the micromachined pressure sensor array in a Mach 3.0 flow without the shock.}

The fringe contrast for this test was calculated for each sensor, and this is presented in Figure 182 along with the raw optical gaps determined by the spectral interrogation system. There is a clear correlation between the absolute value of fringe contrast and the RMS fluctuations of the gap signal. As the tunnel starts and the diaphragm is excited to resonance, the fringe contrast is lost due to spectral smearing. The consequence, as predicted in the simulation above, is large errors in determining the gap, which appears as a large noise content. In addition, during the run, the connection between contrast and variations in the gap estimate can be seen. In Figure 182a, which is of the upstream sensor, the fringe contrast, although low, does not bottom out and remains above the 0.10 level for the majority of the test. In fact, the only noise in the gap estimate seen during the steady part of the run is at the beginning when the contrast is below 0.10. The downstream sensor, results in Figure 182b, has lower fringe contrast and in turn has more noise, or error, in the gap estimates. The contrast remains below 
0.10 for the steady part of the test, yet the system never loses complete track of the gap. For correlation purposes, these tests were split into sections to examine. The variances of the gap and correction factor were calculated for before/after the test, during startup/shut-down, and then during the actual tests. These sections provided separate fringe contrast and variance points for later correlation. This procedure was repeated for all the pressure sensor tests.

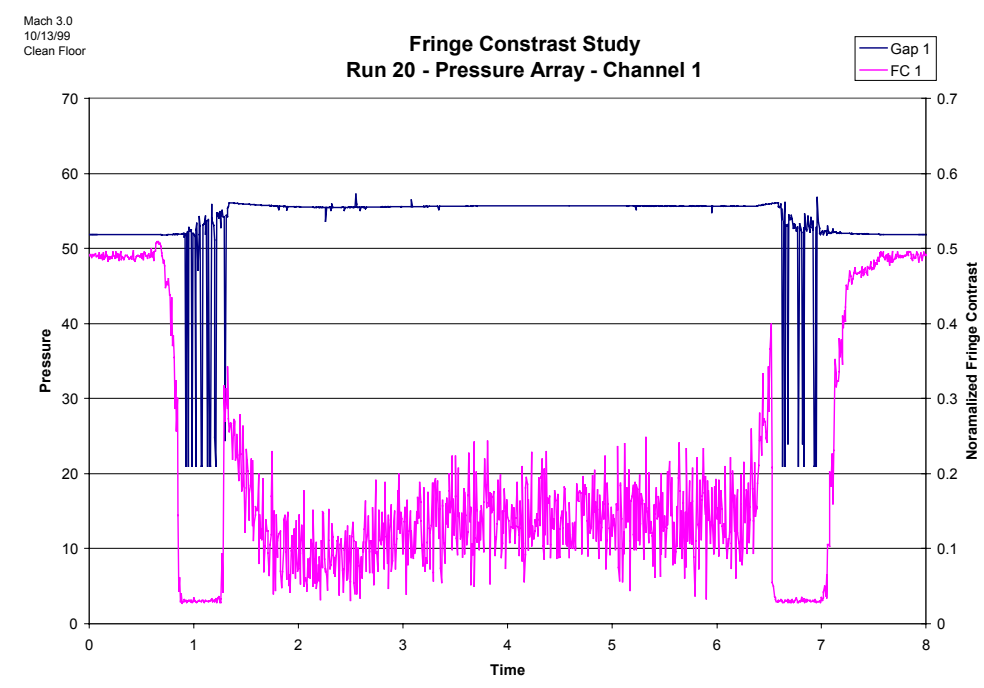

(a)

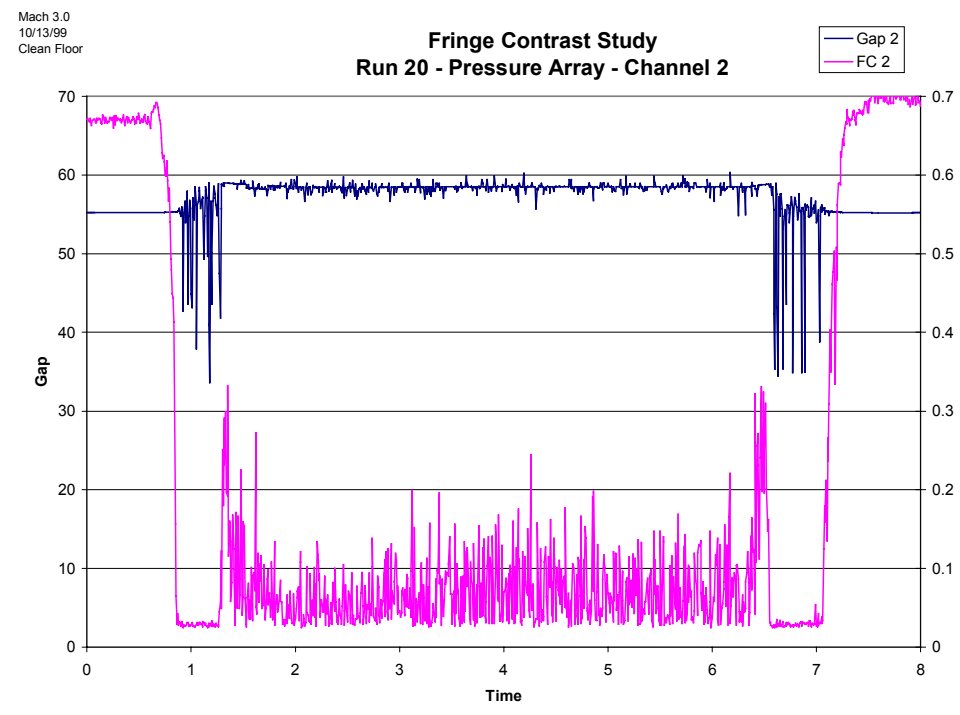

(b)

Figure 182. Comparison of fringe contrast to change in gap estimate for Run 20 of the micromachined pressure array in a Mach 3.0 flow without the shock, a) upstream pressure sensor and b) downstream pressure sensor. 
Below, in Figure 183, the high noise case of pressure measurements in a shock/boundary layer interaction is presented again. Clearly the sensors are smearing during the entire test, as the shock excited the diaphragm into resonance. As stated in Chapter 4, a close examination of the results will show that the first sensor which is ahead of the shock tracks the true pressure rather well, even with the high noise. The downstream sensor is almost always smeared, with only intermittent points in the correct range.

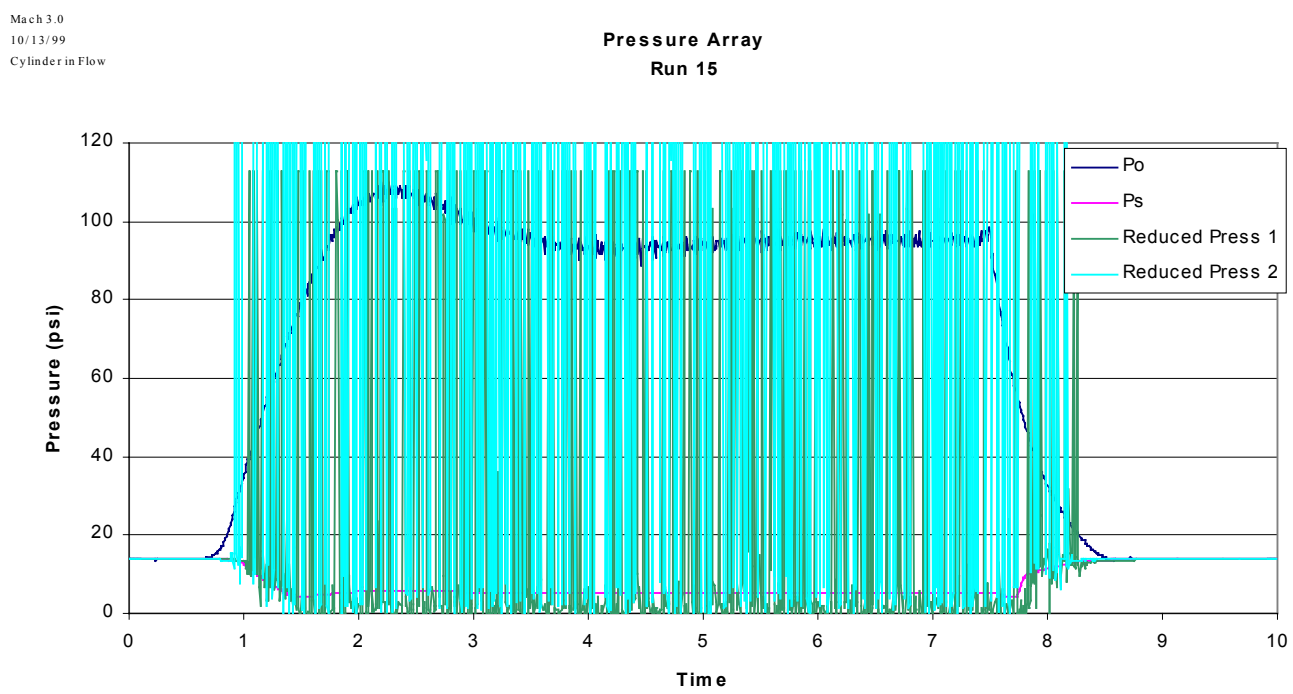

\section{Figure 183. Run 15 of the micromachined pressure sensor array in a Mach 3.0 flow with the shock.}

The fringe contrast-gap estimate comparison plots are shown in Figure 184. For both cases, the fringe contrast remains almost completely a Gaussian during the entire test, indicating that the signal has been completely smeared and accounting for the high noise content in the gap estimation. The straight line seen in the output for the gap is not a true estimate but the signal hitting a rail placed in the signal processing system to limit such errors. 


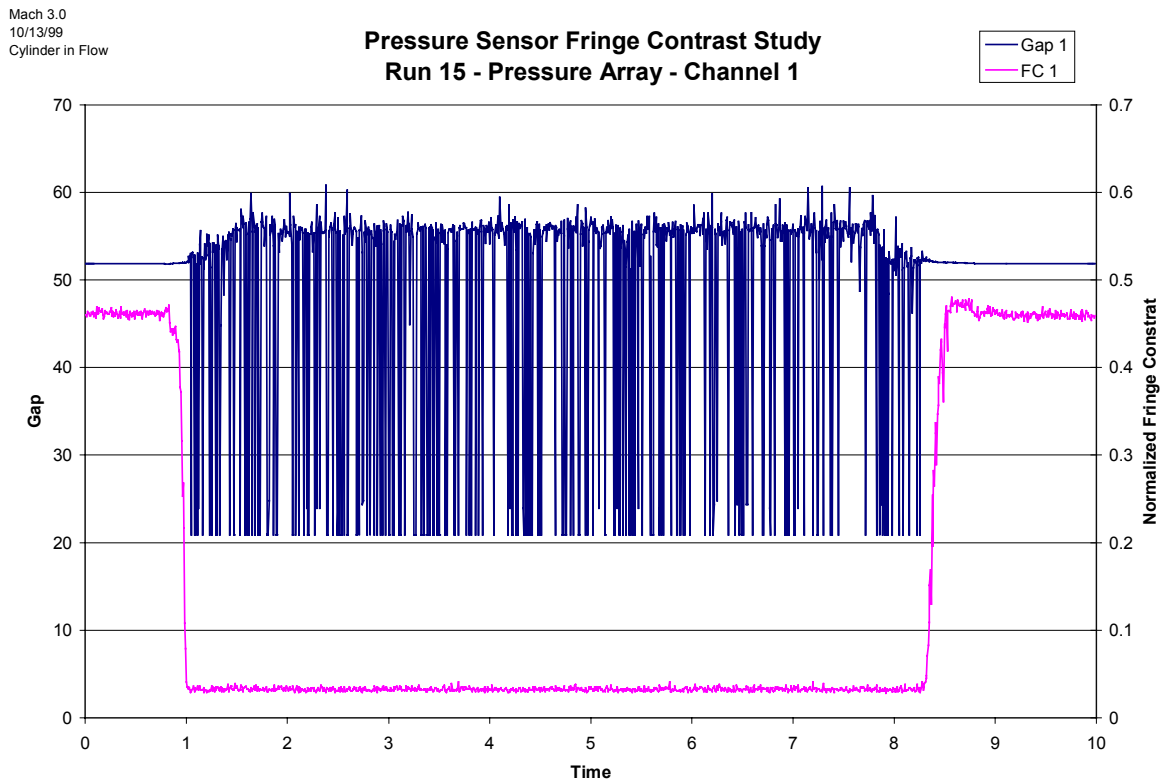

(a)

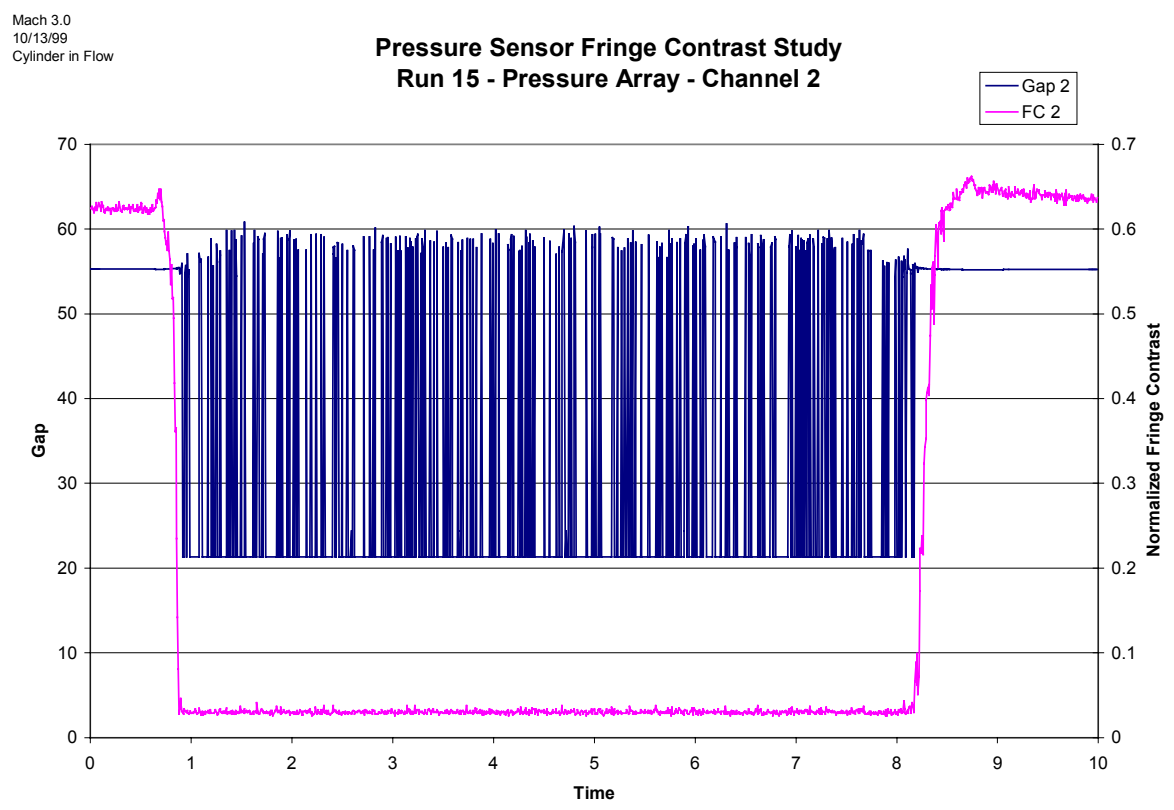

(b)

Figure 184. Comparison of fringe contrast to change in gap estimate for Run 15 of the micromachined pressure array in a Mach 3.0 flow with the shock, a) pressure sensor upstream of shock and b) pressure sensor downstream of shock.

Also of interest is the comparison of the fringe contrast and the correction factor for the algorithm. As stated above, a correlation between this correction and flow 
conditions had been suspected, but not confirmed. It is clear from the examination of Figure 185 and similar tests, that the loss of fringe contrast produces large variation in the correction factor, which can be more of a problem than the algorithm without the correction.

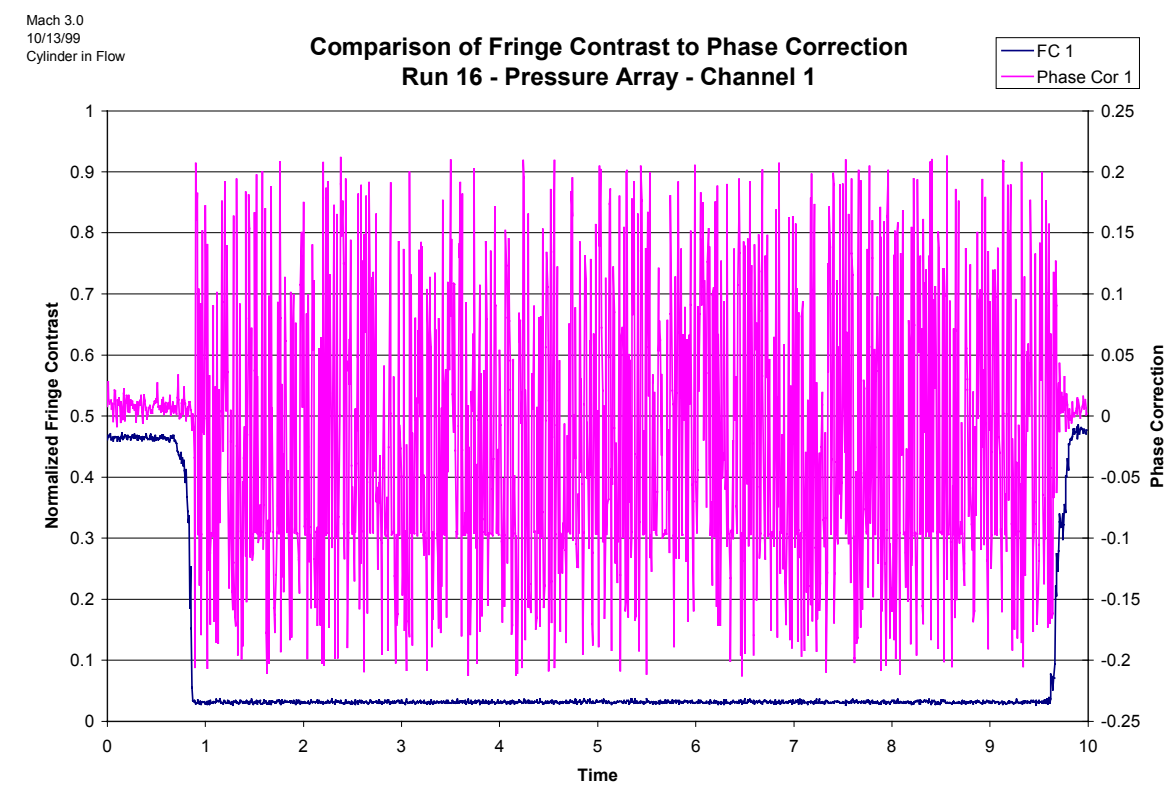

Figure 185. Comparison of fringe contrast and algorithm correction factor in Run 20 of the pressure sensor array in a Mach 3.0 flow.

The variance of the gap estimate and the correction factor were calculated, as described, for all the pressure sensor tests in sections and plotted against the absolute value of normalized fringe contrast. Figure 186 and Figure 188 present the results on a logarithmic chart. There is a clear trend as the fringe contrast decreases to everincreasing fluctuation in the gap estimate and correction factor. The points on the plot are sectioned off to the conditions that produced them. The more unsteady the flow is, such as start up or oscillating shock, the higher the error is. The plot of the correction factor variance to fringe contrast also demonstrates the same trend. Figure 187 shows the fringe contrast at which the errors in the gap estimate increase, approximately 0.030 . This value appears to be well below the predicted value of 0.10 . However, the extreme variances at the lower fringe contrasts (over 6 orders of magnitude greater than the 200 $\mathrm{pm}$ resolution of the system) skew the interpretation. In fact, the variance of the gap estimate at the 0.10 point is over 1000 times the resolution. 


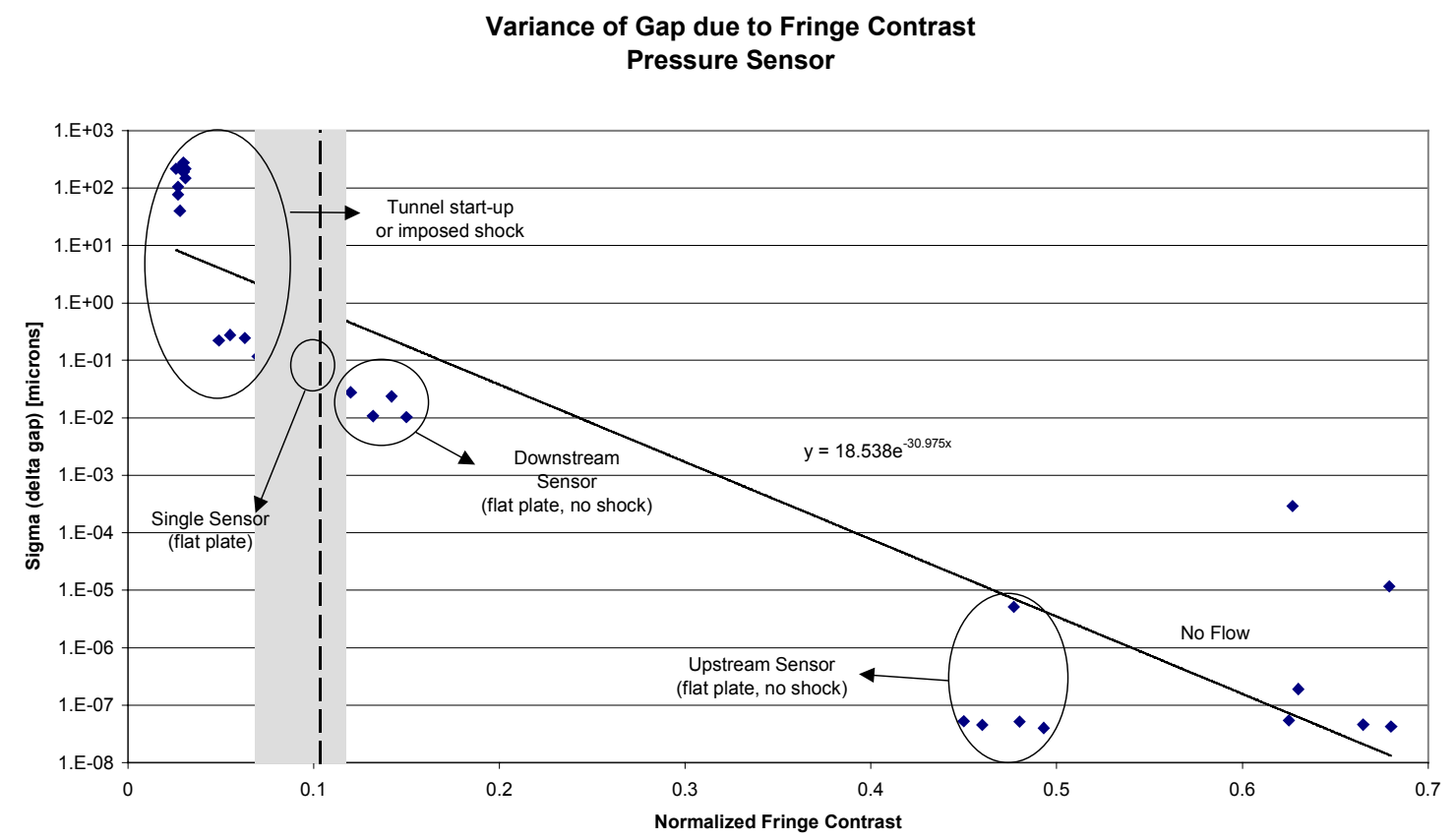

Figure 186. Semi-log plot of fringe contrast vs. variance of gap change for pressure sensor in various flow conditions.

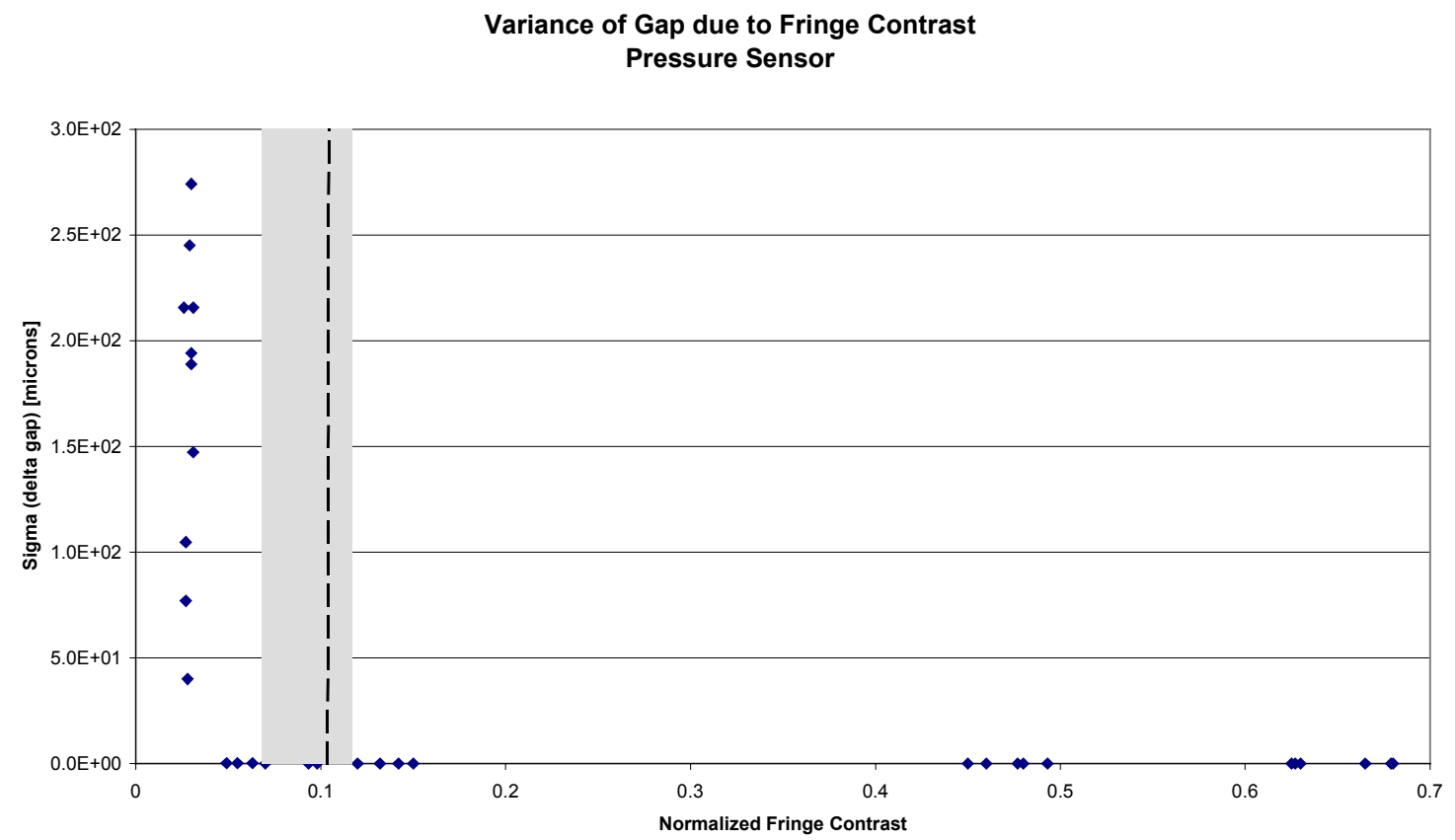

Figure 187. Linear plot of fringe contrast vs. variance of gap change for pressure sensor in various flow conditions showing fringe contrast cut-off. 


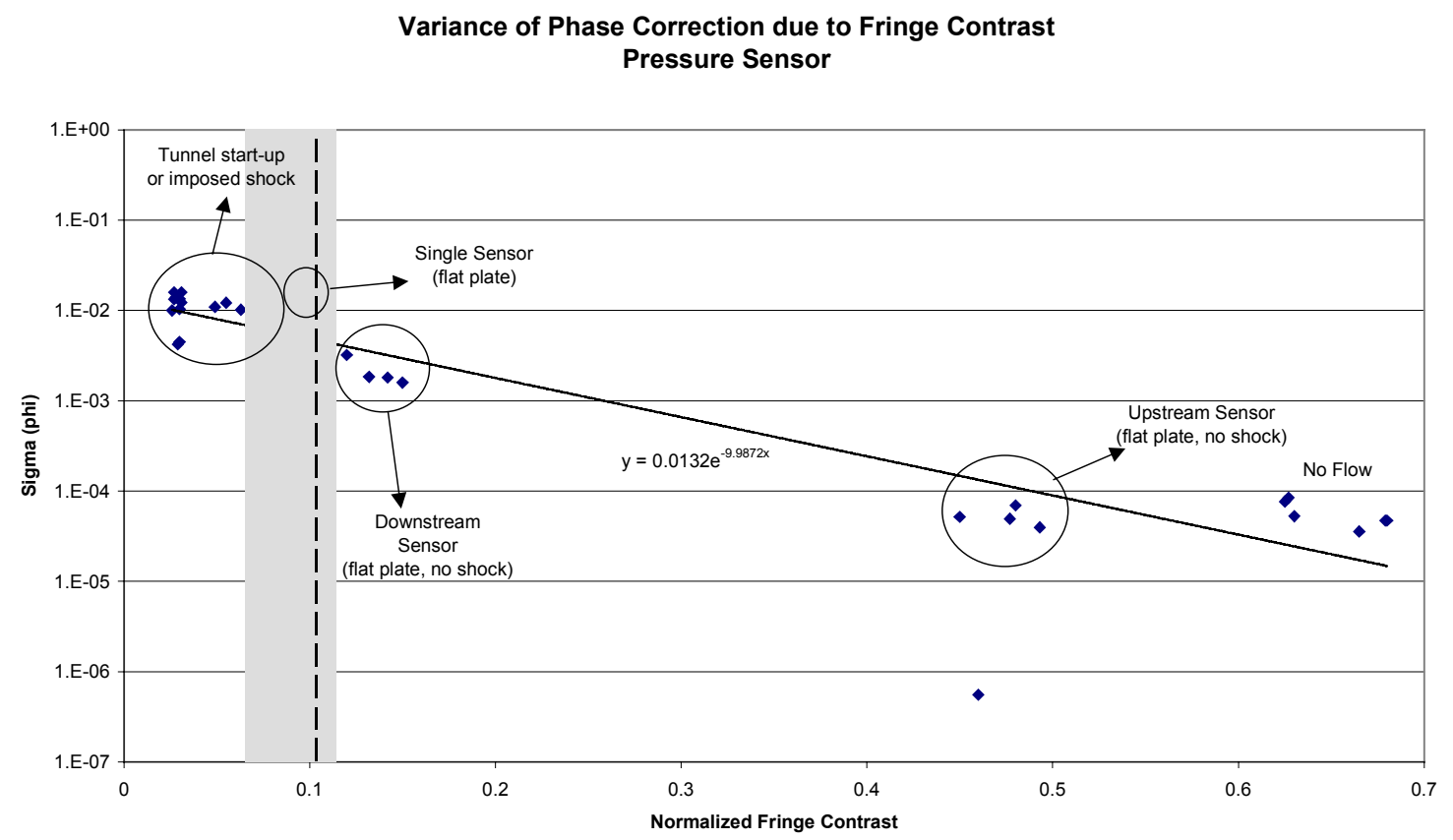

Figure 188. Plot of fringe contrast vs. variance of algorithm correction factor for pressure sensor in various flow conditions.

Cross-correlation of the change in gap to fringe contrast and the fringe contrast to the correction factor, $\phi$, were calculated for each pressure test. For the majority of the cases, a high degree of correlation exists, as seen in Table 10.

Table 10. Correlation of gap change, fringe contrast change, and correction factor change for the pressure sensor.

\begin{tabular}{|c|c|c|c|c|c|}
\hline Sensor & Description & Test & Channel & $\begin{array}{c}\text { Correlation } \\
\text { (Agap, fringe contrast) }\end{array}$ & $\begin{array}{c}\text { Correlation } \\
(\phi, \text { fringe contrast })\end{array}$ \\
\hline Pressure & Mach 3 & 15 & 1 & 0.1715 & 0.1715 \\
\hline \multirow[t]{11}{*}{ Array } & Shock & & 2 & 0.6820 & 0.8119 \\
\hline & & 16 & 1 & 0.0905 & 0.1073 \\
\hline & & & 2 & 0.5238 & 0.7049 \\
\hline & Mach 3 & 18 & 1 & 0.1816 & 0.0091 \\
\hline & No shock & & 2 & 0.4047 & 0.5368 \\
\hline & & 19 & 1 & 0.1481 & 0.0036 \\
\hline & & & 2 & 0.2785 & 0.5878 \\
\hline & & 20 & 1 & 0.2315 & 0.0143 \\
\hline & & & 2 & 0.4566 & 0.6179 \\
\hline & & 21 & 1 & 0.2003 & 0.0825 \\
\hline & & & 2 & 0.4084 & 0.6195 \\
\hline
\end{tabular}


In summary, the correlation between the fringe contrast and errors in the gap estimate has been established. In addition, the aerodynamic input in reference to the errors has been explained. The contrast becomes less as the unsteadiness of the flow increases, which drives the sensor toward resonance. The error in the gap estimate becomes excessive near an absolute fringe contrast of 0.10 , which is the level predicted by the simulations.

\subsubsection{Skin Friction Sensors}

Also examined in detail were the tests of the Version 5a skin friction sensor in an attempt to determine a link between the aerodynamic energy and the error sources. As described above, various tests of increasing flow energy were performed, indicating that the noise of the output is correlated with this flow energy. Figure 189, presents the results of a test of the skin friction sensor in a Mach 2.4 flow at a total pressure of 345 $\mathrm{kPa}(50 \mathrm{psi})$. The magnitude of the results are about $40 \%$ high, but the important fact here is that the fluctuation of the gap estimate output shown here as shear level is low.

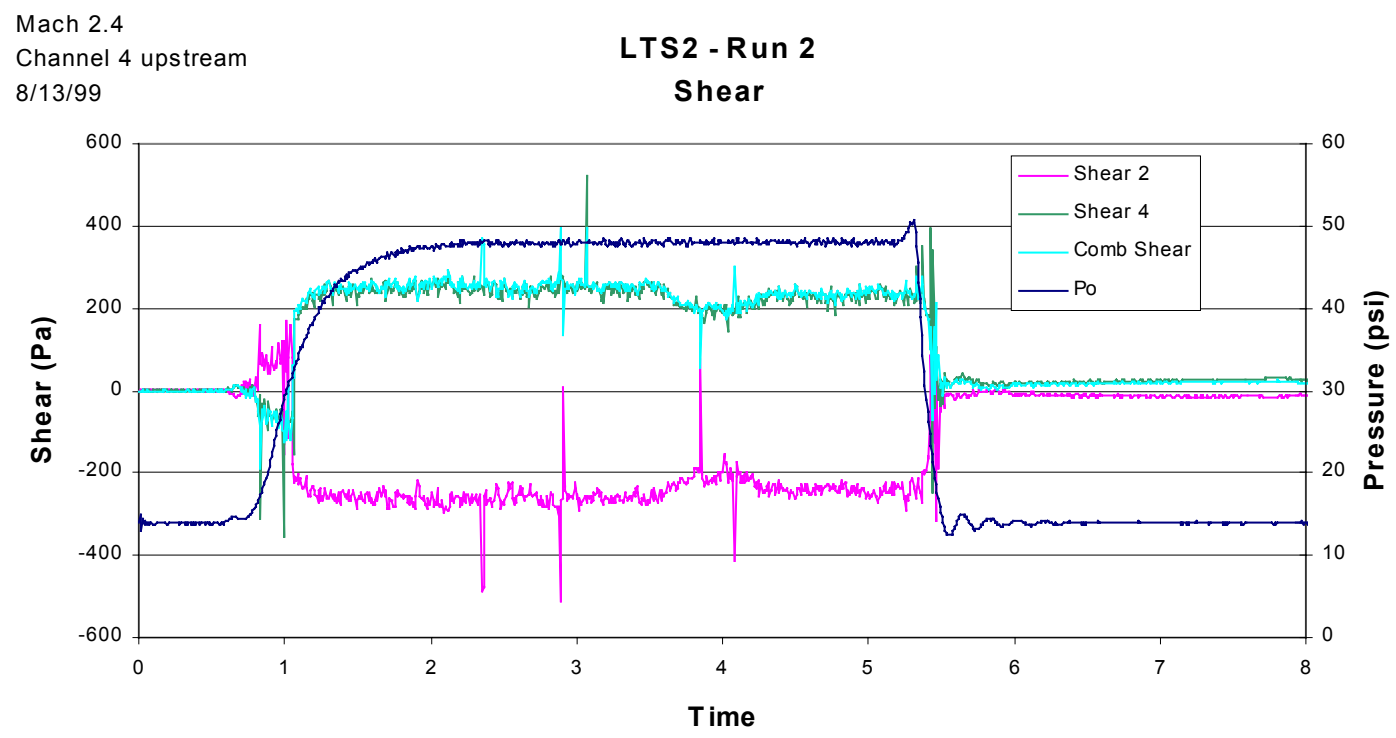

\section{Figure 189. Run 2 of the Version 5a skin friction sensor in a Mach 2.4 flow at a total pressure of $345 \mathrm{kPa}(50 \mathrm{psi})$.}

As before with the pressure sensor, the gap estimate output for both channels of the skin friction sensor is plotted in Figure 190 with the fringe contrast so that a possible correlation can be examined. As can be seen, there is not a large loss of contrast during 
the tests $(\mathrm{FC} \approx 0.7)$, and in turn the fluctuation of the gap estimate is low. The simulations performed above demonstrate that the absolute gap error is small for such a high fringe contrast. As before, each test is split up in sections, and the fringe contrast and the variance of the gap estimate are calculated.

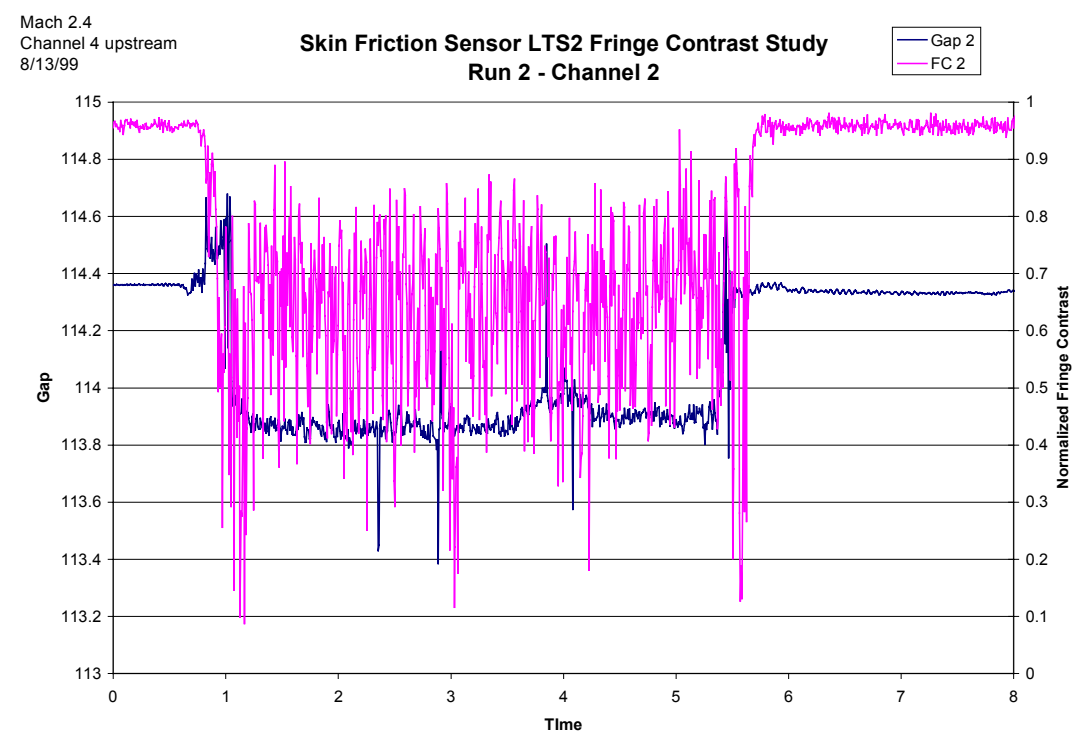

(a)

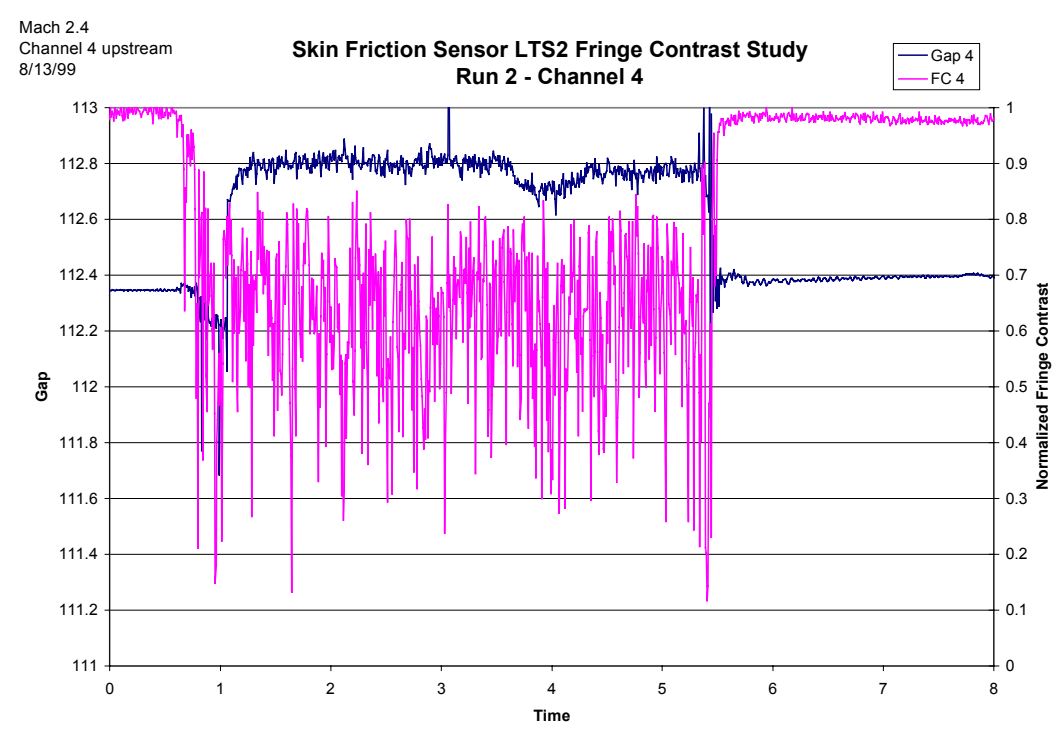

(b)

Figure 190. Fringe contrast change compared to change in gap estimate for Run 2 of the Version 5a skin friction sensor in a Mach 2.4 flow at a total pressure of 345 kPa (50 psi), a) channel 2 and b) channel 4. 
The same procedure was followed again for a moderate noise case, Mach 3.0 and a total pressure of $520 \mathrm{kPa}$ (75 psi), as shown in Figure 191. The fringe contrast/gap estimate comparison for this flow, Figure 192, shows a reduction of the contrast to approximately 0.30. It can also be observed that the fringe contrast and the gap graphs for both channels are rather correlated, in that a change in one produces a change in the other.

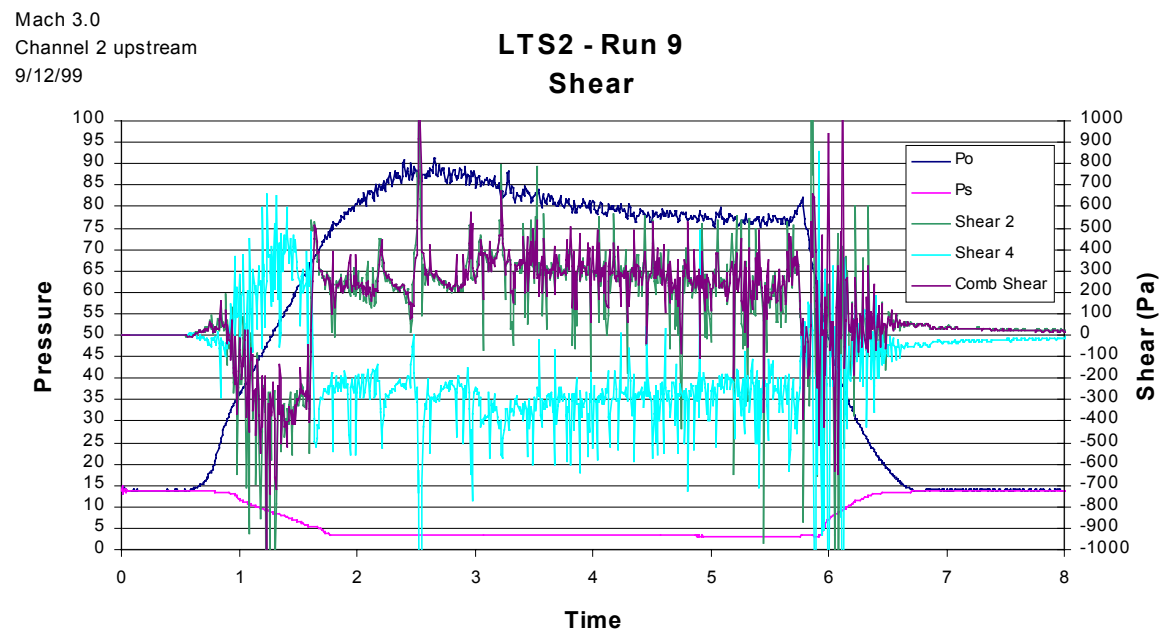

Figure 191. Run 9 of the Version 5a skin friction sensor in a Mach 3.0 flow at a total pressure of $520 \mathrm{kPa}(75 \mathrm{psi})$.

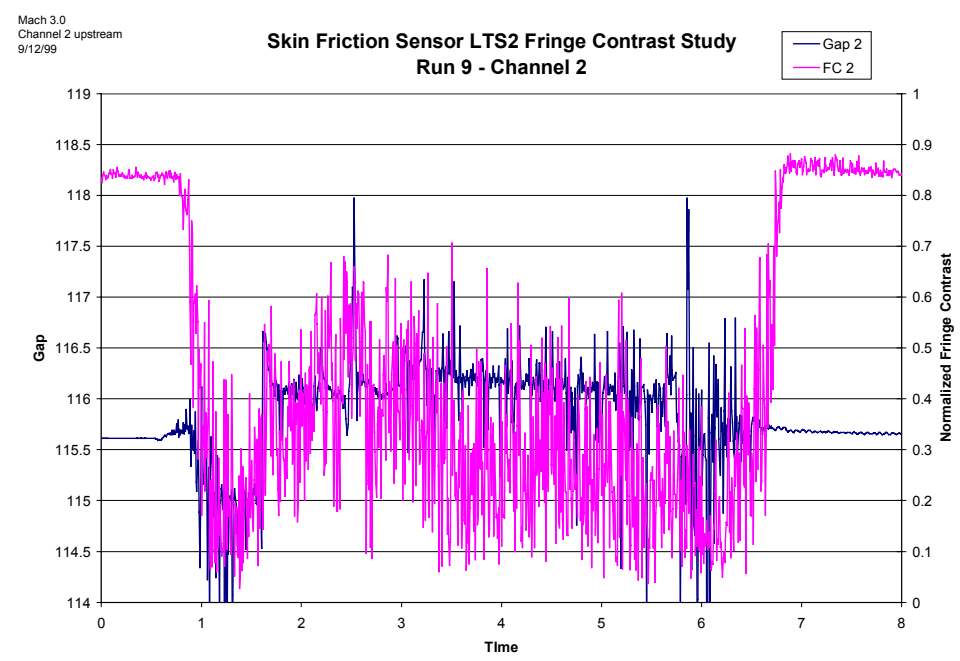

(a) 


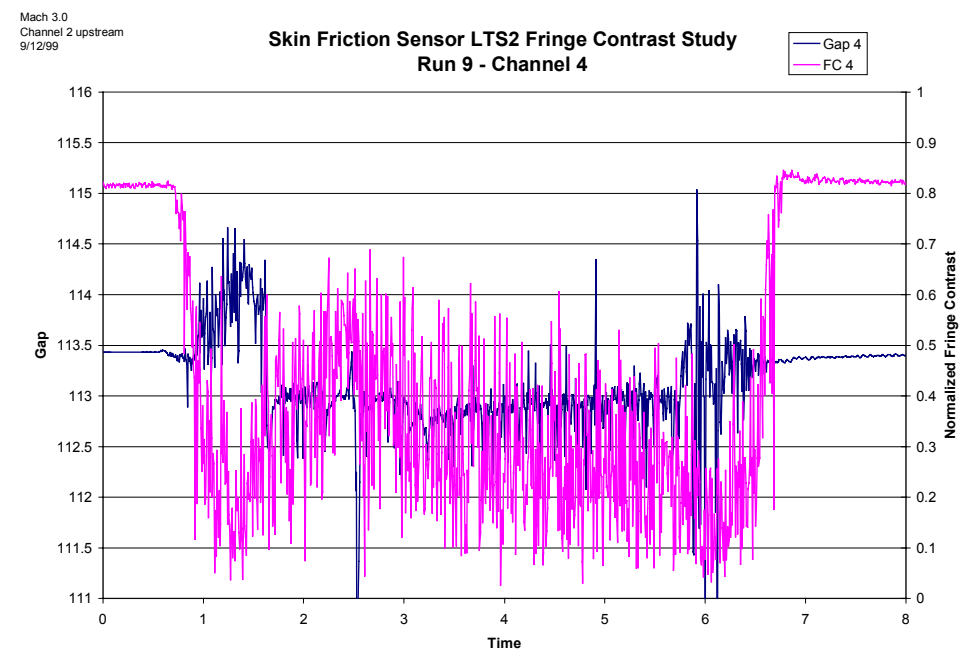

(b)

Figure 192. Fringe contrast change compared to change in gap estimate for Run 9 of the Version 5a skin friction sensor in a Mach 3.0 flow at a total pressure of $520 \mathrm{kPa}$ (75 psi), a) channel 2 and b) channel 4.

Finally, a case of high noise was examined which occurred in Mach 3.0 flow with a total pressure of $750 \mathrm{kPa}(110 \mathrm{psi})$. The results are in Figure 193. There is clearly too much noise in this signal to obtain useful results. Comparisons of the fringe contrast and gap estimate for both channels of the sensor are presented in Figure 194. As can be seen, the contrast is around 0.10 for the entire test. As has been seen numerous times, fringe contrast of this magnitude is a possible problem for these sensors, at least in a tunnel environment.

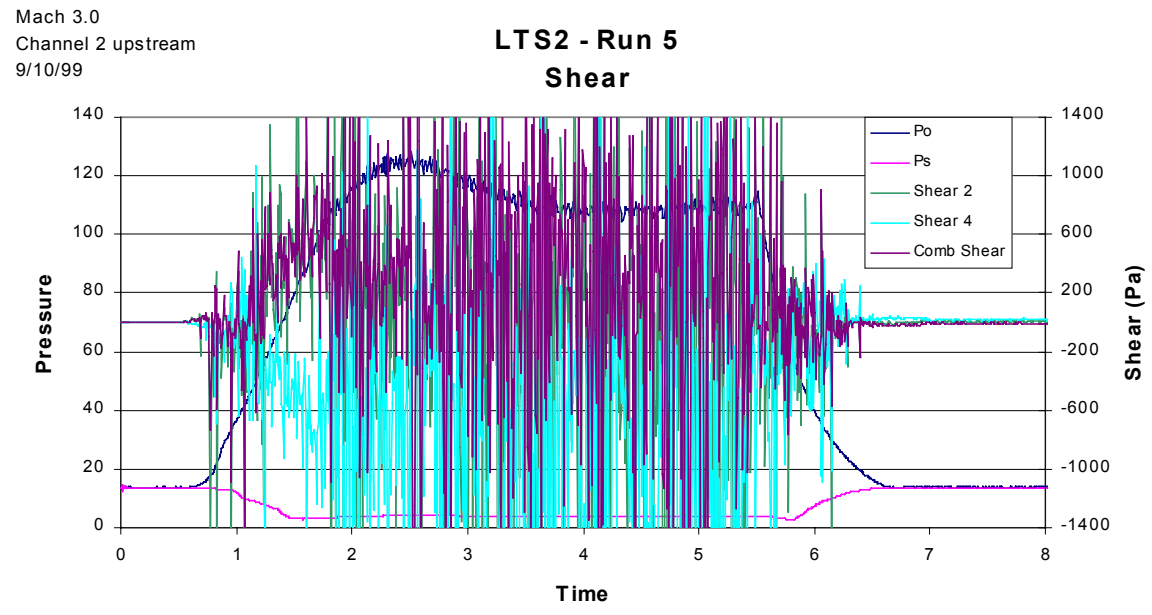

Figure 193. Run 5 of the Version 5a skin friction sensor in a Mach 3.0 flow at a total pressure of $750 \mathrm{kPa}(110 \mathrm{psi})$. 


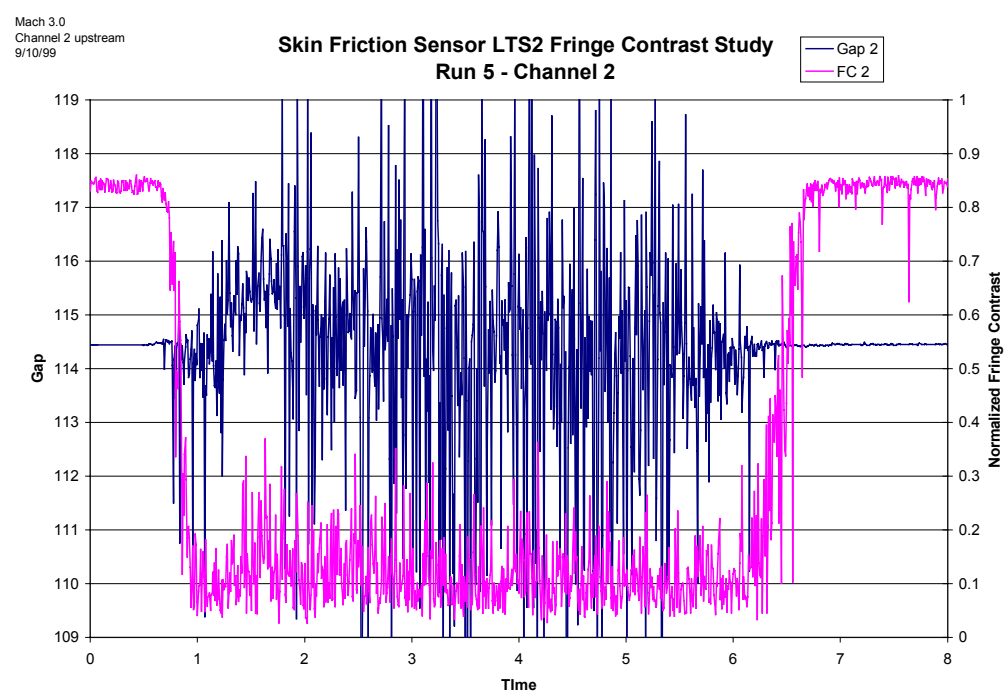

(a)

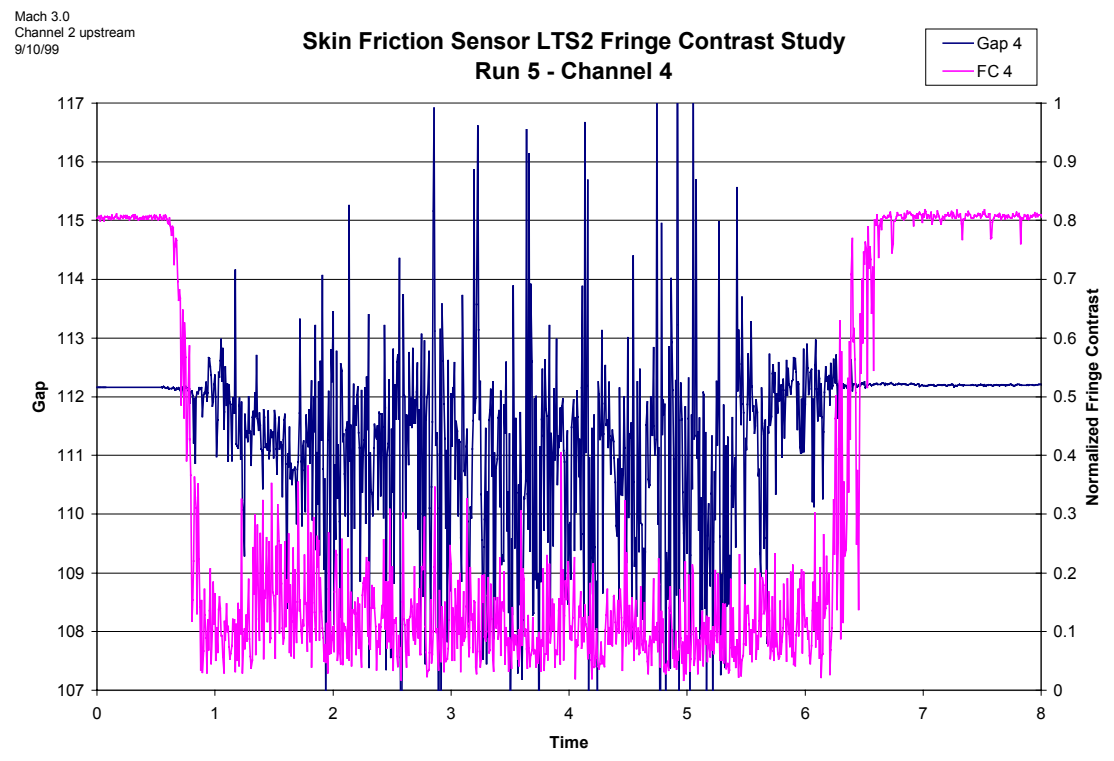

(b)

Figure 194. Fringe contrast change compared to change in gap estimate for Run 5 of the Version 5a skin friction sensor in a Mach 3.0 flow at a total pressure of $750 \mathrm{kPa}$ (110 psi), a) channel 2 and b) channel 4.

Again, the fringe contrast for this case is compared with the correction factor in the algorithm, as presented in Figure 195. As before, the loss of fringe contrast, around 0.10 , produces a large fluctuation in the correction factor. 


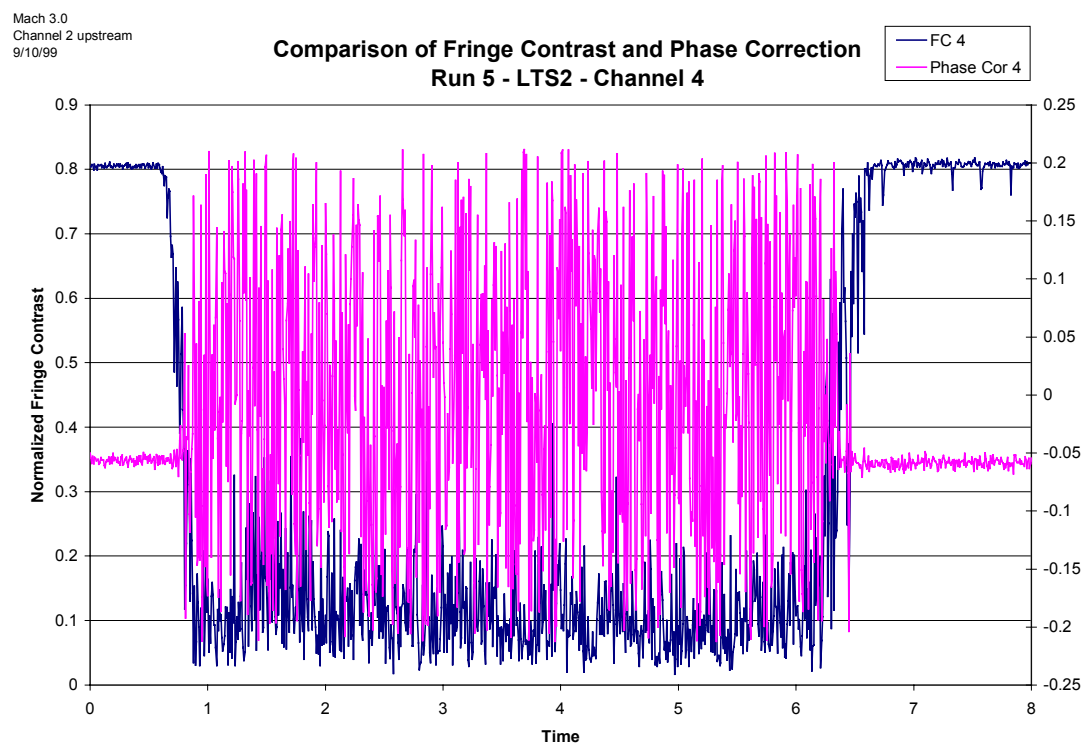

Figure 195. Comparison of fringe contrast and algorithm correction factor estimate for Run 5 of the Version 5a skin friction sensor in a Mach 3.0 flow at a total pressure of $750 \mathrm{kPa}(110 \mathrm{psi})$ demonstrating the correlation of fringe contrast and phase correction RMS variation.

There appears to not only be a correlation between the variance of the correction factor and the fringe contrast, but the absolute value of the correction and the fringe contrast as well. Changes in fringe contrast occur with changes in the correction. This is a concern, because if the changes in the correction factor are significant, those changes will appear as a alteration in the gap estimate, appearing as unexplained phenomenon. Figure 196 presents results for a test of the Version 6 skin friction sensor in which the correlation between the fringe contrast and the correction factor can clearly be seen. 


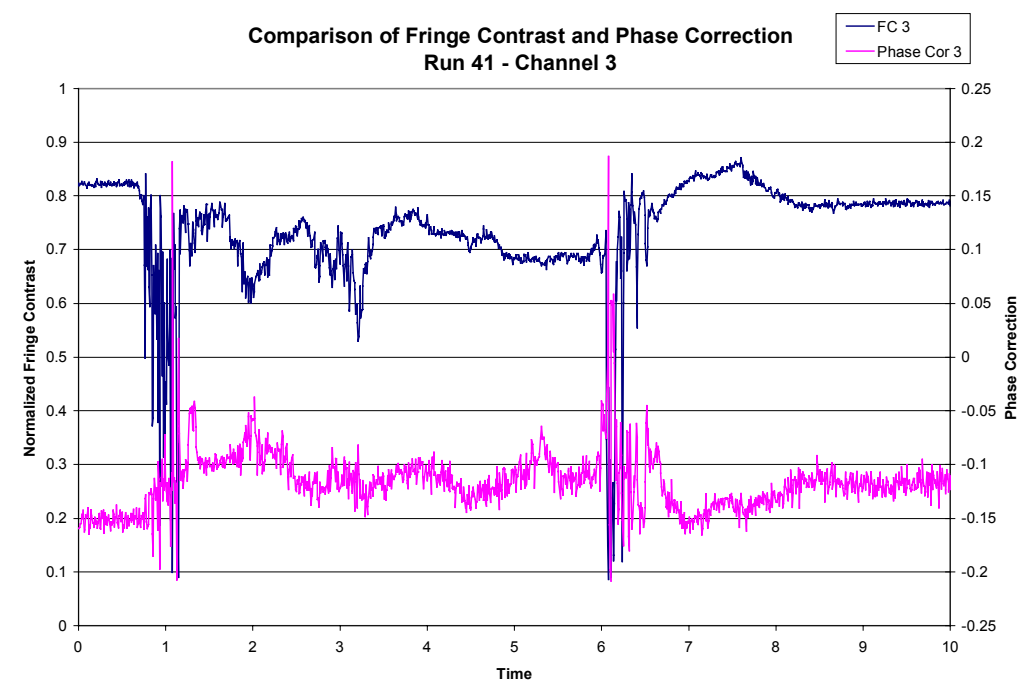

(a)

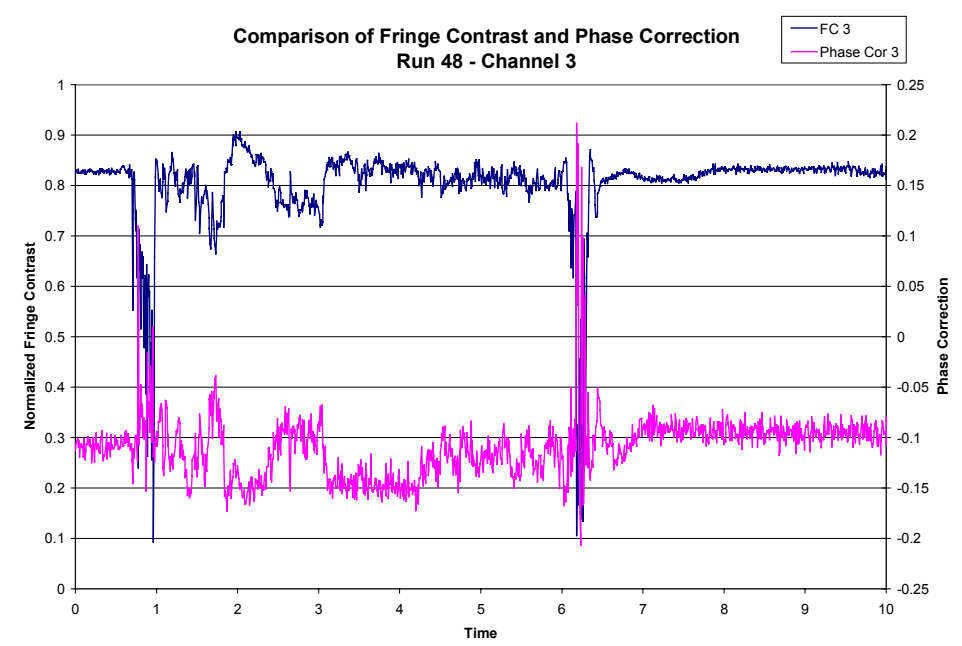

(b)

Figure 196. Comparison of fringe contrast and algorithm correction factor for channel 3 of the Version 6 skin friction sensor in a Mach 3.0 flow at a total pressure of $240 \mathrm{kPa}(35$ psi) showing the correlation of the two, a) Run 41 and b) Run 48.

There has been a continual concern during the project about unexplained variation in the shear output during a tunnel test. For example, a steady test would have what looked like widow peaks in the results. These artifacts could not be explained by aerodynamic input, so a sensor/optic cause was examined. As mentioned above, the correction factor in the algorithm of the signal processing system is a concern considering 
the correlation between the changes seen in Figure 196. A clear depiction of artifacts in the gap estimate being caused by changes in fringe contrast is presented in Figure 197.

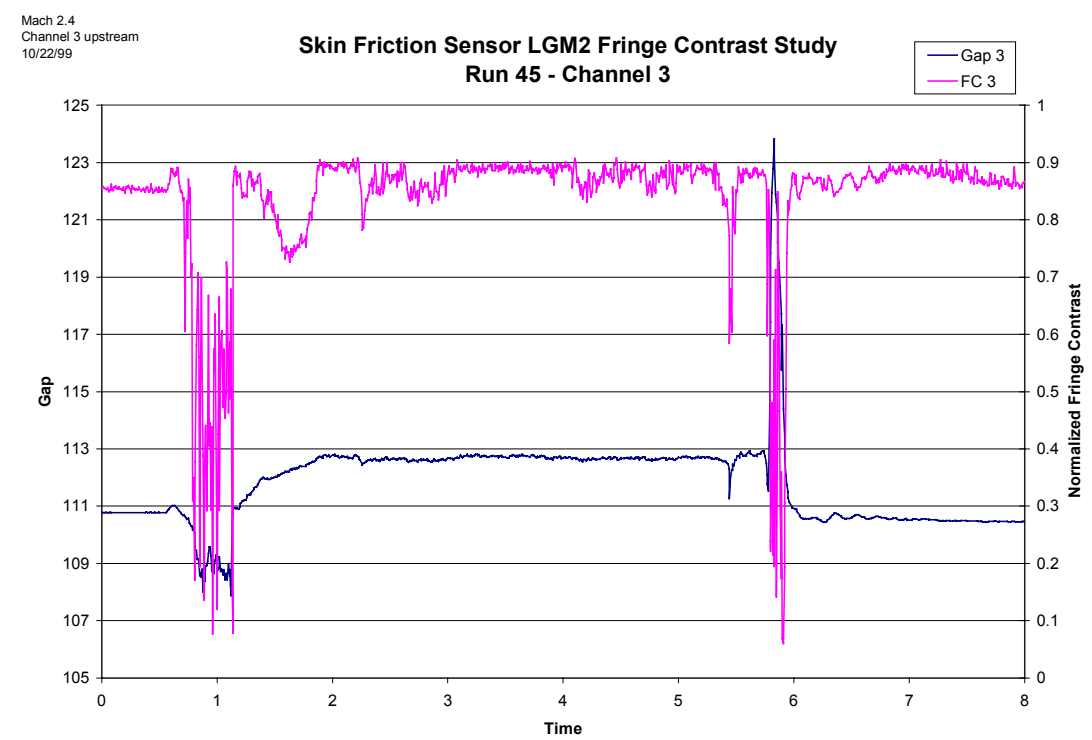

Figure 197. Clear depiction of correlation of gap artifacts and changes in fringe contrast factor for channel 3 of the Version 6 skin friction sensor in a Mach 3.0 flow at a total pressure of $240 \mathrm{kPa}(35 \mathrm{psi})$.

As with the pressure sensors, a plot was constructed comparing the normalized fringe contrast and variance of the gap signal and the correction factor for the Version 5a skin friction sensor. The results are presented in Figure 198 and Figure 200. There is a clear correlation between the fringe contrast and the variance of the gap estimate. As with the pressure sensor, the fringe contrast decreases and in turn the fluctuation of the gap estimate becomes significant as the aerodynamic energy of the flow increased, from the Mach 1.92 (345 $\mathrm{kPa}[50 \mathrm{psi}]$ ) case to the Mach 3.0 (750 kPa [110 psi]) case. As before, the fluctuations, and therefore the errors, become large as the fringe contrast approaches 0.10, as clearly demonstrated in Figure 199.

In fact, to demonstrate the connection between the energy of the flow and the error, the conditions for the center of each circle in Figure 198 was used to calculate dynamic pressure as an indicator of the energy in the flow and then plotted against fringe contrast. As Figure 201 shows, the relationship between the energy of the flow and the loss of fringe contrast is linear for the skin friction sensor. Because the reduction of fringe contrast has to be linked to increasing error both through computation and experiment, a relationship exists between increasing the energy of the flow and 
fluctuations (errors) in the gap estimate, confirming what was seen in Figure 189, Figure 191, and Figure 193. As tests from the Virginia Tech and Wright Pat tunnels have been included, the correlation is sensor and not facility dependant.

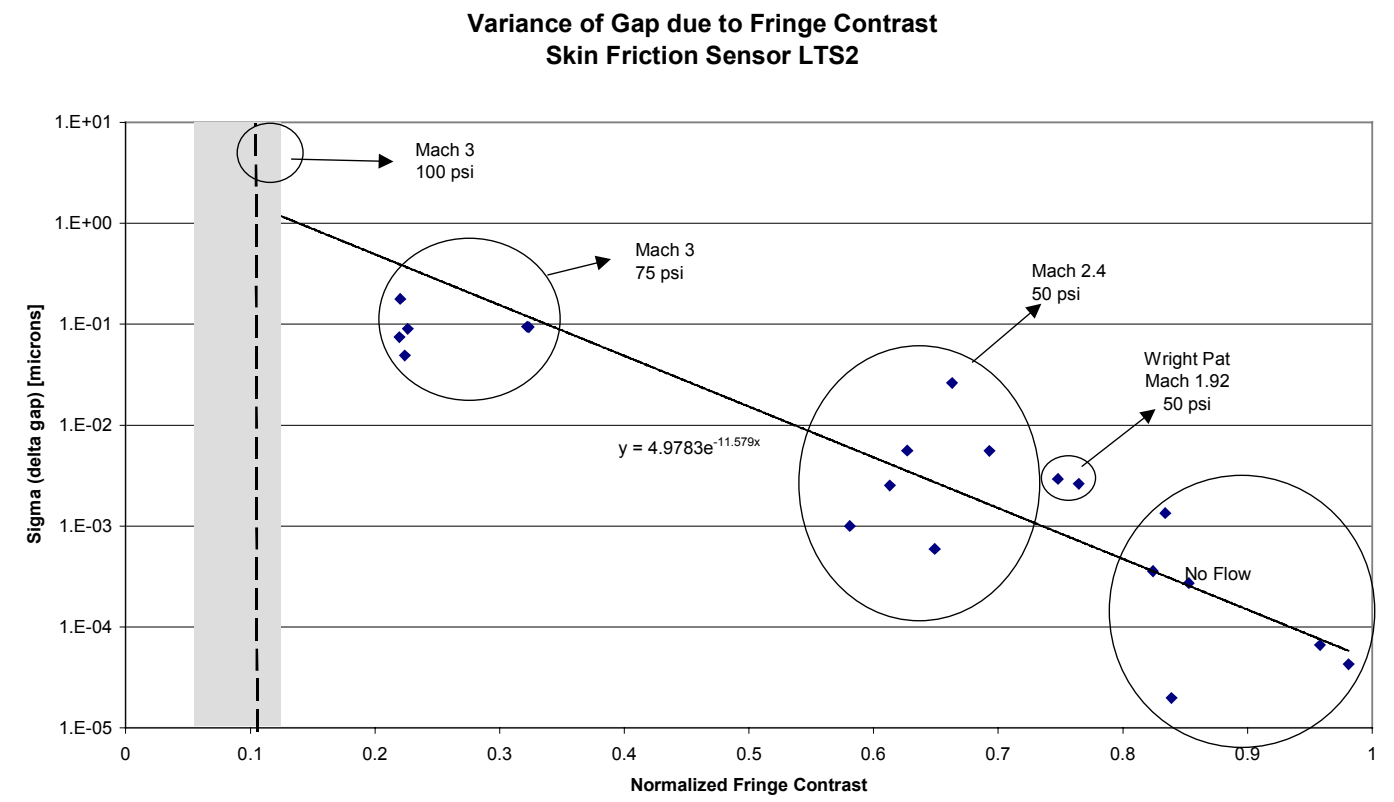

Figure 198. Semi-log plot of fringe contrast vs. variance of gap change for the Version 5a skin friction sensor for various flow conditions.

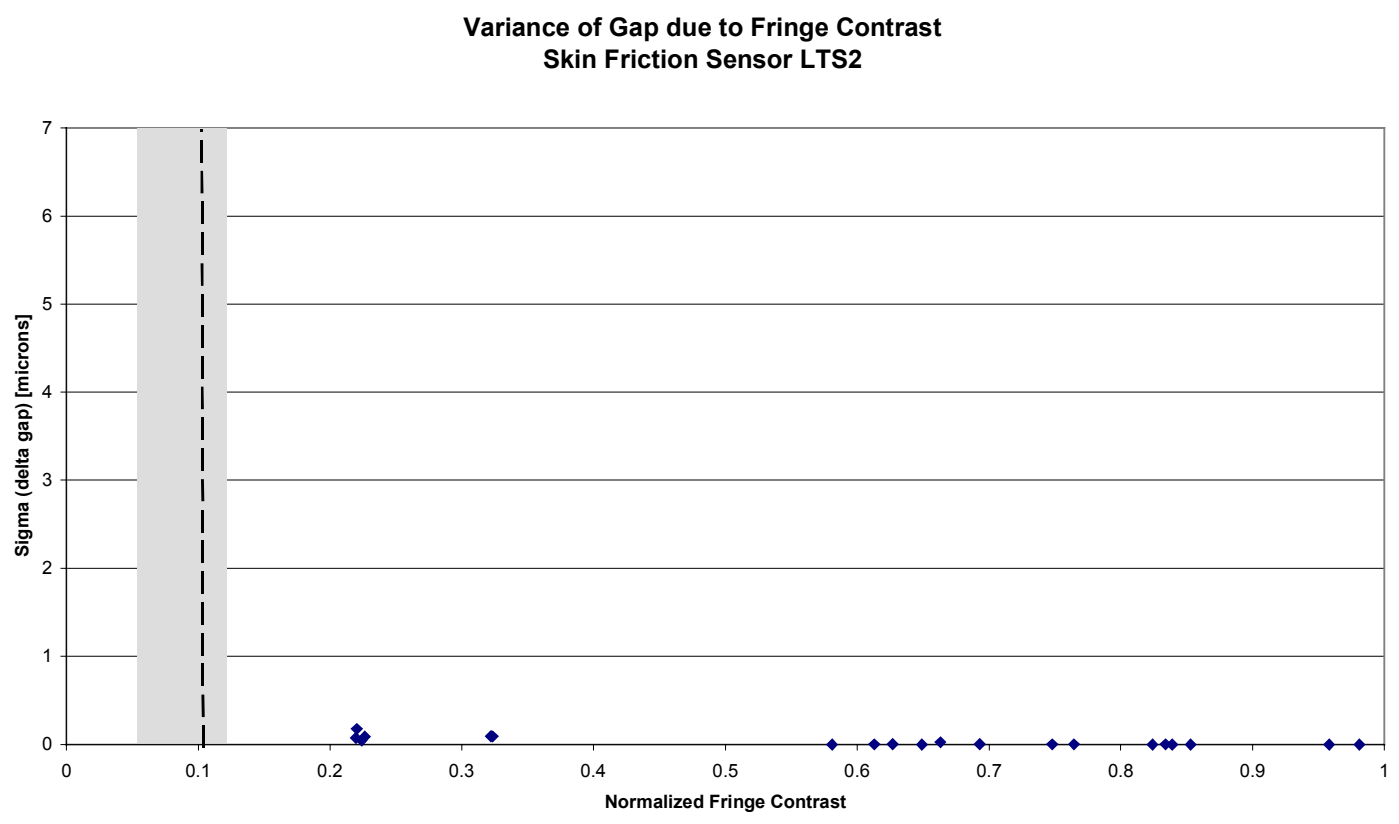

Figure 199. Linear plot of fringe contrast vs. variance of gap change for the Version 5a skin friction sensor for various flow conditions showing fringe contrast cut-off. 


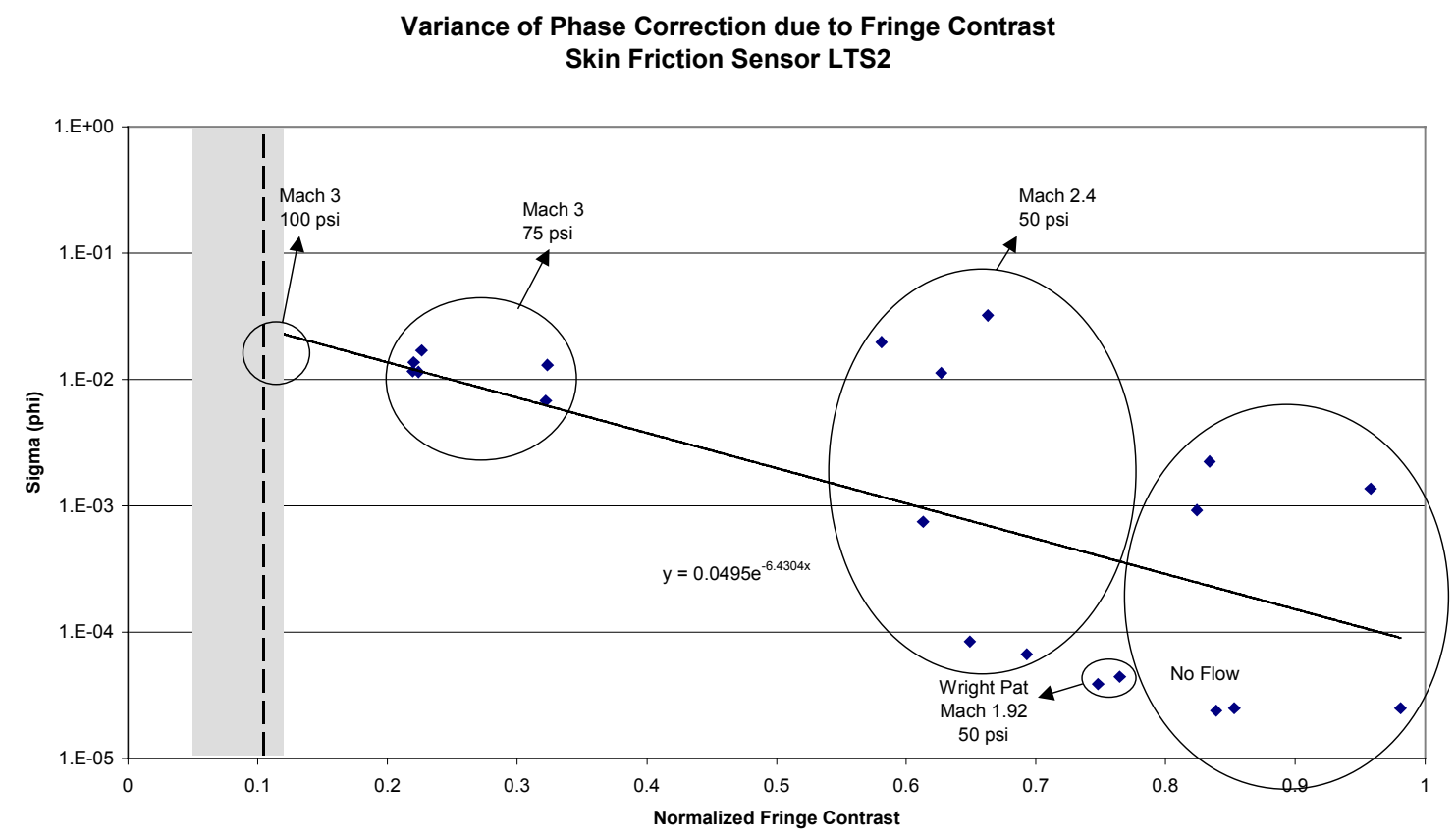

Figure 200. Plot of fringe contrast vs. variance of algorithm correction factor for the Version 5a skin friction sensor for various flow conditions.

Effect of Dynamic Pressure on Fringe Contrast for Skin Friciton Sensor LTS2

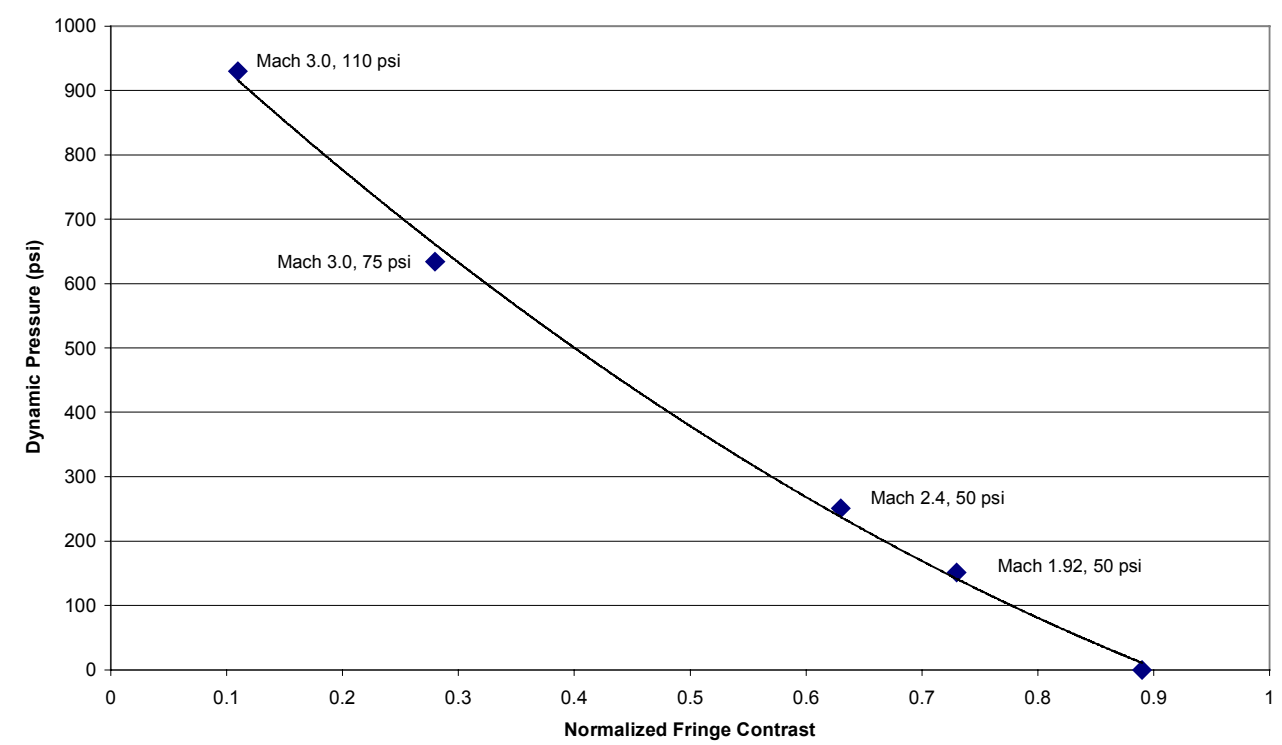

Figure 201. Reduction in the Version 5a skin friction sensor fringe contrast due to energy of the flow as measured by dynamic pressure. 
Finally, as with the pressure sensor, the correlation between the fringe contrast and the gap estimate and the fringe contrast and the correction factor was examined for each test. The results of the calculations are presented in Table 11. There is a high correlation between these factors, indicating that fringe contrast plays a large role in the correction factor and in turn the gap estimate.

In summary, it has been demonstrated that there is a link between the energy in the flow and the fluctuations (errors) in the gap estimate for the skin friction sensor. This result confirms visual inspection of experimental results and simulations. A strong correlation between the fringe contrast and the algorithm correction factor has been established. This correlation is so strong as to affect the gap estimates. It is not known why this correlation between the correction factor and fringe contrast exists.

Table 11. Correlation of gap change, fringe contrast, and correction factor change for the skin friction sensor.

\begin{tabular}{|c|c|c|c|c|c|}
\hline Sensor & Description & Test & Channel & $\begin{array}{c}\text { Correlation } \\
\text { (4gap, fringe contrast) }\end{array}$ & $\begin{array}{c}\text { Correlation } \\
\text { ( }, \text { fringe contrast })\end{array}$ \\
\hline Skin Friction & Mach 2.4 & 2 & 2 & 0.7287 & 0.3884 \\
\hline Sensor & $\mathrm{Po}=345 \mathrm{kPa}$ & & 4 & 0.7526 & 0.2141 \\
\hline Version 5a & & 3 & 2 & 0.7147 & 0.1779 \\
\hline & & 4 & 2 & 0.7711 & 0.3560 \\
\hline & & & 4 & 0.3877 & 0.3692 \\
\hline & & 5 & 2 & 0.7376 & 0.3015 \\
\hline & & & 4 & 0.7614 & 0.3256 \\
\hline & Mach 3.0 & 5 & 2 & 0.3740 & 0.6169 \\
\hline & $\mathrm{Po}=750 \mathrm{kPa}$ & & 4 & 0.3937 & 0.3227 \\
\hline & & 6 & 2 & 0.0931 & 0.6278 \\
\hline & & & 4 & 0.3052 & 0.2677 \\
\hline & Mach 3.0 & 9 & 2 & 0.1472 & .05421 \\
\hline & $\mathrm{P}=520 \mathrm{kPa}$ & & 4 & 0.3422 & 0.2289 \\
\hline & & 10 & 2 & 0.2569 & 0.5661 \\
\hline & & & 4 & 0.5443 & 0.2446 \\
\hline & & 11 & 2 & 0.4981 & 0.5397 \\
\hline & & & 4 & 0.2717 & 0.1882 \\
\hline & & 12 & 2 & 0.4168 & 0.5512 \\
\hline & & & 4 & 0.1752 & 0.2140 \\
\hline & Mach 1.92 & 3 & 2 & 0.4932 & 0.0888 \\
\hline & Po=345 kPa & 6 & 2 & 0.5128 & 0.0827 \\
\hline
\end{tabular}




\subsection{Chapter 6}

\section{Conclusions and Recommendations}

\subsection{Conclusions}

The first operational, interferometric, fiber optic, skin friction sensor was developed in this work. Measurements with the new sensor were made in a laminar incompressible flow and in various supersonic flows. The sensor performed flawlessly in the incompressible flow, and had intermittent success in the supersonic flows. The data indicates that as the energy in the flow increases, more noise is present in the sensor output. This fact has been attributed to vibration of the beam, which causes a loss of fringe contrast in the spectra used by the optical signal processing system to determine the sensor output. Loss of contrast, and its link to vibration of the beam, can be seen most clearly in the case in which the sensor housing contained no viscous fluid. Without the fluid to dampen the beam, the signal from the system is sharply degraded. When the fluid is added to the same sensors and run at identical conditions, the results and fringe contrast improve. For a damped sensor, the results indicate that manufacturing and sealing issues are very important for accurate results.

The micromachined, fiber optic pressure sensor was also evaluated for supersonic flows. The sensor operated without significant temperature drift while flush mounted in a supersonic flow. This feat would be difficult with a piezoresistive pressure transducer. It is clear for this sensor that a certain level of aerodynamic energy input will prevent it from functioning properly. The resonant frequency of the sensor diaphragm is excited at certain flow conditions, causing large gap movements. As with the skin friction sensor, the spectrometer in the optical signal processing system does not have the necessary speed to accurately determine a fast moving optical gap.

A fiber optic heat flux sensor was constructed and tested in the lab. The results demonstrate that such a sensor is possible. Improved fabrication methods and development of a calibration procedure are still required if such a sensor is to be used outside of the laboratory. 
An extensive numerical and computational study was performed to determine the effects of spectral smearing, angle misalignment, reflector roughness, and their combined effects on the accuracy of the spectral interrogation signal processing system. Each of these error sources manifests itself in the reduction of fringe contrast in the return spectra from the sensor. It has been demonstrated by experiment and calculation that if the fringe contrast drops below a certain level, the noise of the signal processing system increases greatly, sometimes to the point of losing a usable signal. With the current optical signal processing unit and algorithm, a normalized fringe contrast below 0.10 will likely lead to large errors. These errors are dependent on many factors, including the optical path length and are not biased in any direction. Therefore, for any given condition, it can not be determined whether the error is positive or negative. For an active sensor, this increase in error and lack of bias will appear as increasing noise on the sensor signal.

Successful numeric simulations of both the pressure and skin friction sensors were developed that demonstrated the increasing error of the system as gap speed increases, or conversely as the fringe contrast decreases. As the dynamic pressure of a flow increases, the more energy it imparts to the sensors, increasing the speed of the gap movement. The simulations also demonstrated that this gap speed is the dominant source for both the loss of fringe contrast and the increase in error.

Finally, examination of the aerodynamic data for both the pressure and skin friction sensors establishes a correlation between change in fringe contrast and artifacts in output signals. Also, the noise of the wind tunnel data has been shown to be dependent on the absolute value of the normalized fringe contrast. In addition, a correlation was found between the change in fringe contrast and a correction factor in the signal processing algorithm. This correlation was not expected, but can be seen as the effect of the low frequency pedestal signal on the higher frequency interference pattern. A correlation between this correction factor and the noise of the signal had been noted in the past, but the cause could not be determined. This analysis demonstrates that changes in the fringe contrast imperceptible to the eye cause a change in this correction factor and therefore in the gap determined by the algorithm.

This aerodynamic data clearly shows a correlation between the dynamic pressure of the flow, and the variance of gap estimate with the skin friction sensor. This correlation is sensor dependent and is caused by a coupling of the flow and the 
mechanical properties of the sensor. Therefore, a change in the mechanical design will change this correlation, allowing sensors to be designed for any flow conditions. Also, as this is a total system problem, the solution could be found in increasing the speed of the signal processing systems, which will have a similar effect as changing the mechanical design. Dynamic pressure was used for the skin friction sensors, but any flow phenomenon which would increase the input energy to the sensor, such as shock interactions or separation, would cause the reduction in fringe contrast and, in turn, the increase in the variance of the gap estimate. The use of other flow phenomenon was used when examining the pressure sensor data, and also demonstrated the correlation.

It should be noted that these results are for a very specific type of fiber optic sensors, extrinsic Fabry-Perot, using an existing spectral interrogation, fiber optic signal processing system. The total system limitations developed above are specific to those fiber optic sensor type and that current system. The use of alternative signal processing systems, or those that may be developed in the future, would obviously change the limitations, or possibly remove them all together for all conceivable flow situations. In addition, the use of other fiber optic sensor types, such as Bragg or long period grating, may allow the development of sensor for all environments with current technology. However, the best engineering judgement was to develop Fabry-Perot sensors with the current signal processing system with the resulting limitations which have been developed.

\subsection{Recommendations}

First, an effort should be made to improve the speed of the spectral interrogation signal processing unit. Much of the sensor limitations could be solved from the electronics end. A faster spectrometer is needed to decrease the integration time of the CCD array. The faster spectrometer will allow faster movements by the internal parts of the sensor without degrading the accuracy. The speed increase could be accomplished by installing a more expensive CCD array that has a faster integration time. In addition, more computational power would permit increased resolution of the system at higher speeds. Development of a hybrid spectral interrogation/dual wavelength system would 
also provide a superior system for aerodynamic measurements, incorporating absolute gap input with the increased speed of the dual wavelength system.

Improvements needed for the skin friction sensor are many. First, the concept of designing for larger optical gap movements should continue. The sensor size does not have to be increased greatly to produce significantly more design gap movement. Secondly, the quality of the reflector should be improved, either by placing an optically flat surface on the back of the sensing head or by plating the beam until the surface roughness is below an acceptable tolerance limit. Thirdly, improved damping methods must be employed to reduce the vibration of the head with the goal being to remove the filling fluid. Placing a rubber sheet across the top of the sensor is perfect for low temperature environments. For higher temperatures a piezoelectric shunt or magnetorestrictive material may provide the necessary damping. As was seen with the Version 6 skin friction sensor, a large design did not have as much noise as a smaller design in the same flowfield. It is possible that the internal fluid can damp better with the lower natural frequency.

In addition, improvements need to be made in the sealing and durability of the sensor. The sealing of the housing around the base should be improved to allow the removal and re-installation of the housing while keeping its seal. Although the sensor does not break as easily as it did at the beginning of the study, better design of the fibersensor interface could reduce the number of lost/broken channels. More work needs to be done to increase the temperature limit of the sensor. Damping that allows the removal of the damping fluid will decrease temperature sensitivity and in turn increase the temperature limit. Also, work must continue in finding and perfecting high temperature adhesives or attachment procedures. By increasing the temperature service ceiling of the fiber optic sensor, one will be able to improve significantly upon conventional strain gages.

As for micromachining the skin friction sensor, there are several avenues which have promise and can be explored. The v-groove concept is inexpensive, meaning that it can be developed on a small budget. If the resources are available, the low-profile, surface micromachined sensor will work and has few fabrication questions, but there are unknown errors associated with the concept. Solving the micromachining problem may 
be the only way to inexpensively fabricate these skin friction sensors for a larger user base.

The fiber optic pressure sensor is a promising sensing platform for many aerodynamic situations. However, the resonant frequency is too easily excited in supersonic flows. The vibration of the pressure sensor in these flows can be decreased in several ways. First, the sensor could be recessed from the flow by a small hole, aerodynamically damping the input noise. Second, for lower temperature measurements, a thin layer of polymer could be coated on the sensor to absorb the mechanical vibration energy of the diaphragm. Third, improvements of the resolution of the signal processing system would permit the use of a thicker diaphragm, providing the same pressure sensitivity but at a much higher natural frequency. On a practical point, the sensor would be smaller and easier to package if it were constructed round instead of square. This circular fabrication is more expensive, but the total sensor cost, including the fabrication of the housing, will probably be less. Also, using gap division multiplexing, an algorithm and calibration procedure, needs to be developed to utilize the reflection within the diaphragm to ascertain the temperature of the pressure sensor and provide some level of temperature compensation. Improving the temperature ceiling for accurate operation of the sensor will allow it to compete with piezoresistive pressure sensors for most aerospace measurements.

The work on the fiber optic heat flux sensor should probably be abandoned. Competing technology, such as the surface thermocouple, can make faster, cheaper, and more accurate measurements than the fiber optic version. The material mismatch between typical aerospace surfaces and the silicon of the sensor will always cause a temperature mismatch and, therefore, an error. A niche area for the fiber optic sensor still exists in regimes where thermocouples can not operate, such as within high EMI fields.

Finally, the correlation between the fringe contrast and the correction factor in the algorithm needs to be investigated further. An improvement on the correction factor may be found in this work, improving stability and accuracy of the optical signal processing system. In addition, future sensors should be designed to keep the fringe contrast as high as possible. This may be accomplished by adjusting the reflectors and/or the medium in the gap to produce the higher contrast. 


\section{Bibliography}

${ }^{1}$ Schetz, J., "Direct Measurement of Skin Friction in Complex Fluid Flows", Applied Mechanical Review, vol. 50, part 2, pp. S198-S203, Nov. 1997

2 Nitsche, W., Haberland, C. and Thunker, R., "Comparative Investigations of the Friction Drag Measuring Techniques in Experimental Aerodynamics, " ICAS -84-2.4.1, 14th ICAS Congress, 1984

${ }^{3}$ Wooden, P. A., Hull, G. H., "Correlation of Measured and Theoretical Heat Transfer and Skin Friction at Hypersonic Speeds Including Reynolds Analogy", AIAA Paper 905244, Oct. 1990

4 Brown, G., "Theory and Application of Heated Films for Skin Friction Measurement", Proc. of the 1967 Heat Transfer and Fluid Mechanics Institute, pp. 361-381, 1967

${ }^{5}$ Bellhouse, B., Schultz, D., "The Measurement of Fluctuating Skin Friction in Air with Heated Thin-Film Gauges", Journal of Fluid Mechanics, vol. 32, part 4, pp. 675-680, 1968

6 Bellhouse, B., Schultz, D., "Determination of Mean and Dynamic Skin Friction, Separation, and Transition in Low-Speed Flow with a Thin-Film Heated Element", Journal of Fluid Mechanics, vol. 24, part 2, pp. 379-400, 1966

${ }^{7}$ Evans, T., Smits, A., "Measurement of the Mean Heat Transfer in a Shock WaveTurbulent Boundary Layer Interaction", Journal of Experimental Thermal and Fluid Sciences, vol. 12, no. 1, pp. 12-87-97, Jan. 1996

${ }^{8}$ Owen, P., Ormerod, A., "Evaporation from the Surface of a Body in an Airstream (With particular reference to the chemical method of indicating boundary-layer transition.)", ARC R\&M 2875, 1951

${ }^{9}$ Sherwood, T., Trass, O., "Sublimation Mass Transfer through Compressible Boundary Layers on a Flat Plate", Journal of Heat Transfer, 82, C4, pp. 313-324, 1960

${ }^{10}$ Winter, K.G.,"An Outline of the Techniques Available for the Measurement of Skin Friction in Turbulent Boundary Layers," Progress in Aerospace Sci., Vol. 18, pp. 1-57, 1977

11 Gupta, R., "A New Device for Skin-Friction Measurements in Three-Dimensional Flows", AIAA Journal, vol. 13, no. 2, pp. 236-238, 1975

12 Tanner, L.H., Blows, L. G., "A Study of the Motion of Oil Films on Surfaces in Air Flow, with Application to the Measurement of Skin Friction", Journal of Physics E:Scientific Instruments, Vol. 9, pp. 194-202, Mar. 1976

${ }^{13}$ Wideman, J., Brown, J., Miles, J., Ozcan, O., "Skin-Friction Measurements in ThreeDimensional, Supersonic Shock-Wave/Boundary-Layer Interaction", AIAA Journal, vol. 33, no. 5, pp. 805-811, 1995

${ }^{14}$ Kim, K., Settles, G., "Skin Friction Measurements by Laser Interferometry in Swept Shock Wave/Turbulent Boundary-Layer Interactions, AIAA 88-0497, $26^{\text {th }}$ Aerospace Sciences Meeting, Reno, NV, Jan. 1988 
15 Klein, E., Margozzi, A., "Exploratory Investigation on the Measurement of Skin Friction by Means of Liquid Crystals", $11^{\text {th }}$ Israel Annual Conf. On Aviation and Astronautics, Tel Aviv, Mar. 1969

${ }^{16}$ Gaudet, L., Gell, T. G., "Use of Liquid Crystals for Qualitative and Quantitative 2-D Studies of Transition and Skin Friction", ICIASF ' $89-13^{\text {th }}$ International Congress on Instrumentation in Aerospace Simulation Facilities, vol. 43, pp. 66-81, 1989.

17 Paros, J., "Application of the Force-Balance Principle to Pressure and Skin Friction Sensors", $16^{\text {th }}$ Annual Technical Meeting, Proc. Inst. Environmental Sciences, pp. 363368,1970

18 Mehregany, M., DeAnna, R., Reshotko, E., "Microelectromechanical Systems for Aerodynamics Applications,”, AIAA Paper 96-0421, Jan. 1996

${ }^{19}$ Padmanabhan, A., Golberg, H., Schmidt, M., Breuer, K., "A Silicon Micromachined Sensor for Shear Stress Measurements in Aerodynamic Flows", AIAA 96-0422, 34 Aerospace Sciences Meeting, Reno, NV, Jan. 1996

20 MacArthur, R., "Transducer for Direct Measurement of Skin Friction in the Hypersonic Shock Tunnel”, CAL Report 129, 1963

${ }^{21}$ Chadwick, K., DeTurris, D.J. and Schetz, J.A., "Direct Measurements of Skin Friction in Supersonic Combustion Flowfields," J. Eng. Gas Turbines and Power, Vol. 115, No. 3, pp. 507-514, 1993

${ }^{22}$ M. Novean, J. A. Schetz, D. Hazelton, and R. Bowersox, "Skin Friction Measurements in Short Duration, High Enthalpy Flows", AIAA 95-6109, April 1995

${ }^{23}$ DeTurris, D., Schetz, J. A., Hellbaum, R. F., "Direct Measurements of Skin Friction in a SCRAMjet Combustor", AIAA Paper 90-2342, July 1990

${ }^{24}$ Acharya, M., Bornstein, J., Escudier, M.P., Vorkurka, V., "Development of a Floating Element for the Measurement of Surface Shear Stress", AIAA Journal, Vol. 23, pp. 410415, Mar 1985

${ }^{25}$ Schetz, J.A. and Fuhs, A.E. (Eds.), Handbook of Fluid Dynamics and Fluid Machinery, John Wiley \& Sons, New York, pp. 935-961, 1996

${ }^{26}$ Doebelin, E., Measurement Systems: Application and Design, $4{ }^{\text {th }}$ edition, Mcgraw-Hill Publishing, pp. 437-524, 1990

27 Morris, M., Donovan, J., Kegelmman, J., Schwab, S., Levy, R., and Crites, R., "Aerodynamic Applications of Pressure Sensitive Paint," AIAA Journal Vol. 31, No. 3, March 1993

${ }^{28}$ Winslow, N. A., Carroll, B. F., and Setzer, F. M., "Frequency Response of Pressure Sensitve Paints," AIAA Paper No. 96-1967, 27th AIAA Fluid Dynamics Conference, June 1996

${ }^{29}$ Carroll, B. F., Abbitt, J. D., Lukas, E. W., and Morris, M. J., "Step Response of Pressure-Sensitive Paints," AIAA Journal, Vol. 34, No. 3, pp 521-526, March 1996 
${ }^{30}$ Hubner, J. P., Abbitt, J. D., and Carroll, B. F., "Pressure Measurements on Rotating Machinery Using Lifetime Imaging of Pressure Sensitive Paint," AIAA Paper No. 962934, 32nd AIAA/ASME/SAE/ASEE Joint Propulsion Conference, July 1996

31 Vollan, A., and Alati, L., "A New Optical Pressure Measurement System," 14 International Congress on Instrumentation in Aerospace Simulation Facilities, Paper No. 10, Rockville, MD, Oct. 27-31, 1991

32 Engler, R. Hartmann, K., and Schulze, B., “Aerodynamic Assessment of an Optical Pressure Measurement System (OPMS) by Comparison with Conventional Pressure Measurements in a High Speed Wind Tunnel," $14^{\text {th }}$ International Congress on Instrumentation in Aerospace Simulation Facilities, Paper No. 17, Rockville, MD, Oct. 27-31, 1991

${ }^{33}$ Chan, M., Collins, S., Smith, R., "A micromachined pressure sensor with fiber optic interferometric readout", Sensors and Actuators, A43, pp. 196-201, 1994

${ }^{34}$ Iwamoto, K., Kamata, I., "Pressure sensor using optical fibers", Applied Optics, 29(3), pp. $375-378,1990$

${ }^{35}$ Schetz, J.A. and Fuhs, A.E. (Eds.), Handbook of Fluid Dynamics and Fluid Machinery, John Wiley \& Sons, New York, pp. 1053-64, 1996

${ }^{36}$ Kidd, S., Sinha, P., Barton, J., Jones, J., "Miniature Fast Response Fibre Optic fabryPerot Temperature Sensors", Applied Optics Digest, Proc. Applied Optics and OptoElectronics, p.41-42, IoP Publishing Ltd, Nottingham, Sept. 1990

${ }^{37}$ Singh, P., Spencer, J., Humphries, G., Jones, G., Dancaster, J., Pinnock, R., Dean, E., Simpson, J., "A Whitelight Interferometric Techniques for Monitoring Temperature", Eleventh International Conference on Optical Fiber Sensors, vol.1, 1996, pp. 344-7

${ }^{38}$ Vatell website, http://www.g3.com/vatell/

39 "Standard Test Method for Measuring Extreme Heat-Transfer Rates from High-Engery Environments Using a Transient, Null-Point Calorimeter", American Society for Testing and Materials Standard E 598-77

${ }^{40}$ Cook, Felderman, AIAA Journal, Vol. 4, 1966, pp. 561-562

${ }^{41}$ Diller, T.E., "Evaluation of numerical methods for determining heat flux with a null point calorimeter.”, University report, Virginia Tech. Blacksburg, VA

${ }^{42}$ Walker,G., Scott, E., "Evaluation of Estimation Methods for High Unsteady Heat Fluxes for Surface Measurements”, Virginia Tech report, 1996

43 Walker, G. "Estimation of non-uniform heating rates from surface temperature measurements." Ph.D. Dissertation, Virginia Tech, 1996

${ }^{44}$ Beck, J., Blackwell. B., St Clair, C, Inverse Heat Conduction: Ill-posed Problems, Wiley, 1985. 
${ }^{45}$ Chadwick, K., DeTurris, D.J. and Schetz, J.A., "Direct Measurements of Skin Friction in Supersonic Combustion Flowfields," J. Eng. Gas Turbines and Power, Vol. 115, No. 3, pp. 507-514, 1993

${ }^{46}$ M. Novean, J. A. Schetz, D. Hazelton, and R. Bowersox, "Skin Friction Measurements in Short Duration, High Enthalpy Flows", AIAA 95-6109, April 1995

${ }^{47}$ DeTurris, D., Schetz, J. A., Hellbaum, R. F., "Direct Measurements of Skin Friction in a SCRAMjet Combustor", AIAA Paper 90-2342, July 1990

${ }^{48}$ Chadwick, Kenneth, An Actively Cooled Floating Element Skin Friction Balance for Direct Measurement in High Enthalpy Supersonic Flows, Dissertation, Aerospace and Ocean Engineering Department, Virginia Polytechnic Institute and State University, 1991.

${ }^{49}$ Murphy, K., Gunther, M., Vengsarkar, A., and Claus, R. O., "Quadrature phase-shifted, extrinsic Fabry-Perot optical fiber sensors," Optics Letters, Vol. 16, No. 4, pp. 173-275, February 1991

${ }^{50}$ Claus, R.O., Gunther, M.F., Wang, A., Murphy, K.A., "Extrinsic Fabry-Perot Sensor for Strain and Crack Opening Displacement Measurements form -200 to 900 C", Smart Material Structures, Vol. 1, pp. 237-242, 1992

51 Jones, M., Bragg Grating Sensor Interrogation Using In-line Dual Mode Fiber Demodualtor, Thesis, Electrical Engineering Department, Virginia Polytechnic Institute and State University, 1996

52 Stroman, J, Optical Fibers, Engineered Materials Handbook, vol. 4, ASM International, p. 410, 1991

${ }^{53}$ Lui, K., Measures, R., "Signal Processing Techniques for Interferometric Fiber-Optic Strain Sensors", Journal Of Intelligent Materials Systems and Structures, vol. 3, pp. 432461, July 1992

${ }^{54}$ Dakin, J., and Culshaw, B., Optical Fiber Sensors: Principles and Components, Artech House, Boston, MA, 1988.

${ }^{55}$ Lefevre, H., "White Light Interferometry in Optical Fiber Sensors", Photonetics

${ }^{56}$ Zuliani, G., Hogg, D., Liu, K., Measures, R., "Demodulation of a Fiber Fabry-Perot Strain Sensor Using White Light Interferometry", Fiber Optic Smart Structures and Skins IV, SPIE, vol. 1588, pp. 308-313, 1991

57 Wavering, Thomas, Optical Path Length Multiplexing of Optical Fiber Sensors, Thesis, Electrical Engineering Department, Virginia Polytechnic Institute and State University, 1998

${ }^{58}$ Koch, A., Ulrich, R., "Displacement Sensor with Electronically Scanned White-Light Interferometer”, Fibre Optic Sensors IV, SPIE, vol. 1267, pp. 126-133, 1990

59 Ezbiri, A., Tatam, R., "Passive Signal Processing of Miniature Fabry-Perot Interferometric Sensors Using a Dual-Wavelength Source”, $10^{\text {th }}$ Optical fibre Sensors Conference, pp. 335-338, 1994 
60 Gelmini, E., Minoni, U., Docchio, F., "Tunable, Double-Wavelength Heterodyne Detection Interferometer for absolute-Distance Measurement", Optics Letters, vol. 19, no. 3, pp. 213-215, 1994

61 Russler, P., Micromachined, Fiber Optic Pressure Sensor Development, internal document, Luna Innovations

62 Di Giovanni, Mario, "Flat and Corrugated Diaphragm Design Handbook", Marcel Dekker, Inc., New York, 1982

${ }^{63}$ Beer, F., Johnston, E., Mechanics of Materials, McGraw-Hill, 1981, p. 598

${ }^{64}$ Cannon, R, Dynamics of Physical Systems, McGraw Hill, 1967

65 Mehregany, M., DeAnna, R., Reshotko, E., "Microelectromechanical Systems for Aerodynamics Applications,", AIAA Paper 96-0421, Jan. 1996.

66 Stefanescu, S., DeAnna, R., Mehregany, M., "Experimental Performance of a Micromachined Heat Flux Sensor", AIAA 97-3034, 33 ${ }^{\text {rd }}$ AIAA/ASME/SAE/ASEE Joint Propulsion Conf., Seattle, WA, Jul. 1997

67 Haritonidis, J., "The Measurement of Wall Shear Stress", Advances in Fluid Mechanics Measurements, Springer-Verlas, pp. 229-261, 1989

${ }^{68}$ Allen, J.M.,"Systematic Study of Error Sources in Supersonic Skin-Friction Balance Measurements," NASA TN D-8291,1976

69 Allen, J.M.,"Improved Sensing Element for Skin-Friction Balance Measurements," AIAA Journal, Vol. 18, No. 10, pp. 1342-1345, 1980

${ }^{70}$ O’Donnell, F.B., Westkaemper, J.C., "Measurement of Errors Caused by Misalignment of Floating Element Skin Friction Balances”, AIAA Journal, Vol. 3, No. 1, pp. 163-165, 1965

${ }^{71}$ Frei, D., Thomann, H., "Direct Measurement of Skin Friction in Turbulent Boundary Layers with a Strong Adverse Pressure Gradient", Journal of Fluid Mech., Vol. 101, pp. $79-95,1980$

72 Hirt, F., Zurfluh, U., Thomann, H., "Skin Friction Balances for Large Pressure Gradients", Experiment in Fluids, Vol. 4, pp. 296-300, 1986

${ }^{73}$ Brown, K.C., Joubert, P.N., "The Measurement of Skin Friction in Turbulent Boundary Layers in Adverse Pressure Gradients”, Journal of Fluid Mech., Vol. 35, Part 4, pp. 737757,1969

${ }^{74}$ Schetz, J., private communications, 1997

${ }^{75}$ Voisinet, R., “Temperature Step Effect on Direct Measurement of Skin Friction Drag”, AIAA 78-0779, 1978.

76 Westkaemper, J. C., "Step Temperature Effects on Direct Measurements of Drag", AIAA Journal, Vol. 1, No. 7, pp. 1708-1710, Jul 1963

${ }^{77}$ Debieve, J-F., Dupont, P., Smith, D., Smits, A., “A Supersonic Turbulent Boundary Layer Subjected to Step Change in Wall Temperature" 
78 Bellhouse, B., Schultz, D., "Determination of Mean and Dynamic Skin Friction, Separation, and Transition in Low-Speed Flow with a Thin-Film Heated Element", Journal of Fluid Mechanics, vol. 24, part 2, pp. 379-400, 1966

${ }^{79}$ Pope, R., "Skin-Friction Measurements in Laminar and Turbulent Flows Using Heated Thin-Film Gages", AIAA Journal

${ }^{80}$ Mukerji, D., Eaton, J., Mofat, R., Elkins, C., "A 2-D Numerical Study of the HeatIsland Effect for Button-Type Heat Flux Gages", \%th ASME/JSME Joint thermal engineering Conference, San Diego, CA, March, 1999

${ }^{81}$ Fuller, E. private communications, 1999

82 Inger, G.R. and Gnoffo, P.G., "Analytical and Computational Study of Wall Temperature Jumps in Supersonic Flow” AIAA Paper 99-0226, Reno, Jan. 1999.

${ }^{83}$ Inger, G., private communications, 1999

${ }^{84}$ Pulliam, W., Wilson, M., "Microfabricated, Low Maintenance Skin Friction Sensor for Highly Transient Applications Project Summary", SBIR Phase I program final report, Contract No. NAS1-98067, NASA Langley Research Center, Oct. 1998

${ }^{85}$ Madou, M., Fundamentals of Microfabrication, CRC Press, 1997, p. 49

${ }^{86}$ Petersen, K., "Silicon as a Mechanical Material", Proc. of the IEEE, vol. 70, no. 5, pp. 420-457, May 1982

${ }^{87}$ Madou, p. 150

${ }^{88}$ Mlcak, R., private communication, 1998

89 Wavering, Thomas, Optical Path Length Multiplexing of Optical Fiber Sensors, Thesis, Electrical Engineering Department, Virginia Polytechnic Institute and State University, 1998

90 J. A. Schetz, Boundary layer Analysis, Prentice Hall, Englewood Cliffs, NJ, p. 214, 1993

${ }^{91}$ Chadwick, K., DeTurris, D.J. and Schetz, J.A., "Direct Measurements of Skin Friction in Supersonic Combustion Flowfields," J. Eng. Gas Turbines and Power, Vol. 115, No. 3, pp. 507-514, 1993

${ }^{92}$ D. DeTurris, J. A. Schetz, and R. F. Hellbaum, "Direct Measurements of Skin Friction in a SCRAMjet Combustor," AIAA Paper 90-2342, July 1990.

93 J. A. Schetz, V. A. Vinogradov, A. Marshakov, and V. Petrov, "Direct Measurements of Skin Friction in a Scramjet Combustor," AIAA 93-2443, June 1993.

${ }^{94}$ M. Novean, J. A. Schetz, D. Hazelton, and R. Bowersox, "Skin Friction Measurements in Short Duration, High Enthalpy Flows," AIAA 95-6109, April 1995.

${ }^{95}$ Magill, S., Study of a Direct Measuring Skin Friction Gage with Rubber Compounds for Damping, Aerospace Engineering Department, Virginia Polytechnic Institute and State University, 1999

${ }^{96}$ Steve Poland, private communication, 1997 
${ }^{97}$ Jason Borinski, private communication, 2000

98 Pulliam, W., "Error Analysis of a Moving Gap of an Extrinsic Fabry-Perot Interferometric Fiber Optic Wall Shear Stress Sensor Utilizing a White Light Interferometric Demodulation System", Luna Innovations internal document, 1998

${ }^{99}$ Optics for Research, products sheet, 1993 
Appendix A

Comprehensive Testing Data

October 1996

Version 1 skin friction sensor

Phase I Skin Friction Sensor
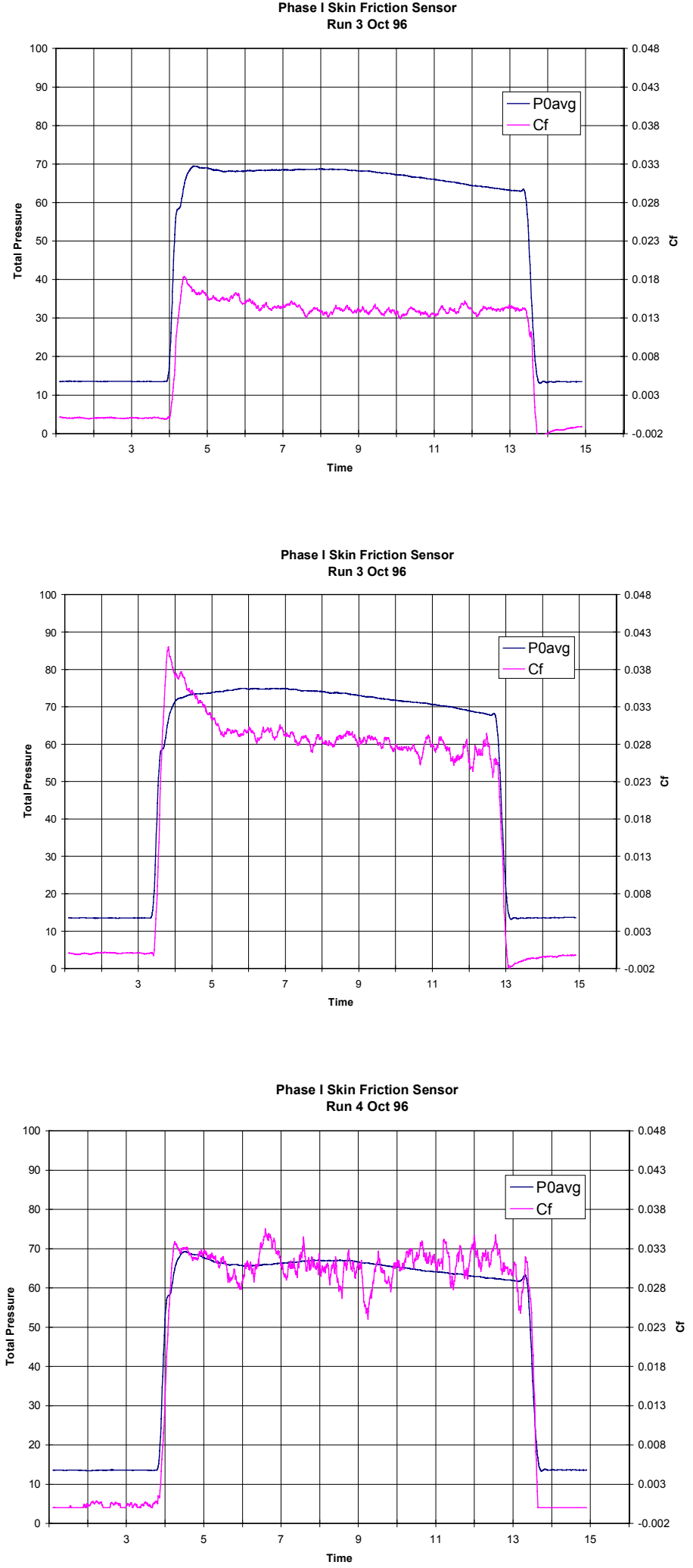
Appendix A

Comprehensive Testing Data

November 1996 Tests

Version 1 skin friction sensor

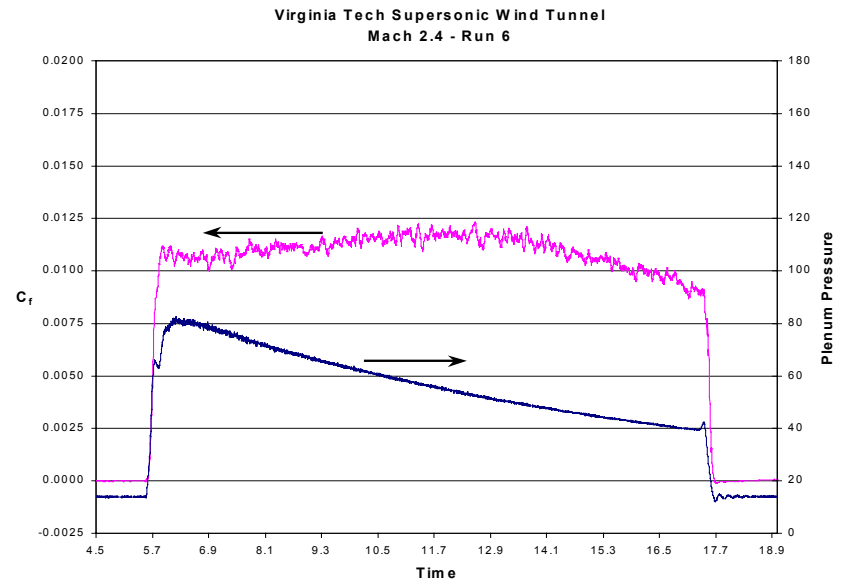

Version 1 sensor-pressure results

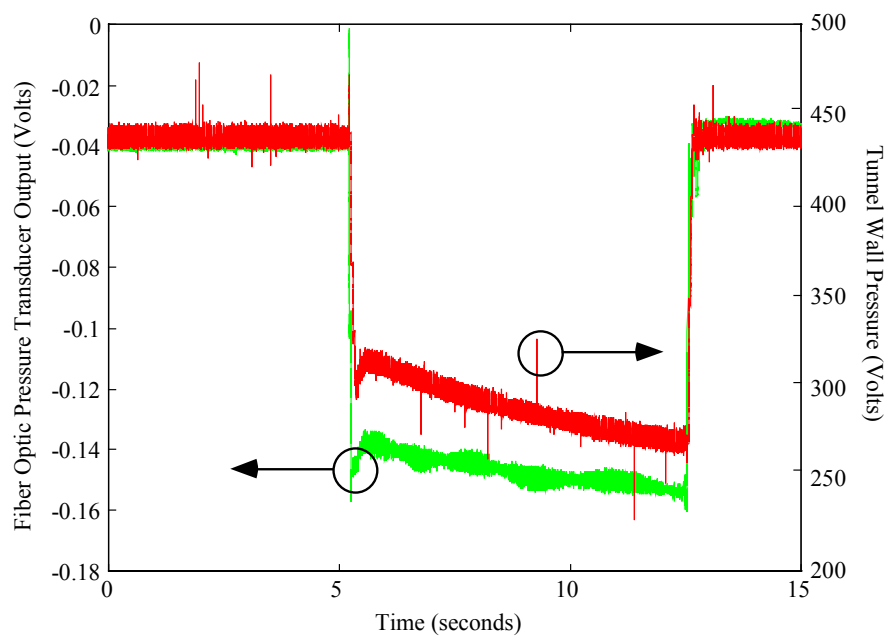

December 1996 Tests

Version 1 skin friction sensor

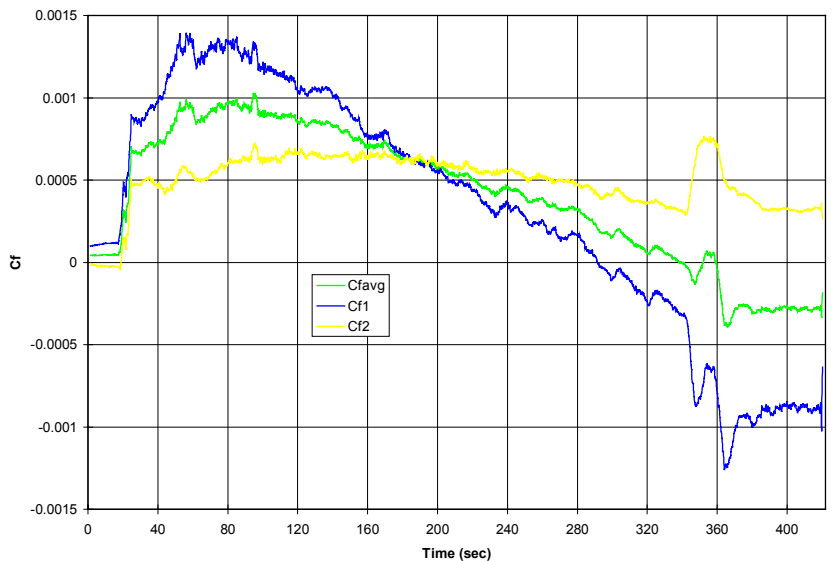


Appendix A

Comprehensive Testing Data

July 1997 Tests

Version 2 skin friction sensor
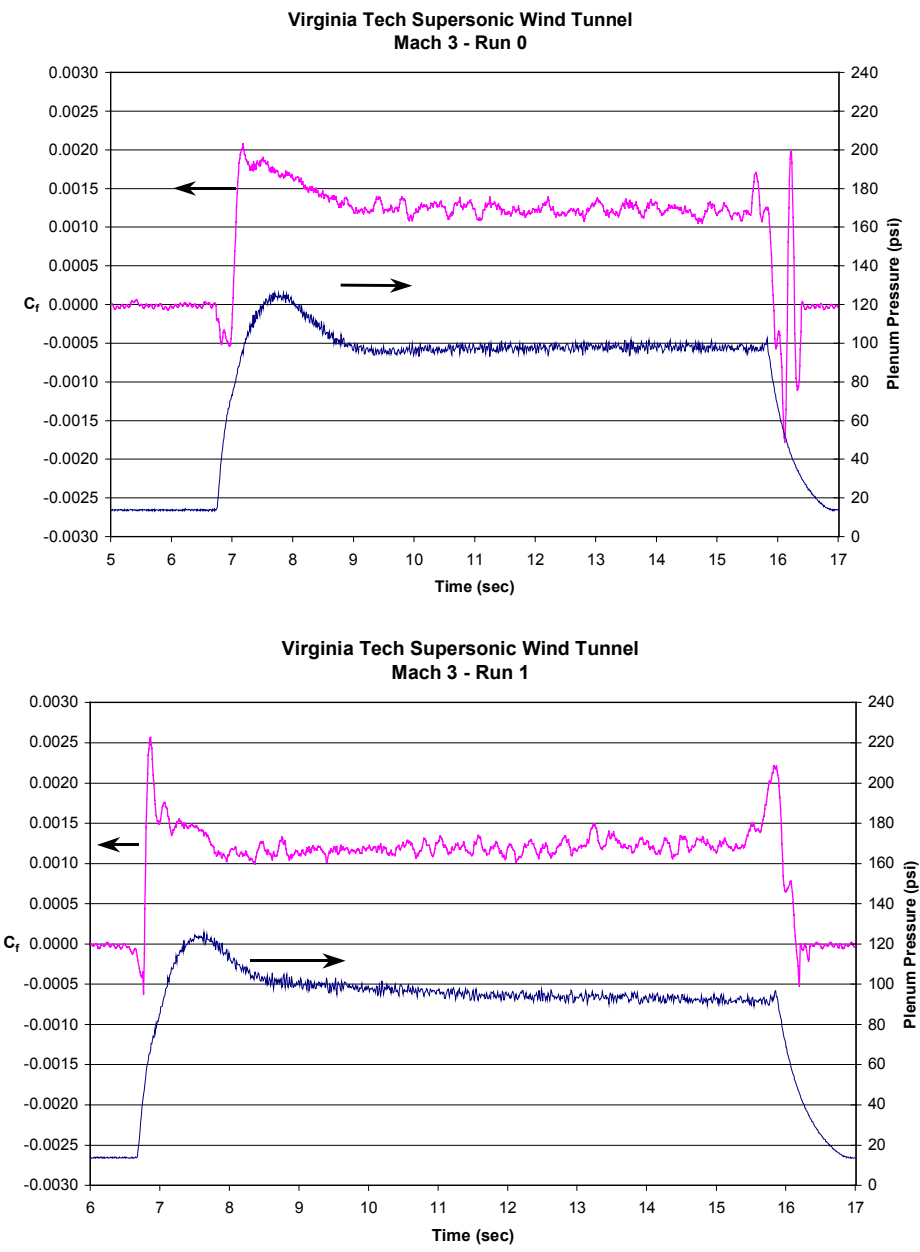

March 1999 Tests

Version 3 skin friction sensor

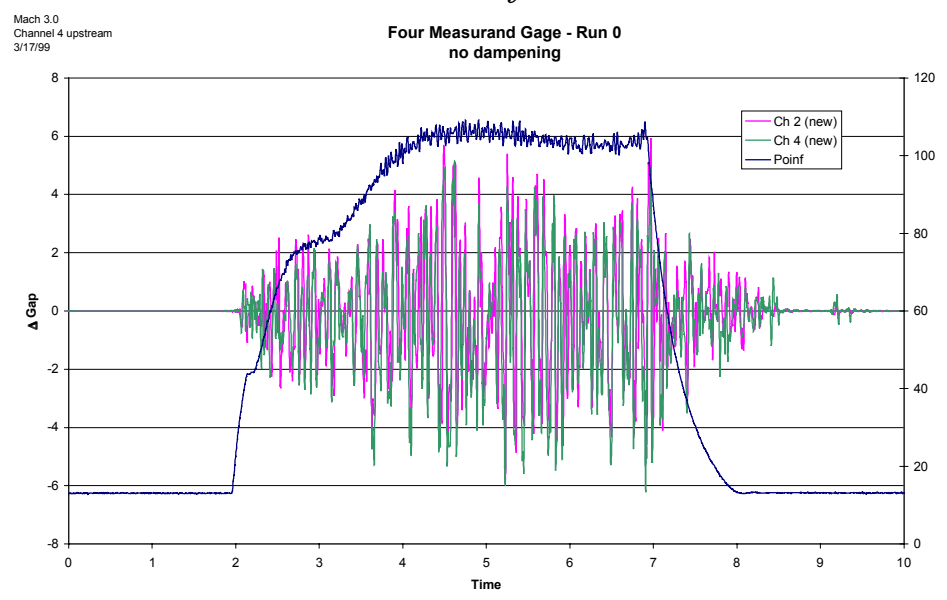


Appendix A

Comprehensive Testing Data
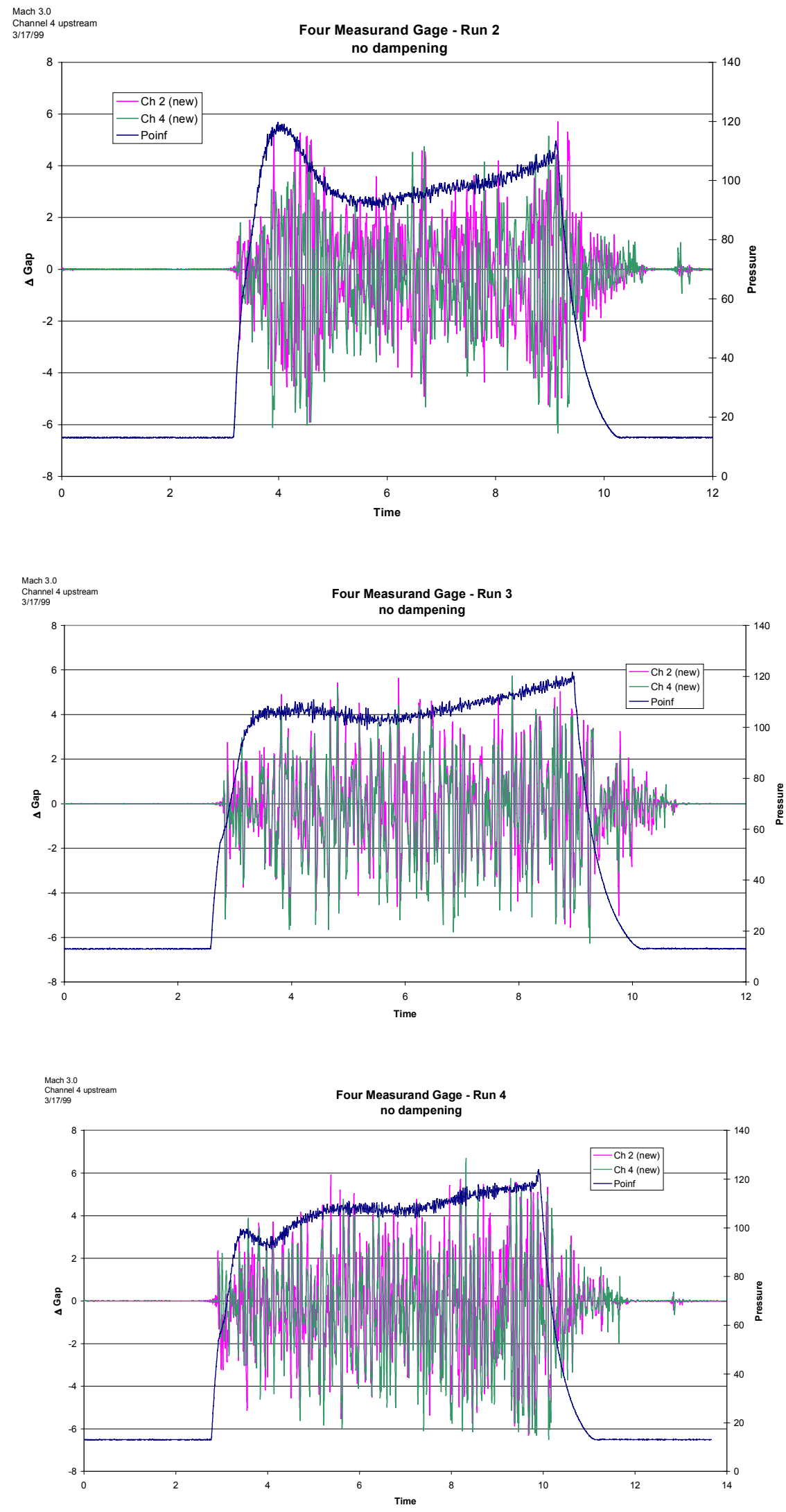
Appendix A

Comprehensive Testing Data
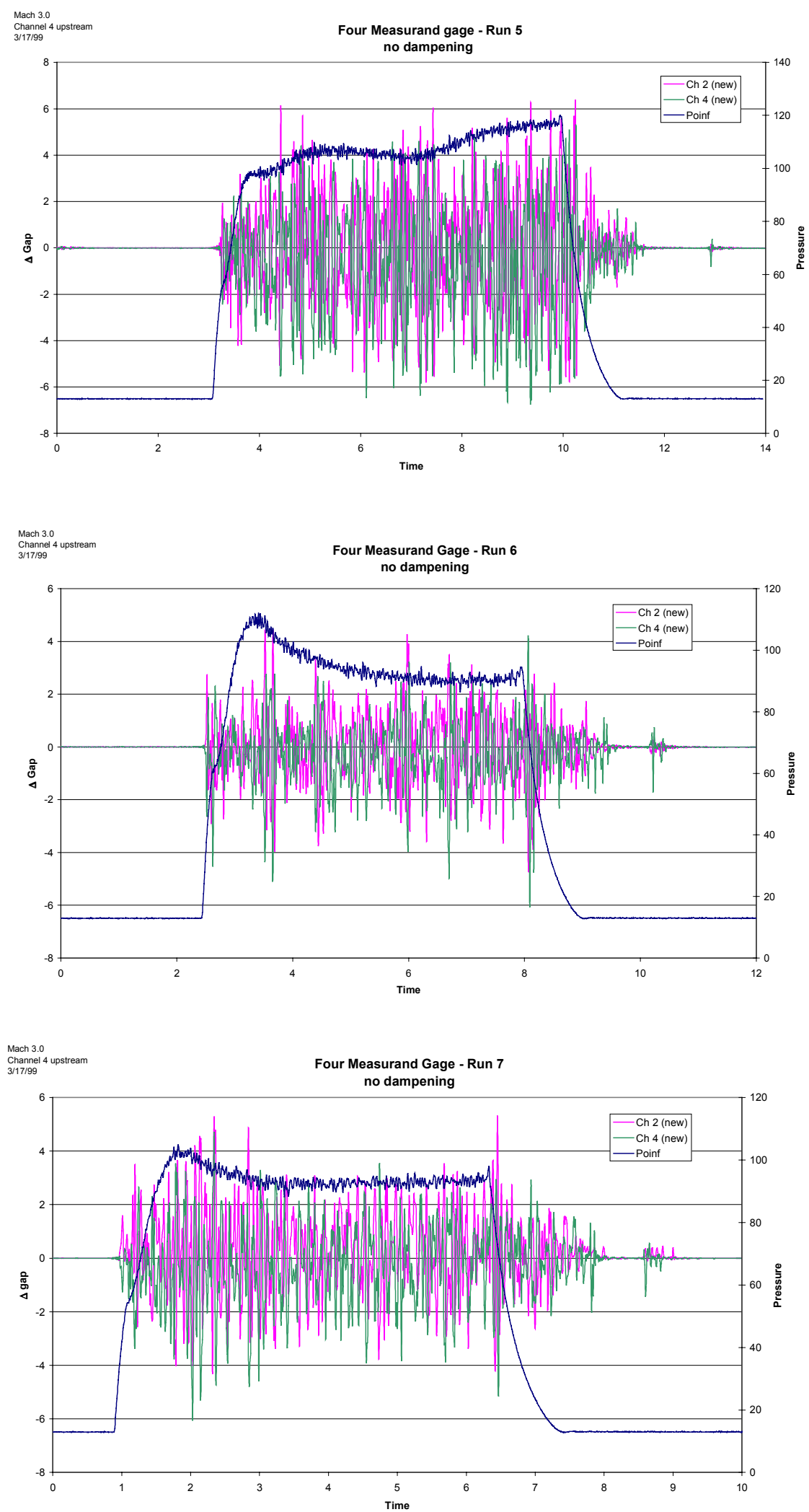
Appendix A

Comprehensive Testing Data
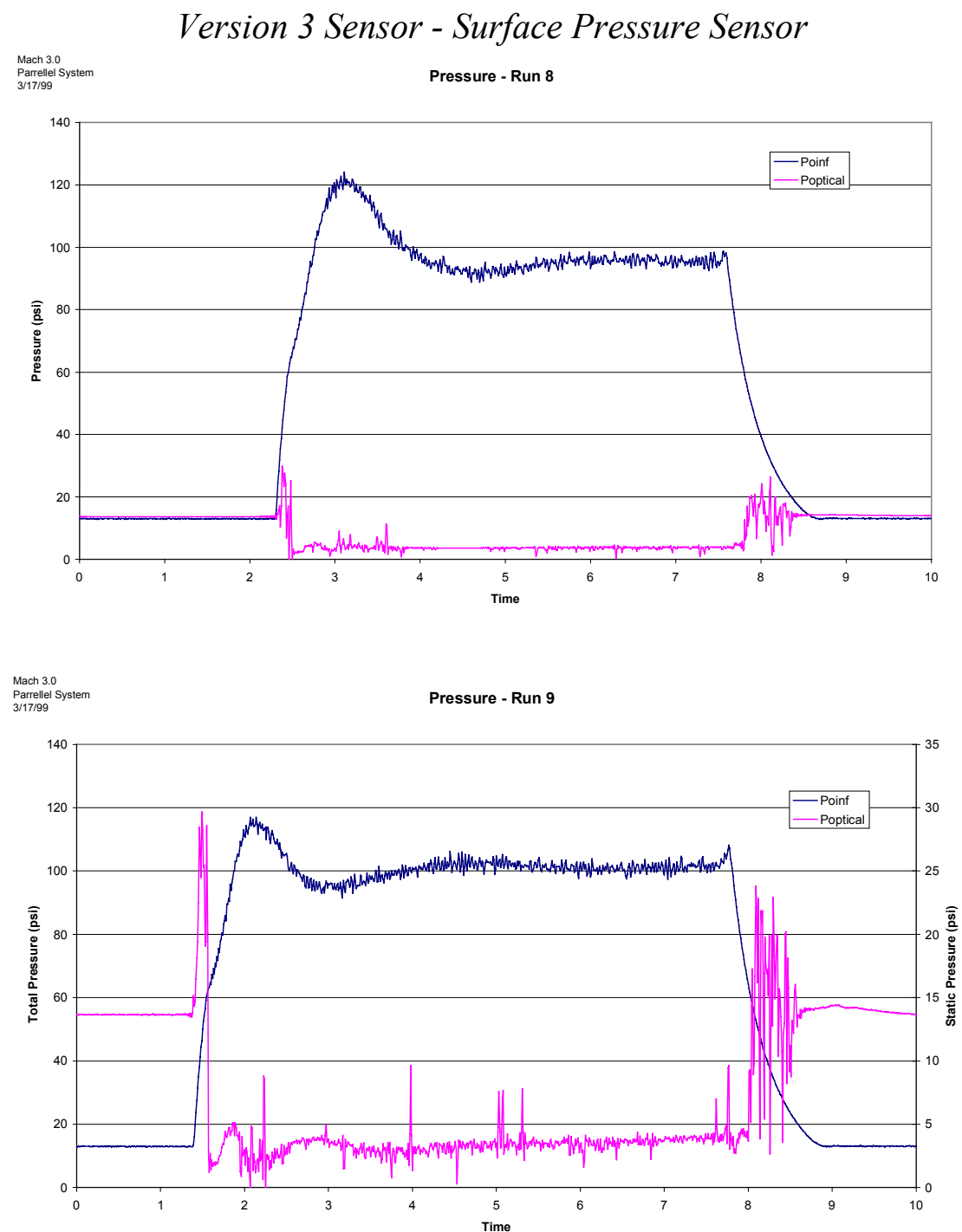

Version 4 skin friction sensor

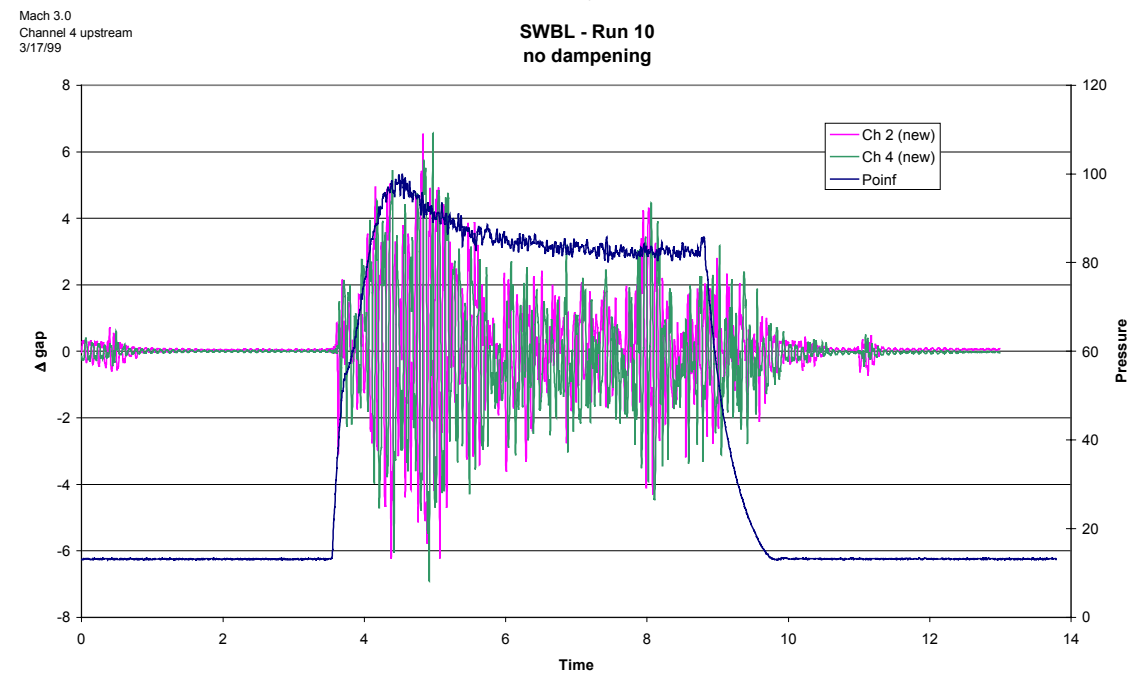


Appendix A

Comprehensive Testing Data
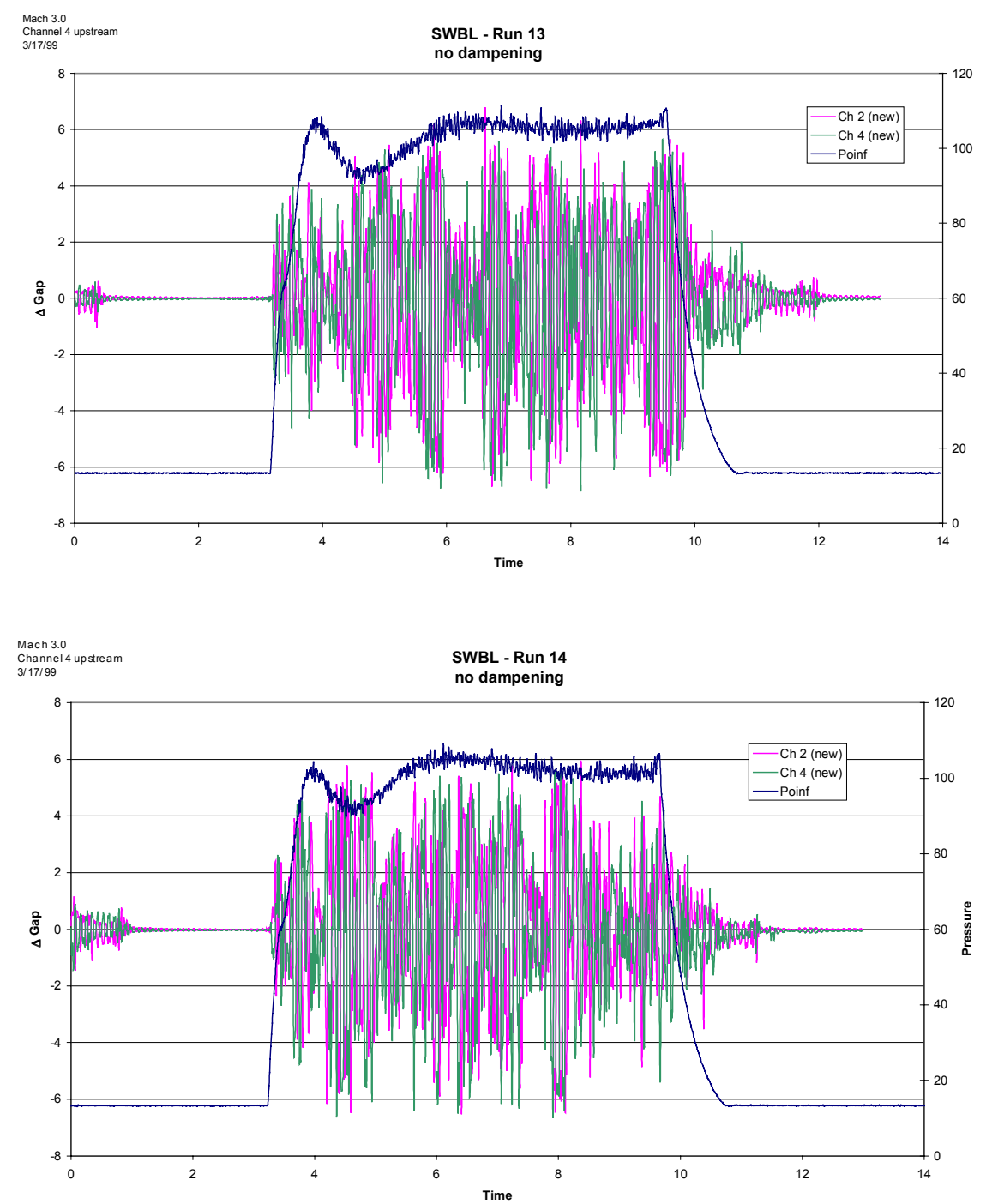

Version 3 skin friction sensor (oil-filled)

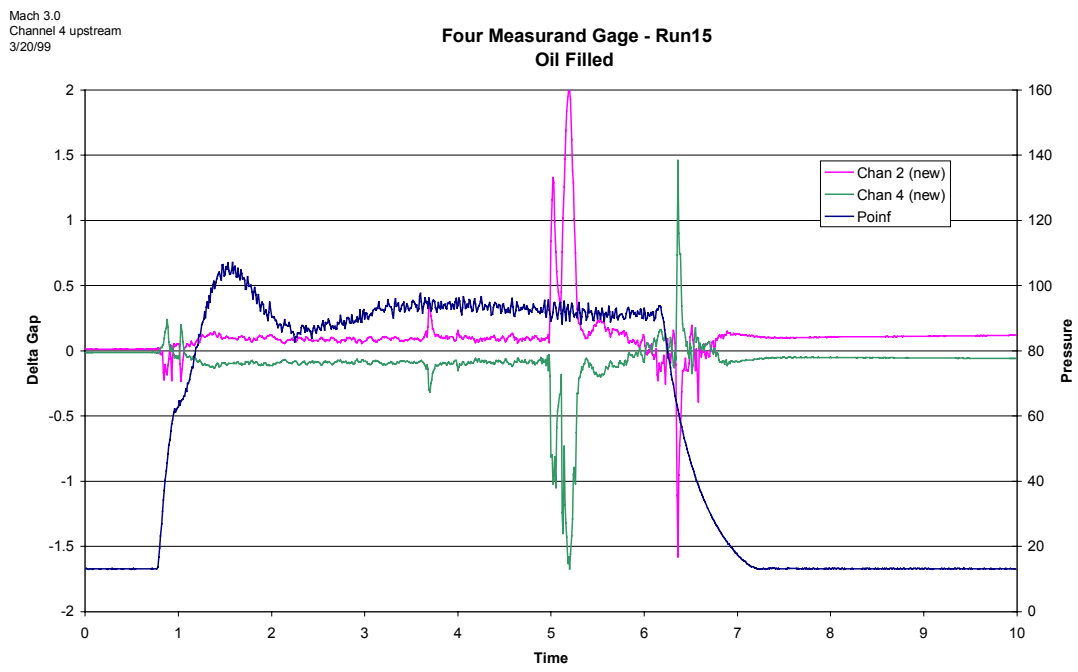


Appendix A

Comprehensive Testing Data

Mach 3.0
Channel 4

Channel
3/20199

Four Measurand Gage - Run16

Oil Filled
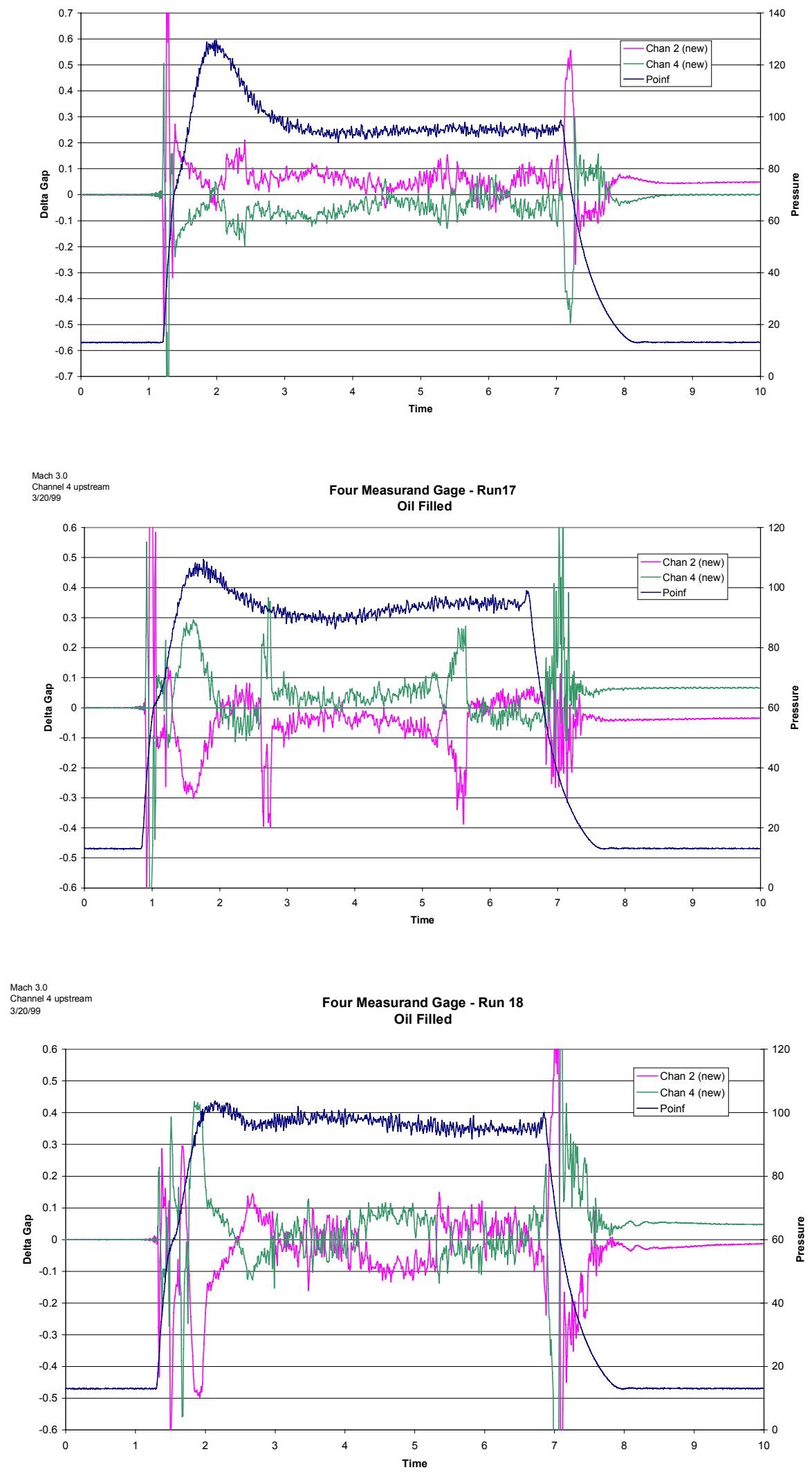
Appendix A

Comprehensive Testing Data

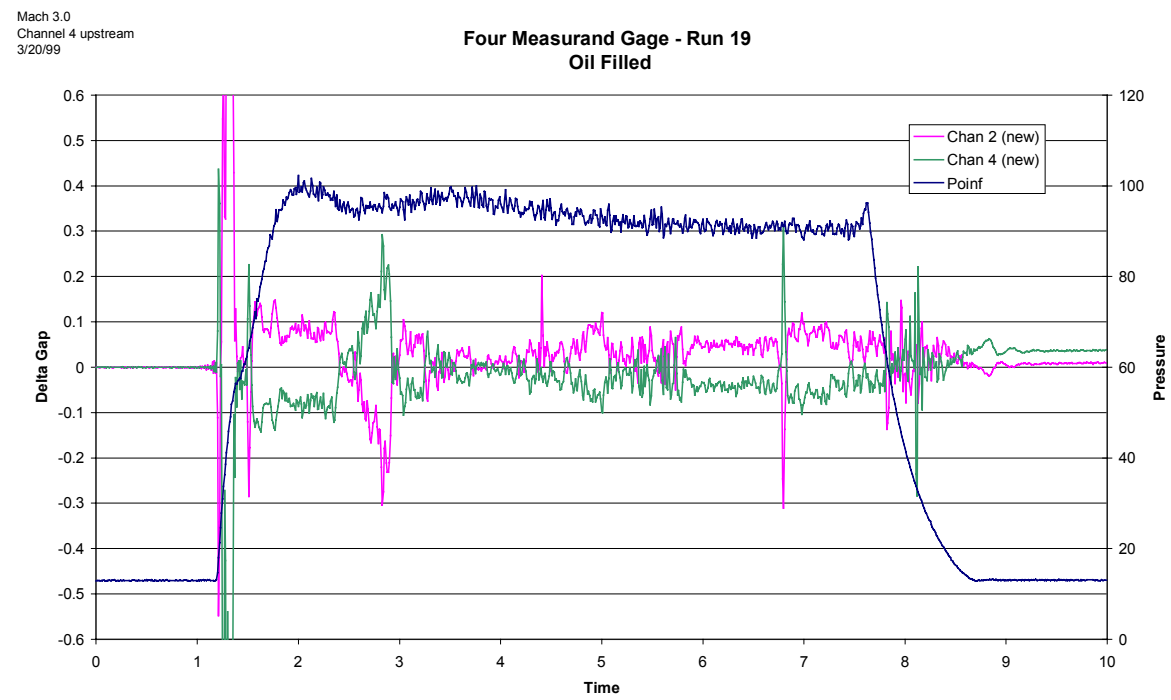

\section{April 1999 Pressure Tests}
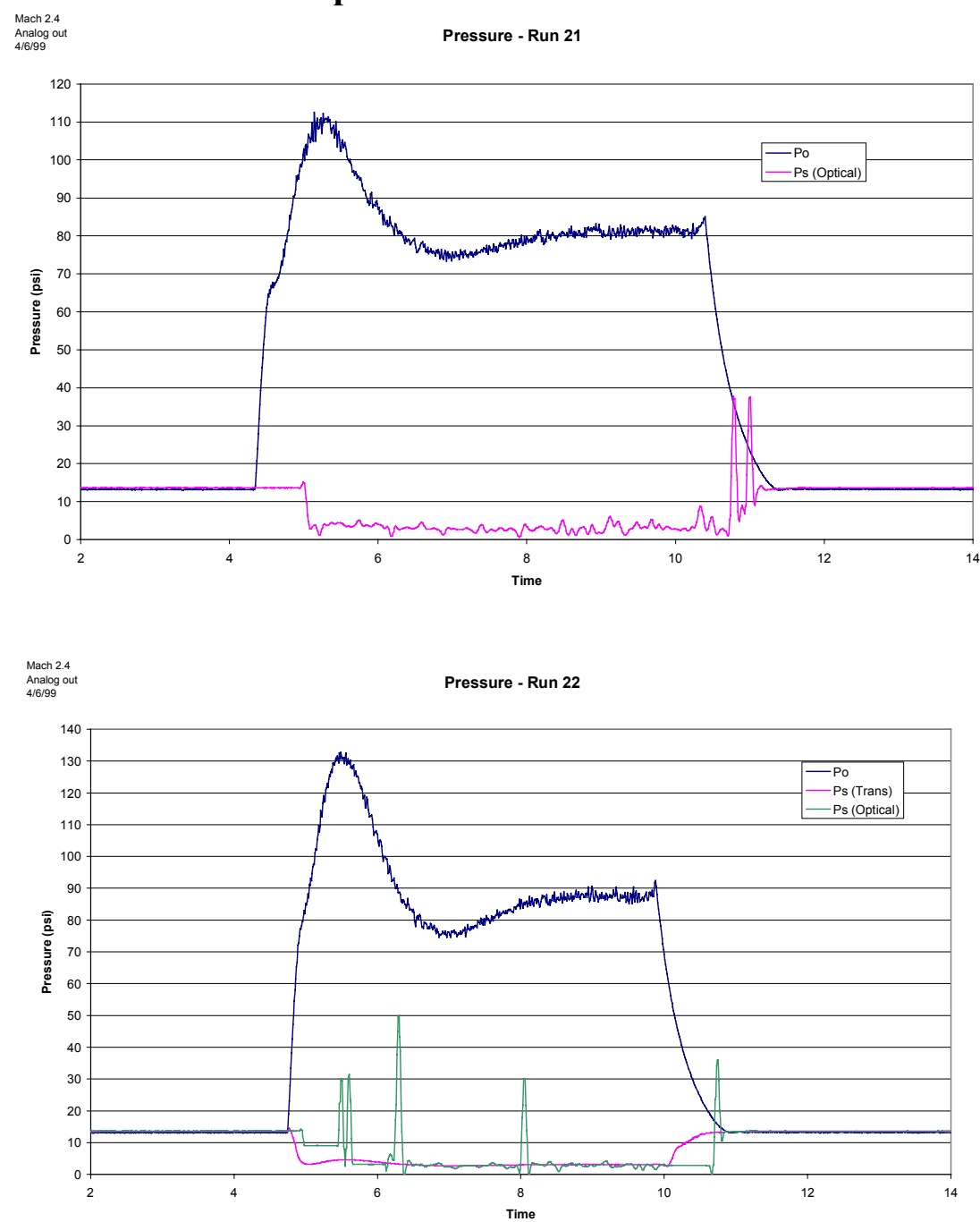
Appendix A

Comprehensive Testing Data
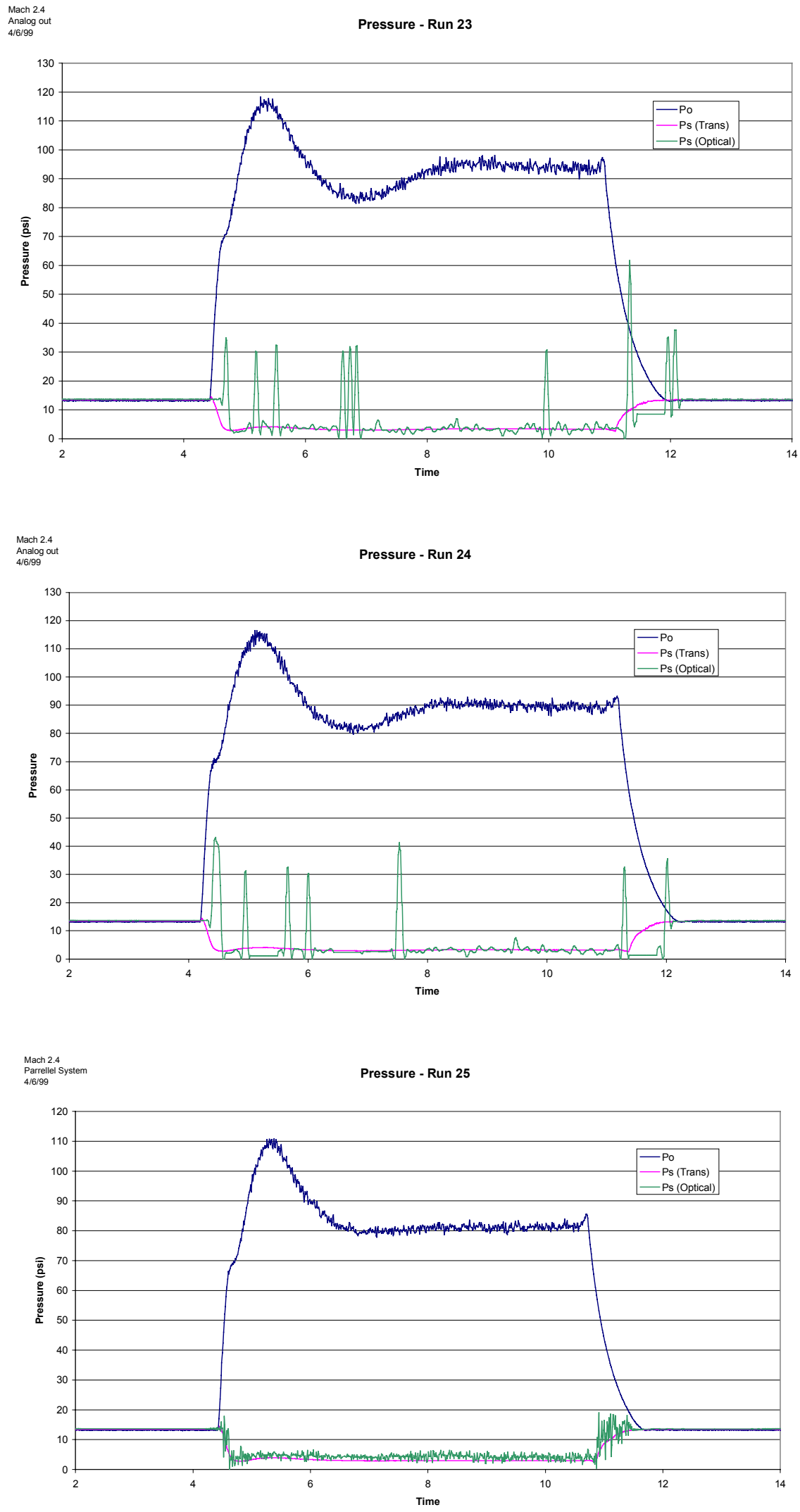
Appendix A

Comprehensive Testing Data

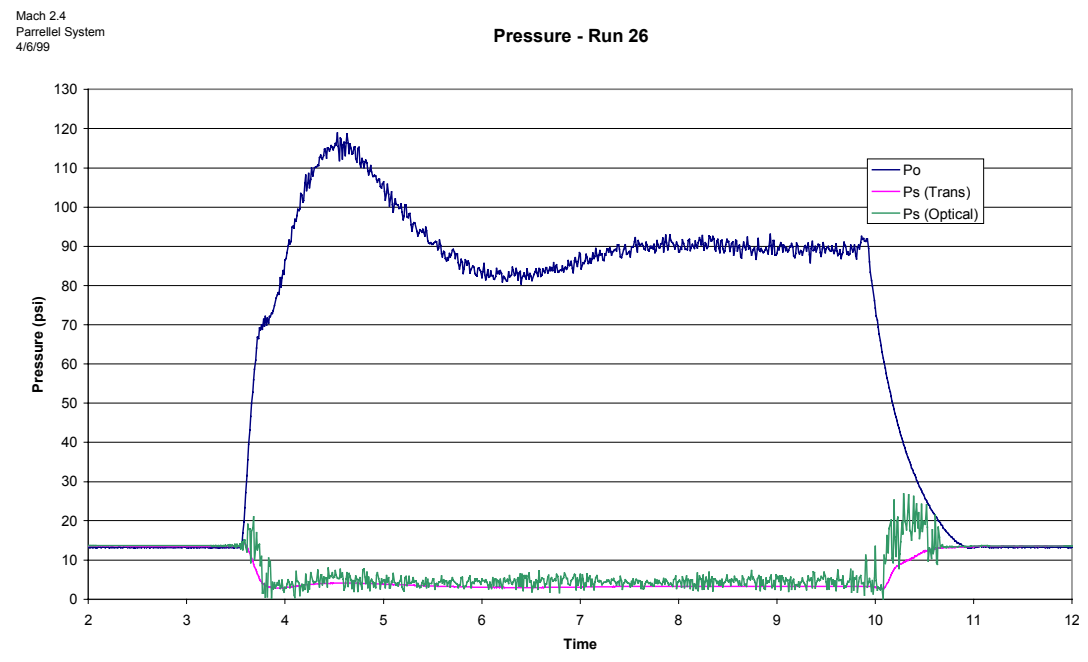

May 1999 Tests

Version 4 skin friction sensor
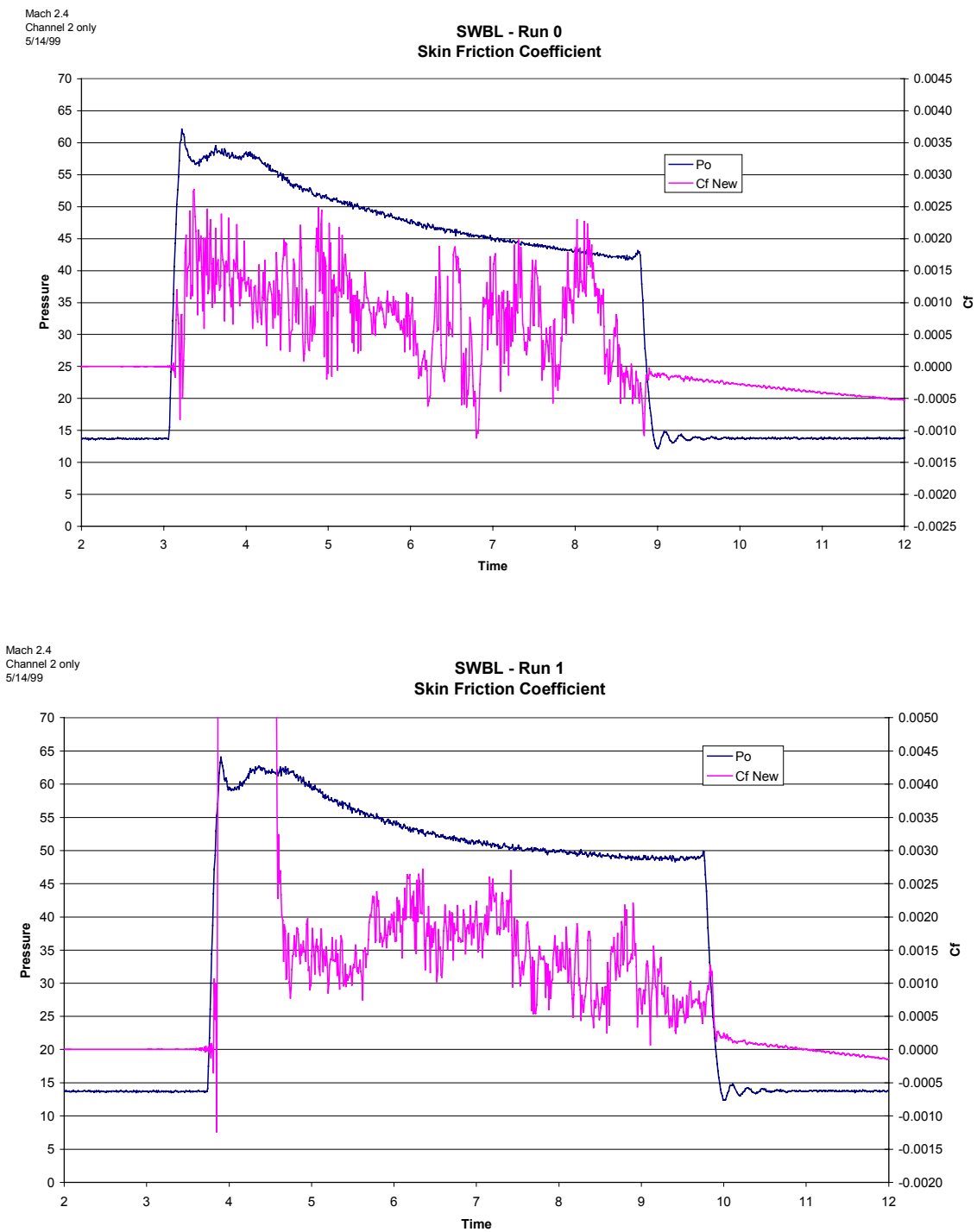
Appendix A

Comprehensive Testing Data
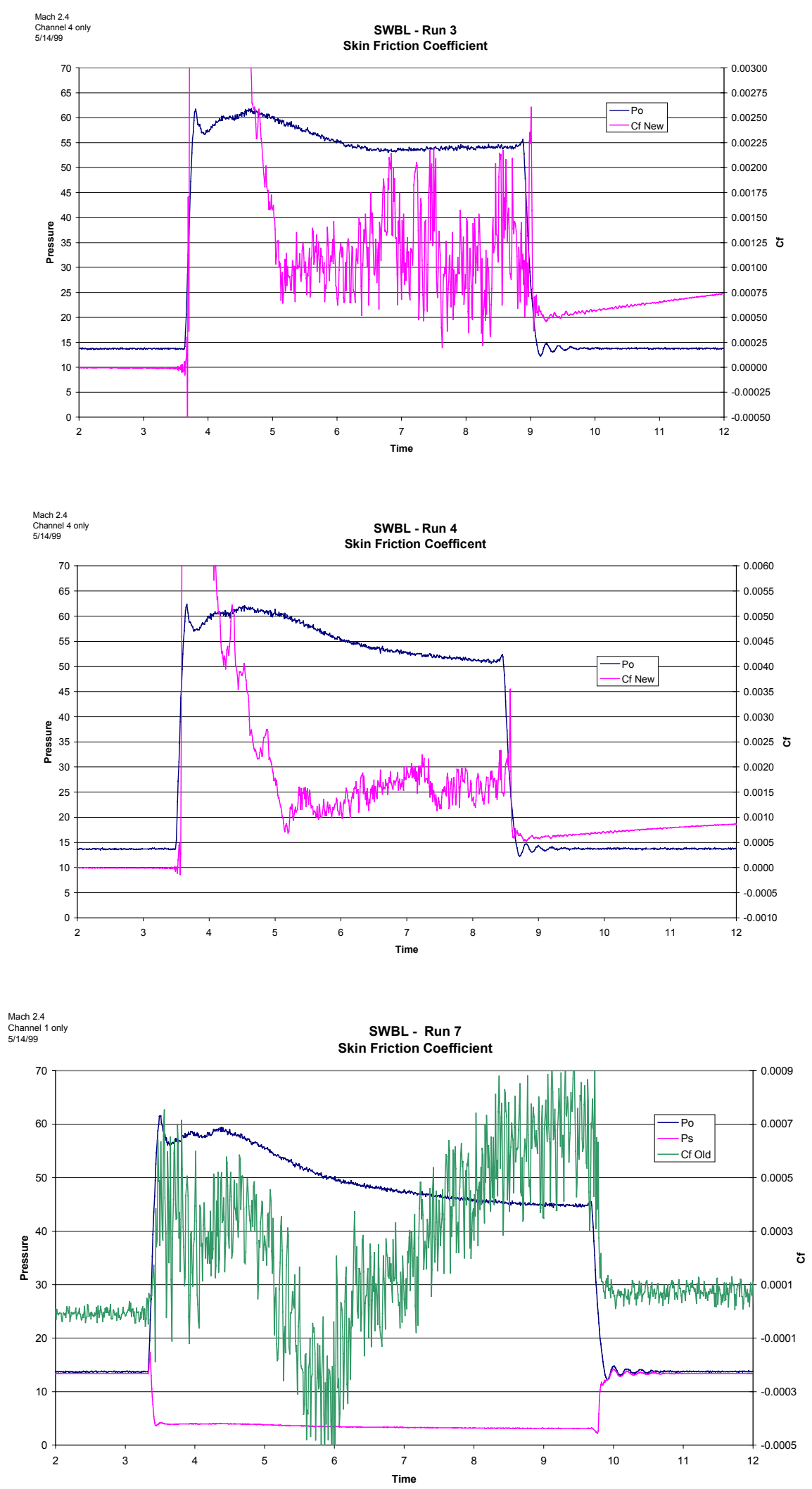
Appendix A

Comprehensive Testing Data
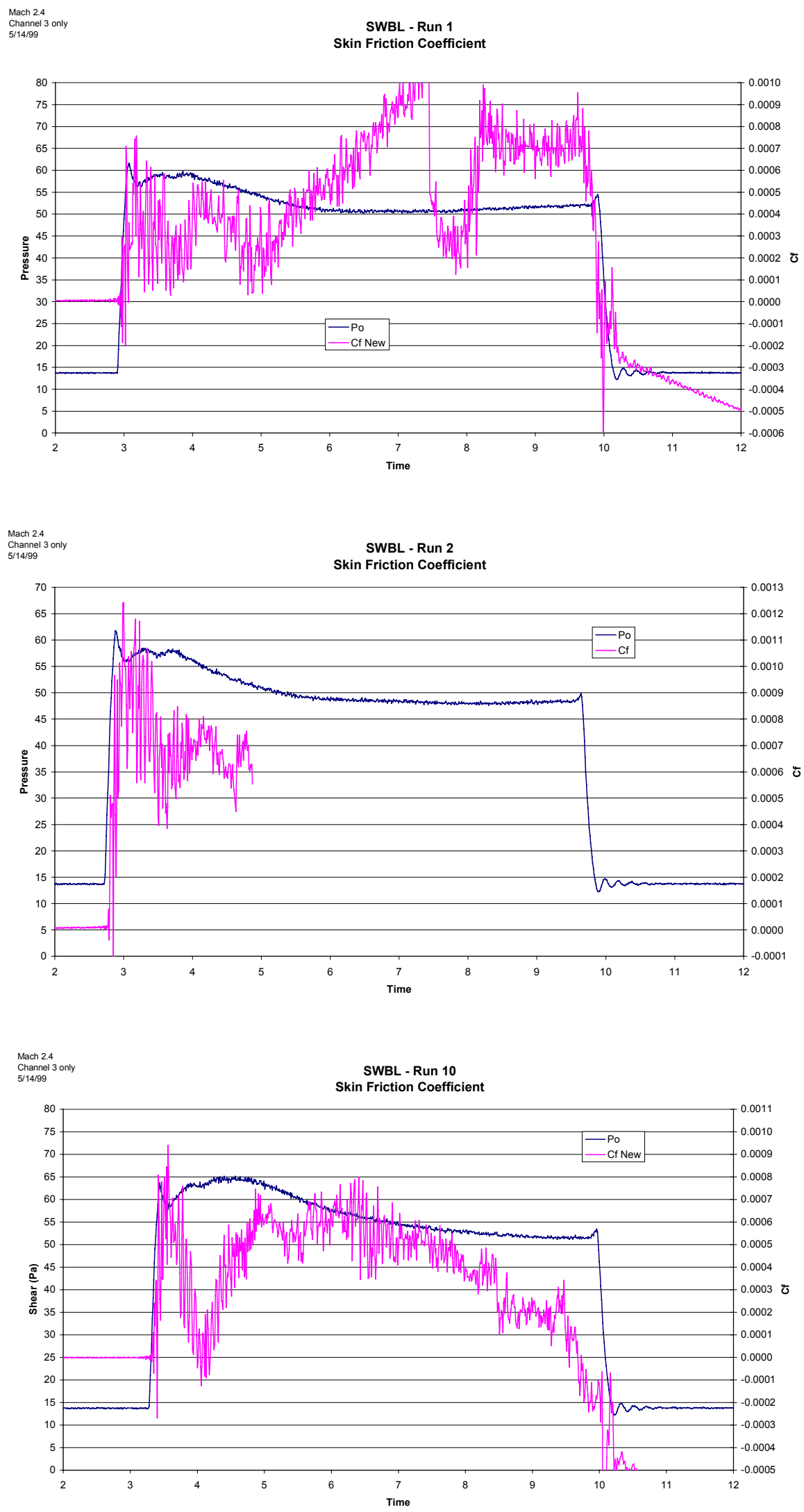
Appendix A

Comprehensive Testing Data
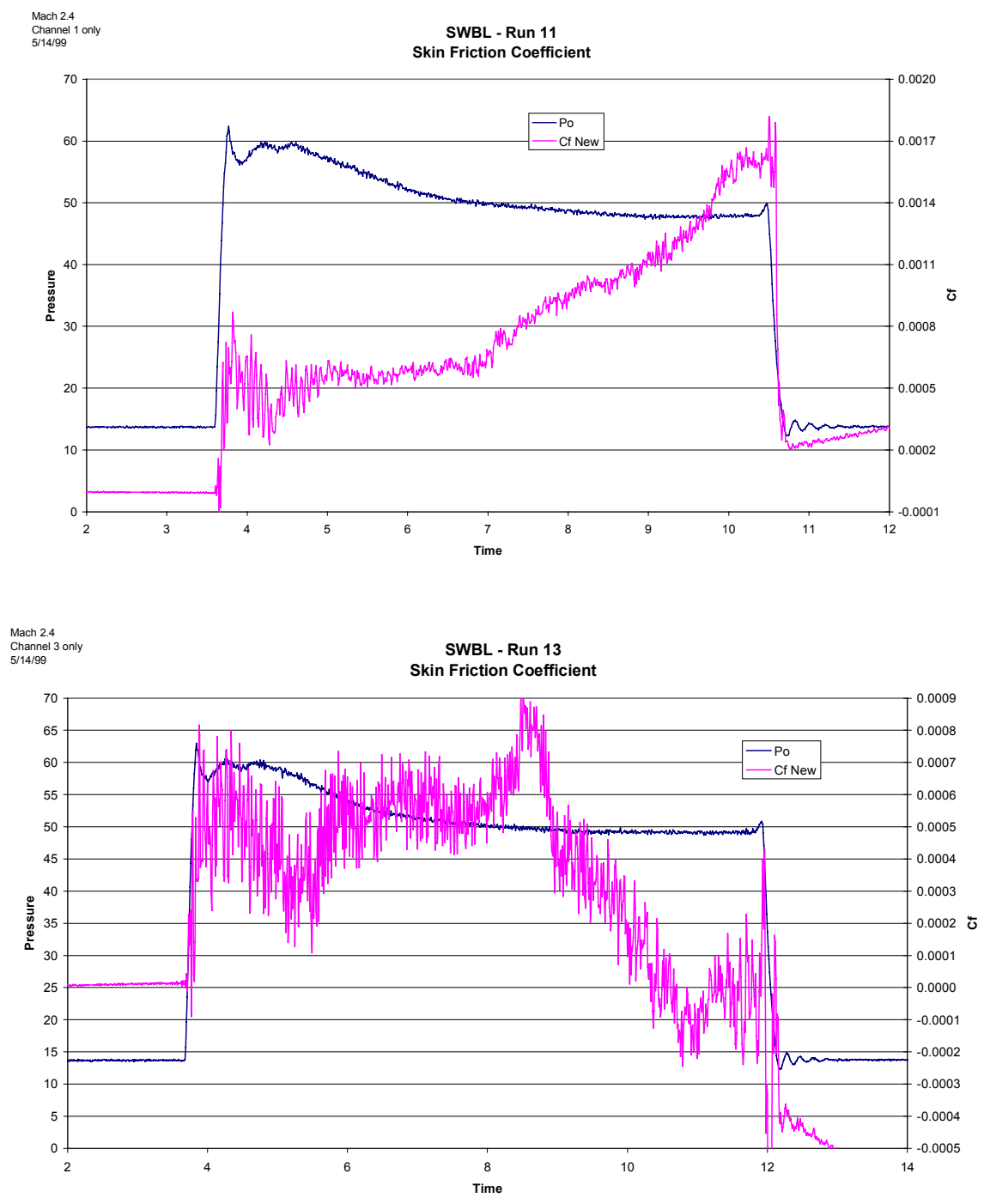

June 1999 Tests

Version 4 skin friction sensor

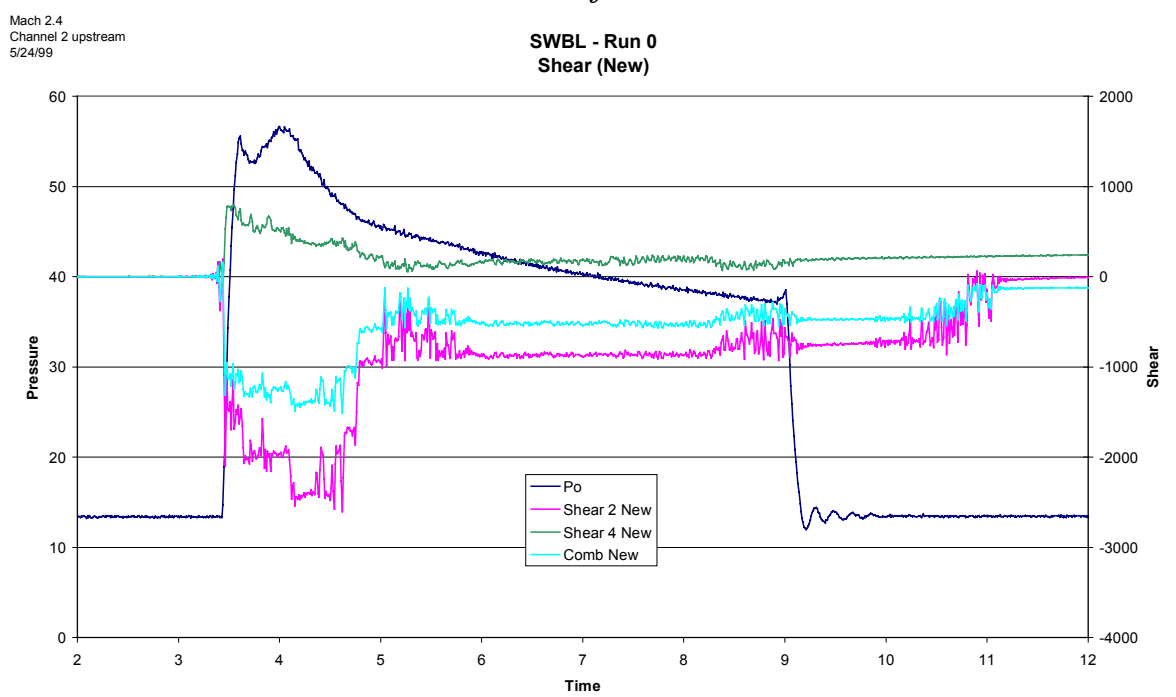


Appendix A

Comprehensive Testing Data
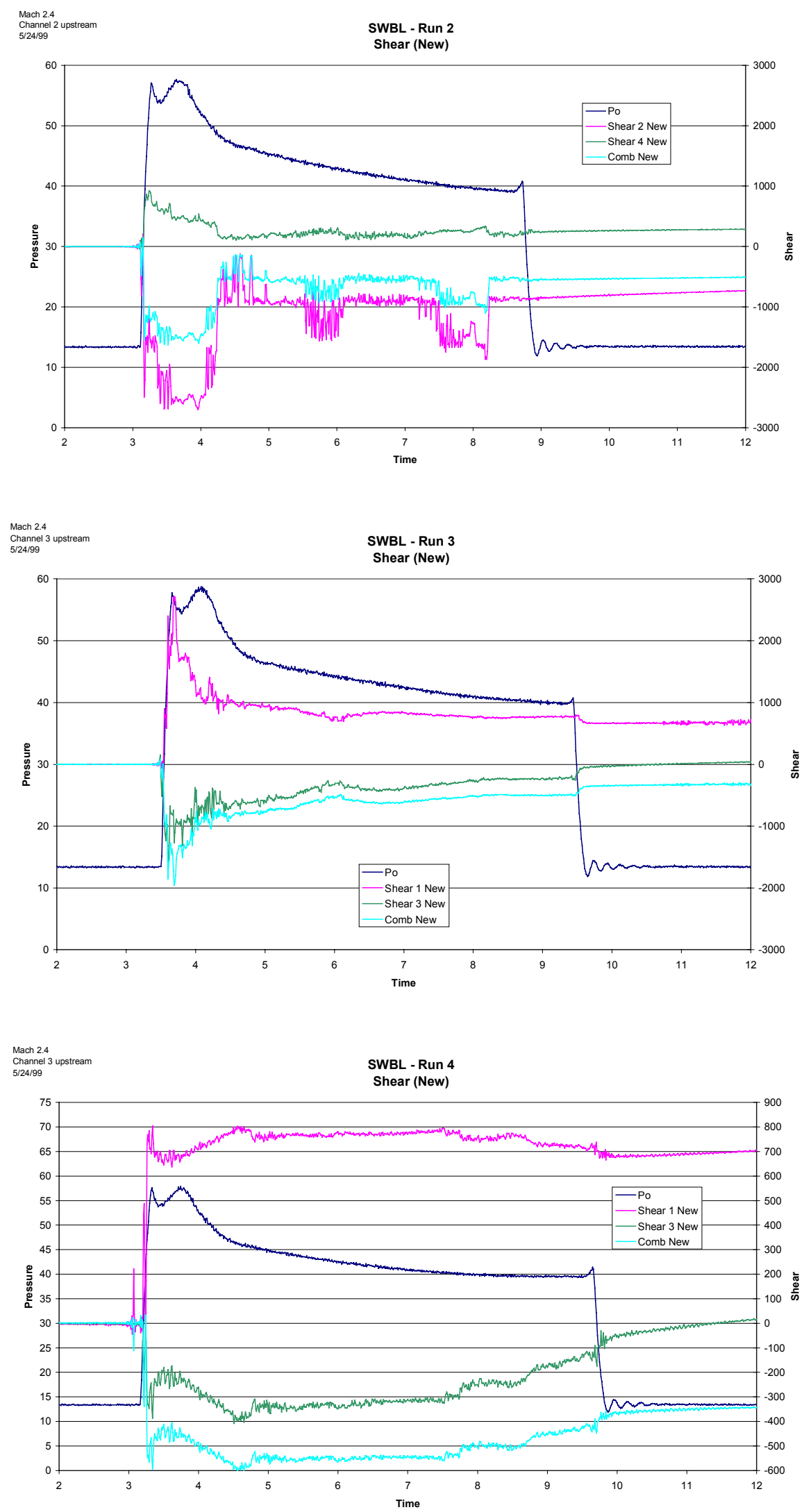
Appendix A

Comprehensive Testing Data
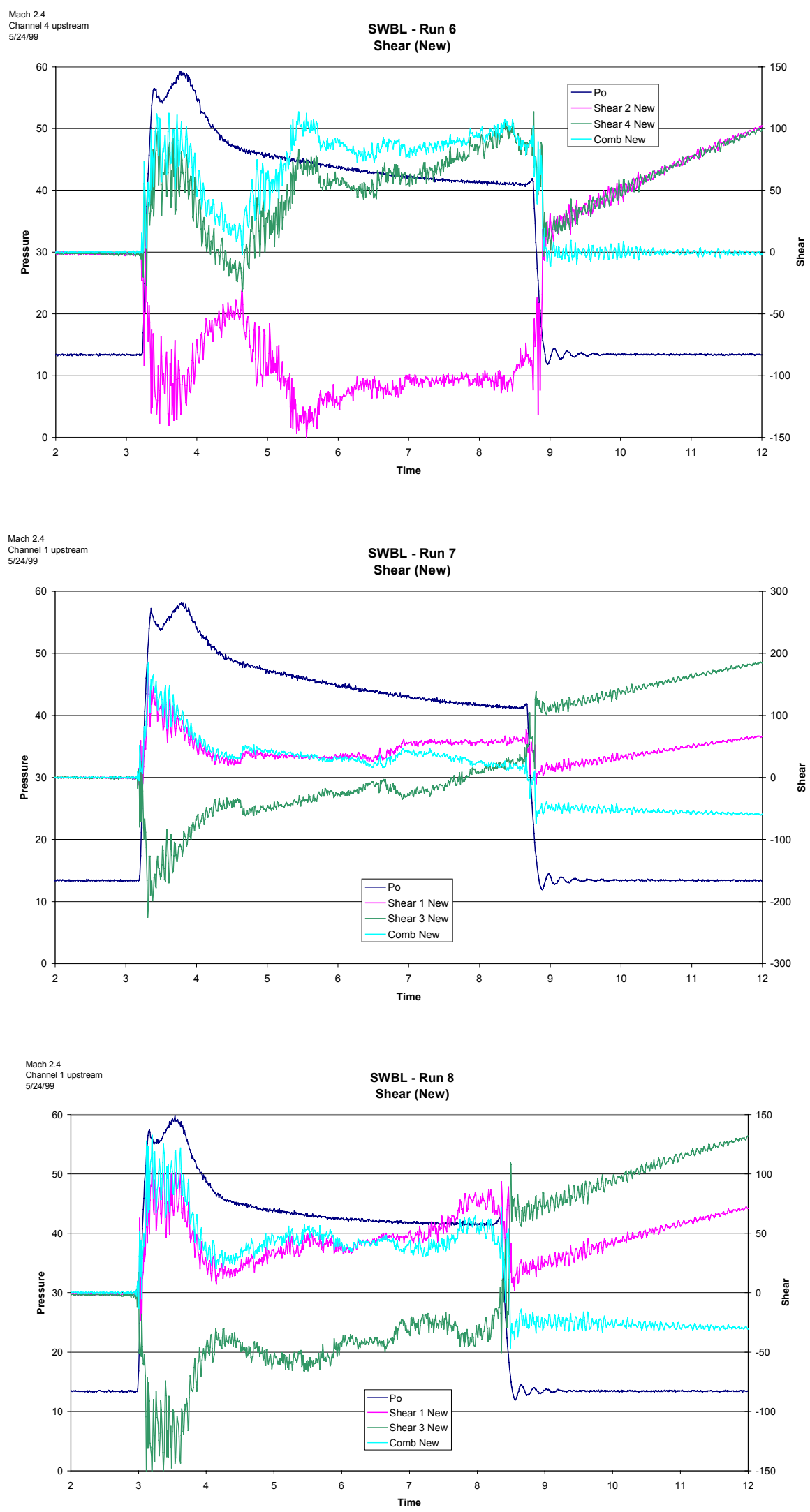
Appendix A

Comprehensive Testing Data
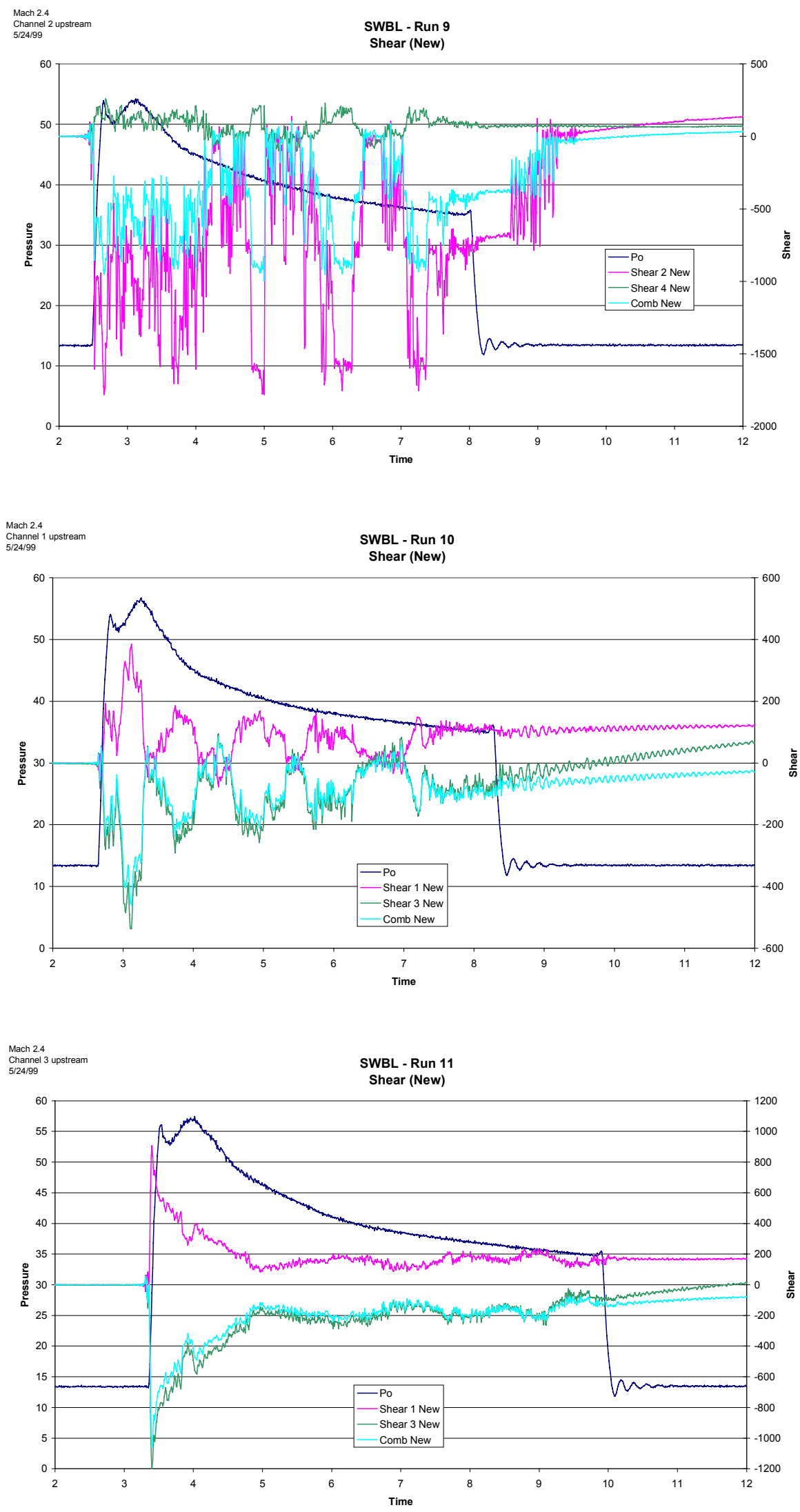
Appendix A

Comprehensive Testing Data

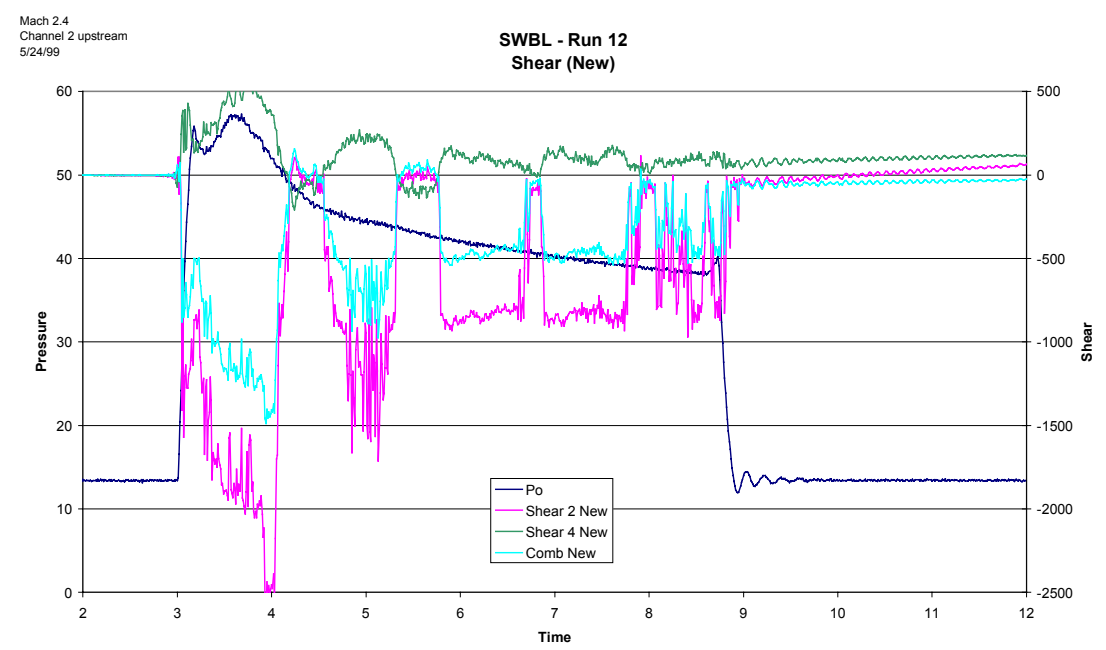

June 1999

Version 4 skin friction sensor - Glycerin Flow Tests Glycerin Flow
Channel 1 upstream
Ful Electrons
July 28,1999 $\quad$ SWBL - Run 1
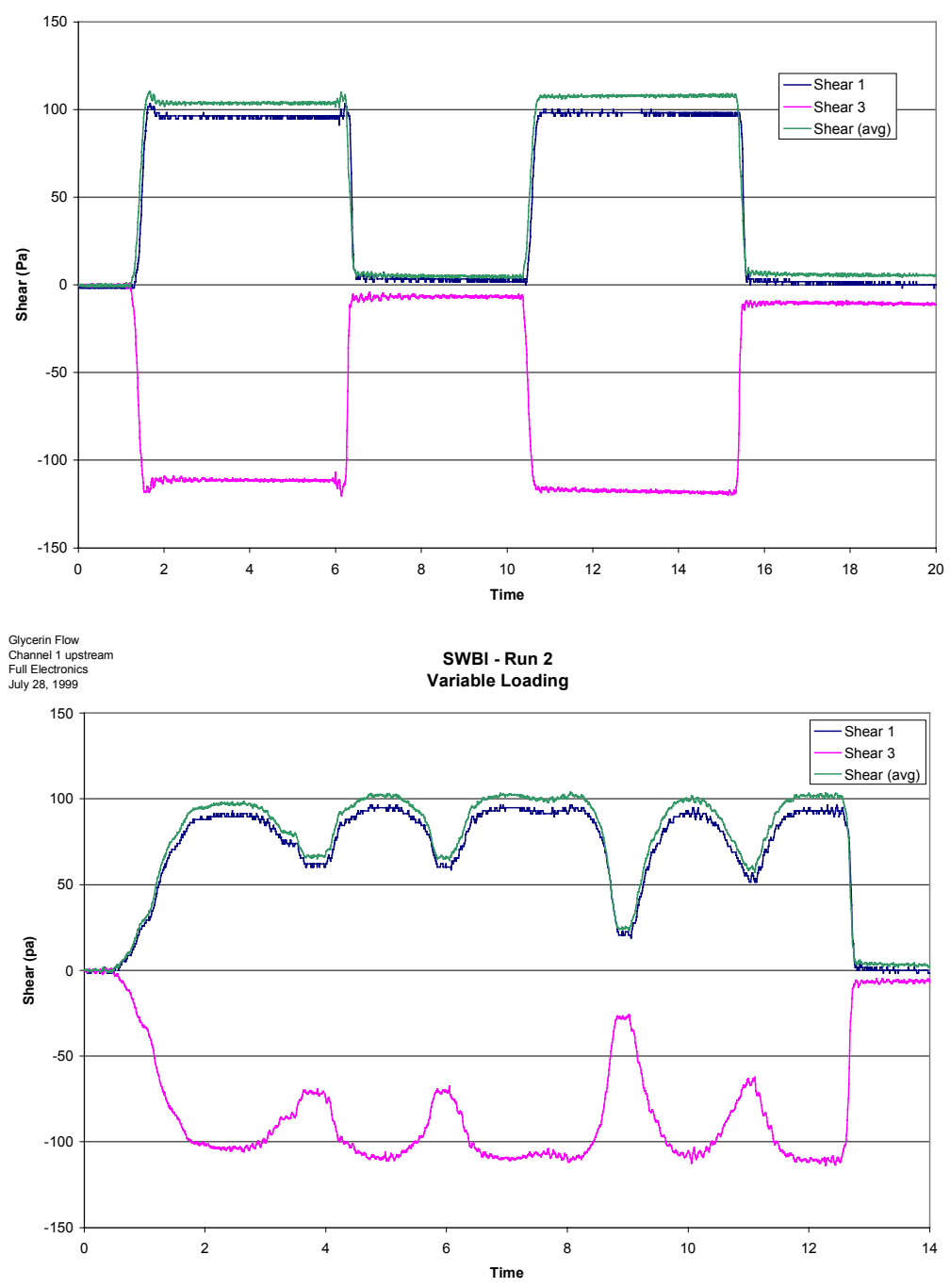
Appendix A

Comprehensive Testing Data
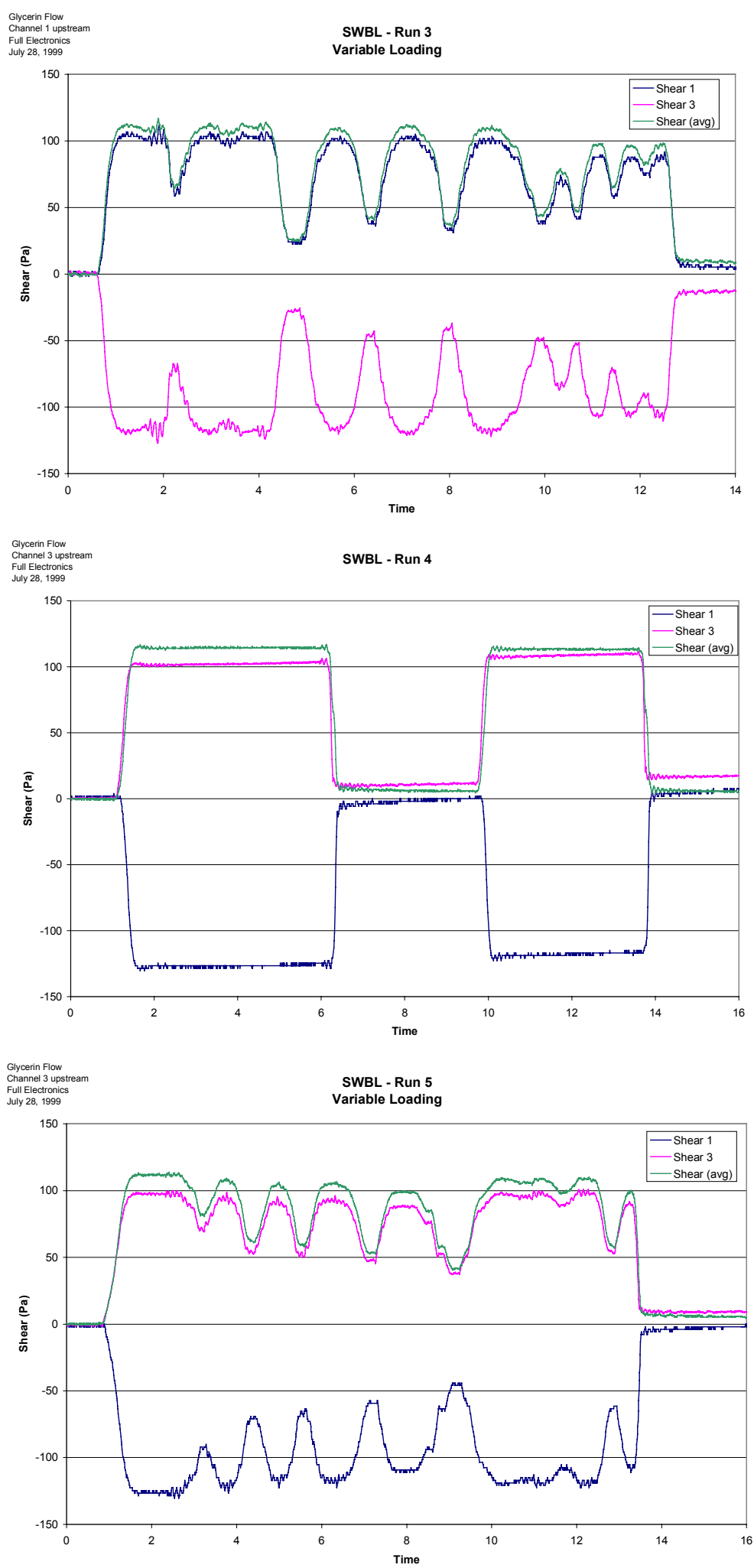
Appendix A

Comprehensive Testing Data

August 1999 Tests

Version 5 a skin friction sensor
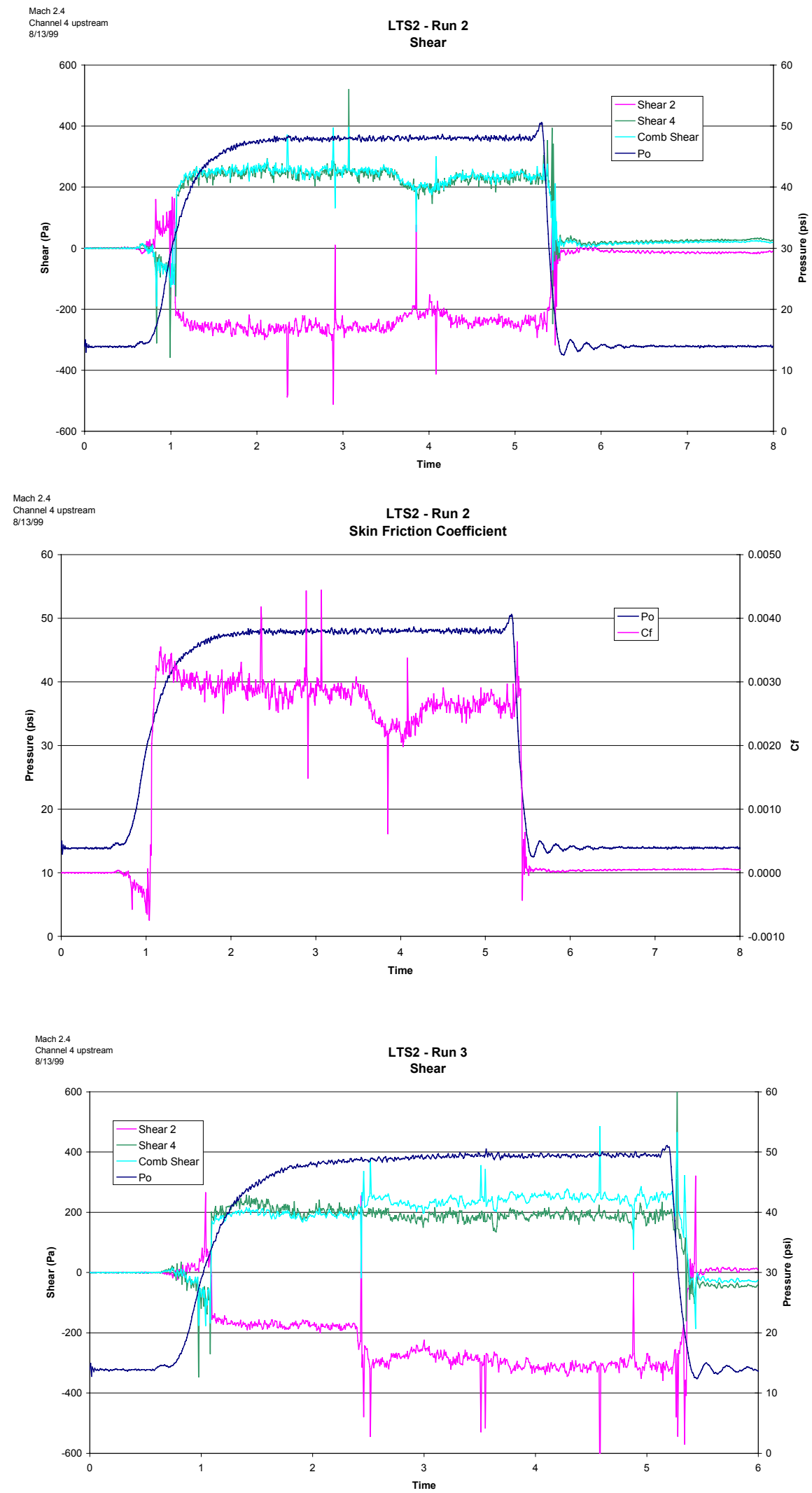
Appendix A

Comprehensive Testing Data
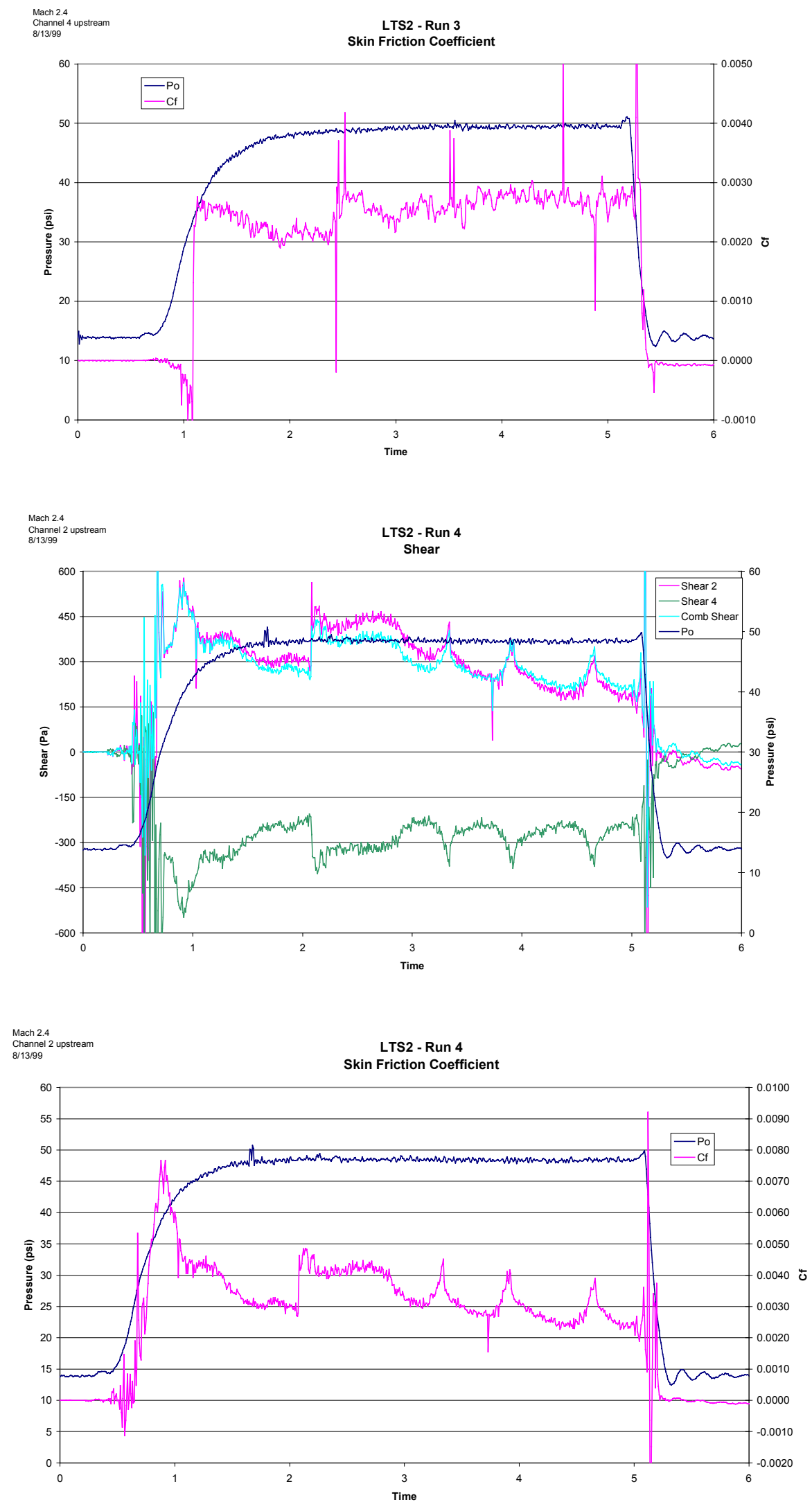
Appendix A

Comprehensive Testing Data
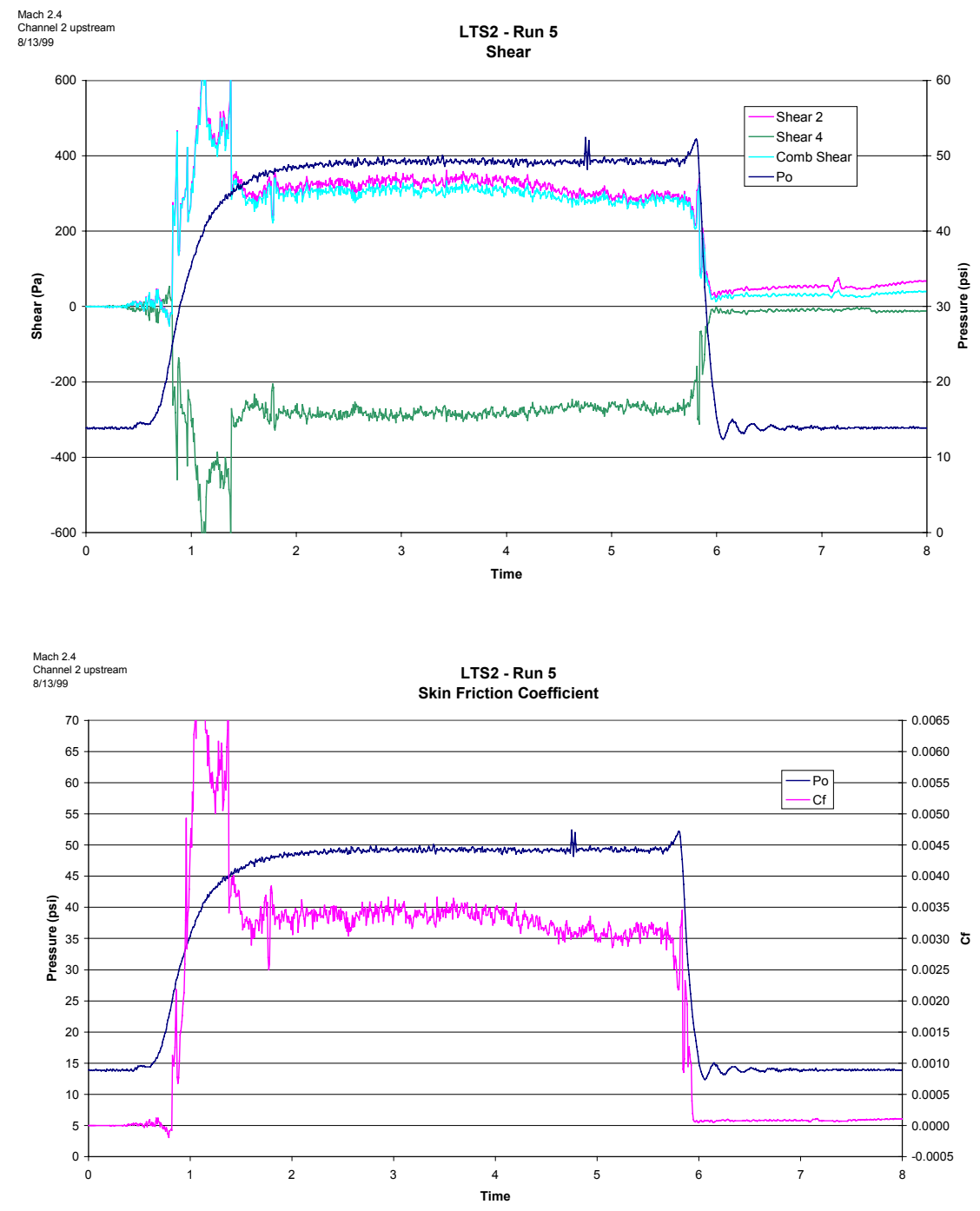

September 1999 Tests

Version 3 skin friction sensor

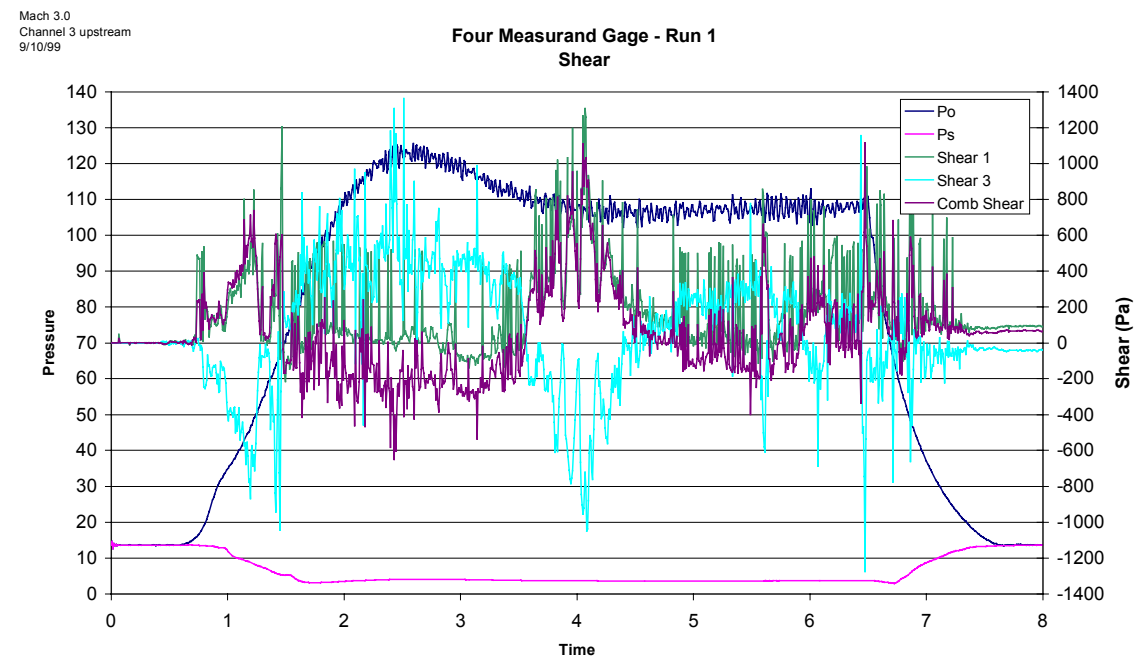


Appendix A

Comprehensive Testing Data

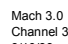
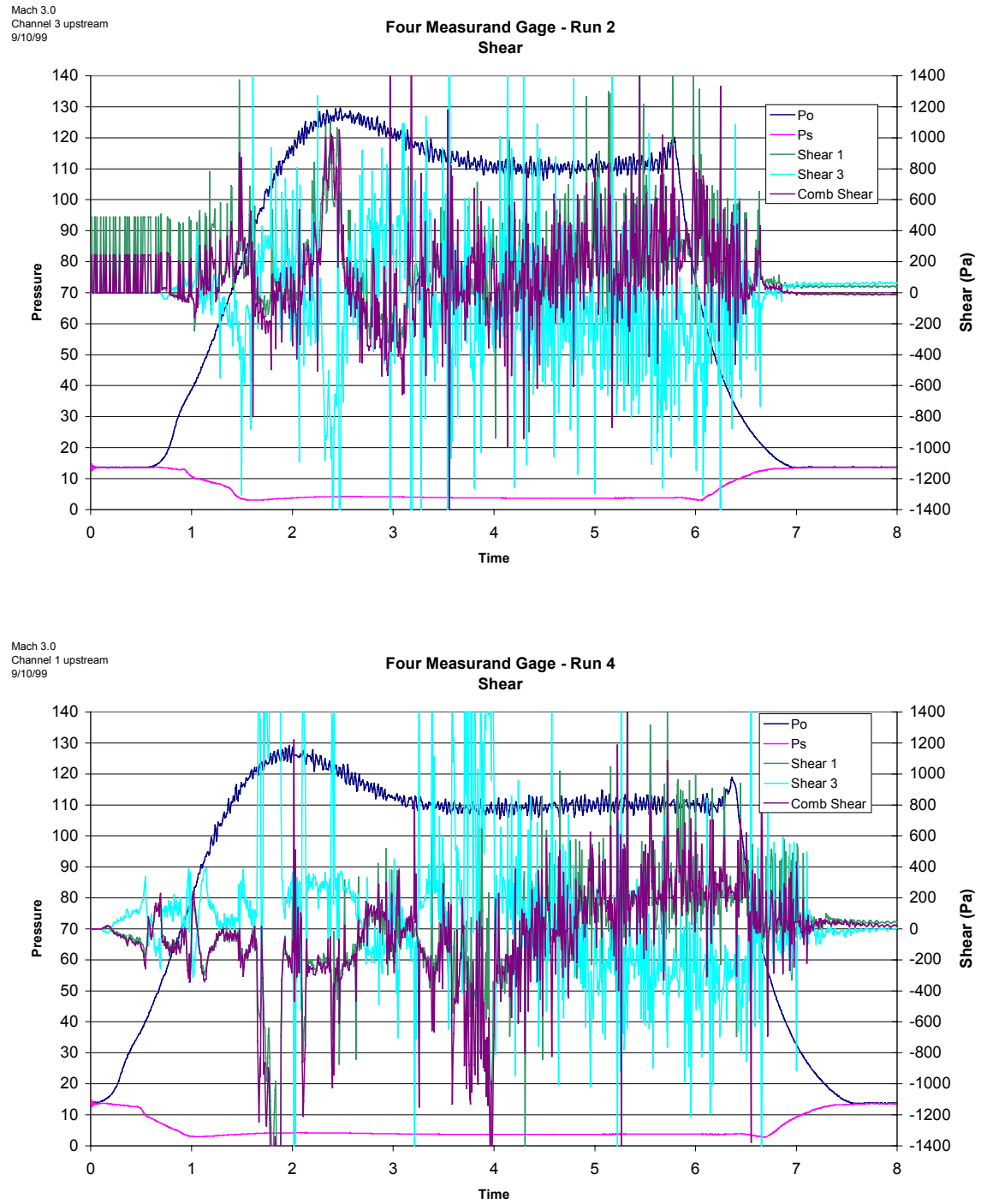

Version 5 a skin friction sensor

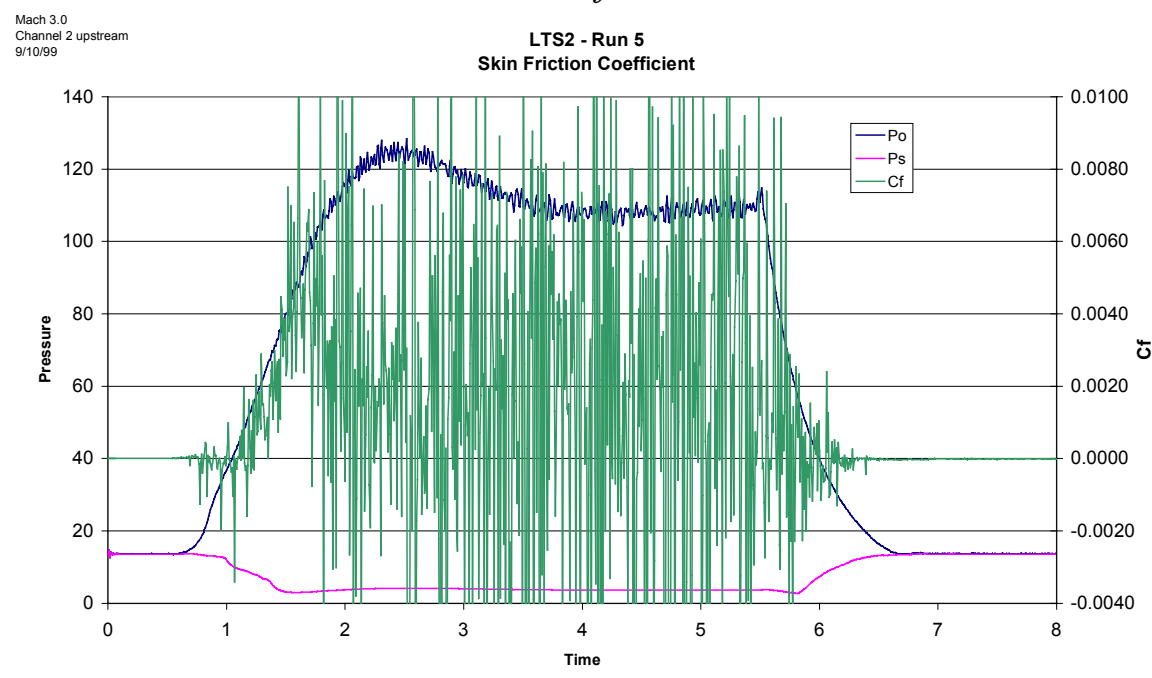


Appendix A

Comprehensive Testing Data
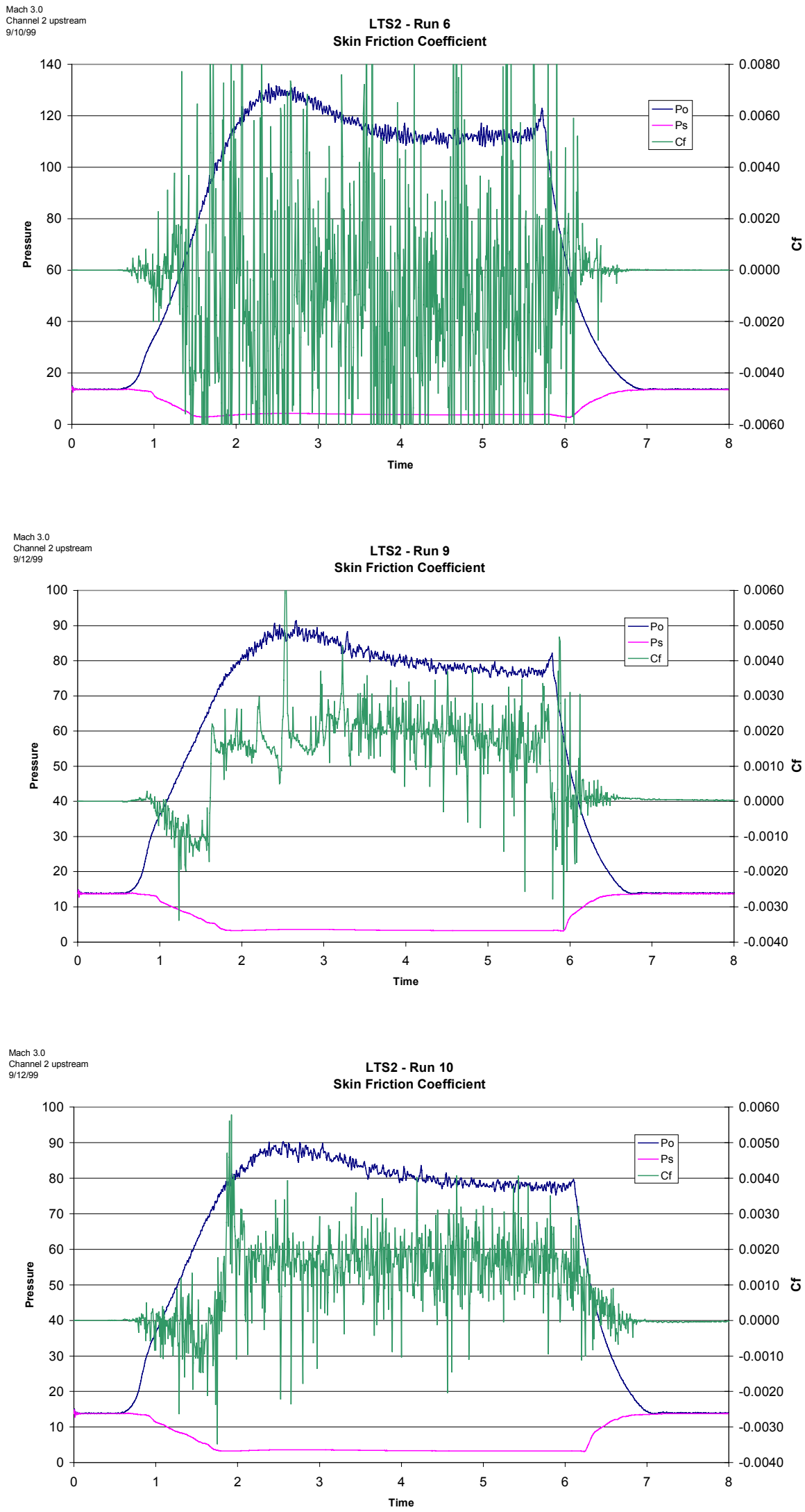
Appendix A

Comprehensive Testing Data
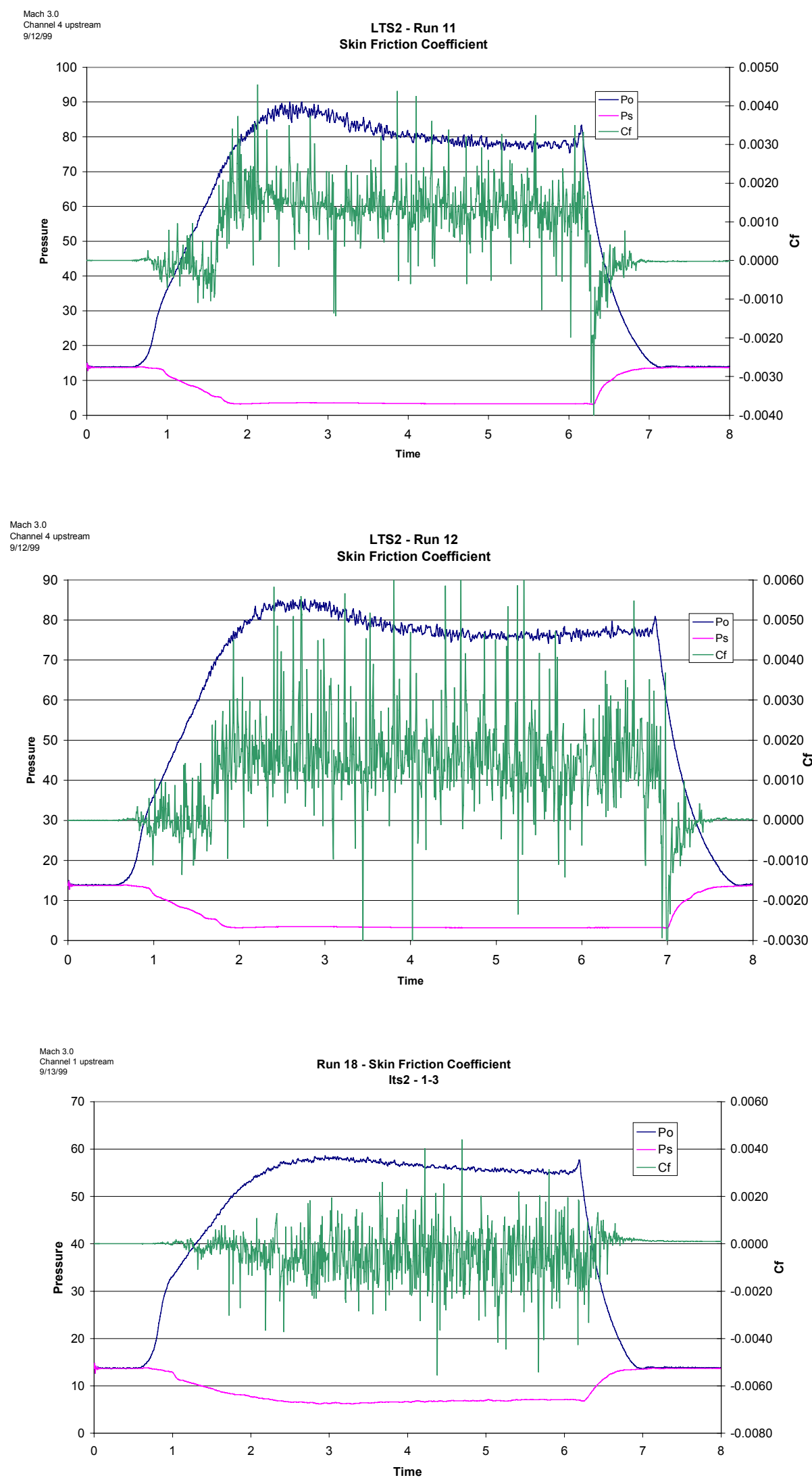
Appendix A

Comprehensive Testing Data
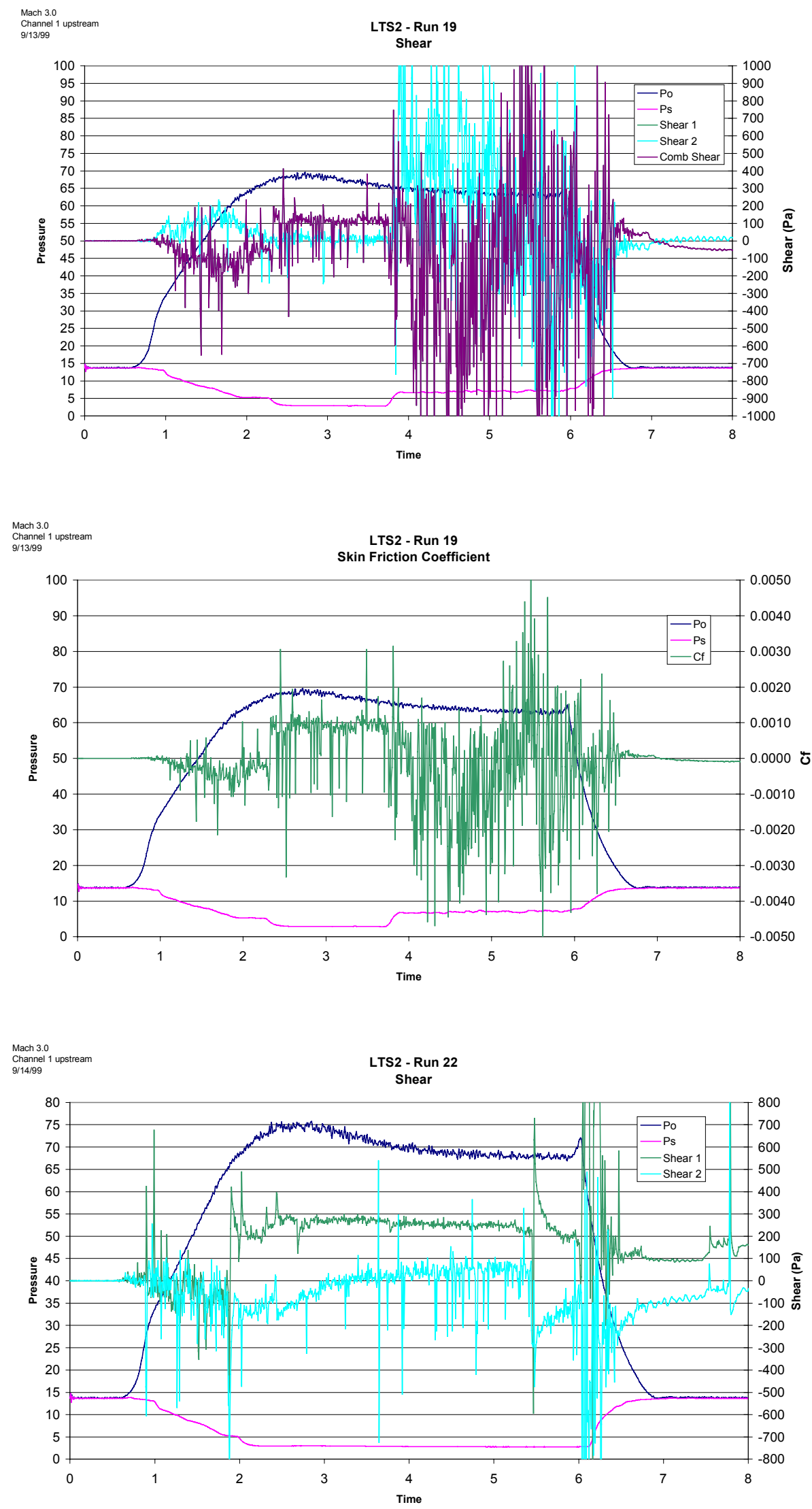
Appendix A

Comprehensive Testing Data
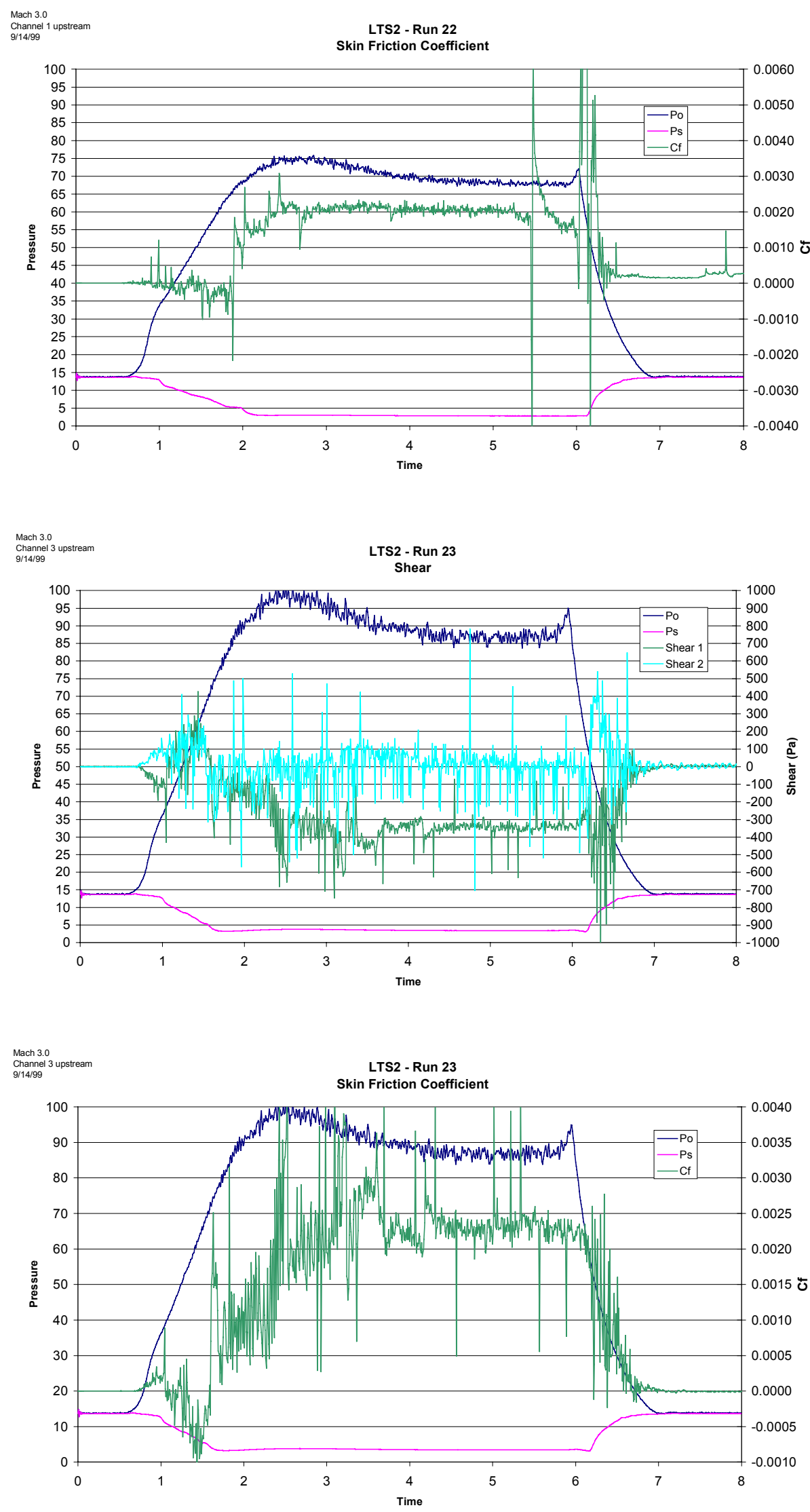
Appendix A

Comprehensive Testing Data

Version $5 b$ skin friction sensor
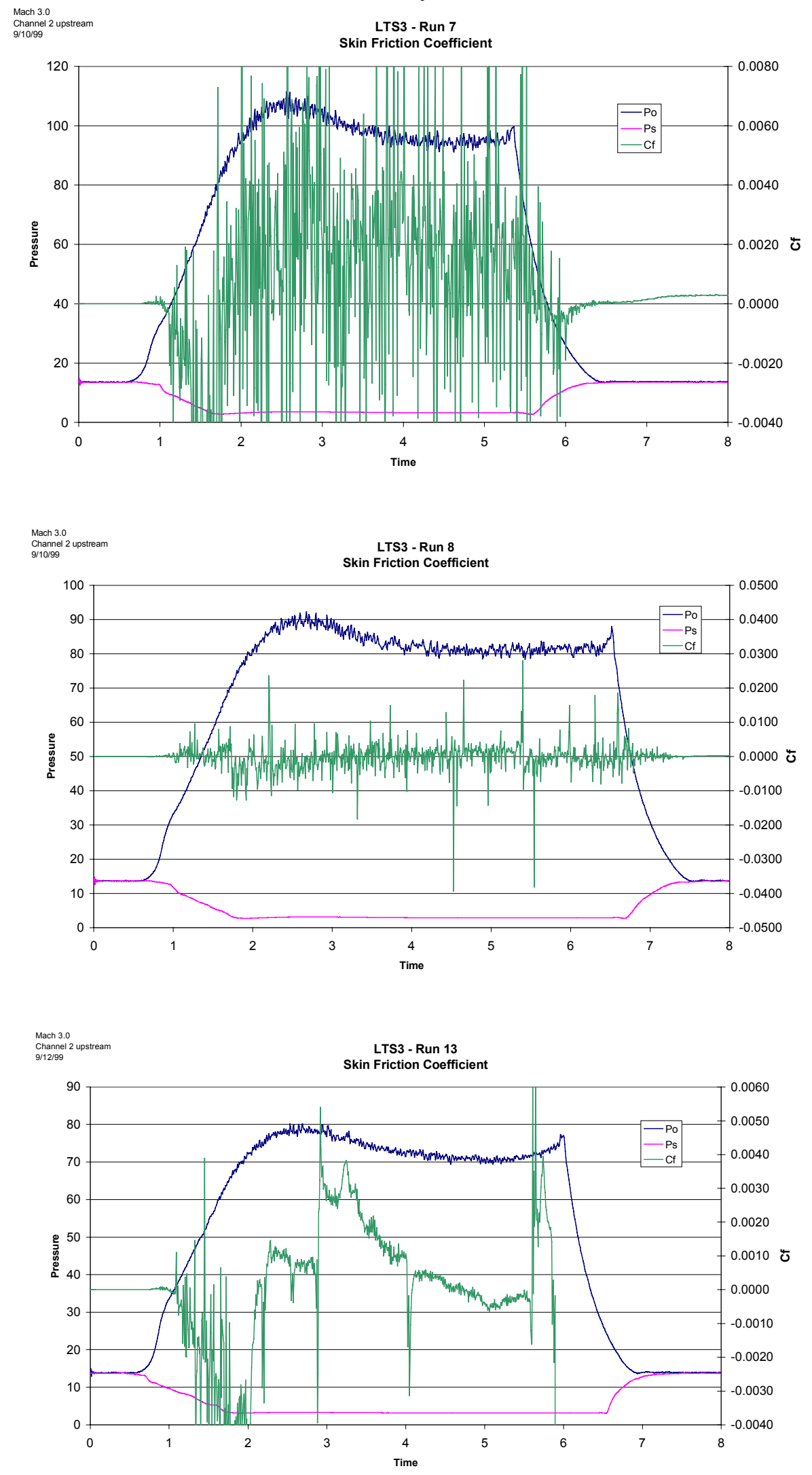
Appendix A

Comprehensive Testing Data

Mach 3.0
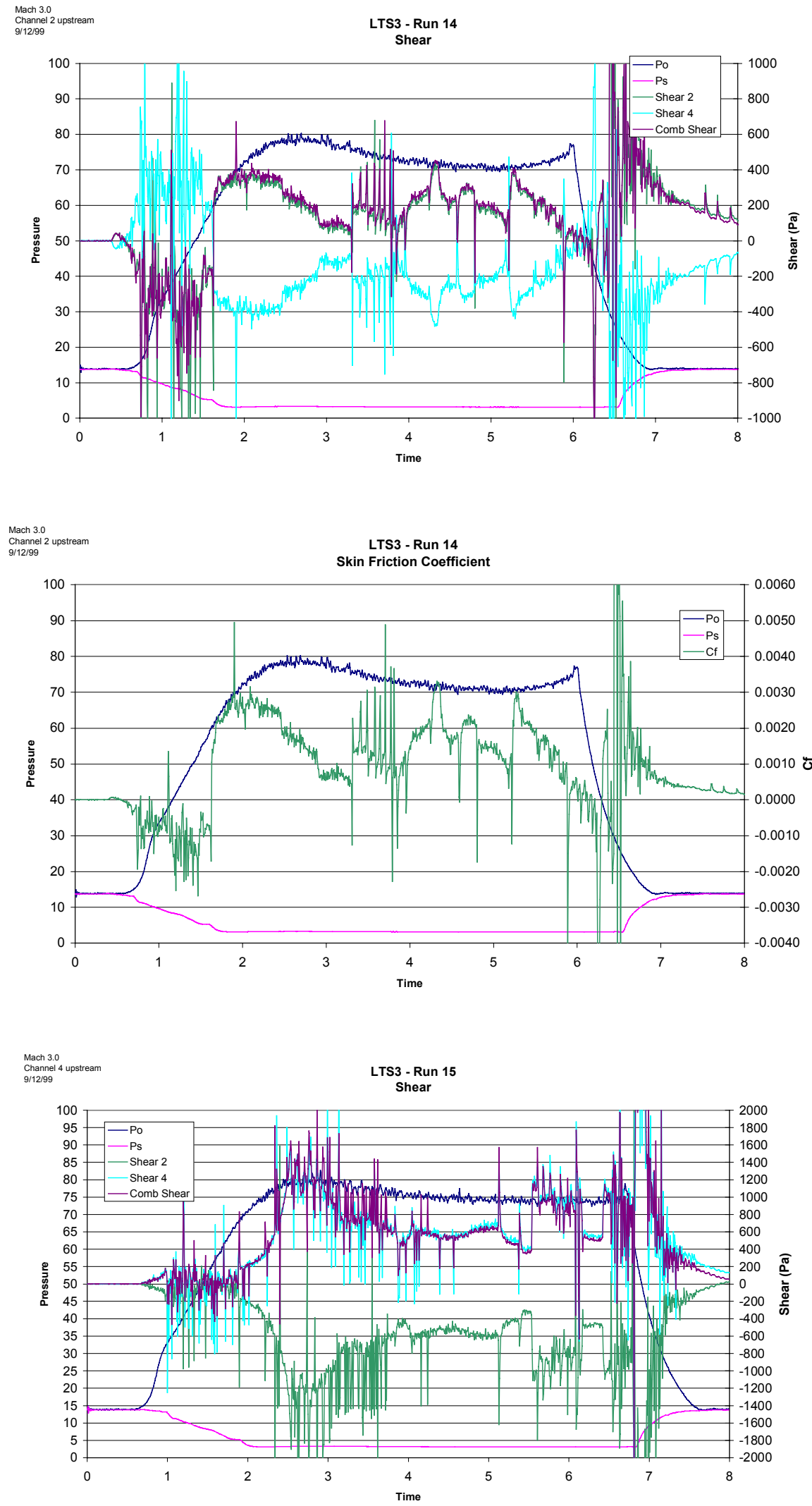
Appendix A

Comprehensive Testing Data
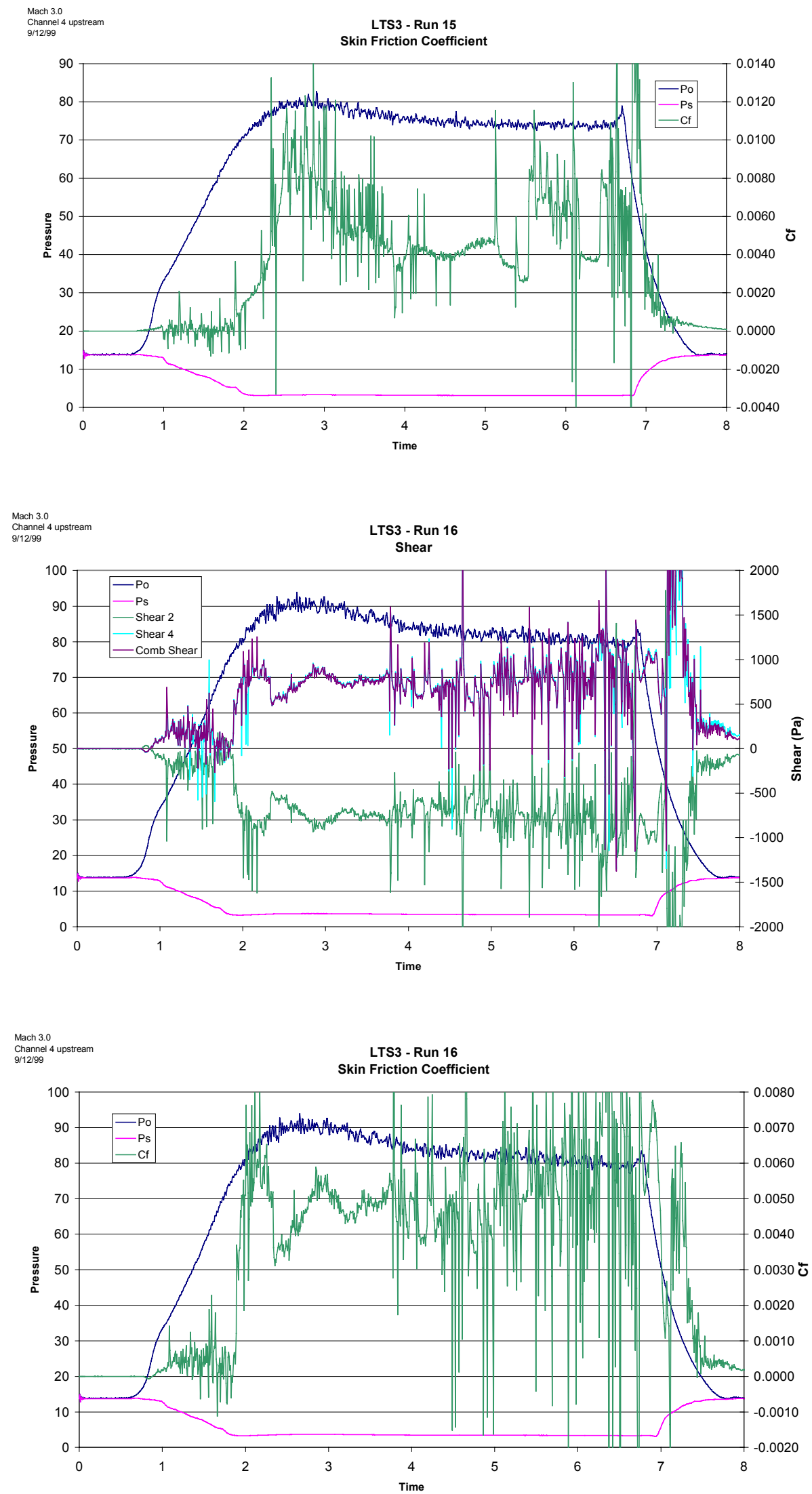
Appendix A

Comprehensive Testing Data
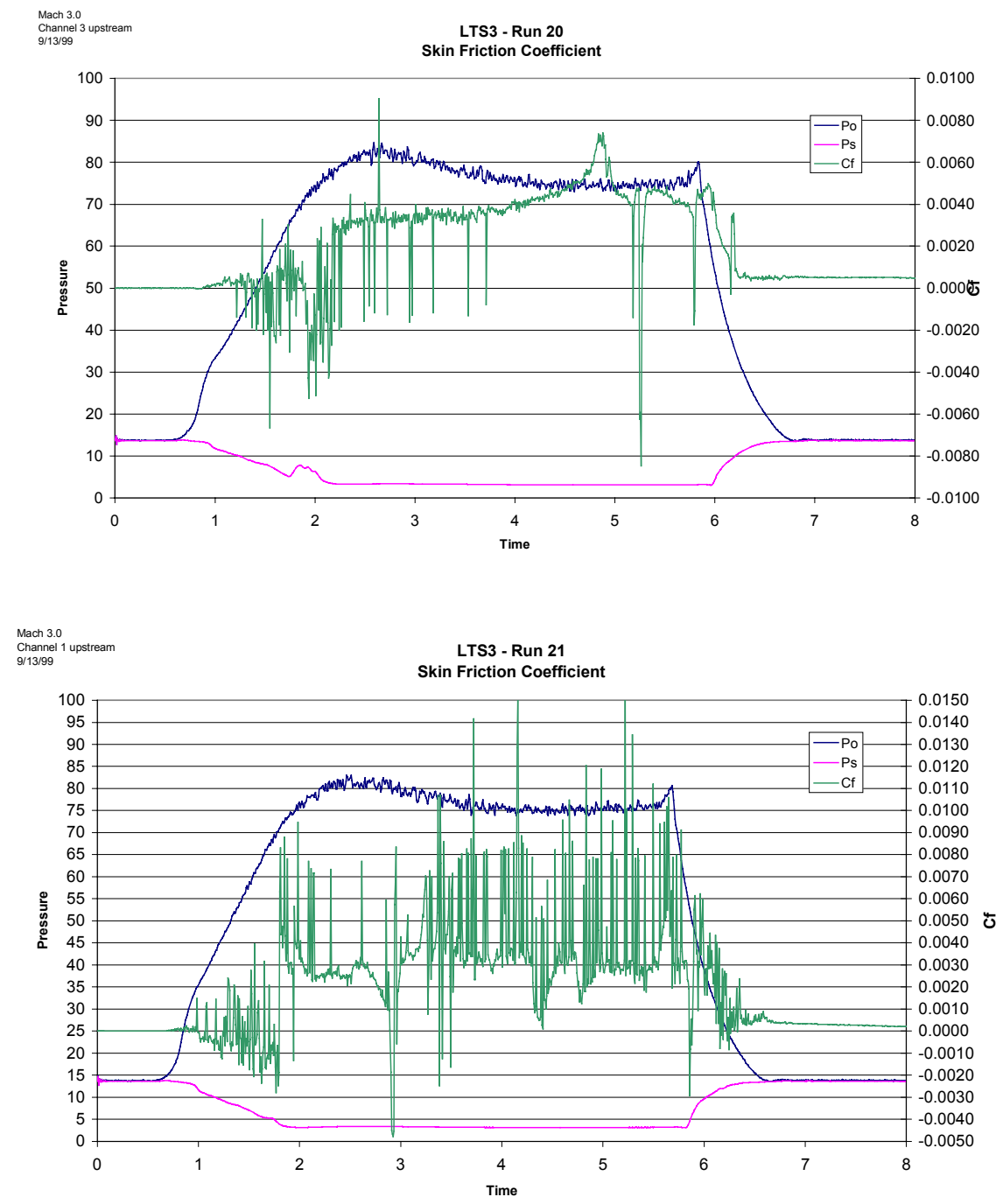

October 1999 Tests

Pressure Array Tests

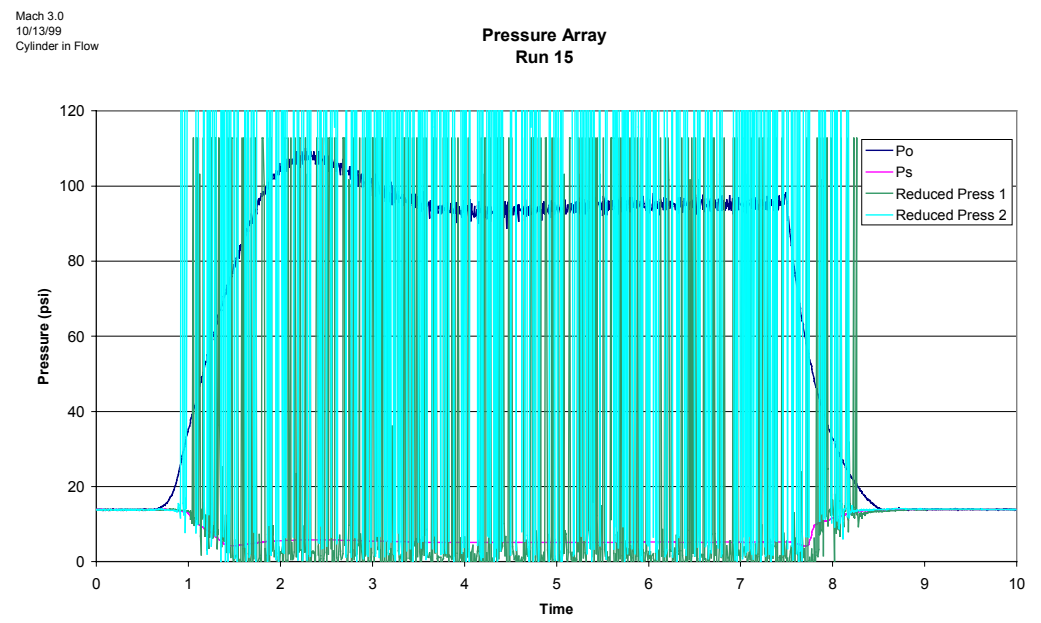


Appendix A

Comprehensive Testing Data
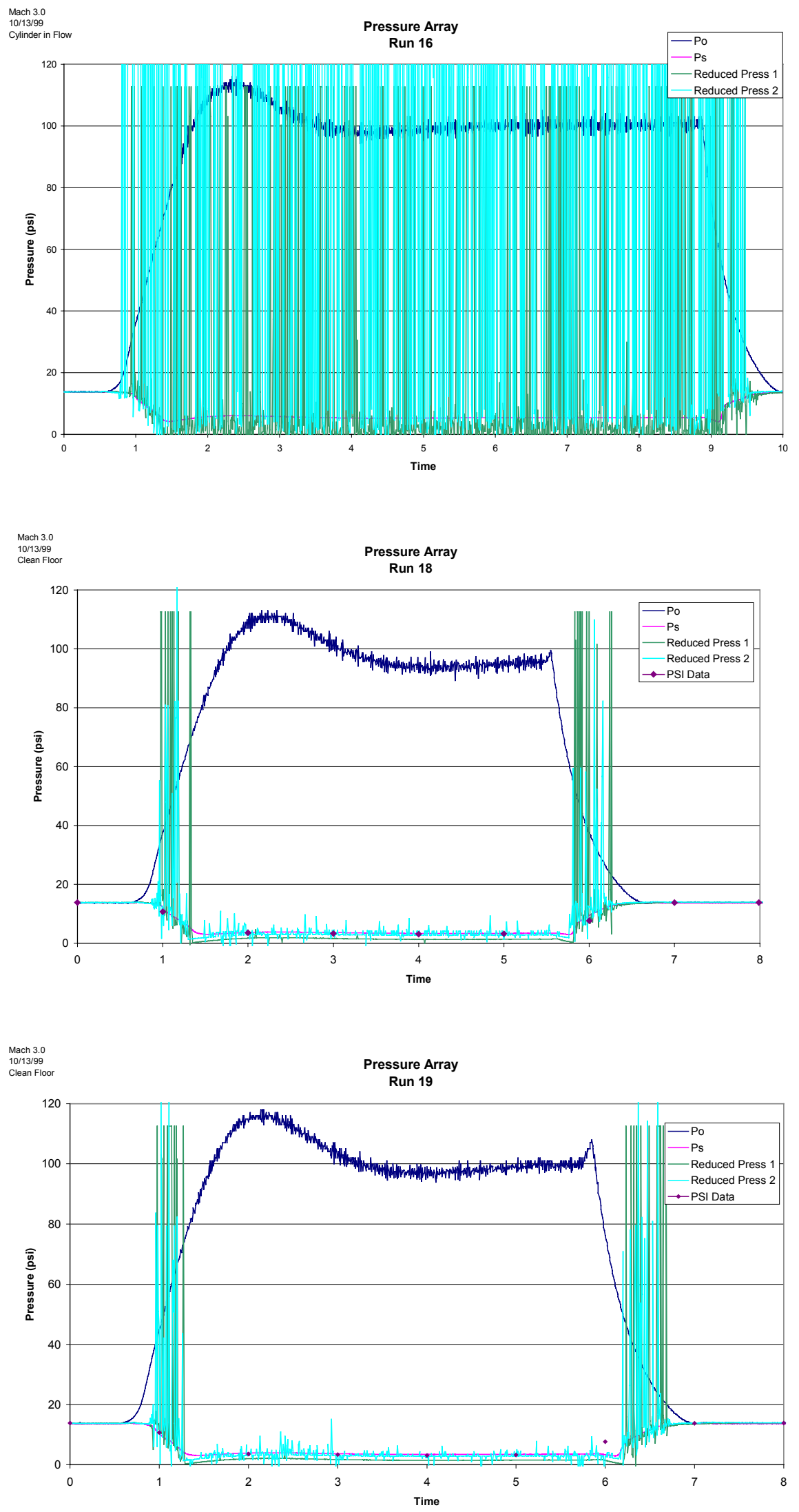
Appendix A

Comprehensive Testing Data

Mach 3.0
$10 / 1399$
Clean Flo

Pressure Array

Run 20

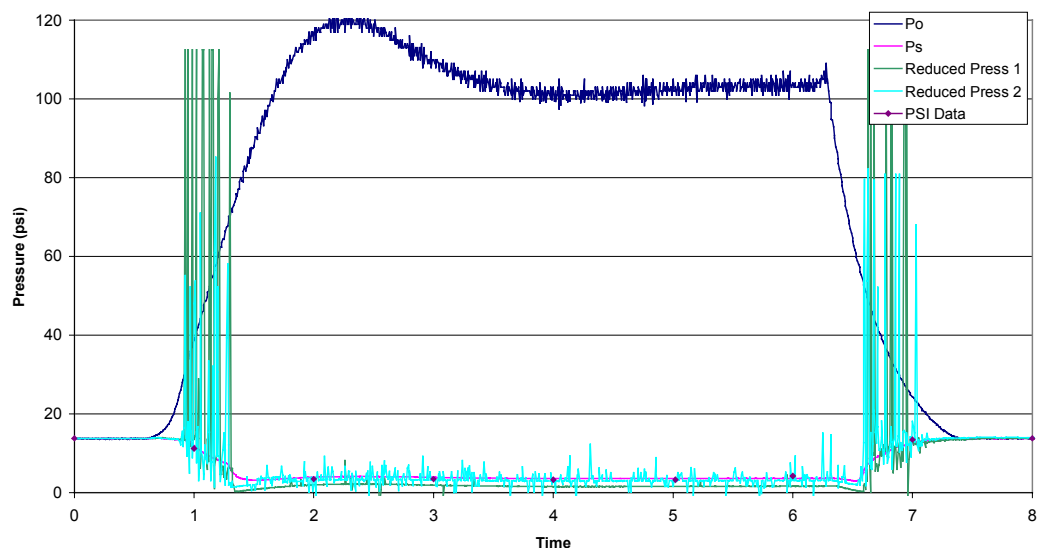

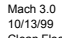

Pressure Array

Run 21

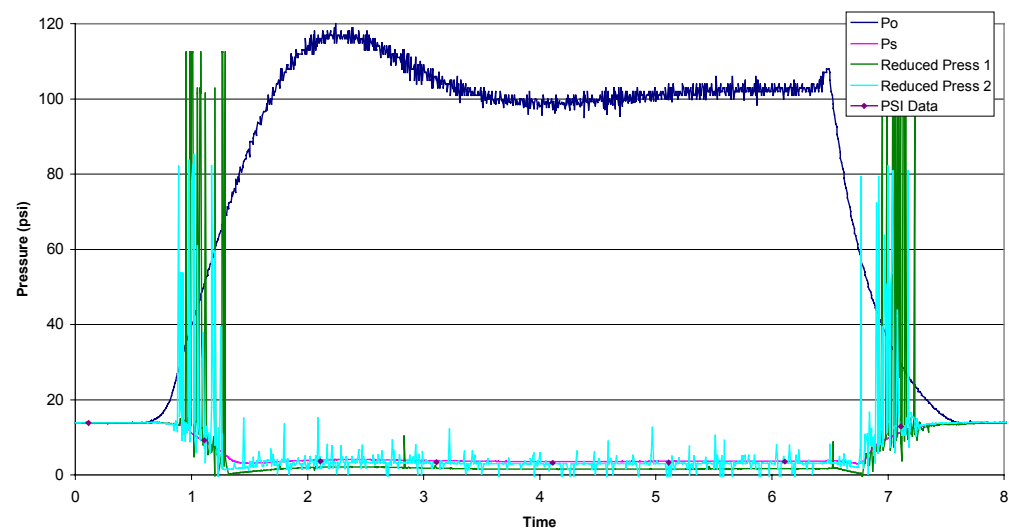

Version 6 skin friction sensor

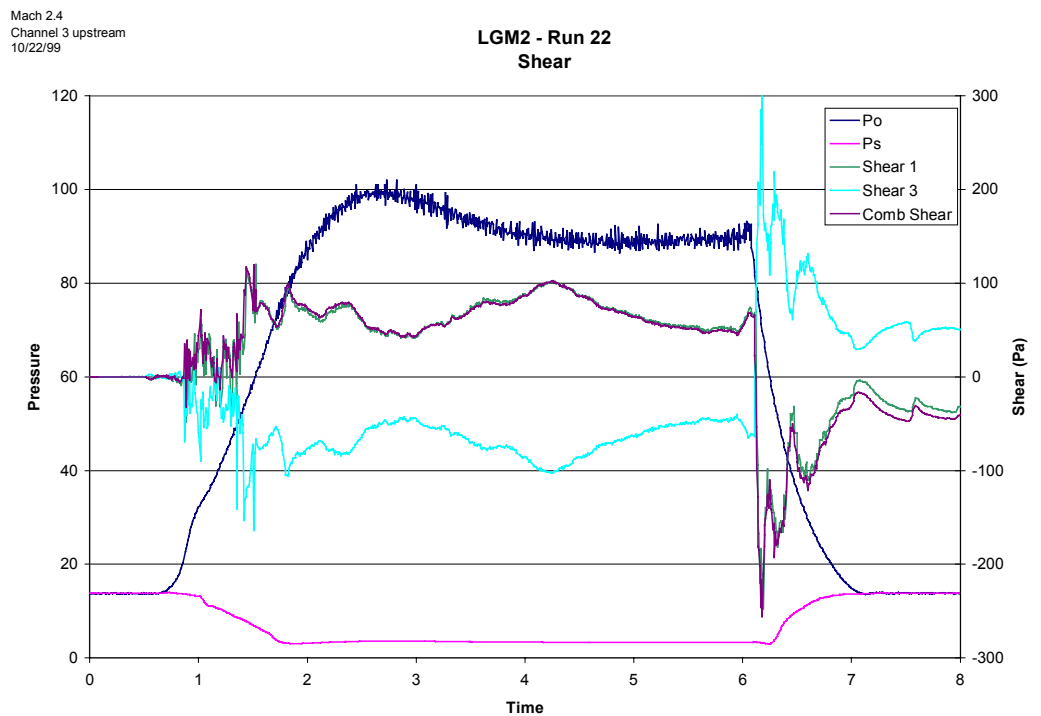


Appendix A

Comprehensive Testing Data
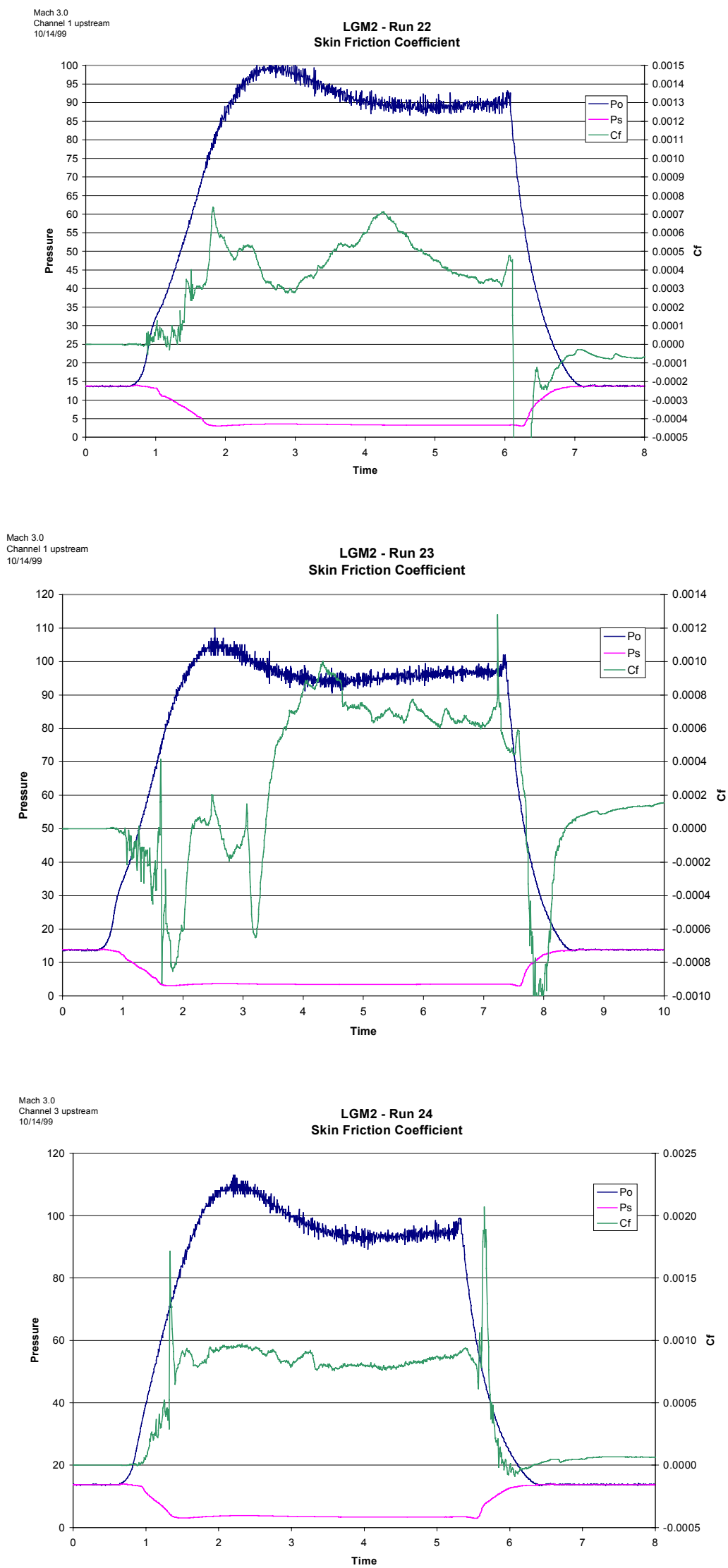
Appendix A

Comprehensive Testing Data
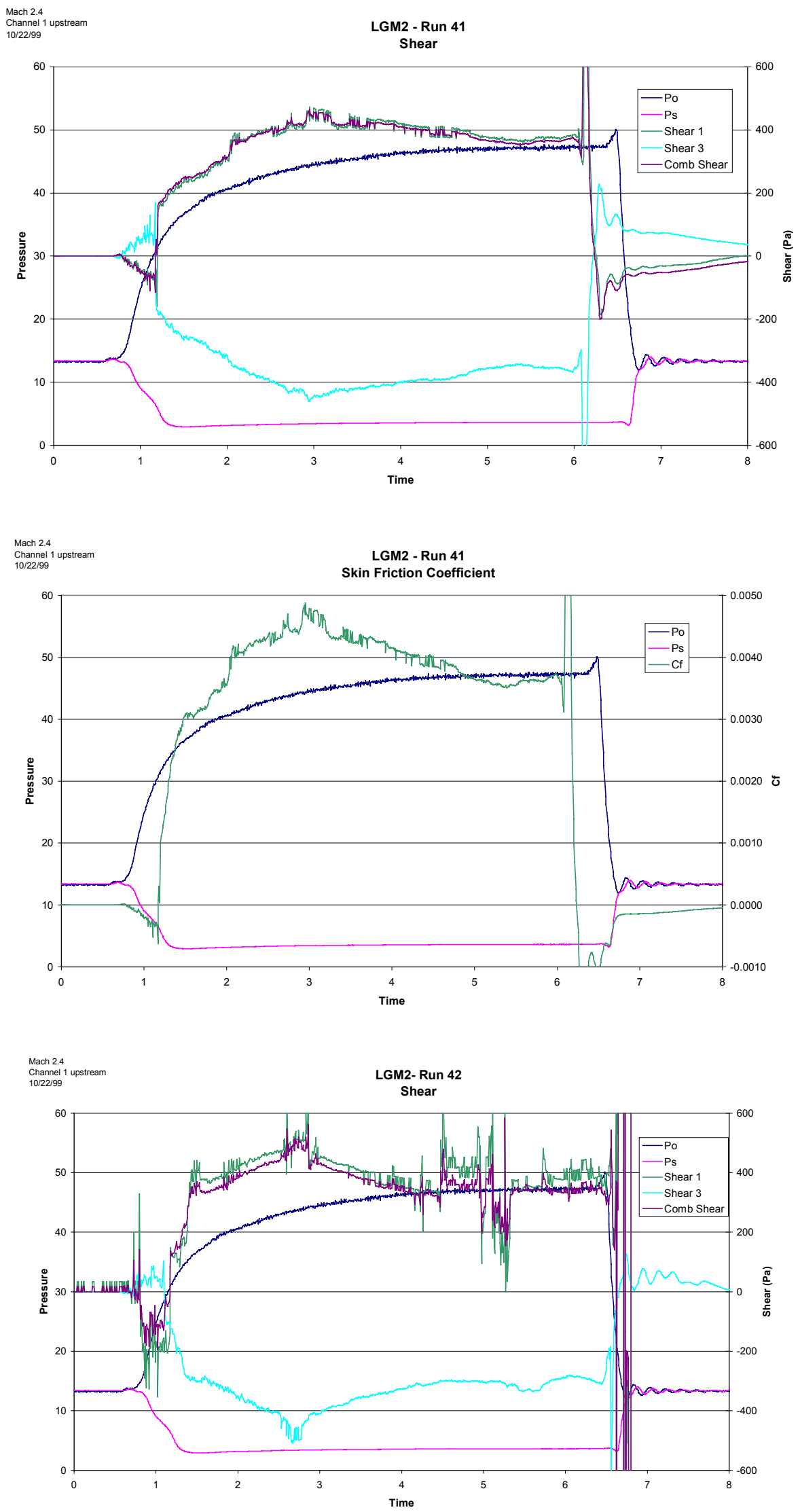
Appendix A

Comprehensive Testing Data
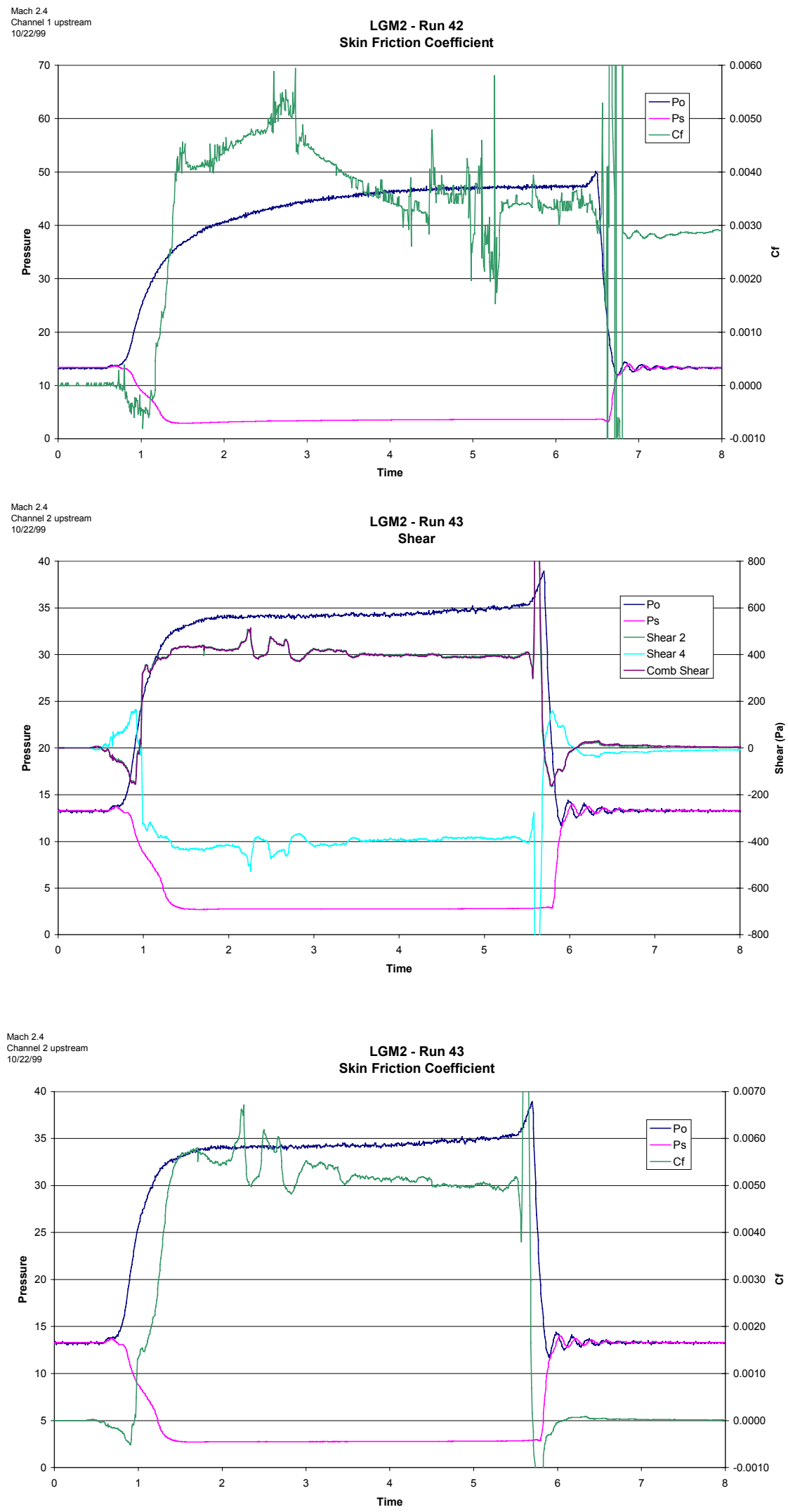
Appendix A

Comprehensive Testing Data
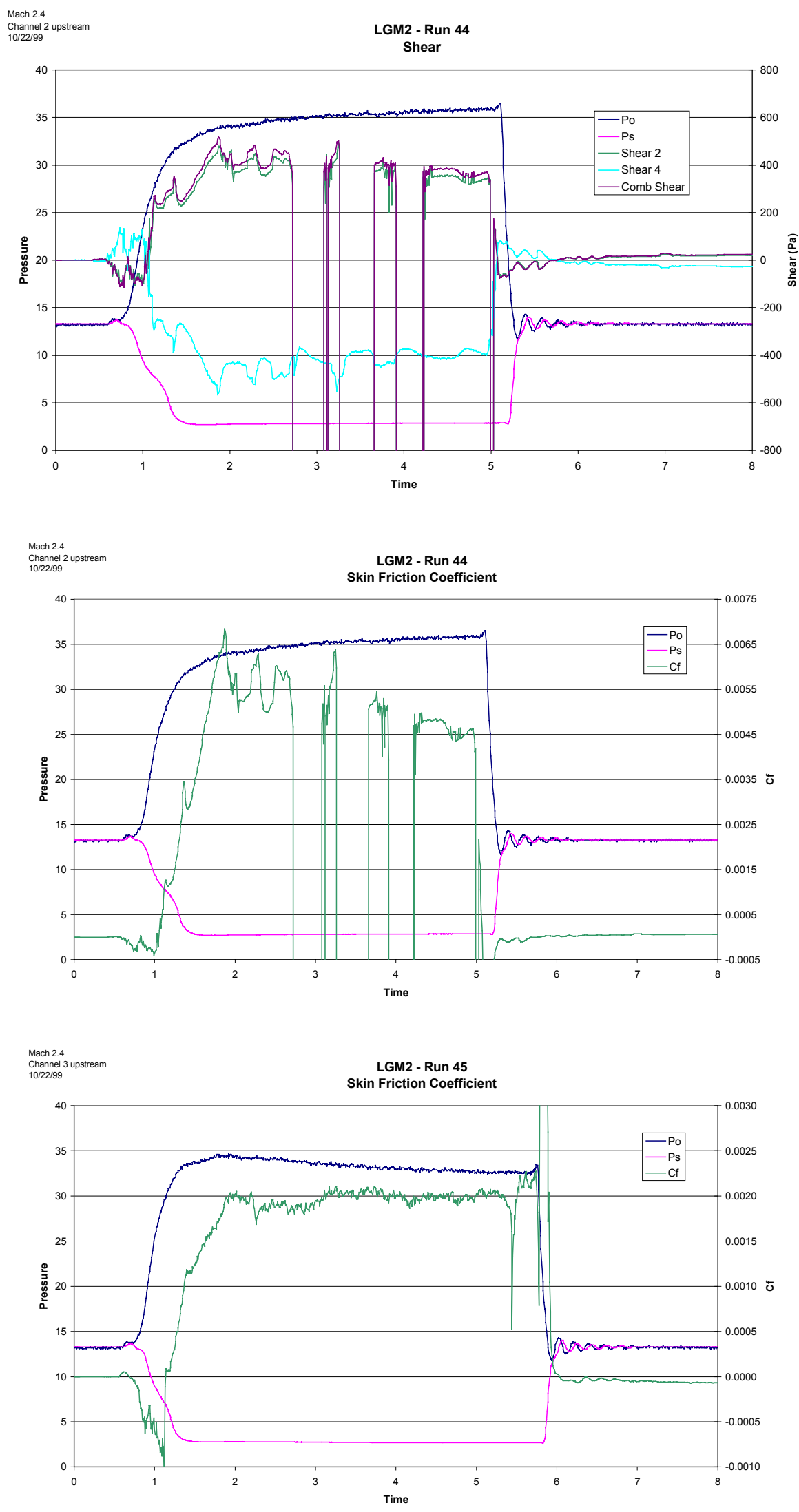
Appendix A

Comprehensive Testing Data
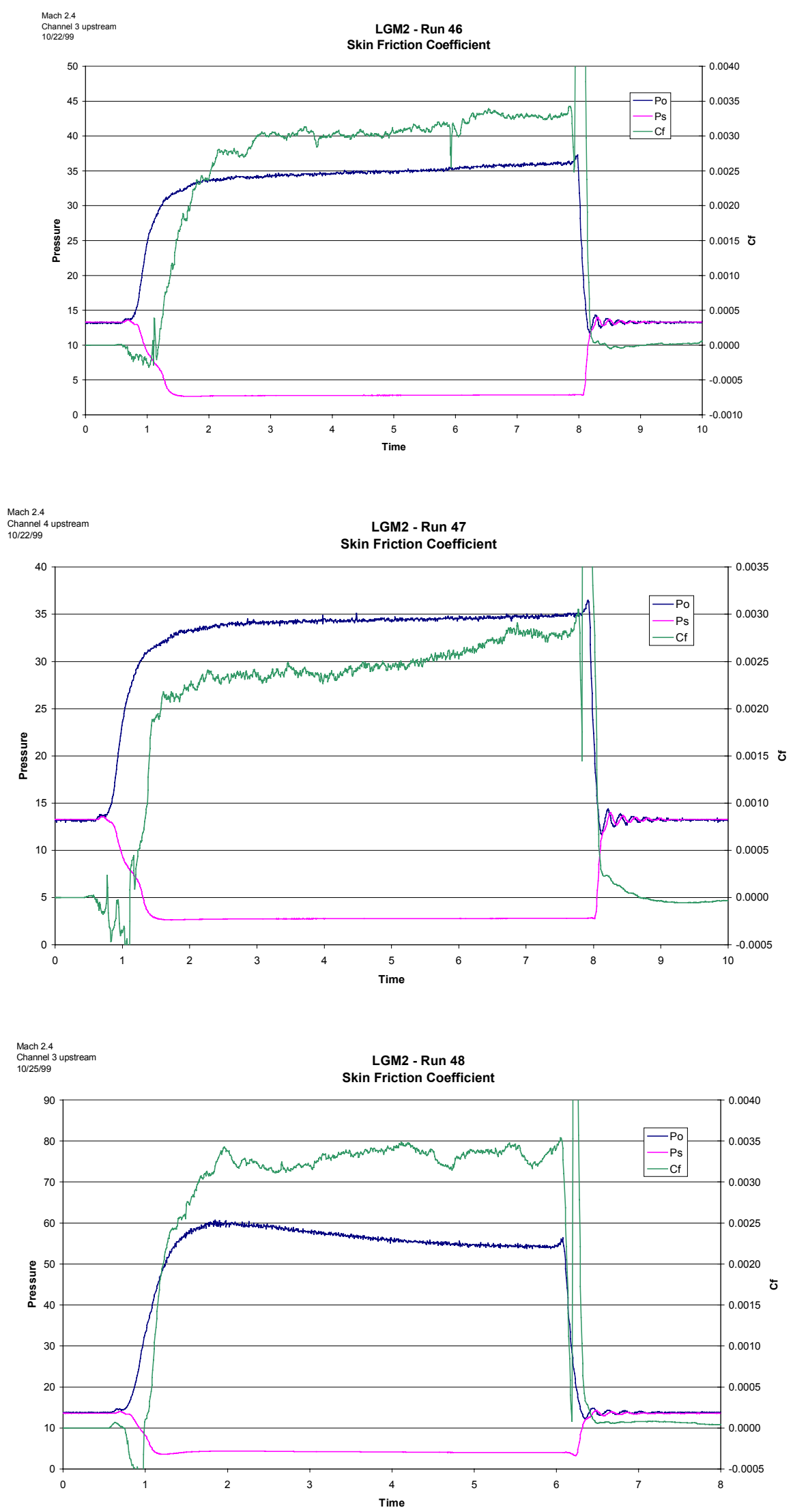
Appendix A

Comprehensive Testing Data

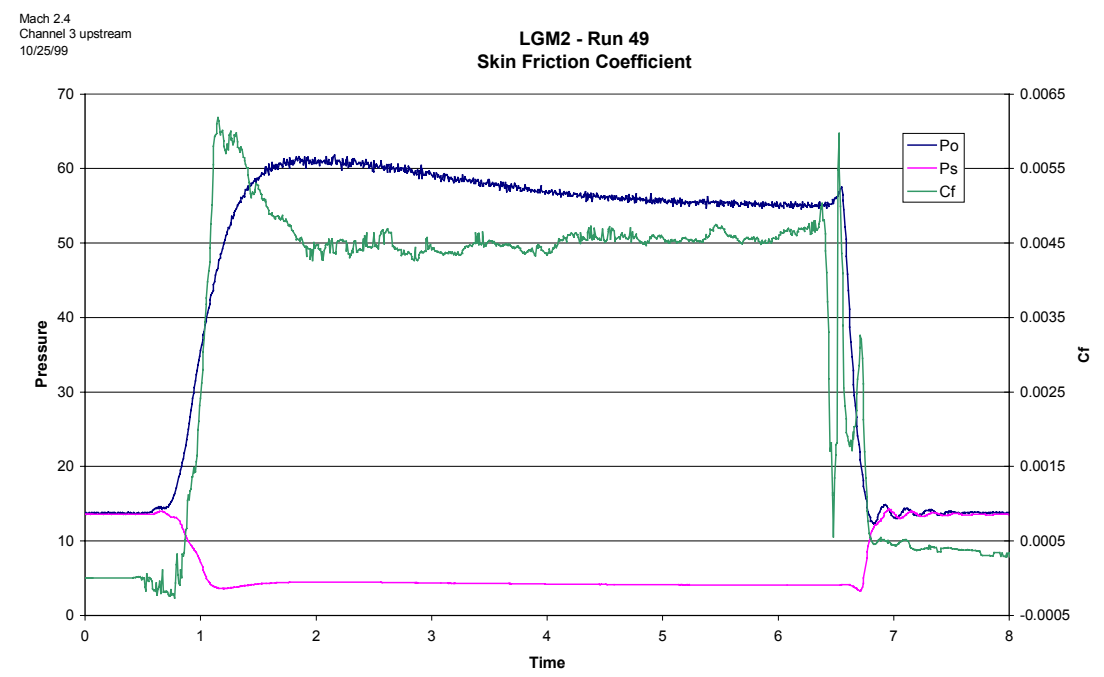

January 2000 Wright Pat Tests

Version 5 a skin friction sensor tests
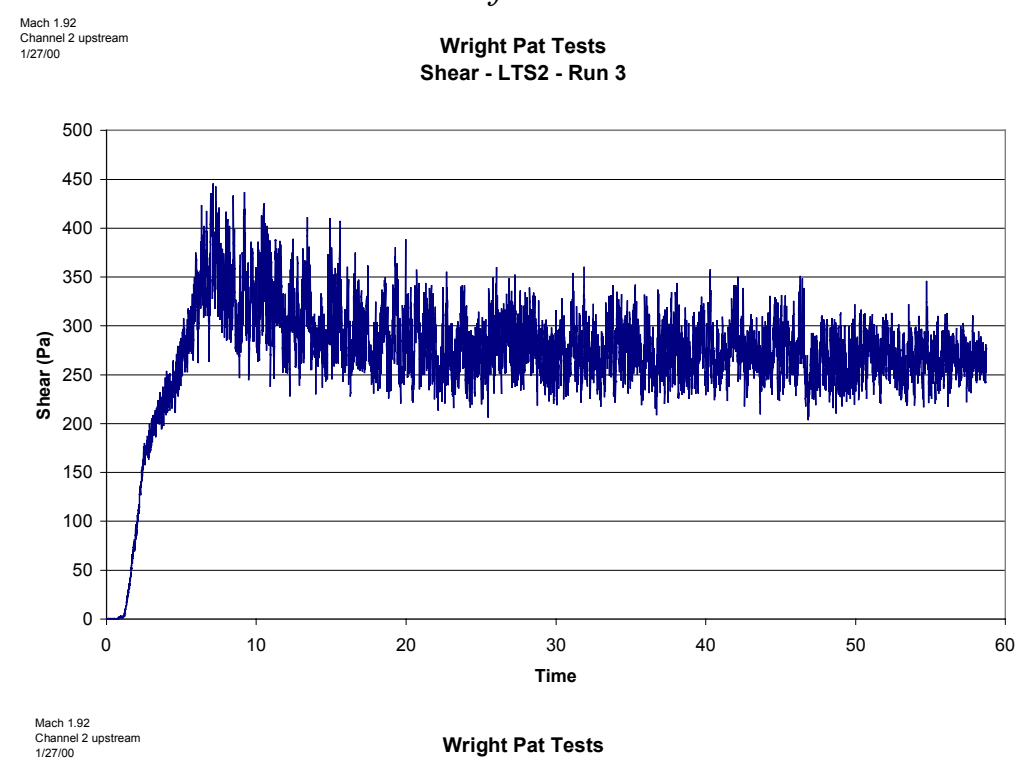

Skin Friction Coefficient - LTS2 - Run 3

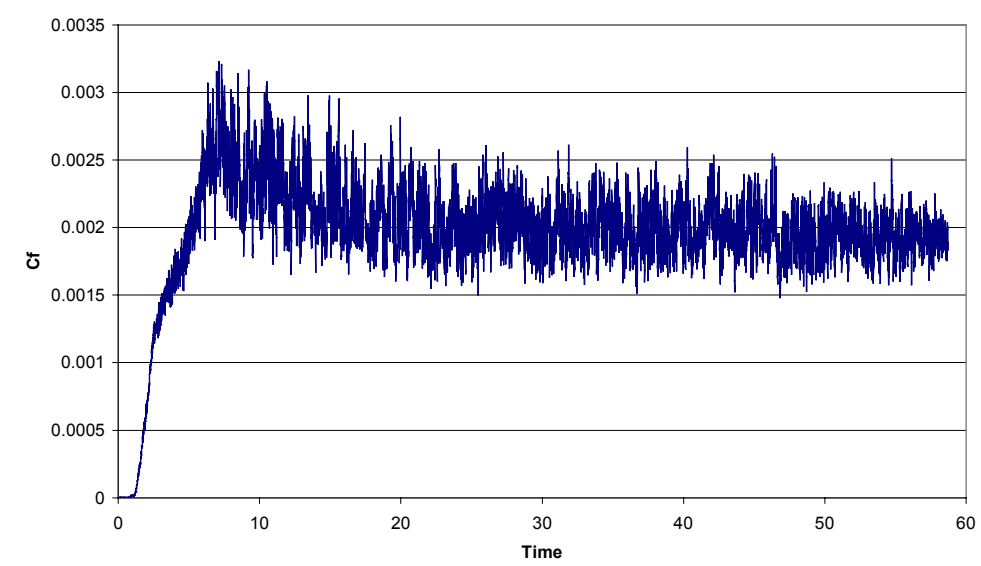


Appendix A

Comprehensive Testing Data

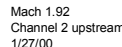

Wright Pat Tests

Shear - LTS2 - Run 6

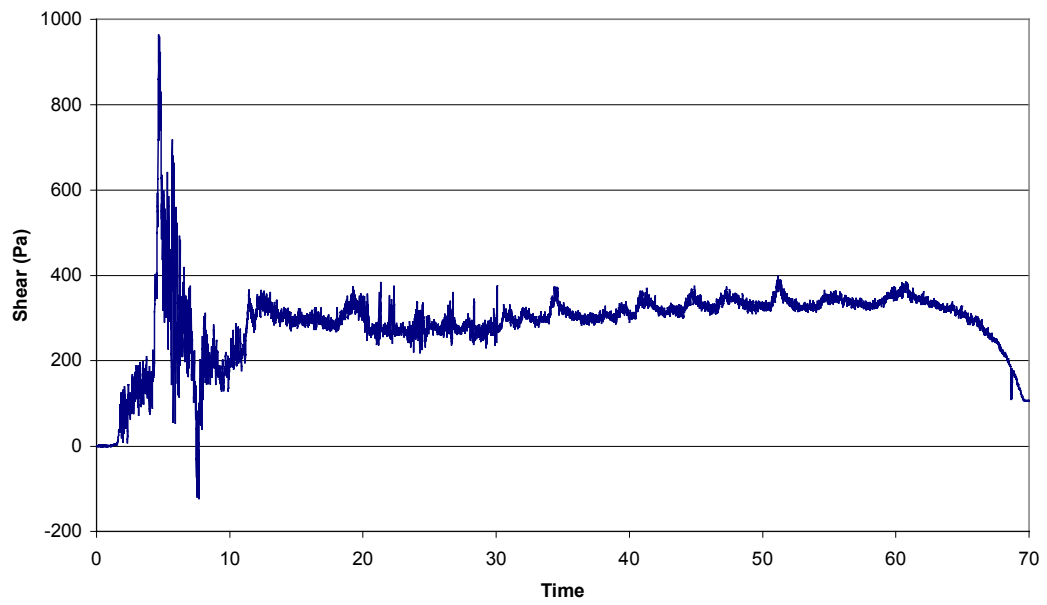

Wright Pat Tests

Skin Friction Coefficient - LTS2 - Run 6

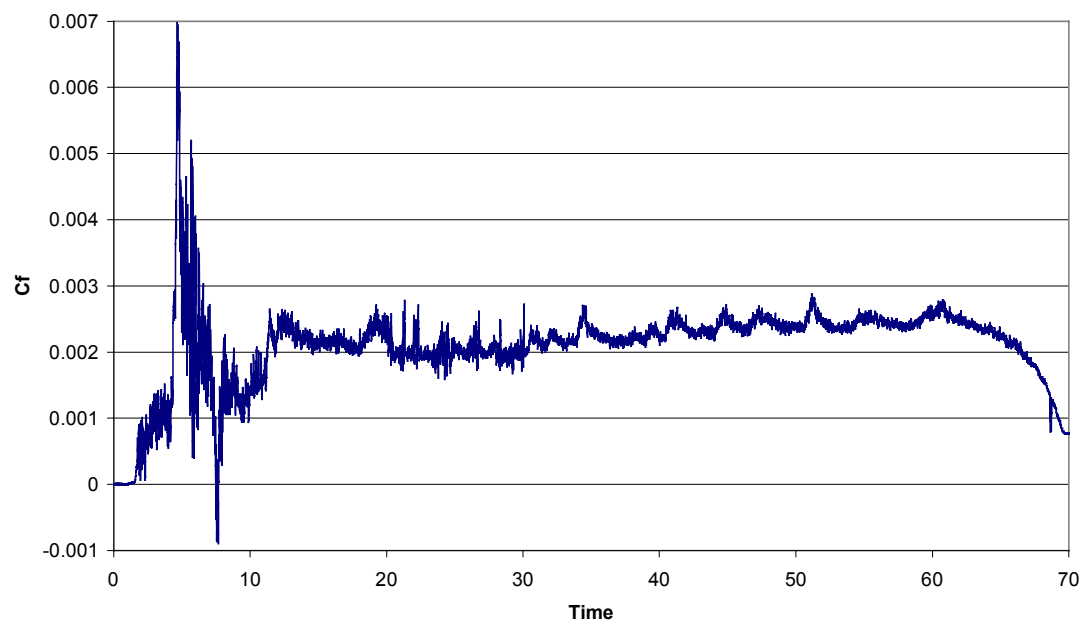


Appendix B

Calibrations

Shear calibration:

\section{Version 1 skin friction sensor}

Phase I Skin Friction Sensor Shear Calibration

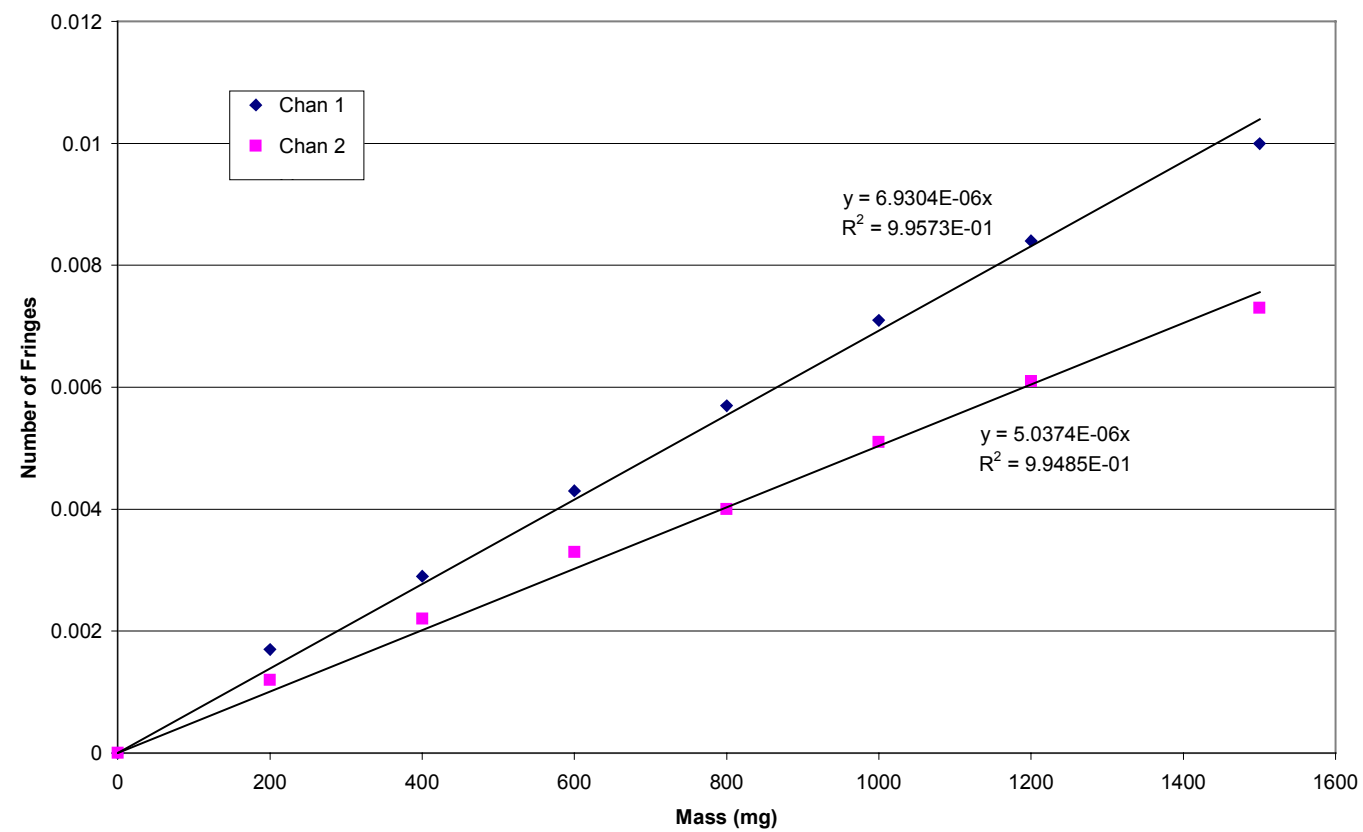

Pressure sensor calibration:

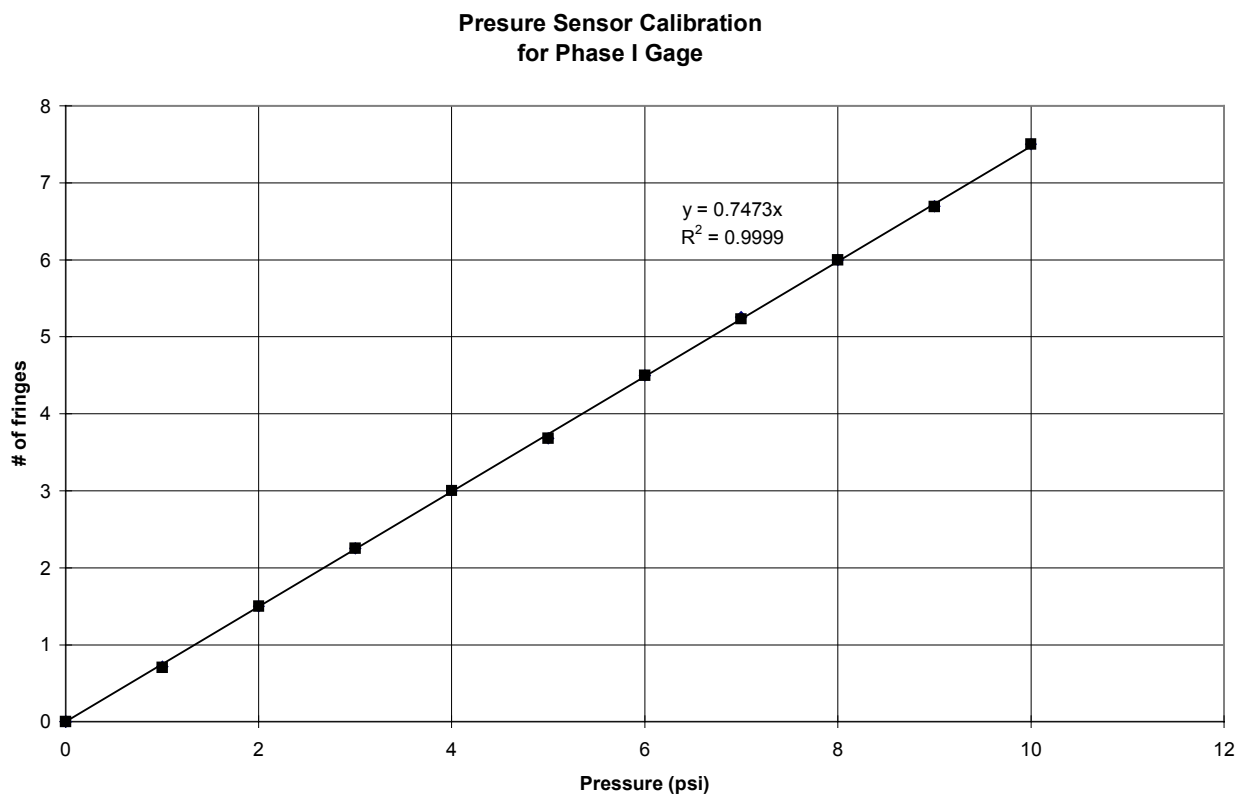


Appendix B

Calibrations

Shear calibration with oil:

\section{Version 2 skin friction sensor}

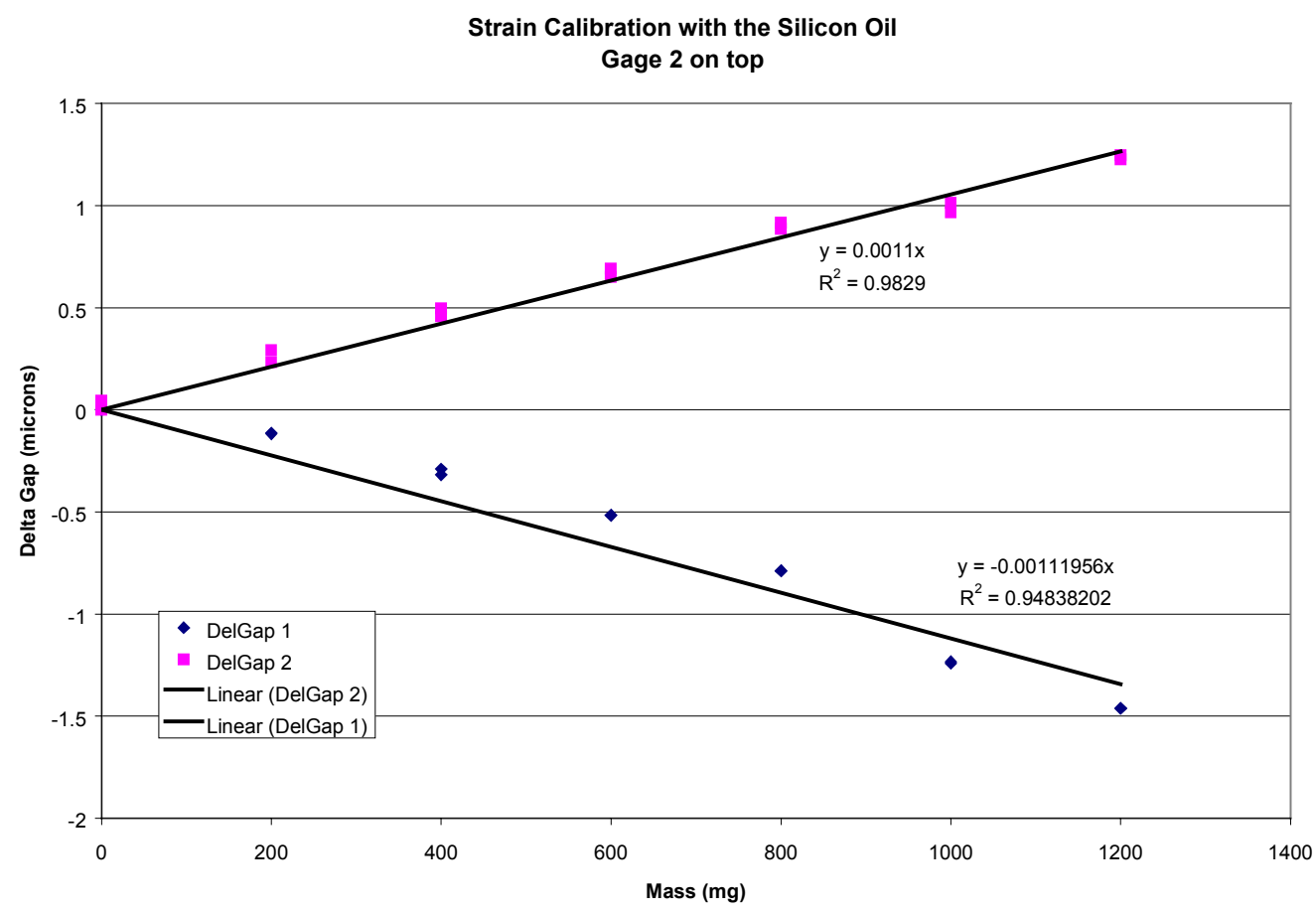

Temperature calibration without oil:

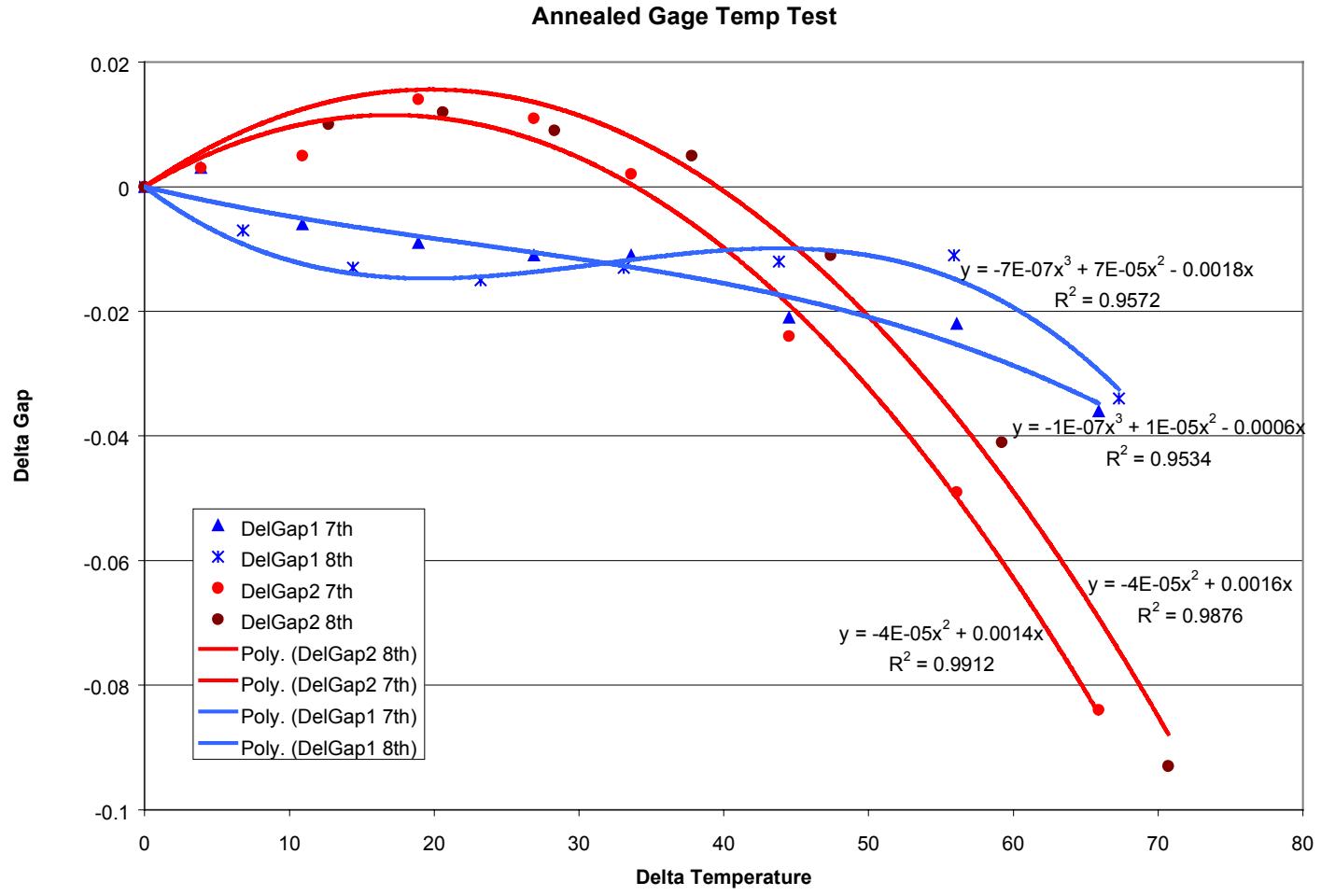


Appendix B

Calibrations

Shear calibration without oil:

\section{Version 3 skin friction sensor}

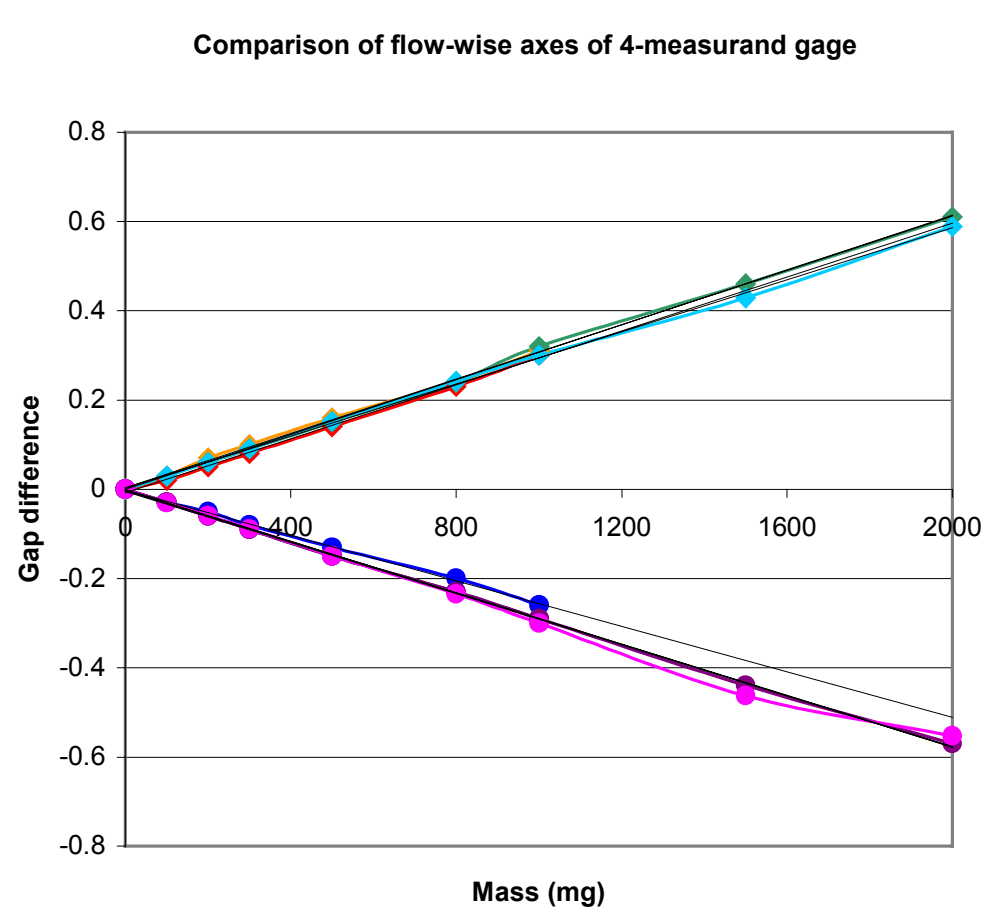

2T

$y=3.021 E-04 x-8.000 E-03$

$y=3.071 E-04 x-6.198 E-04$

$y=2.915 E-04 x+3.139 E-03$

avg slope $=3.012 \mathrm{e}-4$

4C

$y=-2.550 E-04 x-1.500 E-03$

$y=-2.883 E-04 x-2.000 E-03$

$y=-2.865 E-04 x-2.932 E-03$

$y=-2.865 E-04 x-5.026 E-03$

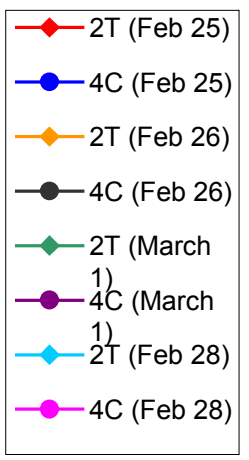

avg slope $=\mathbf{- 2 . 8 6 6 e - 4}$

Shear calibration with oil:

Calibration of oil-filled 4-measurand gage

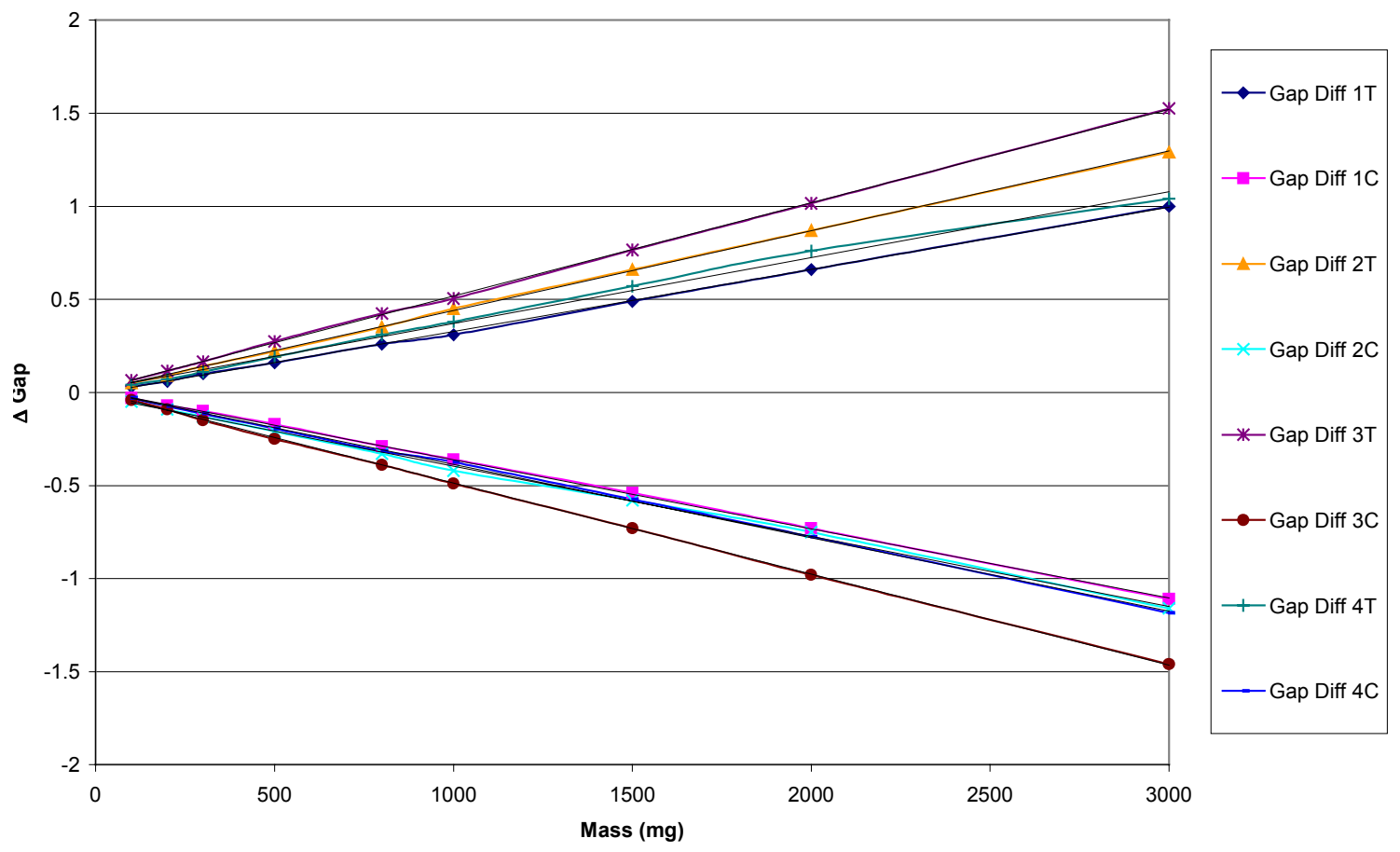


Temperature calibration without oil:

$\Delta T$ vs. $\Delta$ Gap for 4-measurand gage

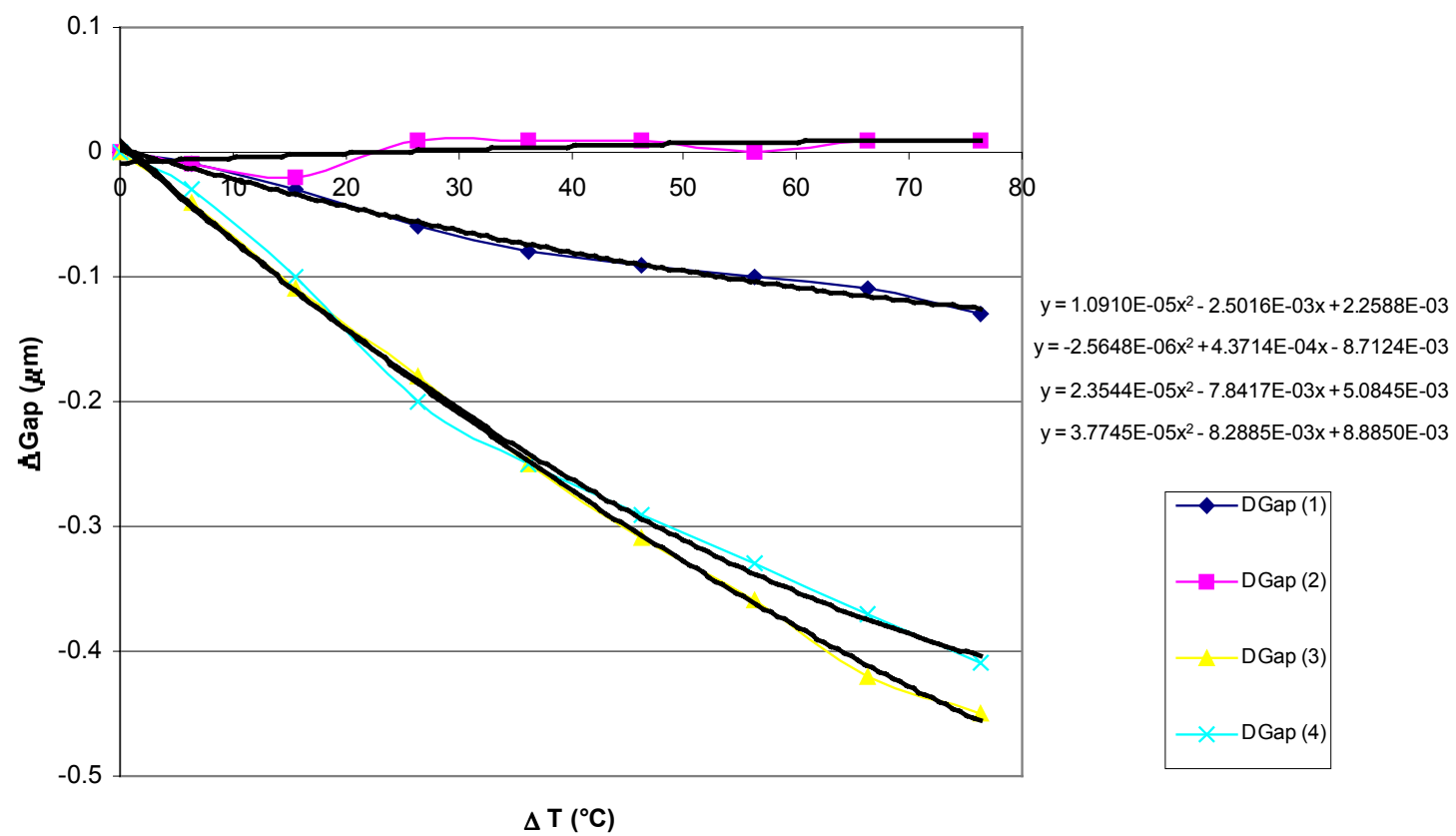

Pressure Sensor calibration:

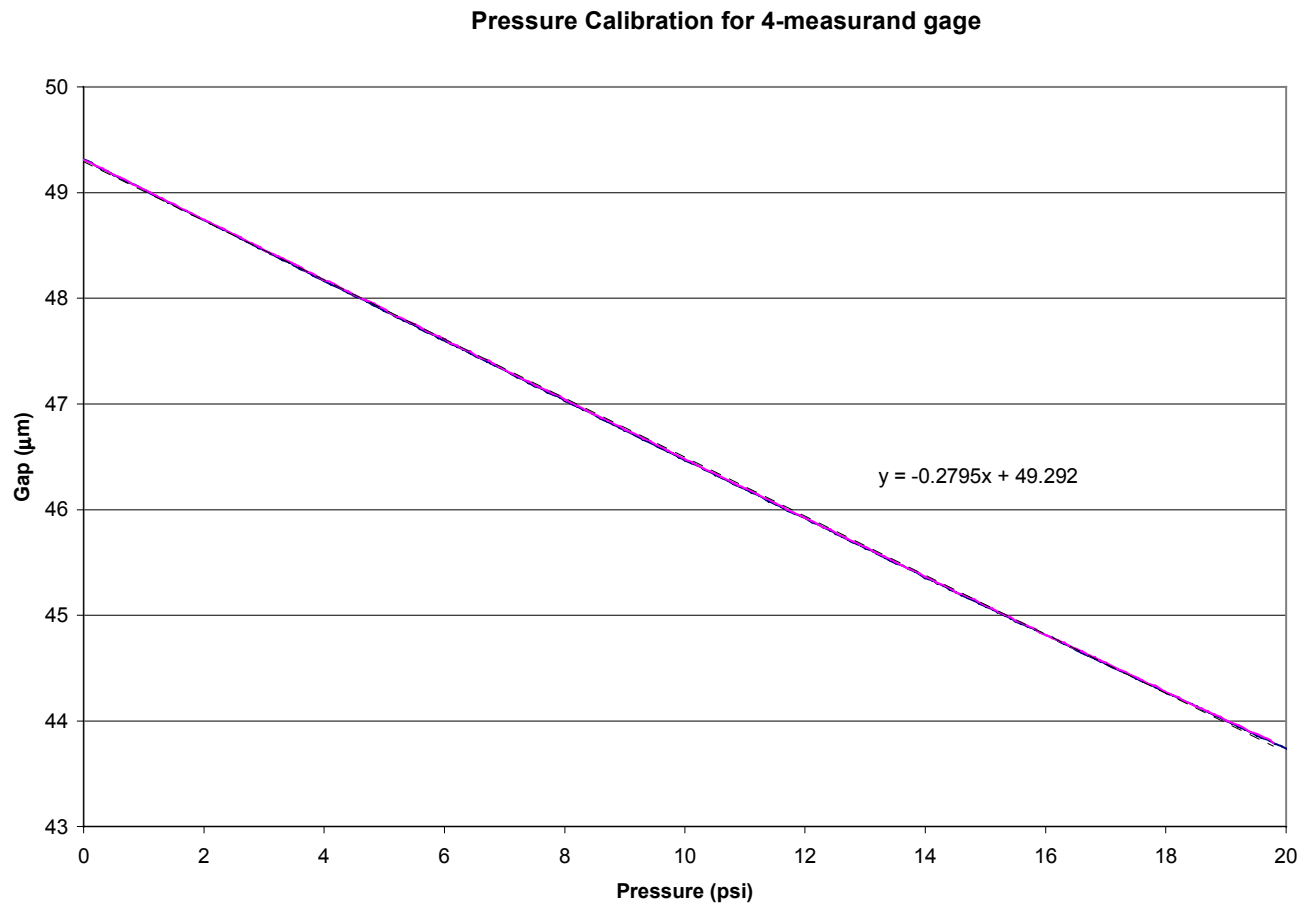




\section{Version 4 skin friction sensor}

\section{Shear calibration without glycerin:}
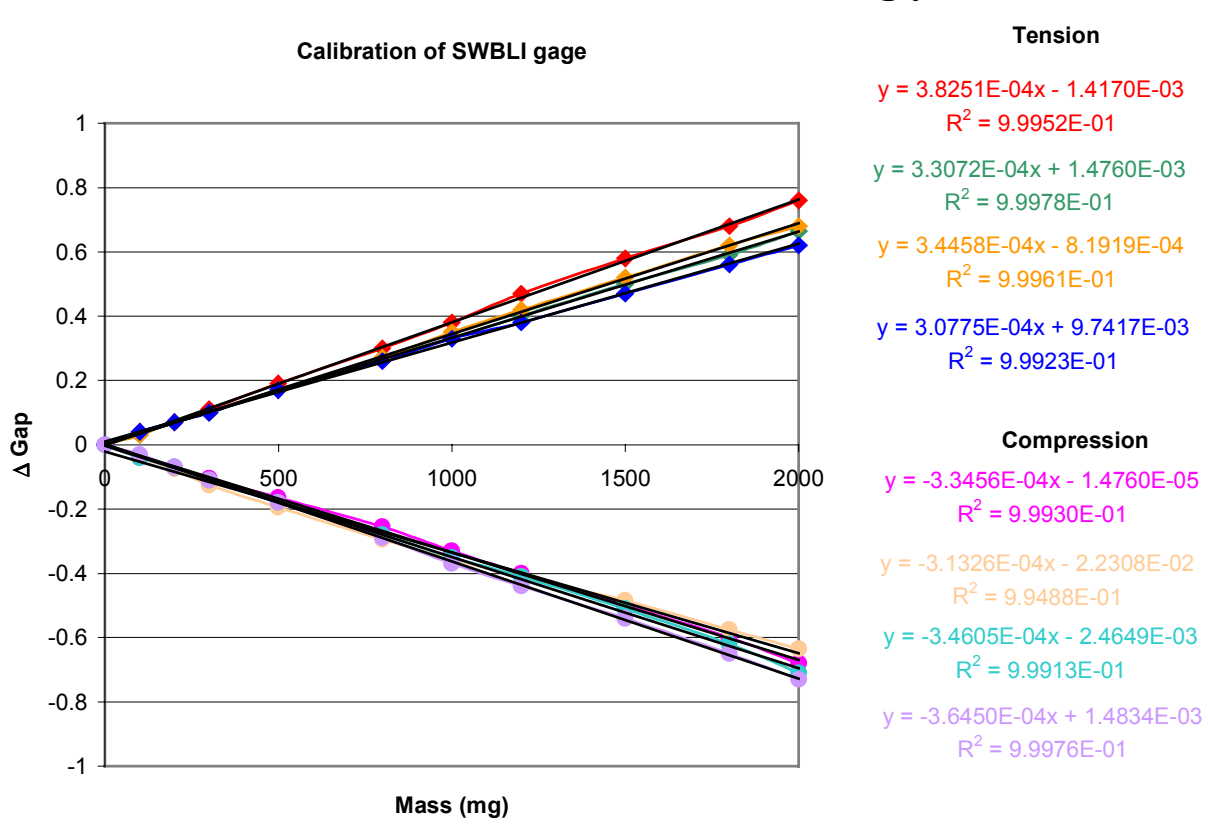

Shear calibration with glycerin:

\section{Calibration of oil-filled SWBL gage}
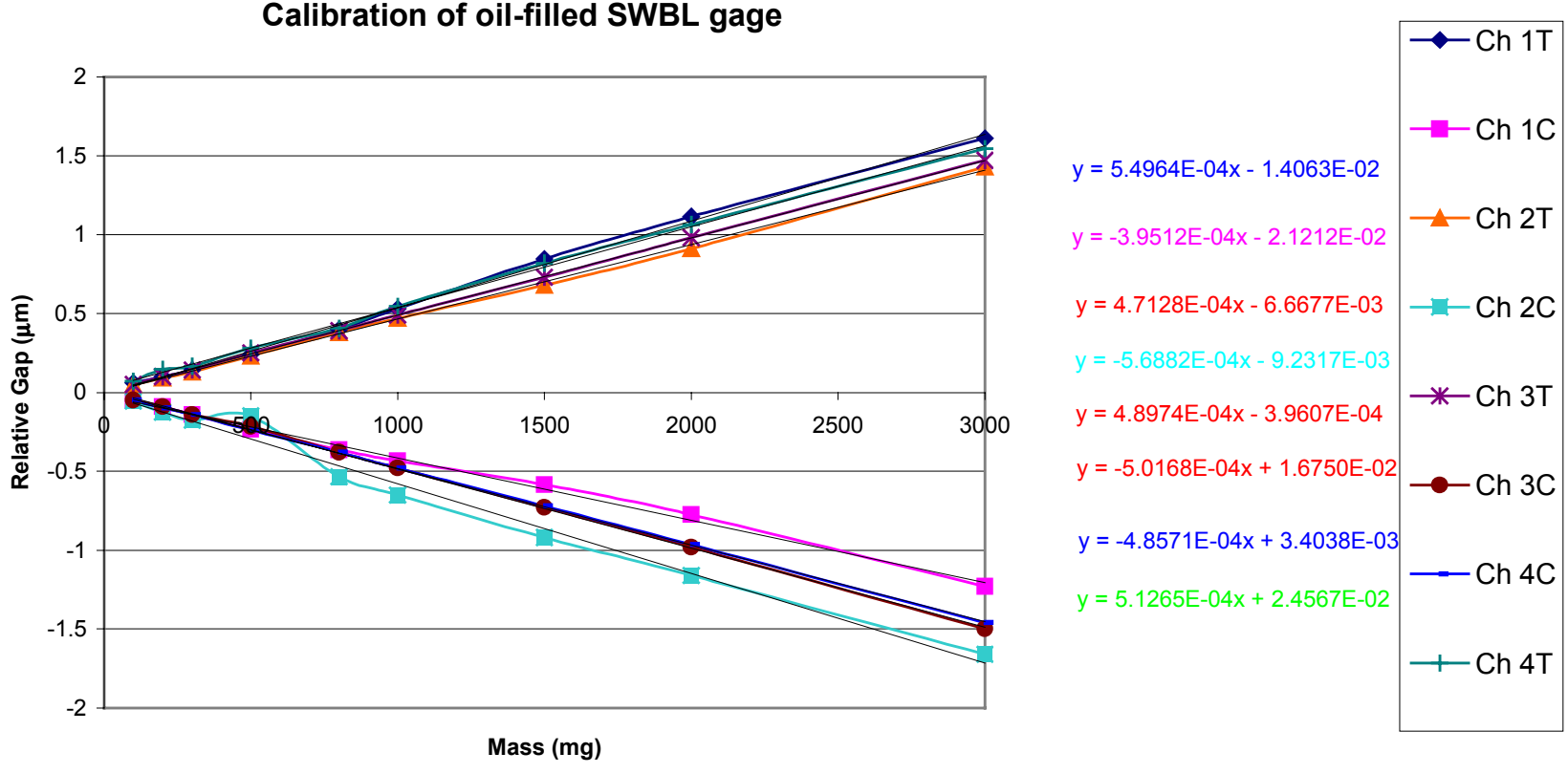
Appendix B

Calibrations

Temperature calibration without glycerin:

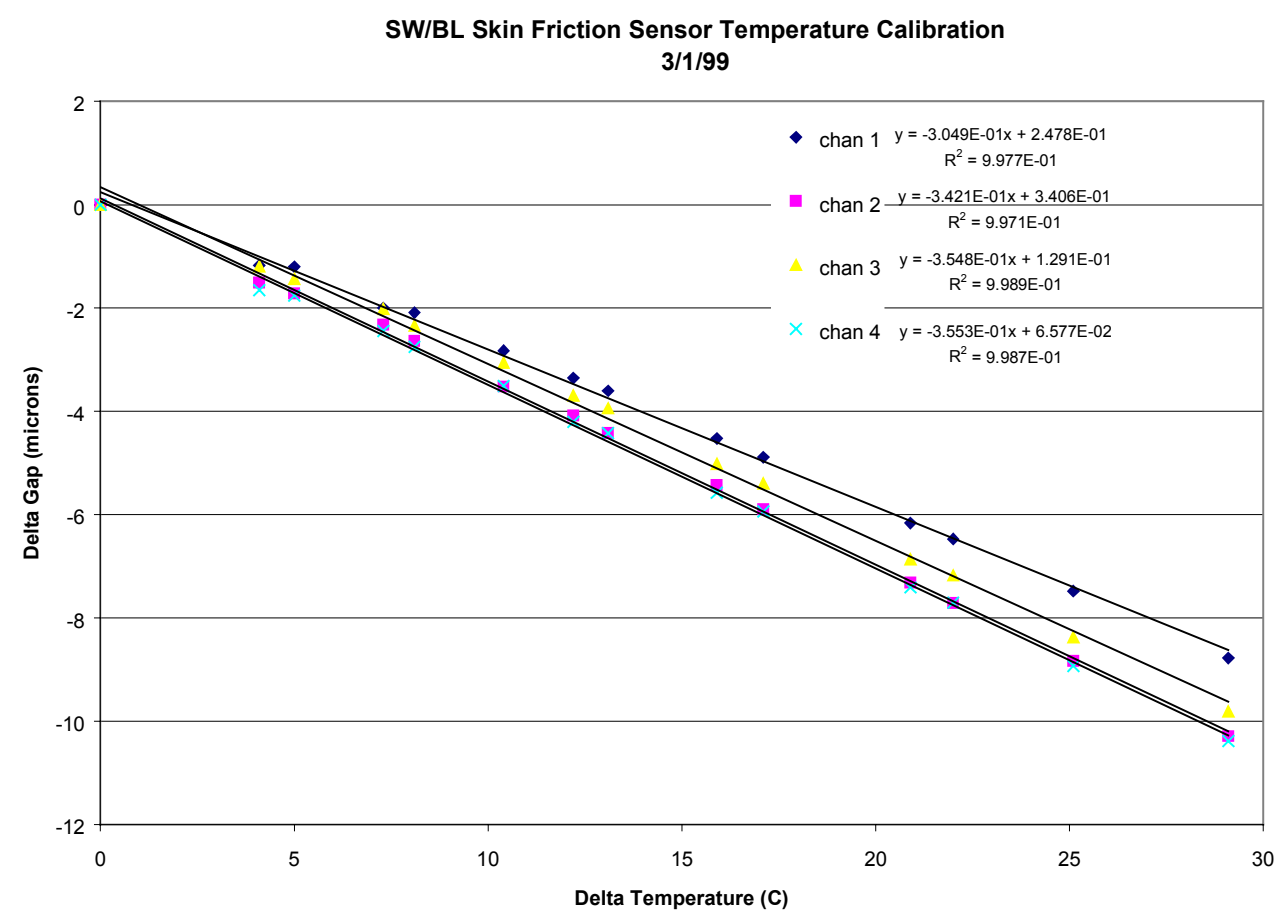

Version 5 a skin friction sensor

Shear calibration without glycerin:

Calibration of new s.f. sensor \#2

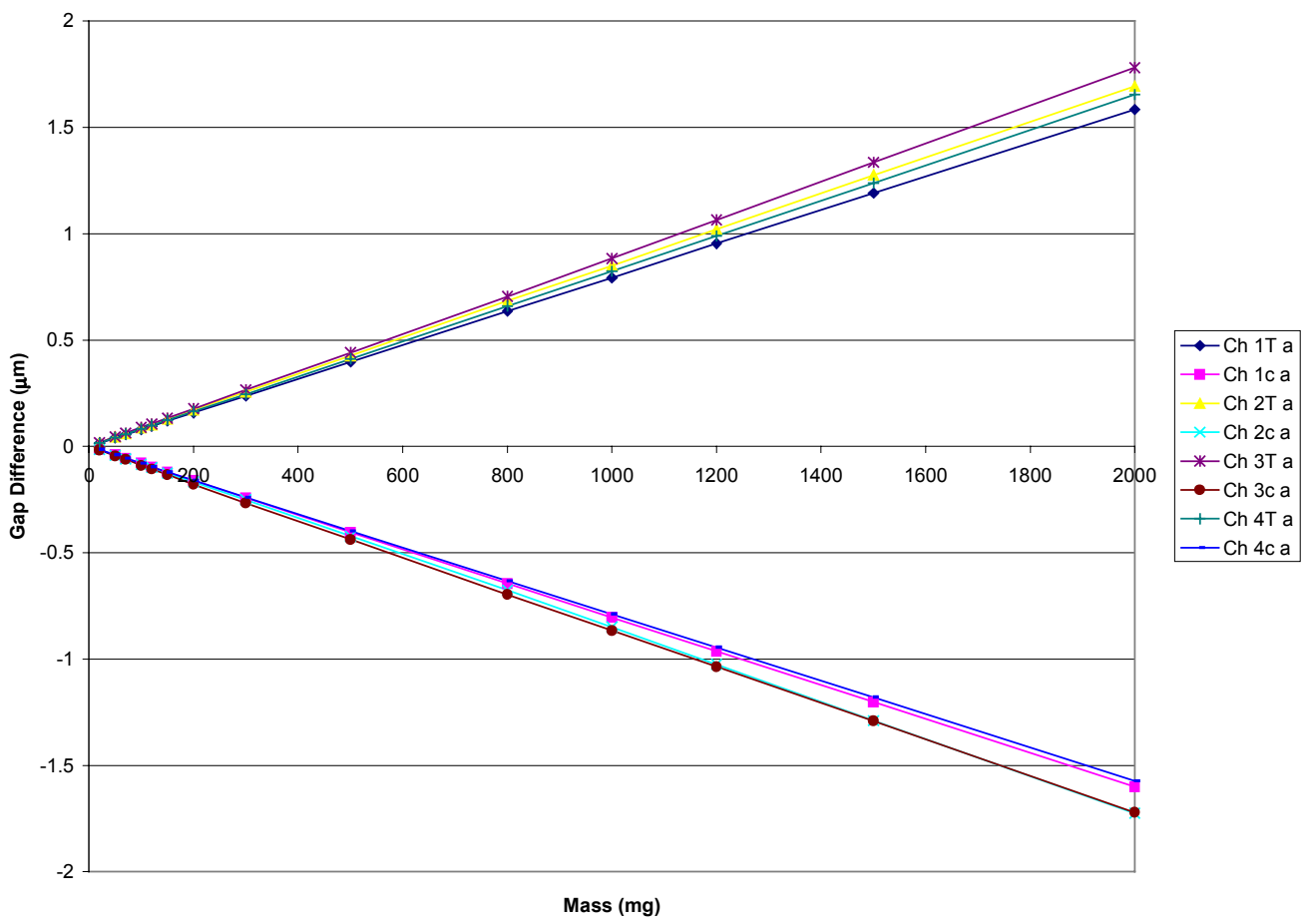


Shear calibration with glycerin:

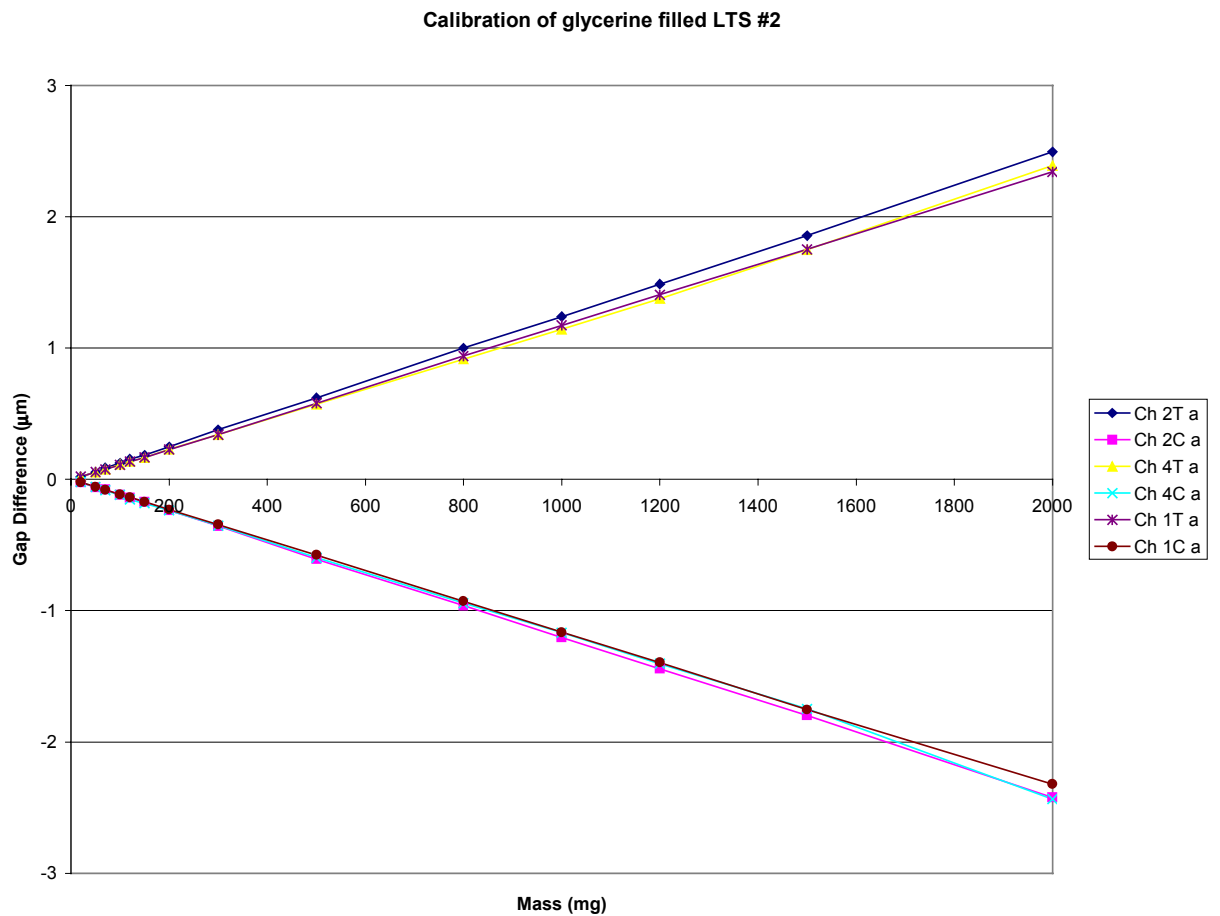

Temperature calibration without glycerin:

Temperature test of LTS s.f. gage

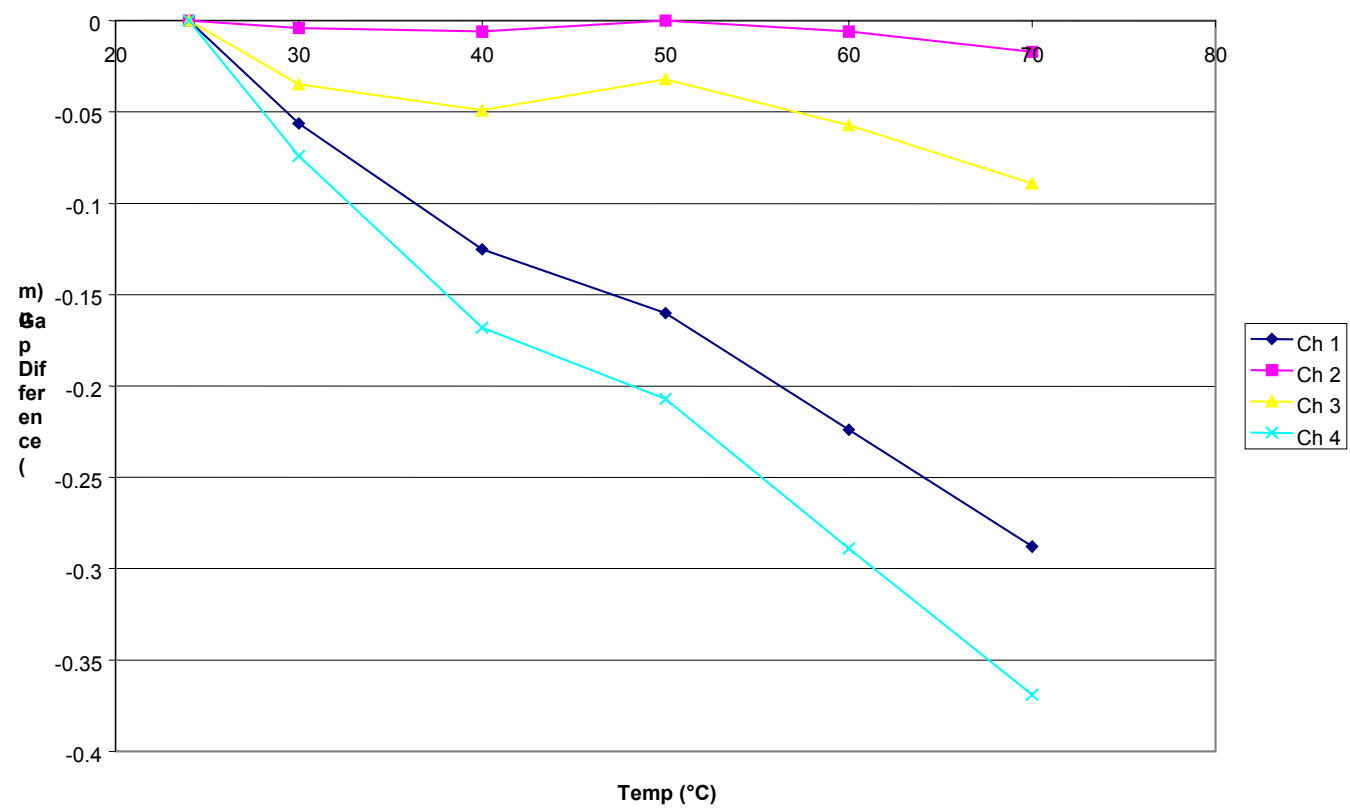


Appendix B

Calibrations

\section{Version $5 b$ skin friction sensor}

Shear calibration without glycerin:

Calibration of LTS \#3 without glycerine

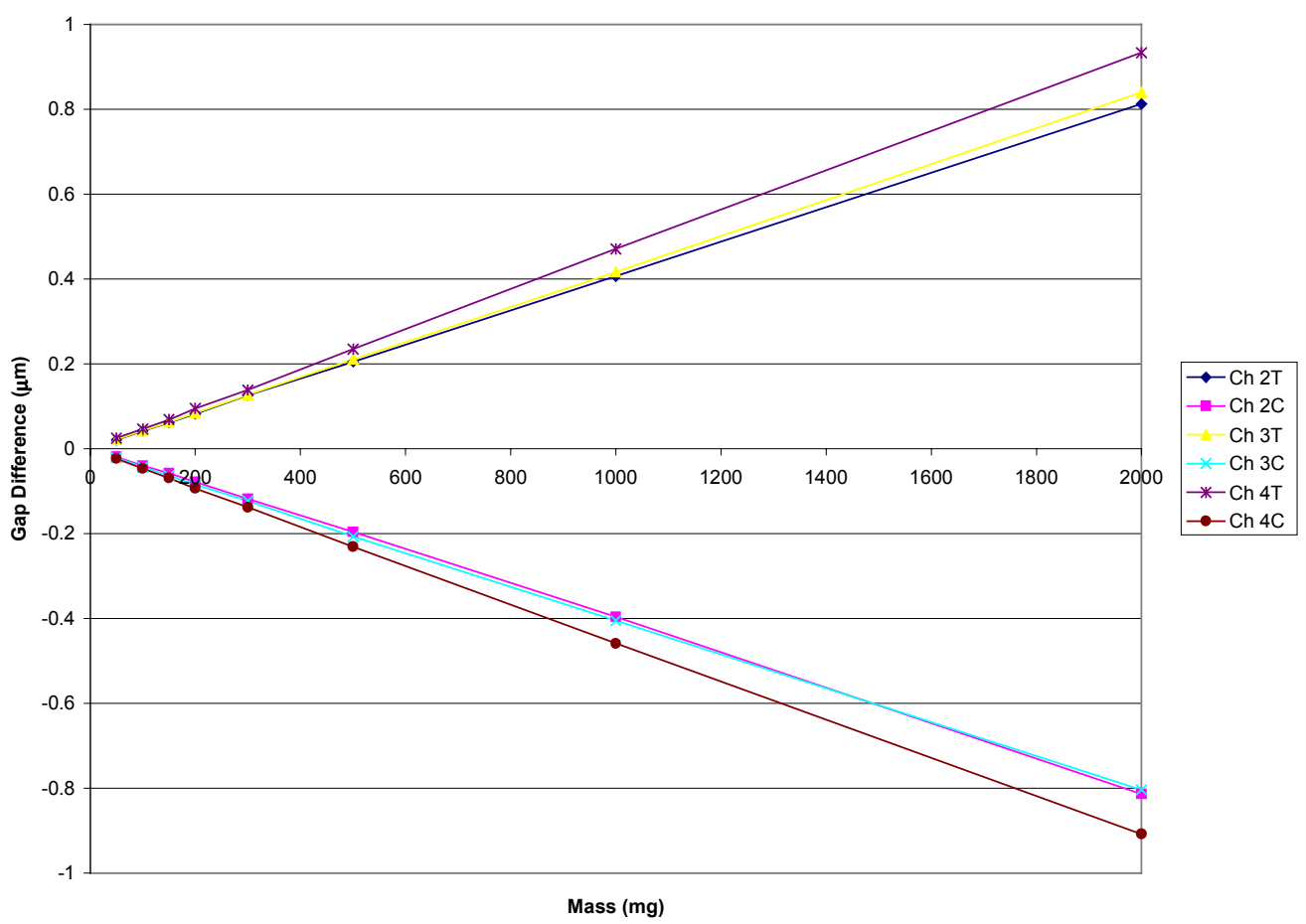

Shear calibration with glycerin:

Calibration of LTS \#3 with glycerine

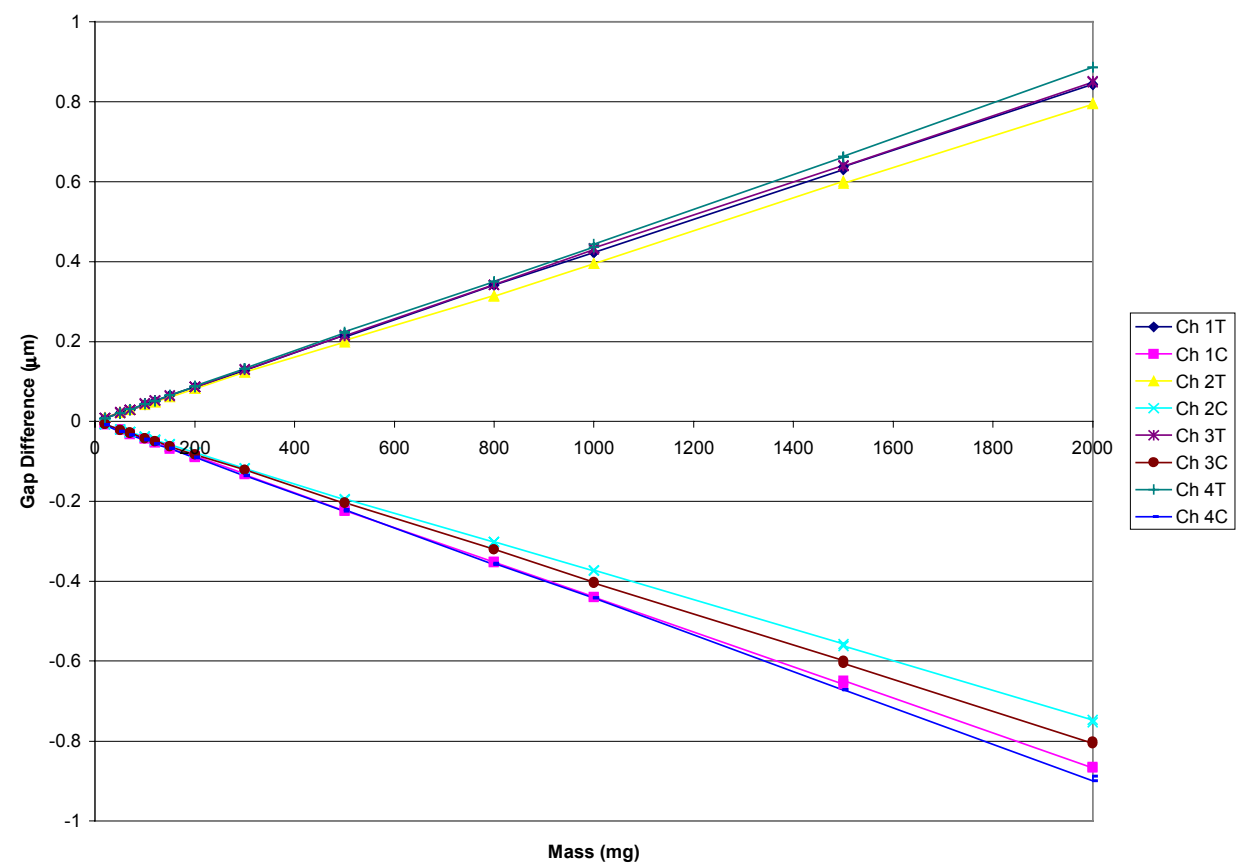


Appendix B

Calibrations

\section{Version 6 skin friction sensor}

Shear calibration without glycerin:

Calibration of LGM \#2 without glycerine

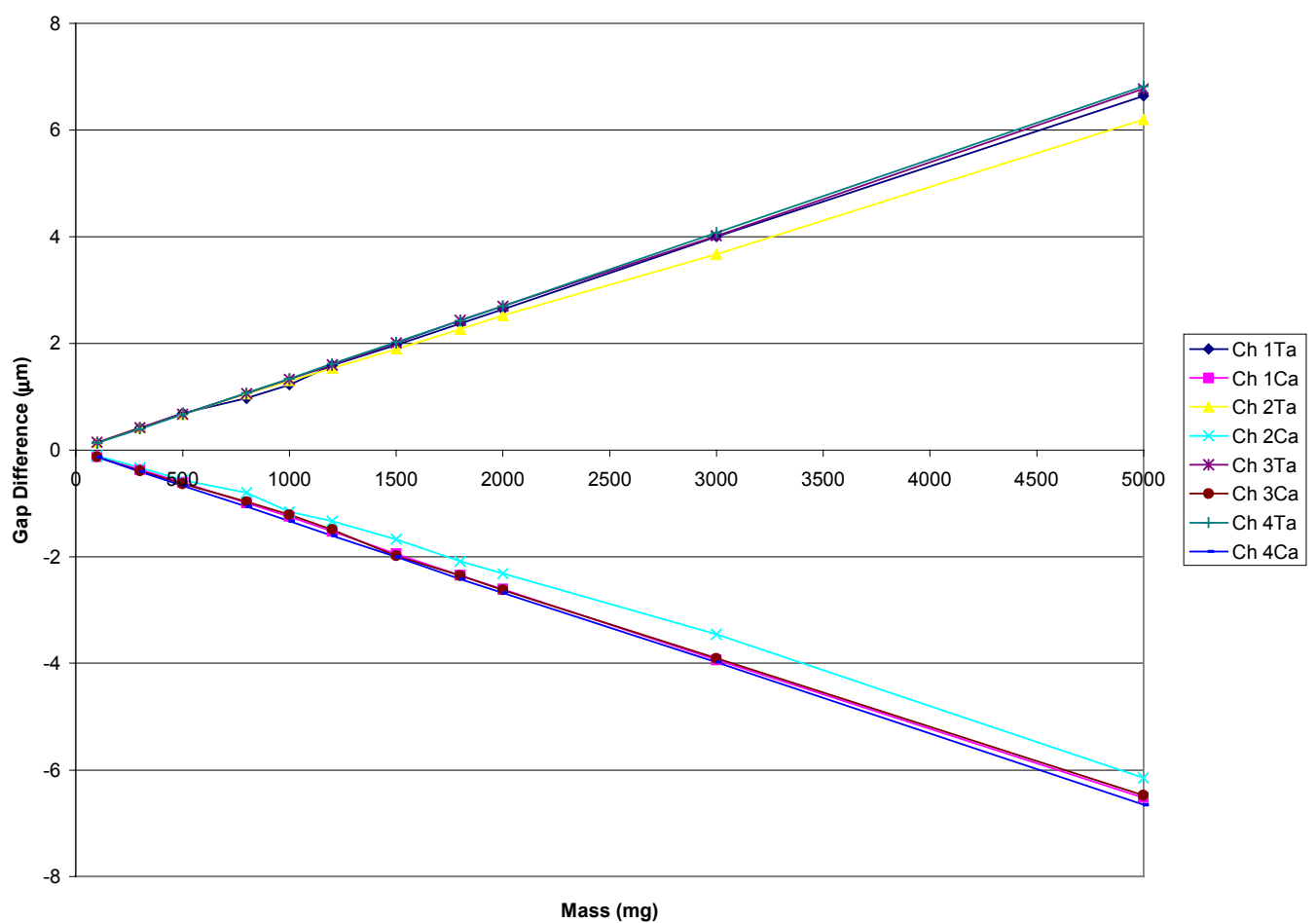

Shear calibration with glycerin:

Calibration of glycerine filled LGM \#2

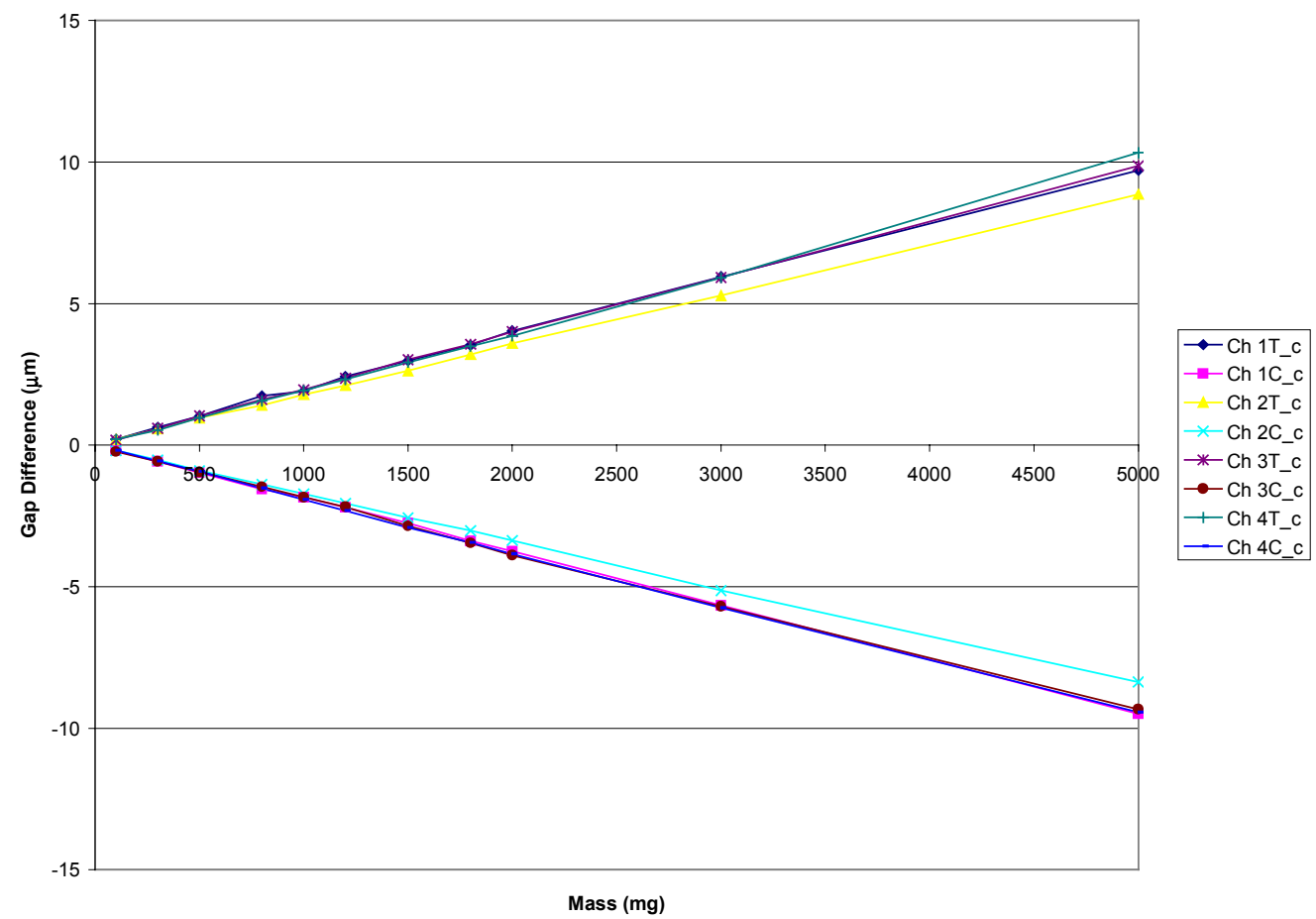


Appendix B

Calibrations

\section{Pressure array calibration}

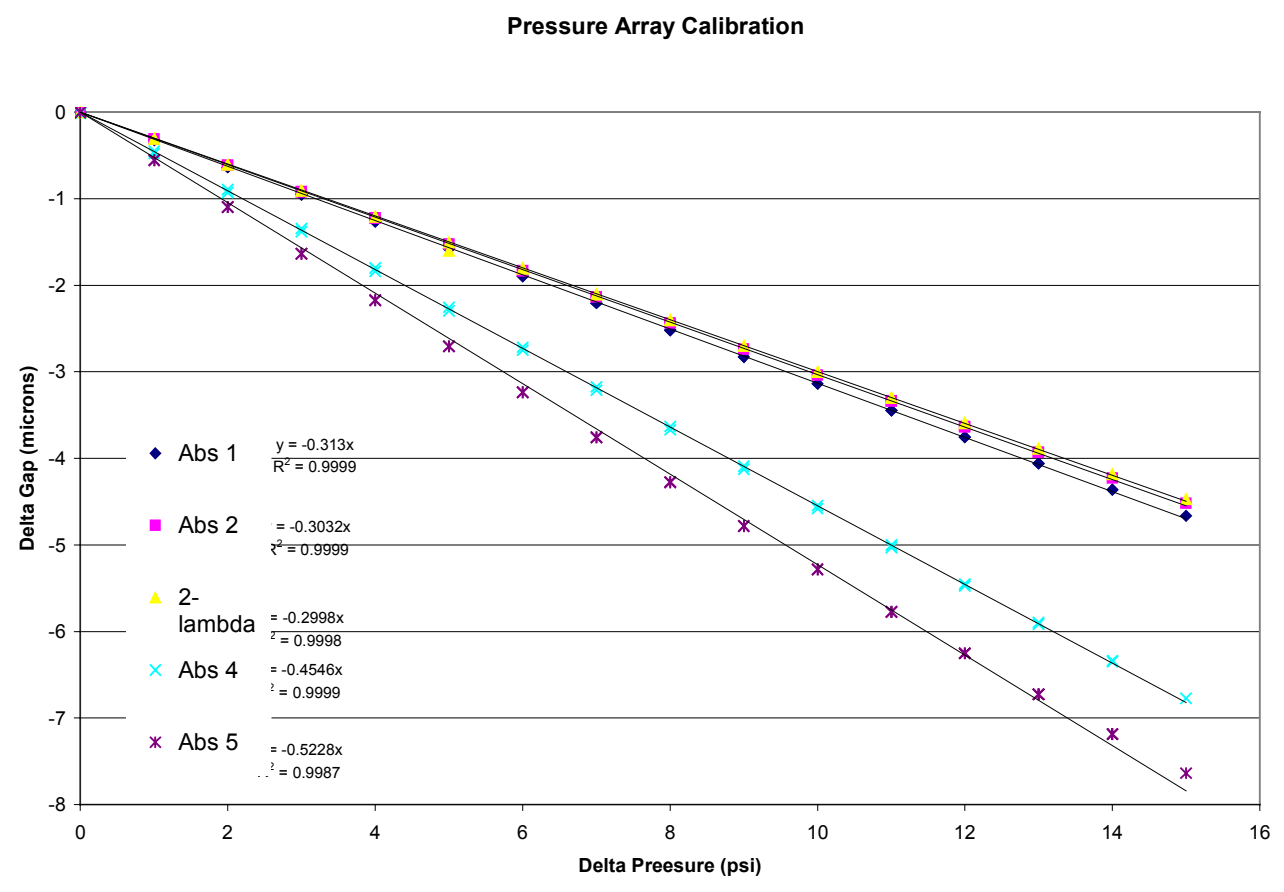

Cone Pressure calibration

Calibration of Pressure Sensors for Cone Experiment

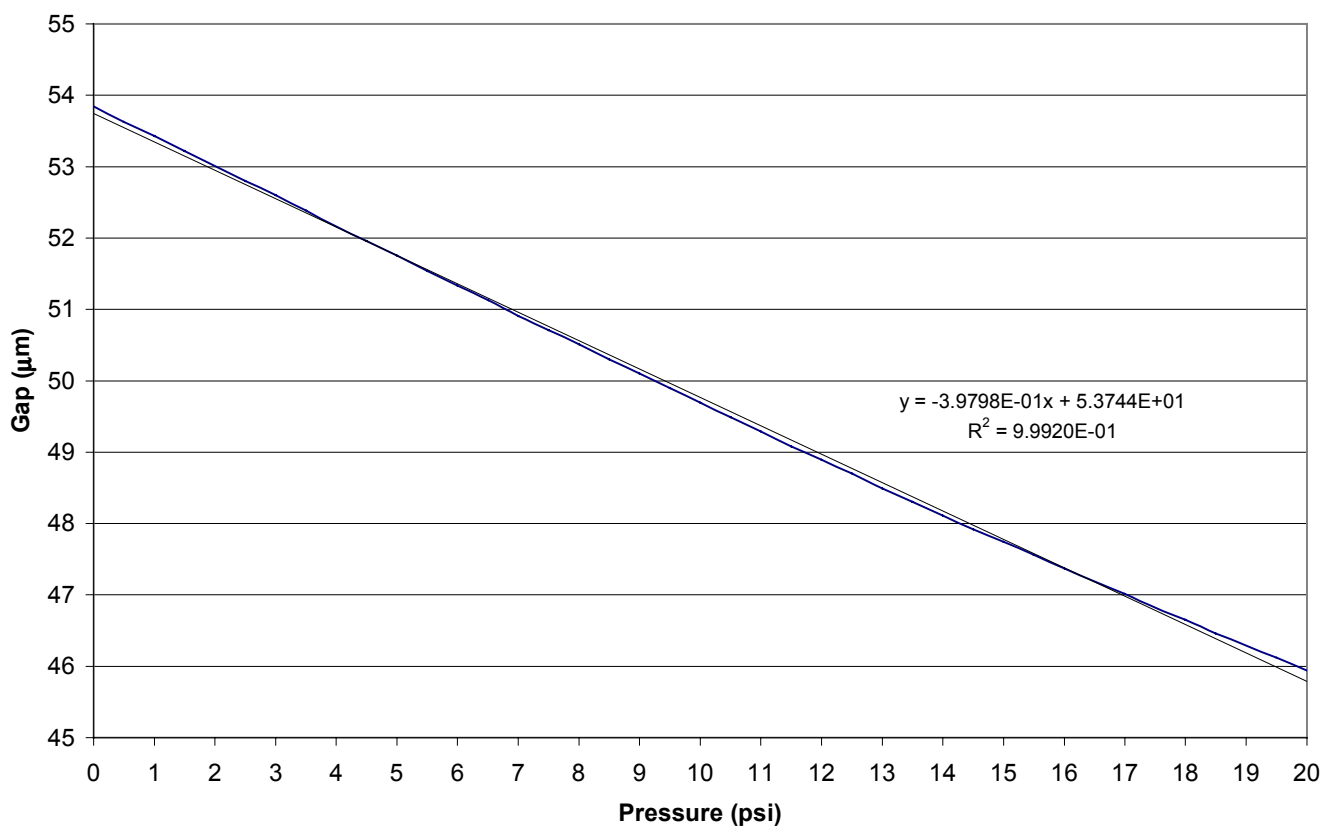




\section{Version 1 skin friction sensor}
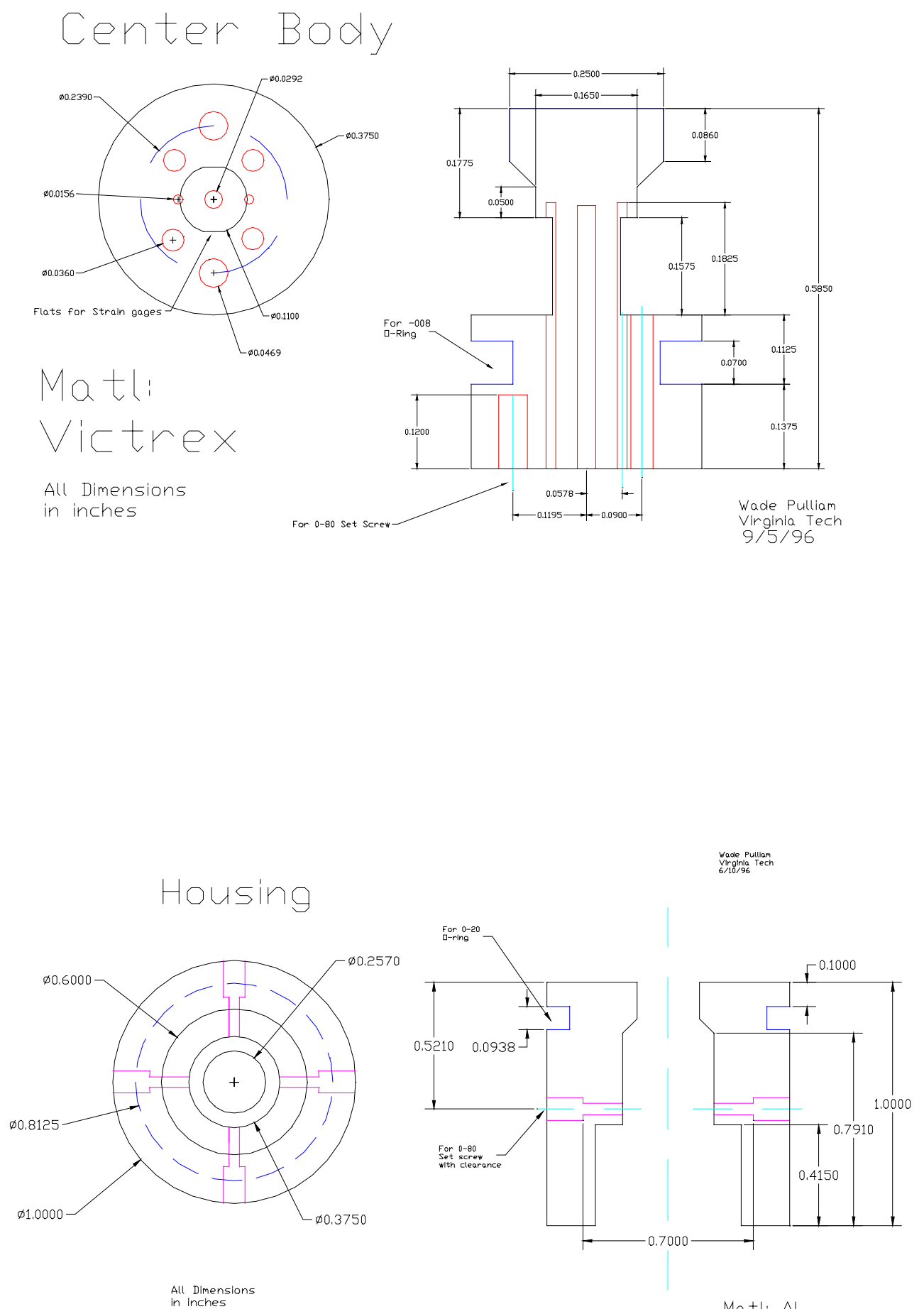

All Dimension
in inches

Mati: AL 
Appendix C

Mechanical Drawings

\section{Version 2 skin friction sensor}

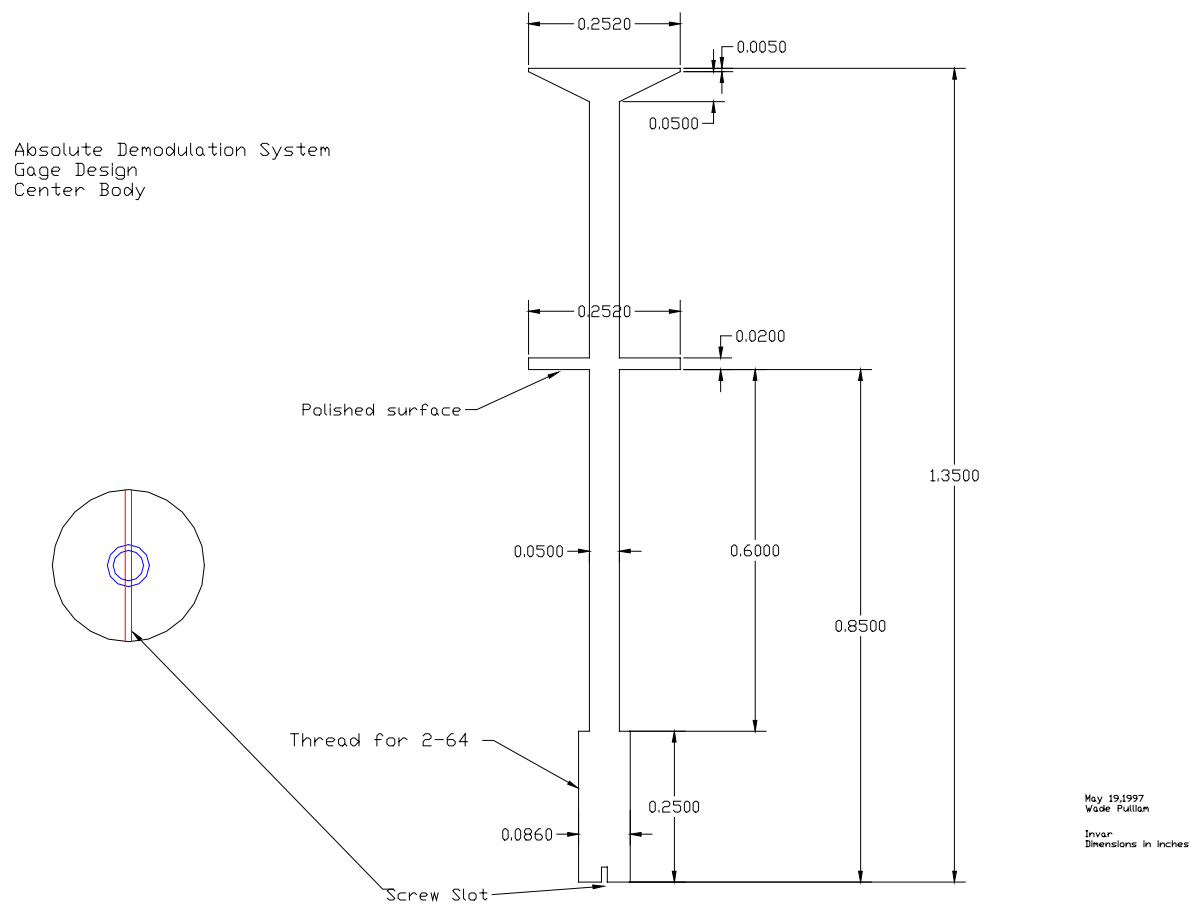

Alosolute Demodulation System

Gage Design

Fiber Holder

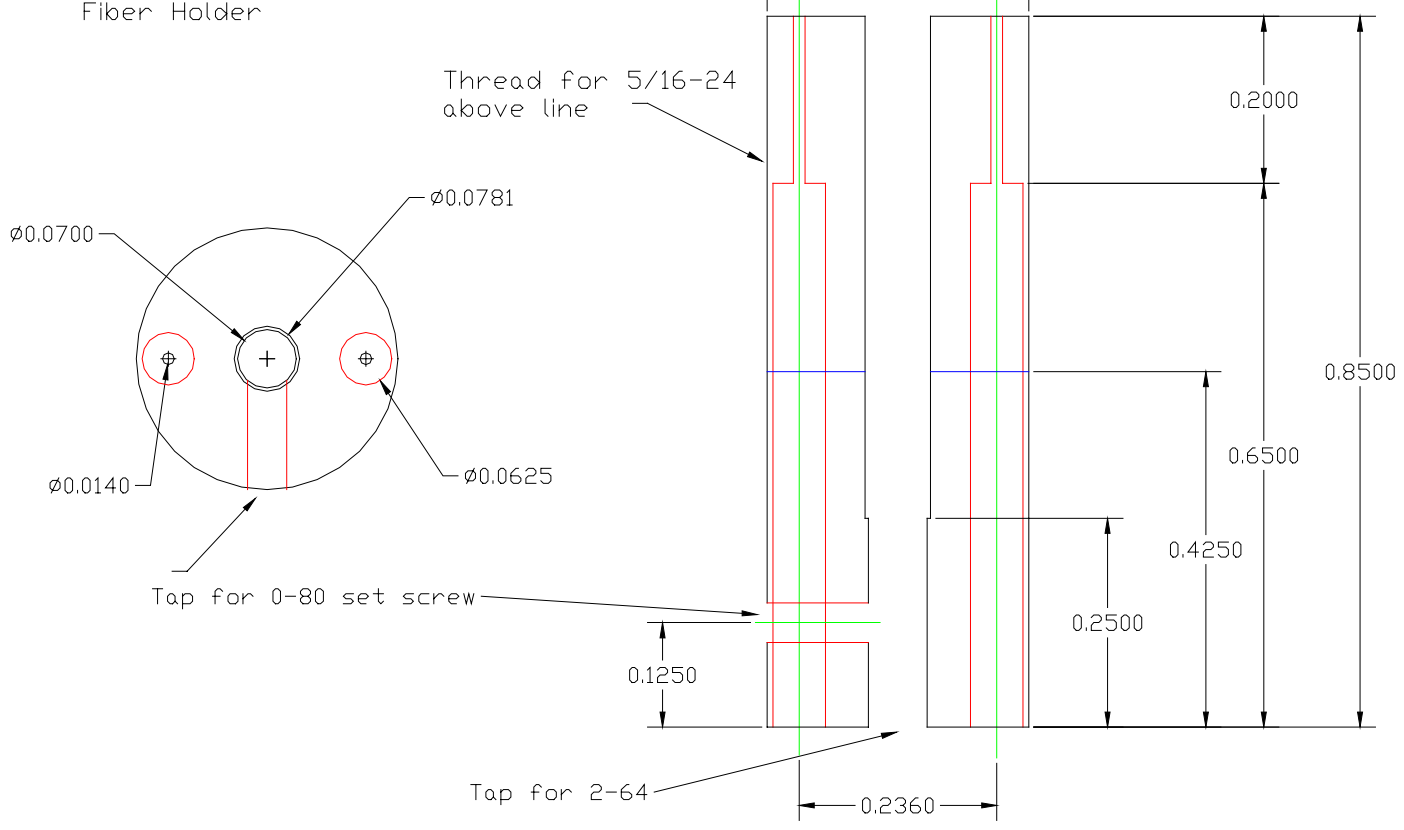

May 19,1997
wade Pullian

Invar
Dimensions in inches 
Appendix C

Mechanical Drawings

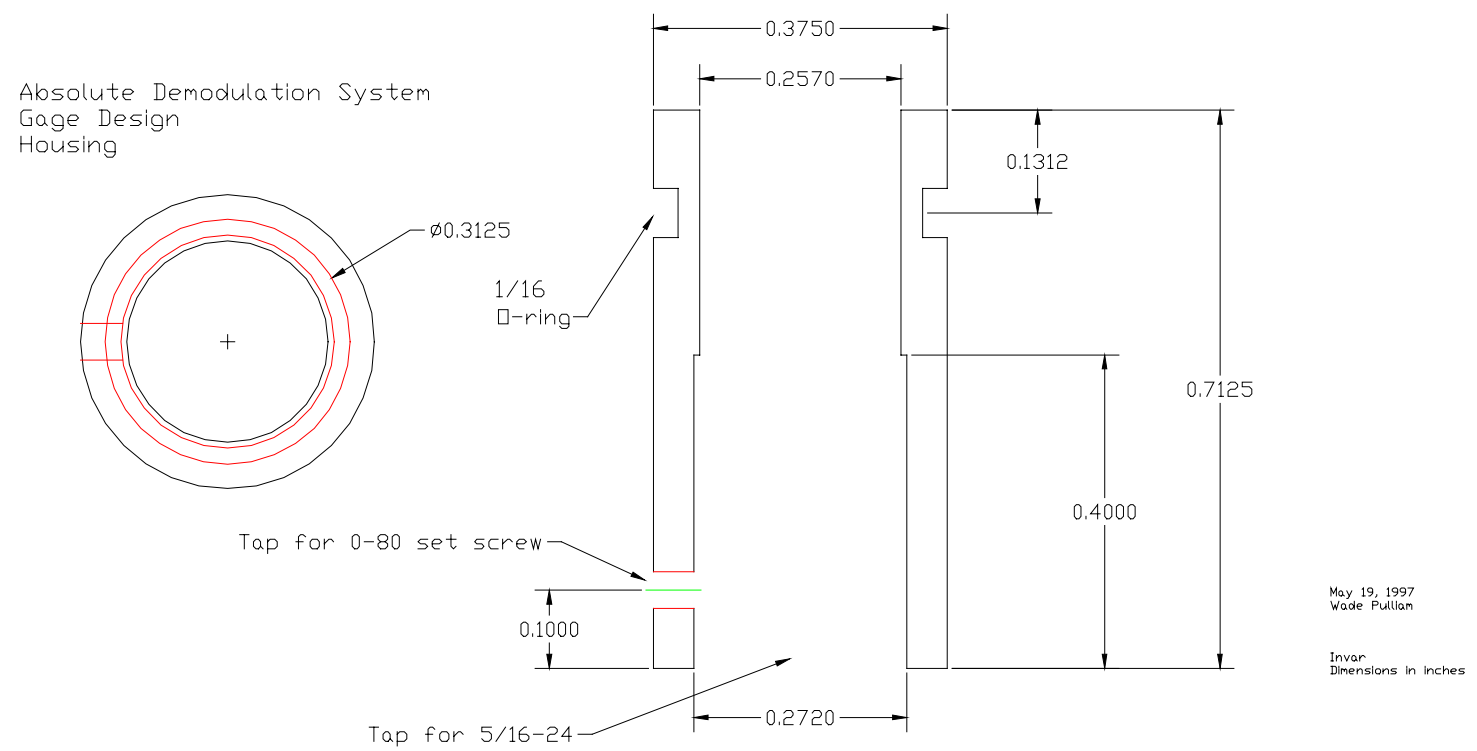




\section{Version 3 skin friction sensor}
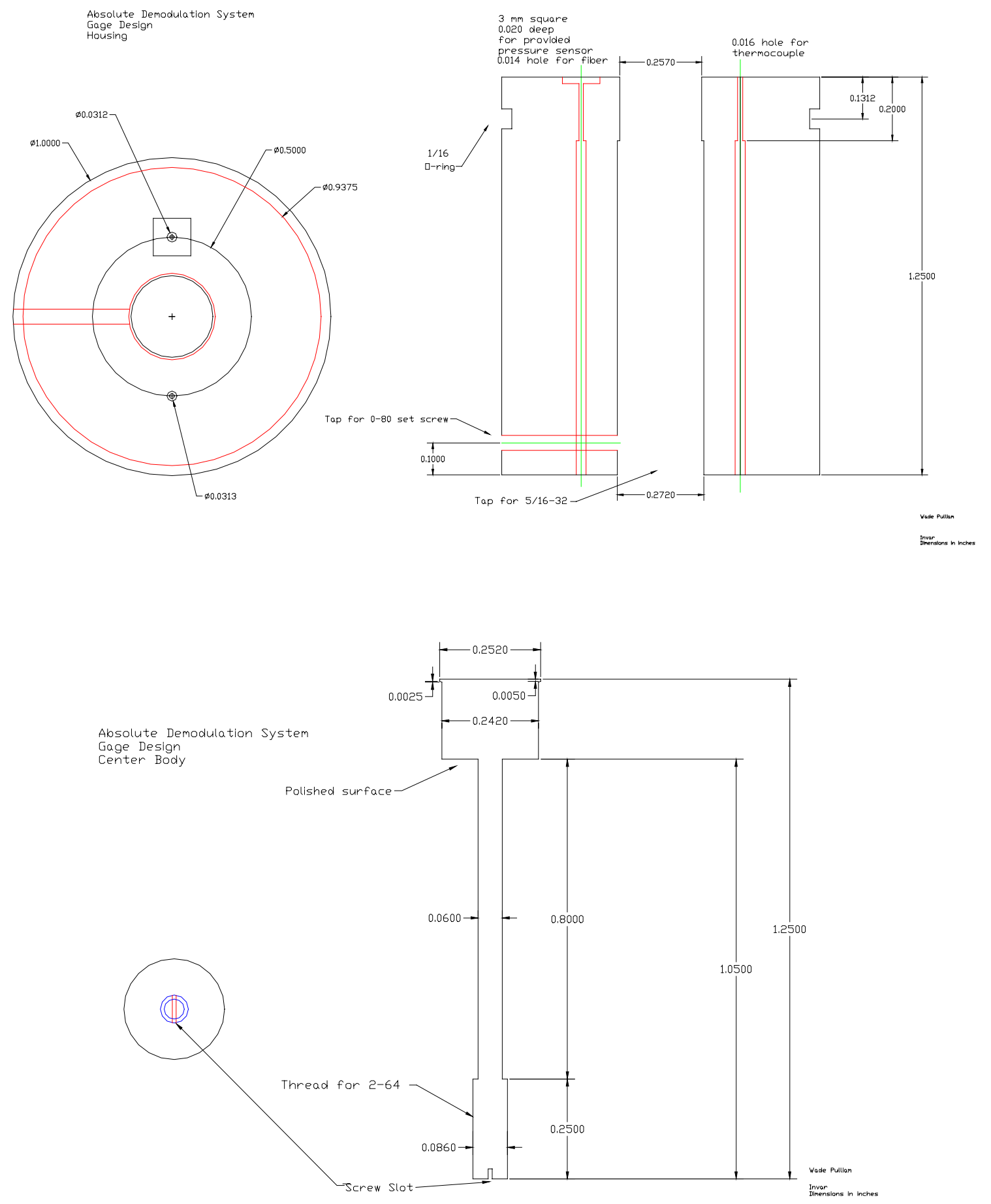
Appendix C

Mechanical Drawings

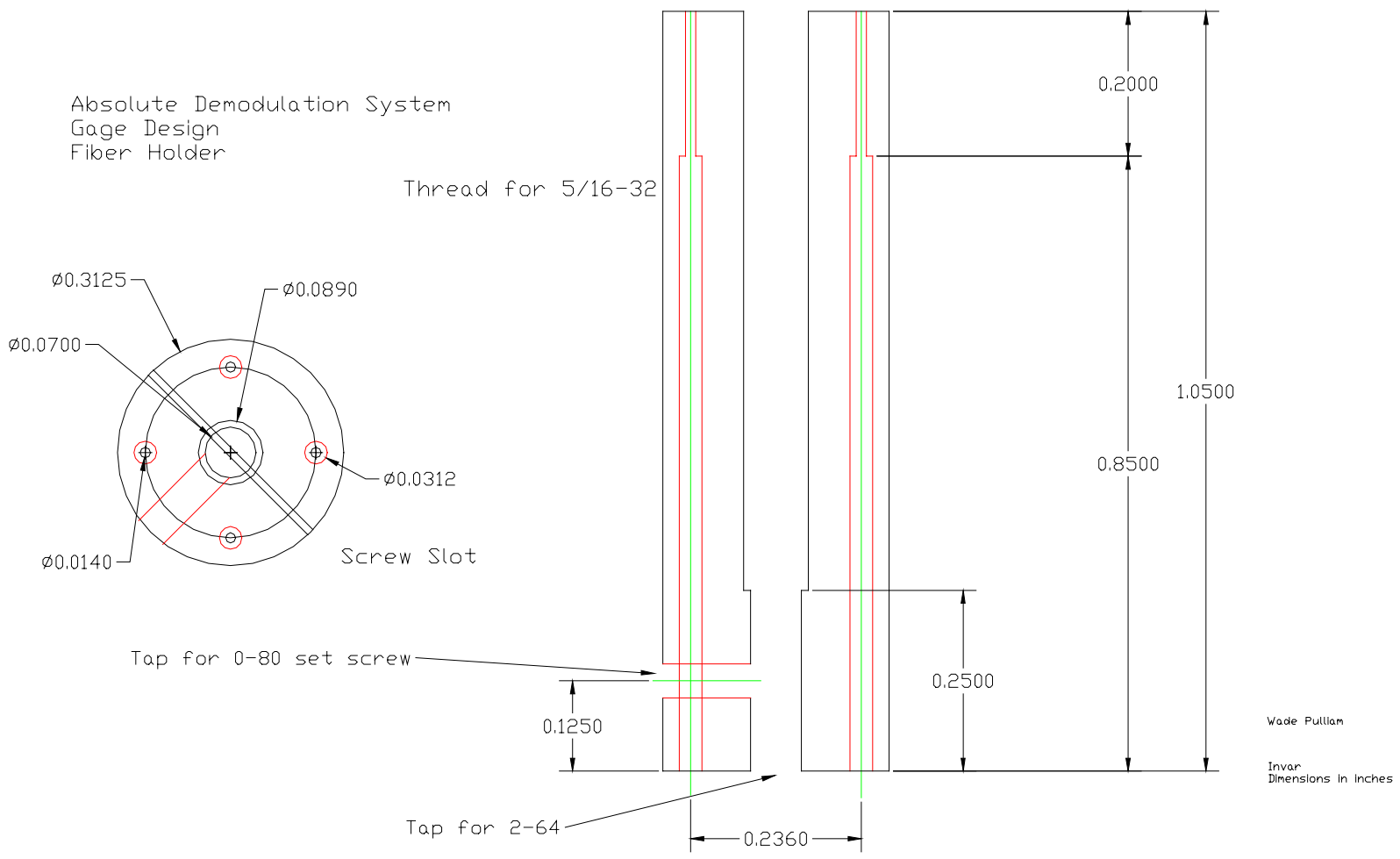


Appendix C

Mechanical Drawings

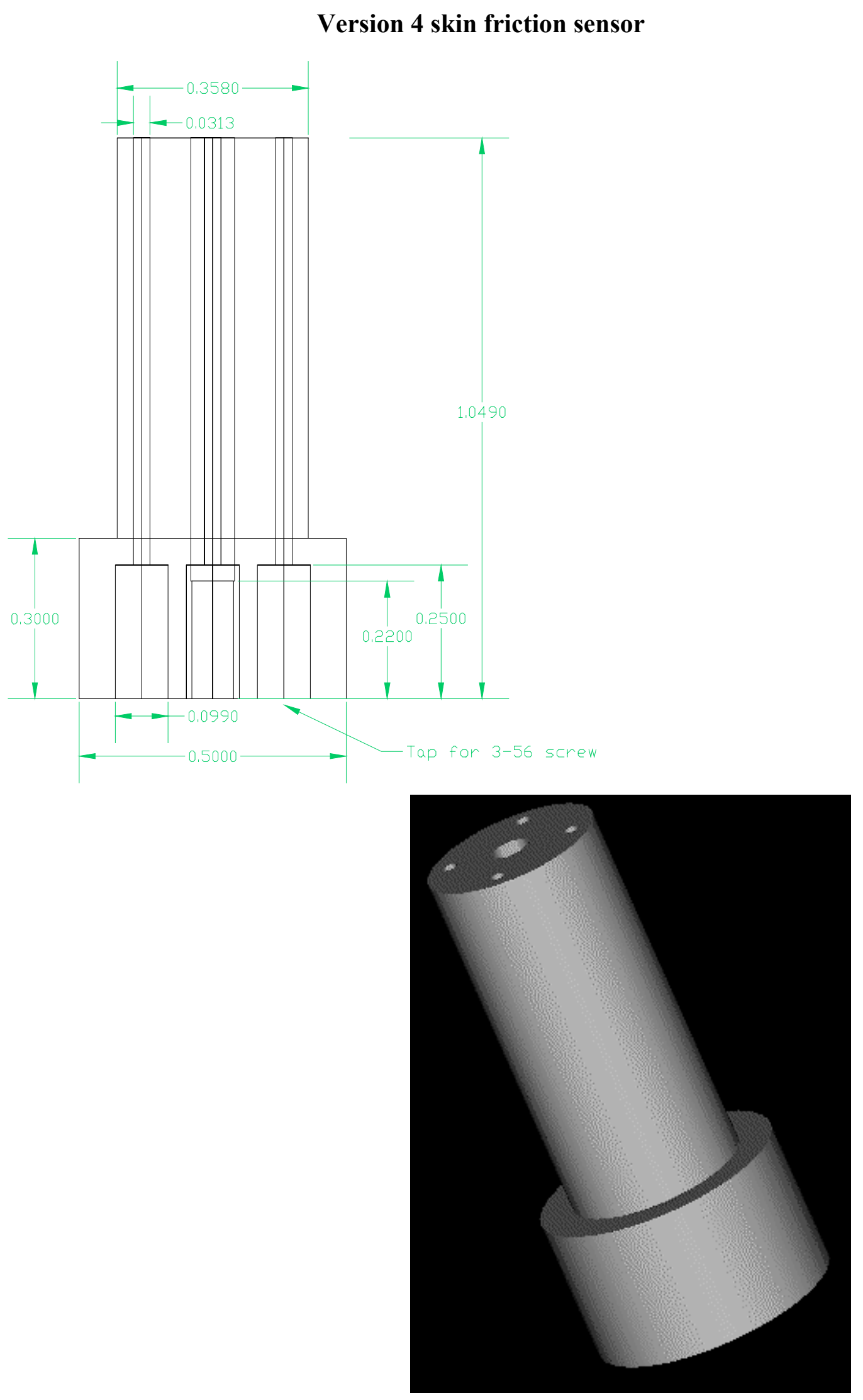


Appendix C

Mechanical Drawings
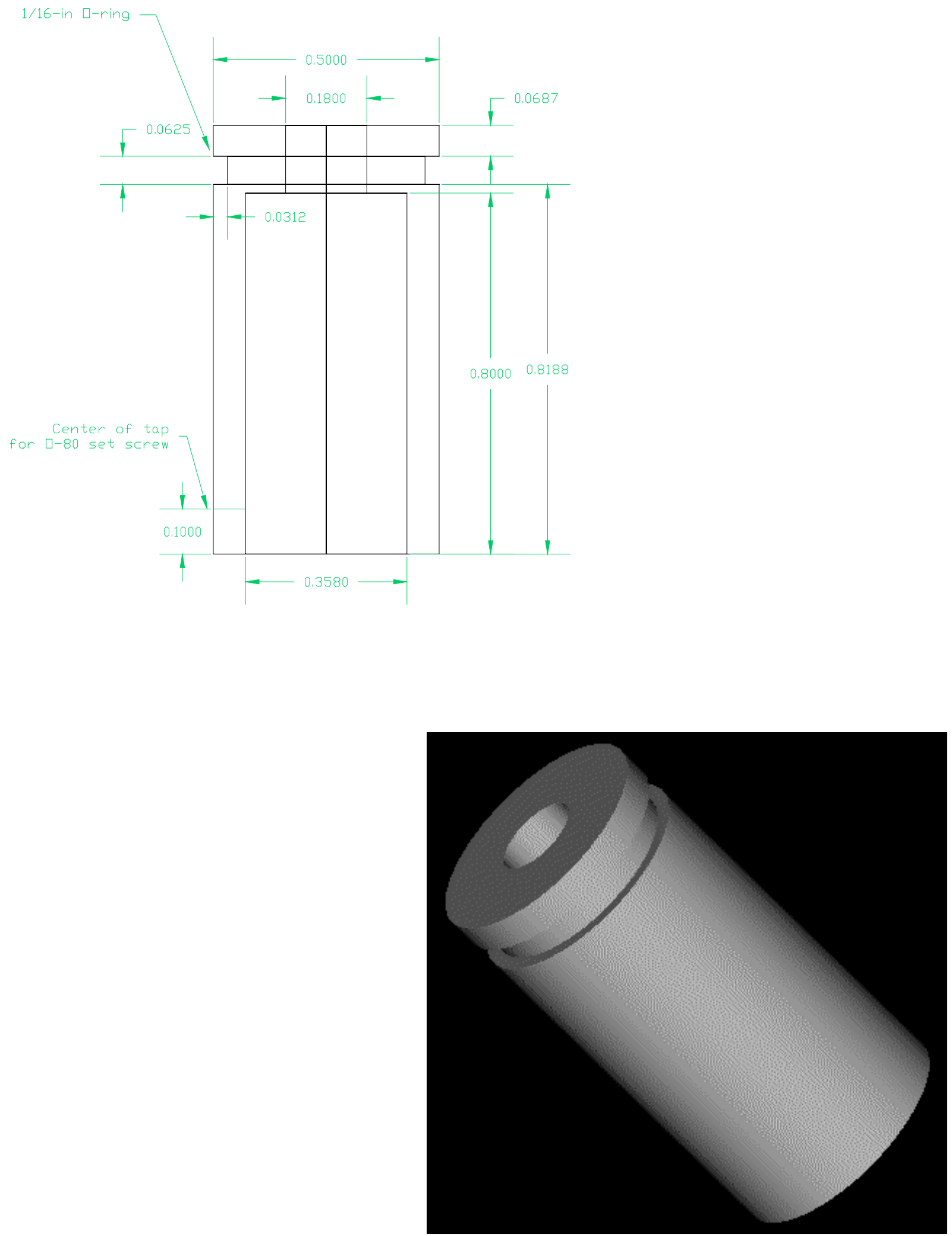
Appendix C

Mechanical Drawings
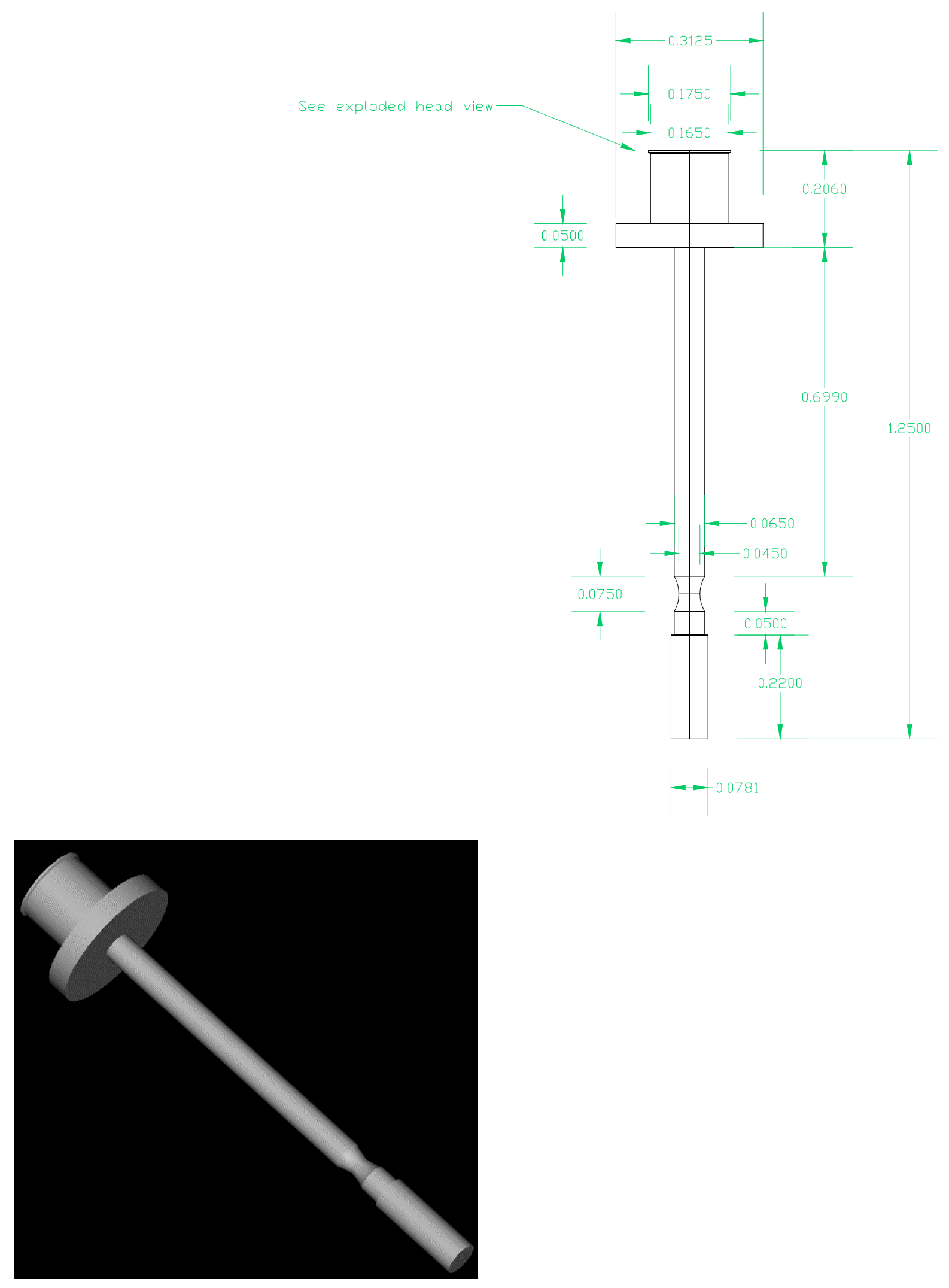


\section{Version 5 skin friction sensor}
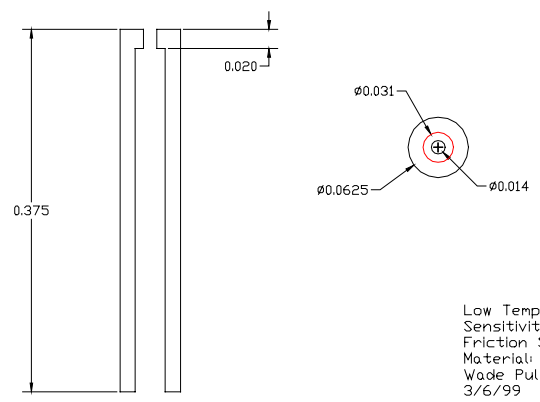

Low Temperature

Friction Sensar Materlahl Invar
Wode Pulliam
$3 / 6 / 99$
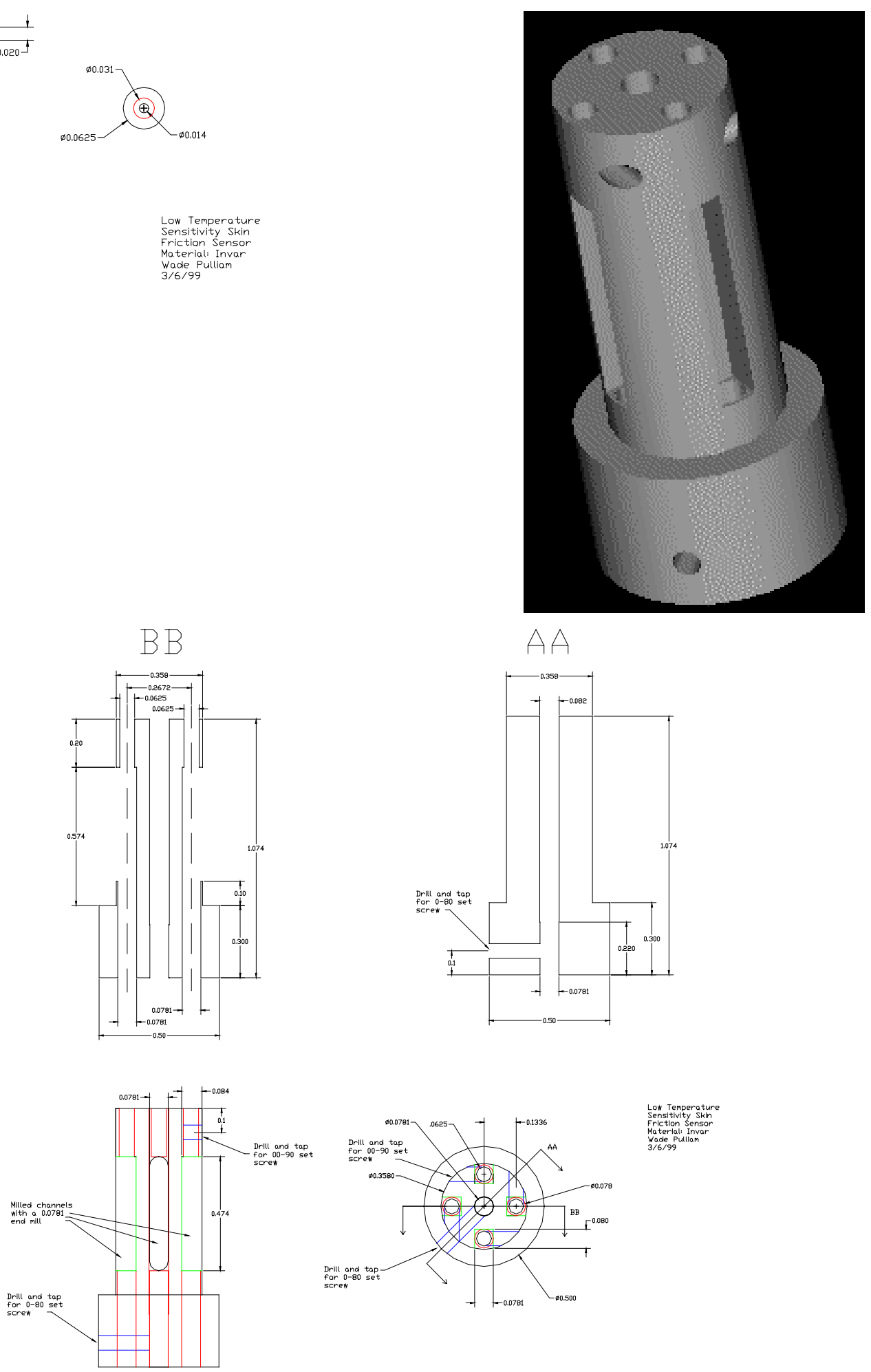

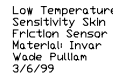



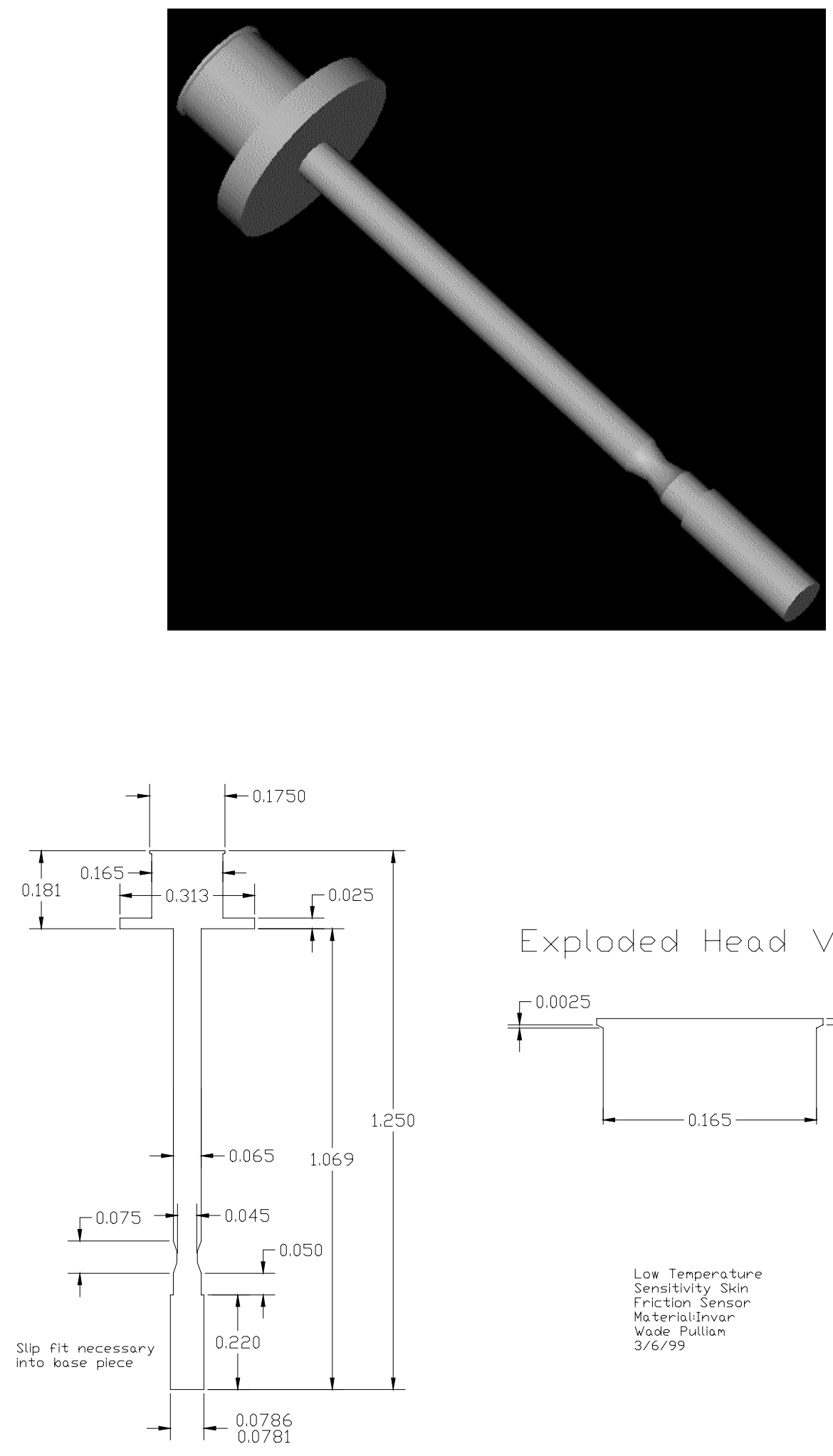

Exploded Head View

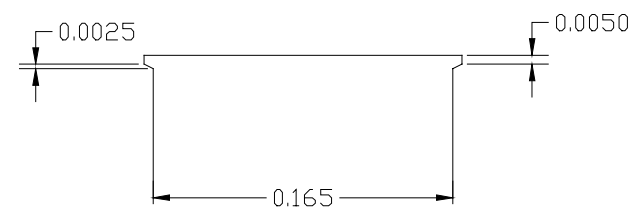

Low Temperature

Sensitivity Skin

Friction Sensor

Wade Pulliam

3/6/99 

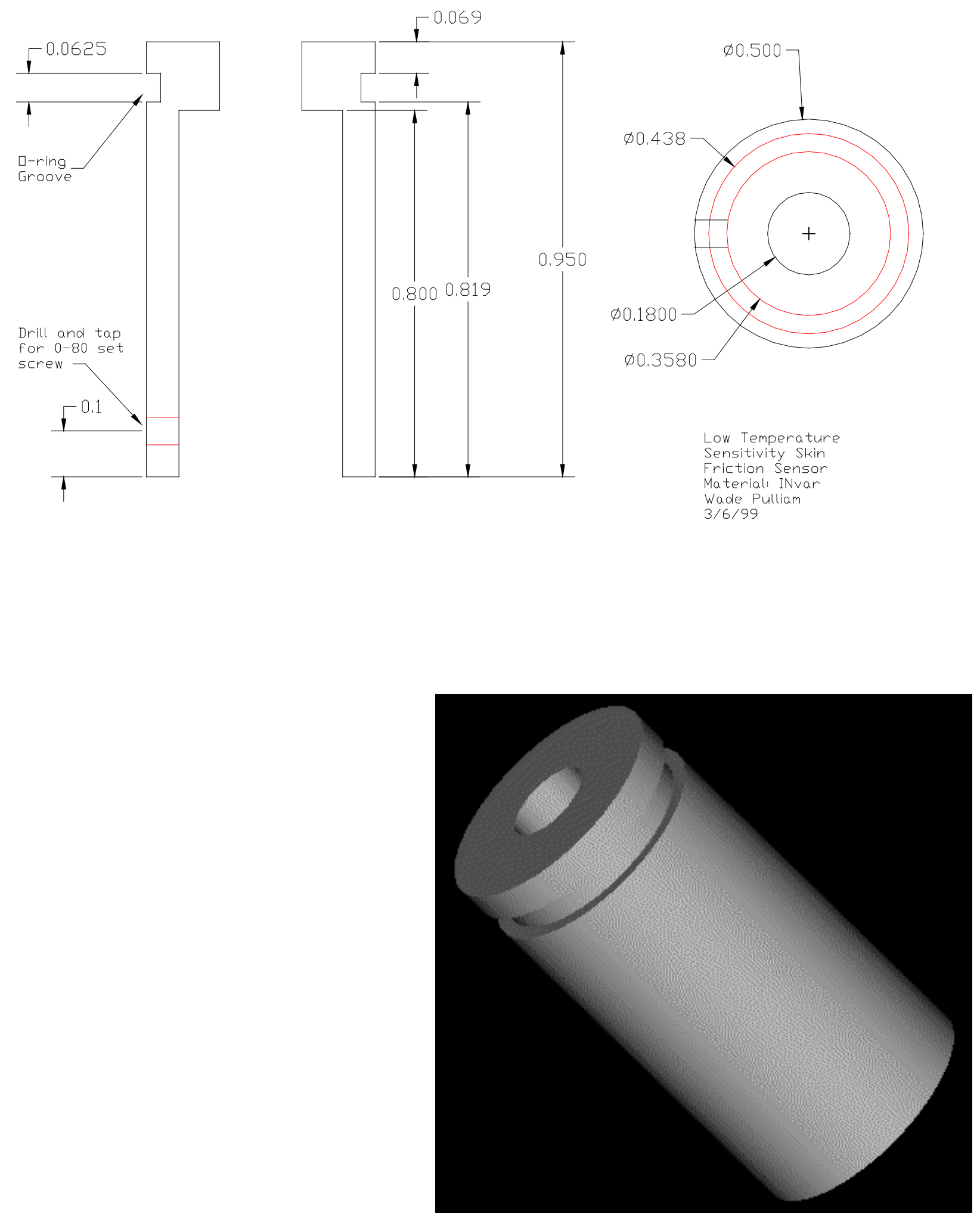


\section{Version 6 skin friction sensor}
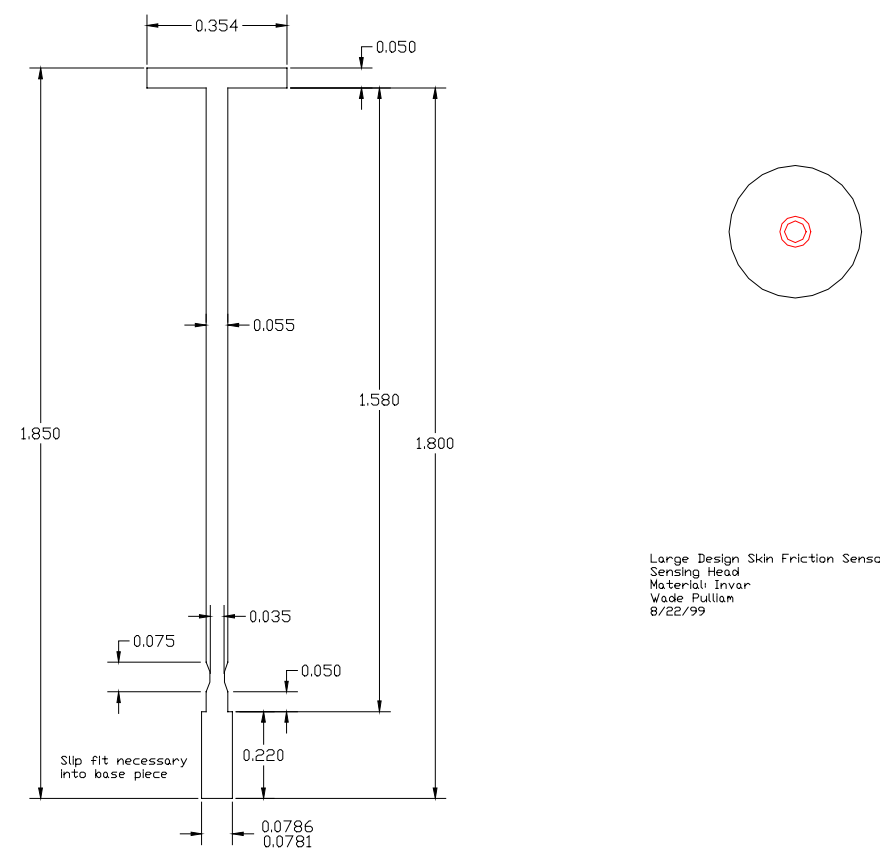

Large Design Skin Friction Sensor
Sensing Head

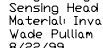

vade Pullla
$8 / 22 / 99$
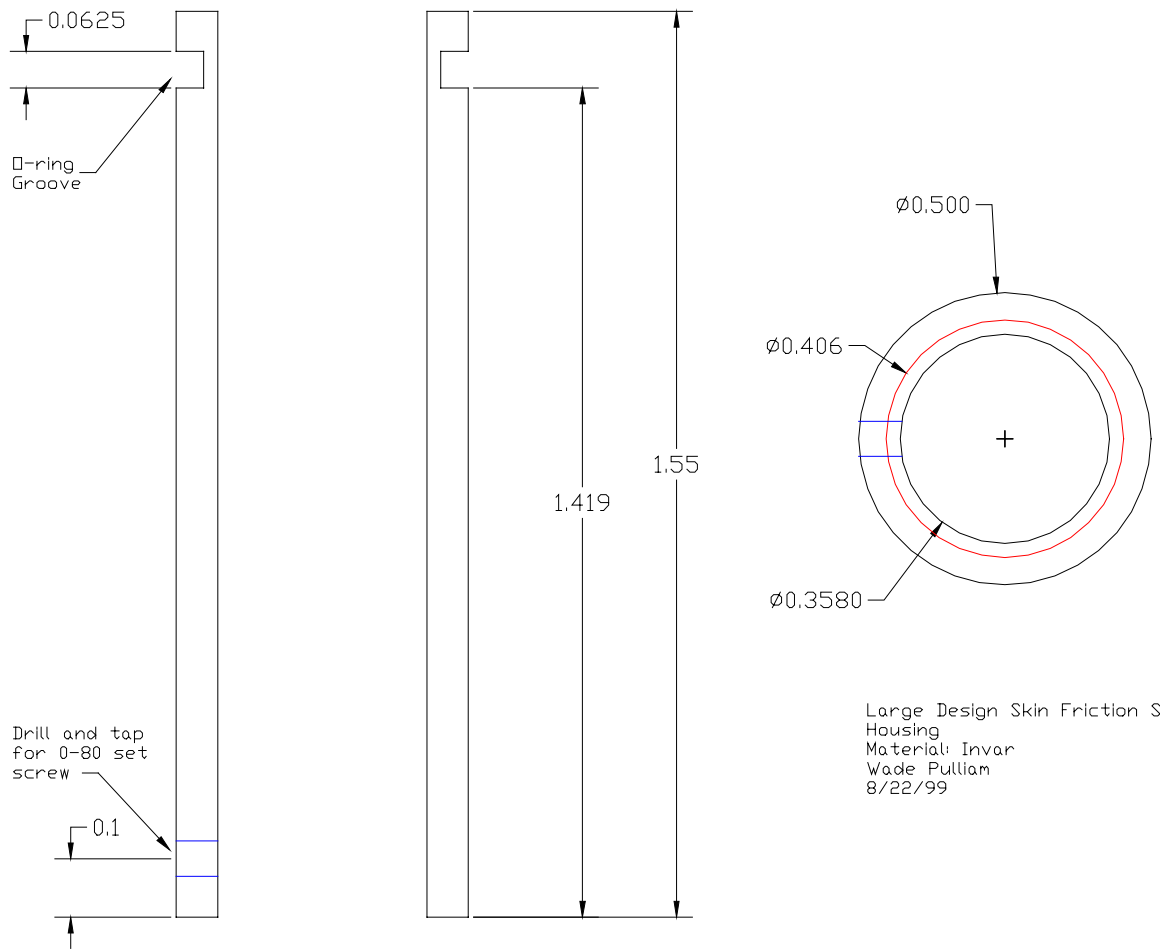

Large Design Skin Friction Sensar Housing

Wade Pulliam 
Appendix C

Mechanical Drawings
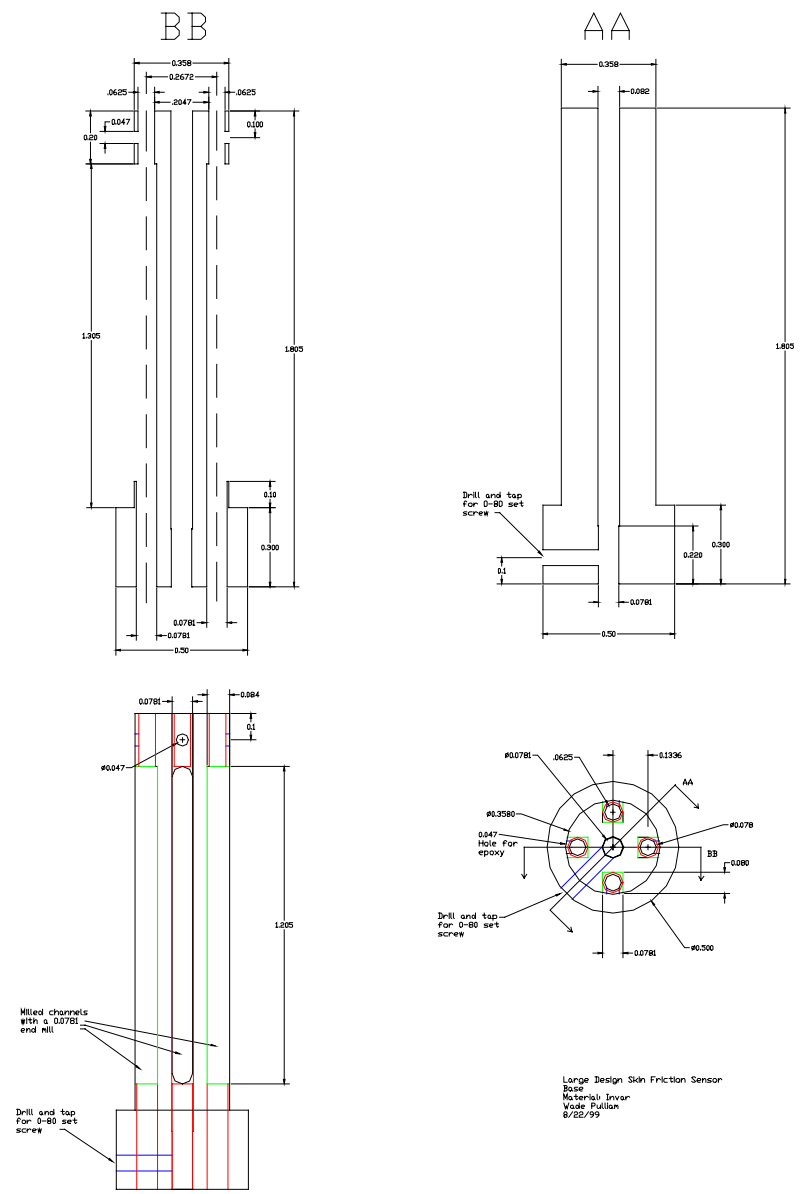


\section{Calculation of the Disturbance Field Induced by a Temperature Jump Patch on a Flat Plate in Supersonic Laminar Flow}

By George Inger

\section{Nomenclature}

$\mathrm{C}_{\mathrm{R}}=$ Chapman-Rubesin parameter $=\mu_{\mathrm{w} 1} \mathrm{~T}_{\mathrm{e}} / \mathrm{T}_{\mathrm{w} 1} \mu_{\mathrm{e}}$

$\mathrm{C}_{\mathrm{Q} 1}=0.315\left[1+0.196\left(1-\mathrm{P}_{\mathrm{R}}\right)\right]$

$\mathrm{C}_{\mathrm{Q} 2}=0.73 \sqrt{3} \Gamma\left(\frac{1 / 3}{3}\right) K^{1 / 3} \operatorname{Re}_{L}^{1 / 8} \beta^{1 / 4} / 2 \pi C_{R}^{1 / 8} \lambda^{1 / 4} \sqrt{\left(T_{w} / T_{e}\right)}$

$\mathrm{D}=$ length of temperature jump "patch" (Figure D1)

$\hat{\mathrm{D}}=\hat{\mathrm{x}}$ with $\mathrm{x}$ replaced by $\mathrm{D}$

$\mathrm{I}_{\mathrm{p}}, \mathrm{I}_{\tau}=$ Integrals appearing in the theory - see Equations D9 and D14, respectively

$\mathrm{L}=\mathrm{x}_{0}$, the origin of the $\mathrm{x}$-coordinate located at the patch front edge (Figure D1)

$\hat{\mathrm{L}}=\hat{\mathrm{x}}$ with $\mathrm{x}$ replaced by $\mathrm{L}$

$\mathrm{M}_{\mathrm{e}}=$ external inviscid flow Mach number at $\mathrm{x}_{\mathrm{o}}$

$\mathrm{p}=$ static pressure

$\Delta_{\mathrm{p}}=$ interactive pressure rise $=\mathrm{p}-\mathrm{p}_{\mathrm{e}}$

$\mathrm{P}_{\text {ref }}=$ reference pressure using relevant scaling parameters $=0.729 \mathrm{MM}_{\mathrm{e}}^{2} \lambda^{1 / 2} C_{R} p_{e} / \beta^{1 / 2} \operatorname{Re}_{L}^{1 / 4} P_{R}^{1 / 3} K^{2 / 3}$

$\mathrm{P}_{\mathrm{R}}=$ Prandtl number

$\operatorname{Re}_{\mathrm{L}}=$ Reynolds number at $\mathrm{x}_{\mathrm{o}}=\rho_{\mathrm{e}} \mathrm{U}_{\mathrm{e}} \mathrm{L} / \mu_{\mathrm{e}}$

$\dot{q}_{w}=$ heat transfer rate per unit area

$\Delta \dot{q}_{w}=$ interactive perturbation in heat transfer

$\mathrm{T}_{\mathrm{w} 1}=$ known background wall temperature (Figure D1)

$\Delta \mathrm{T}_{\mathrm{w} 1}=$ prescribed wall temperature jump on patch D (Figure D1)

$\mathrm{u}=$ streamwise velocity

$\mathrm{x}=$ streamwise distance measured from front edge of patch (Figure D1)

$\hat{\mathrm{x}}=$ scaled distance variable $=(\mathrm{x} / \mathrm{L}) \lambda^{5 / 4} \beta^{3 / 4} \operatorname{Re}_{L}^{3 / 8} / C_{R}^{3 / 8}\left(T_{w} / T_{e}\right)^{3 / 2}$

$\beta=\sqrt{M_{e}^{2}-1}$

$\gamma=$ specific heat ratio

$\Gamma(1 / 3), \Gamma(2 / 3), \Gamma(4 / 3)=$ Standard gamma functions

$\mathrm{K}=$ universal constant occurring in triple deck theory $=0.827$

$\lambda=$ Blasius similarity velocity profile parameter $=0.332$ for a flat plate

$\mu=$ coefficient of viscosity

$\rho=$ density

$\tau_{\mathrm{w}}=$ wall shear stress 
$\Delta \tau_{\mathrm{w}}=$ interactive perturbation of wall shear stress

$\underline{\text { Subscripts }}$

$\mathrm{e}=$ undisturbed inviscid flow at $\mathrm{x}_{\mathrm{o}}$

$\mathrm{o}=$ non-interactive value (usually taken at $\mathrm{x}=\mathrm{x}_{\mathrm{o}}=\mathrm{L}$ )

$\mathrm{w}=$ conditions on the wall surface

\section{Problem Definition}

Consider a 2-D steady laminar flow of an ideal gas along a flat plate, where there is a local "patch" $\mathrm{D}$ of wall temperature change $\Delta \mathrm{T}_{\mathrm{w}}$ located a distance $\mathrm{L}=\mathrm{x}_{\mathrm{o}}$ downstream of the leading edge of the plate (Figure D1). The external inviscid flow is supersonic and the local streamwise distance $\mathrm{x}$ is from the front edge of the patch. The local disturbance fields along $\mathrm{x}$ at the surface are functions of the parameters $\mathrm{D} / \mathrm{L}, \mathrm{M}_{\mathrm{e}}, \Delta \mathrm{T}_{\mathrm{w}} / \mathrm{T}_{\mathrm{w}}$ and Reynolds number $\mathrm{Re}_{\mathrm{L}}$. These include disturbances in pressure, shear stress and heat transfer that are generated by the temperature jump.

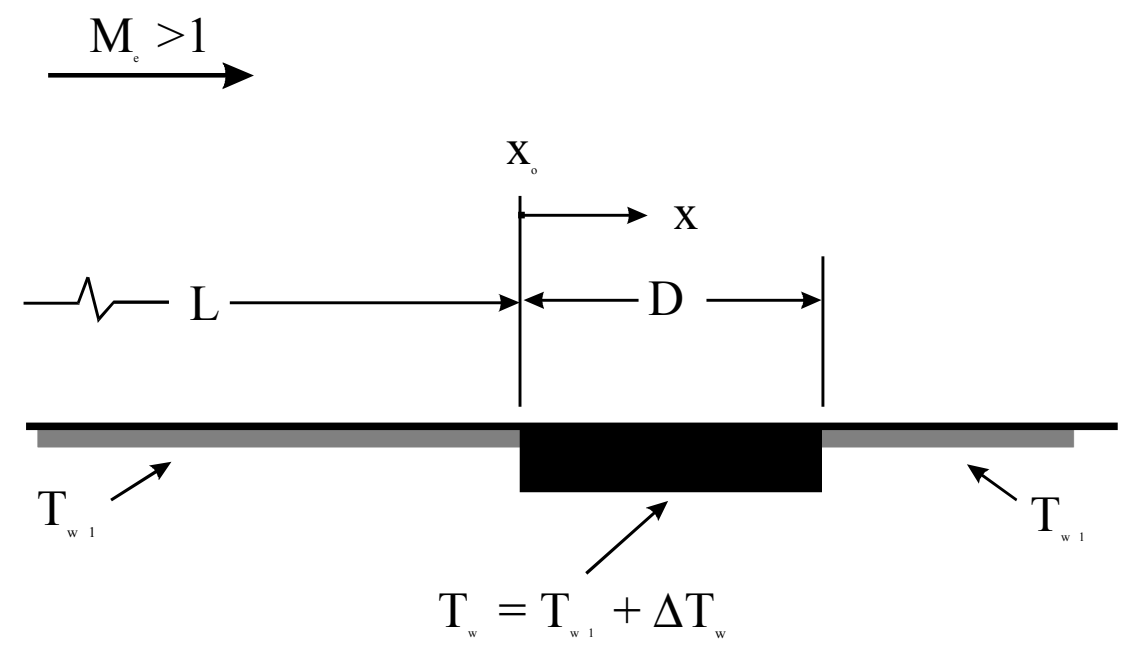

Figure D1. Flow over a flat plate with local patch

\section{Analysis}

Pressure Distribution

As described in detail in Inger (1999) the front edge of the temperature jump produces a pressure perturbation field (compression) upstream equal to,

$$
\frac{\Delta p}{p_{\text {ref }}}=\frac{3}{4}\left(\frac{\Delta T_{w}}{T_{w 1}}\right) e^{K \hat{x}}
$$

where $\mathrm{K}=0.827$ and $\hat{x}$ is negative. On the other hand, downstream there occurs a wakelike pressure decay given by, 


$$
\frac{\Delta p}{p_{\text {ref }}}=\frac{\sqrt{3}}{2 \pi}\left(\frac{\Delta T_{w}}{T_{w 1}}\right) I_{p}(\hat{x})
$$

when,

$$
I_{p}(\hat{x}) \equiv \frac{\left(t+t^{-1 / 3}\right) \mathrm{e}^{-\mathrm{K} \hat{x} t} \mathrm{dt}}{\mathrm{t}^{8 / 3}+t^{4 / 3}+1}
$$

( $t$ is a dummy variable of integration). These solutions are qualitatively illustrated in Figure D2. Although the pressure is continuous across the jump, its corresponding streamwise gradient is not.

Figure D2. Pressure gradient

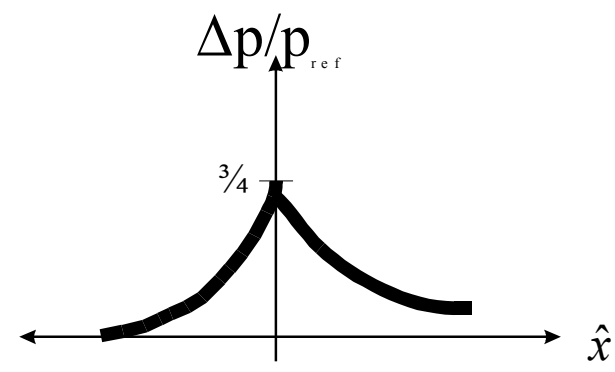

The pressure field is opposed by the corresponding (expansive) disturbance generated by the rear edge of the patch at $\mathrm{x}=\mathrm{D}$ when the $\Delta \mathrm{T}_{\mathrm{w}}$ is "turned off." The latter is governed by,

$$
\frac{\Delta p}{p_{r e f}}=-\frac{3}{4}\left(\frac{\Delta T_{w}}{T_{w 1}}\right)\left(1+\frac{D}{L}\right)^{-1 / 4} \mathrm{e}^{\mathrm{K}(\hat{\mathrm{x}}-\hat{\mathrm{D}})}
$$

in the region $\mathrm{x} \leq \mathrm{D}$ and,

$$
\frac{\Delta p}{p_{\text {ref }}}=\frac{-\sqrt{3}}{2 \pi}\left(\frac{\Delta T_{w}}{T_{w 1}}\right)\left(1+\frac{D}{L}\right)^{-1 / 4} I_{p}(\hat{x}-\hat{D}) \quad \text { (D5), }
$$

in the wake region $\mathrm{x}>\mathrm{D}$ following the rear edge of the patch. This subtractive effect has a streamwise shift in its effective origin relative to Equations (D1) owing to the finite (and arbitrary) length D of the patch. Thus, the net surface perturbation field is,

upstream of the patch front edge $(\mathrm{x}<0)$,

$$
\frac{\Delta p}{p_{\text {ref }}}=\frac{3}{4}\left(\frac{\Delta T_{w}}{T_{w 1}}\right)\left[e^{K \hat{x}}-\left(1+\frac{D}{L}\right)^{-1 / 4} e^{K(\hat{x}-\hat{D})}\right]
$$

anywhere on the patch $(0 \leq \mathrm{x} \leq \mathrm{D})$, and,

$$
\frac{\Delta p}{p_{\text {ref }}}=\frac{\Delta T_{w}}{T_{w 1}}\left[\frac{\sqrt{3}}{2 \pi} I_{p}(\hat{x})-\frac{3}{4}\left(1+\frac{D}{L}\right)^{-1 / 4} e^{K(\hat{x}-\hat{D})}\right] \quad \text { (D7), }
$$


downstream of the patch rear edge $(x>D)$,

$$
\frac{\Delta p}{p_{r e f}}=\frac{\sqrt{3}}{2 \pi}\left(\frac{\Delta T_{w}}{T_{w 1}}\right)\left[I_{p}(\hat{x})-\left(1+\frac{D}{L}\right)^{-1 / 4} I_{p}(\hat{x}-\hat{D})\right]
$$

This behavior is qualitatively illustrated in Figure D3. The pressure perturbation field on the patch itself lies in the wake behavior of the patch front edge minus the upstream influence effect from the patch near edge. Consequently, there is an intermediate station on the patch where the two effects just cancel and the net local pressure disturbance is zero.

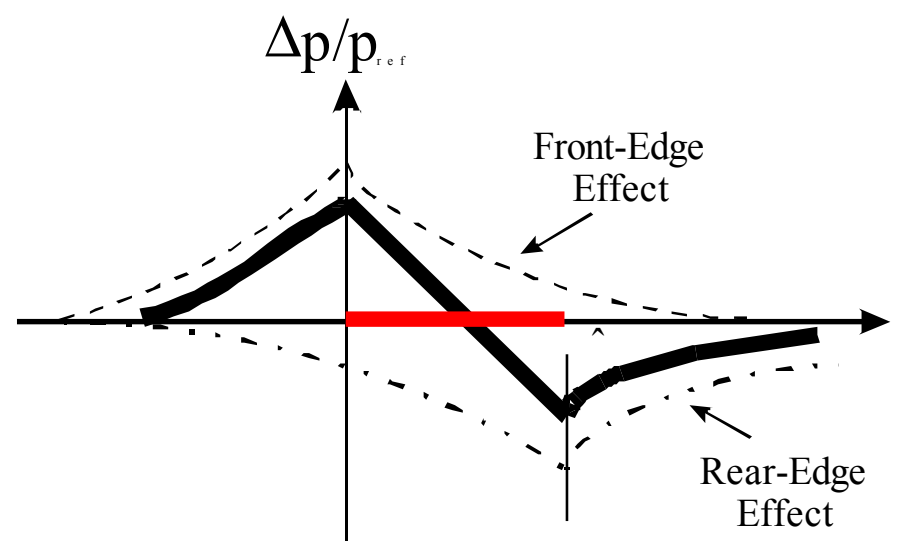

Figure D3. Pressure gradient

The relatively complicated integral $I_{p}(\hat{x})$ may be satisfactorily approximated in practice by a simpler fractional power law-type of expression,

$$
I_{p}(\hat{x}) \cong\left[\frac{2}{\sqrt{3} \pi}+\frac{(K \hat{x})^{2 / 3}}{\Gamma(2 / 3)}\right]^{-1} \quad \text { (D9). }
$$

Thereby rendering the entire pressure field solution in closed analytical form.

\section{Shear Stress Distribution Field}

This is similar to the pressure distribution field calculations, again taking care to subtract the effect of the patch rear edge from the perturbation generated by the front edge. Based on theory, the following results are obtained,

upstream of patch front edge $(\mathrm{x}<0)$,

$$
\frac{\Delta \tau_{w}}{\tau_{w}(L)}=-\frac{3}{4} P_{R}^{-1 / 3}\left(\frac{\Delta T_{w}}{T_{w 1}}\right)\left[e^{K \hat{x}}-\left(1+\frac{D}{L}\right)^{-1 / 2} e^{K(\hat{x}-\hat{D})}\right] \quad \text { (D10), }
$$

anywhere on the patch $(0 \leq x \leq D)$, and, 


$$
\frac{\Delta \tau_{w}}{\tau_{w}(L)}=\left(\frac{\Delta T_{w}}{T_{w 1}}\right) P_{R}^{-1 / 3}\left[\frac{\sqrt{3}}{2 \pi} I_{\tau}(\hat{x})+\frac{3}{4}\left(1+\frac{D}{L}\right)^{-1 / 2} e^{K(\hat{x}-\hat{D})}\right]
$$

downstream of the patch rear edge $(\mathrm{x} \geq \mathrm{D})$,

$$
\frac{\Delta \tau_{w}}{\tau_{w}(L)}=\frac{\sqrt{3}}{2 \pi}\left(P_{R}^{-1 / 3}\right)\left(\frac{\Delta T_{w}}{T_{w 1}}\right)\left[I_{\tau}(\hat{x})-\left(1+\frac{D}{L}\right)^{-1 / 2} I_{\tau}(\hat{x}-\hat{D})\right]
$$

where,

$$
I_{\tau}(\hat{x}) \equiv{ }_{0}^{\infty} \frac{t^{1 / 3}-e^{-K \hat{x} t} d t}{t^{8 / 3}+t^{4 / 3}+1}
$$

Again, this integral can be well-approximated by a more traceable expression based on fractional power law behavior,

$$
I_{\tau}(\hat{x}) \approx\left[\frac{2 \sqrt{3}}{\pi}+\frac{\left.(K \hat{x})^{4 / 3}\right]^{-1}}{\Gamma(4 / 3)}\right.
$$

According to Equation D12, the front and rear edge-generated disturbance fields turn out to reinforce rather than cancel each other on the patch; consequently, the skin friction is increased everywhere along the patch by a local increase in the wall temperature. Note also that unlike the pressure, the disturbance shear stress behavior is not continuous across the patch front edge $(x=0)$. This is a result of the discontinuity of the pressure gradient there (see Figure D2 and Figure D3).

\section{Local Heating}

Treatment of this is analogous to the shear except for a few details.

Upstream of patch front edge $(\mathrm{x}<0)$,

$$
\frac{\Delta \dot{q}_{w}}{\dot{q}_{w}(L)}=-\frac{3}{4} C_{Q 1}\left(\frac{\Delta T_{w}}{T_{w 1}}\right)\left[e^{K \hat{x}}-\left(1+\frac{D}{L}\right)^{-1 / 2} e^{K(\hat{x}-\hat{D})}\right] \quad \text { (D15), }
$$

anywhere on the patch $(0 \leq \mathrm{x} \leq \mathrm{D})$, and

$$
\frac{\Delta \dot{q}_{w}}{\dot{q}_{w}(L)}=\left(\frac{\Delta T_{w}}{T_{w 1}}\right)\left[\frac{\sqrt{3} C_{Q 1}}{2 \pi} I_{\tau}(\hat{x})+\frac{3}{4} C_{Q 1}\left(1+\frac{D}{L}\right)^{-1 / 2} e^{K(\hat{x}-\hat{D})}-\left[1+\frac{C_{Q 2}}{(K \hat{x})^{1 / 3}}\right]\left(\frac{T_{w 1}}{T_{w A D}-T_{w 1}}\right)^{7}(\mathrm{D} 16),\right.
$$

downstream of the patch rear edge $(x \geq D)$,

$$
\frac{\Delta \dot{q}_{w}}{\dot{q}_{w}(L)}=\left(\frac{\Delta T_{w}}{T_{w 1}}\right)\left[\frac{\sqrt{3} C_{Q 1}}{2 \pi} I_{\tau}(\hat{x})+C_{Q 1} I_{\tau}\left(1+\frac{D}{L}\right)^{-1 / 2}-\left(\frac{T_{w 1}}{T_{w A D}-T_{w 1}}\right)\left[1+\frac{C_{Q 2}}{(K \hat{x})^{1 / 3}}-\left\langle\frac{1+C_{Q 2}[K(\hat{x}-\hat{D})]^{1 / 3}}{\left(1+\frac{D}{L}\right)^{1 / 2}}\right]^{7}\right.\right.
$$

Like the shear stress, the heat transfer perturbation is discontinuous across the patch front edge. 


\section{Net Normal Perturbation Force (per unit depth)}

$$
F_{N}=D p_{r e f}^{1}\left(\frac{\Delta p}{p_{r e f}} d(x / d) \quad\right. \text { (D18), }
$$

substituting Equations D7 and D9 yields,

$$
F_{N}=\frac{3}{4} D p_{r e f}\left(\frac{\Delta T_{w}}{T_{w 1}}\right)\left[{ }_{0}^{1} \frac{d \rho}{1+a \rho^{2 / 3}}-\frac{e^{-K \hat{D}} e^{1} e^{K \hat{D} \rho} d \rho}{\left(1+\frac{D}{L}\right)^{1 / 4}}\right.
$$

in terms of the parameter $a \equiv \sqrt{3} \pi(K \hat{D})^{2 / 3} / 2 \Gamma(2 / 3)$ and the dummy integration variable $\rho=x / D$. Introducing $x^{2}=\rho^{2 / 3}$ and using standard integral tables,

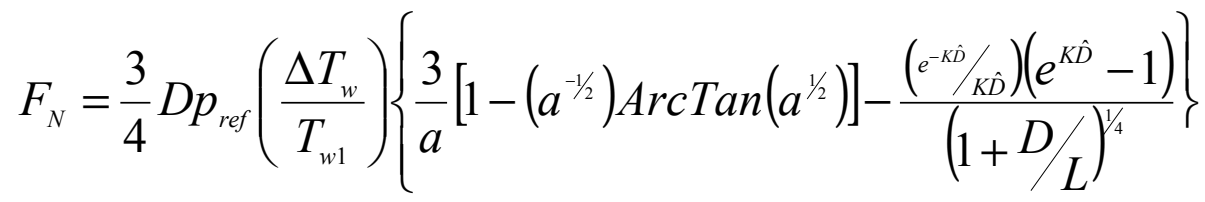

In the limiting case of a small patch sign with $\mathrm{D} / \mathrm{L}<<1$, this formula predicts that the bracket equals,

$$
\{\}=-\frac{3 a}{5}+(1+2 K \hat{L}) \frac{D / L}{4}+\ldots
$$

since $\mathrm{a} \sim(\mathrm{D} / \mathrm{L})^{2 / 3}$, this suggests that the net force in the leading order of approximation is dominated by the pressure gradient in the wake of the patch front edge disturbance and is slightly negative (suction).

\section{Net Tangential Perturbation Force (per unit depth)}

The shear stress disturbance produces the total net force,

$$
F_{T}=\tau_{w_{0}}(L) D_{0}^{1}\left(\frac{\Delta \tau_{w}}{\tau_{w_{0}}}\right) d\left(\frac{x}{D}\right) \quad(\mathrm{D} 22)
$$

substituting Equations D11 and D14 yields, 


$$
F_{T}=\frac{3}{4} D \tau_{w_{o}}\left(\frac{\Delta \tau_{w}}{\tau_{w 1}}\right)\left[\frac{1}{3}{ }_{0} \frac{d \rho}{1+b \rho^{4 / 3}}+\frac{e^{-K \hat{D}}{ }^{1} e^{-K \hat{D} \rho} d \rho}{\left(1+\frac{D}{L}\right)^{1 / 2}}\right.
$$

in terms of the parameter $b=\pi(K \hat{D})^{4 / 3} / 2 \sqrt{3} \Gamma(4 / 3)$ when $\rho \equiv \mathrm{x} / \mathrm{D}$. Introducing $\rho^{4 / 3}=\mathrm{x}^{4}$

and then consulting standard integral tables,

$$
F_{T}=\frac{3}{4} D \tau_{w_{0}}\left(\frac{\Delta T_{w}}{T_{w 1}}\right)\left\{\frac{b^{-3 / 4}}{4 \sqrt{2}}\left[\ln \left(\frac{1+b^{1 / 2}-\sqrt{2} b^{1 / 4}}{1+b^{1 / 2}+\sqrt{2} b^{1 / 4}}\right)+2 \operatorname{ArcTan}\left(\frac{\sqrt{2} b^{1 / 4}}{1+b^{1 / 2}}\right)\right]+\frac{\left(e^{-K \hat{D}} / K \hat{D}\right.}{(1+D / L)^{1 / 2}}\right\}
$$

In the limiting case $\mathrm{D} / \mathrm{L}<<1$ for very small patches, the bracket here passes over to the expression

$$
\{\} \cong \frac{4}{3}-\frac{D}{2 L}(1+K \hat{L})-\frac{b}{7}+\ldots
$$

so that to the leading order of this approximation the shear force is positive downstream).

\section{Total Integrated Heat Transfer Perturbation}

Proceeding in a similar way as before and substituting Equations A14 and A16,

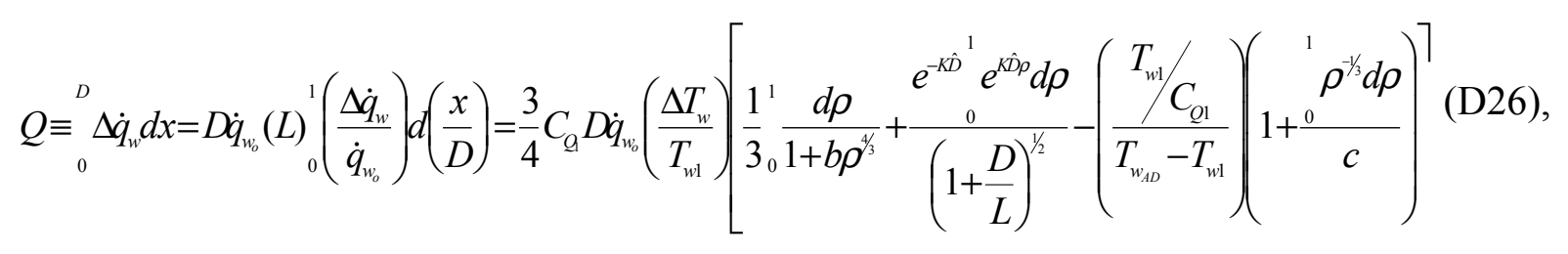

when $c \equiv(K \hat{D})^{1 / 3}$ and the remaining terms in the bracket are the same as in Equation D24. Evaluating the integrals,

$$
Q=\frac{3}{4} C_{Q 1} D \dot{q}_{w_{o}}\left(\frac{\Delta T_{w}}{T_{w 1}}\right)\left\langle\{\}-\left(\frac{T_{w 1} / C_{Q 1}}{T_{w_{1 D}}-T_{w 1}}\right)\left[1+\frac{3 / 2}{(K \hat{D})^{1 / 3}}\right\rangle\right.
$$

where the \{\} is the same bracket that appears in Equation D24. Considering the "small patch limit" $\mathrm{D} / \mathrm{L} \ll<1$ in view of Equation D25 and the fact that $K \hat{D}=(K \hat{L}) D / L$, 
Equation D27 predicts that in the leading order of approximation the total heating perturbation is negative (i.e., a reduction) and proportional to (3/2) $\mathrm{D}^{2 / 3}$. 


\section{VITA}

Wade Joseph Pulliam was born May 14, 1970 in Bremerton, Washington. After graduating from Loyola High School in Towson, Maryland in June of 1988, he entered Virginia Polytechnic Institute and State University as an undergraduate. Upon receiving a dual degree in aerospace and ocean engineering in 1992, the author continued his education in the Aerospace Engineering Department at Virginia Tech as a student of Dr. Joseph Schetz. He became active in the Graduate Student Assembly and the Graduate and Professional Student Association of Virginia during his first few years of graduate studies. After research into the effects of shock and wake passing on turbine blade, skin friction measurements in scramjet combustors, and aerodynamics of magnetically levitated trains and receiving his Masters of Science in 1995, Wade began his work into fiber optic sensors for aerodynamic measurements that lead to this dissertation.

In 1996, the author began work for Luna Innovations, a fiber optic research company in Blacksburg, Virginia. There, he has worked on developing fiber optic sensors for many environments including: pressure and skin friction sensors for flight and extremely high temperatures, skin friction sensors for highly transient flows, force sensor for laser combustion experiments, and integrated micro-device control system using neural nets. 